\title{
Das bisschen Haushalt
}

Zur Kontinuität traditioneller Arbeitsteilung in Paarbeziehungen ein europäischer Vergleich 


\section{Ruth Abramowski}

Das bisschen Haushalt 

Ruth Abramowski

\section{Das bisschen Haushalt}

Zur Kontinuität traditioneller

Arbeitsteilung in Paarbeziehungen ein europäischer Vergleich

Budrich Academic Press

Opladen • Berlin • Toronto 2020 
Bibliografische Information der Deutschen Nationalbibliothek

Die Deutsche Nationalbibliothek verzeichnet diese Publikation in der Deutschen Nationalbibliografie; detaillierte bibliografische Daten sind im Internet über

http://dnb.d-nb.de abrufbar.

(C) 2020 Dieses Werk ist beim Verlag Barbara Budrich erschienen und steht unter der Creative Commons Lizenz Attribution-ShareAlike 4.0 International (CC BY-SA 4.0): https://creativecommons.org/licenses/by-sa/4.0/

Diese Lizenz erlaubt die Verbreitung, Speicherung, Vervielfältigung und Bearbeitung bei Verwendung der gleichen CC-BY-SA 4.0-Lizenz und unter Angabe der UrheberInnen, Rechte, Änderungen und verwendeten Lizenz.

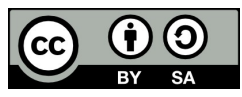

Dieses Buch steht im Open-Access-Bereich der Verlagsseite zum kostenlosen Download bereit (https://doi.org/10.3224/96665008).

Eine kostenpflichtige Druckversion kann über den Verlag bezogen werden. Die Seitenzahlen in der Druck- und Onlineversion sind identisch.

$\begin{array}{ll}\text { ISBN } & 978-3-96665-008-3 \text { (Paperback) } \\ \text { eISBN } & 978-3-96665-982-6 \text { (PDF) } \\ \text { DOI } & 10.3224 / 96665008\end{array}$

Umschlaggestaltung: Bettina Lehfeldt, Kleinmachnow - www.lehfeldtgraphic.de Druck: Books on Demand GmbH, Norderstedt Printed in Europe 


\section{Inhalt}

Abbildungs- und Tabellenverzeichnis...................................................... 9

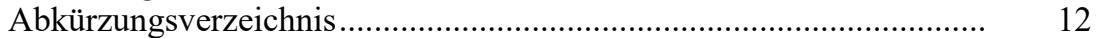

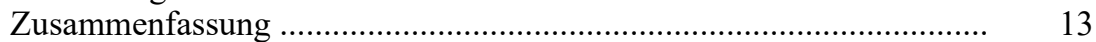

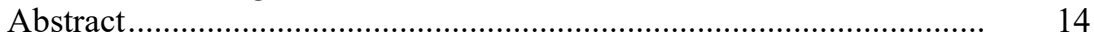

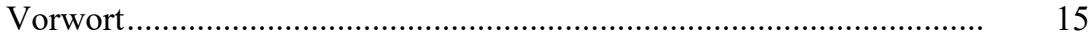

\section{Problemstellung, Stand der Forschung und}

Forschungslücken

1. Einleitung: Ein Paradox , ,liberal-egalitaristischer“ Einstellungen und traditioneller innerhäuslicher Arbeitsteilungsarrangements in Paarbeziehungen?

2. Traditionelle innerhäusliche Arbeitsteilungsarrangements und partnerschaftliche Machtungleichgewichte im ,paradoxemanzipierten" 21. Jahrhundert.

2.1. Forschungserkenntnisse zur Norm-/Realitätsdiskrepanz.... 25

2.2. Forschungserkenntnisse über die Determinanten der innerhäuslichen Arbeitsteilung.

2.3. Machtstrukturen als erklärende Elemente der innerhäuslichen Arbeitsteilung

2.4. Die Grenzen ökonomischer (Macht-)Ressourcenansätze...

2.5. Regimetypologische Erkenntnisse aus der Wohlfahrtsstaatsforschung: „Multiple Equilibria“

2.6. Erkenntnisse über „Desperate Housewives“?

2.7. Empirische Befunde: Ein Vergleich der Indizes zur Messung von Gender(un)gleichheiten.

2.8. Kritik am Forschungsstand: Ein Plädoyer für einen mehrdimensionalen Machtansatz

Zur Herleitung einer Theorie der mehrdimensionalen Machtverhältnisse in Paarbeziehungen

3. Ein Spannungsverhältnis zwischen mikro- und makrosoziologischen Theorien zur Erklärung innerhäuslicher Arbeitsteilungsarrangements

3.1. Mikrosoziologische Argumentation

3.2. Zum dynamischen Wechselverhältnis von

(gesamtgesellschaftlicher) Struktur und (individueller) Handlung - das Mikro-Makro-Problem im Allgemeinen .. 
3.3. Zur integrativen Funktion von Familie und Geschlecht zwischen den Machtdimensionen - der Masterstatus nach Levy zur Lösung des Mikro-Makro-Problems .

3.4. Makrosoziologische Argumentation: Zum Verständnis von Gender-Ungleichheiten im sozialstrukturellen Kontext.

4. Ein multidimensionaler Ansatz:

Der Capability Approach nach Amartya K. Sen

„Freiheit - Gleichheit - Gerechtigkeit?““

4.1. Einführung zur Ausgangsproblematik einer ressourcenorientierten Messung sozialer Ungleichheiten ..

4.2. Zur Dichotomie eines auf Regeln und eines auf Realisierung konzentrierten Verständnisses von Gerechtigkeit.....

4.3. Sens Argumentation in Abgrenzung zu Rawls.................. $\quad 97$

4.4. Kritik an der Sozialwahltheorie nach Arrow ..................... 103

4.5. Functionings (Funktionsweisen) und Capabilities (Befähigungen)............................................. 105

4.6. Das Freiheits-Gleichheit-Dilemma.................................... 108

4.7. Ein Anwendungsbeispiel des Capability Approachs zur Work-Life-Balance.

4.8. Eigene Erweiterung: Macht im Capability-Ansatz als Fähigkeit und Befähigung

5. Zur systematischen Ausklammerung der innerhäuslichen Arbeitsteilung in der Wohlfahrtsstaatsforschung

5.1. Die „Power Resource School“" nach Esping-Andersen und Korpi und ihre feministische Kritik.

5.2. Feministische Kritik an Mainstream-Typologien................ 131

5.3. Weiterentwicklung der ,Faces of Inequality“ “....................

5.4. Das Pendant zur Power Resource School:

Ein kulturalistischer Ansatz zur Sozialpolitik und der Entwicklung von „Care Arrangements“...

5.5. „Between Equalization and Marginalization“: Diversität und Dynamik von Teilzeitarbeitsmodellen im historischen Entwicklungsprozess unterschiedlicher moderner Gesellschaften

5.6. Hakims Präferenztheorie: Die Diversität der Präferenzen für Teilzeiterwerbsmodelle zur Vereinbarung von Familie und Beruf......

5.7. Weiterführende feministische Kritik von Ostner.................. 153 
6. Ein Abriss: Reziprozität, Liebe und Solidarität.

6.1. „Ungleiche“ Liebe und „egalitäre“ Partnerschaft:

Koppetschs Differenzierung zwischen Liebe und

Partnerschaft.

6.2. Reziprozität, Wohltätigkeit und moralischer Absolutismus - ,etwas gegen nichts“(Gouldner).

6.3. Solidaritätstypen nach Bengtson

7. Zwischenfazit der eigenen Argumentation:

Für einen Arbeitsteilungspluralismus

8. Familiensoziologische Machtansätze ,revisited“

8.1. Übersicht zur Entwicklungsgeschichte einer

Soziologie der Machtverhältnisse in Paarbeziehungen: die

Klassiker familiensoziologischer Machtansätze

8.2. Zum Konkurrenzverhältnis von Machtkonzepten und Austauschtheorien....

8.3. Aktuelle machttheoretische Ansätze

9. Die Typologie der Machtverhältnisse in Paarbeziehungen: Macht als mehrdimensionale Begriffskonstruktion

9.1. Macht als Chance, den eigenen Willen durchzusetzen (Mikro)

9.2. Macht als multidimensionales Kräfteverhältnis (Mikro/Makro)

9.3. Zur Konzeptualisierung der Typologie der

Machtverhältnisse: Die Dimensionen der Macht in Paarbeziehungen

9.4. „Bringing Power Back In“: Die Verteidigung des Machtansatzes

9.5. Zusammenfassende theoretische Modellkonzeption

9.6. Hypothesengenerierung................................................... 205

10. „Trouble in Regime Typologies“:

Eine auf länderspezifischen Eigenarten von ,genderrelevanten

Policies" basierende Fallauswahl

10.1. Divergierende Rahmenbedingungen der Arbeitsteilung: "genderrelevante Welfare Policies"

10.2. Ein historischer Abriss über die Gegensätze der gesellschaftlichen Konfliktlinien zwischen kontinentaleuropäischen und sozialdemokratischen Ländern 
10.3. Das „konservative Regime“ kritisch hinterfragt -

Zur inneren Diversität .................................................. 220

10.4. Ein interessanter „Mischtypus“ - die Niederlande............. 234

10.5. Das ,postsozialistische Regime“" kritisch hinterfragt ......... 241

10.6. „Sozialdemokratisch skandinavisch?“ Zur institutionellen Vielfalt skandinavischer Länder.

\section{Empirische Untersuchungen}

11. Datenbasis: Generations and Gender Programme (GGP).

11.1. Zur methodisch bedingten NUTS1-Regionenanalyse mit theoretischem Mehrwert.

11.2. Stichprobenbildung

11.3. Operationalisierung der innerhäuslichen Arbeitsteilung

11.4. Beschreibung der unabhängigen Variablen

12. Methodische Erläuterungen der Mehrebenenanalyse

12.1. Grundlagen der Mehrebenenanalyse

12.2. Zur Analyse von Paneldaten im Rahmen von Mehrebenenmodellen

13. Darstellung und Diskussion der Ergebnisse

13.1. Deskriptive Darstellung der innerhäuslichen Arbeitsteilung im Ländervergleich

13.2. Deskriptive Darstellung der innerhäuslichen Arbeitsteilung im NUTS1-Regionen-Vergleich

13.3. Klassische OLS-Regressionsmodelle zur Erklärung der innerhäuslichen Arbeitsteilung im Länder- und Regimevergleich

13.4. Mehrebenenanalyse der innerhäuslichen Arbeitsteilung im NUTS1-Regionen-Vergleich (GGS Welle 1).

13.5. Dynamischer Traditionalismus? Eine Panelanalyse mit Mehrebenenmodellen zur innerhäuslichen Arbeitsteilung (GGS Welle 1 und 2)

13.6. Abschließende Diskussion:

Empirische Mehrebenenanalysen im theoretischen

Diskurs des „Power-Capability Approachs“

14. Fazit und Ausblick: Power matters?

Literaturverzeichnis 


\section{Abbildungs- und Tabellenverzeichnis}

\section{Abbildungen}

Abbildung 1: $\quad$ Veränderungsrichtungen der

Arbeitsteilungen im Haushalt............................... 25

Abbildung 2: $\quad$ Nash-Lösung eines kooperativen

Abbildung 3: $\quad$ Zusammenfassende Darstellung des

Capability Approachs......................................... 125

Abbildung 4:

Typologie der Machtverhältnisse .

Abbildung 5:

Erwartete innerhäusliche

Abbildung 6:

Arbeitsteilungsarrangements nach Regimetypen .

205

NUTS 1 Regionen in Europa

Abbildung 7:

Die Grundidee der Mehrebenenanalyse -

ein Makro-Mikro-Zusammenhang....

Abbildung 8:

Zusammenhang zwischen Variablen der

Mikro- und Makroebene

Abbildung 9:

Differenzierung des Random-Intercept- und

Abbildung 10:

Random-Slope-Modells

Abbildung 11:

Dreiebenenstruktur der Panelstudie des GGP

Zum Prinzip der Residuen- und

Varianzzerlegung in Mehrebenenmodellen

Abbildung 12: $\quad$ Mittelwerte der Hausarbeiten im

Ländervergleich, GGS Welle 1

Abbildung 13:

Mittelwerte der Hausarbeiten im regionalen

Vergleich (NUTS1), GGS Welle 1....

Abbildung 14: Zusammenfassende Darstellung des

Power-Capability Approachs

Abbildung 15: $\quad$ Entwicklung der Fertilitätsrate im

Ländervergleich, 1988-2016.

Abbildung 16:

Fertilitätsrate im NUTS1-Regionenvergleich, 2008 .

Abbildung 17:

Teilzeiterwerbsquoten von Frauen (\%) im

Ländervergleich, 2001-2017.

Abbildung 18:

Anteil von Kindern im Alter zwischen 0-2 in öffentlichen Betreuungseinrichtungen (\%), 2001-2015.

Abbildung 19:

Gesamte bezahlte Elternzeit (einschließlich Mutterschutz, Vaterschaftsurlaub und Elternzeit) nach Geschlecht in Wochen, 1988-2016 
Abbildung 20:

Abbildung 21:

Abbildung 22:

Abbildung 23:

Abbildung 24:

Abbildung 25:

Abbildung 26:

Abbildung 27:

Abbildung 28:
Political Empowerment (\%), 2008 und 2016 .......

Bruttoinlandsprodukt (KKS), 2001-2016.

Absolute Armut im Sinne der

Einkommensarmutsgrenze von 1,90 US\$ (\%), 2001-2016.

Quote der von Armut bedrohten Personen nach

Armutsgefährdungsgrenze (\%), 2001-2017.........

Rate der erheblichen materiellen Deprivation

(\%), 2003-2017

423

Von Armut und sozialer Ausgrenzung bedrohte

Bevölkerung (\%), 2003-2017

Gini-Index, 2001-2016

425

Gender Pay Gap (Differenz des Stundenlohns zwischen den Geschlechtern), 2001-2016

426

Gender Pay Gap (Differenz der

Monatseinkommen), 2001-2016

\section{Tabellen}

Tabelle 1:

Tabelle 2:

Tabelle 3:

Tabelle 4:

Tabelle 5:

Tabelle 6:

Tabelle 7:

Tabelle 8:

Tabelle 9:

Tabelle 10:
Indizes zur Messung von Genderungleichheiten - ein Vergleich der EU 28

Beispiel zum Kinderbetreuungs- und

Zusammenfassung der mikrosoziologischen

Ansätze

Gesamtübersicht der Theorien zur

innerhäuslichen Arbeitsteilung

Faktorenbeispiele für Functionings und

Capabilities

Familienpolitik

Theoretischer Bezugsrahmen zur Erklärung von innerhäuslichen Arbeitsteilungsarrangements...... Zum „Spiel von Positionswechseln“: Übersicht zur hypothetischen Erklärungsleistung der theoretischen Paradigmen und ihrer Machttypen. Determinanten der innerhäuslichen Arbeitsteilung 
Tabelle 11:

Tabelle 12:

Tabelle 13:

Tabelle 14:

Tabelle 15:

Tabelle 16:

Tabelle 17:

Tabelle 18:

Tabelle 19:

Tabelle 20:

Tabelle 21:

Tabelle 22:

Tabelle 23:

Tabelle 24:

Tabelle 25:

Tabelle 26:

Tabelle 27:

Tabelle 28:

Tabelle 29:

Tabelle 30:

Zusammenfassende Darstellung der familialistischen Entwicklung in Österreich und Deutschland. 235

Zusammenfassende Darstellung der

familialistischen Entwicklung in Frankreich und

Belgien

Aktuelle Mitgliedsländer und Fallzahlen des

GGS.

Fallauswahl

276

Indikatoren der abhängigen Variable

282

Operationalisierung der unabhängigen Variablen 289

Übersicht der Messzeitpunkte des GGS ............... 293

Mittelwerte der Individualvariablen pro Land, erste Welle und zweite Welle

Kontextfaktoren für den

NUTS1-Regionenvergleich

Integrierte Mehrebenengleichungen für

Querschnittsdaten.

Mittelwerte der Hausarbeiten im regionalen

Vergleich (NUTS1), GGS Welle 1

Klassische OLS-Regression zur Erklärung der innerhäuslichen Arbeitsteilung durch

Länderunterschiede

Separate, nach Regimetypen differenzierte, klassische OLS-Regressionen zur Erklärung der innerhäuslichen Arbeitsteilung durch

Individualvariablen

Mehrebenenanalyse der innerhäuslichen

Arbeitsteilung im NUTS1-Regionen-Vergleich

(GGS Welle 1)

Eine Panelanalyse mit Mehrebenenmodellen zur innerhäuslichen Arbeitsteilung im NUTS1-

Regionenvergleich (GGS Welle 1 und 2).

Gewinn-Verlust-Tabelle nach Rawls

Familialismustypologie nach Leitner (2003, 2013)

Übersicht der Hypothesen von Korpi (2000)

407

Entwicklung der direkten familialistischen

Strukturen in Österreich

408

Anteil von Kindern im Alter zwischen $0-2$ in

öffentlichen Betreuungseinrichtungen in \%

(Versorgungsquote in der Kindertagesbetreuung) 
Tabelle 31: $\quad$ Versorgungsquoten der 0- bis 2-Jährigen in Polen, Tschechien und Ungarn

Tabelle 32: $\quad$ Gesamte bezahlte Elternzeit nach Geschlecht in

Tabelle 33: $\quad$ Zusammenfassende Übersicht der Hypothesen und Ergebnisse der Mehrebenenanalyse im NUTS1-Regionen-Vergleich (GGS Welle 1).......

Tabelle 34: Zusammenfassende Übersicht der Hypothesen und Ergebnisse der Panelanalyse mit Mehrebenenmodellen (GGS Welle 1 und 2)........

\section{Abkürzungsverzeichnis}

$\begin{array}{ll}\text { ADM } & =\text { Arbeitsgemeinschaft Deutscher Marktforschungsinstitute } \\ \text { CAPI } & =\text { Computer Assisted Personal Interview } \\ \text { EIGE } & =\text { European Institute for Gender Equality } \\ \text { FIML } & =\text { Full-Information-Maximum-Likelihood-Methode } \\ \text { GDI } & =\text { Gender-related Development Index } \\ \text { GEI } & =\text { Gender Equality Index } \\ \text { GEM } & =\text { Global Gender Gap Index } \\ \text { GGG } & =\text { Generations and Gender Programme } \\ \text { GGP } & =\text { Generations and Gender Survey } \\ \text { GGS } & =\text { Intraklassenkorrelationskoeffizient } \\ \text { GII } & =\text { International Labour Organization } \\ \text { ICC } & =\text { International Standard Classification of Education } \\ \text { ILO } & =\text { Level 1/Ebene 1 } \\ \text { ISCED } & =\text { Level 2/Ebene 2 } \\ \text { KKS } & =\text { Nomenclature des unités territoriales statistiques } \\ \text { L1 } & =\text { Task-Participation-Index } \\ \text { L2 } & =\text { United Nations Development Programme } \\ \text { NUTS } & =\text { Wnited Nations Economic Commission for Europe } \\ \text { REML } & =\end{array}$




\section{Zusammenfassung}

Familiensoziologische Studien vernachlässigen in ihren Erklärungen über die Aufteilung der Routine-Hausarbeiten von Paaren zunehmend den Machtaspekt in Paarbeziehungen, was zum Anliegen dieser Dissertation führt „Bringing Power Back In“. Eine der Hauptursachen dieser Nachlässigkeit könnte die aufkommende „liberale Egalitarismus-Ideologie“ sein, die kritisch hinterfragt und umfassend diskutiert wird. Der theoretische Stellenwert von Macht in Paarbeziehungen wird unter Bezug auf KlassikerInnen zur Soziologie von Machtverhältnissen im Rahmen des Capability Approachs skizziert, der es ermöglicht, ein „Power-Capability Set“ auf unterschiedlichen Dimensionen zu eruieren und zu einer Typologie von Machtverhältnissen zu verdichten. Dass Machtverhältnissen und innerhäuslichen Arbeitsteilungsarrangements eine Dynamik zugrunde liegt, welche die zeitliche Struktur des Lebenslaufs betrifft, wird durch eine prozesshafte Betrachtung unterschiedlicher Zeitpunkte berücksichtigt. Die Anforderungen einer mehrdimensionalen Perspektive von Macht werden methodisch anhand eines Mehrebenendesigns, das auf den Daten der ersten beiden Wellen des „Generations and Gender Surveys“ basiert, umgesetzt. Ein Vergleich von europäischen NUTS1Regionen als Kontexteinheiten steht im Zentrum der Analyse, um die Fragmentiertheit von strukturellen, kulturellen und politischen Rahmenbedingungen zum Ausdruck zu bringen, die sich in innerhäuslichen Arbeitsteilungsarrangements widerspiegeln, sowie eine Überwindung des ,nationalstaatlichen Containermodells“ zu erreichen. Das Ziel dieser Dissertation besteht darin, einen Beitrag zur Erklärung innerhäuslicher Arbeitsteilungsarrangements mittels des theoretisch hergeleiteten „Power-Capability Sets“ zu leisten. Die Ergebnisse belegen, dass innerhäusliche Arbeitsteilungsarrangements nach wie vor sehr stark geschlechtsspezifisch segregiert sind - RoutineHausarbeiten werden in sämtlichen europäischen Regionen mehrheitlich von Frauen ausgeführt. Zwischen den Regionen besteht jedoch eine hochsignifikante Kontextvarianz: 6,2\% der innerhäuslichen Arbeitsteilung gehen auf Unterschiede zwischen den Kontexteinheiten zurück. Insgesamt zeichnet sich ein komplexes Wechselspiel aus individuellen, kulturellen und institutionellen „Power-Capabilites“ zur Erklärung der Ausführung familialer Hausarbeiten $\mathrm{ab}$.

Schlagwörter: Power-Capability Approach, traditionelle innerhäusliche Arbeitsteilung, Egalitarismuskritik, Zeitverlauf, methodologischer Nationalismus, europäischer Regionenvergleich (NUTS1) 


\section{Abstract}

Scholarship has demonstrated a waning focus on the power aspect of couple relationships in family sociological studies regarding the division of labor in routine housework. The focus on 'liberal egalitarian ideology' has been considered one of the major reasons for this. This dissertation 'brings the power back in' through a critical engagement and discussion of the 'liberal egalitarian ideology.' The theoretical significance of power in couple relationships is outlined with reference using classics on the sociology of power relations in the context of the capabilities approach, which enables a "power-capability set" on different dimensions. Thus, a typology of power relations is developed. The fact that power relations and the division of household tasks arrangements are based on a dynamic concerning the temporal structure of the life-course is taken into account with a process-related consideration. The dissertation is based on a multi-level design which incorporates a multidimensional perspective of power using data from the first two waves of the "Generations and Gender Surveys." A comparison of European NUTS1 regions as contextual units is at the center of the analysis to express the fragmented nature of structural, cultural and political frameworks reflected in domestic division of labor as well as to overcome the "national-state container model." The dissertation contributes to the explanation of division of household tasks through a theoretically derived "power-capability set." The results of the analysis show that domestic work-sharing arrangements are still very strongly segregated in gender-specific ways - routine housework is usually carried out by women across all European regions. However, there is a highly significant context variance between the regions: $6.2 \%$ of the distribution of housework is due to differences between the context units. The dissertation thus provides an explanation of the familial division of household tasks by incorporating a complex interplay of individual, cultural, and institutional "power-capabilities" approach.

Keywords: Power-capabilities approach, traditional division of household labor, criticism of egalitarianism, life course, methodological nationalism, regional comparison (NUTS1) 


\section{Vorwort}

Die vorliegende Monographie wurde unter dem Titel „Bringing Power Back In: Zur Kontinuität traditioneller innerhäuslicher Arbeitsteilungsarrangements in Paarbeziehungen - Ein europäischer Vergleich" an der Universität Salzburg als Dissertation eingereicht (Änderungen, die für die Publikation vorgenommen wurden, sind überwiegend stilistischer Art. Ferner wurden einige wenige inhaltliche Ergänzungen vorgenommen). Während meiner dortigen Anstellung als wissenschaftliche Mitarbeiterin wurden mir die erdenklich besten Rahmenbedingungen geboten, meine Dissertation erfolgreich abzuschließen.

Mein besonderer Dank gilt meinem Doktorvater und Betreuer Prof. Dr. Beat Fux, ohne den die vorliegende Dissertation nicht in dieser Form zustande gekommen wäre. Seine ermutigenden und konstruktiven Hinweise haben mir stets dazu verholfen, einen umfangreichen theoretischen Bezugsrahmen für die Erklärung der innerhäuslichen Arbeitsteilung zu entwickeln und hierbei niemals einen kritischen Zugang zur Thematik zu verlieren.

Ebenso möchte ich meiner Nebenbetreuerin Prof. Dr. Ilona Ostner aufs herzlichste für die zahlreichen Diskussionen und äußerst hilfreichen kritischen Anregungen danken. Eine bessere Betreuung als ich sie von Prof. Dr. Beat Fux und Ilona Ostner erhalten habe, hätte ich mir nicht wünschen können. Beide waren und sind mir stets ein Vorbild.

Prof. Dr. Kornelia Hahn und Prof. Dr. Kyoko Shinozaki haben mich immer wieder während meiner Präsentationen in diversen Kolloquien durch ihre Nachfragen und ihre konstruktiven Kommentare inspiriert, auch hierfür möchte ich mich ausdrücklich bedanken.

Ein besonderer Dank gilt auch meinen AbteilungskollegInnen: Ich danke Hemma Zmugg für das Lektorieren meiner Dissertation sowie Dr. Alexander Seymer und Prof. Dr. Wolfgang Aschauer für ihre methodischen Denkanstöße. Desirée Wilke und Alan Schink (mit Jana Nopper) haben mich nicht nur durch ihre inhaltlichen Anmerkungen beruflich unterstützt, sondern sind auch ein unverzichtbarer Teil meines privaten Netzwerkes geworden.

Für den persönlichen Beistand und das Verständnis für meine hohe Arbeitsintensität danke ich insbesondere meiner Familie und meinem Freundeskreis.

In tiefster Verbundenheit danke ich meinem Freund, Christfried Dornis, für die unendliche Geduld, die er in den vergangenen Jahren hatte und das Einfühlungsvermögen, das er mir für das Verfassen meiner Dissertation entgegengebracht hat. Er hat mir die notwendige Kraft und den Beistand gegeben, diese Arbeit zu schreiben. 
Brigitte und Jürgen Dornis danke ich ganz herzlich für die zahlreiche soziologische Literatur, die sie mir zur Verfügung gestellt haben und die anregenden inhaltlichen Diskussionen.

Meine Eltern, Sigrid und Eckhard Abramowski, die nicht minder am Lektorieren und Diskutieren der Inhalte beteiligt waren, haben immer an mich und meine Fähigkeiten geglaubt. Sie haben mich auf meinem Lebensweg begleitet und mich stets darin ermutigt, mich nach eigenem Willen zu entfalten. Nicht zuletzt möchte ich ihnen dafür diese Dissertation widmen.

Bremen im April 2020

Ruth Abramowski 


\section{Problemstellung, Stand der Forschung und Forschungslücken}

\section{Einleitung: Ein Paradox ,liberal-egalitaristischer“ Einstellungen und traditioneller innerhäuslicher Arbeitsteilungsarrangements in Paarbeziehungen?}

Die Vorstellungen über eine traditionelle, kleinbürgerliche Familie und die Organisation des Zusammenlebens zwischen Mann und Frau - zwischen innerhäuslicher Hausarbeit und außerhäuslicher Erwerbsbeteiligung - haben sich in westlichen Gesellschaften im Laufe der letzten Jahre als Folgen gesamtgesellschaftlicher Veränderungen, insbesondere der Modernisierung, gewandelt. Frauen wird seither zunehmend die Chance geboten, erwerbstätig zu werden, auch wenn eine Segregation des Arbeitsmarktes ${ }^{1}$ sowie branchenspezifische Lohn-unterschiede vorhanden sind - Tendenz sinkend. Dennoch hat sich die Chancengleichheit der Geschlechter im Bildungs- und Erwerbswesen in den vergangenen Jahren enorm verbessert (vgl. Schulz/Blossfeld 2006: 23). Die von Bourdieu (2005) attestierte „männliche Herrschaft“ scheint auf den ersten Blick obsolet geworden zu sein. Eine zunehmende Integration in das Erwerbsleben führt dazu, dass Frauen nicht mehr ausreichend Zeit haben, sämtliche Bereiche im Haushalt zu übernehmen - es gilt die Organisation des Alltags umzustrukturieren. Veränderungen der häuslichen Aufgabenverteilung werden durch die Berufstätigkeit der Frau vorausgesetzt (vgl. Held/Levy 1974: 143). Alltägliche innerhäusliche Aufgabenbereiche, wie die Geldverwaltung oder die Ausübung der Haushaltspflege, werden scheinbar neu ausgehandelt. Demzufolge wird häufig erwartet, dass Frauen zunehmend Aufgaben übernehmen, die nach konservativen Vorstellungen eher von Männern ausgeübt wurden und zugleich, dass Männer im Gegensatz zum traditionellen Bild der bürgerlichen Kleinfamilie in die Ausführung der alltäglichen Haushaltstätigkeiten integriert werden. Insbesondere wird prognostiziert, dass die Bildungs-, Erwerbs- und Karrierechancen von Frauen zu einer Enttraditionalisierung führen, d. h. innerhäusliche Aufgabenbereiche zu gleichen Teilen von Männern wie Frauen übernommen werden (vgl. Schulz/Blossfeld 2006: 23). Im Gegensatz dazu waren Frauen in früheren Jahrzehnten vornehmlich aufgrund von geringeren Bildungsqualifikationen wie auch gesellschaftlichen Werten und Normen dazu ,ver-

1 Arbeitsmarktsegregation meint eine Stereotypisierung der Berufsfelder in weibliche/männliche Domänen. 
pflichtet', sich um den Haushalt sowie die Kindererziehung zu kümmern. Aktuell wird oftmals erwartet, dass ein Wertewandel - der Trend zur Egalität in Paarbeziehungen - stattfindet. Doch inwieweit sind moderne Erwartungen des gleichberechtigten liberalen Zusammenlebens und seiner Organisation bisher in der Bezugsgruppe „Familie“ institutionalisiert? Oder sind es nach wie vor, trotz teils veränderter Rahmenbedingungen, Geschlechterideologien, die die Verhaltensweisen des zwischenmenschlichen Zusammenlebens beeinflussen?

Gemäß dem aktuellen Forschungsstand ist insofern eine Diskrepanz zwischen Einstellungs- und Verhaltensebene festzustellen, als liberalegalitaristische Einstellungen in Bezug auf Arbeitsteilungsarrangements zwischen den Geschlechtern meist vertreten werden, zugleich jedoch eine traditionelle innerhäusliche Arbeitsteilung ausgeführt wird.

Prognostiziert werden immer wieder symmetrische Entwicklungen (Annahme einer zunehmenden Erwerbstätigkeit der Frau mit einhergehender Enttraditionalisierung) der Gleichberechtigung, die mit einem Abbau geschlechtsspezifischer Arbeitsteilungen im Haushalt verbunden werden (vgl. Schulz/Blossfeld 2006: 23). Im Gegensatz zu den Erwartungen zeigen die Ergebnisse von Schulz und Blossfeld (2006), dass häusliche Arbeitsteilungen, bei denen der Ehemann mehr Hausarbeiten als seine Frau übernimmt, die absolute Ausnahme sind (vgl. Schulz/Blossfeld 2006: 36). Auch die Analysen von Huinink/Feldhaus $(2008)^{2}$ und Kelle $(2011)^{3}$ bestätigen diesen Trend der traditionellen innerhäuslichen Arbeitsteilung.

Seit den Prognosen symmetrischer Entwicklungen ist in Vergessenheit geraten, dass traditionelle Aufgabenverteilungen trotz der Erwerbstätigkeit von Frauen und damit verbundene Machtkonstellationen in Paarbeziehungen auch in ,modernen“ Gesellschaften von Bedeutung sind. Obwohl die zunehmende Berufstätigkeit von Frauen Arbeitsteilungsarrangements in Paarbeziehungen zweifellos tangieren, so ist doch der Einfluss erstaunlich gering (vgl. Krüger/Levy 2000: 381; Klaus/Steinbach 2002: 21). Folglich ist die steigende Frauenerwerbsquote ,nur die eine Seite der Medaille“, die nicht per se auf die innerhäusliche Arbeitsteilung übertragen werden kann (Kersten 2016: 104). „The core aspects of the sexual division of labor remain: Women perform most domestic work whether or not they work for pay, while men do very little domestic work“ (Orloff 1993a: 313). Selbst in Fällen von DoppelKarriere-Paaren erweist sich die Hausarbeit meist als Aufgabenbereich der Frauen (vgl. Cesinger et al. 2012: 28; Kersten 2016: 106). Soziologische

2 „Entgegen der immer mehr zu vernehmenden Norm von Geschlechteregalität in Lebensformen und Gesellschaft, zeigt die bisherige Forschung, dass die Positionen und Rollen der Partner in allen Lebensformen nach wie vor in geschlechtstypischer Weise variieren“ (Huinink/Feldhaus 2008: XXX).

$3,[\ldots]$ die traditionelle Arbeitsteilung sowie normenbasierte Rollenerwartungen [können] keineswegs als obsolet für die jüngeren Kohorten betrachtet werden“ (Kelle 2011: 55). 
empirische Analysen und theoretische Erklärungsansätze geschlechtsspezifischer Machtverhältnisse sind seither zurückgegangen (vgl. Löw 2009: 7). „Nicht die Gegenstände der Analyse sind verschwunden, sondern ihre Benennung in Machtkategorien“ (Löw 2009: 7).

(Macht-)Ungleichheit im Haushalt ist jedoch ein Faktum, das in Folge der modernen Lebensumstände westlicher Industrieländer häufig unterschätzt wird und mehr Auswirkungen hat, als dies meist angenommen wird. Einige Analysen weisen zunehmend auf Machtungleichheiten und Benachteiligungen von Frauen im Familienleben hin, beispielsweise die Studien von Christine Wimbauer (2003) $)^{4}$ und Yvonne Lott (2009). Laut den Ergebnissen von Lott (2009) führt ein gleiches oder höheres Einkommen von Frauen nicht zu mehr Macht in der Paarbeziehung; vielmehr bestehen Machtungleichgewichte unabhängig des von ihnen erzielten Einkommens (vgl. Lott 2009: 327). Dieser Umstand ist für Wimbauer (2003) der Komplexität multidimensionaler Machtverhältnisse geschuldet. Wimbauer beschreibt das Phänomen der Diskrepanz zwischen Einstellungs- und Verhaltensebene als das Fortbestehen strukturierter Ungleichheiten zwischen den Geschlechtern, deren Ursachen auf differenzierten Ebenen ${ }^{5}$ von Makrostrukturen und mikrosoziologischer Ebene zu verorten sind.

Die dargestellten Entwicklungen beziehen sich insbesondere auf zentraleuropäische Länder, namentlich Deutschland und Österreich. Fraglich ist, inwiefern Traditionalisierungsprozesse der Verhaltensebene in weiteren europäischen Ländern vorherrschend sind, respektive welche Rolle nationale Differenzen im europäischen Kontext spielen. Bisherige makrosoziologische Studien zum Thema ,Genderungleichheiten' weisen darauf hin, dass im europäischen Vergleich drastische Unterschiede zwischen den Ländern bestehen, jedoch eine Kategorisierung der Gemeinsamkeiten von süd/osteuropäischen Staaten und nord-/westeuropäischen Staaten vorzufinden ist. Während west- und nordeuropäische Länder in der Regel eine hohe Geschlechtergleichheit aufweisen, sind für süd- und osteuropäische ${ }^{6}$ Länder deutliche Defizite der Geschlechtergleichheit vorzufinden (vgl. Plantenga et al. 2009: 30; World Economic Forum 2014: o. S.). Im Hinblick auf die inner-

4 „Selbst wenn Frauen quantitativ über mehr Geld verfügen als Männer, kann dies qualitativ als weniger wert definiert werden, und dies wird möglich, weil die Bedeutungszuschreibung von Geld von den jeweiligen Liebeskonzepten, den individuellen Ressourcen und den institutionellen Arrangements, also den gesellschaftlichen Rahmenbedingungen beeinflusst werden“ (Wimbauer 2003: 283).

5, ,[...] auf Ebene gesellschaftlicher Makrostrukturen der Wirtschaftsordnung und des Wohlfahrtsstaates, auf intermediärer Ebene von Arbeitsorganisationen - in Form von strukturellen Begrenzungen, hegemonialen Deutungen und interaktiven Praktiken - und auf der mikrosoziologischen Ebene von Individuen-in-Beziehungen“ (Wimbauer 2012: 102).

6 „During the post-1989 period, most if not all Central and Eastern European countries witnessed a considerable decline in women's labor force participation, in many cases accompanied by a reversion to more traditional gender role attitudes and gender relations" (Schmitt/Trappe 2010: 262). 
häusliche Arbeitsteilung ist festzustellen, dass trotz der genannten Unterschiede in europäischen Ländern Frauen deutlich mehr innerhäusliche Routinetätigkeiten übernehmen als Männer (vgl. Meuwly et al. 2011: 37; Batalova/Cohen 2002: 743; Schmid/Schön-Bühlmann 2003: 131; Fuwa 2004: 764; Wengler et al. 2009: 67; Dörfler/Wernhart 2016: 72) - gleichwohl ist der „housework gender gap“ in den nordeuropäischen Ländern vergleichsweise am geringsten, in den konservativen Ländern am größten und die liberalen englischsprachigen Länder bilden auf dieser Skala die Mittelkategorie (vgl. Sayer 2010: 34). Darüber hinaus variieren geschlechterkulturelle Leitbilder zwischen europäischen Ländern (vgl. Pfau-Effinger 2005: 340).

Die Beharrlichkeit traditioneller Verhaltensmuster zu erklären, ist keineswegs eine neue wissenschaftliche Königsdisziplin; Studien zur innerhäuslichen Arbeitsteilung sind bereits seit den 60ern en vogue. In Bezug auf die Frage, wie sich innerhäusliche Arbeitsteilungsarrangements entwickeln werden, konkurrieren die soziologischen Forschungsperspektiven jedoch miteinander. Ein zentrales Forschungsdesiderat ist ferner, dass sich weder Traditionalisierung noch Enttraditionalisierung der innerhäuslichen Arbeitsteilung logisch ableiten lassen: Mischformen (Lewis 20017) und länderspezifische Unterschiede (wie z. B. Esping-Andersens ,multiple equilibria approach“8) sind in Erwägung zu ziehen, um die innerhäusliche Arbeitsteilung in unterschiedlichen Kontexten erklären zu können, anstatt Gefahr zu laufen, lineare Entwicklungen $\mathrm{zu}$ schlussfolgern. Je nach Forschungsperspektive dienen Gendertheorien als Garant für eine fortschreitende Traditionalisierung (der Identitätsformationsansatz nach Bielby/Bielby (1989), der Gender-Display-

„Male-Breadwinner Model“: Mann = Vollzeit erwerbstätig, Frau = Hausfrau;

„Dual-Breadwinner Model 1“: Mann Vollzeit erwerbstätig, Frau kurzzeitig Teilzeit erwerbstätig und erbringt Hausarbeiten (ggf. Haushaltshilfe durch Verwandte);

„Dual-Breadwinner Model 2“: Mann = Vollzeit erwerbstätig, Frau = langfristig Teilzeit erwerbstätig, Hausarbeiten werden von anderen Familienmitgliedern übernommen oder ausgelagert;

„Dual-Breadwinner Model 3“: Mann und Frau sind beide Teilzeit erwerbstätig und teilen sich die Hausarbeit; „Dual-Career Model: Mann und Frau sind beide Vollzeit erwerbstätig, Hausarbeiten werden ausgelagert;

„Single-Earner (Lone-Mother Family) Model“: Ein-Eltern Familie mit Kindern und erwachsener Person, die voll-, teilzeiterwerbstätig oder nicht erwerbstätig ist, Hausarbeit wird entweder allein erbracht oder im Falle der Erwerbstätigkeit ausgelagert (Lewis 2001: 157).

8 Traditional equilibrium means that ,[...] the male is the breadwinner, and, the female, the homemaker $[\ldots]$. Unstable equilibria are associated with periods of equilibrium transition and manifest the absence of broadly shared agreement of what is ,propper ${ }^{*}$ behaviour [...] [and the] egalitarian equilibrium [...] entails partnerships based on two full-time employed spouses who engaged in a gender-symmetric allocation of child care and housework" (Esping-Andersen et al. 2013: 3). Während sich laut den Ergebnissen von Esping-Andersen im „Vorreiterland“ Dänemark das ,egalitarian equilibrium“ durchgesetzt hat, das mit Homogenität und Genderge-rechtigkeit in der innerhäuslichen Arbeitsteilung einhergehen würde, würde in Spanien das ,traditional equilibrium“ und in Großbritannien das „unstable equilibrium“ dominieren (vgl. Esping-Andersen et al. 2013: 3). 
Ansatz nach Brines (1994) respektive der Gender-deviance-neutralizationAnsatz nach Schneider (2012) und die Honeymoon-Hypothese nach Künzler (1994)), Rational Choice Ansätze hingegen prognostizieren Enttraditionalisierungsprozesse (die ökonomische Theorie der Familie nach Becker (1981), die ökonomische Verhandlungstheorie nach Ott (1992), die Theorie des sozialen Tauschs nach Blau (1964) und der Time-Availability-Ansatz nach Coverman (1985)) - in beiden Paradigmen wird unzulänglicher Weise der Prozess weitgehend als linear unterstellt. Dieser Widerspruch wird zum Anlass genommen, eine Typologie der Macht zu entwickeln, die die divergierenden Theorien in einen aus dem Machtansatz hergeleiteten Zusammenhang stellt und hierbei ihre Kontextabhängigkeit berücksichtigt. „Bringing Power Back In" heißt die Devise, wird doch die Benennung der Machtkategorien innerhalb der Familiensoziologie zunehmend vernachlässigt! Die zentrale Ausgangsannahme ist, dass die innerhäusliche Arbeitsteilung durch Machtverhältnisse beeinflusst wird, wobei Macht als ein latentes, dispositives, komplexes, soziales Phänomen erachtet wird, was eine mehrdimensionale Betrachtungsweise unumgänglich erscheinen lässt. Ein theoretischer Mehrwert ist insofern in der multidimensionalen Perspektive einer Verknüpfung von soziologischen, austauschtheoretischen, ökonomischen und makrosoziologischen Theorien $\mathrm{zu}$ finden. Ferner können vier zentrale Argumente für dieses Dissertationsvorhaben festgehalten werden:

1. Der zunehmenden Ausklammerung des Machtansatzes als Folge einer oberflächlich erscheinenden kulturellen Gendergleichheit tritt eine theoretisch fundierte Typologie der Macht entgegen, wodurch die Diskrepanz zwischen soziologischen Rational-Choice-Ansätzen und Gendertheorien aufgelöst werden kann. Ende der 70er Jahre konnte Held (1978) feststellen, dass familiensoziologische Machtansätze lediglich auf die Eltern-Kind-Beziehung bezogen wurden und, dass das Mann-Frau-Verhältnis aufgrund des strukturell-funktionalisitischen Familienmodells als horizontal begriffen wurde. So konnte Held die Ausklammerung der Macht aus der Paarbeziehung kritisieren. Heutzutage findet eine Ausklammerung insofern statt, als innerhäusliche Arbeitsteilungsarrangements nunmehr nahezu ausschließlich über mikrosoziologische Rational-Choice oder Gendertheorien erklärt werden. Insbesondere in der Ökonomie dominieren theoretische Ansätze der sozialen Austausch- und Ressourcentheorie sowie das CooperativeBargaining-Modell zur Erklärung partnerschaftlicher Arrangements (vgl. Lott 2009: 330).

2. Ein langwieriges Forschungsdesiderat einer mangelnden mehrdimensionalen Betrachtungsweise, das sowohl auf einen Mangel an theoretischer Verknüpfung der Ansätze als auch auf einen hohen methodischen Anspruch zurückzuführen ist, kann entschlüsselt werden. Obwohl die Analysen von Teilaspekten von Genderungleichheiten, 
Macht in Paarbeziehungen und traditionellen Rollenbildern in der Forschungspraxis durchaus gängig sind, bleiben ihre Perspektiven doch zumeist auf die Mikro- oder Makroebene begrenzt, ohne ihre fundamentale Wechselwirkung zu berücksichtigen. Studien, die MultilevelAnalysen durchführen, sind in diesem Forschungsfeld eher selten.

3. Vergleiche der empirischen Messungen von Genderungleichheiten durch Indizes, wie z. B. den Gender-related Development Index, den Gender Inequality Index oder den Global Gender Gap Index, erfassen auf europäischer Ebene lediglich geringfügige Unterschiede zwischen den Ländern, weil sie als Messinstrumente für weltweite Erhebungen $\mathrm{zu}$ allgemein konstruiert sind. Es gilt weitere adäquate Indikatoren zu identifizieren, durch die europäische Unterschiede konstatiert werden können.

4. Die empirisch zu beobachtende Diskrepanz zwischen Einstellungsund Verhaltensebene, ferner die Beständigkeit traditioneller Arbeitsteilungsarrangements kann erklärt werden.

In Anknüpfung an den bisherigen Forschungsstand und unter Berücksichtigung der Forschungsdefizite stellt sich die, für die vorliegende Dissertation zentrale, Forschungsfrage: Inwiefern determinieren Machtstrukturen und Empowerment die innerhäusliche Arbeitsteilung in Paarbeziehungen im Kontext europäischer Regionen? Hieraus ergeben sich weitere forschungsleitende Fragestellungen, die fortführend analysiert werden:

- Warum ist die innerhäusliche Arbeitsteilung in Paarbeziehungen überwiegend traditionell?

- Welcher Zusammenhang besteht zwischen Machtstrukturen und innerhäuslicher Arbeitsteilung in Paarbeziehungen?

- Wie stark determinieren sozietale und kulturelle Dimensionen der Macht die innerhäusliche Arbeitsteilung?

- Wie entwickeln sich (Machtstrukturen und) innerhäusliche Arbeitsteilungsarrangements in Paarbeziehungen im Zeitverlauf im europäischen Vergleich?

- Wie entwickelt sich das Verhältnis zwischen außerhäuslicher Erwerbsbeteiligung und innerhäuslicher Hausarbeit?

Zur Beantwortung der Forschungsfragen werden die Daten der ersten beiden Erhebungswellen des ,Generations and Gender Programmes' aufbereitet, mittels einer Mehrebenenanalyse auf vermutete Einflussfaktoren getestet und im Zeitverlauf regime-, länder- sowie regionen-vergleichend (NUTS1) analysiert. Da nicht nur Unterschiede zwischen Regimen und zwischen Ländern bestehen, sondern sich diese durch eine innere institutionelle Fragmentiertheit ökonomischer, familienpolitischer und kultureller Rahmenbedingungen der innerhäuslichen Arbeitsteilungsarrangements auszeichnen, wird für die 
zentrale Bedeutung eines regionalen Vergleichs plädiert - auch, um den methodologischen Nationalismus zu überwinden.

Die vorliegende Dissertation ist wie folgt aufgebaut: Zunächst wird ein Überblick des aktuellen Forschungsstandes gegeben. Anschließend wird der theoretische Bezugsrahmen skizziert und daraus die Typologie der Machtverhältnisse in Paarbeziehungen sowie resultierende Hypothesen abgeleitet. Der empirische Teil dieser Dissertation wird durch eine Darstellung der zu untersuchenden Daten des „Generations and Gender Programmes“ eingeleitet. Weiterführend werden methodische Aspekte der Mehrebenenanalyse erläutert, die in der Darstellung der univariaten Ergebnisse und multivariaten Ergebnisse der Mehrebenenanalysen münden. Abschließend werden im letzten Kapitel die zentralen Ergebnisse diskutiert, ein Resümee gezogen sowie ein Ausblick in Form von zukünftigen Forschungsanreizen zur Thematik der innerhäuslichen Arbeitsteilung eröffnet. 

2. Traditionelle innerhäusliche Arbeitsteilungsarrangements und partnerschaftliche Machtungleichgewichte im ,,paradoxemanzipierten“ 21. Jahrhundert

\subsection{Forschungserkenntnisse zur Norm-/Realitätsdiskrepanz}

In den vergangenen Jahrzehnten sind Prozesse der Modernisierung in europäischen Ländern vorherrschend. Gendergleichheit ist als politisches Ziel und als Wertvorstellung in den Erwartungen der Individuen verankert. Wird jedoch die innerhäusliche Arbeitsteilung in Paarbeziehungen im Kontext europäischer Länder miteinander verglichen, ist, wie bereits erläutert, eine Diskrepanz zwischen egalitären Einstellungen und traditionellen innerhäuslichen Verhaltensweisen zu beobachten: Nach wie vor wird trotz einem Gleichheitsideal die Mehrheit der Hausarbeiten von Frauen übernommen, wobei Männer lediglich eine „Helfer-Rolle“ einnehmen - ,[...] the pradox between egalitarian values and inegalitarian practices“ (Bühlmann et al. 2010: 49). Unter ,traditionaler Arbeitsteilung' wird hierbei die Ausführung von unbezahlten Hausarbeiten verstanden, die überwiegend von der Frau übernommen werden, , partnerschaftliche/egalitäre Arbeitsteilung' heißt, dass beide PartnerInnen zu gleichen Teilen die Hausarbeiten übernehmen und von einem ,Rollentausch' ist auszugehen, wenn der Mann die Hausarbeiten überwiegend ausführt (s. Abbildung 1).

Abbildung 1: Veränderungsrichtungen der Arbeitsteilungen im Haushalt

\begin{tabular}{|l|}
\hline Stark traditional \\
Frau erledigt die \\
Hausarbeit allein \\
\hline
\end{tabular}

Partnerschaftlich Beide Partner engagieren sich zu gleichen Teilen

\section{Rollentausch}

Mann erledigt die

Hausarbeit allein

Enttraditionalisierung

Traditionalisierung

Quelle: Grunow et al. 2007: 163. 
Bereits in den 70er Jahren wurden erste Diskrepanzen zwischen Einstellungsund Verhaltensebene von Schweizern/Schweizerinnen im Rahmen der Studie „Die Stellung der Frau in Familie und Gesellschaft" von Thomas Held und René Levy (1974) festgestellt (vgl. Held/Levy 1974: 254). Nicht die Situation, sondern die Wahrnehmung der Situation hat sich verändert. „Die Ideologie, die Frau sei gleichberechtigt, ist stärker als die Tatsache, dass Unterschiede fortbestehen" (Held/Levy 1974: 2). Obwohl Einstellungsmuster der Gleichberechtigung vertreten werden und Diskrimination von Frauen nicht mehr wahrgenommen wird, sind konträre Verhaltensmuster zu beobachten. Held/Levy bezeichnen dieses soziale Phänomen als „Norm/Realitätsdiskrepanz" (Held/Levy 1974: 254). Jane Lewis betitelt die Gleichstellungsnorm in Bezug auf die Vollzeit-Erwerbstätigkeit auch als ,adultworker-Norm“ bzw. als ,[...] "adult-worker model family," whereby it is assumed that all adults are in the labor market" (Lewis 2001: 154).

Obwohl sich diese Ergebnisse von Levy/Held auf die 70er Jahre beziehen, zeigt sich, dass die Norm-/Realitätsdiskrepanz bzw. Diskrepanz zwischen Einstellungs- und Verhaltensebene immer noch eine hoch aktuelle Thematik ist, was Bühlmann et al. mit dem „Paradox Between Egalitarian Values and Inegalitarian Practices" beschreiben (Bühlmann et al. 2010: 49). Heutzutage würden in Deutschland beispielsweise $45 \%$ der Väter gerne die Elternzeit wahrnehmen, doch beträgt laut Daten des Statistischen Bundesamtes (2017) die Elternzeitquote von Vätern, deren jüngstes Kind unter drei Jahren ist, nur 2,7\% (vgl. Dechant/Schulz 2014: 588; Statistisches Bundesamt 2018a: o. S.). Auch in anderen europäischen Ländern würde die Mehrheit der Väter gerne die Arbeitsstunden zu Gunsten der Familie reduzieren und sogar drohende Gehaltskürzungen in Kauf nehmen, woraus Hobson/Fahlén die Bedeutung des Capability Ansatzes betonen, um die Freiheit zur Erreichung einer ausgeglichenen Work-Life-Balance verstehen zu können ${ }^{9}$. Doch all diesen Wünschen zum Trotz zeigt die Empirie ein anderes Bild. Männer und Frauen nehmen in Haushalt und Familie unterschiedliche Rollen ein: „Für Männer sind Familie und Arbeitsmarkt nach dem UND-Prinzip organisiert. Für Frauen [mit Kindern und erwerbstätigem Partner] hat die Arbeitsmarktbeteili-

9 Hobson und Fahlén (2009), deren theoretische Argumentation auf dem Capability Approach von Sen basiert, analysieren die ,agency inequalities“ der Work-Life-Balance von europäischen Vätern. Mittels der Agency Ungleichheiten wird Bezug auf die Diskrepanzen zwischen Normen/Werten und dem sozialen Handeln von Vätern genommen sowie zwischen Policies und den Capabilities, diese zu nutzen. Hobson/Fahlén (2009) kommen zum Ergebnis, dass Unterschiede der Capabilities und Agency der Work-Life-Balance zwischen alten und neuen Mitgliedsländern der EU fortbestehen, die jeweils unterschiedliche Wohlfahrtstypen repräsentieren. Insgesamt möchte die Mehrheit der Väter dennoch die Arbeitsstunden zu Gunsten der Familie trotz drohender Gehaltskürzungen reduzieren, woraus für Hobson/Fahlén die Bedeutung des Capability Ansatzes resultiert. Aus dem Capability Approach wird ein multidimensionales Konzept eines Capability Sets für die Erklärung der Work-Life-Balance abgeleitet, das individuelle Faktoren, kulturelle Faktoren und institutionelle Faktoren berücksichtigt. 
gung den Status eines Zusatzprogramms oder Lückenfüllers und ist strukturell weniger festgelegt" (Baumgartner/Fux 2004: 110). Zahlreiche Ergebnisse zeigen, dass partnerschaftliche Rollenbilder gemäß dem Muster der innerhäuslichen Traditionalisierung ausgeführt werden (vgl. Schulz/Blossfeld 2006: 46; Huinink/Feldhaus 2008: 4; Lott 2009: 327; Nitsche/Grunow 2016: $80)^{10}$.

„Zwar können heute, angesichts des langsam gender-egalitär werdenden gesetzlichen Rahmens und der toleranter gewordenen sozialen Normen, Paare die Gestaltung ihres Zusammenlebens relativ frei wählen und leichter wieder auseinander gehen, doch führt die Ausübung ihrer „Freiheit“" erstaunlich häufig zur Reproduktion traditionaler Muster während der Phasen der gemeinsamen Haushaltsführung“" (Krüger/Levy 2000: 381, Hervorhebungen im Original; die Verf.).

\subsection{Forschungserkenntnisse über die Determinanten der innerhäuslichen Arbeitsteilung}

Während zu Beginn einer Paarbeziehung meist egalitäre Arbeitsteilungsarrangements zu beobachten sind, wird mit zunehmender Paarbeziehungsdauer und insbesondere mit der Geburt des ersten Kindes die Arbeitsteilung traditioneller (vgl. Kaufmann 1990: 49; Krüger/Levy 2000: 386; Levy/Ernst 2002: 120; Levy et al. 2002: 24; Schulz/Blossfeld 2006: 43; Bühlmann et al. 2010: 62; Lott 2012: 6; Berghammer/Neuwirth 2013: 17; Zabel/Heintz-Martin 2013: 663; Dechant/Schulz 2014: 593ff.; Dechant/Blossfeld 2015: 373). Kaufmann beschreibt diesbezüglich, wie durch die Geburt des ersten Kindes eine neue Lebensphase des Paares entsteht, in der die neuen Eltern „Teil eines neuen Wertesystems und neuer Handlungsabläufe sind" (Kaufmann 1999a: 78). Weiterführend steht die traditionelle innerhäusliche Arbeitsteilung häufig in engem Zusammenhang mit einem Wandel des Beschäftigungsverhältnisses bzw. -status: nach Ende des Mutterschutzes sind Frauen meist nur noch in Teilzeit erwerbstätig (vgl. Baumgartner/Fux 2004: 113; Vogel 2009: 170; Hobson 2011: 160; Esping-Andersen et al. 2013: 2; Statistisches Bundesamt 2015: o. S.), wobei ,[...] maternal employment is higher in countries that combine comprehensive childcare policies with an available and affordable private care market" (Flynn 2017: 260). Die zunehmende sowie immer häufiger ununterbrochene Erwerbsbeteiligung von Frauen ist in der Empirie meist auf einen hohen Anteil an Teilzeitbeschäftigung zurückzuführen (vgl. Leitner et al. 2004: 14). Hingegen sind Väter im Laufe der gesamten Erwerbsbiographie meist in Vollzeit tätig (vgl. Baumgartner/Fux

10 In West- Deutschland ist das Muster der Traditionalisierung deutlich stärker ausgeprägt als in Ost-Deutschland (vgl. Klärner/Keim 2011: 121; Pfau-Effinger/Smidt 2011: 219). 
2004: 113). Im europäischen Vergleich sind 2013 die höchsten Teilzeitquoten von Männern mit knapp $10 \%$ in Schweden vorzufinden, während beispielsweise nur 7,7\% der Männer in Österreich und 5\% der Männer in Frankreich teilzeiterwerbstätig sind (vgl. Dörfler/Wernhart 2016: 37). In der Schweiz sind sechs von sieben Vätern vollerwerbstätig, doch trifft ein derartiges Erwerbspensum nur auf eine von sieben Müttern zu (vgl. Baumgartner/Fux 2004: 111). Insbesondere in West-Deutschland ist ein Trend des ,modifizierten Breadwinner-Modells‘ zu beobachten, nach dem der Mann Vollzeit erwerbstätig ist, während die Frau Teilzeit erwerbstätig ist ${ }^{11}$ und sich um die Hausarbeiten kümmert (vgl. Trappe et al. 2015: 238f.) $)^{12}$. Bereits Baumgartner/Fux konnten feststellen, dass das Vollzeit-Teilzeit-Modell selbst in späteren Lebensphasen nach dem Auszug von Kindern aus dem Elternhaus das häufigste ist, bevorzugt wird und eine geschlechtsspezifische Rollenverteilung bewirkt (vgl. Baumgartner/Fux 2004: 120). Selbst in Fällen von Doppel-Karriere-Paaren (egalitäres Erwerbsmodell nach Baumgartner/Fux (2004)) $)^{13}$ sind Hausarbeiten i. d. R. Zuständigkeitsbereiche von Frauen oder werden an Dritte ausgelagert (vgl. Solga/Wimbauer 2005: 20f.; Wimbauer 2012: 301) ${ }^{14}$. Es ist darauf hinzuweisen, dass auch die Motive der Teilzeitarbeit zu differenzieren sind: nach der Standbein-Spielbein-Formation wird der familiären Fürsorge oberste Priorität zugewiesen (,Standbein“), hingegen wird die Teilzeitarbeit als „Spielbein“ genutzt. Von diesem Typus sind teilzeitarbeitende Frauen zu unterscheiden, die mittels ihrer Erwerbstätigkeit versuchen, sowohl dem Beruf als auch der Familie eine gleiche Bedeutung beizumessen (vgl. Fux/Baumgartner 1998: 6; Baumgartner 2003: 29f.). Die genderspezifische Segregation der inner- und außerhäuslichen Arbeitsteilung sowie ihre Wechselwirkung bezeichnen Levy et al. (2002) auch als „modernization of family traditionalism” (Levy et al. 2002: 31). Anstelle eines Verschwindens des traditionellen Modells haben Levy et al. (2002) eine Modernisierung des Familientraditonalismus festgestellt, die die Widerstandsfähigkeit traditioneller Elemente zum Ausdruck bringt und die zu

11 Entspricht dem ZweiverdienerInnenmodell: Frau - Teilzeit, Mann - Vollzeit.

12 Es gilt zu beachten, dass die Erwerbsquote von Frauen deutlich mit ihrem Alter, der Familiengründung und der Anzahl der Kinder in einem Kausalzusammenhang steht (vgl. Held/Levy 1974: 79).

13 „Unter Dual Career Couples (DCCs) fassen wir Paare, in denen beide Partner eine hohe Bildung und Berufsorientierung besitzen sowie eine eigenständige Berufslaufbahn verfolgen. Im Unterschied dazu bezeichnen Zwei-Verdiener-Paare Paare, in denen beide Partner in irgendeiner Form erwerbstätig sind und Frauen oft keiner professionellen Tätigkeit nachgehen“ (Solga/Wimbauer 2005: 9; Hervorhebungen im Original; die Verf.).

14 Für einen Vergleich zwischen „At-Home und Breadwinner Eltern“ s. Chesley/Flood (2017). Sie analysieren, ,[...] how time availability, relative earnings, and gender shape time use in couples. [To summerize, mothers do more child care than similarly situated fathers and] comparisons point to the importance of distinguishing among gender-normative housework tasks and accounting for differences in engagement on work and nonwork days" (Chesley/Flood 2017: 511). 
einem großen Teil auf den außerfamiliären Kontext und seine Funktionsweise zurückzuführen ist (vgl. Levy et al. 2002: 31). Wesentlich ist, dass Arbeitsteilungsmuster zwischen inner- und außerhäuslicher Erwerbsbeteiligung prozesshaft zu betrachten sind, d. h. je nach Lebensphase variieren können, wodurch eine Berücksichtigung von individuellen Erwerbsverläufen unumgänglich ist. Vornehmlich das Ausmaß an Teilzeitarbeit scheint von der jeweiligen Familienphase abhängig zu sein. Doch trotz der subjektiven Alltagsbeobachtung einer vermehrten Zunahme von Vätern, die ihre Kinder im öffentlichen Raum (Spielplatz etc.) betreuen, konnten Baumgartner/Fux (2004) kein strukturelles Äquivalent dieser Entwicklung finden (vgl. Baumgartner/Fux 2004: 126). Gleichwohl wird eine zunehmende Beteiligung von Vätern an der Kinderbetreuung erwartet, sodass nicht nur vollzeiterwerbstätige Frauen, sondern auch vollzeiterwerbstätige Männer einem Interrollenkonflikt zwischen Familie und Beruf ausgesetzt sein können, weshalb die Perspektive der Analyse auf beide Geschlechter und nicht ausschließlich auf die Frau gerichtet werden sollte. Für schweizerische Väter konnte im Rahmen der Tarzan-Studie (2016) nachgewiesen werden, dass sie durchschnittlich im Verlauf der Woche 46,2 Stunden für die Erwerbstätigkeit, 12,7 Stunden für Familienarbeit (administrative Tätigkeiten, Haushalt, Kochen und gemeinsames Essen) und 4,9 Stunden für die Zweisamkeit mit dem Kind aufwenden (zum Vergleich sind es am Wochenende 2,3 Stunden für die Erwerbstätigkeit, 9,9 Stunden für die Familienarbeit und ebenfalls 4,9 Stunden für die Zweisamkeit mit dem Kind) (vgl. Stamm 2016: 9). Folglich ist die Erwerbstätigkeit nach wie vor ein Bereich, in den Männer einen Großteil ihrer zeitlichen Ressourcen investieren. Dennoch versuchen sie, auch familiäre Verpflichtungen wahrzunehmen, gleichwohl diesen ein deutlich geringeres Ausmaß als der Erwerbstätigkeit zukommt.

Im Rahmen dieser Dissertation wird unterstellt, dass innerhäusliche Arbeitsteilungen ein Ausdruck von unterschiedlichen Machtverhältnissen in der Paarbeziehung sind. Demzufolge werden innerhäusliche Arbeitsteilungen als zu erklärendes Ereignis durch mehrdimensionale Machtkonstellationen analysiert. Welche aktuellen Erkenntnisse zur Machtthematik können dem Forschungsstand entnommen werden?

\subsection{Machtstrukturen als erklärende Elemente innerhäuslicher Arbeitsteilung}

Macht ist nicht nur ein Faktum, das auf Eliten und ihre Verzweigungen bezogen werden kann, sondern ferner in den alltäglichsten Situationen von Paaren vorzufinden ist. Jede soziale Beziehung ist durch Machtkonstellationen geprägt. Gerade in intimen Beziehungen spielt die soziale Einflussnahme auf 
das Verhalten des Partners/der Partnerin eine zentrale Rolle. So kann von einer engen Beziehung erst dann gesprochen werden, wenn das Verhalten von Ego positive oder negative Konsequenzen für Alter hat (vgl. Grau 2004: o. S.). Häufig wird Macht über den Zugang zur Machtressource „Geld“ operationalisiert. In den 1980ern und 1990ern erwiesen Untersuchungen, dass innerhalb von Paarbeziehungen eine beträchtliche Ungleichheit der Geldarrangements besteht und sein Zugang für Frauen begrenzt ist (vgl. LudwigMayerhofer et al. 2006: 212). Einen wesentlichen Beitrag zur Erforschung von Machtverteilungen in Paarbeziehungen lieferte Pahl (1983) ${ }^{15}$, dem zufolge Frauen Machtdefiziten ausgesetzt sind, wenn das zu verwaltende Einkommen hoch ist. Auf diesen Ergebnissen baute Yvonne Lott ihre Studie (2009) auf. Sie analysierte das Erwerbseinkommen als Machtfaktor in Paarbeziehungen. Dabei wurde das theoretische Konzept „Macht“ differenziert in die Kategorien „,ausführend“/,dirigierend“ und über die Einkommensverwaltung und Entscheidungsmacht als Indikatoren gemessen (vgl. Lott 2009: 327). Lotts deskriptive Analysen wie auch die multinominal logistischen Regressionsmodelle zeigten, dass Machtungleichgewichte zu Ungunsten der Frau unabhängig von ihrem Einkommen bestehen (vgl. Lott 2009: 227). Deshalb sollten zukünftige Studien Normen und Werte berücksichtigen, anhand derer Theorien entwickelt werden, die nicht ausschließlich dem Rational-Choice-Paradigma folgen. Ihrer Argumentation entsprechend könnte sich das klassische Rollenkonzept des „Mannes als Ernährer“ bzw. eine Geschlechterideologie als dominanter Faktor von Machtungleichheiten in Paarbeziehungen erweisen. Das traditionelle Ernährermodell (male breadwinner model) sieht vor, dass der Mann erwerbstätig ist, während die Frau der Kindererziehung und Haushaltsaufgaben nachgeht (vgl. Kelle 2011: 1). In einer weiterführenden Studie (2012) analysierte Lott erneut, welche Faktoren Macht in Paarbeziehungen beeinflussen, wobei sie zwischen ,financial and non-financial Power" differenzierte und zusätzlich untersuchte, welche Faktoren einen Machtkonstellationswechsel hervorrufen können (vgl. Lott 2012: 252). Financial-Power operationalisierte sie über die Einkommensverwaltung und non-financial Power über den sozialen Einfluss: Ist eine Person in der Lage, die Einstellung und das Verhalten seines Partners/seiner Partnerin zu beeinflussen, verfügt sie über non-financial power. Den Ergebnissen zufolge ist die financial power von der Einkommenshöhe abhängig: unabhängig vom Geschlecht verwaltet und entscheidet der/die PartnerIn mit dem höheren Einkommen über finanzielle Angelegenheiten in der Partnerschaft. Männer fühlen sich jedoch in ihrer männlichen Identität verletzt, wenn die Rolle des Familienernährers/der Familienernährerin von ihrer Frau ausgeführt wird. Im

15 Pahl (1983) hat die Verbundenheit von Macht und Geld, dem er sowohl eine ökonomische als auch eine soziale Bedeutung zuschreibt, in Ehen erforscht. „Work on inequality, and on the distribution of power and advantage, has pointed to money as a key element" (Pahl 1983: 237). 
Hinblick auf die Hausarbeit wird festgestellt, dass die Identität von Männern auch bei einem überwiegenden Anteil häuslicher Arbeiten nicht verletzt wird, sofern es sich um „männliche Aufgaben“, wie beispielsweise Handwerksoder Gartenarbeit handelt. Wird ihnen jedoch die Kinderbetreuung zugeteilt, die als typisch „weibliche“ Aufgabe gesellschaftlich verankert ist, wird erneut ihre männliche Identität angegriffen. Demzufolge sei es ratsam, zwischen verschiedenen ,typisch männlichen“ und ,typisch weiblichen Hausarbeiten" zu differenzieren. Außerdem zeigen die Ergebnisse von Lott (2012), dass sich Machtkonstellationen in Paarbeziehungen ändern können, wobei insbesondere eine Veränderung des Beschäftigungsstatus, die Heirat und die Geburt des ersten Kindes einen Wechsel begünstigen (vgl. Lott 2012: 252).

\subsection{Die Grenzen ökonomischer (Macht-)Ressourcenansätze}

Den Ergebnissen von Schulz/Blossfeld (2006), Grunow et al. (2007), Lott (2009) und Nitsche/Grunow (2016) ${ }^{16}$ zufolge ist der ökonomische Ressourcenansatz nicht adäquat, innerhäusliche Arbeitsteilungen und Machtungleichheiten in Paarbeziehungen zu erklären. Im Fall von Grunow et al. (2007) ist zu konstatieren, dass zwar - mit Verweis auf Schulz/Blossfeld (2006) - Ressourcenkonstellationen innerhalb des Paares keinen signifikanten Effekt auf eine stärkere Beteiligung des Mannes an den Hausarbeiten hervorrufen, jedoch Traditionalisierungsprozesse scheinbar trotzdem von ökonomischen Ressourcen beeinflusst werden.

„In Übereinstimmung mit den Ergebnissen der neueren, internationalen Arbeitsteilungsforschung finden wir, dass Paare, in denen beide Partner etwa gleich viel verdienen, ein deutlich geringeres Traditionalisierungsrisiko haben als Paare, in denen der Mann deutlich mehr verdient als die Frau. Verdient die Frau jedoch mehr als ihr Partner, verringert sich das Traditionalisierungsrisiko dadurch nicht weiter. Es gelingt den Frauen also offenbar nicht, diesen ökonomischen Vorteil in einen Verhandlungsvorteil umzusetzen. Das ist ein Hinweis darauf, dass die entscheidungstheoretische Bedeutung ökonomischer Ressourcen geschlechtsspezifisch asymmetrisch vorstrukturiert ist“" (Grunow et al. 2007: 177f.).

16 Nitsche/Grunow (2016) betrachten die innerhäusliche Arbeitsteilung anhand der pairfamDaten für Deutschland im Längsschnitt. Ihr zentrales Ergebnis der separaten Analysen für Paare ohne Kinder und Paare, die während der Panelwellen Eltern geworden sind, ist, dass die Hausarbeit vornehmlich durch Genderideologien beeinflusst wird: eine egalitäre Genderideologie führt zu einer egalitären innerhäuslichen Arbeitsteilung. Rational Choice Indikatoren konnten weder für Paare ohne Kinder, noch für Paare, die während des Panels ihr erstes Kind bekommen haben, ein signifikantes Ergebnis erzielen (vgl. Nische/Grunow 2016: $88)$. 
Reaktionsweisen von Männern seien nicht ressourcengesteuert, sondern geschlechtsspezifisch, weshalb asymmetrische Gender-Theorien zur Erklärung hinzugezogen werden sollten - so der Tenor von Schulz/Blossfeld (2006). Schulz/Blossfeld verweisen auf eine Überhöhung von Ressourcenkonstellationen und ökonomischen Kalkülen als essentielle Elemente der Arbeitsteilungsarrangements. Im Gegenteil bestätigen sie, dass „Normen, Rollen, Identitäten und Trägheiten“" innerfamiliäre Arbeitsteilungen am besten erklären (Schulz/Blossfeld 2006: 46). Schulz/Blossfeld sind der Auffassung, dass das Bildungsniveau nicht als ökonomische Einkommensressource, sondern ,als Indikator für liberale Einstellungen und Geschlechterrollenorientierungen zu interpretieren ist" (Schulz/Blossfeld 2006: 37). An dieser These wird Kritik insofern geübt, als weder Einstellungen noch Werte/kulturelle Leitbilder in ihrer Analyse berücksichtigt werden, die unter Umständen einen Erklärungsgehalt aufweisen könnten. Die Annahme, ein hoher Bildungsstand sei gleichzusetzen mit einer liberalen Einstellung, wird unzureichend geprüft. Demzufolge sind weitere Indikatoren erforderlich, die Einstellungsmuster sowie Werte messen.

Konträr zu den Thesen des ökonomischen Paradigmas sind weitere Hypothesen bekannt, die die geschlechtsspezifische Aufgabenverteilung möglicherweise adäquater beschreiben könnten, beispielsweise die Kompensationshypothese nach Brines (1994): wenn eine Frau die Rolle als Ernährerin übernimmt, dann werden sich die Haushaltstätigkeiten besonders traditionalisieren. Je größer die finanzielle Abhängigkeit des Mannes von seiner Frau ist, desto weniger beteiligt sich der Mann an der Hausarbeit („Display Modell") (vgl. Brines 1994: 682). „Weiblichkeit“ wird nach Brines Argumentation mit finanzieller Abhängigkeit vom Mann verbunden. Ist dieses Verhältnis umgekehrt, d. h. der Mann ist finanziell von der Frau abhängig, so fühlt er sich in seiner Geschlechtsidentität angegriffen und muss diese Frustration über eine traditionelle Haushaltsführung kompensieren. Ein neuerer Ansatz, der die Idee von Brines aufgreift, ist die ,gender deviance neutralization" Perspektive. Die zentrale These ist ebenso, dass eine gender-atypische Erwerbsarbeit durch gender-typische Hausarbeiten kompensiert wird. Schneider (2012) argumentiert, ,,[...] that men who do "women's work" and women who do "men's work" in the labor market may seek to neutralize their gender deviance by doing male- and femaletyped work at home" (Schneider 2012: 1029). Auch Busch-Heizmann/Bröckel (2015) bestätigen durch ihre auf den Daten des Sozio-ökonomischen Panels (1993-2011) basierende Analyse einer Kompensation geschlechtsuntypischer Berufstätigkeit durch weiblich konnotierte Hausarbeiten (vgl. Busch-Heizmann/Bröckel 2015: 475). Was passiert, wenn in Paaren aus unterschiedlichen Milieus nicht der Mann der Familienernährer, sondern die Frau die Familienernährerin ist, erforschen Koppetsch/Speck (2015). Ausgerechnet das individualisierte Milieu, das sich durch eine starke kulturelle Orientierung an der Geschlechtergleichheit kenn- 
zeichnet, ist jenes, welches durch eine starke Doppelbelastung der Frau durch Beruf und Hausarbeiten auffällt (für eine detaillierte Erläuterung dieser Studie s. Kapitel 3.1.2.3.).

Eine modifizierte Version, die ,gender deviance neutralization“ Perspektive zu überprüfen, greift McClintock (2017) auf. Sie kommt im Gegensatz zu Brines (1994) und Schneider (2012) zu dem zentralen Ergebnis, dass eine genderatypische Erwerbsbeteiligung zu einer genderatypischen innerhäuslichen Arbeitsteilung führt und insofern kein Kompensationseffekt vorliegt. Sowohl Frauen als auch Männer würden Hausarbeiten im Sinne ihrer Erwerbstätigkeit ausführen: diejenigen, die „männliche Berufe“ ausüben übernehmen auch ,männliche Hausarbeiten“ und diejenigen, die „,weibliche Berufe“ ausüben übernehmen im Haushalt „weibliche Tätigkeiten“ (vgl. McClintock 2017: 494) ${ }^{17}$. Anders formuliert: genderstereotype Erwerbstätigkeiten führen zur Übernahme von genderstereotypen Hausarbeitstätigkeiten und genderatypische Erwerbstätigkeiten führen zur Übernahme von genderatypischen Hausarbeitstätigkeiten. Der Einfluss der ,gender deviance neutralization" überschattet McClintock zufolge alternative Erklärungsansätze und Modellspezifikationen: „In particular, by assuming a quadratic association, researchers may impose, rather than test, gender deviance neutralization" (McClintock 2017: 475).

König/Langhauser (2016) finden heraus, dass eine berufliche Selbstständigkeit und Autonomie zu einer geringeren Übernahme der Haushaltstätigkeiten von Männern führen, hingegen für selbstständige Frauen das Gegenteil zutrifft. Relative Ressourcen, Bargaining-Theorie und Zeitbudget-Ansätze seien für die Erklärung der von Frauen übernommenen Hausarbeiten, die selbstständig erwerbstätig sind, weniger relevant als für die Erklärung der von selbstständigen Männern übernommenen Haushaltstätigkeiten (vgl. König/Langhauser 2016: 289).

Auch der Identitätsformationsansatz nach Bielby und Bielby (1989) betont eine asymmetrische Entwicklung traditioneller Rollenbilder. Ihnen zufolge basieren Geschlechterrollen auf gesellschaftlichen Normen und Werten, wobei ihr Wandel nur sehr langsam erfolgen kann. Selbst in modernen Gesellschaften seien Frauen noch für die Hausarbeit und die Kindererziehung zuständig (vgl. Bielby/Bielby 1989: 786). Die „Honeymoon-Hypothese“ bzw. das „Trägheitsgesetz“ besagt letztlich, dass die Arbeitsteilung im Haushalt mit der Dauer der Ehe sogar zunehmend traditioneller wird (vgl. Künzler 1994: 108; Schulz/Blossfeld 2006: 32; Langfeldt 2008: 84). Zu Beginn der

$17,[. .$.$] there is no evidence that men or women compensate for occupational gender atypical-$ ity through housework. Instead, occupational gender atypicality is associated with weaker adherence to gender-stereotypical patterns of housework performance, particularly for men. Both men and women perform gendered housework consistent with their gendered occupations - those employed in predominately male occupations do the most male chores and those employed in predominately female occupations do the most female chores" (McClintock 2017: 494). 
Paarbeziehung sei eine große Bereitschaft des Mannes zur Beteiligung an der Hausarbeit vorhanden, doch im Laufe der Zeit verfestigen sich geschlechtstypische Aufgaben durch den Alltag, d. h. traditionelle Arbeitsteilungen schleichen sich langsam ein (vgl. Schulz/Blossfeld 2006: 32).

Festzuhalten ist, dass die mikrosoziologische Argumentation zur Erklärung traditioneller Rollenbilder in zwei Theorietraditionen differenziert werden kann: symmetrische Ansätze des Rational-Choice-Paradigmas und soziologische asymmetrische Ansätze der Gendertheorien.

Die vorliegende Arbeit baut u. a. auf den Ergebnissen von Schulz/Blossfeld (2006) und Lott (2009) auf, setzt ihre Anregungen der theoretischen Konzeption mit einem Fokus auf Werte und Normen um, wobei geschlechtsspezifische Rollenkonzepte sowie damit verbundene Machtkonstellationen im Zeitverlauf analysiert werden.

Auffallend ist, dass die oben angeführten Studien Konzepte von Macht und traditionellen Rollenbildern weitestgehend aus mikro- und in Teilen aus mesosoziologischer Perspektive betrachtet haben; vorwiegend strukturelle Aspekte der makrosoziologischen Sichtweise, aber auch kulturelle Leitbilder (im Falle der ökonomischen Theorien und im Fall eines fehlenden adäquaten Indikators von Schulz/Blossfeld) wurden nur unzureichend berücksichtigt. ${ }^{18}$ Es gilt jedoch den Handlungsspielraum mitzudenken.

\subsection{Regimetypologische Erkenntnisse aus der Wohlfahrtsstaatsforschung: „Multiple Equilibria“}

Im Gegensatz zu Becker, der davon ausgeht, dass ein Bedeutungsverlust der Ehe aus der veränderten ökonomischen Rolle von Frauen einschließlich steigender Erwerbspartizipation resultiert, wodurch der Nutzen der Ehe abnimmt (Becker erachtet den Nutzen aus der ehelichen Spezialisierung als den wichtigsten Vorteil von Paarbeziehungen) ${ }^{19}$ und im Gegensatz zur These Van de

18 ,Zentral erscheint [...], dass bisher häufig nur der Einfluss von Partnerschaftsmerkmalen auf die je individuellen Lebens- und Erwerbsverläufe von in Partnerschaften lebenden Personen - zumeist der Frauen - betrachtet wurde (z. B. Blossfeld/Drobnič 2001). Mit dieser gängigen Individualbetrachtung werden Partner und Kinder jedoch nur als Kontextvariablen behandelt. Das Paar als Handlungseinheit und die Verflechtung der Erwerbsverläufe der Partner als Produkt gesellschaftlich strukturierter Geschlechterbiografien sowie innerpartnerschaftlicher Beziehungs- und Koordinierungsarrangements werden so nur unzureichend berücksichtigt - oder wie bei den austauschtheoretischen und haushaltsökomischen Erklärungen ,gesetzt““ (Solga/Wimbauer 2005: 17, Hervorhebungen im Original; die Verf.).

$19, \ldots[. .$.$] the gain from marriage is reduced by a rise in the earnings and labor force participa-$ tion of women and by a fall in fertility because a sexual division of labor becomes less advantageous“" (Becker 1981: 353). 
Kaas (1987) und Lesthaeghes (1992) des zweiten demographischen Übergangs, der aufgrund eines Wertewandels von postmaterialistischen Werten, Selbstverwirklichung und Individualisierung zum Bedeutungsverlust von Ehe und Paarbeziehungen führt, argumentiert Esping-Andersen ,[that] the dynamics are driven by the revolution of women's roles" (Esping-Andersen 2016: 10, Herbvorhebungen durch die Verf.). Sein zentrales Argument ist,

„[...] that the revolution will, in its early stages, indeed provoke fewer births, more singlehood, and less stable partnerships. The key turning point comes when both men and society at large adapt to women's new roles. It is when a new family equilibrium emerges, becomes stable, and enjoys broad normative acceptance that we will see stronger inclinations to partner and marry, more enduring relationships, and also a return to fertility levels that match citizens' actual ideals [ushaped dynamics thesis]“" (Esping-Andersen 2016: 10).

Genderegalität wird über die Zustimmung des Items ,[...] when jobs are scarce, men should have more right to a job than women" erhoben (EspingAndersen 2016: 54). In Ländern wie Deutschland, Italien oder Spanien, in denen eher konservative Gendernormen bestehen, ist das ,less family scenario“ dominant, in Ländern mit egalitären Gendernormen hingegen lebt die Familie wieder auf (Esping-Andersen 2016: 10). Ein stabiles ,gender egalitarian equilibrium" würde ferner zu höherer Fertilität und stabileren Paarbeziehungen, d. h. „more family“ führen (Esping-Andersen 2016: 16). Konträr dazu wurde über Jahrzehnte argumentiert, dass die Erwerbstätigkeit von Frauen negativ mit der Fertilitätsrate korreliert ist (vgl. Esping-Andersen 2016: 14). Das „less family and more individualism scenario“, das VertreterInnen des Postmodernismus als Wertewandel bezeichnen, stellt nach Esping-Andersen lediglich eine Übergangsphase dar (Esping-Andersen 2016: 99). Diese ist geprägt vom „multiple equilibria“ - eine Phase, in der eine Ambiguität von Normen und Unsicherheiten bestehen. Ziel EspingAndersens ist zu zeigen, wie das ,[...] old male breadwinner Family equilibrium was replaced by a new gender symmetric one“, wobei er das „less family and more individualism scenario" als Zwischenphase erachtet (EspingAndersen 2016: 99).

Warum sind auch in den Ländern, die Esping-Andersen als genderegalitär bezeichnet, die innerhäuslichen Tätigkeiten eher traditionell organisiert? Führt dieses stabile ,gender egalitarian equilibrium“ $\mathrm{zu}$,,more family“ und damit auch wieder zur traditionellen innerhäuslichen Arbeitsteilung? Fragwürdig ist außerdem, ob der „Employment Fertility Nexus“ (im ,gender egalitarian equilibrium“: je mehr Erwerbstätigkeit von Frauen, desto mehr Geburten) nicht nur, wie im Falle der Analysen Esping-Andersens, im länderspezifischen Kontext, sondern auch im Rahmen von Regionen beobachtet werden kann. Aus feministischer Perspektive fällt die von Esping-Andersen 
befürwortete Norm der Erwerbspartizipation ${ }^{20}$ auf: Esping-Andersen argumentiert aus rationalen Gründen (Erwerbstätigkeit von Frauen verhilft, soziale Ausgrenzung und Armut zu vermeiden (vgl. Esping-Andersen 2002: 94)), warum Gendergerechtigkeit von Relevanz ist und, dass die Erwerbsarbeit ein zentraler Faktor zur Erreichung der Gendergerechtigkeit sei, der „Wille“ von Frauen bleibt jedoch unberücksichtigt - viele Frauen arbeiten im Dienstleistungssektor ${ }^{21}$ und/oder unter prekären Bedingungen. „Das skandinavische oder »moderne« - Modell [ist] eine staatlich gestützte weibliche Dienstleistungsgesellschaft" (Langan/Ostner 1991: 306). Zwar hat sich durch die Integration von Frauen in die Erwerbstätigkeit das Machtverhältnis zwischen Frauen und Männern geändert, jedoch geht es immer noch - obgleich auf eine andere Art und Weise - auf Kosten von Frauen (vgl. Langan/Ostner 1991: 309). ,[...] there is, realistically, a limit to female life course masculinization" (Esping-Andersen 2002: 95). Es ist nicht verwunderlich, dass Ostner anlässlich des aktuellen 39. Kongresses der Deutschen Gesellschaft für Soziologie an Ulrike Prokops (1976) Idee der „Verselbstständigung politischer Strategien" erinnert, da sie die Besonderheit des Alltagslebens der Frauen, einschließlich seiner Potentiale und Ambivalenzen, weibliche Wünsche wie auch Verluste außer Acht ließen, die im Zusammenspiel mit der fortschreitenden Ökonomisierung des weiblichen Lebensbereichs auftraten. Wird die Argumentation von Esping-Andersen weiterführend interpretiert, kann geschlussfolgert werden, dass ,aus Frauen keine Männer gemacht“ werden könnten, weshalb es aus Esping-Andersens Perspektive zu unterstützen sei, dass Frauen in ,weiblichen Berufen“, was unter Umständen unter prekären Bedingungen (Niedriglöhne, befristete Arbeitsverhältnisse, Teilzeit- und Minijobs etc.) heißen kann, arbeiten. Darüber hinaus wird eine Fokussierung auf „das Männliche“ ersichtlich, ferner eine scheinbare Maskulinisierung weiblicher Lebensläufe:

,$[\ldots]$ ongoing change in gender behaviour is producing an increasingly ,masculine" profile of female biographies [...] simultaneously, the male life course becomes more ,feminine ${ }^{6}$. [...] if we want more gender equality our policies may have to concentrate on men's behaviour" (Esping-Andersen 2002: 68).

$\mathrm{Zu}$ kritisieren ist eine im engsten Sinne normative Perspektive: erstens, dass vorab bestimmte Bereiche als primär männlich deklariert werden und zweitens, dass, wie bereits erwähnt, die Interessen von Frauen als männlich unterstellt werden, unabhängig davon, ob es sich um ,family-centered women“,,career-centered women" oder ,dual-role mothers"22 handelt (Esping-

20 „Lifetime employment is now practically the norm among North American and Scandinavian women. And indicators are that the same norm is now being embraced throughout the advanced countries" (Esping-Andersen 2002: 88).

21, ,...] rising female employment is triggered by the emergence of the service economy“ (Esping-Andersen 2002: 68).

22 Diese Typen gehen auf Hakims ,Key Issues in Women's Work' (1996) zurück. 
Andersen 2002: 72). Das skandinavische Regime wird als zu erstrebende Norm zugrunde gelegt. Beispielsweise erfolgt eine Analyse des employment rate gap als prozentuale Abweichung diverser Länder (Schweden, Österreich, Belgien, Frankreich, Deutschland, Italien, Niederlande, Spanien, Großbritannien, USA) von Dänemark - stellvertretend für skandinavische Länder - als Norm. Die Vereinbarkeit von Familie und Beruf soll möglichst durch „frauenfreundliche" Policies, wie Kindertagesbetreuungsangebote, bezahlten Mutter- und Vaterschaftsurlaub oder Vorkehrungen im Falle einer Arbeitsunfähigkeit aufgrund kranker Kinder, erreicht werden (vgl. Esping-Andersen 2002: 94). Auffallend ist jedoch die ,negative Diskussion“ der Familie:

„Im Mittelpunkt stehen Multidysfunktionalitäten: zu wenig Kinder, zu wenig erwerbstätige Mütter und ganz aktuell das Bildungsversagen der Familie. Diese Diagnosen münden inzwischen immer öfter in der Forderung nach »Entfamilisierung « der Familie [Esping-Andersen ist ein Vertreter dieser Argumentation]. [...] Dem Familienversagen könne man, so das Fazit, nur durch konsequente Entfamilisierung $[\ldots]$ begegnen. Auf diese Weise könne die neue sozialinvestive Sozialpolitik das Projekt des Egalitarismus verwirklichen. Sie soll das vor allem aus Gründen der Effizienz tun“ (Ostner 2004a: 90).

Die vorliegende Dissertation erhebt den Anspruch, eine Sensibilität für Pluralismus und Differenzierung zu entwickeln: Nicht die Fragen, ,[...],Wie zufrieden ist diese Frau [oder dieser Mann]?‘ oder, Über wie viel Ressourcen kann sie [oder er] verfügen?' Es ist vielmehr die Frage ,Was ist sie [oder er] tatsächlich fähig (able to) zu tun und zu sein?““, die im Vordergrund steht (Nussbaum 2003: 18, Hervorhebungen im Original; die Verf.). Hierin liegt die stärkere Fokussierung auf den Handlungsspielraum als auf die letztlich von den Individuen gewählte Alternativen begründet. Es wäre ebenso anmaßend und unterkomplex, einen spezifischen Lebensweg als den besten zu definieren, wie eine „Skandinavisierung“ Europas (im Sinne des skandinavischen Regimes als eine zu begrüßende Norm sämtlicher europäischer Regime ungeachtet ihrer historisch und kulturell bedingten Unterschiede ${ }^{23}$ ) zu verfechten.

$23 \mathrm{Zu}$ verweisen sei hier auf die Argumentation von Peter Baldwin: „Mit der simplen Unterscheidung zwischen dem ,institutionellen“ und dem „residualen“ Wohlfahrtsstaat lässt sich die Tatsache, dass der Rest der Welt die Perfektion des Schwedischen Modells nicht erreicht hat, nur negativ erklären: Aufgrund der Abwesenheit der nötigen Voraussetzungen, insbesondere einer starken und gut organisierten Arbeiter[Innen]bewegung, war die Herausbildung eines solidarischen Wohlfahrtsstaatsmodells nicht möglich. Einer solchen Sichtweise ist der Gedanke fremd, dass verschiedene Staaten ähnliche Probleme auf unterschiedliche Weise zu lösen suchen. Macht dann die Frage noch Sinn, ob das skandinavische Modell auch anderswo anwendbar ist oder jemals sein wird? Wenn nicht, wenn andere Wohlfahrtsstaaten anders und nicht einfach nur unvollkommene Versionen des skandinavischen Modells sind, die irgendwann vielleicht, nach einem Regime sozialpolitischer Steroide, auf nordische Standards gemästet werden, warum sollte man das sozialdemokratische Modell dann noch derart ernst nehmen? Warum kann man es nicht als eine Variante, und 


\subsection{Erkenntnisse über „Desperate Houswives“?}

Cranney und Miles (2016) gehen der Fragestellung, ,[...] how satisfied stayat-home mothers are with their work relative to employed mothers" auf den Grund (Cranney/Miles 2016: 1). Basierend auf der Datengrundlage des General Social Survey (1972-2012) und mittels logistischer Regressionsverfahren können die Autoren zeigen, dass in den USA sowohl nicht-erwerbstätige als auch berufstätige Mütter eine hohe Zufriedenheit während des Analysezeitraums mit ihrer Tätigkeit aufweisen. Dies deutet darauf hin, dass beide Arten von Arbeit selbstverwirklichende Erfahrungen darstellen (vgl. Cranney/Miles 2016: 15). Im Zeitverlauf hat sich die Zufriedenheit von erwerbstätigen und nicht-erwerbstätigen Müttern weitgehend angenähert, seit 1996 sind die Unterschiede der Zufriedenheit der beiden Gruppen nicht mehr signifikant. Cranney und Miles führen diese Entwicklung auf einen kulturellen Wandel zurück: Der Anstieg des ,intensive mothering“ und kulturelle Rahmenbedingungen haben den Wert und die Zufriedenheit des ,stay at home mothering“ vornehmlich für Frauen aus der Mittel- und Oberschicht erhöht (vgl. Cranney/Miles 2016: 17). Es scheint eine Neubewertung des ,intensive mothering“ zu bestehen. Durch den kulturellen Wandel sei die Wahlfreiheit der Hausfrauenrolle und, so das mutmaßlich bedeutendere Ergebnis, die Zufriedenheit von nicht-erwerbstätigen Müttern gestiegen (vgl. Cranney/Miles 2016: 17).

„[...] the SAHMs of today are not unfulfilled, desperate housewives, but rather are just as satisfied with their work as are employed mothers. [...] most important of all, this study directs much needed attention to SAHMs [Stay-at-home mothers], who make up a significant portion of the population but who have largely been neglected in the social scientific literature. SAHMs do not appear to be going anywhere and deserve further study" (Cranney/Miles 2016: 18).

Während sich Cranney/Miles (2016) aus einer kulturtheoretischen Perspektive auf den Vergleich der Zufriedenheit erwerbstätiger und nichterwerbstätiger Mütter fokussieren, konzentrieren sich Studien, die eine ländervergleichende Betrachtung verfolgen, häufig auf strukturelle Faktoren der Makroebene. Doch wie können strukturelle, kulturelle und individuelle Faktoren konstatiert werden, wenn die Analysen i. d. R. auf eindimensionale Betrachtungen begrenzt sind? Ferner plädieren Alexander/Welzel (2014) dafür, dass Machtstrukturen (in diesem Fall Empowerment) als reflexiver Prozess analysiert werden müssen. „Women's empowerment is a coevolutionary process“ (Alexander/Welzel 2014: 17).

zwar eine relativ randständige, innerhalb des breiten Spektrums von Sozialpolitikentwicklung betrachten“" (Baldwin, P. 2003: 51)? 
Inwiefern können Gender(un)gleichheiten in Europa gemäß dem Forschungsstand beobachtet werden? Nachfolgend werden aktuelle Ergebnisse ländervergleichender Indizes zur Messung von Genderungleichheiten aus überwiegend makrosoziologischer Perspektive skizziert.

\subsection{Empirische Befunde: Vergleich der Indizes zur Messung von Gender(un)gleichheiten}

GDI: Der Gender-related Development Index (GDI) ist ein vom ,United Nations Development Programme، (UNDP) konzipierter Index zur weltweiten Messung von Genderungleichheiten, mittels dem die drei Schlüsseldimensionen Gesundheit (Lebenserwartung), Bildungszugang (durchschnittliche Anzahl an Schuljahren) und Lebensstandard (Brutto-Einkommen pro Kopf) jeweils differenziert nach Geschlecht erfasst werden (vgl. UNDP 2015: o. S.). Aufgrund des Forschungsinteresses an europäischen Ländern wird auf eine weltweite Darstellung der GDI-/GII und GGG-Werte verzichtet, lediglich die Werte der 28 EU-Länder sind in Tabelle 1 aufgeführt. Je kleiner der GDI-Indexwert (Wertebereich 0 bis 1), desto größer der Unterschied zwischen Männern und Frauen, d. h. je größer die Genderungleichheit. Werden die GDI-Werte für das Jahr 2013 miteinander verglichen, fällt auf, dass alle europäischen Länder sehr ähnliche Werte aufweisen; sie schwanken zwischen min. 0,935 (Österreich) und max. 1,042 (Estland). Folglich sind keine größeren Unterschiede zwischen den europäischen Ländern festzustellen, die Gendergleichheit in Europa bewegt sich gemäß diesem Index auf sehr hohem Niveau. Der GDI ist ein Nachfolger des Human Development Indxes (HDI), der vor allem durch Amartya Sens Denkanstöße dazu konzipiert wurde, die Entwicklungsstände unterschiedlicher Länder theoretisch fundierter miteinander vergleichen zu können als dies mit dem bloßen Vergleich des Bruttoinlandsprodukts möglich war (für einen umfassenden Überblick der Messprobleme und Schwierigkeiten des Bruttoinlandsprodukts als Indikator für Wohlstand s. Stiglitz et al. (2009)). Ergänzend wurde der GDI entwickelt, der dieselben Indikatoren enthält wie der HDI, jedoch zwischen den Geschlechtern differenziert. Kritikfähig ist, dass der GDI zwar den Entwicklungsstand eines Landes als geschlechtsspezifischen Index abzeichnet, jedoch eine Messung von Genderungleichheiten weitere geschlechtsspezifische Indikatoren erfordert. Ergänzend zum GDI wurde vom UNDP ein weiterer Index zur Messung von politischen sowie ökonomischen Teilhabechancen von Frauen entwickelt, der Gender Empowerment Measure Index (GEM). Der GEM ist ein Index zur Messung von Genderungleichheit, der politische Teilhabe (Anteil an Parlamentssitzen von Frauen), ökonomische Teilhabe (Anteil von Verwaltungsbeamtinnen/Managerinnen, Frauen in technischen Berufen) und 
Einkommensressourcen (Einkommenslücke Frauen/Männer) beinhaltet. Doch auch eine Betrachtung von Ungleichheiten, die ausschließlich auf Teilhabechancen basiere, sei noch nicht spezifisch genug, Genderungleichheiten empirisch zu messen:

„[...] a main criticism of the GDI index is that it does not measure gender equality in itself, but a combination of gender equality and levels of achievement. A similar argument applies to the GEM" (Plantenga et al. 2009: 21).

GII: Aufgrund der geübten Kritik am GDI und GEM wurde (2010) der Gender Inequality Index (GII) zur weltweiten Messung der Benachteiligung von Frauen entwickelt, der folgende Dimensionen beinhaltet: Gesundheit und Reproduktion (Müttersterblichkeitsrate, Fertilitätsrate), Empowerment (Anzahl an Parlamentssitzen, die von Frauen besetzt sind, Anteil an Personen, die einen weiterführenden Bildungsabschluss erreichen, d. h. Sekundarstufe II und höher) und Teilhabe am Arbeitsmarkt (Erwerbsquote) (vgl. UNDP 2015: o. S.). Im Wertebereich sind Werte zwischen 0 und 1 vertreten, wobei ein Wert von 0 als max. Gendergleichheit, ein Wert von 1 als max. Ungleichheit interpretiert werden kann.

2013 streuen die GII-Werte in Europa zwischen 0,021 (Slowenien) und 0,32 (Rumänien), d. h. es sind lediglich geringfügige Unterschiede zwischen den Ländern zu erkennen, insgesamt ist auch gemäß diesem Index ein hohes Gleichheitsniveau zu verzeichnen.

GGG: Der Global Gender Gap Index (GGG) wird vom , World Economic Forum' erhoben und dient ebenfalls zur weltweiten Messung von Genderungleichheiten in folgenden Teilbereichen:

- Ökonomische Teilhabe/Chancen (Verhältnis der Erwerbsquote Männer/Frauen, Verhältnis Einkommen Männer/Frauen, Verhältnis von Führungspositionen Frauen/Männer, Verhältnis Fachkräfte Männer/Frauen und Frauen in Führungspositionen),

- Bildung (Verhältnis Alphabetisierungsrate Frauen/Männer, Verhältnis Primar-/Sekundar-/Tertiäre-Bildungs-Rate Männer/Frauen),

- Gesundheit/Überlebenschancen (Verhältnis Männer/Frauen bei Geburt, Verhältnis der Lebenserwartung Männer/Frauen) und

- politische Teilhabe (Empowerment) (Verhältnis der Parlamentssitze Frauen/Männer, Verhältnis Männer/Frauen in Ministerien, Verhältnis von weiblichen Staatsoberhäuptern zu männlichen in den vergangenen 50 Jahren) (vgl. World Economic Forum: 4).

Wie auch im Falle des GDI können die GGG-Werte zwischen 0, d. h. max. Ungleichheit und 1, d. h. max. Gleichheit streuen. In Ungarn $(\mathrm{GGG}=0,6759)$ ist gemäß dieser Messung die Genderungleichheit am stärksten ausgeprägt, während in Finnland (GGG $=0,8453$ ) der höchste GGG-Wert vorzufinden ist. Folglich sind auch hier nur geringe Unterschiede zwischen den Ländern 
festzustellen, insgesamt ist ein hohes Gleichheitsniveau zu verzeichnen, gleichwohl summa summarum geringere Werte als diejenigen des GDI erreicht werden. Auffallend ist jedoch, dass süd- und osteuropäische Länder (mit wenigen Ausnahmen, beispielsweise Bulgarien) geringere Werte aufweisen als west- und nordeuropäische Länder.

GEI: In Anbetracht der Diskussion über die oben aufgeführten Indizes wird vom ,European Institute for Gender Equality ' der Gender Equality Index (GEI) erhoben.

Kritik üben Plantenga et al. (2009) an den zu groben Abstufungen bisheriger Gender-Indizes, da wesentliche Gender-Aspekte weltweit erhoben werden, folglich Unterschiede europäischer Staaten unberücksichtigt bleiben würden.

„Existing indices are [...] not appropriate because they do not focus exclusively on gender (in)equality and have not been created to be used at the European level" (Plantenga et al. 2000: 19).

Während sich beispielsweise die Messung der Genderungleichheit des GDI eher auf die menschliche Entwicklung konzentrieren würde, sei die Messung des GEM auf die Möglichkeiten von Frauen beschränkt (politische Teilhabe, ökonomische Teilhabe, Macht über ökonomische Ressourcen - Einkommen). Kurz: die Unterschiede zwischen europäischen Ländern werden aufgrund der zu groben Skalierungen missachtet. Demzufolge würde mittels dieses Indexes das Ziel verfolgt, einen ,EU Gender Equality Index ‘ zu entwickeln, mit dem relevante europäische Genderaspekte konstatiert werden können. Eine Analyse anhand von Wohlstand und allgemeiner Ungleichheit sei für den europäischen Raum nicht ausreichend, um Genderaspekte aufzuzeigen. Ein adäquater Index sollte nicht nur ein Ranking zwischen den Mitgliedsstaaten beinhalten, sondern zusätzlich die (Un-)Gleichheit zu einem Zeitpunkt analysieren, unter Berücksichtigung wie schnell/langsam ein Land eine ,gendergleiche' Gesellschaft verkörpert (Berücksichtigung von Zeitpunkt und Zeitspanne).

Mit diesem GEI-Index werden Benachteiligungen von Frauen anhand der folgenden sechs Bereiche ermittelt:

- Work (Erwerbsquote und Arbeitsbedingungen, differenziert nach Geschlecht),

- Money (finanzielle Ressourcen/ökonomische Situation differenziert nach Geschlecht),

- Knowledge (Bildungszugang, Segregation differenziert nach Geschlecht),

- Power (politische (Anzahl an Parlamentssitzen von Frauen) und ökonomische Macht (GesetzgeberInnen, Amtspersonen, ManagerInnen etc. operationalisiert über die internationale standardisierte Klassifizierung der Berufe (ISCO)), 
- Time (Verhältnis von Arbeitszeit, Erziehung/Betreuung und Freizeit),

- Health (Gesundheitszustand und Zugang zu Krankenversorgungseinrichtungen) (vgl. EIGE 2015: 12).

Der Wertebereich dieses Indexes liegt zwischen 1 und 100, wobei 1 als absolute Ungleichheit, 100 als eine absolute Gendergleichheit interpretiert werden kann.

Die Ergebnisse des GEI 2015 zeigen, dass im Vergleich zu den anderen Indizes die Gender-ungleichheit deutlich ausgeprägter zu sein scheint, als bisher angenommen - das Niveau insgesamt ist drastisch niedriger.

Süd-/ost-europäische Länder schneiden mit GEI-Werten kleiner als 40 am schlechtesten $\mathrm{ab}$, mittel- und zentraleuropäische Länder erreichen mittlere GEI-Werte zwischen 40 und 60 und nordeuropäische Länder weisen die größte Gleichheit $(\mathrm{GEI}=60+)$ auf. Insgesamt sind eindeutige Unterschiede zwischen den Ländern zu erkennen; in Rumänien ist aufgrund eines GEIWertes von 33,7 ein deutliches Defizit der Gendergleichheit festzustellen, während in Schweden $(74,2)$ das höchste Ausmaß der Gendergleichheit zu beobachten ist. Nachfolgend sind die einzelnen Werte klassifiziert dargestellt:

Niedrig:

$<40$ : $\quad$ Rumänien, Slowakei, Portugal, Griechenland, Bulgarien, Kroatien

\section{Mittel:}

$40-50$ : Litauen, Italien, Ungarn, Polen, Tschechien, Zypern, Malta, Lettland, Estland

50-60: Österreich, Spanien, Luxemburg, Deutschland, Frankreich, Irland, Slowenien, Vereinigtes Königreich, Belgien

Hoch:

60+: $\quad$ Niederlande, Dänemark, Finnland, Schweden

Zusammenfassend ist festzuhalten, dass gemäß dem GEI große Differenzen zwischen den Mitgliedstaaten der EU hinsichtlich der Gleichheit/Ungleichheit zwischen Männern und Frauen vorhanden sind sowie eine Spaltung zwischen Süd-/Osteuropa und Nord-/Westeuropa (mit wenigen Ausnahmen) zu bestehen scheint.

Anhand der Betrachtung der Indikatoren, die in den dargestellten Indizes enthalten sind, fällt eine überwiegend auf strukturellen Faktoren basierende Analyse auf. Weiterhin gilt es die europäische Ebene zu diskutieren, auf der mittels des GDI, des GII und des GGG nur geringfügige Unterschiede zwischen den Ländern zu konstatieren sind. Der GEI erweist sich als einziger Index, europäische Differenzen drastisch darzulegen, jedoch ist hier ein Mangel an kulturellen Aspekten, die eine Erklärungsleistung für Genderungleichheiten erbringen könnten, vorzufinden; auch eine Unterscheidung zwischen ,Fähigkeiten und Teilhabechancen' (deren Bedeutung wird im weiteren Verlauf durch Argumente von Sen hervorgehoben) ist nur unzureichend 
berücksichtigt, wäre jedoch in Anbetracht der theoretischen Argumentation als Mehrwert zu erachten.

Batalova/Cohen haben 2002 versucht, die innerhäusliche Arbeitsteilung (ein Index aus folgenden Tätigkeiten: Wäsche waschen, Lebensmittel einkaufen, Krankenpflege von Familienmitgliedern und Management bzw. Entscheidung, was es zu Essen gibt) von Paarbeziehungen u. a. mittels des GEM als Makrofaktor zu erklären (ihr primäres Ziel ist jedoch, die These eines egalisierenden Einflusses von vorehelichen Kohabitationserfahrungen auf die innerhäusliche Arbeitsteilung $\mathrm{zu}$ überprüfen). Batalovas/Cohens zentrale Ergebnisse sind, dass erstens in allen 22 untersuchten Ländern (weltweite Erhebung) Frauen mehr Hausarbeiten ausführen als Männer. Zweitens sei die Arbeitsteilung von Paaren, die bereits vor der Ehe zusammengewohnt haben, deutlich egalitärer organisiert und drittens sei die innerhäusliche Arbeitsteilung umso egalitärer, je höher die nationale Kohabitationsrate sei (dieser Effekt ist jedoch nur für Länder vorzufinden, die ohnehin hohe Gendergleichheitswerte aufweisen) (vgl. Batalova/Cohen 2002: 743). Interessant ist, dass in keinem der untersuchten Länder egalitäre innerhäusliche Arbeitsteilungsmuster dominant sind. Selbst in Ländern, in denen hohe GEM-Werte festgestellt werden können, werden die Hausarbeiten eher traditionell ausgeführt, gleichwohl egalitärere Werte als in Ländern mit ausgeprägter Genderungleichheit beobachtet werden können. ${ }^{24}$ Sind derartige Ergebnisse auf die heutige Zeit übertragbar?

24 Der Index der innerhäuslichen Arbeitsteilung umfasst in dieser Studie einen Wertebereich von min. 1 (Frau erledigt Hausarbeiten allein) bis max. 5 (Mann erledigt Hausarbeiten allein), d. h. ein Wert $=3$ würde eine egalitäre Arbeitsteilung bedeuten. Dies wird in keinem Land erzielt, der maximale erreichte Wert der innerhäuslichen Arbeitsteilung beträgt 2,3 (Norwegen und Schweden). 
Tabelle 1: Indizes zur Messung von Genderungleichheiten - ein Vergleich der EU 28

\begin{tabular}{|c|c|c|c|c|}
\hline EU 28 & GDI (2013) & GII (2013) & GGG (2014) & GEI (2015) \\
\hline Österreich & 0,935 & 0,056 & 0,7266 & 50,2 \\
\hline Zypern & 0,940 & 0,136 & 0,6741 & 44,9 \\
\hline Malta & 0,954 & 0,220 & 0,6707 & 46,8 \\
\hline Griechenland & 0,959 & 0,146 & 0,6784 & 38,3 \\
\hline Luxemburg & 0,961 & 0,154 & 0,7333 & 55,2 \\
\hline Italien & 0,962 & 0,067 & 0,6973 & 41,1 \\
\hline Deutschland & 0,962 & 0,046 & 0,7780 & 55,3 \\
\hline Irland & 0,965 & 0,115 & 0,7850 & 56,5 \\
\hline Niederlande & 0,968 & 0,057 & 0,7730 & 68,5 \\
\hline Tschechien & 0,969 & 0,087 & 0,6737 & 43,8 \\
\hline Portugal & 0,970 & 0,116 & 0,7243 & 37,9 \\
\hline Rumänien & 0,973 & 0,320 & 0,6936 & 33,7 \\
\hline Belgien & 0,977 & 0,068 & 0,7809 & 58,2 \\
\hline Spanien & 0,985 & 0,100 & 0,7325 & 53,6 \\
\hline Kroatien & 0,987 & 0,172 & 0,7075 & 39,8 \\
\hline Frankreich & 0,989 & 0,080 & 0,7588 & 55,7 \\
\hline Dänemark & 0,989 & 0,056 & 0,8025 & 70,9 \\
\hline Vereinigtes Königreich & 0,993 & 0,193 & 0,7383 & 58,0 \\
\hline Bulgarien & 0,994 & 0,207 & 0,7444 & 38,5 \\
\hline Ungarn & 0,998 & 0,247 & 0,6759 & 41,6 \\
\hline Slowakei & 1,000 & 0,164 & 0,6806 & 36,5 \\
\hline Schweden & 1,004 & 0,054 & 0,8165 & 74,2 \\
\hline Finnland & 1,006 & 0,075 & 0,8453 & 72,7 \\
\hline Slowenien & 1,006 & 0,021 & 0,7443 & 57,3 \\
\hline Polen & 1,010 & 0,139 & 0,7051 & 43,7 \\
\hline Lettland & 1,033 & 0,222 & 0,7691 & 46,9 \\
\hline Litauen & 1,036 & 0,116 & 0,7208 & 40,2 \\
\hline Estland & 1,042 & 0,154 & 0,7017 & 49,8 \\
\hline
\end{tabular}

Quellen: United Nations Development Programme 2015: o. S., World Economic Forum 2014: o. S., EIGE 2015: o. S. 


\subsection{Kritik am Forschungsstand: Ein Plädoyer für einen mehrdimensionalen Machtansatz}

Viele Studien analysieren Teilaspekte von Genderungleichheiten, Macht in Paarbeziehungen und traditionellen Rollenbildern, doch bleiben die Perspektiven meist auf die Mikro- oder Makroebene begrenzt, ohne ihre fundamentale Wechselwirkung zu berücksichtigen. Einige Ausnahmen, die sich einer mehrdimensionalen Perspektive widmen, sind:

- die theoretische Integration der Determinanten der innerhäuslichen Arbeitsteilung in einem Mehrebenenmodell von Röhler et al. (2000), deren Ansatz noch einer empirisch quantitativen Überprüfung bedarf.

- Lam et al. (2002) ${ }^{25}$, die die intraindividuelle Veränderung der innerhäuslichen Arbeitsteilung amerikanischer Ehepaare im Zeitverlauf betrachten, doch ebenfalls kulturelle Kontextindikatoren außer Acht lassen und Macht auf einen ökonomischen Teilaspekt begrenzen (Im Unterschied zu Schulz/Blossfeld (2006) und Grunow et al. (2007), die eine Verfestigung traditionaler Arbeitsteilungsarrangements im Eheverlauf westdeutscher Paare feststellen, weisen die Ergebnisse von Lam et al. darauf hin, dass der Anteil der Ehefrau an der Hausarbeit im Zeitverlauf sinkt. Es stellt sich insofern die Frage, wie diese Diskrepanz zwischen amerikanischen und deutschen Paaren zu Stande kommt, weshalb es von Bedeutung ist, kulturelle Kontextindikatoren in die Analysen einzubeziehen.).

- die Studien von Bühlmann et al. (2010) und Gupta et al. (2010), die zumindest die Kontextabhängigkeit von Gendertheorien und ökonomischen Ressourcenansätzen erkannt haben.

- Hook (2010), die Hausarbeitssegregation anhand von Mehrebenenmodellen analysiert und herausfindet, dass die landesspezifischen Kontexteffekte einer langen Arbeitszeit und einer langen Elternzeit die Beteiligung von Männern an der Hausarbeit verringern. In Ländern ${ }^{26}$, in denen öffentliche Kinderbetreuungseinrichtungen zur Verfügung stehen und auch Männern die Möglichkeit gegeben wird, die Elternzeit wahrzunehmen, übernehmen Frauen weniger Hausarbeiten. Hook betont daher die Relevanz von nationalen Kontexteffekten (vgl. Hook 2010: 1480).

25 „This study examined how the division of household labor changed as a function of marital duration and whether within-couple variation in spouses' relative power and availability were linked to within-couple variation in the division of labor" (Lam et al. 2002: 944).

26 Es wird ein Vergleich von 18 Ländern mittels der Daten der „Multinational Time Use Study" durchgeführt: Australien, Belgien, Bulgarien, Dänemark, Deutschland (differenziert nach Ost und West), England, Finnland, Frankreich, Italien, ehem. Jugoslawien, Kanada, Österreich, Polen, Schweden, Tschechien, Ungarn, USA 
- die Mehrebenenanalyse von van der Lippe et al. (2011), die die Persistenz der Genderungleichheiten in der Zeitverwendung von Männern und Frauen zwischen bezahlter und unbezahlter Arbeit nicht nur anhand von individuellen, sondern insbesondere anhand von ökonomischen, politischen und kulturellen Rahmenbedingungen als Makroindikatoren analysieren. Für den Ländervergleich von insgesamt 17 Ländern ${ }^{27}$ wird auf die Daten des „Multinational Time Use Archivs““ zurückgegriffen.

,[...] men and women in highly developed economies and in countries with high rates of child-care facilities do more paid work, although they spend less time on paid work after having children. With respect to the influence of culture, it appears that highly educated and married women in masculine cultures do less paid work, and that married women also do more housework, than their counterparts in more feminine cultures" (van der Lippe et al. 2011: 164).

- die Mehrebenenanalyse von Kersten (2016) für Schweizer Kantone, die neben individuellen auch politische, kulturelle und wirtschaftliche Bestimmungsgründe als kantonale Kontextfaktoren in die Erklärung der innerhäuslichen Arbeitsteilung integriert. Obwohl Hausarbeit und Erwerbstätigkeit als reziprok erachtet werden, lässt sich Kerstens Ergebnissen zufolge die zunehmende Erwerbstätigkeit von Frauen nicht per se auf die innerhäusliche Arbeitsteilung übertragen, weil die $\mathrm{Zu}$ sammenhänge je nach kantonalen Kontexten variieren (vgl. Kersten 2016: 84).

- die Mehrebenenanalyse von Nitsche/Grunow (2016), die für deutsche Paare auf der ersten Ebene die Zeitabhängigkeit (Paneldaten) und auf der zweiten Ebene die Paare analysieren, jedoch keine makrosoziologischen Kontextindikatoren berücksichtigen und

- die Strukturgleichungsmodelle von Carlson/Lynch (2017) ${ }^{28}$. Trotz der dargestellten Ausnahmen sind Studien, die Mehrebenenanalysen durchführen, in diesem Forschungsfeld eher selten. So kritisieren Carlson/Lynch (2017) zu Recht, dass

„All of these perspectives [relative resources, gender display, deviance neutralization hypotheses and autonomy theory] help explain variations in spouses' housework performance, but are limited by the use of cross-sectional data

27 Belgien, Dänemark, Deutschland (gesamt sowie differenziert nach Ost und West), England, Frankreich, Italien, ehem. Jugoslawien, Kanada, Niederlande, Norwegen, Österreich, Polen, Tschechien, Ungarn, USA

28 Carlson/Lynch (2017) überprüfen mittels Strukturgleichungsmodellen eine reziproke Kausalbeziehung zwischen Einkünften und Hausarbeiten - den Einfluss vom Einkommen auf die Hausarbeit und umgekehrt, den Einfluss von Hausarbeit auf das Einkommen. Da diese Analyse der Reziprozitätsannahme durchaus ein Forschungsdesiderat darstellt, ist dieser Ansatz als innovativ zu erachten. Sie finden heraus, , ,...] that wives' personal earnings and housework are reciprocally related; her earnings have a stronger effect on housework than vice versa. For husbands, time in routine housework affects earnings only" (Carlson/Lynch 2017: 199). 
and single-direction, single-equation models (e.g., OLS regression) to examine the earnings-housework relationship. This is problematic given that an entirely separate body of research suggests reverse causality — that time spent in housework has a negative effect on both men's and women's earnings" (Carlson/Lynch 2017: 201).

Darüber hinaus wird eine Benennung der Machtkategorien als zunehmend obsolet erachtet. Die systematische Ausklammerung von Machtkonzepten aus der Familiensoziologie scheint ein Forschungsdesiderat zu sein, dass konjunkturellen Schwankungen erlegen ist: Bereits in den 70er Jahren wurde von Held festgestellt, dass

„[...] in der neueren Literatur zur Problematik der ehelichen Macht immer wieder die gleiche Klage über das Ungenügen der Machtkonzepte auftaucht und immer wieder neue Maße, Konzepte und Typologien entwickelt, aber nur selten Versuche unternommen werden, die existierenden Daten über den Zusammenhang zwischen Außensystem und Familien- bzw. Ehestruktur theoretisch zu integrieren“ (Held 1978: 80, Hervorhebungen im Original; die Verf.).

Im Rahmen feministischer Bewegungen und spätestens nach Helds Herausgabe seiner Soziologie der ehelichen Machtverhältnisse (1978) konnten theoretische Machtkonzepte einen Aufschwung erfahren, doch ist aktuell erneut eine, vermutlich auf Modernisierungseffekte zurückführende, Erosion zu verzeichnen. Um diesem Trend entgegenzuwirken ist ,[...] an einem strukturellen, d. h. an Schicht- oder Statustheorien orientierten Machtbegriff auch gerade für diesen Bereich" festzuhalten (Held 1978: 80).

Ausgehend von dieser Kritik werden für diese Dissertation folgende Ziele festgelegt:

1. Die Sichtung des Forschungsstands relevanter Studien, die Ansatzpunkte aus der bisherigen Nutzung der Macht innerhalb der Soziologie bieten, um diese einzelnen Ansätze miteinander verknüpfen zu können, d. h. letztlich die theoretische Erklärungsleistung innerhäuslicher Arbeitsteilung zu erhöhen;

2. eine Typologie der Macht zu entwickeln;

3. Macht als Mehrebenenkonstrukt mit einer Benennung einander ergänzender Machtkategorien zu verstehen, d. h. eine Mehrebenenanalyse durchzuführen, die u. a. Dimensionen, sozietaler Empowerment ${ }^{*}$ (Makro-) und Machtkategorien in Paarbeziehungen (Mikro-) umfasst;

4. die innerhäusliche Arbeitsteilung in Paarbeziehungen im europäischen Kontext zu erklären

(bezüglich des ersten und zweiten Forschungsziels s. Tabelle 8: Theoretischer Bezugsrahmen zur Erklärung von innerhäuslichen Arbeitsteilungsarrangements). 



\section{Zur Herleitung einer Theorie der mehrdimensionalen Machtverhältnisse in Paarbeziehungen}

\section{Ein Spannungsverhältnis zwischen mikro- und makrosoziologischen Theorien zur Erklärung innerhäuslicher Arbeitsteilungsarrangements}

Erklärungsansätze der innerhäuslichen Arbeitsteilung in Paarbeziehungen können, wie bereits erläutert, nach mikrosoziologischer und makrosoziologischer Perspektive unterschieden werden (vgl. Wimbauer 2012: 88). Zunächst werden akteurzentrierte mikrosoziologische Ansätze, weiterführend strukturzentrierte makrosoziologische Ansätze dargestellt. Im Anschluss werden diese Ansätze diskutiert sowie fortführend weiterentwickelt, d. h. zu Machtkategorien typologisiert. Ziel ist, aufbauend auf dem Forschungsstand eine möglichst umfassende Erklärung für Arbeitsteilungsarrangements in Paarbeziehungen zu generieren. Bestehende theoretische Ansätze werden skizziert und in Form einer zusammenfassenden Analyse des Inhaltes zu Machtkategorien gebündelt, woraus die im Rahmen dieser Dissertation entwickelte Typologie der Macht resultiert.

\subsection{Mikrosoziologische Argumentation}

\subsubsection{Symmetrische (ökonomische) Theorien: Becker, Ott, Coverman, Blau}

Ökonomische Handlungstheorien gehen davon aus, dass eine Veränderung der Geschlechterrollen symmetrisch erfolgt, d. h. dass die zunehmende Berufstätigkeit der Frau eine zunehmende Beteiligung der Männer an der Hausarbeit bewirkt (vgl. Schulz/Blossfeld 2006: 29). Die Arbeitsteilung würde gemäß dieser Annahme im Falle einer Erwerbstätigkeit beider PartnerInnen „partnerschaftlicher“, d. h. egalitärer organisiert werden (s. Abbildung 1). Ist nur die Frau erwerbstätig, würde sogar ein Rollentausch stattfinden, d. h. der Mann wäre allein für die Hausarbeit zuständig (Enttraditionalisierung). Im Gegensatz dazu würde die Arbeitsteilung traditionell erfolgen, sofern die Frau keiner Erwerbstätigkeit nachgehen würde, d. h. dass alle Hausarbeiten 
von der Frau allein ausgeführt werden würden (vgl. Grunow et al. 2007: $163)$.

\subsubsection{Die ökonomische Theorie der Familie (New Home Economics, Gary Becker) - >Spezialisierung als Motto der innerhäuslichen Arbeitsteilung -}

Im Gegensatz zur klassischen Ressourcentheorie, die von einer individuellen Nutzenmaximierung der Individuen eines Haushaltes ausgeht, betrachten die New Home Economics den Haushalt als Einheit der Nutzenmaximierung, d. h. alle Mitglieder eines Haushaltes versuchen gemeinsam eine Nutzenmaximierung des Haushaltes zu erzielen. Die Funktion eines Haushaltes wird mit der Funktion eines Unternehmens verglichen, wobei eine Nutzenmaximierung durch eine Optimierung der Zeitallokation der Mitglieder erreicht wird. Die ,ökonomische Wohlfahrt [einer Person] hänge von ihrem über die Zeit verteilten Konsum von Objekten ihrer Wahl ab, die als Güter (commodities) bezeichnet werden“ (Becker 1982: 130). Commodities werden durch die Verarbeitung von Marktgütern (goods) im Haushalt produziert, wofür eine Investition von Zeitressourcen erforderlich ist, und dienen der Nutzenmaximierung des gesamten Haushaltes.

Grundlegende Idee ist, dass alle Familienmitglieder, die sich einen Wohnsitz teilen, durch die gemeinsame Nutzung haushaltsöffentlicher Güter Kosten einsparen und dadurch ihren individuellen Wohlstand erhöhen (vgl. Galler/Ott 1990: 112). Einzelne Mitglieder des Haushaltes haben komparative Vorteile, die durch die Spezialisierung des Humankapitals erworben werden. Die neue ökonomische Theorie versucht folglich, Interaktionsprozesse zwischen den Haushaltsmitgliedern, die sich auf Entscheidungen zwischen „Kindern, Heirat, Arbeitsteilung zwischen Hausarbeit und Investitionen in marktbezogene oder nicht-marktbezogene Fähigkeiten, Schutz von Mitgliedern vor Gefahren, Intergenerationstransfers zwischen den Mitgliedern und so weiter" beziehen, ursächlich zu erklären (Becker 1982: 187). Als Begründer der neuen Familien- und Haushaltsökonomik ist Gary S. Becker bekannt (vgl. Ott 1998: 63). Seine ökonomische Theorie der Familie besagt, dass bei einem gemeinschaftlichen Wohnsitz beide PartnerInnen den Nutzen ihres Haushalts gemeinsam maximieren, wobei die Aufgabenverteilung nach Fähigkeiten/Ressourcenkonstellationen, d. h. im Sinne einer Spezialisierung erfolgt.

„Everyone with a greater comparative advantage in the market than his member's would specialize completely in the market, and everyone with a greater comparative advantage in the household would specialize completely there" (Becker 1981: 33). 
Derjenige/diejenige mit höheren Humankapitalressourcen beruflicher Tätigkeiten würde sich auf die Erwerbsarbeit (Marktarbeit) spezialisieren und derjenige/diejenige mit ausgeprägteren komparativen Vorteilen der Kinderbetreuung/Hausarbeit auf den Haushalt, um den Haushaltsnutzen insgesamt zu maximieren (vgl. Becker 1981: 33). Ergo: Je höher das erwerbsspezifische Humankapital, desto höher die Opportunitätskosten der Hausarbeitszeit - es gilt die Opportunitätskosten möglichst gering zu halten. Folglich ist die Spezialisierung abhängig von der Ausprägung des Humankapitals respektive wer sich in welchem Aufgabenbereich spezialisiert, sei abhängig von den komparativen Vorteilen der PartnerInnen zu Ehebeginn. Im Zeitverlauf wird sich gemäß der Spezialisierungsargumentation die Arbeitsteilung zunehmend polarisieren. Die traditionelle Arbeitsteilung des Mannes als Familienernährer und der Frau als Hausfrau bzw. Mutter sei ein Resultat der unterschiedlichen Humankapitalinvestitionen. Während der Ausführung der Tätigkeiten steigt für die Frau das haushaltsspezifische Humankapital, für den Mann das erwerbsspezifische Humankapital, wodurch die traditionale Arbeitsteilung im Eheverlauf zunehmend verfestigt wird. Aufgrund von traditionellen Werten und Normen waren Frauen in der Vergangenheit eher im häuslichen Arbeitsfeld engagiert und Männer erwerbstätig (vgl. Schulz/Blossfeld 2006: 26). Dem Rational-Choice-Modell Beckers zufolge müsste sich jedoch die Rollenverteilung umkehren, wenn Frauen über höhere Bildungs-, Erwerbs-, und Einkommensvorteile verfügen. Doch belegen aktuelle Ergebnisse nicht, dass eine Zunahme des Modells des Rollentauschs aus der Folge der Bildungsexpansion resultiert. Hingegen zeigt sich, dass Frauen in der Regel Männer mit gleichem (Bildungshomogamie) oder höherem Bildungsniveau bevorzugen und qualifizierte Frauen häufig alleinstehend bleiben, weil sie aufgrund normativer Erwartungsstrukturen nicht „nach unten heiraten“ (Schulz/Blossfeld 2006: 26). Andererseits tendieren auch Männer dazu, Frauen mit ähnlichen Merkmalen, wie beispielsweise Intelligenz, Ausbildung und physischem Kapital, als Partnerin zu wählen (vgl. Becker 1982: 259).

Bezugnehmend auf die Kinderbetreuung erwähnt Becker, dass die Arbeitsteilung zwar überwiegend auf eine Nutzenmaximierung durch Spezialisierung der PartnerInnen zwischen Markt- und Haushaltssektor zurückzuführen ist, jedoch auch biologische Unterschiede zwischen den Geschlechtern zu berücksichtigen sind.

„Although the sharp sexual division of labor in all societies between the market and household sectors is partly due to the gains from specialized investments, it is also partly due to intrinsic differences between sexes. [...] Women not only have a heavy biological commitment to the production and feeling of children, but they also are biologically committed to the care of children in other, more subtile ways. Moreover, women have been willing to spend much time and energy caring for their children because they want their heavy biological investment in production to be worthwhile". In addition, a mother can more readily feed and 
watch her older children while she produces additional children than while she engages in most other activities“"(Becker 1981: 37f.).

Obwohl zunächst geschlechtsneutral argumentiert wird, dass eine Spezialisierung je nach Humankapital auf die Erwerbstätigkeit oder die unbezahlte Reproduktionsarbeit erfolgt, werden weiterführend auch biologische Voraussetzungen in Betracht gezogen, die zu einer größeren Beteiligung von Frauen an der Hausarbeit führen würden.

Notburga Ott spricht auch von einem „Teufelskreis ökonomischer Rationalität“, wobei sie zugleich kritisiert, dass die Zirkularität ${ }^{29}$ zwischen geschlechtsspezifischer innerhäuslicher Arbeitsteilung, gefolgt von einem Einkommensnachteil für Frauen und dadurch bedingter erneuter Reproduktion geschlechtsspezifischer innerhäuslicher Arbeitsteilung von Becker nicht explizit dargestellt wird (Ott 1999: 173; siehe auch Ott 2001: 131).

Nicht nur die biologische Argumentation, sondern auch die mangelnde Berücksichtigung kultureller Aspekte, Werte und Normen, wird häufig zum Anlass genommen, Beckers ökonomische Theorie der Familie zu kritisieren. Ott bemängelt folglich die unterschiedliche Entwicklung in verschiedenen Ländern als einen blinden Fleck in Beckers Theorie (vgl. Ott 2001: 133). Auch Bühlmann et al. (2010) kritisieren insofern zu Recht, dass

„The micro-economic theory of family considers the division of labour within the couple as the result of a relatively mechanic calculation of optimal family utility, independent of values and meanings given subjectively to activity" (Bühlmann et al. 2010: 51).

\subsubsection{Die ökonomische Verhandlungstheorie (Notburga Ott)}

Ökonomische Verhandlungstheorien betrachten familiäre Entscheidungen als permanenten Verhandlungsprozess, in dem jede/r TeilnehmerIn seine individuellen Interessen durchzusetzen versucht (vgl. Galler/Ott 1990: 112). Grundprinzip ist das Prinzip der Rationalität: ,[...] the allocation of scarce resources based on the principle of utility maximization" (Ott 1992: 196). Die ökonomische Verhandlungstheorie nach Notburga Ott (1992) basiert auf den Grundannahmen der New Home Economics, jedoch wird eine spieltheoretische Erweiterung vorgenommen. Kritisiert werden die New Home Economics insofern, als jede Entscheidungsfindung nicht gesamtfamiliär, hingegen als individueller Aushandlungsprozess betrachtet werden solle, der nicht ausschließlich durch rationales Verhalten erklärt werden könne, vielmehr im Kontext affektiver Beziehungen zu betrachten sei; nicht nur materielle, son-

29 „Die geschlechtsspezifische innerfamiliäre Arbeitsteilung führt aufgrund unternehmerischen Rentabilitätskalküls zu Benachteiligung von Frauen am Arbeitsmarkt - zu geringeren Löhnen und schlechteren Arbeitsbedingungen. Diese wiederum schreiben aufgrund familialer Nutzenüberlegungen die geschlechtsspezifische innerfamiliale Arbeitsteilung fest" (Ott 1999: 172). 
dern auch immaterielle Güter würden zur Nutzenmaximierung beitragen. ${ }^{30}$ Die Familie könne nicht als fixe Einheit betrachtet werden, da sich die $\mathrm{Zu}-$ sammensetzung der Mitglieder und ihre Beziehungen untereinander wandeln können. Folglich sei die Erwartung, dass über alle familiären Entscheidungen ein innerfamiliärer Konsens bestehe, nicht realitätsgetreu, Aushandlungsprozesse müssten viel eher individuell angesehen werden (vgl. Ott 1989: 97f.). Auch die dynamische Perspektive gelte es zu verfolgen, denn die neoklassische Theorie erfasse ein unzureichendes, statisches Bild:

„[...] sie zeigt einen Circulus vitiosus auf, der sich ständig reproduziert, und keine Änderung erwarten läßt [sic!]. Weder die Entstehung dieser Situation noch ihre Veränderung kann mit diesen Ansätzen erklärt werden" (Ott 1999: 174).

Der neoklassische Ansatz wird von Ott erweitert, indem 1. der Begriff der Arbeit nicht nur auf die Erwerbs-, sondern auch auf die Hausarbeit bezogen wird, 2. eine dynamische Perspektive eingenommen wird und 3. die Verbindlichkeit familialer Vereinbarungen berücksichtigt wird.

Ad 1. Arbeit wurde in der traditionellen Ökonomie auf Erwerbsarbeit bezogen, doch ist diese Perspektive unzulänglich, ,[...] da es doch auch Frauen, die nicht am Arbeitsmarkt auftreten, an Arbeit nicht fehlt" (Ott 1999: 169). Markt- und Hausarbeit unterscheiden sich in der Art der Transaktion. Die Austauschlogik der Marktarbeit ist ein ,[...] direkter, bilateraler Austausch von Leistung und [meist monetärer] Gegenleistung“", die der Hausarbeit eine ,[...] langfristige Austauschbeziehung, [ein] Austausch von vielen verschiedenen Gütern" (Ott 1999: 175). Wie bereits von Becker angenommen, wird der private Haushalt als eine ,ökonomische Institution“31 angesehen, die durch eine langfristige Kooperation eine höhere Wohlfahrtsproduktion für alle Mitglieder erzielen soll, als dies durch Marktbeziehungen der Fall wäre, da durch die familiale Gemeinschaft des Haushalts Transaktionskosten verringert werden (vgl. Ott 1999: 176). Durch die langfristige Kooperation in

30 „The model developed describes the allocation of scarce resources based on the principle of utility maximization. Certainly, there are factors in the family - in particular of an affective nature - which can hardly be explained by rationality, or only in less fruitful, tautological manner. However, this should be not lead us to deny rational behavior in the family as such. If affections do not crowd out all rational behavior, we should observe some systematical reactions which can be described by an economic model" (Ott 1992: 196).

31 Die Vorstellung von der Familie respektive dem privaten Haushalt als „ökonomische Institution" kann ausdifferenziert werden in Produktions-, Konsum- und Versicherungsgemeinschaft: „Indem Familienmitglieder eine Produktionsgemeinschaft bilden, können dann auch bei der Produktion von Gütern mit hohen Transaktionskosten durch Spezialisierung der Haushaltsmitglieder komparative Produktionsvorteile genutzt werden. Darüber hinaus können von der Familie als Konsumgemeinschaft durch den gemeinsamen Konsum oder Gebrauch haushaltsöffentlicher Güter [z. B. Auto, Wohnung, Waschmaschine etc.] Effizienzgewinne sowie einfache Größenvorteile (economies of scale) erzielt werden. Als Versicherungsgemeinschaft bietet schließlich die Familie materielle wie immaterielle Absicherung von Risikofällen wie Krankheit, Arbeitslosigkeit und im Alter“ (Ott 1999: 177, Hervorhebungen im Original; die Verf.). 
der Haushaltsgemeinschaft ergeben sich ferner Vorteile für die Mitglieder in Form eines höheren Wohlfahrtsniveaus, das sie allein nicht erreichen würden. Insofern betont Ott ein individuelles Interesse an der gemeinsamen Haushaltsführung (vgl. Ott 1999: 177). Die Verteilung des Kooperationsgewinns zwischen den Haushaltsmitgliedern wird durch spieltheoretische Verhandlungsmodelle beschrieben. Spieltheoretische Verhandlungsmodelle

,[...] gehen davon aus, daß [sic!] die Verteilung des Kooperationsgewinns das Ergebnis eines Verhandlungsprozesses ist, dessen Verlauf vom Interesse beider PartnerInnen an einer kooperativen Lösung abhängt. Da dieses Interesse je nach Attraktivität der Alternativen außerhalb der Familie unterschiedlich ist, bestimmen diese die Verhandlungsstärke der PartnerInnen. Änderungen in den externen Alternativen haben dann Änderungen der Verhandlungsmacht in der Familie zur Folge und führen so zu einer veränderten innerfamilialen Wohlfahrtsverteilung“ (Ott 1999: 177).

Dieses kooperative Spiel lässt sich graphisch darstellen (s. Abbildung 2):

Abbildung 2: Nash-Lösung eines kooperativen Verhandlungsproblems

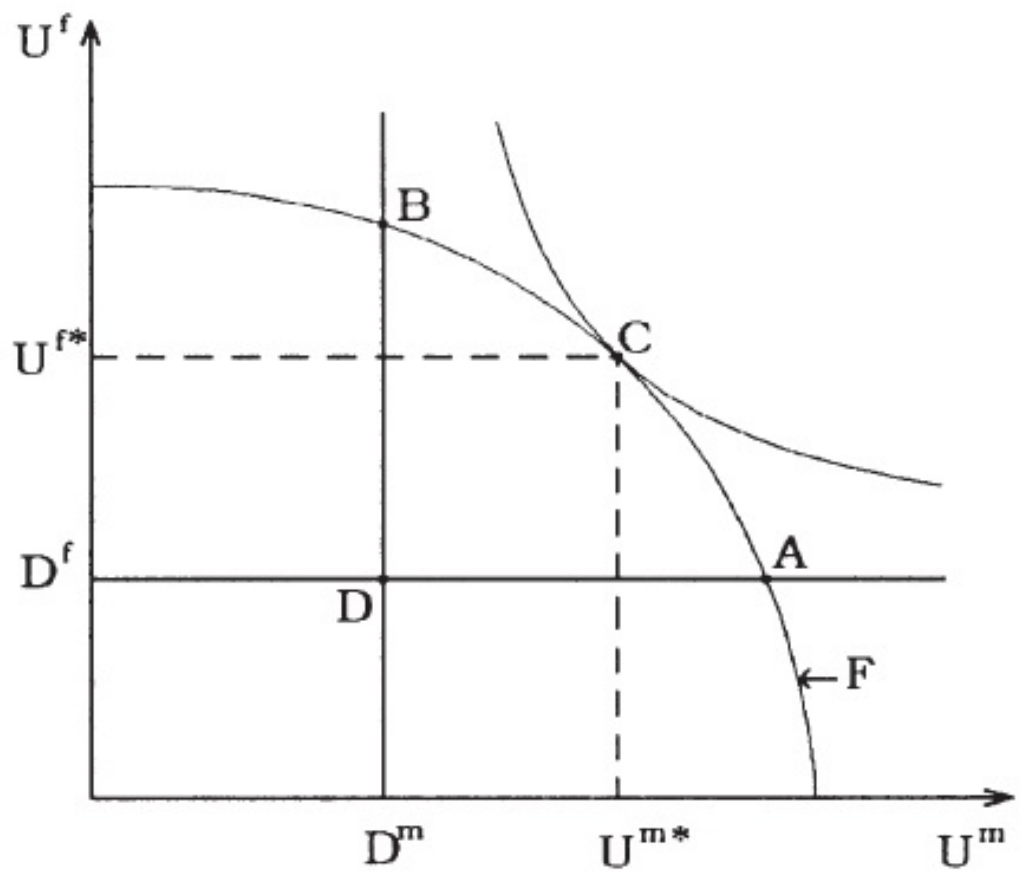

Quelle: Ott 1999: 177. 
$\mathrm{D}^{\mathrm{m}}$ und $\mathrm{D}^{\mathrm{f}}$ sind die jeweiligen Nutzenniveaus, die von beiden PartnerInnen außerhalb des gemeinsamen Haushalts erreicht werden könnten; $U^{f^{*}}$ und $U^{m^{*}}$ sind die Nutzenniveaus bei gemeinsamer Haushaltsführung. Die Nutzenkombination $\left(\mathrm{U}^{\mathrm{f}}, \mathrm{U}^{\mathrm{m}^{*}}\right)$ verdeutlicht, dass beide PartnerInnen ihr externes individuelles Nutzenniveau durch die kooperative gemeinsame Haushaltsführung verbessern können. Sofern sich die Situation ergibt, dass beide PartnerInnen über vergleichbar externe Alternativen verfügen, wird nach der kooperativen Spieltheorie eine egalitäre Aufteilung des Kooperationsgewinns, d. h. mittlere Lösung zwischen A und B verfolgt, die nicht an den Rändern liegt (nicht Punkt A oder B) (vgl. Ott 1999: 177f.).
„Dies folgt aus der Tatsache, daß [sic!] die Kooperation beider Partner zur zu- sätzlichen Wohlfahrtsproduktion notwendig ist und damit beide in gewissen Um- fang über das Drohpotential der Nichtkooperation verfügen. Welche Verhand- lungsmacht sich [aus dem Drohpotential der Nicht-Kooperation] ergibt, hängt je- doch von den externen Alternativen ab, da die Drohung mit Nichtkooperation umso glaubwürdiger ist, je höher das Nutzenniveau D' [das Nutzenniveau, das jeweils außerhalb der Familie erreicht werden kann] ist. Unter den Annahmen des Nash-Spiels ergibt sich eine innerfamiliale Verteilung, bei der das Produkt der individuellen Zugewinne maximiert wird. Das mit der besten externen Alter- native verbundene Nutzenniveau D' hängt dabei von den jeweils individuell zur Verfügung stehenden Ressourcen und insbesondere von der individuellen Ein- kommenserzielungskapazität ab“ (Ott 1999: 178).

Folglich entsteht eine zirkuläre Entwicklung: „Damit wirken sich Entscheidungen zur innerfamilialen Arbeitsteilung auch auf die individuelle Ressourcenausstattung aus, was wiederum

Rückwirkungen auf die Verteilungssituation in der Familie hat" (Ott 1999: 178).

Ad 2. Innerfamiliale Arbeitsteilung als dynamischer Prozess: Im Unterschied zu Becker, der die Spezialisierung je nach komparativen Vorteilen der PartnerInnen auf Markt- oder Hausarbeit als maximalen Haushaltsnutzen erachtet, kritisiert Ott, dass Spezialisierung auch immer den Verzicht auf eine andere, nicht gewählte Form von Humankapital bedeutet (vgl. Ott 1999: 178). Aus einer dynamischen Perspektive sei dies ineffizient, weil die Art des (haushalts- oder erwerbsspezifischen) Humankapitals eine unterschiedliche Bedeutung in unterschiedlichen Lebensphasen einnimmt. Beispielsweise resultiert im Falle einer Trennung eine unterschiedliche Bedeutung der Humankapitalarten. Während eine Spezialisierung auf die Marktarbeit das individuelle Einkommen steigert, das auch nach der Trennung erhalten bleibt, hat der Verzicht auf haushaltsspezifisches Humankapital nahezu keine Bedeutung (vgl. Ott 1999: 179). Hieraus ergeben sich wiederum Rückwirkungen auf die Verteilung des Kooperationsgewinns in der Familie. Es besteht ein unterschiedlicher Anreiz, die Beziehung aufrecht zu erhalten. 
„Für den auf Marktarbeit spezialisierten Partner verbessern sich im Laufe der Zeit die Alternativmöglichkeiten aufgrund der steigenden Einkommenskapazität, während sie sich für den auf Hausarbeit spezialisierten Partner verschlechtern. Entsprechend dem Gedanken kooperativer Verhandlungen, wonach der Zugewinn gegenüber den jeweiligen Alternativen "fair" geteilt wird, bedeutet dies bei erneuten Verhandlungen eine Umverteilung der internen Wohlfahrtsverteilung zugunsten des Partners mit den besseren Alternativen, da sich dessen Verhandlungsposition verbessert“" (Ott 1999: 180).

Wenn nach dieser Umverteilung trotzdem noch eine individuelle Wohlfahrtssteigerung für beide PartnerInnen aus dem gemeinsamen Haushalt möglich ist, ergeben sich keine Auswirkungen. Doch fällt der Wohlfahrtsgewinn geringer aus, ergibt sich die Situation des Gefangenendilemmas (vgl. Ott 1999: 180). Es könnte sein, dass sich für den/die PartnerIn mit nunmehr schlechteren externen Alternativen und folglich einer schlechteren Verhandlungsposition ein Wohlfahrtsniveau ergibt, das unterhalb der Ausgangssituation des Status Quo liegt (vgl. Ott 1999: 180). Rational handelnde Akteure würden sich auf diese Situation nicht einlassen, weshalb es ad 3. eines Vertrages bedarf, der ein aus dem Haushalt resultierendes, für beide PartnerInnen maximierendes Wohlstandsniveau regelt, auch wenn sich die Verhandlungsposition eines Partners/einer Partnerin verändert. Vornehmlich die Entscheidung für ein Kind ist ein derartiges Szenario (zur graphischen Visualisierung der Fertilitätsentscheidung siehe Ott 2001: 134): Eine Realisierung des Kinderwunsches würde theoretisch angenommen, wenn daraus ein positiver Nettonutzen trotz der anfallenden Kosten resultieren würde. Meist ist für einen der beiden PartnerInnen diese Entscheidung mit einer Verringerung der Erwerbsarbeit verbunden, wodurch seine/ihre Verhandlungsposition geschwächt wird. Im Falle von späteren Verhandlungen läuft diese Person Gefahr, unter das Anfangsniveau abzusinken, weshalb aus dieser Perspektive die Realisierung des Kinderwunsches irrational ist. Folglich ist eine Lösung, die für beide eine Verbesserung darstellt, erforderlich, weshalb eine implizite vertragliche Vereinbarung abgeschlossen wird, die derartige Verteilungsverhandlungen ausschließt. Insofern kann die traditionelle innerhäusliche Arbeitsteilung als ein impliziter Vertrag interpretiert werden, gemäß dem die Frau zwar eine Verschlechterung ihrer externen alternativen Möglichkeiten akzeptiert, ihr jedoch im Gegenzug ein konstanter Anteil der Wohlfahrtsproduktion lebenslänglich versprochen wird (vgl. Ott 1999: 181; Ott 2001: 134f.). Problematisch ist, dass aufgrund der langfristigen Austauschsituation ein Anreiz zum Vertragsbruch des Partners/der Partnerin mit den höheren externen Alternativen gesetzt wird. Eine auf der affektuellen Beziehung beruhende Verbindlichkeit des Vertrages wird von Ott angezweifelt, weil die emotionale Beziehung nicht im Sinne einer ökonomischen Tauschbeziehung, sondern als eigene Austauschbeziehung erachtet werden muss. Die Tauschlogik emotionaler Beziehungen lässt in der Regel nur einen gegenseitigen Aus- 
tausch emotionaler Zuwendung zu, der nicht durch andere Leistungen kompensiert werden kann. Ökonomische Abhängigkeiten innerhalb der Paarbeziehung verletzen dieses Prinzip, wodurch die Paarbeziehung belastet werden kann (vgl. Ott 1999: 183). Um der Vertragsverletzung zu entgehen, wird ein strategisches Verhalten der Person, die zu überwiegenden Teilen die Hausarbeiten übernimmt, erwartet, indem sie ihre Erwerbsarbeit nicht gänzlich aufgibt. Zugleich wird resultierend aus dem Verhandlungsprozess erwartet, dass sich auch der/die andere an der Hausarbeit beteiligt, um eine zu asymmetrische Situation zu vermeiden. Insofern sei eine maximale Spezialisierung unter diesen Bedingungen nicht als Idealfall zu betrachten (vgl. Ott 1999: 181). Ferner ergibt sich im Gegensatz zu Becker eine innerhäusliche Arbeitsteilung, die aus rationalen Kalkülen beider PartnerInnen bestenfalls egalitär organisiert wird. Dass die Vorteile der Spezialisierung abnehmen, ist Ott zufolge auf die wirtschaftliche Entwicklung zurückzuführen:

„Economic development has reduced the family gains in an essential way. First, market substitutes have been provided for many traditional household goods. Due to this trend and the increase in female wages, household production has become more and more inefficient and, in turn, gains from intrafamily specialization have decreased“"(Ott 1992: 197).

So werden beispielsweise die Opportunitätskosten einer Spezialisierung auf haushaltsspezifisches Humankapital durch verbesserte Einkommenschancen erhöht, in ihrer Zubereitung aufwendige Mahlzeiten werden durch Fertiggerichte ersetzt, die Eigenproduktion von Bekleidung ist aufgrund günstiger Preise von Bekleidung nicht mehr rentabel und durch technische Haushaltsgeräte wird die Verrichtung von Hausarbeiten zunehmend einfacher, weshalb keine spezifischen Kenntnisse mehr erforderlich sind (vgl. Ott 1999: 184). Folglich kann Hausarbeit in überwiegenden Teilen durch Marktarbeit substituiert werden. Im Falle der Spezialisierung resultiert hieraus die schlechtere Verhandlungsposition des/der auf Hausarbeit spezialisierten Partners/Partnerin, weil durch eine Auflösung des Haushalts der/die auf Marktarbeit spezialisierte PartnerIn die ehemals innerhäuslich verrichteten Tätigkeiten des anderen durch Marktsubstitute ersetzen kann (vgl. Ott 1999: 187). Der traditionelle intrafamiliale Vertrag kann heutzutage als asymmetrischer als in der Vergangenheit und damit als unrentabel angesehen werden (vgl. Ott 1992: 198).

„Generell läßt [sic!] sich also feststellen, daß [sic!] die wirtschaftliche Entwicklung zu einer Reduzierung der Gewinne aus gemeinsamer Haushaltsführung geführt hat. Die formale Ehe bietet daher heutzutage nur geringe materielle Vorteile, woraus eine geringere Heiratsneigung resultiert, insbesondere da die nichtmateriellen, affektiven Aspekte des Zusammenlebens mittlerweile auch ohne formale Eheschließung realisiert werden können. Die hohen Opportunitätskosten der Kindererziehung übersteigen deren Nutzen, was den Geburtenrückgang zumindest zum Teil erklären kann“"(Ott 1999: 186f.). 
Durch den wirtschaftlichen Wandel entsteht vermehrt die Situation des Gefangenen-Dilemmas, weil beide PartnerInnen auch einzeln ein hohes Wohlfahrtsniveau erreichen, weshalb der Kooperationsgewinn gering ausfällt (vgl. Ott 1999: 188).

„Bei fehlender Verbindlichkeit familialer Verträge resultiert dann unter solchen Bedingungen ein einseitig hohes Risiko für den [/die] auf Hausarbeit spezialisierte[n] Partner[In]. Sinkende Geburtenziffern und eine steigende Erwerbsbeteiligung von Frauen müssen dann als rationale Reaktion auf eben diese individuellen Risiken gesehen werden. Sofern aber asymmetrische Vereinbarungen in der Familie getroffen werden, führen tatsächliche und vermeintliche Vertragsbrüche zu vermehrt nichtkooperativem Verhalten, was sich letztendlich auch in steigenden Scheidungsziffern niederschlägt" (Ott 1999: 188).

Schlussfolgerung Otts ist der Bedarf einer Familienpolitik, die die Vereinbarung von Beruf und Familie für beide Geschlechter ermöglicht, um die individuelle Entscheidungsfreiheit zu erhöhen.

Im Hinblick auf die Arbeitsteilung ist wesentlich, dass Hausarbeiten von Frauen und Männern als unangenehm/lästig empfunden werden, weshalb jede/r PartnerIn versucht, diese Tätigkeiten zu meiden. Die Arbeitsaufteilung erfolgt über machtbezogene Verhandlungen. Derjenige/diejenige, der/die über bessere Einkommenschancen verfügt, genießt einen Machtvorteil gegenüber ihrem Partner/seiner Partnerin und kann seine/ihre Interessen im Verhandlungsprozess durchsetzen (vgl. Schulz/Blossfeld 2006: 27).

„Spezialisierung auf Markt- und Hausarbeit führt via Training-on-the-Job zur Akkumulation von unterschiedlichem Humankapital, das sowohl die späteren Produktionsmöglichkeiten des gemeinsamen Haushalts als auch die individuellen Einkommenserzielungskapazitäten bestimmt, die wiederum die Exit-Optionen und damit die interne Verhandlungsstärke beeinflussen. Dabei erhöht nur das marktförmige Humankapital die gemeinsamen, wie die individuellen Produktionsmöglichkeiten gleichermaßen, während haushaltsspezifisches Humankapital überwiegend nur im ganz speziellen Haushaltskontext verwertet werden kann. Die Exit-Option des auf Hausarbeit spezialisierten Partners verschlechtert sich damit und damit die interne Verhandlungsstärke" (Ott 2001: 133).

Beispielsweise wird im Falle einer Asymmetrie der Einkommen aufgrund von Opportunitätskosten entschieden, dass der/die PartnerIn mit dem niedrigeren Einkommen die Hausarbeiten übernimmt, während der/die andere die Erwerbstätigkeit reduziert oder unterbricht (vgl. Ott 1992: 185). Außerdem verfolgen alle Familienmitglieder das Interesse der Fortführung des Haushaltes, um ihr Wohlstandsniveau zu erhalten, sofern außerhalb der Familie keine größeren Anreize der Wohlstandsmaximierung bestehen, wodurch Machtunterschiede hingenommen werden, aber auch kooperatives Verhalten möglich wird (vgl. Galler/Ott 1990: 113). Im Falle einer Chancengleichheit des Einkommens (Einkommenshomogamie) und der Erwerbstätigkeit würden die Hausarbeiten kooperativ von Frau und Mann zu gleichen Teilen ausgeführt 
(s. u. Nash-Gleichgewicht). Zentral ist hierfür die spieltheoretische Annahme des Gefangenen-Dilemmas ${ }^{32}$, das auf familiäre Entscheidungen (im unten aufgeführten Beispiel auf die Fertilitätsentscheidung: die Wahrscheinlichkeit einer Realisierung des Kinderwunsches ist umso geringer, je höher die Opportunitätskosten) übertragen wird. Zur Erläuterung wird nachfolgend das Gefangenen-Dilemma anhand eines vereinfachten, idealtypischen Beispiels (ohne Berücksichtigung von staatlicher finanzieller Förderung, Betreuungsmaßnahmen etc.) illustriert: Ein Paar möchte dem dringlichen Wunsch, ein erstes Kind zu bekommen, nachgehen. Fraglich ist jedoch, ob diese Entscheidung finanziell tragbar ist und wer die Kinderbetreuung übernehmen würde. Derzeit sind beide PartnerInnen Vollzeit berufstätig und erzielen jeweils ein Einkommen von 2000 Euro (Einkommenshomogamie). Wenn der Mann erwerbstätig bleibt, beträgt sein Einkommen weiterhin 2000 Euro und die Frau verdient nichts; wenn beide Teilzeit erwerbstätig sind verdient jede/r PartnerIn 1000 Euro; ist die Frau weiterhin vollzeiterwerbstätig und der Mann für die Kindererziehung zuständig, beträgt ihr Einkommen 2000 Euro, der Mann verdient nichts.

Tabelle 2: Beispiel zum Kinderbetreuungs- und Einkommensdilemma

\begin{tabular}{lll}
\hline & Betreuung & Keine Betreuung \\
\hline Betreuung & 1000,1000 & 0,2000 \\
Keine Betreuung & 2000,0 & 2000,2000 \\
\hline
\end{tabular}

Quelle: Eigene Darstellung.

Rational wäre die Option, dass sich beide PartnerInnen gegen ein Kind entscheiden, folglich keiner die Betreuung übernimmt, jedoch ist diese nicht stabil, da sich ein/e einzelne/r PartnerIn einen affektuellen Vorteil verschaffen könnte, in dem er/sie die Beziehung beendet („Drohpotential der NichtKooperation") und den Kinderwunsch mit einem anderen Partner/einer anderen Partnerin erfüllt (Ott 1999: 178), um den mit der Fertilität verbundenen „Nutzen aus emotionaler Verbundenheit" zu erreichen (Ott 2001: 38).

Im Sinne des Nash-Gleichgewichtes ist die Handlungsalternative, dass beide PartnerInnen die Betreuung übernehmen und Teilzeit erwerbstätig werden, optimal, da kein/e PartnerIn einen finanziellen Machtvorteil genießen würde. Hieraus ergibt sich ein weiterer Unterschied zur ökonomischen Theorie der Familie nach Becker, weil im Sinne der Verhandlungstheorie durch die ressourcenbasierte Homogamie kooperatives, egalitäres Verhalten

32 Die Wahrscheinlichkeit des Gefangenendilemmas steigt mit der Einkommenshomogamie und sinkt aufgrund von Opportunitätskosten mit zunehmender Asymmetrie der Einkommen (vgl. Ott 1992: 186). 
ermöglicht wird (und durch die Bildungsexpansion sowie eine Zunahme der Erwerbsbeteiligung von Frauen verstärkt werden müsste). Als Verfechter der Spezialisierung argumentiert Becker hingegen mit einer Polarisierung der Arbeitsteilung, die von der Ressourcenkonstellation beider PartnerInnen zum Paarbeziehungsbeginn abhängig ist („Frau > Mann“ würde gemäß Becker zu einem Rollentausch der innerhäuslichen Arbeitsteilung führen und „Mann > Frau“ zu einer traditionellen Hausarbeitsteilung).

Würde im Gegensatz zum aufgeführten Beispiel der Mann einen Einkommensvorteil gegenüber der Frau genießen und erfolgt die Geburt des ersten Kindes, wird ein impliziter Vertrag zwischen den PartnerInnen geschlossen, der der Frau einen Wiedereinstieg in das Berufsleben ermöglichen soll, sobald eine Betreuung des Kindes (mit zunehmendem Alter) nicht mehr notwendig ist. Außerdem würde der Mann versprechen, ihre geschwächte Verhandlungssituation nicht auszunutzen und ihr ,eine lebenslang fixierte Verteilung der Kooperationsgewinne“ garantieren (Ott 2001: 135). Problematisch ist, dass sich der Wert des erwerbsspezifischen Humankapitals mit Dauer der Erwerbsunterbrechung verringert, wodurch eine Abnahme der Verhandlungsmacht der Partnerin resultiert. Die Partnerin unterbricht die Erwerbstätigkeit im Vertrauen auf den impliziten Vertrag, doch erfährt der Partner einen Anreiz durch die Vertragsbrechung, da Leistung und Gegenleistung zeitlich verzögert sind und er hieraus einen individuellen Machtvorteil suggeriert. Folglich kann sie aufgrund ihrer geschwächten Verhandlungsmacht die Durchsetzung des Vertrages nicht mehr erzwingen - die traditionelle Arbeitsteilung verstärkt sich selbst (vgl. Ott 1989: 104f.; Ott 2001: 135; Lois 2008: 57).

Wäre das Einkommen der Frau höher als das des Mannes, wäre auch eine Erwerbsunterbrechung des Mannes denkbar, doch aufgrund der geschlechtsspezifischen Lohnsätze sei diese Form der Arbeitsteilung in den meisten Paarbeziehungen ineffizient (vgl. Ott 1989: 105).

Zusammenfassend erhöhen das Einkommen und die Erwerbstätigkeit die Verhandlungsmacht und führen dazu, dass der/die PartnerIn mit den niedrigeren Ressourcen die Hausarbeit übernimmt. Für die Erklärung innerfamiliärer Entscheidungen gilt es Ott (1992) zufolge vornehmlich die Opportunitätskosten und das Gefangenendilemma zu berücksichtigen.

\subsubsection{Der Time-Availability-Ansatz (Shelley Coverman)}

Gemäß dem Time-Availability-Ansatz von Stafford et al. (1977) sowie Perrucci et al. (1978) wird die Aufteilung der Hausarbeit durch das Zeitkontingent erklärt. Je nach Ausmaß und Zeitaufwand der Erwerbstätigkeit ist die verfügbare Zeit, die für Hausarbeiten investiert werden könnte, begrenzt. Der/die PartnerIn mit dem geringeren Zeitaufwand der Erwerbstätigkeit würde überwiegend die Hausarbeiten übernehmen. Auffallend ist, dass an dieser 
Stelle nur das Angebot an Hausarbeitszeit, jedoch nicht die Nachfrage an Hausarbeit Bestandteil der theoretischen Erklärung ist. Eine Erweiterung dieses Ansatzes, der Demand/Response-Capacity-Ansatz, erfolgte durch Coverman (1985), indem einerseits Faktoren, die das Ausmaß der Hausarbeitszeit erhöhen (z. B. Anzahl der Kinder) und andererseits Faktoren, die Haushaltsmitglieder hindern, bestimmte Tätigkeiten zu übernehmen (z. B. Erwerbstätigkeit) berücksichtigt werden (vgl. Coverman 1985: 84; Künzler 1999: 237). „Generally, wives' employment status, number or presence of children and, less frequently, number of hours spent in market work are treated as indicators of time availability“" (Coverman 1985: 84).

\subsubsection{Die Theorie des sozialen Tauschs ( Peter M. Blau)}

„The theory conceptualizes social relations in terms of exchange processes. Mutual bonds emerge in social interaction as persons who incur obligations reciprocate, but the imbalance resulting from unilateral benefits engenders superior status" (Blau 1989: 16).

Der Theorie des sozialen Tauschs nach Peter M. Blau zufolge sollten ökonomische Theorien durch die Annahme ergänzt werden, dass soziale Austauschbeziehungen von einzelnen Personen oder Gruppen durch Macht, Autoritätskonstellationen, normative Kontrolle und kulturelle Legitimation beeinflusst, vielmehr in einen Kontext von Makrostrukturen eingebettet sind (vgl. Münch 2002: 63). Im Unterschied zur rein ökonomischen Tauschtheorie wird vornehmlich auch sozialer Austausch thematisiert, wobei der soziale Austausch den Fokus von Blaus Austauschtheorie bildet. „Austauschprozesse beschränken sich nicht nur auf ökonomische Märkte - soziale Austauschprozesse sind allgegenwärtig“ (Blau 2005: 127). Dem wirtschaftlichen und sozialen Austausch gemein sind die Reziprozitätsannahme der Erwartung, für eine Leistung eine Gegenleistung zu erhalten und das Prinzip des abnehmenden Grenznutzens: beispielsweise würden zwei FreundInnen mit zunehmender Dauer der Freundschaft immer weniger auf ein Fortbestehen ihrer Beziehung drängen oder der Ratschlag eines/einer KollegIn sei solange von Nutzen, bis das Problem gelöst ist. Alle nach der Problemlösung folgenden Ratschläge sind nicht mehr von vergleichbar hohem Wert wie der erste Ratschlag (vgl. Blau 2005: 129). Interaktion wird als sozialer Austausch konzeptualisiert, dessen Grundlage ein Streben nach Belohnungen ist, die nur im Rahmen von Interaktionen erzielt werden können (vgl. Blau 2005: 125).

Doch geht Blau keineswegs davon aus, dass jede soziale Handlung als sozialer Austausch zu verstehen ist, was er an einem Beispiel, dass ein Mann anderen Menschen Geld gibt, verdeutlicht (vgl. Blau 2005: 128f.): Die erste Möglichkeit ist, dass der Mann Geld gibt, weil er bedroht wird, d. h. ein Austausch des Geldes für sein Leben stattfindet. Zweitens könnte er das Geld aus altruistischen, wohltätigen Gründen spenden, um sein Gewissen zu beruhi- 
gen, das von ihm verlangt, anderen zu helfen ohne dafür Dankbarkeit zu erwarten, was als ein Austausch des Geldes gegen die interne Anerkennung seines Über-Ichs gedeutet werden kann. Drittens könnte das Geld aufgrund eines unkontrollierten Impulses sinnlos ausgegeben werden. Alle drei Varianten werden von Blau nicht als sozialer Austausch erachtet, weil im ersten Fall anstatt eines sozialen Austauschs physischer Zwang ausgeübt wird; im zweiten Fall würde Blau die Übereinstimmung mit verinnerlichten Normen vom sozialen Austausch ausschließen und im dritten Fall wird ein irrationaler Antrieb angenommen, der ebenfalls keinen sozialen Austausch darstellt. Eine vierte Möglichkeit ist, dass der Mann Obdachlosen Geld gibt, da er sich über den ihm hierfür entgegengebrachten Dank freut, wobei Blau hierin einen sozialen Austausch „Geld gegen Dankbarkeit“ sieht.

„Das Konzept des Austauschs zielt auf freiwillige soziale Handlungen ab, die von belohnenden Reaktionen anderer abhängig sind und die eingestellt werden, wenn die erwartenden Reaktionen nicht eintreffen“ (Blau 2005: 128f.).

Soziale Beziehungen werden als reziproke Austauschprozesse immaterieller und materieller Belohnungen analysiert, wobei statusbedingte Vorteile berücksichtigt werden. In Paarbeziehungen erwartet beispielsweise Ego, sofern er eine Leistung für Alter erbringt, dass er dafür irgendwann (auch über gröBeren Zeitraum hinweg) eine Gegenleistung erhält (vgl. Schulz/Blossfeld 2006: 28). Es kann sich hierbei sowohl um einen Austausch rein immaterieller als auch um einen Austausch materieller gegen immaterielle Güter handeln. Ferner wird dem sozialen Austausch eine Reziprozitätsannahme zugrunde gelegt. Dabei wird die Gegenleistung des sozialen Tausches nicht formal festgelegt, sondern basiert auf Vertrauen, weil der/die Begünstigte über den Gegenstand der Gegenleistung und den Zeitpunkt der Gegenleistung entscheidet (vgl. Blau 1964; 2008: 91ff.). ${ }^{33}$ Der zentrale Unterschied zum ökonomischen Austausch ist, dass die Verpflichtung nicht im Vorfeld von beiden TauschpartnerInnen festgelegt wird, sondern zwar eine grundsätzliche Erwartungshaltung über eine (vom Zeitpunkt des Tauschs entkoppelte) Gegenleistung besteht, die Ausgestaltung dieser Gegenleistung jedoch unspezifiziert bleibt. Dies muss so sein, weil andernfalls eine präzise Festlegung der Verpflichtung die soziale Bedeutung des Tauschs zerstören würde und in einen ökonomischen Tausch umwandeln würde (vgl. Blau 2005: 129f.). Die Ehe wird insofern als ein stabiles Partnerschaftsverhältnis betrachtet, das die Erwartungssicherheit in Form von Vertrauen auf die Erfüllung des Tausches auch in unbestimmter Zeit erhöht.

33 „Social exchange differs in important ways from strictly economic exchange. The basic and most crucial distinction is that social exchange entails unspecified obligations. [...] Social exchange [...] involves the principle that one person does another a favor, and while there is a general expectation of some future return, its exact nature is definitely not stipulated in advance“" (Blau 1964; 2008: 93, Hervorhebungen im Original; die Verf.). 
Im Sinne des Rational-Choice-Paradigmas wird soziales Handeln als ein Streben nach Nutzenmaximierung und Belohnungen betrachtet, Kosten werden so gering wie möglich gehalten. Individuen versprechen sich von sozialen Beziehungen Vorteile; die Beziehungen werden aufrechterhalten, sofern sie als lohnend empfunden werden (vgl. Blau 2005: 125). Innerhalb von Austauschprozessen entsteht nach Blau die „Differentation of power“ (Blau 1964; 2008: 115). Machtunterschiede resultieren aus Abhängigkeitsverhältnissen, sofern ein Akteur von den Belohnungen eines anderen abhängig ist, $d$. h. nicht eine entsprechende Gegenleistung erbringen kann, die Belohnung nicht über eine andere Quelle beziehen kann, gewaltsam erzwingen kann oder nicht in der Lage ist, seine Bedürfnisse zu ändern (vgl. Blau 1964; 2008: 119ff.). Die Machtausübung wird vom „Unterworfenen in Bezug auf soziale Normen der Fairness beurteilt" (Blau 2005: 135). Wird die Ausübung der Macht als fair empfunden, erhält der machtausübende Akteur Anerkennung, woraus ein sekundärer Austauschprozess resultiert: Fairness der Machtausübung gegen gesellschaftliche Anerkennung. Folglich kann sekundärer Austausch einerseits im Falle der Fairness zur Legitimation respektive weiterführend zur Organisation der Machtausübung führen, andererseits auch dem Widerstand einer Opposition im Falle unfairer Machtausübung ausgesetzt sein (vgl. Blau 2005: 135). Blau nimmt außerdem an, dass soziales Handeln durch „Mediating Values in Complex Structures“ begrenzt ist, jedoch innerhalb dieser Grenzen beliebig nach Belohnungen gestrebt wird, wofür Austauschprozesse erforderlich sind (Blau 1964; 2008: 253).

Im Rahmen der innerhäuslichen Arbeitsteilung erhöhen strategische Ressourcen wie Einkommen und durch Erwerbstätigkeit erworbenes Humankapital das Machtpotential, weil diese auch in andere Beziehungen transferiert werden können und folglich als Exitandrohung einer Beendigung der Beziehung dienen, wodurch der/die schwächere PartnerIn dazu bewegt werden kann, die Hausarbeiten zu übernehmen. Demzufolge werden die gleichen Vorhersagen wie im ökonomischen Verhandlungsmodell prognostiziert (vgl. Schulz/Blossfeld 2006: 29).

Obwohl Blau den Versuch unternimmt, die rein ökonomischen Annahmen der Austauschtheorie durch eine Kontextualisierung von Makrostruktu$\operatorname{ren}^{34} \mathrm{zu}$ erweitern, erfolgt eine Schwerpunktsetzung der Machtmittel auf ökonomische Ressourcen, weil diese auch in andere Beziehungen übertragen werden können. Die Abwesenheit der vier Alternativen („Gegenleistung“, „Belohnung über einen anderen Akteur beziehen“, „Zwang“ und ,auf Bedürfnis verzichten“) definiert die Bedingungen der Macht (vgl. Blau 1964;

34 ,Requirements of Power: Indifference to what others offer, monopoly over what others need, law and order, materialistic and other relevant values

Structural Implications: Exchange and distribution of resources, competition and exchange rates, organization and differentation, ideology formation“" (Blau 1964; 2008: 124, Hervorhebungen im Original; die Verf.). 
2008: 119). Kann ein/e AkteurIn im Sinne eines Austauschs auf die Belohnung durch eine Gegenleistung reagieren, die vom anderen Tauschpartner/von der anderen Tauschpartnerin benötigt wird, ist das Machtpotential beider TauschpartnerInnen auf einer Ebene anzusetzen. Beispielsweise könnte finanzielle Unterstützung gegen die Verrichtung der Hausarbeiten innerhalb einer Paarbeziehung getauscht werden. Jedoch ist der Austausch von materiellen (z. B. erwerbsspezifisches Einkommen) und immateriellen (z. B. Hausarbeit) Gütern mit unterschiedlichen Positionen verbunden. ,[...] häusliche-familiäre Macht [kann] nicht in öffentlich-gesellschaftliche Macht transformiert werden“ (Held 1978: 43). Während Frauen vornehmlich die Produktion von Gebrauchswerten (Hausarbeit) zugeschrieben wird, wird Männern die Produktion von Tauschwerten zugeschrieben. Doch existiert im Unterschied zu Tauschwerten kaum ein Markt für Gebrauchswerte (Ausnahme: außerhäusliche Hausarbeiten und Pflegesektor), sodass der Hausarbeit nahezu kein Tauschwert zukommt (vgl. Held 1978: 43). Die familiäre Aktivitätspalette ist , [...] objektivierenden Bewertungsmaßstäben entzogen [..] und außerdem privat und nicht öffentlich realisiert" (Krüger/Levy 2000: 391). Abhängigkeiten resultieren hieraus, wobei im Falle einer Trennung der Paarbeziehung eine Rückkehr in die Erwerbstätigkeit für den/die ehemals im Haushalt tätige/n PartnerIn nach langer Unterbrechung nur schwer möglich ist, während es für denjenigen/diejenige, der/die sich in der Erwerbsarbeit spezialisierte, einfacher ist, Hausarbeiten auszuführen.

„Ihre vornehmliche Fixierung auf den familialen Binnenraum bringt die Frau gegenüber dem Mann strukturell in eine schwächere Bewältigungsposition, welches auch immer die konkreten Positionen und Ressourcen sein mögen" (Krüger/Levy 2000: 391).

Folglich ist eine Fokussierung ,[...] auf die Partizipation an unterschiedlichen Institutionen und den dort eingenommenen Positionen“ zu bedenken, weshalb das Machtkonzept im Rahmen einer allgemeinen Statustheorie entwickelt werden muss (Krüger/Levy 2000: 382). Blaus grundlegende Annahme ist, dass soziale Beziehungen durch Tauschprozesse entstehen, in denen sich die TauschpartnerInnen Belohnungen aus der Beziehung erhoffen. Problematisch ist nach der Argumentation von Münch (auch Koppetsch sowie Simmel würden ähnlich argumentieren), dass die Institution des Schenkens nicht utilitaristischen Prinzipien unterworfen ist. Die Erwartung einer Gegenleistung beruht nicht auf dem Sinne eines Gabentauschs, sondern lediglich auf dem Sinne eines Partnerschaftstauschs (s. Argumentation von Koppetsch im Kapitel 6.1.). Blau bezeichnet hingegen beide Tauschformen als sozialen Tausch, dem die Rationalität zugrunde liegt. Auch die Voraussetzung der rationalen Wahl, die freie Wahl, trifft nur sehr begrenzt auf das Schenken zu (vgl. Münch 2002: 69). 
„Hier scheint Blau ein unabsichtliches Opfer seiner Entscheidung geworden zu sein, soziale Vereinigung in erster Linie als Austausch von Belohnungen zu analysieren. Er verfehlt hier seine Absicht, über die ökonomischen Schranken von Homans" Austauschtheorie hinauszugehen" (Münch 2002: 69).

Darüber hinaus müssen wir uns noch expliziter als Blau, ,[...] der Tatsache bewusst sein, dass jeder einzelne soziale Austausch, der in einer Gesellschaft stattfindet, mit [...] makro-strukturellen Kräften vermischt ist" (Münch 2002: 86). Diesen Aspekt befürwortet auch Blau durch seine Darlegung über den Schwerpunkt seiner Austauschtheorie auf face-to-face-Beziehungen, die durch eine Fokussierung auf komplexe Strukturen ergänzt werden sollte (vgl. Blau 2005: 136).

Die ökonomische Theorie der Familie, die ökonomische Verhandlungstheorie, der Time-Availability-Ansatz und die Theorie des sozialen Austauschs gehen davon aus, dass

„[...] eine Veränderung der Geschlechterrollen symmetrisch erfolgt, d. h. dass eine wachsende Beteiligung der Frauen an der Einkommenserzielung [...] nicht nur einen Rückzug der (Ehe-)Frauen aus der Hausarbeit, sondern auch eine wachsende Beteiligung der (Ehe-)Männer an der Hausarbeit herbeiführt" (Schulz/Blossfeld 2006: 29, Hervorhebungen im Original; die Verf.).

Das (biologische) Geschlecht leistet diesen Ansätzen zufolge nahezu keine Erklärung für häusliche Arbeitsteilungen. Doch konträr zu diesen Annahmen zeigen Ergebnisse ausgewählter Analysen (Erickson (2005); Schulz/Blossfeld (2006); Grunow et al. (2007); Lott (2009); Lott (2012)), dass kulturellbedingten Geschlechterideologien, Werten und Normen eine zentrale Bedeutung zur Erklärung von häuslicher Arbeitsteilung und Machtkonstellationen zukommt. Ferner ist anhand dieser Studien zu konstatieren, dass eine zunehmende Berufstätigkeit der Frau sowie eine Angleichung des bildungsspezifischen Qualifikationsniveaus der Geschlechter nicht zwangsläufig mit einer Enttraditionalisierung einhergehen, sondern die Frau trotz ihrer Erwerbstätigkeit den Haushalt und die Kinderbetreuung übernimmt.

Oppositär zu den ökonomischen Theorien erfassen soziologische asymmetrische Gender-Theorien das (erlernte) Geschlecht als zentrale Komponente. Im Sozialisationsprozess und in der Identitätsfindung werden Geschlechterrollen/-identitäten erworben, die maßgeblich für die innerfamiliäre Arbeitsteilung sind, wobei Frauen im Sinne des Interrollenkonflikts durch Beruf und Familie in doppelter Weise belastet sind (vgl. Schulz/Blossfeld 2006: 29). Diese Erklärungsansätze prognostizieren im Gegensatz zur ökonomischen Argumentation, dass traditionelle Rollenbilder auch aktuell von Bedeutung sind und unter bestimmten Voraussetzungen, wie beispielsweise mit zunehmender Ehedauer, sogar an Bedeutung gewinnen können. 


\subsubsection{Asymmetrische (Gender-)Theorien}

\subsubsection{Der Doing-Gender-Ansatz: Brines, Bielby/Bielby, Honeymoon-Effekt}

Ausgangspunkt der konstruktivistischen Gender-Ansätze ist, ,[...] dass sie die Unterscheidung von Natur und Kultur respektive von Sex und Gender nicht ihrerseits fortschreiben, sondern als Bestandteil einer reflexiven sozialen Praxis begreifen [wechselseitige Konstruktion], die beides zugleich hervorbringt" (Wetterer 2004: 122). Die Konstruktion des Geschlechts (Natur) selbst basiert nicht nur auf der biologischen Konstruktion, sondern ist auch eine Konsequenz der kulturell bestimmten, sozialisierten Übernahme des sozialen Geschlechts (Gender) im Sinne eines prozesshaften ,Werdens ${ }^{6}$ : als Rolle der Frau und Rolle des Mannes. ${ }^{35}$ Diese Diskussion der Geschlechtskonstruktion wurde insbesondere auch von Judith Butler (2009) angeführt und ist als Doing-Gender-Ansatz bekannt. ${ }^{36}$ Anthropologisch wird von Butler eine wechselseitige Konstruktion von biologischem und sozialem Geschlecht angenommen. „Weiblich“ ist kein feststehender Begriff, sondern sozial konstruiert oder um es in Butlers Worten auszudrücken „,verworren und unfixiert".

„Precisely because "female" no longer appears to be a stable notion, its meaning is as troubled and unfixed as "woman," and because both terms gain their troubled significations only as relational terms, this inquiry takes as its focus gender and the relational analysis it suggest. Further, it is no longer clear that feminist theory ought to try to settle the questions of primary identity in order to get on with the task of politics. Instead, we ought to ask, what political possibilities are the consequence of a radical critique of the categories of identity" (Butler 1999: 29).

Statt eines Versuches, die Identitätsfrage durch feministische Theorien zu beantworten, gilt es nach Butler vielmehr zu diskutieren, welche (politischen) Möglichkeiten aus einer radikalen Kritik der geschlechtsspezifischen Identitätskategorien resultieren.

„Attempting to explain why biological sex remains the primary predictor of household labor allocation, gender theorists have suggested that husbands and wives perform family work in ways that facilitate culturally appropriate construction of gender" (Erickson 2005: 337).

36 „Was ich als das »eigene« Gender bezeichne, erscheint manchmal als etwas, dessen Urheber ich bin oder das ich sogar besitze. Die Bedingungen, die das eigene Gender kreieren, liegen jedoch von Anfang an außerhalb meiner selbst, wurzeln außerhalb meiner selbst in einer Sozialität, die keinen einzelnen Urheber kennt (und die Idee der Urheberschaft selbst grundlegend in Frage stellt)“(Butler 2009: 9). 
Erstmals wurde die Begrifflichkeit „social construction of gender“ von Kessler/McKenna (1978) entwickelt, die neben der biologischen Perspektive des Geschlechts untersuchten, inwiefern Kinder die Regeln der Zweigeschlechtlichkeit erlernen. Im Laufe der Sozialisation respektive Identitätsbildung werden Geschlechterrollenbilder erworben und sind auf der Verhaltensebene in der innerfamiliären Arbeitsteilung ersichtlich (vgl. Schulz/Blossfeld 2006: 29). Es werden dauerhafte normative Erwartungsstrukturen gebildet (Normen-orientierter Ansatz), die dazu führen, dass Männer sich nicht an der Ausführung des Haushalts beteiligen und Frauen sich trotz ihrer Erwerbstätigkeit für die Hausarbeit verantwortlich fühlen. Geschlechtsbezogene Rollenbilder sind fest in den gesellschaftlichen Erwartungen verankert (vgl. Kelle 2011: 9). Geschlechtsidentitäten werden im Alltag ständig produziert und reproduziert, wobei die Ausführung der Hausarbeit oder ihre Verweigerung die Funktion der Produktion der Geschlechtsidentität erfüllt. Die Typisierung der Aufgabenbereiche ist abhängig vom kulturellen Hintergrund. Nach traditionellem Rollenbild produzieren Frauen ihre Weiblichkeit mit Aufgaben, wie beispielsweise Kochen, Bügeln, Waschen; Männer hingegen versuchen ihre Männlichkeit durch die Ablehnung „weiblicher" Tätigkeiten und die Ausführung „männlicher" Aufgaben, wie z. B. Handwerken, zu demonstrieren (vgl. Kelle 2011: 9). Die Ergebnisse von Erickson (2005) zeigen, dass die soziale, kulturell-bedingte GenderKonstruktion eher die häusliche Arbeitsteilung bestimmt als das biologische Geschlecht (vgl. Erickson 2005: 337).

Brines hat 1994 den Doing-Gender-Ansatz durch die Kompensationshypothese ergänzt: Die zunehmende Chancengleichheit von Frauen und Männern in der Erwerbsarbeit bestärkt traditionelle Rollenbilder der innerfamiliären Arbeitsteilung (vgl. Schulz/Blossfeld 2006: 30).

VertreterInnen der ökonomischen Austauschtheorie argumentieren, dass die ökonomische Abhängigkeit durch unbezahlte Hausarbeit kompensiert wird; VertreterInnen des Doing-Gender-Ansatzes behaupten, dass die Ausführung/nicht-Ausführung von Hausarbeiten ein Ausdruck von männlicher/weiblicher Identität ist (vgl. Brines 1994: 652). Brines verknüpft beide Perspektiven miteinander und kann belegen, dass je mehr der Mann ökonomisch von seiner Frau abhängig ist, desto weniger Hausarbeit wird von ihm übernommen (Frauen hingegen agieren nach dem ,dependency model“). ${ }^{37}$ Während die Ausführung von Hausarbeiten zur Identitätskonstruktion von Weiblichkeit dient, dient die Erwerbstätigkeit des Mannes als Hauptverdiener zur Identitätskonstruktion von Männlichkeit. Solange die Frau finanziell vom

37 „Wives respond in ways consistent with the dependency model [with rules of economic exchange: unpaid labor for a share of the husband's income]. The same is not true for husbands. Regardless of whether economic patterns of support between wife and husband are measured contemporaneously or over a period of years, dependent husbands do less housework the more they depend on their wives income" (Brines 1994: 682). 
Mann abhängig ist, ist die Geschlechtsidentität nicht gefährdet, wodurch auch eine Beteiligung des Mannes an der Hausarbeit möglich ist. Kehrt sich jedoch das Abhängigkeitsverhältnis um, d. h. ist der Mann finanziell von seiner Frau abhängig, ist die Identitätskonstruktion von Männlichkeit und Weiblichkeit gefährdet. Diese Normverletzung führt zu einem verstärkt rollenkonformen, d. h. traditionellen Verhalten: Um die Erwartungsenttäuschung des Mannes zu kompensieren, müssen berufstätige Frauen erst recht häusliche Aufgaben ausführen, damit Männer sich subjektiv wieder „männlich fühlen“. Umgekehrt werden Männer aus Partnerschaften, in denen Männer die Rolle des Familienernährers und Frauen die Rolle der Hausfrau übernehmen, eher im Haushalt unterstützend agieren, weil ihr subjektives Männlichkeitsempfinden bereits durch ihre Berufstätigkeit ausgedrückt wird.

\section{Der Identitätsformationsansatz (Bielby/Bielby)}

Bielby/Bielby (1989) haben den Identitätsformationsansatz entwickelt und nehmen an, dass der Prozess der Rollenübernahme zur Entwicklung einer Rollenidentität führt, wobei die Identitätsfindung von Männern und Frauen Unterschiede im Hinblick auf Hausarbeit und Erwerbstätigkeit aufweist, ferner der Prozess der Identitätsformation nach Geschlecht variiert:

„We find that for both men and women, engagement in work and family roles leads to identification with those roles. However, the process of identity formation differs for men and women in ways that correspond to gender-based differentiation in household and workplace activities" (Bielby/Bielby 1989: 776).

Gemäß den Annahmen dieses Ansatzes werden familiäre Rollenbilder wie auch Berufsrollen durch gesellschaftliche Werte und Normen bestimmt. Nach der These von Bielby/Bielby ist weder der „Scarcity-Ansatz“, nach dem Individuen entweder eine Arbeitsidentität oder eine Familienidentität entwickeln, noch der „Multiplicity-Ansatz“, nach dem Individuen multiple Rollen übernehmen und folglich fähig sind, diverse Identitäten zu entwickeln, die gleichermaßen einen Verpflichtungsgrad aufweisen können (eine starke Verpflichtung gegenüber Familie und Beruf) geeignet zu erklären,

,[...] how men and women distribute commitments to work and family roles. Behaviors in these realms [work and family] are shaped by a sex-based division of labor, and the values placed on those behaviors are prescribed by sex role norms. [...] we expect that wives employed outside the home balance dual role identities by trading one off against the other. In contrast, for men, contemporary normative expectations for the "husband" and father roles still do not include fully shared responsibility and involvement in household and childcare activities. Furthermore, a husband's role in the workplace is consistent with his family obligations as "provider"“" (Bielby/Bielby 1989: 777).

Frauen neigen aufgrund von Gendernormen dazu, sich stärker mit ihrer Familie zu identifizieren und Männer mit ihrer Erwerbsarbeit (vgl. Bielby/Bielby 
1989: 785). Hausarbeiten seien Aufgabenbereiche von Frauen, deren Übernahme zur Konstruktion der Familienidentität der Frau beiträgt.

„[...] we allow for a reciprocal relationship between work and family identity, and test empirically whether women and men differ in their properties to ,trade off" one form of identity against the other" (Bielby/Bielby 1989: 778).

Berufs- und Familienidentitäten sind für Frauen als konkurrierendes Wechselwirkungsverhältnis zu begreifen, dessen Balance es auszuloten gilt, weshalb meist eine Doppelbelastung zwischen Beruf und Familie resultiert. Eine verstärkte Berufsidentität würde aufgrund der normativen Erwartungen zu einem Interrollenkonflikt, insbesondere von Müttern, führen. Die Verdrängung traditioneller Rollenbilder vollzieht sich nur sehr langsam, wodurch die häusliche Arbeitsteilung im Sinne ,vergangener“ Erwartungsstrukturen fortgeführt wird. So wird nach Bielby/Bielby (1989) Frauen heute noch die Erwartung der Ausführung ihrer Hausfrauen- und Mutterrolle zugeschrieben, selbst wenn sie über hohes Humankapital und Einkommensressourcen verfügen (vgl. Schulz/Blossfeld 2006: 30). Dem Interrollenkonflikt zwischen berufstätiger Karrierefrau, Hausfrau und Mutter wird eine zentrale Bedeutung beigemessen, denn die Familienidentität darf von der Frau nicht vernachlässigt werden, wobei ihre Ausführung normativ über gesellschaftliche Werte „kontrolliert" wird. Hingegen werden die Verpflichtungen der Geschlechterrolle des (Ehe-)Mannes mit seiner erwerbsspezifischen Ernährerrolle als erfüllt betrachtet. Das zentralste Ergebnis der quantitativen Überprüfung des Identitätsformationsansatzes mittels LISREL (Linear Structural Relations System) ist:

„Married working women give precedence to family in balancing work and family identities. In contrast, married men may have the discretion to build identification with work and family roles without trading one off against the other" (Bielby/Bielby 1989: 786).

Ein Rollentausch ist diesem Ansatz zufolge das unwahrscheinlichste Ergebnis eines sozialen Wandels (vgl. Schulz/Blossfeld 2006: 31).

\section{Der Honeymoon-Effekt}

Die „Honeymoon-Hypothese“ bzw. das „Trägheitsgesetz“ besagt, dass mit zunehmender Ehedauer die Arbeitsteilung im Haushalt traditionaler wird (vgl. Künzler 1994: 108; Schulz/Blossfeld 2006: 32; Langfeldt 2008: 84). Zu Beginn einer Paarbeziehung zeigen sich Männer noch als engagierte und unterstützende „Hausmänner“, doch im Laufe der Zeit veralltäglicht sich die Beziehung, indem gesellschaftliche Erwartungsmuster einer traditionalen Arbeitsteilung ,schleichend“ übernommen werden (vgl. Schulz/Blossfeld 2006: 32).

Zusammenfassend ist zu konstatieren (s. Tabelle 3), dass symmetrische und asymmetrische Ansätze jeweils ausgehend von einer modernisierungs- 
theoretischen Perspektive argumentieren, die eine zunehmende Erwerbsbeteiligung von Frauen unterstellt. Der zentrale Unterschied der Paradigmen ist, dass einerseits VertreterInnen symmetrischer Ansätze eine Entwicklung zur egalitären Arbeitsteilung von Doppelkarrierepaaren als Möglichkeit zur Vereinbarkeit von Beruf und Familie, andererseits VertreterInnen asymmetrischer Ansätze eine innerhäusliche Traditionalisierung trotz der zunehmenden Erwerbstätigkeit von Frauen prognostizieren. Beide Paradigmen haben eine Akteurszentriertheit gemein: sie argumentieren als Handlungstheorien überwiegend auf der Mikroebene. Mit der Konzentration auf mikrosoziologische Erklärungen des familialen Binnensystems ist ,[...] oft eine Abkehr von einer makrosoziologischen Familientheorie verbunden [...], welche die familiären Strukturen als abhängige begreift" (Held 1978: 71). Weitgehend ausgeblendet wird die (historische) Situations- respektive Kontextabhängigkeit, die für die Bestimmung von Handlungsmöglichkeiten von großer Bedeutung ist.

„[...] resource theories or role theories mostly ascertain the asymmetry of occupational assets and roles, often without testing their assumptions by empirically examining contextual or historical variations. How specific structures of the labour market or welfare policies contribute to the postulated asymmetries and how transformations and variations of these structures moderate the degree and nature of the resource- and role hierarchies is rarely shown" (Bühlmann et al. 2010: 51).

Soziale Handlungen von Individuen können nicht als isoliert erachtet werden, sondern sind in eine strukturelle Argumentation einzubetten. Darüber hinaus wird der Entstehungsprozess der Rollenbilder von HandlungstheoretikerInnen weitgehend ausgeklammert, dessen Erklärung ebenso einer Berücksichtigung von Struktur sowie einer Kontextualisierung bedarf (s. „Cultural lag“ im Kapitel 7.).

„[...] all of them are more or less clearly limited to a micro-social level (interpersonal interactions, exchanges or negotiations) as they focus on various mechanisms of individual or interpersonal behavior. This behavior is seen as motivated by such factors as norm conformism, identity expression or interest, and - at least implicitly - as being acted out in a given context that sets different incentives for various behavioral alternatives. The context itself, i.e., the social-structural setup of the environment in which people and couples live, appears as a " given » outside the explanatory models, as these barely focus on the context's structure and its sex-discriminating features“" (Levy et al. 2002: 4). 


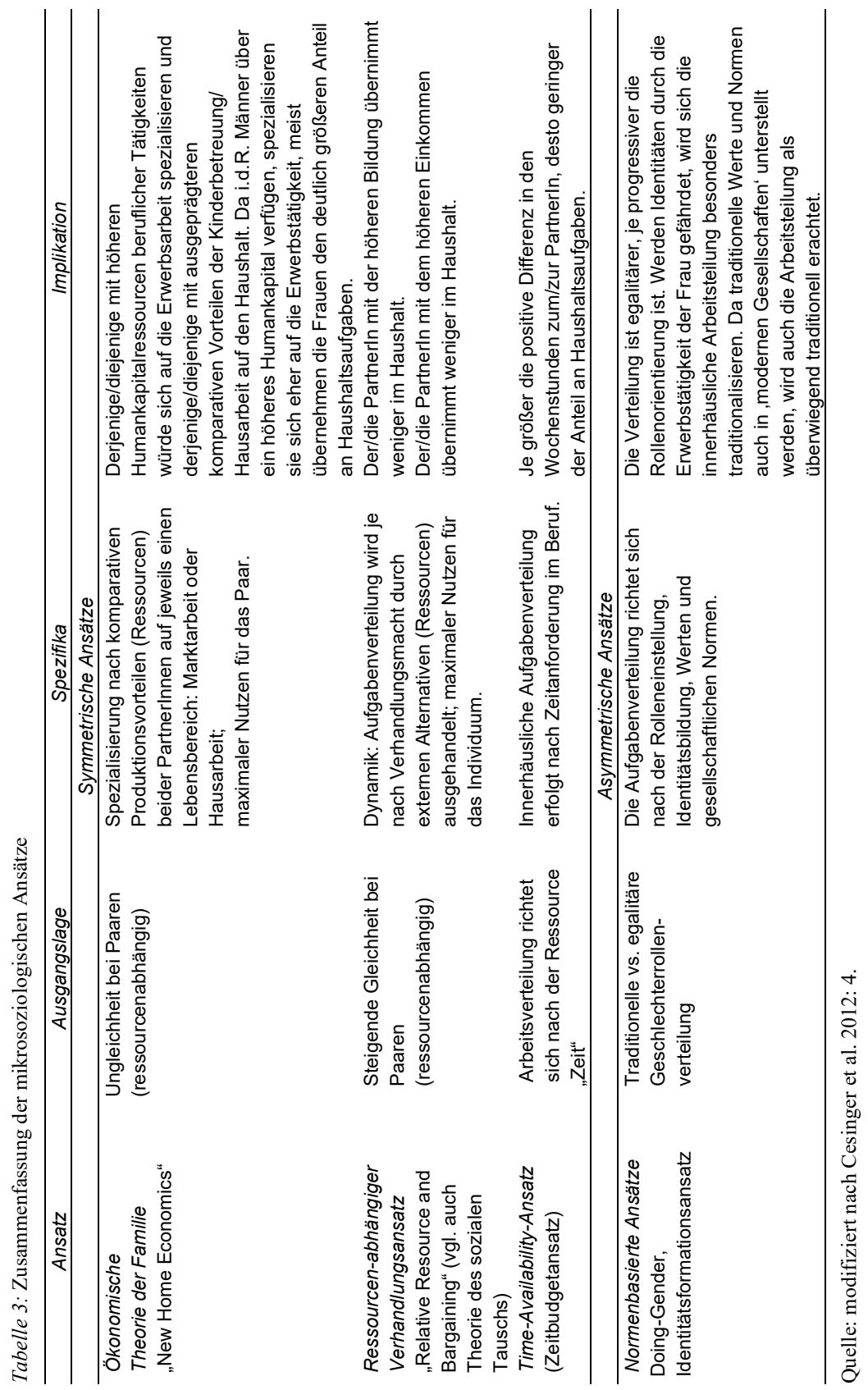




\subsubsection{Der alltagspraktische Ansatz innerhäuslicher Arbeitsteilung nach Jean-Claude Kaufmann}

Jean-Claude Kaufmann widmet sich der innerhäuslichen Arbeitsteilung (im Spezifischen dem Umgang mit der schmutzigen Wäsche und dem Bügeln) aus einer qualitativen Perspektive, indem er verstehende, leitfadengestützte Paarbefragungen (das „verstehende Interview“ ist ein von ihm entwickeltes methodisches Verfahren) durchführte. Für ihn kommt dem Ideal der Gleichberechtigung eine wesentliche Bedeutung zu:

„Die Macht der Veränderung, welche zur Neudefinition der Ehe treibt, ist die Idee der Gleichheit zwischen den Geschlechtern, welche fest im Prozeß [sic!] der gesellschaftlichen Demokratisierung verwurzelt ist. Sie befiehlt den Individuen zu handeln“ (Kaufmann 1994: 292).

Kaufmann (1994; 1999a) begründet die Diskrepanz zwischen dem Gleichheitsideal und der traditionellen innerhäuslichen Arbeitsteilung - für ihn die Diskrepanz zwischen Ideen und Gesten - mit der Alltagspraxis (,,den elementarsten Haushaltspraktiken") und sieht die Gleichheit der Geschlechter als eine der größten Herausforderungen ${ }^{38}$ der aktuellen Epoche an. Zu Zeiten des bürgerlichen Familienmodells erfüllte die Frau ihre Rollen als Hausfrau sowie Mutter und erhielt für ihre häuslichen Fähigkeiten Anerkennung durch ihren Mann (vgl. Kaufmann 1994: 190). Doch heute kann sich dieses Kapital auch ,[...] klammheimlich in ein defizitträchtiges Negativkapital verwandeln" (Kaufmann 1994: 190). Indem Frauen ihre Kompetenz im Haushalt und ihre hohen Ansprüche zum Ausdruck bringen, tappen sie in eine Falle, „[...] welche dazu treibt, sich im Widerspruch zu dem zu verhalten, was man eigentlich will [Gleichheitsideal]" (Kaufmann 1994: 190). Unterschiedliche Verhaltensweisen und Ansprüche führen dazu, dass der Besitzer/die Besitzerin des größeren „Negativkapitals“ dazu gebracht wird, mehr im Haushalt zu tun und der Besitzer/die Besitzerin des geringeren „Negativkapitals“ wird mit Nicht-Anerkennung der Mehranstrengung reagieren, weil er/sie nur nach dem Maßstab des eigenen Verhaltens urteilt (vgl. Kaufmann 1994: 191). Das Problem liegt gemäß Kaufmann in der Veränderung ehelicher Rollen, die einen kontinuierlichen Autonomiezuwachs erfahren. Früher waren eheliche

38 „Die Gleichheit der Geschlechter ist eine der größten Herausforderungen unserer Epoche. Ein Ziel, welches allzuoft als Kampf zwischen Männern und Frauen dargestellt worden ist, welche freie Urheber ihrer Handlungen seien. Aber die Akteure sind nicht frei, und der größte Teil ihres Verhaltens wird ihnen von der Gesellschaft aufgezwungen. Der gesellschaftliche Zwang zieht seine Kraft aus dem Umstand, daß [sic!] er nicht von außen kommt, sondern in jedem von uns auf sehr persönliche und konkrete Weise verkörpert ist, in scheinbar harmlosen Verhaltens- und Handlungsweisen, welche uns zu dem machen, was wir sind. Die Analyse zeigt auf diese Weise, daß [sic!] das Zentrum des Widerstandes gegen die Geschlechtergleichheit in der Familie liegt, zu Hause, bei den elementarsten Haushaltspraktiken. Und insbesondere bei denjenigen, die am meisten Frauensache sind: beim Umgang mit der Wäsche“ (Kaufmann 1994: 293). 
Pflichten und Schulden gesellschaftlich festgelegt, während heute beide PartnerInnen autonom entscheiden können, welche Bemessungsregeln sie verfolgen möchten. Grundlage hierfür bieten Diskrepanzen des Verhaltenskapitals, das sich in Negativkapital für denjenigen verwandelt, der über einen Überschuss verfügt und umso negativer wird, je mehr er/sie davon besitzt (vgl. Kaufmann 1994: 191).

Warum es wichtig ist, sich mit der Hausarbeit zu beschäftigen, obwohl uns die Hausarbeit auf den ersten Blick als das Trivialste überhaupt erscheint? Die zentrale These Jean-Claude Kaufmanns ist,

„[...] daß [sic!] es vielleicht nichts Wichtigeres als diesen Haushaltskleinkram gibt, mit dem wir Tag für Tag beschäftigt sind. [...] - Tag für Tag erschaffen sich Menschen mit diesen [alltäglichen Routinen] und tausend anderen Gesten aufs neue die Grundlage eines Systems von ungeheurer Komplexität, ein Ordnungsund Klassifikationssystem, das jedem Ding seinen genauen Platz innerhalb einer größeren Ordnung zuweist, die, trotz ihrer scheinbar geringen Bedeutung, die Grundlage der Zivilisation bildet” (Kaufmann 1999a: 19).

Auch Kaufmann sieht die Überwindung einer einseitigen Perspektive zwischen Individuum oder Gesellschaft, ferner zwischen strukturtheoretischen Ansätzen oder theoretischen Ansätzen, die die Freiheit des Individuums betonen, als eine der größten Herausforderungen für die Soziologie an - es gelte den Graben zu überwinden und eine wechselseitige Perspektive einzunehmen (vgl. Kaufmann 1999a: 59).

\subsubsection{Eine Differenzierung nach Milieus: Was passiert, ,wenn der Mann} kein Ernährer mehr ist?“ (Koppetsch/Speck)

Wie schon bei der Erläuterung des Display-Ansatzes von Brines (1994) zum Ausdruck gebracht wurde, ist die atypische Umkehrung des finanziellen Abhängigkeitsverhältnisses in Paarbeziehungen eine interessante Forschungsthematik, um Aushandlungsprozesse der Geschlechterarrangements zu analysieren. Familienernährerinnen sind Frauen, die 60\% des Gesamteinkommens beider PartnerInnen verdienen. Dies sind gemäß der SOEP-Daten (2007) 9\% in Deutschland (vgl. Brehmer et al. 2010: o. S.); die Bilanz des Projekts „Familienernährerinnen“ (Kooperationsprojekt aus dem Bundesministerium für Familie, Senioren, Frauen und Jugend und dem Deutschen Gewerkschaftsbund) zeigt eine steigende Tendenz (vgl. BMFSFJ 2013: o. S.). Koppetsch/Speck (2015) beziehen ihre Studie auf Familienernährerinnen verschiedener Milieus, die einen prekär erwerbstätigen oder arbeitslosen Partner haben und untersuchen,

„[...] was es für Weiblichkeit und Männlichkeit sowie für das Geschlechterarrangement in [heterosexuellen] Paarbeziehungen bedeutet, wenn der Mann kein Ernährer mehr ist - differenziert nach sozialen Milieus. Kommt es zu einer Krise in der Paargemeinschaft? Werden Geschlechterarrangements neu ausgehandelt, 
und welche Rolle spielen dabei milieuspezifische Leitbilder sowie Muster der Lebensführung?" (Koppetsch/Speck 2015: 15f.).

Wie teilen sich heterosexuelle Paare in Deutschland (die Befragten stammen im Wesentlichen aus dem Rhein-Main-Gebiet und Berlin (vgl. Koppetsch/Speck 2015: 257)) die Arbeit auf, wenn der Mann nicht der Familienernährer ist bzw. die Frau über ein höheres Einkommen verfügt, während ihr Partner Geringverdiener oder arbeitslos ist? Koppetsch/Speck (2015) führten eine qualitative Studie durch, die 29 leitfadengestützte Interviews mit Paaren (zunächst Einzel-, anschließend Paarinterviews) aus unterschiedlichen Milieus (traditional, individualisiert oder familistisch) sowie Beobachtungsprotokolle der Interviewsituationen beinhaltet (vgl. Koppetsch/Speck 2015: 257). In Anlehnung an die Grundsätze der objektiven Hermeneutik nach Oevermann basierte die Auswertung auf dem Prinzip der Fallrekonstruktion (vgl. Koppetsch/Speck 2015: 260). Diese folgt der Annahme, ,[...] dass der Text des Gesagten mehr bedeutet als der Sprecher typischerweise subjektiv meint, und eignet sich insofern besonders gut zum Aufdecken latenter $\mathrm{Zu}-$ sammenhänge“" (Koppetsch/Speck 2015: 260f.). Das individualisierte Milieu umfasst ,[...] die gebildete Mittelschicht mit urbanem Lebensstil (Akademikermilieu)", die nach Gleichberechtigung und Selbstverwirklichung streben (Koppetsch/Speck 2015: 38). Die These könnte folglich lauten: Wenn der Mann arbeitslos wird, dann wird er sich aufgrund der Orientierung an der Gleichberechtigung um den Haushalt und die Kinder kümmern, solange die Frau ihrer Erwerbstätigkeit nachgeht. Konträr zu dieser Annahme zeigt sich jedoch anhand der zentralen Ergebnisse von Koppetsch/Speck (2015), dass ausgerechnet im individualisierten Milieu genau das Gegenteil eintritt: die traditionelle Arbeitsteilung verstärkt sich, indem die Frau nun durch die Haushaltstätigkeiten und die Erwerbstätigkeit doppelt belastet ist. Eine egalitäre Arbeitsteilung erfolgt überraschender Weise am ehesten im familistischen, wertkonservativen Milieu, während im individualisierten Milieu die Selbstverwirklichung des Mannes im Vordergrund steht. Obwohl im wertkonservativen Milieu die traditionellen Rollenbilder am ehesten aufgelöst werden, stellen Koppetsch/Speck (2015) fest, dass auch in diesem Fall keine faktische Gleichberechtigung erreicht wird - Frauen und Männer könnten einfach nicht aus ihrer Rolle. Der Anstieg der Familienernährerinnen beruht einerseits auf der Bildungsexpansion einschließlich eines Anstiegs berufstätiger Hochschulabsolventinnen und andererseits auf einem Wandel der Erwerbsgesellschaft, der eine unfreiwillige Beschäftigung von Männern in prekären Beschäftigungsverhältnissen hervorbringt. Ob diese Entwicklungen auch zu einem Wandel des Geschlechterverhältnisses in Familie und Paarbeziehungen führen, betrachten die AutorInnen aufgrund ihrer Befunde skeptisch (vgl. Koppetsch/Speck 2015: 235). Hierfür spiele es auch keine Rolle, ob egalitäre oder traditionale Geschlechterrollenverständnisse von den Paaren vertreten werden (vgl. Koppetsch/Speck 2015: 236f.). 
Das individualisierte Milieu folgt dem egalitären Partnerschaftskode (für eine Differenzierung der Partnerschaftskodes der Liebe und dem egalitären Partnerschaftskode s. Kapitel 6.1. Diese Thematik von Koppetsch wird im Anschluss an die vergleichende Wohlfahrtsstaatsforschung nochmals aufgegriffen und vertieft.) mit einem Schwerpunkt auf der Gleichheit der Geschlechter und vertritt Werte wie Selbstverwirklichung und Autonomie (vgl. Koppetsch/Speck 2015: 37f.). Männer dieses Milieus inszenieren sich als „,cool“ und gefühlslos - „Coolness“ dient als eine alternative Form von Männlichkeit (vgl. Koppetsch/Speck 2015: 68). Diese Gefühlslosigkeit beschreiben die Autorinnen mit der „männlichen Bindungsmacht“ im Sinne Eva Illouz (2011), dessen Ursache ein Ungleichgewicht auf dem Partnermarkt ist.

„Je stärker die Frauen ökonomisch und hinsichtlich ihres Bildungsstatus aufholen, desto deutlicher wird, dass attraktive Männer, das heißt solche mit entsprechender »Ausstrahlung « und Bildung, nun aus einem größeren Pool interessierter Frauen auswählen können“ (Koppetsch/Speck 2015: 72).

Nun müssten die Frauen stärker um die tendenziell weniger bindungswilligen Männer werben. Hieraus resümieren Koppetsch/Speck (2015):

„Für Frauen entsteht damit insgesamt eine ungünstige Marktsituation bei der Partner[Innen]wahl, und genau das ist die Voraussetzung jener neuen Form männlicher Bindungsmacht, die sich in einer Verknappung emotionaler Zuwendung ausdrückt. Vermutlich wird diese Strategie für Männer umso wichtiger, je weniger sie im Paargeschehen auf andere Machtressourcen zurückgreifen können. Sie wirkt als stumme Drohung, die Beziehung im Konfliktfall auch beenden zu können. Und sie ist ein wesentlicher Grund dafür, dass männliche Hegemonie selbst in Beziehungen, in denen die Frauen die Rolle der Ernährerin einnehmen, bestehen bleibt. Die Konsequenz ist, dass der Gleichheitsanspruch im individualisierten Milieu oft nicht durch ein partnerschaftliches Arrangement der Verteilung von Aufgaben und Belastungen unter Bedingungen der Zeitknappheit eingelöst, sondern durch Statuskonkurrenz zwischen den Geschlechtern unterlaufen wird“ (Koppetsch/Speck 2015: 72f.).

Innerhalb des traditionalen Milieus (traditionales HandwerkerInnen- und ArbeiterInnenmilieu) besteht eine „hierarchische Sphärentrennung“ und ein „ritueller Patriarchalismus“ (Koppetsch/Speck 2015: 38). Die „Normalbiographie“" (in folgender Reihenfolge: Schule-Ausbildung-ErwerbstätigkeitHochzeit-Hausbau-Kinder) gilt als erstrebenswertes Modell dieses Milieus. Geschlechterrollen werden nicht hinterfragt, sondern sind eindeutig traditionell festgelegt: Der Mann ist Familienernährer, die Frau für Familie und Haushalt verantwortlich. Dies gilt auch für Fälle, in denen die Frau vor der Geburt des ersten Kindes erwerbstätig war. Sofern sie nach der Geburt erwerbstätig ist, ist meist eine Teilzeitbeschäftigung mit geringem Zuverdienst vorzufinden. In dem Moment, in dem er seine Erwerbstätigkeit verliert und sie Familienernährerin ist, besteht die Forderung, dass er im Haushalt unter- 
stützend agiert. Insofern konstatieren Koppetsch/Speck (2015) für dieses Milieu einen kleinen Machtgewinn für Frauen, wenn Männer einer prekären Erwerbssituation ausgesetzt sind. Frauen können beispielsweise fortan Kaufentscheidungen treffen und ihren Partner zu Haushaltstätigkeiten und Jobsuche auffordern. Ihre Familienernährerinnenposition wird jedoch nur unter der Bedingung akzeptiert, dass es sich hierbei um eine vorübergehende Phase handelt. Es wird von der Familie betont, dass der Mann nichts für seine Situation kann und sich diese auch bald ändern würde. Ein langfristiger Wandel der Geschlechterrollen ist folglich auch in diesem Milieu nicht zu erwarten.

Das familistische Milieu (das Segment der mittleren Dienstleistungsberufsgruppen, Beamten und Sozialberufe: pädagogische Berufe, Pflege- und Gemeindeberufe) zeichnet sich durch Werte der Gemeinschaft und Solidarität aus (vgl. Koppetsch/Speck 2015: 38). Die Familie hat oberste Priorität, sie steht an erster Stelle und ist wichtiger als eine berufliche Karriere. Erwerbstätigkeit ist lediglich ein pragmatisches Mittel, um den Lebensstandard der Familie zu sichern. Diesem Milieu gelingt es am ehesten die traditionellen Rollenbilder zu überwinden. Doch diese Überwindung ist keine Folge einer Orientierung an der Geschlechtergleichheitsnorm, sondern eine Folge der Vorstellung von Familie als komplementäre, arbeitsteilige Gemeinschaft (vgl. Koppetsch/Speck 2015: 136). Innerhalb dieses Milieus verfügen Frauen häufig über eine höhere Qualifikation als ihre Partner, wodurch eine atypische Arbeitsteilung akzeptiert werden kann. Paare dieses Milieus hätten nicht mit der im individualisierten Milieu dominanten Falle der Frauen zu kämpfen, weil sie sich keinen Partner suchen, der nach Selbstverwirklichung strebt.

Obgleich die Studie von Koppetsch/Speck (2015) interessante Befunde anhand empirischer Fallbeispiele aufzeigen kann (wobei das Ergebnis, dass es ausgerechnet das individualisierte Milieu ist, dem am wenigsten Gleichberechtigung gelingt, nichts grundlegend Neues ist, weil bereits Hochschild (1997), Koppetsch/Burkart (1999) und Wimbauer (2012) von ähnlichen Befunden berichten), darüber hinaus sehr populär und medienwirksam wurde, sind dennoch ein paar wichtige Kritikpunkte nicht auszublenden. Die Hauptkritik von Motakef/Wimbauer (2015) bezieht sich auf die Milieuklassifizierung: Die Milieus wurden ex ante definiert (sie basieren auf einer früheren Studie von Koppetsch/Burkart (1999)) und finden im Datenmaterial eine scheinbare Bestätigung, doch hätten sie aus dem Datenmaterial heraus kategorisiert werden sollen. Durch diese Vorgehensweise blieben wichtige Einsichten im Verborgenen. Kritisch zu hinterfragen ist außerdem, dass lediglich drei Milieus beschrieben werden und andere Milieus vollkommen unthematisiert bleiben. Auch die Homogenität der Milieus ist fragwürdig: Wenn PartnerInnen aus verschiedenen Milieus stammen - wie kann dann ihre Zusammenstellung, die doch so milieuhomogen erscheint, begründet werden? Warum schließen sich eine berufliche Selbstverwirklichung und eine Familien- 
orientierung überhaupt aus (vgl. Motakef/Wimbauer 2015: o. S.)? Der symmetrischen ökonomischen Theorietradition zufolge wäre eine egalitäre Erwerbs- und Einkommenskonstellation der Paare die Ausgangsbedingung einer egalitären innerhäuslichen Arbeitsteilung. Diese Argumentation kann jedoch nicht von Koppetsch/Speck (2015) nachgezeichnet werden, weil keines ihrer befragten Paare diese Voraussetzung erfüllt, stattdessen atypische finanzielle Abhängigkeitsverhältnisse zwischen den PartnerInnen vorliegen. Motakef/Wimbauer (2015) erörtern weiterführend eine überraschend widerspruchsfreie Argumentation. Beispielsweise führen sie die von Koppetsch/Speck (2015) vertretene These auf, dass das Private einer Änderungsresistenz unterliege, hingegen sich die öffentliche Sphäre zunehmend an einer Gleichheit orientiere. Wimbauer konstatierte bereits (2003), dass es deutliche Anzeichen gäbe, die auf die Annahme ,[...] einer wechselseitigen und komplexen Verschränkung von Privatheit und Öffentlichkeit in ihren für das Normalfamilienmodell konstatierten Grenzziehungen" hinweisen (Wimbauer 2003: 264, Hervorhebungen im Original; die Verf.). Ferner ist es nicht verwunderlich, dass Motakef/Wimbauer (2015) dieses Argument in Bezug auf ihre Kritik an Koppetsch/Speck (2015) aufführen:

\begin{abstract}
„Aber sprechen die vielen von ihnen auch angesprochenen Studien zur vertikalen und horizontalen Segregation der Arbeitsmärkte und die weiterhin das Ernährermodell stützenden sozialpolitischen Regelungen wie das Betreuungsgeld nicht ebenfalls, vielleicht sogar noch stärker als im Privaten, für eine solche Änderungsresistenz auch in der Erwerbssphäre und in der Familien- und Sozialpolitik? Ungleichheitssoziologisch ließe sich hinzufügen, dass mit dem Fokus auf das Private und das ,Innengeschehen' der Paare die zahlreichen ungleichheitsrelevanten gesellschaftlichen Strukturen aus dem Blick geraten sind. Hier wäre es fruchtbarer gewesen, auch die Wechselwirkungen ungleichheitskonstitutiver Mechanismen aus Erwerbssphäre, Sozialstaat und Familie herauszuarbeiten. Wenn also in den meisten Studien zu prekären Erwerbskonstellationen das Private ganz ausgeblendet wird, wird es hier überhöht" (Motakef/Wimbauer 2015: o. S.).
\end{abstract}

Es ist Anliegen dieser Dissertation eben diese Wechselwirkungen herauszuarbeiten.

\title{
3.2. Zum dynamischen Wechselverhältnis von (gesamtgesellschaftlicher) Struktur und (individueller) Handlung - das Mikro-Makro-Problem im Allgemeinen
}

Das Mikro-Makro-Problem wird soziologisch auch als das ,[...] Verhältnis von Struktur und Handlung, von Individualismus und Kollektivismus, von Determinismus und Voluntarismus, von Strukturalismus und Subjektivismus 
[...] diskutiert" (Heidenreich 1998: 229). Es können drei idealtypische Positionen differenziert werden: Erstens kann der Handlung, zweitens der Struktur oder drittens dem Wechselwirkungsverhältnis zwischen Handlung und Struktur Vorrang eingeräumt werden. Indessen ist zu berücksichtigen, dass „[...] a theoretically powerful sociological analysis must pay attention to both structural and micro-level issues" (Blossfeld 1996: 181). Vornehmlich zwei Fragestellungen sind für das Mikro-Makro-Problem von Relevanz: Wie beeinflussen strukturelle und institutionelle Bedingungen (Situationsrahmungen) der Makroebene Werte und Einstellungen von Individuen (vgl. Heidenreich 1998: 231)? Und: Wie führt das individuelle Handeln zu einer neuen sozialen Ordnung? Weder gesellschaftlich voraussetzungslose Handlungen noch eine Unabhängigkeit der Gesellschaft von sozialen Handlungen können als zusammenhangslos gedacht werden, sondern sind als zwei reflexive, sich wechselseitig reproduzierende Ebenen zu betrachten (vgl. Heidenreich 1998: 231).

„Mit dem Einbezug von Einstellungen und Werten zu den strukturellen Bedingungen hinzu soll eine einseitig strukturdeterminierte Betrachtung einerseits und eine voluntaristische auf Präferenzen beruhende Sichtweise andererseits vermieden werden“ (Baumgartner 2008: 15).

Die Dynamik beruht auf der Reproduktion, dass einerseits individuelles Handeln durch die Institutionalisierung von Verhaltensregeln einer sozialen Ordnung geprägt ist, andererseits auf der Interpretationsfreiheit beruht, diese Regeln nicht als absolute Indoktrination, vielmehr als individuellen Handlungsspielraum zu erachten. Wäre diese Reproduktion nicht gegeben, wäre deviantes Verhalten niemals möglich. „Struktur darf nicht mit Zwang gleichgesetzt werden: sie schränkt Handeln nicht nur ein, sondern ermöglicht es auch“; d. h. Akteure sind reflexionsfähig (Giddens 1992: 78). Giddens begreift im Rahmen seiner Theorie der Strukturierung den dynamischen Prozess als „Dualität von Struktur“ - ein produktiver Lösungsansatz der MikroMakro-Problematik (Giddens 1992: 77).
„Konstitution von Handelnden und Strukturen betrifft nicht zwei unabhängig voneinander gegebene Mengen von Phänomenen - einen Dualismus -, sondern beide Momente stellen eine Dualität dar. Gemäß dem Begriff der Dualität von Struktur sind die Strukturmomente sozialer Systemen sowohl Medium wie Er- gebnis der Praktiken, die sie rekursiv organisieren“" (Giddens 1992: 77).

Wesentlich ist Giddens Differenzierung zwischen Struktur(en), System(en) und Strukturierung: Strukturen sind (latente) „Regeln oder Ressourcen“, Systeme basieren auf ,,reproduzierte(n) Beziehungen zwischen Akteuren oder Kollektiven, organisiert als soziale Praktiken" und Strukturierung implementiert die prozesshafte Dynamik von Strukturen, die sich verändern können, d. h. „die Reproduktion sozialer Systeme bestimmen“ (Giddens 1992: 77). Akteure verfügen über ein (teils mehr, teils weniger implizites) Wissen von 
Strukturen, zugleich auch über ein Handlungsvermögen, das eine Restrukturierung sozialer Systeme hervorrufen kann.

Verbunden mit dem Mikro-Makro-Problem ist auch die Frage, wie individuelle Entscheidungen entstehen. Seit den 1980er Jahren ist eine „Explosion“ der „Theorie der rationalen Entscheidung (engl. Rational Choice)“ festzustellen, die insbesondere auf VertreterInnen wie James S. Coleman (1986) und Hartmut Esser (1990) zurückzuführen ist. Blossfeld kritisiert an diesen Theorien, dass der Rahmen (,frame“) der Handlung zwar vorausgesetzt und als gegeben betrachtet, jedoch zu wenig reflektiert wird. Stattdessen würden Rational-Choice-Ansätze zu sehr danach streben, universale Theorien des Handelns zu generieren. Blossfeld schlägt vor, eine Präzision der Hypothesen mit geringerem Abstraktionsniveau vorzunehmen, eine stärkere Ausrichtung auf empirische Probleme zu fokussieren sowie die Entscheidungskontexte einschließlich des zeitlichen Wandels zu berücksichtigen.

„Thus, rational choice protagonists' endeavour to find universal theories comes at a high price: theorizing does not arise out of concrete empirical problems any more and the suggested more specific hypotheses often become more or less arbitrary. However, it is exactly these specific hypotheses that are of particular interest in any empirical study. They help the researcher to understand the situation or to predict individuals' concrete actions, and they are the 'variables' that typically have to be assessed through empirical research" (Blossfeld 1996: 185, Hervorhebungen im Original; die Verf.).

Jede ertragreiche soziologische Analyse muss sowohl Struktur als auch Handlung reflektieren, doch nicht in einem statischen, sondern - so seine zentrale Schlussfolgerung - in einem zeitbezogenen, historisch-dynamischen Sinne. Der Zeitfaktor sei die wesentliche Komponente zwischen Mikro- und Makroebene. Ein Makro-Mikro-Rahmen muss die spezifischen historischen Strukturen und Prozesse identifizieren, die den Wandel einer bestimmten Gesellschaft dominieren und er muss die kausalen Mechanismen spezifizieren, die es ermöglichen, das Zusammentreffen von bewusst handelnden Individuen mit dem ,flow of history“ als eine Reihe von Wahlprozessen zu verfolgen. Im Hinblick auf die empirische Analyse ist eine Errungenschaft, dass Längsschnittdaten durch entsprechende „neue“ Methoden analysiert werden können (Blossfeld führt diesbezüglich die „Event history analysis“ an, doch auch Mehrebenenmodelle für Paneldaten sind in diesem Zusammenhang zu erwähnen), um Kausalität in ihrer zeitlichen Abfolge zu modellieren.

„Any macro-micro framework must recognize that time matters in this relationship. It must identify the particular historical structures and processes which dominate the changes occurring in a given population and it has to specify the causal mechanisms that allow us to trace the encounters of intentionally acting individuals with the flow of history as a series of choice processes. An important advance in this respect has been that longitudinal data can be studied by new statistical methods in a stepwise time-related fashion" (Blossfeld 1996: 198). 
Das Verständnis über ein dynamisches Wechselverhältnis von Mikro- und Makroebene ist ein relevanter Schritt einer mehrdimensionalen Konzeption von Machtverhältnissen, wie auch Krüger/Levy (2000) verdeutlichen.

\subsection{Zur integrativen Funktion von Familie und Geschlecht zwischen den Machtdimensionen - der Masterstatus nach Levy zur Lösung des Mikro-Makro-Problems}

Ein Ansatz, der eine biographisch-dynamische Perspektive einnimmt und die Teilhabe im Lebenslauf an unterschiedlichen Institutionen berücksichtigt, ist der von Krüger/Levy entwickelte Masterstatus. Dieser wird durch bestimmte biographische Ereignisse, beispielsweise die Geburt des ersten Kindes, ausgelöst und führt zu einer Präferenzzuweisung von Frauen auf den familialen Bereich (einschließlich sämtlicher damit verbundener Haushaltstätigkeiten) und von Männern auf das außerhäusliche Berufsleben. Die Dominanzordnung des Masterstatus schließt die Teilhabe an anderen sozialen Feldern nicht aus, doch werden die Präferenzen denen des Masterstatus-entsprechenden Bereiches untergeordnet.

„Die Verbindung von Lebenslauf- und Familienforschung führt uns entsprechend $\mathrm{zu}$ der zentralen These, dass einerseits die Institutionenstrukturierung des Lebenslaufs ohne Berücksichtigung der Familie nicht angemessen analysiert werden kann, und andererseits die Reproduktion traditionaler Familienmuster nicht ohne die Vernetzung von Familie mit den übrigen Institutionen des Lebenslaufs zu erklären ist. Als latente Brückenfunktion zwischen Familie und den übrigen Institutionen des Lebenslaufs vermuten wir die soziale Strukturierung der Kategorie Geschlecht. Um diese in ihrer ,irgendwie“ immer wieder auferstehenden Wirkung auf Geschlechterordnungen erfassen zu können, greifen wir auf ein Konzept zurück, das Lebensläufe unter ihrem Teilhabe-Aspekt an sozialen Feldern analysiert" (Krüger/Levy 2000: 382).

Der Begriff der Familie birgt die Forderung einer Verknüpfung zwischen Individuen und institutionenorientierten Lebensläufen: Innerhalb der Familie finden individuelle Aushandlungsprozesse statt, doch sind diese nicht isoliert von, sondern verknüpft mit außerfamilialen sozialstrukturellen Bedingungen $\mathrm{zu}$ verstehen ${ }^{39}$ - Familie ist der Ort, an dem die Erwartungen außerfamiliärer sozialer Felder sowie innerfamiliärer kombiniert werden. Es handelt sich um eine mikrosoziale Organisationsform, die auch meso- und makrosozial strukturiert ist (vgl. Krüger/Levy 2000: 391). Doch laufen mit Geschlecht und

39 Siehe hierzu auch die Argumentation von Orloff (1993a): „The sexual division of labor in caretaking and domestic work within institutions other than the family must also be considered“"(Orloff 1993a: 313). 
Familie verbundene strukturelle Handlungszwänge Gefahr, als individuelle Privatentscheidung (Verdeckungsphänomen) verschleiert zu werden, sodass eine dynamische Analyse einschließlich der Institutionalisierung geschlechtsdifferenter Ordnungsprinzipien sowie subjektiver Interpretationsmöglichkeiten unumgänglich ist, um diese Strukturblindheit zu meiden (vgl. Krüger/Levy 2000: 389). Mittels der Relevanz der Verknüpfung zwischen Lebenslaufs- und Familiensoziologie wird fokussiert,

„[...] dass einerseits die Institutionenstrukturierung des Lebenslaufs ohne Berücksichtigung der Familie nicht angemessen analysiert werden kann, und andererseits die Reproduktion traditionaler Familienmuster nicht ohne die Vernetzung von Familie mit den übrigen Institutionen des Lebenslaufs zu erklären ist" (Krüger/Levy 2000: 382).

Dem Geschlecht kommt hierbei eine Brückenfunktion zwischen Familie und außerfamiliären Institutionen zu (vgl. Krüger/Levy 2000: 382). Zum einen divergiert die Gewichtung der Teilhabe an verschiedenen sozialen Feldern nach Geschlecht (z. B. nach traditionalem Modell ein dominanter Familienstatus der Frau bei parallelem dominanten Erwerbsstatus des Mannes) ${ }^{40}$, zum anderen dient Geschlecht als zur Strukturkategorie gewordener Geschlechterordnung von Aktivitätsprofilen (z. B. institutionalisierte männlich und weiblich dominierte Berufsfelder) (vgl. Krüger/Levy 2000: 382f.). Der Masterstatus $^{41}$ ist als theoretisches Konstrukt für die Verknüpfung von Mikro- und Makroebene von Krüger/Levy (2000) entwickelt worden: So ist das Geschlecht als Masterstatus sowohl in der Gewichtung von Aktivitätsprofilen als Verfestigung geschlechtsspezifischer Territorialität (individuell), als auch in der Übernahme von geschlechtsdifferenten Zuständigkeitsregeln (strukturell) zu suchen (vgl. Krüger/Levy 2000: 383). Die Rollenübernahme von Frauen und Männern erfolgt weitgehend nach dem Kriterium Geschlecht, sodass nur wenige Möglichkeiten für Aushandlungsprozesse wahrgenommen werden (vgl. Baumgartner/Fux 2004: 109). Während der Arbeitsmarkt als struktureller Rahmen des männlichen Masterstatus fungiert, gilt nach wie vor die Familie als struktureller Rahmen des weiblichen Masterstatus, obwohl die Teilnahme am Arbeitsmarkt von Müttern in westlichen Industrieländern im Lauf der letzten Jahrzehnte zugenommen hat (vgl. Krüger/Levy 2000: 392; Levy et al. 2002: 30; Baumgartner/Fux 2004: 110). Ferner ist gemäß der Gender-integrierten Lebenslauftheorie der Lebenslauf keineswegs ge-

40 „Für sie - nicht für ihn - verschiebt Familienpartizipation die Existenzgrundlage und wird zu einem per Eigenarbeit nicht abzusichernden Risiko der Lebensführung, während sie in der männlichen Biographie die Kontinuität der Partizipationsabfolge nicht tangiert“ (Krüger/Levy 2000: 383).

41 Masterstatus wird nach Levy et al. (2002) definiert als , ,[...] meaning the (socially determined) dominance of one status area over the others in an individual's participation profile, and to apply this definition to the complementarity of sex-specific master-statuses of the partners in a couple" (Levy et al. 2002: 30). 
schlechtsneutral, sondern variiert zwischen den Geschlechtern: der weibliche ist (nach wie vor) mehr als der männliche Lebenslauf einer Komplexität der Institutionenstrukturierung ausgesetzt, die durch lebens-laufzyklische Unterbrechungen der Erwerbstätigkeit (u. a. durch Geburten von Kindern) verstärkt wird (vgl. Krüger/Levy 2000: 394) (auch wenn ein Entwicklungstrend $\mathrm{zu}$ verzeichnen ist, der die diskontinuierliche Erwerbsbeteiligung von Frauen durch eine kontinuierliche ablösen könnte (vgl. Leitner/Ostner 2000: 213f.)). Personen mit einer kontinuierlichen Berufsorientierung haben einen arbeitsmarktstrategischen Vorteil („Arbeitszeit-Hypothese"), wobei es sich in der Regel um erwerbstätige Männer handelt (vgl. Kreckel 1993: 53). Ein Zusammenhang zwischen familialer Situation und Erwerbspensum ist für die Männer gering; im Gegensatz dazu ist die Familie für Frauen das Hauptmotiv, Teilzeit oder nicht erwerbstätig zu sein (vgl. Baumgartner/Fux 2004: 114f.). ,[...] men's employment careers are steady and tendencially ascending, women's careers are broken and static or descending" (Levy et al. 2002: $31)$.

„[...] der geschlechtsdifferente Masterstatus [überformt] das Ensemble der Statuspositionen im Lebenslauf [...] und [bringt] insbesondere Verknüpfungen hervor [...], die gesellschaftliche und individuelle Verhältnisse als geschlechtsspezifische Hierarchisierung und partizipative Ungleichheit im Lebenslauf verfestigen. Die negativen Folgen betreffen, aufgrund der ungleichen Chancen in der Nutzung von Bildung und Beruf v.a. den weiblichen Lebenslauf und werden in einzelnen Phasen subjektiv besonders virulent“" (Krüger/Levy 2000: 387).

Kritik üben Bühlmann et al. (2010) am Master Status Konzept insofern, als dass durch die biographische Betrachtung des Lebenslaufs zwar der Versuch angestrebt wird, den Wandel der Arbeitsteilung historisch zu erfassen, jedoch die Wertvorstellungen der involvierten AkteurInnen nicht erfasst würden, die für den historischen Wandel von zentraler Bedeutung sind. Darüber hinaus würden Kontextfaktoren, wie Arbeitsmarktbedingungen oder Wohlfahrtsstaatsleistungen, in der empirischen Überprüfung des theoretischen Modells nicht einbezogen (vgl. Bühlmann et al. 2010: 51). Bevor weiterführende Genderungleichheitsaspekte der makrosoziologischen Argumentation skizziert werden, sind in Tabelle 4 die bereits diskutierten Theorien zur innerhäuslichen zusammengefasst. 


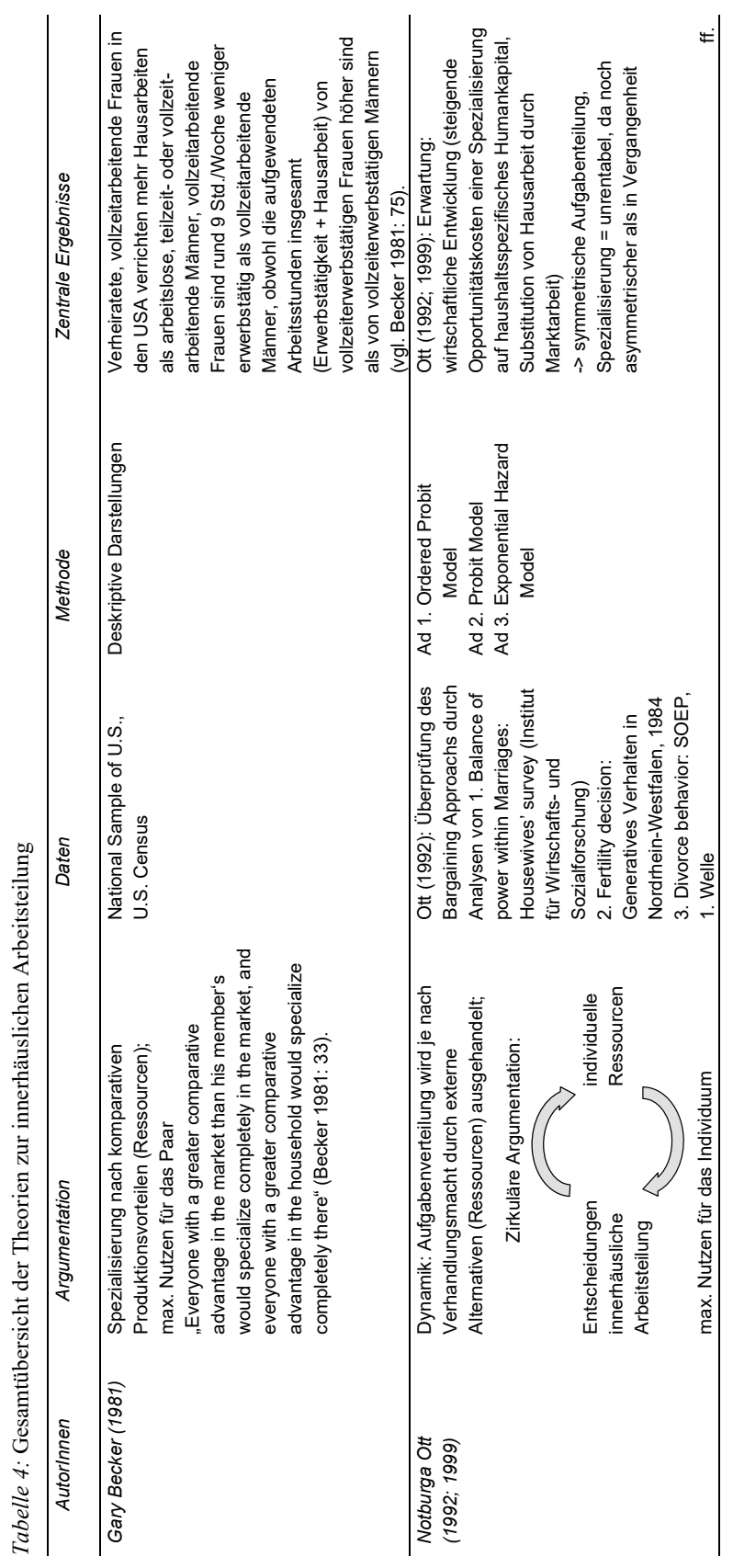




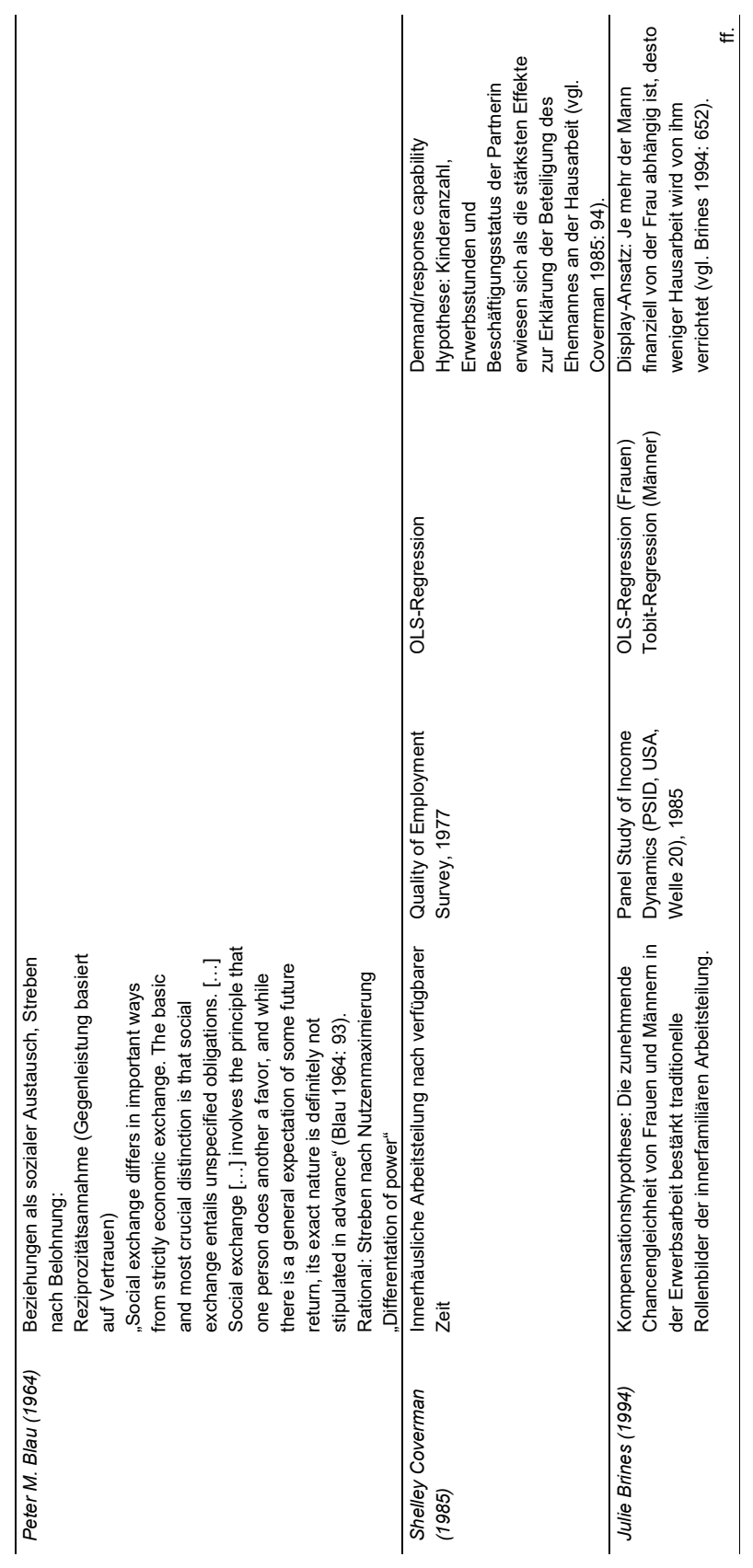




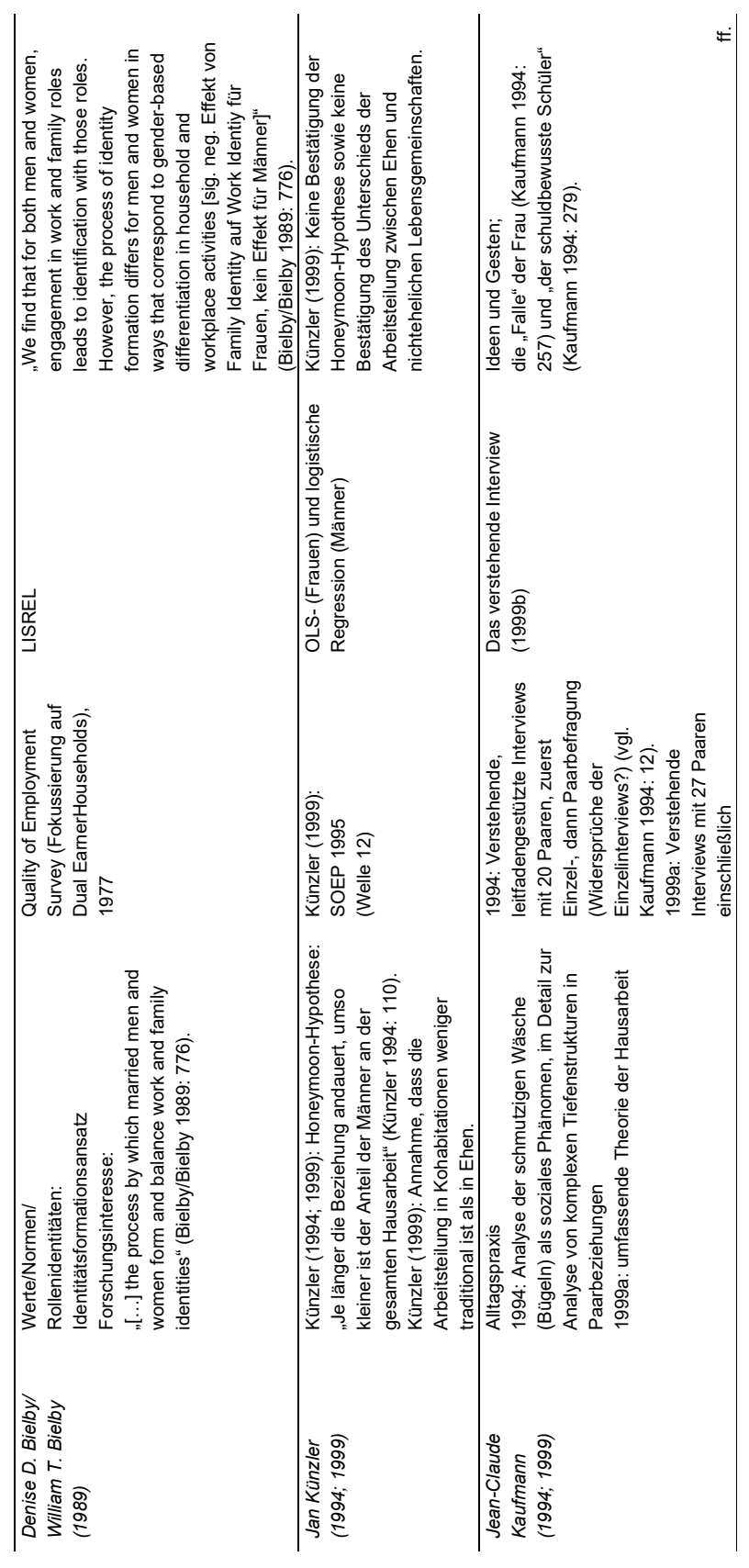




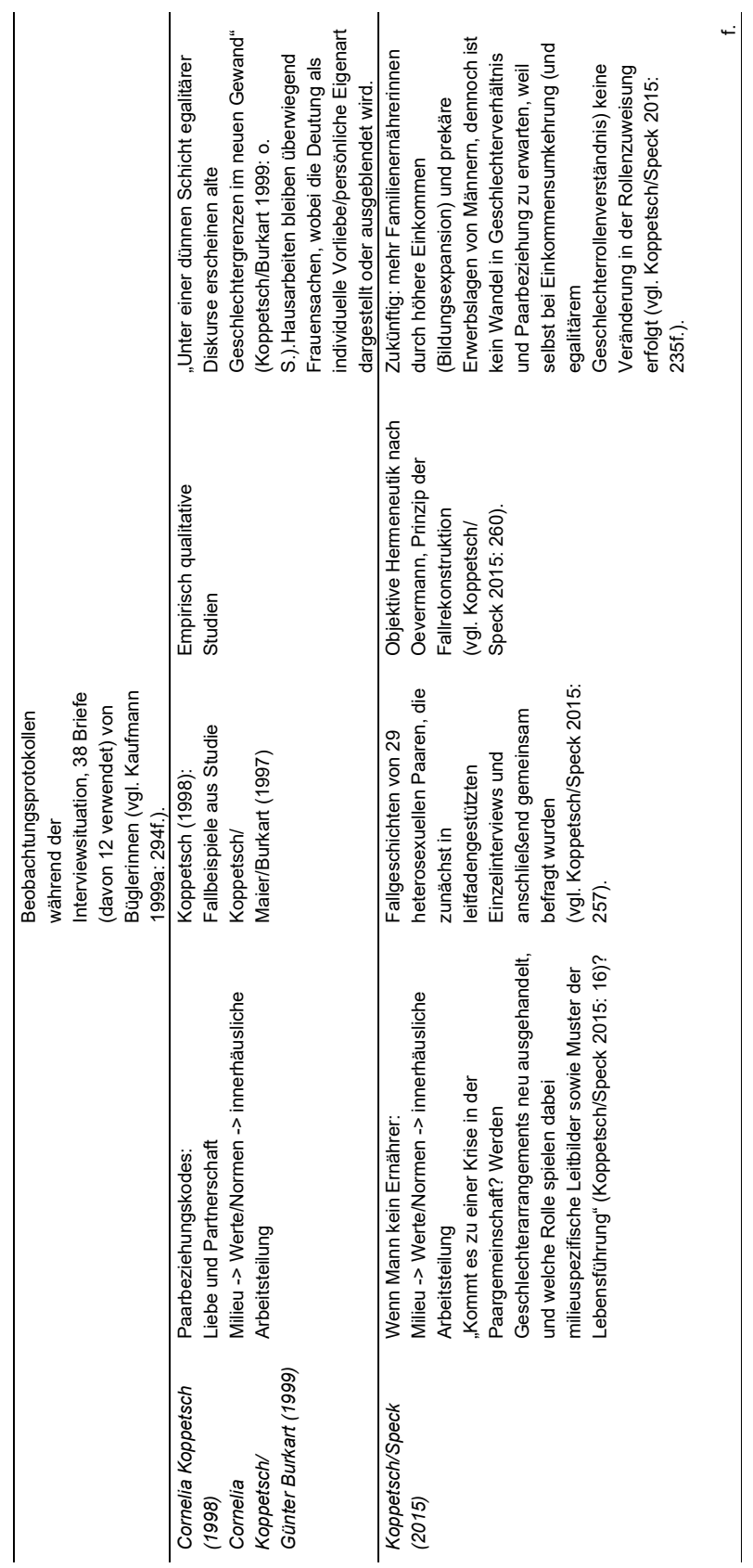




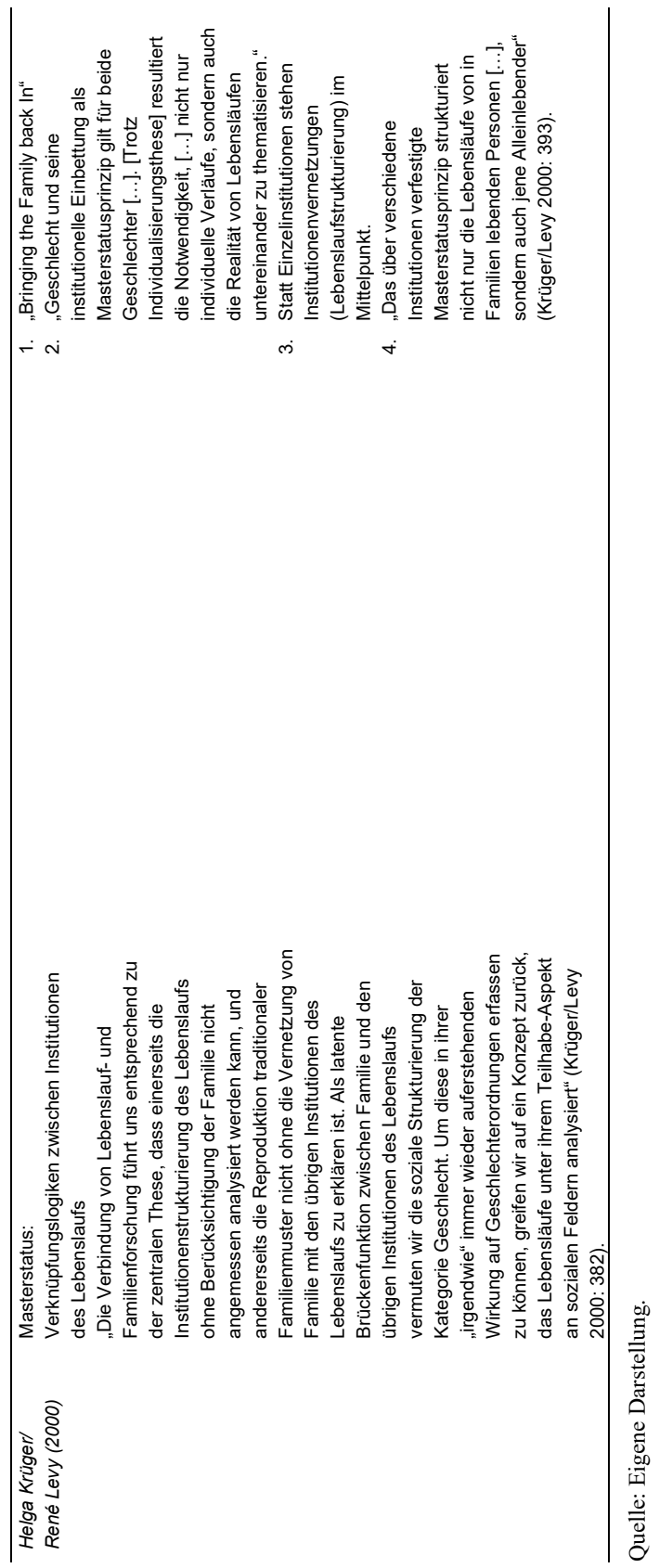




\subsection{Makrosoziologische Argumentation: Zum Verständnis von Gender-Ungleichheiten im sozialstrukturellen Kontext}

Nehmen wir an, es gäbe ein Paar XY; die Frau verfüge über einen höheren Bildungsabschluss als ihr Partner, bis zur Geburt des ersten Kindes auch über ein höheres Einkommen, beide teilen egalitäre Einstellungen und vollziehen eine partnerschaftliche Arbeitsteilung. Nach der Geburt des ersten Kindes würde das Paar gerne die egalitäre Arbeitsteilung weiterführen, doch gäbe es staatliche Regulierungen, die es Müttern bis zu einem Alter des Kindes von sieben Jahren erschweren würden, wieder erwerbstätig zu werden. So wäre die Auswahl an Handlungsmöglichkeiten, d. h. der Handlungsspielraum des Paares durch vorherrschende Normen drastisch eingeschränkt.

Dieses Beispiel ist zwar hypothetisch, soll jedoch verdeutlichen, dass mikrosoziologische Argumentationen wissenschaftlich sehr wertvoll sind, aber ausschließlich eine Teilerklärung liefern können. Nicht nur die Fähigkeiten und Ressourcenkonstellationen einzelner Personen, sondern auch die Entscheidungsfreiheit, aus einer Anzahl an Möglichkeiten diejenige auszuwählen, die Person X als subjektiv beste Handlungsalternative erachtet (Handlungsspielraum), gilt es für soziologische Erklärungen zu berücksichtigen. Um Genderungleichheiten adäquater messen zu können, ist nicht nur die Handlungsalternative, die eine Person letztlich wählt, auch die Handlungsalternative, die sie gerne wählen würde, ist mitzudenken.

Ferner gilt es, den makrosoziologischen Handlungsrahmen (respektive die „"harten“ sozialen Bedingungen") zu berücksichtigen, der sich unter dem Deckmantel ,sozialer Ungleichheiten“ formiert (Kreckel 1993: 57).

Soziale Ungleichheit ist dort gegeben,

„[...] wo die Möglichkeit des Zugangs zu allgemein verfügbaren und erstrebenswerten sozialen Gütern und/oder zu sozialen Positionen, die mit ungleichen Macht- und/oder Interaktionsmöglichkeiten ausgestattet sind, dauerhafte Einschränkungen erfahren und dadurch die Lebenschancen der betroffenen Individuen, Gruppen oder Gesellschaften beeinträchtigt bzw. begünstigt werden“ (Kreckel 2004: 17).

Soziale Ungleichheiten begrenzen den Handlungsrahmen - in der Definition von Kreckel als Möglichkeit des Zugangs, weiterführend als Einschränkung der Lebenschancen bezeichnet - zu sozialen Gütern und/oder sozialen Positionen. An dieser Stelle wird deutlich, dass Kreckels Definition aus makrosoziologischer Perspektive erfolgt, weil die Handlungsmöglichkeiten von Akteuren „einer strukturellen Argumentation untergeordnet werden“ (Kreckel 1993: 57). Doch schließt Kreckel die Erklärungsleistung sozialpsychologischer und sozialtheoretischer Argumente nicht grundsätzlich aus, jedoch ordnet er sie strukturellen Bedingungen unter, worin für ihn die Begründung 
liegt, sie außer Acht zu lassen (vgl. Kreckel 1993: 57). Die geschlechtsspezifische Trennung zwischen Reproduktions- und Produktionssphäre sieht Kreckel (im Durkheimschen Sinne) als historische Strukturtatsache und Folge des modernen Kapitalismus (vgl. Kreckel 1993: 57). „Das Alltagsleben der Menschen spielt sich in der Polarität zwischen privater Haushaltsführung und kapitalistisch oder bürokratisch organisierter Erwerbstätigkeit ab" (Kreckel 1993: 57). In Folge von Modernisierungsprozessen, einer Angleichung von Bildungschancen zwischen den Geschlechtern und einer, durch die Tertiarisierung hervorgebrachten, zunehmenden Integration von Frauen in die Erwerbstätigkeit (trotz Arbeitsmarksegregation) entsteht eine „doppelte Vergesellschaftung“. Das Konzept der ,doppelten Vergesellschaftung“ wird zu Recht von Kreckel durch seine Kritik an Regina Becker-Schmidt (1987) - die „doppelte Vergesellschaftung“ von Arbeitskraft zwischen Familien- und Berufsorientierung nicht nur auf den weiblichen Teil der Bevölkerung zu beziehen, sondern auf alle Gesellschaftsmitglieder - modifiziert (vgl. Kreckel 1993: 58). „Die “doppelte Vergesellschaftung" gilt in der bürokratischkapitalistischen Gesellschaft für beide Geschlechter" (Kreckel 1993: 58, Hervorhebungen im Original; die Verf.). Dennoch ist zu erwarten, dass insbesondere Frauen von den Folgen der ,doppelten Vergesellschaftung“ betroffen sind,

,[...] weil man [...] davon ausgehen muß [sic!], daß [sic!“"] das strukturell bedingte Machtgefälle zwischen Produktions- und Reproduktionssphäre solange fortbesteht, wie marktwirtschaftliche Verhältnisse herrschen“" (Kreckel 1993: 61).

Die explizite Betonung eines unabdingbaren Wechselwirkungsverhältnisses von Makro- und Mikrokosmos wird im Gegensatz zu Kreckel nachfolgend durch die Argumentation von Sen hervorgehoben. Sens Capability Approach stellt die Rahmentheorie der vorliegenden Arbeit dar. Zuvor steht noch eine Begriffsklärung von Gender-Ungleichheiten aus: Gender-Ungleichheiten sind als eine spezifische Form sozialer Ungleichheiten zu verstehen, die beinhalten, wie die Zugangsmöglichkeiten zu verschiedenen Bereichen (Bildung, Arbeitsmarkt etc.) zwischen den Geschlechtern variieren (vgl. Plantenga et al. 2009: 22; Arpino et al. 2015: 2). Ferner können Genderungleichheiten als ein Mangel an Verwirklichungschancen verstanden werden. $\mathrm{Zu}$ betonen ist das Kreckel-Argument eines übergeordneten Bezugsrahmens:

„Wenn man also davon ausgehen muss, dass lokale, regionale, nationale und internationale Ungleichheitsverhältnisse einerseits eng miteinander verbunden, andererseits durch politische Grenzziehungen voneinander getrennt sind, so bedeutet dies, dass ihre sozialwissenschaftliche Erforschung unter einem ,gemeinsamen begrifflichen Dach" stattfinden muss, also: innerhalb eines übergreifenden konzeptionellen Bezugsrahmens" - für Kreckel das Dach der globalen Ungleichheit (Kreckel 2006: 5, Hervorhebungen im Original; die Verf.). 
In der vorliegenden Dissertation wird keineswegs der Versuch angestrebt, eine ,globale Welttheorie“ zu entwerfen, doch zumindest wird ein übergeordneter theoretischer Bezugsrahmen im europäischen Kontext entwickelt, ohne kulturspezifische Detailinformationen zu vernachlässigen. Auch Kreckels Forderung zur Verpflichtung der Soziologie sozialer Ungleichheit ,[...] ihre Forschungen als Mehrebenen-Analysen anzulegen, die nicht an nationalen (und auch nicht an Schengener) Grenzen halt machen" wird nachgegangen (Kreckel 2006: 32). Dies zeigt sich in einem nationenübergreifenden theoretischen Rahmen, wobei die Detailinformationen durch die Analyseeinheiten von NUTS1-Regionen abgebildet werden. Es gilt, und hier argumentiert Kreckel überzeugend, den „methodologischen Nationalismus ${ }^{42}$ “ bzw. das „nationalstaatliche Containermodell“ zu überwinden. Auch „Amartya Sen [weist] die für die neuere Ungleichheitsforschung sehr einflussreiche Gerechtigkeitstheorie von John Rawls in ihre Schranken, indem er ihr ,nationalen Partikularismus“" attestiert“ (Kreckel 2006: 6).

42 „Lange Zeit unterstellte die Gesellschaftstheorie als ihre Untersuchungseinheit den Nationalstaat; die Begriffe „Gesellschaft" und „Kultur" bezogen sich unreflektiert auf das, was man als abgegrenzte, unabhängige und relativ homogene Einheiten wahrnahm, die sich durch nationale Grenzen, Institutionen und Gesetze konstituierten. Dementsprechend ging das theoretische Nachdenken von der unhinterfragten Annahme aus, dass sich Nation, Territorium, Gesellschaft und Kultur nahtlos ineinander fügen“ (Beck/Grande 2010: 189). Beck/Grande plädieren folglich für eine Überwindung des methodologischen Nationalismus durch eine „kosmopolitische Wende“, deren Kern sich folgendermaßen auszeichnet: „In der Zweiten Moderne muss die soziologische und politikwissenschaftliche Theoriebildung die ganze „Pluralität“ der Modernisierungspfade durchschreiten, die westlichen und nicht-westlichen Erfahrungen und Perspektiven mitsamt ihren Dependenzen, Interdependenzen und Interaktionen einbeziehen. [...] [Es handelt sich um] einen Ansatz, der die Variationen der Moderne und ihre globalen Interdependenzen als Ausgangspunkt theoretischer Überlegungen und empirischer Forschung nimmt“ (Beck/ Grande 2010: 190). 


\section{Ein multidimensionaler Ansatz: Der Capability Approach nach Amartya K. Sen „Freiheit - Gleichheit - Gerechtigkeit?“}

\subsection{Einführung zur Ausgangsproblematik einer ressourcenorientierten Messung sozialer Ungleichheiten}

Der Nobelpreisträger von 1998, Amartya Sen (Ökonom und Philosoph), übt Kritik am neoklassischen Ansatz und betont die Relevanz von Normen sowie Rollenzuschreibungen, wodurch er der feministischen Kritik am neoklassischen Ansatz durchaus nahe steht (vgl. Ott 1999: 167). Sen konnte mit seinem Befähigungsansatz „Capability Approach“ die Verknüpfung von Befähigungen und dem Chancenaspekt der Freiheit theoretisch herleiten. Darüber hinaus konnte er zur Messung individueller Vorteile anhand der Befähigung einer Person, diejenige Handlungsalternative zu wählen, die sie am meisten schätzt (bzw. die Befähigung das Leben zu führen, das die Person für sich selbst am besten bewertet), beitragen (vgl. Sen 2010a: 259). Sein Vorwurf ist insbesondere an die Mikroökonomie gerichtet und beinhaltet, dass bisherige Messungen von sozialen Ungleichheiten, im Spezifischen Armutskonzepte, ausschließlich auf Ressourcen basieren, d. h. die Freiheit einer Person, diese oder jene Handlungsalternative zu wählen, ausblenden. Wohlfahrt bedeutet in der Theorie von Sen mehr als das bloße Verfügen über Ressourcen, sie impliziert die Freiheit der Auswahlmöglichkeiten diverser Lebensentwürfe.

„Der Befähigungsansatz konzentriert sich auf das Leben, das Menschen führen können, und nicht auf ihre Ressourcen, das heißt den Besitz - oder die Nutzung von Bedarfsgütern, über die sie verfügen“" (Sen 2010a: 281).

Beispielsweise sei eine Person, die über finanziellen Reichtum verfüge, jedoch aufgrund von körperlichen Einschränkungen nicht jede beliebige Handlungsalternative auswählen könne, benachteiligt. Auch wenn ein/e RollstuhlfahrerIn das gleiche Einkommen wie ein/e Nicht-RollstuhlfahrerIn erhalten würde, wäre der/die RollstuhlfahrerIn im Vergleich zu einem/einer NichtRollstuhlfahrerIn nicht gleichermaßen befähigt, dieselben Dinge zu tun bzw. dasselbe Ausmaß an Mobilität zu erreichen. Diese Form der Benachteiligung, die Einschränkung des Handlungsspielraumes, müsse zentraler Bestandteil der Messung von sozialen Ungleichheiten werden anstatt Ungleichheiten ausschließlich auf die Einkommensthematik zu begrenzen ${ }^{43}$.

43 Etwas differenzierter verdeutlicht Sen seine Argumentation des Befähigungsansatzes mittels der folgenden drei Szenarien eines Menschen mit körperlicher Behinderung (vgl. Sen 2010a: 332f.): 
Mit seinem Argument in „Development as Freedom“ - „Development can be seen, it is argued here, as a process of expanding the real freedoms that people enjoy" (Entwicklung heißt für Sen Freiheit respektive die Ausweitung von Verwirklichungschancen. Die Frage nach der Entwicklung ist für Sen folglich immer verbunden mit der Frage, inwiefern Menschen befähigt werden) - wendet sich Sen explizit gegen Gerechtigkeitstheorien, die Entwicklung in einem engeren Sinne auf die Verteilung von Grundgütern beziehen, wie vornehmlich die Gerechtigkeitstheorie nach John Rawls (Sen 1999: 3). Rawls und Sen sind sich darin einig, dass der Freiheit eine hohe Bedeutung zukommt, doch hat für Sen Freiheit einen höheren Stellenwert als das Verfügen über Grundgüter. Messgrößen, die häufig als Indikatoren für Entwicklung herangezogen werden, wie das Bruttoinlandsprodukt, sind für Sen eher ein Mittel zum Zweck, doch, so seine These, ist Freiheit mehr als das. Freiheit hänge auch von anderen Determinanten, wie z. B. Bildung, Gesundheit oder politischen Rechten (beispielsweise ein Recht auf freie Meinungsäußerung), ab (vgl. Sen 1999: 3).

„If freedom is what development advances, then there is a major argument for concentrating on that overarching objective, rather than on some particular means, or some specially chosen list of instruments. Viewing development in terms of expanding substantive freedoms directs attention to the ends that make development important, rather than merely to some of the means that, inter alia, play a prominent part in the process" (Sen 1999: 3).

Zudem werden durch die Erfassung des Bruttoinlandsprodukts keinerlei Aussagen über die Einkommensverteilung getroffen. Normative Gerechtigkeitstheorien fordern ,[...] die Gleichheit von etwas, von etwas, das in der jeweiligen Theorie als besonders wichtig gilt" - für Rawls z. B. die Grundgüter (Sen 2010a: 318, Hervorhebungen im Original; die Verf.). Wir müssten die

1. Person $\mathrm{x}$ bekommt keine Hilfe von anderen und kann daher das Haus nicht verlassen.

2. Person $x$ erhält Unterstützung von einem Pflegedienst und kann daher zu jeder Zeit das Haus verlassen.

3. Person $x$ verfügt über DienstbotInnen, die dem Befehl gehorchen müssen, Person $x$ jeder Zeit einen Ausgang aus dem Haus zu ermöglichen.

Gemäß dem Befähigungsansatz werden die zweite und dritte Situation nicht weiter differenziert, da es in diesem Beispiel um die Perspektive der Person mit Behinderung und nicht um die Freiheit der DienstbotInnen geht. Beide Szenarien stehen im Gegensatz zum ersten Szenario, weil sie die Befähigung der Person x, das Haus verlassen zu können, darstellen. Im ersten Szenario ist diese Befähigung nicht gegeben, weshalb die Person in dieser Hinsicht unfrei ist. „Diesen Unterschied versucht der Befähigungsansatz zu erfassen, und dass man ihn anerkennt, ist folgenschwer sowohl im Allgemeinen wie für politische Maßnahmen im Besonderen. Wollten wir die Fälle 1 und 2 ohne weiteres in die gleiche Schublade namens Nicht-Freiheit stecken [wie es der republikanische Ansatz tun würde], würden wir damit auf die Ansicht zusteuern, dass die Einrichtung von Sozialfürsorge oder eine hilfreiche Gesellschaft keinen Unterschied für die Freiheit eines Menschen im Umgang mit seinen Behinderungen oder Handicaps machen kann. Für eine Theorie der Gerechtigkeit wäre das eine ungeheure Lücke“ (Sen 2010a: 333). 
fundamentale Ähnlichkeit jeglicher normativer Gerechtigkeitstheorien erkennen, die allesamt für „Gleichheit in irgend einem Bereich plädieren“ und die Vorrangstellung des für ihren relevanten Gleichheitsbereichs durchzusetzen versuchen, wobei sie die Gleichheitsforderungen konkurrierender Gerechtigkeitstheorien als weniger relevant erachten und folglich nicht gelten lassen (Sen 2010a: 322, Hervorhebungen im Original; die Verf.). Ferner ist es Sen zufolge notwendig, eine Theorie der Gerechtigkeit in einem weiteren Sinne zu entwickeln.

„Sie soll klären, wie wir verfahren können, wenn wir Fragen der Erweiterung von Gerechtigkeit und Beseitigung von Ungerechtigkeit in Angriff nehmen wollen; sie hat nicht das Ziel, Antworten auf die Frage nach dem Wesen vollkommener Gerechtigkeit zu bieten. Dadurch unterscheidet sie sich deutlich von den Theorien der Gerechtigkeit, die in der gegenwärtigen politischen und Moralphilosophie das Feld beherrschen" (Sen 2010a: 9).

Sen erwähnt drei Aspekte, wie sich sein Ansatz der Gerechtigkeit von den klassischen Gerechtigkeitstheorien unterscheiden sollte:

1. Im Gegensatz zu den normativen Theorien der Gerechtigkeit der politischen Philosophie sollte sich die Theorie nicht ausschließlich auf die Bestimmung vollkommen gerechter Gesellschaften konzentrieren (vgl. Sen 2010a: 9).

Die Theorie ,[...] sollte sich nicht ausschließlich auf die Charakterisierung vollkommen gerechter Gesellschaften konzentrieren, wie es in den Theorien der Gerechtigkeit der politischen Philosophie von heute häufig geschieht. Es gibt Zusammenhänge zwischen diesen beiden verschiedenen Zielsetzungen, aber trotzdem sind sie analytisch voneinander entkoppelt. Das Ziel, auf das sich dieses Buch [Die Idee der Gerechtigkeit] bezieht, hat zentrale Bedeutung für Entscheidungen über Institutionen, Verhaltensweisen und andere Determinanten der Gerechtigkeit; und die Ableitung solcher Entscheidungen muss die wichtigste Aufgabe einer Theorie der Gerechtigkeit sein, die als Richtlinie für praxisorientierte Überlegungen dienen soll. Die Behauptung, dass diese vergleichende Arbeit erst möglich sei, nachdem die Aufforderungen der vollkommenen Gerechtigkeit geklärt wurden, diese Behauptung ist ganz und gar falsch [zum Nachweis s. Kapitel 4.]“ (Sen 2010a: 9).

2. Vertritt Sen die These, ,[...] dass mehrere verschiedene [konkurrierende] Gründe der Gerechtigkeit nebeneinander bestehen können [im Sinne einer Wertepluralität], die alle kritischer Prüfung standhalten, aber $\mathrm{zu}$ unterschiedlichen Folgerungen führen [sein berühmtes Gleichnis hierzu ist »drei Kinder und eine Flöte ${ }^{44} \ll$, s. Sen

44 Drei Kinder streiten sich über den Besitz einer Flöte: Das erste Kind, Anne, ist das einzige, das Flöte spielen kann. Das zweite Kind, Bob, ist so arm, dass es kein einziges Spielzeug besitzt und das dritte Kind, Carla, hat die Flöte selbst gebaut (vgl. Sen 2010a: 41). Wie würden Sie entscheiden, welchem Kind die Flöte gehören sollte? Während Bob die meiste Unterstützung von ökonomischen EgalitarierInnen erhalten würde, um seinen Nachteil an ökonomischen Mitteln zu verringern, würde Carla Unterstützung von Libertären erhalten. 
2010a: 41ff.]. [...] Die Pluralität, mit der wir dann enden, wird das Resultat des Vernunftgebrauchs, nicht des Verzichts auf vernünftiges Denken sein“ (Sen 2010a: 10).

3. AnhängerInnen des ,transzendentalen Institutionalismus“ fassen ideale Institutionen als Maßstab für eine vollkommene Gerechtigkeit auf, versäumen aber, einen relationalen Vergleich von mehr oder weniger Gerechtigkeit zu erfassen. Statt Gerechtigkeitstheorien auf die Einrichtung idealer, gerechter Institutionen im Sinne eines ,transzendentalen Institutionalismus“ zu beziehen, sollte die Perspektive auf das Leben, das Menschen führen können, bezogen werden - verfehlen doch alle Gesellschaften das Ideal der Vollkommenheit (vgl. Sen 2010a: 33f.). So würden beispielsweise Rawls Gerechtigkeitsgrundsätze ausschließlich auf die Einrichtung von gerechten Institutionen abzielen. Ferner würde eine vollkommene, makellose Anpassung des Verhaltens von Menschen an die Bedingungen eines Funktionierens der gerechten Institutionen verlangt (vgl. Sen 2010a: 11).

Aber: ,Auch wenn Gerechtigkeitsgrundsätze im hier vorgelegten Ansatz nicht im Hinblick auf Institutionen, sondern im Hinblick auf das Leben und Freiheiten der betroffenen Menschen definiert werden, haben Institutionen dennoch für die Förderung von Gerechtigkeit zwangsläufig eine wichtige instrumentelle Rolle“ (Sen 2010a: 12f.).

Die Grundidee, derer der multidimensionaler Ansatz Sens zugrunde liegt und gleichwohl seine indischen Wurzeln zum Ausdruck bringt, ist die Differenzierung zweier Ausdrücke für Gerechtigkeit - „,niti“ und ,nyaya“ - aus der frühen indischen Rechtslehre.

„Niti bezeichnet die Korrektheit von Institutionen und Verhalten, während nyaya erfasst, was entsteht und wie es entsteht, und besonders darauf achtet, welches Leben Menschen tatsächlich führen können [verwirklichte Gerechtigkeit]. Der Unterschied zwischen beiden Konzepten, [...] hilft uns, zu begreifen, dass es zwei Arten des Gerechtseins gibt, die verschieden, wenn auch nicht unabhängig voneinander sind, und dass die Idee der Gerechtigkeit beide berücksichtigen muss“ (Sen 2010a: 15).

Weil Anne die einzige ist, die die Flöte spielen kann, würde ihr von UtilitaristInnen der Besitz der Flöte zugesprochen, da ihr Vergnügen wahrscheinlich das größte ist (vgl. Sen 2010a: 42). 


\subsection{Zur Dichotomie eines auf Regeln und eines auf Realisierung konzentrierten Verständnisses von Gerechtigkeit}

Kritik übt Sen an der zweiteiligen Gruppierung von führenden aufklärerischen PhilosophInnen respektive an der ,substanzielle[n] Dichotomie hinsichtlich ihrer Auffassung von Gerechtigkeit" (Sen 2010a: 16). Die erste kontraktarische Gruppe des transzendentalen Institutionalismus widmet sich primär ihrer Hauptaufgabe einer Charakterisierung gerechter Institutionen in Form eines hypothetischen Gesellschaftsvertrages (Thomas Hobbes, John Locke, Jean-Jacques Rousseau, Immanuel Kant), während sich die zweite komparative Gruppe (Adam Smith, Marquis de Condorcet, Mary Wollstonecraft, Jeremy Bentham, Karl Marx, John Stuart Mill) dem auf Verwirklichung konzentrierten Vergleich verschiedener Möglichkeiten, ein Leben zu führen, widmet - „Möglichkeiten, die von Institutionen, aber auch tatsächlichen Verhaltensweisen, sozialen Interaktionen und anderen signifikanten Determinanten beeinflusst werden“" (Sen 2010a: 16). Sens Idee der Gerechtigkeit stützt sich zwar nicht ausschließlich, aber dennoch in hohem Ausmaß auf die zweite Tradition. Doch betont Sen nicht nur Unterschiede beider Paradigmen, sondern auch ihre Ähnlichkeiten des Vertrauens auf vernünftiges Denken und ihre Forderungen eines öffentlichen Diskurses (vgl. Sen 2010a: 16). Sen sieht den Ausgangspunkt seiner Idee der Gerechtigkeit folglich im Versuch, ,[...] die Realisierungsvergleiche zu überprüfen, die Fortschritte oder Rückschritte der Gerechtigkeit ins Visier [zu] nehmen“ - die Frage ist nicht, ob eine Gesellschaft gerecht ist, sondern ob sie gerechter oder ungerechter als eine andere ist (Sen 2010a: 37). Für ihn ist beispielsweise die Frage, „Wie kann Gerechtigkeit erhöht werden?“ zentral, während die Frage nach vollkommen gerechten Institutionen im Rahmen seiner theoretischen Annahmen nicht beantwortet werden kann (Sen 2010a: 37). Hieraus erzielt er insofern einen doppelten Effekt, als erstens ein komparativer anstelle eines die Realität überschreitenden Weges eingenommen wird und zweitens anstelle eines ausschließlichen Bezuges auf Institutionen und Regeln tatsächliche Realisierungen im Fokus stehen (vgl. Sen 2010a: 37). Der transzendentale Institutionalismus wird aufgrund mangelnder Realisierbarkeit und Redundanz als problematisch erachtet. Das zentrale Problem der Realisierbarkeit sieht Sen in der Pluralität konkurrierender Grundsätze, ferner in der „Frage, ob sich vielfältige und konkurrierende Begründungen für Gerechtigkeit aufrechterhalten lassen, die sämtliche Ansprüche auf Unparteilichkeit haben und trotzdem voneinander verschieden sind - und einander widerstreiten [s. Bsp. „drei Kinder und eine Flöte“]" (Sen 2010a: 41). Für jedes Argument mag es Begründungen geben, warum es gerecht ist, doch ist es willkürlich und abhängig von der Betrachtungsperspektive, ein Argument über ein anderes zu 
stellen und zu argumentieren, es sei gerecht, während die anderen ungerecht seien (vgl. Sen 2010a: 43). Insofern ist es nicht möglich, etwas als vollkommen gerecht $\mathrm{zu}$ identifizieren. UtilitaristInnen, ökonomische EgalitarierInnen, ArbeitsrechtstheoretikerInnen und Libertäre könnten alle der Auffassung sein, dass es eine vollkommen gerechte Lösung für den Streit der drei Kinder um den Besitz der Flöte gäbe, doch würden sie allesamt eine andere richtige Lösung präferieren (Sen 2010a: 42f.). Die Problematik der Redundanz erläutert Sen am Beispiel der Wahl zwischen zwei Bildern von Picasso und Dalí: Gesetzt den Fall, dass die Festlegung der Mona Lisa als ideales Gemälde für die ganze Welt möglich wäre, helfe uns diese Diagnose dennoch nicht bei der Entscheidung (vgl. Sen 2010a: 44). Allgemeiner formuliert bedeutet dies:

„Wenn eine Theorie der Gerechtigkeit Leitfaden für rationale Wahl von Grundsätzen, Strategien oder Institutionen sein soll, dann ist die Bestimmung von vollkommen gerechten sozialen Regelungen weder notwendig noch hinreichend“ (Sen 2010a: 44) - ,[...] die Festlegung einer transzendentalen Alternative [bietet] keine Lösung für die Aufgabe, zwei nicht-transzendentale Alternativen miteinander zu vergleichen. [...] Stattdessen benötigen wir eine auf öffentlichem Vernunftgebrauch beruhende Einigung über die Rangfolge von Alternativen, die verwirklicht werden können“ (Sen 2010a: 45).

Sen schlussfolgert aus seiner Kritik die These, dass eine Theorie benötigt wird, die sich nicht auf die Bestimmung von gerechten Institutionen und idealen sozialen Regelungen begrenzt, sondern die Perspektive auf das Leben, das Menschen führen - oder nicht führen - können richtet. Weil die Frage, welches Leben Individuen führen möchten, eine höchst subjektive Eigenart menschlicher Lebensführung darstellt, sind die Freiheiten, eine Auswahl zwischen verschiedenen Lebensformen treffen zu können, ebenfalls zu bedenken. Diese Freiheit trägt maßgeblich zum Wohlergehen bei und ist abgesehen vom Wohlergehen - gar darüber hinaus - als wesentlich zu verstehen (vgl. Sen 2010a: 46f.). Der Perspektivenwechsel besteht darin, ,[...] soziale Verwirklichungen nicht nach den Kriterien von Nutzen oder Glück [zu] beurteil[en] (wie Jeremy Bentham und andere Utilitarist[Inn] $\mathrm{en}^{45} \mathrm{emp}$ fehlen), sondern an den Befähigungen oder Chancen [abzulesen], die Menschen tatsächlich haben [s. Unterkapitel Functionings und Capabilities]" (Sen 2010a: 47).

„Die Freiheit der Wahl gibt uns die Möglichkeit zu entscheiden, was wir tun sollten, aber damit zugleich auch die Verantwortung für das, was wir tun - soweit

45 Der Utilitarismus als Indikator des well-beings ist für Sen nicht adäquat, denn: „Welfarism in general and utilitarianism in particular see value, ultimately, only in individual utility, which is defined in terms of some mental characteristic, such as pleasure, happiness, or desire. This is a restrictive approach to taking note of individual advantage in two distinctive ways: (1) it ignores freedom and concentrates only on achievements, and (2) it ignores achievements other than those reflected in one of these mental metrics" (Sen 1992: 6). 
unsere Handlungen frei gewählt sind. Da eine Befähigung das Vermögen ist, etwas zu tun, erfasst die neue Perspektive auch die Verantwortlichkeit, die aus diesem Vermögen - dieser Macht - hervorgeht, und das kann Raum für Pflichtgebote, in weitem Sinne deontologische Forderungen, schaffen. Hier ergibt sich eine Überschneidung zwischen handlungsorientierten Überlegungen und den Implikationen des Befähigungsansatzes, aber nichts unmittelbar mit der utilitaristischen Perspektive Vergleichbares, da diese uns nur die Verantwortung für unser Glück zuschiebt“" (Sen 2010a: 48, Hervorhebungen durch die Verf.).

\subsection{Sens Argumentation in Abgrenzung zu Rawls}

Rawls versteht Gerechtigkeit als Fairness (A Theory of Justice (1971)), wobei Fairness auf dem allgemeinen Grundsatz der Unparteilichkeit ${ }^{46}$ basiert (vgl. Sen 2010a: 82; Sen 2010b: 54). Die Forderung auf Unparteilichkeit erörtert Rawls anhand seiner zentralen Konzeption des Urzustandes, der eine fiktive Situation darstellt, in der alle Beteiligten eine ursprüngliche Gleichheit erfahren, weil sie ihre eigenen Identitäten und die Positionen innerhalb der Gruppe nicht kennen. Umhüllt von einem „Schleier des Nichtwissens“ (ohne Kenntnisse von persönlichen Interessen, Ansichten über das gute Leben und Präferenzen) werden, so die hypothetische Annahme, Gerechtigkeitsprinzipien einstimmig und vernünftig unter gleichen demokratischen Prinzipien gewählt, die weiterführend in der Bestimmung sozialer Institutionen münden.

Da die Entscheidung aufgrund eines Informationsdefizits des Schleiers des Nichtwissens erfolgt, führt Rawls die Maximin-Regel ein, nach der die Beteiligten notwendigerweise gerecht entscheiden. Das Entscheidungskriterium stellt insofern die Maximin-Regel dar, nach der ein ausreichendes Minimum für jeden gewährleistet wird, als diejenige Alternative gewählt wird, die auch für das am schlechtesten gestellte Mitglied der Gesellschaft einen Vorteil erbringt:

„Die Maximin-Regel ordnet die Alternativen nach ihren schlechtesten möglichen Ergebnissen: man soll diejenigen wählen, deren schlechtestmögliches Ergebnis

46 „It can broadly be seen as a demand for impartiality. Rawls' specification on the demands of impartiality is based on his constructive idea of the 'original position', which is central to his theory of 'justice as fairness'. The original position is an imagined situation of primordial equality, when the parties involved have no knowledge of their personal identities, or their respective vested interest, within the group as a whole. Their representatives have to choose under this 'vail of ignorance', that is, in an imagined state of selective ignorance (in particular, ignorance about the distinct personal interests and actual views of a good life what Rawls calls 'comprehensive preferences') and it is in that state of devised ignorance that the principles of justice are chosen unanimously. The principles of justice, in a Rawlsian formulation, determine the basic social institutions that should govern the society they are, we imagine, about to 'create' (Sen 2010b: 54; vgl. Sen 2010a: 82). 
besser ist als das jeder anderen. [...] die Regel lenkt unsere Aufmerksamkeit auf das Ungünstigste, was bei irgendeiner der betrachteten Handlungen geschehen kann, und weist uns an, die Entscheidung in diesem Lichte zu treffen ${ }^{47 \text { “ (Rawls }}$ 1996: 178).

Die gewagte These Rawls ist ferner, dass die politische Konzeption von Gerechtigkeit als Fairness zu einer einstimmigen Entscheidung für die Grundsätze führen müsse:

„Rawls identifies some very specific principles of justice [...], and makes the strong claim that these principles would be unanimous choice that would emerge from the political conception of justice as fairness" (Sen 2010b: 56; vgl. Sen 2010a: 82).

Er argumentiert, dass alle im Urzustand einer ursprünglichen Gleichheit diese Grundsätze wählen würden und es insofern legitim sei, diese als angemessene politische Konzeption von Gerechtigkeit zu begreifen. Erzielt wird durch das Konzept der Fairness als Gerechtigkeit, „,...] [to] identifying appropriate principles that would determine the choice of just institutions needed for the basic structure of a society" (Sen 2010b: 56; vgl. Sen 2010a: 82). Die einstimmige Wahl bezieht sich insofern auch auf die Wahl von Institutionen für die Grundstruktur der Gesellschaft und die Festlegung der politischen Konzeption von Gerechtigkeit:

„So the unanimous choice of these principles of justice does quite a bit of work in the Rawlsian system, which includes the choice of institutions for the basic structure of society, as well as the determination of a political conception of justice, which Rawls presumes will correspondingly influence individual behaviours in conformity with that shared conception" (Sen 2010b: 56, Hervorhebungen im Original; die Verf.; vgl. Sen 2010a: 82).

Rawls beschreibt vier Stufen der Entwicklung sozialer Gerechtigkeit: 1. Wahl der Grundsätze 2. konstitutionelle Stufe: Wahl der Institutionen in Übereinstimmung mit Gerechtigkeitsprinzipien 3. legislative Stufe: Arbeit der Institutionen führt zu Entscheidungen (Gesetzgebung) 4. Schritt für Schritt entwickeln sich vollkommen gerechte gesellschaftliche Ordnungen (vgl. Sen 2010a: 84; Sen 2010b: 56). Große Skepsis äußert Sen an Rawls hinsichtlich der Annahme, dass im Urzustand einstimmig ein einziger Satz von Prinzipien für gerechte Institutionen gewählt würde, denn nach Sen gibt es eine Vielzahl von, unter Umständen auch widersprechenden, Interessen:

47 Rawls führt als Beispiel eine Gewinn-Verlust-Tabelle (s. Anhang Tabelle 26) an, die Geldbeträge im Vergleich zur Ausgangssituation darstellt. Gemäß der Maximin-Regel wird die dritte Entscheidung getroffen, weil in diesem Fall das ungünstigste Ergebnis ein Gewinn von $5 €$ ist, was einen höheren Gewinn darstellt als das schlechtmöglichste Ergebnis der ersten beiden Entscheidungen (ein Verlust von $7 €$ im Falle von Entscheidung 1 bzw. ein Verlust von $8 €$ im Falle von Entscheidung 2) (vgl. Rawls 1996: 178). 
„I have to express considerable scepticism about Rawls's highly specific claim about the unique choice, in the original position, of one particular set of principles for just institutions, needed for a fully just society. There are genuinely plural, and sometimes conflicting, general concerns that bear on our understanding of justice" (Sen 2010b: 56f.; vgl. Sen 2010a: 85).

Folglich kann Unparteilichkeit verschiedene Formen annehmen: „I would argue, reflect the fact that impartiality can make different forms and have quite distinct manifestations" (Sen 2010b: 57; vgl. Sen 2010a: 85). So ist jedes Argument des Flötenbeispiels durch eine allgemeine Theorie begründet, gleichwohl sie unterschiedlichen Grundsätzen folgen (vgl. Sen 2010a: 85; Sen 2010b: 57).

In „Political Liberalism“ (1993) behauptet Rawls als Vertreter des egalitären Liberalismus, dass folgende Grundsätze im Urzustand einstimmig angenommen würden (vgl. Sen 2010a: 87; Sen 2010b: 59):

1. „Erster Grundsatz: Jedermann hat das gleiche Recht auf das umfangreichste Gesamtsystem gleicher Grundfreiheiten, das für alle möglich ist" (Rawls 1996: 336, Hervorhebungen im Original; die Verf.). Gleiche Grundfreiheiten für alle, , ,...] which is compatible with a similar scheme of liberties for all“ (Sen 2010b: 59).

2. „Zweiter Grundsatz: Soziale und wirtschaftliche Ungleichheiten müssen folgendermaßen beschaffen sein:

(a) sie müssen unter der Einschränkung des gerechten Spargrundsatzes den am wenigsten Begünstigten den größtmöglichen Vorteil bringen, und

(b) sie müssen mit Ämtern und Positionen verbunden sein, die allen gemäß fairer Chancengleichheit offenstehen" (Rawls 1996: 336, Hervorhebungen im Original; die Verf.).

Es gelten hierbei folgende Vorrangregeln:

1. „Erste Vorrangregel: Die Gerechtigkeitsgrundsätze stehen in lexikalischer Ordnung; demgemäß können die Grundfreiheiten nur um der Freiheit willen eingeschränkt werden, und zwar in folgenden Fällen:

(a) eine weniger umfangreiche Freiheit muß [sic!] das Gesamtsystem der Freiheiten für alle stärken;

(b) eine geringere als gleiche Freiheit muß [sic!] für die davon Betroffenen annehmbar sein.

2. Zweite Vorrangregel (Vorrang der Gerechtigkeit vor Leistungsfähigkeit und Lebensstandard): Der zweite Gerechtigkeitsgrundsatz ist dem Grundsatz der Leistungsfähigkeit und Nutzenmaximierung lexikalisch vorgeordnet; die faire Chancengleichheit ist dem Unterschiedsprinzip vorgeordnet, und zwar in folgenden Fällen:

(a) eine Chancen-Ungleichheit muß [sic!] die Chancen der Benachteiligten verbessern; 
(b) eine besonders hohe Sparrate muß [sic] insgesamt die Last der von ihr Betroffenen mildern" (Rawls 1996: 336f., Hervorhebungen im Original; die Verf.).

Für die Analyse der Verteilungsgleichheit von Ressourcen erstellt Rawls weiterführend eine Liste an primären Grundgütern: u. a. Ressourcen, Rechte, Freiheiten, Einkommen, Vermögen (vgl. Sen 2010a: 88; Sen 2010b: 60).

\subsubsection{Positive Argumente von Rawls, die Sen teilt}

Sen übt Rawls gegenüber nicht nur negative Kritik, sondern sieht auch folgende der rawlsschen Thesen als ertragreich an:

1. Der Grundgedanke, dass Fairness für eine Gerechtigkeitstheorie zentral ist, ginge über die bisherigen Konzeptionen von Gerechtigkeit hinaus, selbst wenn von Sen die Unparteilichkeit als nicht zweckdienlich erachtet wird ${ }^{48}$.

2. Die besondere Rolle der Objektivität in der praktischen Vernunft.

3. Die Differenzierung zwischen rationalem und vernünftigem Verhalten $^{49}$. Menschen verfügen über ein moralisches Vermögen - „moral power" -, das mit ihrer „,capacity for a sense of justice“ und „for a conception of the good" verbunden ist (Sen 2010b: 63; vgl. Sen 2010a: 91). Im Gegensatz zu einigen Versionen der Rational Choice Theorie geht dieses Verständnis weit über ein rationales Eigeninteresse hinaus.

4. Die Vorrangigkeit der Freiheit hat absolute Priorität - es ist jedoch eine ,extreme form of its total priority“ (Sen 2010b: 62; vgl. Sen 2010a: 91). Freiheit gehört für Rawls zur Liste an Grundgütern, doch darüber hinaus wird ihr ein besonderer Status zugesprochen:

„Giving a special place - a general pre-eminence - to liberty goes well beyond taking note of the importance of liberty as one of many influences on a person's overall advantage. [...] It is a central concern in a person's freedom, touching the most private aspects of personal life, and it is also a basic necessity (for example, in the form of freedom of speech) for the practice of public reasoning, which is so crucial to social evaluation. [...] By separating out the importance of liberty shared by all, Rawls draws attention to a distinction the 'original position' (on which Rawls greatly relies) is adequate for the purpose, this is in no way a rebellion against the basic Rawlsian idea of the foundational priority of fairness in developing a theory of justice" (Sen 2010b: 62; vgl. Sen 2010a: 91).

49 „Aside from enriching the concept of rationality, Rawls also purposes the distinction between being 'rational' and being 'reasonable' in a very useful way, and that is a distinction that will be used fairly extensively in this work [The Idea of Justice]" (Sen 2010b: 63; vgl. Sen 2010a: 91). 
between liberty and other helpful facilities - that is really important to note and pursue" (Sen 2010b: 63f.; vgl. Sen 2010a: 92).

5. Die Notwendigkeit fairer Verfahren im Sinne einer Ausweitung eines Verständnisses für soziale Ungleichheiten. Innerhalb der Sozialwissenschaften werden Ungleichheiten häufig in Bezug auf den Status oder ökonomische Ergebnisse betrachtet, während missachtet wird, dass Ungleichheiten beispielsweise häufig durch Verfahren des Ausschlusses von Ämtern aufgrund von ,race, colour or gender“ ausgelöst werden (vgl. Sen 2010a: 92; Sen 2010b: 64). Insofern wird von Rawls eine Garantie der fairen Chancengleichheit für alle, die sich um Ämter bewerben, gefordert.

6. Das Differenzprinzip, das eine Gleichheit sozialer Regelungen betont und folglich die am schlechtesten Gestellten in das Zentrum der Aufmerksamkeit rückt ${ }^{50}$.

7. Gemäß der Senschen Interpretation von Rawls erkennt Rawls durch seine Konzentration auf Grundgüter die Bedeutung der Freiheit indirekt an, wenn es darum geht, wirkliche - und nicht nur formell zugestandene - Chancen zu geben ${ }^{51}$.

Die notwendige Differenzierung zwischen den Grundgütern, die eine Person besitzt und den Freiheiten, die sie tatsächlich genießt, ist für Sen zentral, wie sich in der Beschreibung zwischen, functionings and capabilities' zeigen wird.

\subsubsection{Argumente von Rawls, die laut Sen überarbeitet werden müssen}

Neben den erörterten Problemen, die Sen zwar als gravierend erachtet, die jedoch durchaus in der Forschungsdiskussion Beachtung gefunden haben und überwunden werden können (absolute Priorität der Freiheit sowie Fokussierung auf die zur Verfügung stehenden Mittel und Vernachlässigung der „Fähigkeiten, Grundgüter in gutes Leben zu konvertieren" (Sen 2010a: 94, Hervorhebung im Original; die Verf.)) konstatiert Sen fundamentale Schwierigkeiten von Rawls Theorie, die bisher wenig Berücksichtigung in der Forschungsdiskussion gefunden haben und neu überdacht werden müssen:

50, ,...] the difference principle indicates the importance of equity in social arrangements so that attention is drawn particularly to the predicament of the worst-off people" (Sen 2010b: 64; vgl. Sen 2010a: 92f.).

$51,,[. .$.$] by focusing on 'primary goods', Rawls gives indirect acknowledgement to the im-$ portance of human freedom in giving people real - as distinct from only formally recognized - opportunity to do what they would like with their own lives" (Sen 2010b: 64; vgl. Sen 2010a: 93). 
1. „Die unausweichliche Relevanz des tatsächlichen Verhaltens“ (Sen 2010a: 95): Sen bemängelt an Rawls die einseitige Konzentration auf gerechte Institutionen ohne gerechten Gesellschaften Aufmerksamkeit zu schenken. Diese könnten sich nicht nur auf Institutionen, sondern auch auf tatsächliche Verhaltensweisen stützen (vgl. Sen 2010a: 95). Seine vorgebrachte Kritik begründet er mit den Prinzipien der indischen Rechtslehre, ferner wirft er Rawls eine Fokussierung auf „niti“ vor, doch auch „nyaya“, das heißt die „Frage [...], was mit Menschen wirklich geschieht" müsse in das Zentrum einer Gerechtigkeitstheorie einbezogen werden (Sen 2010a: 96). Weiterführend thematisiert Sen die Problematik der rawlschen Annahme, dass ,,...] alle Mitglieder einer Gesellschaft irgendwie spontan zu allgemein vernünftigem Verhalten übergehen würden“ (Sen 2010a: 97). Rawls hätte, weil er so differenziert wie kein anderer verdeutlicht hat, dass eine gute Funktionsfähigkeit der Gesellschaft vernünftiges Verhalten jedes Einzelnen voraussetzt, wissen müssen, wie problematisch diese Annahme ist. Folglich können gerechte Institutionen nicht ermittelt werden, wenn zugleich die tatsächlichen Verhaltensmerkmale ausgeklammert werden.

„Rawls' [...] Theorie enthält die formelhafte, drastische Vereinfachung der gewaltigen, vielschichtigen Aufgabe, die Anwendung der Gerechtigkeitsgrundsätze mit dem tatsächlichen Verhalten von Menschen zusammenzubringen; diese Aufgabe ist für den Einsatz der praktischen Vernunft in Fragen der sozialen Gerechtigkeit zentral. Die Vereinfachung ist ungünstig und womöglich ein Versäumnis, da man behaupten kann, dass die Beziehung zwischen sozialen Institutionen und wirklichem - im Gegensatz zu idealem - individuellen Verhalten zwangsläufig für jede Gerechtigkeitstheorie, die das Ziel hat, «Social Choice» zu sozialer Gerechtigkeit zu lenken, entscheidend ist" (Sen 2010a: 98).

2. „Alternativen zum kontraktarischen Ansatz“ (Sen 2010a: 98): Rawls Ansatz ist insofern kontraktarisch, als angenommen wird, dass im Urzustand ein Gesellschaftsvertrag einstimmig beschlossen würde. Die Grenzen dieses Ansatzes belegt Sen in Bezug auf Adam Smith, der im Gegensatz zu Rawls nicht auf einen Gerechtigkeitsvertrag plädiert, sondern den unparteiischen Zuschauer zur Beurteilung der Gerechtigkeit einführt. Dieser Ansatz eröffnet Möglichkeiten, die Rawls in der Form nicht bieten kann (vgl. Sen 2010a: 99): vergleichende Einschätzungen, statt transzendentale Lösungen; Verwirklichungen zu berücksichtigen, statt einer Fokussierung auf die Ansprüche von Institutionen; Unvollkommenheit zuzulassen und zugleich Richtlinien für Probleme der sozialen Gerechtigkeit zu gewähren und die Meinungen außerhalb der VertragspartnerInnen des Gesellschaftsvertrages einzuschließen, ,[...] entweder, um ihre Interessen wahrzunehmen, oder 
um zu vermeiden, dass wir in die Falle einer provinziellen Beschränktheit geraten" (Sen 2010a: 99).

3. „Die Bedeutung globaler Perspektiven“ (Sen 2010a: 99):

„Der Kunstbegriff des fiktiven Urzustands lässt wenig Spielraum, es sei denn, man denkt an einen gigantischen globalen Gesellschaftsvertrag, wie Thomas Pogge und andere in einer «kosmopolitischen» Erweiterung des rawlsschen Begriffs vom Urzustand. In diesem Fall ist es jedoch [aufgrund der Schwierigkeit zu definieren, was gerechte Institutionen sein sollen, wenn konkurrierende Interessen vertreten werden] höchst problematisch, dem rawlsschen Stufenplan zu folgen und für die globale Gesellschaft gerechte Institutionen festzusetzen, das heißt eine Weltregierung zu fordern“" (Sen 2010a: 99).

Gleichwohl muss die Welt jenseits von Nationalstaatsgrenzen für die Einschätzung der Gerechtigkeit innerhalb eines Landes gedacht werden, weil zum einen das, was innerhalb eines Landes passiert, Auswirkungen auf andere Länder hat und zum anderen müssen provinzielle Überzeugungen eines Landes aus einem globalen Blickwinkel betrachtet werden. Die Erfahrungshorizonte anderer können durchaus zur Überprüfung der eigenen hinzugezogen werden.

Sens Arbeit wurde zwar maßgeblich von Rawls geprägt, jedoch ist die Entwicklung des Capability Approachs auch in Auseinandersetzung mit einigen weiteren bedeutenden PhilosophInnen vorangetrieben worden.

\subsection{Kritik an der Sozialwahltheorie nach Arrow}

Die französischen Mathematiker Jean-Charles de Borda und Marquis de Condercet gelten als Begründer der Theorie kollektiver Entscheidungen. Sie befassten sich zu Zeiten der französischen Revolution erstmals mit der Frage, wie individuelle Präferenzen zu kollektiven Entscheidungen aggregiert werden können. Kenneth Arrow griff diese Thematik in den 1950er Jahren auf und konnte im Rahmen seiner Social Choice Theorie darlegen, dass eine Präzision der Minimalbedingungen jeglicher Entscheidungsverfahren erfolgen muss, ferner die Axiome in einer analytischen und strukturierten Rangfolge dargelegt werden müssen (vgl. Sen 2010a: 120). Im Laufe seiner Kritiken an der Theorie kollektiver Entscheidungen entwickelte er das berühmte „Arrow Unmöglichkeitstheorem“, das besagt, „[...] dass kein Verfahren gesellschaftlicher Entscheidung [...] alle Bedingungen gleichzeitig erfüllen kann, die von sozialen Entscheidungen eine vernünftige Sensibilität für die Wünsche der Mitglieder einer Gesellschaft verlangen - selbst wenn einige dieser Bedingungen sehr milde sind“" (Sen 2010a: 120). Die von Arrow for- 
mulierten Axiome (Ausschluss von Diktatur, schwaches Pareto-Prinzip ${ }^{52}$, Transitivität, Unabhängigkeitsprinzip irrelevanter Alternativen, universelle Gültigkeit individueller Präferenzen) führen zu seiner Erkenntnis, dass es nicht möglich, ist aus individuellen Präferenzen eine kollektive Präferenzordnung abzuleiten. Lediglich ein Verzicht des Axioms „Ausschluss von Diktatur" könnte zur Lösung des Dilemmas führen. Sen entwickelt die ursprünglich auf Arrow zurückgehenden Axiome weiter. Sein Theorem der „Unmöglichkeit eines paretianischen Liberalen“ zeigt, , ,...] dass die formalen Bedingungen des Pareto-Optimums womöglich mit einigen Minimalforderungen individueller Freiheit in Konflikt geraten, wenn Menschen beliebige Präferenzen haben können“" (Sen 2010a: 336). Nach Sen hat das Konzept der individuellen Freiheit, auch unabhängig von anderen Entscheidungen treffen $\mathrm{zu}$ können, Priorität. Ob man auf dem Bauch oder auf dem Rücken schlafen möchte, ist beispielsweise eine Entscheidung, in der die Gesellschaft dem Individuum absolute Freiheit gewähren sollte, diese autonom zu treffen, auch wenn die Mehrheit der Gesellschaft davon überzeugt ist, dass es besser ist, auf dem Rücken zu schlafen (vgl. Sen 1970: 152). Sen verdeutlicht seine Kritik an Arrows Axiomen erstmals in seinem Artikel ,The Impossibility of a Paretian Liberal“ (1970) an dem bekannten Beispiel eines erotischen Buchs (Lawrences Lady Chatterley's Lover) und zwei möglichen Lesern, Herrn Prüde und Herrn Lasziv (vgl. Sen 1970: 155). Es bestehen drei, von Sen diskutierte, Möglichkeiten: 1. Herr Prüde liest das Buch, 2. Herr Lasziv liest das Buch oder keiner liest das Buch. Herr Prüde möchte das Buch nicht lesen, doch würde er noch mehr als beim Selbststudium leiden, wenn Herr Lasziv mit Begierde das Buch lesen würde, weil dieser möglicherweise kichern könnte. Lasziv hätte viel Freude dabei das Buch zu lesen, doch wäre es ihm am allerliebsten, wenn der Prüde es lesen würde. Nach liberalen Vorstellungen würde jeder autonom entscheiden: der Prüde würde das Buch nicht lesen, während Lasziv es lesen würde. Doch ist diese Situation konträr zum Pareto-Optimum, weil beide der Meinung sind, dass der Prüde eher das Buch lesen sollte. Im Namen der Freiheit kann jedoch nicht verlangt werden, dass keiner das Buch liest, denn offensichtlich möchte es Lasziv lesen. Andererseits kann im Sinne der Freiheit nicht vom Prüden verlangt werden, das Buch zu lesen, weil er es offensichtlich nicht lesen möchte. Diese beiden Prinzipien würden gegen die Minimalanforderungen der Freiheit verstoßen,

„[... [ also kann keine Wahl getroffen werden, welche die spezifischen Forderungen des gesellschaftlichen Entscheidungsprozesses erfüllt, da jede verfügbare Al-

52 „Das Prinzip [nach Vilfredo Pareto] erklärt einen Zustand für optimal, wenn man ihn nicht so abändern kann, daß [sic!] mindestens ein Mensch besser dasteht, ohne daß [sic!] irgend jemand schlechter dasteht. So ist eine Verteilung einer Gütermenge auf bestimmte Menschen pareto-optimal, wenn es keine Umverteilung gibt, nach der mindestens ein Beteiligter besser und keiner schlechter dasteht" (Rawls 1996: 87f.). 
ternative schlechter ist als eine andere. Daher die Unmöglichkeit, beide Prinzipien gleichzeitig zu erfüllen" (Sen 2010a: 337).

Anstatt lediglich von einer Aggregation individueller Präferenzordnungen auszugehen, ist für Sen die Differenzierung zwischen Funktionsweisen (Functionings) und Befähigungen (Capabilities) zentral, die in unterschiedlichen Formen auftreten, die Lebensweise von Individuen beeinflussen und deren Ausprägung abhängig von der Freiheit ist. ${ }^{53}$

\subsection{Functionings (Funktionsweisen) und Capabilities (Befähigungen)}

\subsubsection{Functionings (Funktionsweisen)}

Functionings (Funktionsweisen) werden definiert als ,[...] the various things a person may value doing or being" (Sen 1999: 75). Functionings sind verwirklichte bzw. erreichte Fähigkeiten und Zustände, die sich auf die von einer Person realisierten Handlungsalternative beziehen und sind insofern konstitutiv für das „well-being“ ${ }^{45}$ respektive Wohlergehen. Für die Transformation von Ressourcen in Functionings werden Conversion Factors (Umwandlungsfaktoren) benötigt. Dies sind ,[...] Faktoren, die für die Umwandlung von Ressourcen in konkrete, individuell unterschiedliche Funktionen notwendig sind" (Schneider 2010: 18). Conversion Factors können sowohl individueller (individuelle Fähigkeiten) als auch gesellschaftlicher (gesellschaftliche Bedingungen) Art sein. Es stellt sich die Frage, wie Individuen ihre Ressourcen nutzen können. Beispielsweise beziehen sich Conversion Factors auf das Ausmaß an Mobilität, das eine Person durch die Ressource eines Autos erreichen kann. Falls die Person nicht Autofahren kann, verfügt sie nicht über die notwendigen individuellen Conversion Factors, das Auto in Mobilität umzu-

53 „Funktionsweisen und Befähigungen sind vielfältig, und so müssen sie sein, da sie mit unterschiedlichen Facetten unseres Lebens und unserer Freiheit zu tun haben" (Sen 2010a: 266).

54 Zur Definition des well-beings und dem Zusammenhang mit functionings: „The well-being of a person can be seen in terms of the quality (the "well-ness," as it were) of the person's being. Living may be seen as consisting of a set of interrelated "functionings," consisting of beings and doings. A person's achievement in this respect can be seen as the vector of his or her functionings. The relevant functionings can vary from such elementary things as being adequately nourished, being in good health, avoidable escapable morbidity and premature mortality, etc., to more complex achievements such as being happy, having selfrespect, taking part in the life of the community, and so on. The claim is that functionings are constitutive of a person's being, and an evaluation of well-being has to take the form of these constituent elements" (Sen 1992: 39, Hervorhebungen im Original; die Verf.). 
wandeln. Weiterhin könnte es sein, dass es Frauen gesetzlich verboten ist, Auto zu fahren (gesellschaftliche Conversion Factors) oder es keine geeigneten Straßen gibt, die eine Infrastruktur für Autos bieten (umweltbezogene Conversion Factors).

Im Gegensatz zu den Funktionsweisen, die sich auf die Realisierung einer konkreten Handlungsalternative beziehen, zielen Capabilities (Befähigungen) auf den Handlungsspielraum, d. h. auf den Chancenaspekt der Freiheit ab sie stehen im Fokus des Capability Approachs. Am Beispiel der Mobilität durch die Ressource eines Autos lässt sich auch der von Sen betonte Freiheitsaspekt verdeutlichen: Anstatt der Mobilität selbst rückt die Wahlmöglichkeit, mobil zu sein, in das Zentrum der Aufmerksamkeit von Freiheit nicht Mobilität, sondern die Wahl zur Mobilität bedeutet nach dieser Perspektive Freiheit. Insofern besteht ein theoretischer Vorrang dessen, was im Sinne der individuellen Freiheit möglich sein könnte im Vergleich zu dem, was tatsächlich verwirklicht wurde. „Befähigungen werden aus Funktionsweisen abgeleitet; sie schließen unter anderem alle Informationen über die Kombinationen ein, die eine Person wählen kann" (Sen 2010a: 263f.). Sen verwendet für sein Freiheitskonzept einen instrumentellen und einen intrinsischen Aspekt: Das Instrumentelle nimmt Bezug auf die Freiheit als Mittel zur Erreichung von Functionings, das Intrinsische bezieht sich auf die freie Wahl von verschiedenen Handlungsoptionen und findet Ausdruck in der Wertschätzung für das selbstbestimmte Handeln. Der instrumentelle und der intrinsische Aspekt bestimmen die „Doings and Beings“, die gemeinsam das Ausmaß an realer Freiheit bilden (vgl. Schneider 2010: 18). Ferner weist Freiheit einen Gelegenheits- (,opportunity aspect") und einen Verfahrensaspekt (,process aspect") auf: Während der Gelegenheitsaspekt Bezug auf das Ausmaß an Verwirklichungschancen (Anzahl, Qualität) nimmt, betont der Verfahrensaspekt den Prozess, der zur Entscheidung führt. Die Aussage von Isaiah Berlin, ,[...] a man's, or a people's, liberty too choose to live as they desire", auf die sich Sen bezieht, vereint den Gelegenheits- und den Verfahrensaspekt - sowohl frei wählen zu können als auch die Subjektivität der Bewertung der Wahlmöglichkeit einzuschließen (Sen 1992: 67). Obwohl im Capability Approach beide Aspekte ihre Bedeutung haben, spielt der Gelegenheitsaspekt eine größere Rolle, da der Capability Approach keine reine Entscheidungstheorie darstellt, sondern eher einen Evaluationsrahmen - das Entscheidungsverhalten bleibt in gewisser Hinsicht eine Black Box (vgl. Bartelheimer et al. 2008: 20). Die Wahlmöglichkeit wird durch den Ausdruck der ,,agency“ hervorgehoben. Das Individuum wird hierbei als ein handelnder „Agent" beschrieben, der möglichst die Freiheit wahrnehmen können sollte, autonom für eigene Ziele zu agieren. 


\subsubsection{Capabilities (Befähigungen) und Handlungsspielräume}

Befähigungen sind nach Sen

,[...] the various combinations of functionings (beings and doings) that the person can achieve. Capability is, thus, a set of vectors of functionings, reflecting the person's freedom to lead one type of life or another. [...] 'the capability set' in the functioning space reflects the person's freedom to choose from possible livings" (Sen 1992: 40).

Das Vermögen, verschiedene Kombinationen von Functionings miteinander vergleichen zu können und diejenigen erreichen zu können, die aus subjektiven Gründen hoch geschätzt werden, ist nach Sens Verständnis die Befähigung (vgl. Sen 2010a: 260f.). Folglich werden Capabilities als Verwirklichungschancen verstanden; es sind die Auswahlmöglichkeiten, die einer Person zur Verfügung stehen. Dies impliziert auch einen bewussten Verzicht auf Functionings: Obwohl ein spezifisches Functionings erreicht werden könnten, wenn es gewollt wäre, ist es ebenso ein Aspekt von Freiheit, bewusst darauf verzichten zu können ohne negativen Konsequenzen ausgesetzt $\mathrm{zu}$ werden. Wesentlich ist lediglich, dass die Möglichkeit bestünde, dieses Functioning erreichen zu können, sofern es aus subjektiven Gründen von Bedeutung wäre. Die Perspektive ist nicht nur auf die gewählte Handlungsalternative, gleichwohl auf das ausgerichtet, was eine Person unabhängig ihres Handlungsspielraumes tun möchte (vgl. Sen 2010a: 263). Unterschiedliche Kombinationen an Functionings, die eine Person mit gegebenen Ressourcen erreichen kann bzw. nicht erreichen kann, bilden ihre Capability, wobei die Gesamtmenge an Verwirklichungschancen als Capability Set bezeichnet wird. Die Capability einer Person hängt ferner von diversen Faktoren ab, weshalb die Umsetzung und Operationalisierung des Capability Approaches vom jeweiligen Forschungsfeld abhängig zu machen ist. Sen bietet hierfür einen allgemeinen umfassenden theoretischen Rahmen. Beispielsweise beschreiben Verkerk et al. (2001), die Sens Theorie auf das Forschungsfeld der "Health-related quality of life" anwenden möchten, die Situation, dass eine körperlich eingeschränkte Person mehr Ressourcen benötigt, um das Functioning der Mobilität zu erreichen als eine körperlich nicht beeinträchtigte Person (Verkerk et al. 2001: 51). Aufgrund des hohen Abstraktionsniveaus dieser Theorie werden ForscherInnen für eine Umsetzung des Capability Approachs stets gezwungen sein, je nach Forschungsperspektive Vereinfachungen vorzunehmen. Im Kontext der weiterführenden Theorie der Machtverhältnisse in Paarbeziehungen wird das Capability Set als Gesamtmenge an Verwirklichungschancen von Machtfähigkeiten definiert, die sich auf unterschiedlichen Ebenen ausdifferenzieren lassen (s. Kapitel 4.8. und 9.3.).

In der Betrachtung des Zusammenhangs zwischen Freiheit und Befähigung wird auch Martha Nussbaums Einfluss deutlich, die mit Sen gemeinsam 
den Capability Approach entwickelte. Sie hat maßgeblich zu den Forschungsfortschritten der Verbindung zwischen der Befähigungsperspektive und klassischen Aristotelischen Konzepten wie der Fähigkeit des gelungenen Lebens bzw. Wohlergehens sowie dem Zusammenhang zwischen Capability und der menschlichen Entwicklung, den Genderstudies und Menschenrechten beigetragen (vgl. Sen 2010a: 23). Inwiefern sich die Denkrichtungen von Sen und Nussbaum unterscheiden, wird im Kapitel 4.6.3. „Die neue Egalitarismuskritik im Überblick" diskutiert.

\subsection{Das Freiheits-Gleichheit-Dilemma}

In einem seiner neueren Werke „The Idea of Justice“ vertritt Sen die These einer Ausweitung der Gerechtigkeitstheorien, da er die Vielfalt als eine zentrale Komponente für die Gerechtigkeitseinschätzung erachtet (vgl. Sen 2010a: 335f.). Die Idee der mehrdimensionalen Pluralität ist für seine Theorie immanent, die er bereits in seinen frühen Arbeiten publik machte. So war schon in „Inequality Reexamined“ seine Ausgangsannahme der Ungleichheitsforschung die Unterschiedlichkeit, anstelle eines absolutistischen Gleichheitsideals. Sein Pluralitätsgedanke in Bezug auf die Freiheit wurde nicht zuletzt mit einem Nobelpreis gewürdigt.

„Wir müssen beachten, dass Gleichheit wie Freiheit viel Inhalt umfassen und mehrdimensional sind. Wir haben allen Grund, uns nicht auf engstirnige, eindimensionale Ansichten von Freiheit und Gleichheit einzulassen, die alle anderen Ansprüche dieser umfassenden Werte ignorieren. Diese Pluralität muss Teil einer Theorie der Gerechtigkeit sein, denn sie muss den vielfältigen Überlegungen gerecht werden, zu denen diese großen Ideen Freiheit und Gleichheit aufrufen" (Sen 2010a: 343).

Innerhalb seines Befähigungsansatzes lautet für Sen die entscheidende Frage „Gleichheit wovon?"; es ginge nicht darum, ,[...] ob wir überhaupt Gleichheit brauchen, in welchem Bereich auch immer" (Sen 2010a: 320). Wie er in „The Idea of Justice“ zeigen möchte, ist Gleichheit zwar wichtig und Befähigung „ein zentrales Merkmal menschlichen Lebens“" (Sen 2010a: 322). Trotzdem widerstrebt ihm eine Forderung nach Befähigungs- oder Chancengleichheit. Als Gründe bringt Sen Folgendes vor:

1. Befähigung ist nur ein Aspekt der Freiheit, der sich lediglich auf den Chancenaspekt der Freiheit, d. h. auf individuelle Begünstigungen bezieht, doch über die Prozesshaftigkeit von Fairness und Billigkeit könne die Befähigung nicht ausreichend informieren (vgl. Sen 2010a: 322). Als Beispiel führt Sen die unterschiedliche Lebenserwartung von Frauen und Männern an. Nur weil Frauen eine höhere Lebenser- 
wartung haben, wäre es nicht vernünftig, ihnen eine schlechtere medizinische Versorgung zukommen zu lassen als Männern, damit eine egalitäre Chancengleichheit der Lebenserwartung für beide $\mathrm{Ge}$ schlechter resultiert (vgl. Sen 2010a: 323). In diesem Fall habe die Fairness im Prozessaspekt eine Vorrangstellung gegenüber der Freiheit, vielmehr verbiete sie sogar eine Vorrangigkeit der Gleichheit der Lebenserwartung.

„Ein ausschließlich auf einen Bereich zentriertes Verständnis von Gleichheitsforderungen (in diesem Fall die ausschließliche Sicht auf Befähigungen) betrachte ich mit der Skepsis, die Teil meiner weitreichenden Kritik an jeder unifokalen Auffassung von Gleichheit ist" (Sen 2010a: 324).

2. Freiheit ist zwar ein wichtiger Aspekt für die Beurteilung persönlicher Begünstigungen und damit auch der Gleichheit, ,[...] aber es kann auch andere Ansprüche an Urteile über Verteilung geben, die womöglich nicht am besten als Forderungen nach gleicher Gesamtfreiheit (in einer klaren Bedeutung) für verschiedene Menschen $\mathrm{zu}$ verstehen sind" (Sen 2010a: 324, Hervorhebungen im Original; die Verf.).

3. Befähigung kann unterschiedlich definiert werden, wobei innerhalb der Definitionen ,[...] auch zwischen Freiheit zum Wohlergehen und Handlungsfreiheit" zu differenzieren ist (Sen 2010a: 324). Befähigungen lassen sich daher nicht immer in eine vollständige Rangordnung bringen, insbesondere, wenn wie im Flötenbeispiel unterschiedliche Typen von Befähigungen zur Verfügung stehen, die allesamt als vernünftig bewertet werden können ${ }^{55}$. Eindeutige Beurteilungen sind daher nicht immer möglich, gleichwohl es uns durch eine partielle Ordnung zumindest ermöglicht ist, extreme Ungleichheiten zu erkennen.

4. Eine Theorie der Gerechtigkeit hat sich nicht nur mit der Gleichheit als einzigem Wert auseinanderzusetzen. Gleichheit ,[...] ist nicht einmal das einzige Gebiet, auf dem die Idee der Befähigung Nutzen hat" (Sen 2010a: 325). So verdiene nicht nur die Verminderung von Chancenungleichheiten Aufmerksamkeit, sondern auch ,[...] die allgemeine Verbesserung der Chancen aller" (Sen 2010a: 325).

Dass ein absolutistisches Gleichheitsverständnis Grenzen hat und darüber hinaus zu weiteren Ungleichheiten führen kann, soll an dieser Stelle mit einer fiktiven Geschichte Simmels, die vielleicht überall auf der Welt spielen könnte, sowie der daran anschließenden von Ostner geführten Debatte um soziale

55 „Eine partielle Ordnung mag ausreichen, um Ungleichheiten in einigen Fällen einzuschätzen, vor allem krasse Ungerechtigkeit zu erkennen, aber sie ergibt in anderen Fällen nicht notwendig klare Beurteilungen. Diese Einwände besagen nicht, dass es nutzlos sei, Aufmerksamkeit auf die Verminderung von Chancenungleichheit zu wenden. Diese Verminderung ist sicherlich eine große Aufgabe, aber man muss auch sehen, dass die Reichweite der Chancengleichheit Grenzen hat und dass sie nur ein Teil der Gerechtigkeitsforderungen ist" (Sen 2010a: 325). 
Ungleichheiten und Ressentiment verdeutlicht werden. Anschließend erfolgt eine Übersicht der neueren Egalitarismusdebatte, innerhalb derer Sens Argumentation und der Bezug zu Martha Nussbaum eingeordnet werden sollen.

\subsubsection{Blühende Rosen und Neid}

Eine fiktive Geschichte Simmels, die vielleicht überall spielen könnte: Während einige BürgerInnen es sich leisten konnten, in ihrem Garten Rosen zu ziehen, wurden Sie von den anderen BürgerInnen, die sich keine Rosenzucht leisten konnten, dafür beneidet. Aufgrund des Benachteiligungsgefühls der Nicht-Rosen-BesitzerInnen formierten sie sich zu einer Revolutionspartei und konnten soziale Gerechtigkeit in Form eines Rechts auf Rosenbesitz erkämpfen.

„So gleichmäßig freilich, wie die Seiten einer mathematischen Gleichung, konn-
ten die Anteile dennoch nicht ausfallen. Immerhin hatte der eine die glücklichere
Hand im Aufziehen der Rosen, der andere ein wenig mehr Sonne, der dritte ein
kräftigeres Pfropfreis; denn die Natur läßt [sic!] sich immer nur ganz ungefähr,
und ohne sich irgendwie zu binden, auf die Symmetrie der menschlichen Pläne
ein“ (Simmel 1989: 170).
"Es ging also nun, so lange es ging; aber eines Tages war die Anpassung voll-
bracht und jene kleinsten Unterschiede in Farbe und Form, in Duft und Reiz der
Rosen, mit denen sich die Natur doch als die letzte Instanz über allen Anglei-
chungsversuchen erweist, erregten den gleichen Haß [sic!] und Neid, denselben
Hochmut auf der einen Seite, dasselbe Gefühl der Enterbtheit auf der anderen“
(Simmel 1989: 171).

Auf Simmels Rosen und die Frage, welchen Stellenwert diese Argumentation innerhalb des hier aufgeführten Machtansatzes einnimmt, wird im Kapitel 9.4. „Bringing Power Back In“ zurückgegriffen.

\subsubsection{Ostners (1998) Ressentimentannahmen zur Geschlechtergleichheit im Feminismus}

Mit Bezug auf Simmel argumentiert Ostner, dass zwar durch ressentimentgeladene Bewältigungsstrategien versucht würde, Gleichheit zwischen den Geschlechtern (wie in Simmels Beispiel zwischen den BesitzerInnen und Nicht-BesitzerInnen der Rosen zu erreichen), doch „Der im Angleichungsprozeß [sic!] immer wieder neu entstehenden Ungleichheit [...] weder Frau noch Mann entkommen [können]" (Ostner 1998: 383). In der Tat, aber eine adäquate Frage liegt auch in der Umkehrung: nicht, ob Frau und Mann der Ungleichheit entkommen können, sondern wie viel Gleichheit und Ungleichheit zugelassen werden kann. Die Spannbreite dazwischen ist groß, mit vielen 
Facetten, unterschiedlichen Kategorien, und keinesfalls als dichotom, sondern relational zu denken.

Ostner ,[...] versucht, die Entstehung von Frauenbewegungen und feministischen Strategien aus dem spannungsreichen Zusammenspiel von Gleichheitspostulat, sozialer Ungleichheit, Angleichungsprozessen und neuer Ungleichheit zu erklären. Das für diese konfliktträchtige Dynamik konstitutive Moment ist der Vergleich, die Möglichkeit, sich mit anderen als potentiell Gleichen zu vergleichen - Ressentiment das Gefühl derjenigen, die meinen schon wieder vergleichsweise schlecht abzuschneiden" (Ostner 1998: 384, Hervorhebungen im Original; die Verf.).

In Anlehnung an die Bezugsgruppentheorie, die von einem Vergleich statusähnlicher Personen bzw. Gruppen ausgeht, erläutert Ostner, dass durch die Ausweitung der Rollen von Frauen ihr Bezugspunkt wechselt, insofern als Männer zunehmend die Referenzgruppe für Frauen darstellen, was zum Ressentiment führe (vgl. Ostner 1998: 389). Durch die moderne, funktional differenzierte Gesellschaft entstehe eben dieser (massenhafte) Vergleich. Allen Mitgliedern würde das gleiche Maß an Freiheit gewährt, wobei Statusunterschiede durch die Argumentation gerechtfertigt würden, dass ungleichheitsrelevante Positionen grundsätzlich allen offen stehen (vgl. Ostner 1998: 387). Häufig würde die moderne Gesellschaft durch Umverteilungsmaßnahmen dafür Sorge tragen, dass Benachteiligte etwas mehr von diesen Maßnahmen profitieren, wodurch ihre Teilhabechancen verbessert werden sollen, um den Prinzipien der modernen Gesellschaft Ausdruck zu verleihen. Für einen kurzen Moment mögen die Ungleichheiten nivelliert zu sein, doch langfristig entstehen neue Ungleichheiten:

„Ehemalige Verlierer[Innen] haben jetzt vielleicht die besseren Karten gezogen; aber ebenso häufig bleiben sie - auf neue Weise und doch schon wieder - Verlierer[Innen]. An dieser Stelle kommt das Ressentiment ins Spiel: der Groll, der die neue Verlierer[Innen]position begleitet und der aktiv gewendet wird. Die moderne Gleichheitslehre und die funktional differenzierte Gesellschaft sind deshalb nicht nur eine Bedingung für die Entwicklung von Ressentiment, sondern auch ihr Ergebnis. Dies gilt vor allem dann, wenn diejenigen, die fürchten, im Vergleich zu verlieren, Gleichheit einfordern - und zwar eine nun abwärts gerichtete Gleichheit, die Angleichung an das Niveau der niedriger Stehenden, Schwächeren, Benachteiligten“ (Ostner 1998: 387, Hervorhebungen im Original; die Verf.).

Der Kampf um Gleichheit, verschärft durch sozialstrukturellen Wandel und ressentimentgeladene Bewältigungsstrategien, führt zu neuen sozialen Ungleichheiten, der Vergleich führt zum Ressentiment - und die Spirale wiederholt sich von vorne. Durch Angleichung als Ausgangspunkt entstehe nach Ostner, wobei sie sich auf Simmel beruft, neue Ungleichheit. Beispielsweise habe die zunehmende Integration von Frauen in die Erwerbsarbeit zu einer Segregation des Arbeitsmarktes geführt (vgl. Ostner 1998: 392). In den USA 
habe sich die Wechselbeziehung von Angleichung und neuer Ungleichheit weiterführend in der für Männer und Frauen bestehenden Angewiesenheit eines zweiten, weiblichen Einkommens zur Existenzsicherung gezeigt (vgl. Ostner 1998: 393).

\begin{abstract}
„Das Zusammenspiel von Entdifferenzierung und neuer sozialer Differenzierung ist immer komplizierter, dynamischer und undurchschaubarer geworden. Es vermochte jedenfalls die Identität der Frauenbewegung in Frage zu stellen: Wer spricht für wen, wenn im Rekurs auf „,die Frau“ - oder schon angemessener: „,der Frauen" Diagnosen erstellt und Forderungen erhoben werden? Wenn sich die weiblichen Lebenslagen und Lebensläufe vervielfältigen? Schließlich hatte die Rede von den „Frauen“ vorausgesetzt, daß [sic!] die Frauen etwas gemeinsam haben [...], das sie strikt von Männern trennte (Ostner 1998: 394, Hervorhebungen im Original; die Verf.).
\end{abstract}

Je mehr Frauen mit den Männern mitziehen konnten, umso weniger galten Männer als Komparatoren und umso deutlicher wurden die Unterschiede zwischen den Frauen. Die Vergleichsgruppe wechselte von den Männern zurück zu den Frauen, wodurch zunehmend die Vielfalt der Frauen betont wurde. „Das Ressentiment begann sich zurückzuwenden und (wieder vermehrt) gegen Frauen zu richten“"(Ostner 1998: 394).

Angleichungsprozesse zwischen Männern und Frauen innerhalb der sozialen Felder „Beruf und Familie“ münden im Verlust der Evidenz

„[...] eine[r] Position, die die früher einmal offenkundige Besonderheit von Frauen ins Positive steigert[e]. Da diese Angleichungen zudem mit neuen Ungleichheiten einhergehen, läuft eine Strategie der Überhöhung der althergebrachten Mann-Frau-Differenz doppelt fehl“" (Ostner 1998: 399, Hervorhebungen im Original; die Verf.).

\title{
4.6.3. Die neue Egalitarismuskritik im Überblick: Equality of What?
}

Die Auffassung von Gerechtigkeit im Sinne gleicher Lebensaussichten für alle wird vom Mainstream der GerechtigkeitsphilosophInnen vertreten. Doch gehen die Auffassungen über die Frage, woran die Gleichheit der Lebenschancen festgemacht werden kann, stark auseinander (vgl. Krebs 2000: 7). Krebs nennt drei Paradigmen der ursprünglich von Sen ins Leben gerufenen „Equality-of-What?-Debatte ${ }^{56 “: ~ W a ̈ h r e n d ~ d i e ~ e i n e n ~ e i n e ~ G l e i c h h e i t ~ u ̈ b e r ~}$ Ressourcen fordern, fordert die zweite Gruppe gleiche Möglichkeitschancen des Wohlergehens, während die letzte Perspektive in Anlehnung an Sen (1992) eine Gleichheit der Verwirklichungschancen von Funktionsfähigkei-

56 „Two central issues for ethical analysis of equality are: (1) Why equality? (2) Equality of what (Sen 1992: 12)? 
ten (,equality of capability to function“) betont (Krebs 2000: 7, Hervorhebungen im Original; die Verf.).

Doch „Gleichheit tauge nicht als Grundlage der Gerechtigkeit“ - so die neue Egalitarismuskritik (Krebs 2000: 8). Dem kritisierten Egalitarismus, dessen Ziel der Gerechtigkeit die Gleichheit darstellt, weshalb er in dieser Forschungsarbeit als absolutistisch bezeichnet wird, ist eine Relationalität der Gerechtigkeit immanent:

,[...] als die Gleichheit der einen mit den anderen [wie in Simmels Beispiel stehen einem/r DorfbewohnerIn Rosen zu, weil andere auch Rosen haben]. Genauer kombiniert der Egalitarismus in der Regel, als sogenannter "pluralistischer Egalitarismus«, ein auf unverdiente Lebensaussichten bezogenes Gleichheitsprinzip mit einem Wohlfahrtsprinzip, das die Erhöhung der allgemeinen Wohlfahrt anstrebt" (Krebs 2000: 8, Hervorhebungen im Original; die Verf.).

Unverdiente Lebensaussichten sollen einschließen, dass Menschen selbst für ihre Situation verantwortlich sein können. Es sei keine Verletzung der Gleichheit, sondern moralisch gerecht, wenn einige viel arbeiten, während andere faul seien, denn für die Folgen ihrer Entscheidungen müssten die Betroffenen selbst aufkommen. Lediglich das, was Menschen nicht durch ihre eigene Leistung erhalten haben, sondern ihnen einfach - aus welchen Gründen auch immer - zufällt (Geschenke, Erbschaften, Gaben der Natur etc.) müsste egalisiert werden (vgl. Krebs 2000: 11).

Im Gegensatz zum Egalitarismus vertritt der Nonegalitarismus die Ansicht, dass Gleichheit kein zentrales Ziel sei, sondern in vielen Fällen lediglich ein Nebenprodukt darstelle (wie z. B. in Bezug auf die Forderung, dass alle Hungernden Essen erhalten sollen). Für NonegalitaristInnen steht die Erfüllung eines absoluten Standards für alle, anstatt einer Forderung auf Gleichheit im Vordergrund (ausreichende Nahrung als eine Bedingung eines menschenwürdigen Lebens) (vgl. Krebs 2000: 15). Krebs unterscheidet diesbezüglich zwischen „egalitären“ und „egalitaristischen“ Gerechtigkeitstheorien, die Gleichheit als zentrales Ziel der Gerechtigkeit fordern. Folglich können nonegalitaristische Theorien egalitärer sein als egalitaristische Theorien. Die neue Egalitarismuskritik würde sich nicht mit der Frage, ob eine Gerechtigkeitstheorie egalitär sein sollte, befassen; dies sei vielmehr das Fundament auf dem alle NonegalitaristInnen aufbauen würden. Es ginge in der neuen Egalitarismuskritik nur um die Frage, ob eine Gerechtigkeitstheorie „egalitaristisch“ sein sollte (vgl. Krebs 2000: 15f.).

„Das »Oder« im Titel dieses Bandes »Gleichheit oder Gerechtigkeit« bezieht sich demnach auch nur auf diese zweite Frage- [...] es enthält die These, dass Gerechtigkeit nicht egalitaristisch, nicht wesentlich als auf Gleichheit abzielend zu verstehen ist (Krebs 2000: 15f., Hervorhebungen im Original; die Verf.).

Krebs fasst vier Kritikpunkte des neuen Egalitarismus gegen den Egalitarismus zusammen: 
1. „Gleichheit nur als Nebenprodukt“: Allgemeinheit würde mit Gleichheit verwechselt, Gleichheit sei nicht Ziel, sondern lediglich ein Nebenprodukt. Erzielt werden sollte stattdessen, dass niemand unter prekären Lebensbedingungen, wie z. B. Hunger, leiden solle, für alle sollten die gleichen Lebensbedingungen bestehen. Der Vorwurf beinhaltet, dass die elementaren Standards der Gerechtigkeit nicht-relational sind und Gleichheit nur als Nebenprodukt implementieren. So wesentlich, wie es der Egalitarismus annimmt, könne Gleichheit nicht sein (vgl. Krebs 2000: 17f.).

Beispielsweise sei Kranken zu helfen, weil sie sich in einer schrecklichen Situation befinden und

,[...] nicht deswegen, weil es anderen schließlich besser geht [...]. Die Gleichheitsterminologie ist redundant. Es geht nichts verloren, wenn man anstelle von: »Alle Menschen sollen gleichermaßen genug zu essen haben« einfach nur sagt: »Alle Menschen sollen genug zu essen haben «" (Krebs 2000: 18, Hervorhebungen im Original; die Verf.).

2. „Inhumanität“: Die Kritik der Inhumanität des Egalitarismus beinhaltet, dass der Egalitarismus nicht in der Lage sei, für alle die Bedingungen eines menschenwürdigen Lebens herzustellen - „Menschen, die an ihrem Elend selbst schuld sind, werden in ihrem Elend allein gelassen“" (Krebs 2000: 21, Hervorhebungen im Original; die Verf.). Diejenigen, die Hilfe brauchen, erhalten sie aus relationalen und nicht aus menschenwürdigen Gründen, wodurch sie zugleich stigmatisiert werden. Über das Ausmaß des Elends entscheidet der Staat, was BürgerInnen entmündigt und in ihrer Privatsphäre verletzt, um an Informationen für die Staatsentscheidung - selbstverdient oder unverschuldet - heranzukommen (vgl. Krebs 2000: 21). Durch die Forderung des Egalitarismus einer Egalisierung unverdienter Lebensaussichten entsteht - akribisch betrachtet - die Folge, dass Menschen, die aufgrund ihrer eigenen Entscheidungen in Not geraten sind, nicht zu helfen ist bzw. sie keinen Anspruch auf Unterstützung haben, weil sie für die Folgen ihrer Entscheidungen Eigenverantwortung tragen sollen. Schließlich gelte es lediglich unverdiente Lebensaussichten zu egalisieren (vgl. Krebs 2000: 22).

3. „Verkennung von Komplexität“: Die Berücksichtigung der Komplexität von Gerechtigkeit ist mangelhaft und wird unterschätzt, da die Gerechtigkeitskultur kulturspezifisch und mit einer Vielzahl an Prinzipien verknüpft ist, wie z. B. Verdienstprinzip, Leistungsprinzip etc. „Angesichts dieser Fülle an Gerechtigkeitsgesichtspunkten [die sich auch gegenseitig überlagern können und daher ein Abwägen erfordern] stellt sich der Egalitarismus mit seiner Ausrichtung auf Gleichheit als falscher Monismus dar" (Krebs 2000: 25).

„Unsere Gerechtigkeitskultur ist damit »so kompliziert wie das Leben selbst«. Der Glaube, man könne diese Kultur im Wesentlichen über ein oder zwei 
Prinzipien, das Gleichheitsprinzip in Kombination mit dem Prinzip der Wohlfahrtssteigerung, einfangen, zeugt von der Philosoph[Inn]enkrankheit der theorieverliebten Überheblichkeit gegenüber der Wirklichkeit. Die Egalitaristen sind zu sehr »Freunde einfacher Verhältnisse«, um der Komplexität unserer Gerechtigkeitskultur gerecht zu werden“ (Krebs 2000: 27, Hervorhebungen im Original; die Verf.).

4. „Nichtrealisierbarkeit“: Ist der Einwand, dass Egalitarismus nicht gänzlich umgesetzt werden kann. ,[...] schon der Versuch einer flächendeckenden Egalisierung [erscheint] wie ein Sandkastenspiel oder eine realitätsfremde Anmaßung“ (Krebs 2000: 30). Mit dem Versuch der Angleichung von Ungleichheiten entstehen, wie auch Ostner argumentiert, Ressentiments und in der Folge neue Ungleichheiten.

VertreterInnen der neuen Egalitarimuskritik geben sich nicht mit minimalistischen Standards eines absolutistischen Egalitarismus zufrieden: die neuen NonegalitaristInnen fordern nicht nur den Schutz der negativen Freiheit aller durch den Staat, sondern der Staat hat auch Sorge dafür zu tragen, dass keiner unter menschenunwürdigen Umständen (wie z. B. Mangel an Nahrung, Obdach, medizinischer Grundversorgung etc.) leben muss. Hierdurch wird die Perspektive des nonegalitaristischen Humanismus auf die Menschenwürde fokussiert. Prinzipien, wie das Verdienstprinzip, das Prinzip der Tauschfreiheit oder das Qualifikationsprinzip werden einem Prinzip auf menschenwürdiges Leben für alle nachgeordnet. Martha Nussbaums aristotelischer Essentialismus vermag eines der bekanntesten Beispiele für eine humanistische Gerechtigkeitstheorie zu sein (vgl. Krebs 2000: 31). In Abgrenzung zu Sen, der eine Klassifikation von gerechten Gesellschaften strikt ablehnt, geht Nussbaum einen Schritt weiter, in dem sie ein minimales Bündel an Voraussetzungen für eine gerechte Gesellschaft fordert. Sie widmet sich ferner dem „guten Leben“ einschließlich der Frage, welche Kriterien erfüllt sein müssen, um ein gutes menschliches Leben führen zu können (vgl. Nussbaum 1999: 188). Diese Frage impliziert die Abgrenzung dessen, was menschlichen Fähigkeiten immanent ist. Insofern lautet die übergeordnete grundlegende Fragestellung, die wir uns laut Nussbaum stellen müssen:

„What are the characteristic activities of the human being? What does the human being do, characteristically, as such - and not, say, as a member of a particular group, or a particular local community" (Nussbaum 1995: 72).

Sen, der viele Forschungstätigkeiten mit Nussbaum geteilt hat, unterscheidet sich in einem zentralen Punkt ${ }^{57}$ von ihr: Während sie in ihrem Werk „Gerech-

57 Ein weiterer Unterschied ist, dass sie „capabilities“ stärker auf Fähigkeiten fokussiert, die sie ausdifferenziert in grundlegende, interne und kombinierte Fähigkeiten (vgl. Nussbaum 2000: 84). Grundlegende Fähigkeiten beziehen sich auf die angeborene Ausstattung einer Basis zur Entwicklung von Fähigkeiten. Interne Fähigkeiten meinen die ausreichenden $\mathrm{Zu}-$ stände einer Person zur Ausübung ihrer erforderlichen Tätigkeiten. Kombinierte Fähigkeiten erfassen die internen Fähigkeiten in Kombination mit ihren äußeren Bedingungen (vgl. 
tigkeit oder das gute Leben“ ein fixes Bündel von zehn universalen Indikato$\operatorname{ren}^{58}$ (z. B. Fähigkeit einer guten Gesundheit, Zugang zu Bildung einschließlich dem Schutz dieser Fähigkeit in Form von gesetzlichen Garantien der Meinungs- und Religionsfreiheit, Zugang zu einer beruflichen Tätigkeit etc.; die vollständige Liste ist im Anhang dargestellt), sogenannten „Central Human Functional Capabilities“ (Nussbaum 2000: 78), fordert (vgl. Nussbaum 1999: 200ff.), würde er als Liberaler die Indikatoren nicht im Vorfeld festlegen $^{59}$ (im Falle der Konzeptionierung des HDI tut er es dennoch) und den

Nussbaum 2003: 22). Nussbaum führt als Beispiel für die internen Fähigkeiten eine nicht beschnittene Frau an, die die interne Fähigkeit besitzt, sexuelle Lust zu verspüren. Eine weitere Frau, die ebenfalls nicht beschnitten ist, aber bereits in jungen Jahren Witwe wurde und der es verboten ist, wieder zu heiraten, besitzt zwar die interne Fähigkeit, nicht aber die kombinierte Fähigkeit sexueller Entfaltung (vgl. Nussbaum 2003: 22).

58 Nussbaum konstatiert zwei Schwellen - die erste, die sich auf ein menschliches Leben bezieht und die zweite, die sich auf ein gutes menschliches Leben bezieht. Auf Basis der zweiten Stufe siedelt sie die zehn Grundfähigkeiten an. Die erste Schwelle meint ,,[...] eine Schwelle der Fähigkeit zur Ausübung von Tätigkeiten, unterhalb derer ein Leben so verarmt wäre, daß [sic!] es überhaupt nicht mehr als menschliches Leben gelten könnte; und eine etwas höher anzusetzende Schwelle, unterhalb deren die für den Menschen charakteristischen Tätigkeiten so reduziert ausgeübt werden, daß [sic!] wir das entsprechende Leben zwar als ein menschliches, nicht aber als ein gutes menschliches Leben bezeichnen würden. Es ist die letztgenannte Schwelle, die uns interessiert, wenn wir uns der Politik zuwenden: Denn wir wollen nicht, daß [sic!] die Gesellschaften ihren Bürgern nur eine minimale Ausübung von Tätigkeiten ermöglichen. Ich glaube mit Aristoteles, daß [sic!] ein politisches System dann gut ist, wenn es jedem einzelnen »die Ausübung tugendhafter Handlungen ermöglicht “" (Nussbaum 1999: 197, Hervorhebungen im Original; die Verf.). Insofern sollte jede Gesellschaft die von Nussbaum aufgeführten zehn Grundfähigkeiten für ihre Mitglieder erzielen. Nussbaum ist davon überzeugt, dass ein Leben, dem eine dieser Grundfähigkeiten fehlt, kein gutes Leben sein kann. Folglich sollte eine Messung der Lebensqualität diese Grundfreiheiten berücksichtigen (vgl. Nussbaum 1999: 200).

59 „Capability assessment can be used for different purposes (varying from poverty evaluation to the assessment of human rights or of human development), and public reasoning and discussion are necessary for selecting relevant capabilities and weighing them against each other in each context. It would be a mistake to build a mausoleum for a "fixed and final" list of capabilities usable for every purpose and unaffected by the progress of understanding of the social role and importance of different capabilities" (Sen 2004: 77). Als Argument bringt Sen hervor:

„What I am against is the fixing of a cemented list of capabilities, which is absolutely complete (nothing could be added to it) and totally fixed (it could not respond to public reasoning and to the formation of social values). I am a great believer in theory. The theory of evaluation and assessment does, I believe, have the exacting task of pointing to the relevance of what we are free to do and free to be (the capabilities in general), as opposed to the material goods we have and the commodities we can command. But pure theory cannot "freeze" a list of capabilities for all societies for all time to come, irrespective of what the citizens come to understand and value. That would be not only a denial of the reach of democracy, but also a misunderstanding of what pure theory can do, completely divorced from the particular social reality that any particular society faces.

Along with the exercise of listing the relevant capabilities, there is also the problem of determining the relative weights and importance of the different capabilities included in the relevant list" (Sen 2004: 78). 
Fokus auf die Betrachtung der kulturspezifischen Entfaltungsmöglichkeiten legt. Insofern vertritt er eine relativistische Position, die jedoch sehr stark auf die Freiheit zur Bestimmung menschenwürdiger Lebensbedingungen bezogen und als multidimensional $\mathrm{zu}$ betrachten ist. Nicht die fundamentale Gleichheit, sondern die Unterschiedlichkeit ist Sen zufolge die Ausgangsannahme ${ }^{60}$. Die Vorteile der Senschen Perspektive hat u. a. auch Reinhard Kreckel zu Recht erkannt:

„In seinem Buch „Inequality reexamined (1992)“ hatte Amartya Sen betont, dass die Ausgangstatsache jeder Ungleichheitstheorie die große Diversität menschlicher Eigenschaften, Wünsche und Lebensbedingungen sein müsse, nicht die Annahme fundamentaler Gleichheit. Der bloße Umstand, dass reale Menschen ,ungleich" seien, sei wenig bemerkenswert. Für Sen lässt sich das FreiheitsGleichheits-Dilemma deshalb nicht einseitig in Richtung auf ein absolutes Gleichheitsideal auflösen, das es gestatten würde, jede Art von Ungleichheit als „,ungerecht“ zu verwerfen. Aus seiner Sicht sind „Handel und Wandel“ und die sich daraus ergebenden Diversitäten und Ungleichheiten ein selbstverständlicher Bestandteil jeden freien menschlichen Zusammenlebens, ebenso wie der zwischenmenschliche Austausch im Gespräch. Soziale Ungleichheit wird erst dann $\mathrm{zu}$ einem nichttrivialen Problem, wenn durch sie die „Freiheit, unterschiedliche Lebensstile zu realisieren", beschnitten wird, weil die Welt in dauerhaft Begünstigte und Benachteiligte aufgeteilt ist. Vor diesem Hintergrund gilt Sens Interesse der Ungleichverteilung von „human capabilities““” (Kreckel 2006: 15, Hervorhebungen im Original; die Verf.).

Kreckel betont durch die Erweiterung der Ungleichheitstheorie von Sen Ungleichheiten nicht nur auf monetäre Ungleichheitsindikatoren zu beziehen, sondern auch Bildungs- und Wissensstände, körperliche und geistige Verfassungen von Menschen sowie materielle und institutionelle Rahmenbedingungen zu erfassen - die Ähnlichkeit zwischen Sens Capabilities und Anthony Giddens (1984) Verständnis von Macht als ,[...] transformative capacity, [...] as the capability of the individual to ,make a difference“" (Giddens, zitiert nach Kreckel 2006: 15).

„Die Ungleichverteilung von „capabilities“ in einem Land, in einem Kontinent oder auch weltweit ist somit im Sinne von Amartya Sen immer auch gleichbedeutend mit einer Ungleichverteilung von Durchsetzungs- bzw. Verwirklichungschancen. Ungleichheit der „Verwirklichungschancen“ verweist also, kurz gesagt, auf das Wirken strukturell verfestigter Machtpotentiale. In diesem Sinne ist Sens Beitrag zur Ungleichheitstheorie deshalb nicht einfach der Beitrag eines Ökonomen, sondern der eines „political economist“, eines Politökonomen, der Fragen von Macht und „empowerment“ nicht ausklammert. Das macht seinen

60 „Human beings differ from each other in many different ways. We have different external characteristics and circumstances. We begin life with different endowments of inherited wealth and liabilities. We live in different natural environments - some more hostile than others. The societies and the communities to which we belong offer very different opportunities as to what we can or cannot do" (Sen 1992: 19f.). 
theoretischen Ansatz für die Soziologie [und insbesondere für diese Dissertation] so besonders interessant und kommt meinem eigenen Verständnis [sowie der Argumentation dieser Dissertation] von strukturierter sozialer Ungleichheit sehr entgegen“" (Kreckel 2006: 15, Hervorhebungen im Original; die Verf.).

Abschließend ist die Rolle der Gleichheit im Nonegalitarismus zusammenzufassen: Zwar hat Gleichheit im Nonegalitarismus nicht oberste Priorität, gleichwohl wird der Gleichheit 1. als Nebenprodukt, 2. als ein Gerechtigkeitsaspekt unter diversen anderen und 3. als Vorbedingung zur Erfüllung humanistischer absoluter Standards (Gleichheit als Mittel zum Zweck) durchaus eine Bedeutung zugesprochen (vgl. Krebs 2000: 32).

Der letzte Punkt, der zugleich ein neues Argument darstellt, setzt voraus,

„dass keine zu große Ungleichheit zwischen den Lebensaussichten der Menschen besteht. [...] Als relationale Vorbedingungen gewisser absoluter Standards haben diese Gleichheiten jedoch nur einen vom Wert dieser Standards abgeleiteten Wert. Sie sind nur Mittel zum Zweck, als Weg zum Ziel, als Teil des Ganzen Geboten“ (Krebs 2000: 32f.).

Welche Erkenntnisse können aus dieser Diskussion für die Familie und insbesondere für die innerhäusliche Arbeitsteilung festgehalten werden? Ein Aspekt, der hierbei im Vordergrund steht, ist die Notwendigkeit Ungleichheiten innerhalb von Haushalten zu erkennen. ,[...] one issue that emerges clearly is the need to recognize inequalities, possibly substantial, within the household" (Sen 1994: 463). Sen übt diesbezüglich Kritik an den Annahmen der "Glued-together Family“ (Annahme der Familie als eine Einheit ohne individuelle Nutzenmaximierung), der "Super-Trader Family“ nach Becker $(1981)^{61}$ und der „Despotic Family“ (Familienoberhaupt trifft Entscheidungen und alle anderen gehorchen): In the standard theory of prices and equilibrium [...], individuals and firms are visible, but definitely no families" (Sen 1994: 452). Keine der speziellen ökonomischen Annahmen eröffnen einen sinnvollen Weg, die traditionellen Ansichten der Preistheorie und Marktallokation beizubehalten. Sie eröffnen zwar interessante Einsichten, die für spezifische Probleme nützlich sein können, aber sie ersparen uns nicht die Notwendigkeit, über die gesamte Frage der Familienökonomie und ihre Implikationen für die traditionelle Preistheorie und Marktallokation erneut nachzudenken (vgl. Sen 1994: 455). Sen schlägt daher vor, den Capability Approach auf die Familie anzuwenden. Das individuelle Wohlergehen wäre hierbei weder in Bezug auf konsumierte Güter noch in Bezug auf die mentale Metrik des Nutzens zu beurteilen, sondern im Hinblick auf die Fähigkeiten von Personen. Es ist eine Perspektive der „Freiheit“ im positiven Sinne: Die Frage, wer was tun kann, steht für ihn im Zentrum, anstatt zu fragen, wer welches

$61, \ldots[. .$.$] individuals are assumed to be relentlessly pursuing their individual utilities, and in$ doing this they enter into trades at implicit prices resulting in marriages and the working of the family“ (Sen 1994: 453). 
Bündel von Waren zur Verfügung hat oder wer wie viel Nutzen erhält. Im Sinne der Capability-Perspektive kann familiales Wohlbefinden vernünftigerweise als eine Funktion dessen gesehen werden, welche positiven Freiheiten die verschiedenen Mitglieder der Familie genießen können (vgl. Sen 1994: 457). Was die Frage einer strikt egalitaristischen Arbeitsteilung anbelangt, äußert sich Nussbaum explizit zu dieser Thematik. Sie differenziert für die Paradigmen, die von getrennten Tätigkeitsbereichen von Männern und Frauen ausgehen, zwei Positionen:

1. Für die erste Position (A) ist grundsätzlich die Norm einer Geschlechtergleichheit von Bedeutung, diese wird jedoch im Sinne einer geschlechtsspezifischen lokalen Spezifikation ausgelegt: Für Männer wie für Frauen gilt zwar derselbe normative Katalog an Fähigkeiten, wobei aber die Tätigkeiten von den Geschlechtern in unterschiedlichen Lebensbereichen ausgeführt werden sollten (vgl. Nussbaum 1999: 219). Nussbaum erachtet diese Position als problematisch, weil „[...] die Zuweisung von »getrennten, aber gleichen « Tätigkeitsbereichen [...] gewöhnlich den Zielen einer dominanten Gruppe [dient] und [...] die Unterdrückung der Machtlosen [zementiert] (Nussbaum 1999: 222).

2. Die zweite Position (B) nimmt zwei geschlechtsspezifische Normen an: Für Männer und Frauen gelten verschiedene Tätigkeitskataloge. Hieran kritisiert Nussbaum u. a., dass die wissenschaftliche Überprüfung nicht möglich ist, da Säuglinge von Geburt an gemäß ihrem Geschlecht unterschiedlich behandelt werden und ihre Emotionen unterschiedlich interpretiert werden. Insofern ist es nicht möglich die Kultur soweit auszuklammern, dass die notwendigen Informationen über die Grundfreiheiten eruiert werden.

Nussbaum argumentiert, dass eine Arbeitsteilung im Haushalt auch über einen längeren Zeitraum völlig vernünftig sei, weil die Haushaltsmitglieder ein unterschiedliches Geschick für spezifische Aufgaben haben, aber:

„Es wäre gegenüber dem aktuellen Zustand in allen bekannten Ländern schon ein großer Fortschritt, wenn die häuslichen Pflichten vom Zeit- und Arbeitsaufwand her gleich verteilt wären. Aber selbst in dieser utopischen Situation kann die Zuweisung von Aufgaben gemäß der traditionellen Rollenverteilung fragwürdig sein, da sie möglicherweise mit einem Mangel an Achtung und Selbstachtung verbunden ist. Wenn zum Beispiel nur alle Mädchen kochen lernen, ist dies nicht ein neutraler Fall von Spezialisierung (so wie das eine Kind Klavier, das andere Klarinette lernt); denn dadurch werden die Stereotype verstärkt, die historisch mit der Tatsache assoziiert sind, daß [sic!] den Frauen weder der Status von Staatsbürgerinnen noch Autonomie zuerkannt wurde. Daher komme ich zu dem Schluß [sic!], daß [sic!] es keine guten Argumente für die Position A gibt und daß [sic!] selbst der kluge Gebrauch von A zur schrittweisen Durchsetzung von gesellschaftlichen Veränderungen mit Vorsicht sowie mit dem Bewußtsein [sic!] zu be- 
trachten ist, daß [sic!] es Normen gibt, die genuinere Gleichheit ermöglichen“ (Nussbaum 1999: 222).

Aufgrund ihrer Kritik an beiden Positionen trifft Nussbaum folgende Schlussfolgerung und betont die Lösung des Gerechtigkeitsproblems - eines durch Ungleichbehandlung verursachten Mangels an Fähigkeiten - von Frauen durch die Konzeption des guten menschlichen Lebens (vgl. Nussbaum 1999: 226):

„Mithin kann die Gesellschaft den Menschen nicht zu einem erfüllten Leben verhelfen, wenn sie die Bereiche [für Frauen die Familie und für Männer die Erwerbstätigkeit] einfach trennt. Sie muß [sic!] danach streben, in jedem einzelnen Menschen die volle Bandbreite menschlicher Fähigkeiten zu entwickeln" (Nussbaum 1999: 225).

\subsubsection{Ein Egalitarismus oder viele Egalitarismen? Varianten des Egalitarismus}

Inwiefern können wir überhaupt von einem einzigen Egalitarismus ausgehen? Knight/Brinton (2017) befassen sich mit der Frage „One Egalitarism or Several?" (Knight/Brinton 2017: 1485). Müssen wir nicht viel mehr von unterschiedlichen Egalitarismen innerhalb Europas ausgehen sowie Egalitarismus als mehrdimensional erachten? Knight/Brinton hinterfragen die von vielen ländervergleichenden Studien vertretene implizite These einer eindimensionalen Dichotomie zwischen Egalitarismus und Traditionalismus. Die zentrale Annahme der Autorinnen ist:

,$[\ldots]$ we suggest that while there may be a general trend across post-industrial Europe toward gender egalitarism in its narrowest sense (i.e., away from traditionalism and the belief in male primacy) there will be a continued plurality of egalitarianisms; some individual will support greater similarity between men and women (less gender essentialist) and others will emphasize the distinct characteristics of men and women (more gender essentialist)" (Knight/Brinton 2017: 1493f.).

Knight/Brinton analysieren 17 europäische Länder und identifizieren mittels einer latenten Klassenanalyse drei Formen des Egalitarismus: den ,liberal egalitarianism“, den ,egalitarian familism“ und den „flexible egalitarianism“ (Knight/Brinton 2017: 1485). Sie stellen fest, dass die europäischen Länder nicht zu einem dominanten egalitären Modell konvergieren, sondern vielmehr durch Varianten des Egalitarismus differenziert bleiben (vgl. Knight/Brinton 2017: 1485).

„[...] the traditional and liberal egalitarian classes comprise the classic bookends of a linear approach to gender egalitarianism. On the one hand, views of male primacy and gender essentialism support traditional family and work values; on 
the other hand, non-gender-essentialist attitudes and disagreement with male primacy are supportive of egalitarian family values and prowork attitudes. However, beyond this dichotomy we identify two classes that evince a more complex attitudinal structure" (Knight/Brinton 2017: 1502).

Wesentlich ist, dass die Autorinnen das Argument des „freedom of choice“ hervorbringen, um die lineare Annahme zwischen Egalitarismus und Traditionalismus zu überwinden. Insbesondere gehen sie davon aus, dass die Kombination von Einstellungen zu Egalitarismus, Essentialismus und individueller Wahl zu geschlechtsspezifischen Einstellungsschemata führen wird, die mit unterschiedlichen Ideologien zusammenhängen und nicht als Varianten entlang eines traditionellen bis egalitären Spektrums verstanden werden können (Knight/Brinton 2017: 1492). Knight/Brinton haben ferner einen mehrdimensionalen Ansatz entwickelt gemäß dem sich GenderrollenEinstellungen nach den Dimensionen „egalitarianism“, „essentialism“ und „individual choice“ unterscheiden lassen, wobei innerhalb Europas die oben genannten Hauptklassen identifiziert wurden (vgl. Knight/Brinton 2017: 1517). Während in den vergangenen Jahrzehnten Zweifel an der impliziten Annahme der Modernisierungstheorie einer Entwicklung vom GenderRollen-Traditionalismus zum Egalitarismus aufgekommen sind, haben Genderungleichheits-WissenschaftlerInnen begonnen, die Kohärenz des Egalitarismus selbst in Frage zu stellen (vgl. Knight/Brinton 2017: 1486).

\subsection{Ein Anwendungsbeispiel des Capability Approachs zur Work-Life-Balance}

Hobson (2011) überträgt den Capability-Ansatz von Sen auf die Diskrepanz zwischen Einstellungen und Verhaltensweisen bezüglich der Work-LifeBalance, deren Ambivalenz sie mittels des ,,agency-gaps“ zu erklären versucht (,,agency achievement" bedeutet nach Sen (1992) das für Handlungsmöglichkeiten zur Verfügung stehende, realisierte individuelle Bündel an Functionings respektive die Realisierung individueller Ziele, unabhängig davon, ob diese zum well-being ${ }^{62}$ beitragen. Ferner ist agency als Handlungsfähigkeit zu verstehen ${ }^{63}$ ).

62 Sens pluralistisches Konzept des well-beings wird im folgenden Zitat deutlich: „There are many fundamentally different ways of seeing the quality of living, and quite a few of them have some immediate plausibility. You could be well off, without being well. You could be well, without being able to lead the life you wanted. You could have got the life you wanted, without being happy. You could be happy, without having much freedom. You could have a good deal of freedom, without achieving much. We can go on" (Sen 1987: 1).

63 „A person's agency achievement refers to the realization of goals and values she has reasons to pursue, whether or not they are connected with her own well-being“" (Sen 1992: 56). 
Agency gap: „New rights and policies for WLB have emerged at the European and national levels - including rights to reduce hours, entitlements to care leaves, and flexibility in working times and workplace. However, there is a gap in the capabilities of individual parents to exercise these rights and utilize these options. The extent of this "agency gap" is dependent on how these entitlements are embedded in different national policy frameworks, mediated through firms/ workplaces, and translated into individual lives and households" (Hobson 2011: 148).

Ihrer Argumentation folgend besteht das agency gap zwischen den Policies und den Möglichkeiten von Individuen, diese zu nutzen bzw. in einer fehlenden Umsetzung politischer Rechte, aufgrund von individuellen, sozietalen und institutionellen Hürden. Capabilities werden auf ,[...] the agency freedom to achive a WLB [Work-Life-Balance]“ übertragen (Hobson 2011: 149). Ferner werden Sens Kategorien des Capability Sets (individual, environmental und societal factors) zu individual, societal und institutional factors modifiziert und bilden nach Hobsons Konzept das Capability Set der Work-LifeBalance im Europäischen Kontext (vgl. Hobson 2011: 157). Auf diese Differenzierung wird im Rahmen der Konzeptualisierung der Typologie von Machtverhältnissen zurückgegriffen, um die Barrieren einer ,agency freedom" - einen Pluralismus innerhäuslicher Arbeitsteilungsarrangements zu erreichen - herzuleiten. Anders formuliert werden die ,agency inequalities“ einer nach wie vor traditionell dominierten innerhäuslichen Arbeitsteilung analysiert. Ein erster Bezug kann zwischen den mikrosoziologischen Theorieansätzen und den Indizes zur Messung von Genderungleichheiten hergestellt werden: insofern als individual factors nach Hobson (2011) überwiegend individuelle Aspekte der symmetrischen ökonomischen Theorien implizieren (u. a. Humankapital, Einkommen, aber auch Gender, Alter), societal factors mittels der Fokussierung auf Normen an asymmetrische Theorien erinnern (Normen, die über Bezugsgruppen, Medien/öffentliche Diskurse und soziale Bewegungen/Mobilisierungen entstehen) und letztlich institutional factors (Policy Level: u. a. (soziale) Rechte, Dienstleistungen der (Kinder)Betreuung) auf einer makrosoziologischen Argumentation beruhen, die vorwiegend für die Konzeption von Genderungleichheitsindizes von Bedeutung ist. Vornehmlich ist die Verknüpfung, dass individuelle und sozietale Faktoren in einen institutionellen Rahmen eingebettet sind, wesentlich (vgl. Hobson 2011: 159). Erste Faktoren einer Differenzierung zwischen Functionings und Capabilities, die aus der bisherigen Argumentation abgeleitet werden können, sind beispielhaft in Tabelle 5 dargestellt.

Zusammenfassend ist festzuhalten, dass Sen an den etablierten Gerechtigkeitstheorien eine Utopie des transzendentalen Institutionalismus kritisiert. Fragen der Gerechtigkeit sollten statt auf gerechte Institutionen auf die Einschätzung sozialer Verwirklichung bezogen werden. Darüber hinaus sieht er eine Stärkung von Gerechtigkeitstheorien in vergleichenden Erwägungen anstelle eines unmöglichen Versuchs, vollkommen gerechte Regelungen fest- 
Tabelle 5: Faktorenbeispiele für Functionings und Capabilities

\begin{tabular}{ll}
\hline Erreichte Functionings & Capabilities \\
\hline Erreichter Bildungsstand & Bildungszugang \\
Ausgeübter Beruf, erzieltes & Zugang zum Arbeitsmarkt \\
Einkommen & Freie Entscheidung zur \\
Kinder & Reproduktion: \\
& Freiheit zur Realisierung/ zur Nicht- \\
& Realisierung des Kinderwunsches \\
Verwirklichte Einstellungen & Recht auf Meinungsfreiheit \\
\hline
\end{tabular}

Quelle: Eigene Darstellung.

zulegen (vgl. Sen 2010a: 437f.). Der Capability-Ansatz verbindet zwei wesentliche Aspekte für die Erklärung sozialer Ungleichheiten miteinander: erstens Functionings (Funktionsweisen), die sich auf das Ergebnis einer spezifischen erreichten Handlung beziehen und zweitens Capabilities (Befähigungen), die die Freiheit der Handlungsmöglichkeiten implizieren. Fraglich ist, warum die Berücksichtigung von Teilhabechancen im Kontext von Genderungleichheiten ein zentraler Aspekt ist, den es zu berücksichtigen gilt? Zur Verdeutlichung der Relevanz erfolgt ein weiteres Beispiel:

Wird die Erwerbslosigkeit zweier Mütter miteinander verglichen, ist die Mutter, die sich freiwillig dazu entschieden hat, erwerbslos zu sein, um sich der Kinderbetreuung zu widmen, ebenso ein ,Opfer ${ }^{6}$ der Erwerbslosigkeit, wie eine, der es gesetzlich untersagt ist, erwerbstätig zu sein. Die Erwerbslosigkeit ist in beiden Fällen eine in die Tat umgesetzte Funktionsweise, doch gibt es einen relevanten Unterschied zwischen den Fällen: während die erste Mutter freiwillig aufgrund ihrer Präferenzen die Handlungsalternative der Erwerbslosigkeit gewählt hat, ist die zweite Mutter insofern benachteiligt, als ihr keine Wahlmöglichkeit zur Verfügung stand; unfreiwillig musste sie ihre Rolle einnehmen (andernfalls wäre sie negativen Sanktionen ausgesetzt). $\mathrm{Ob}$ erreichte Functionings das Ergebnis einer individuellen Wahl darstellen oder auf einen Mangel an Verwirklichungschancen zurückzuführen sind, ist folglich ein zentrales Kriterium für die Bewertung von Gleich- bzw. Ungleichheiten von Functionings. Würde nur die genutzte Funktionsweise der Erwerbslosigkeit gemessen, wäre kein Unterschied der Genderungleichheit zwischen den beiden Müttern festzustellen. Ergo: eine Unterscheidung zwischen ,Handeln" und „Freiheit zum Handeln“ ist erforderlich (Sen 2010a: 265, Hervorhebungen im Original; die Verf.). Doch gibt es systematische Disparitäten der Freiheiten zwischen Frauen und Männern in unterschiedlichen Gesellschaften, die häufig nicht nur auf Einkommensdifferenzen oder Ressourcen begrenzt werden können. Obwohl Lohnunterschiede in vielen Gesellschaften einen konstitutiven Teil von Genderungleichheiten einnehmen, bestehen viele 
andere Bereiche, die ebenso relevant für die Erfassung von Genderungleichheiten sind, wie z. B. die innerhäusliche Arbeitsteilung (vgl. Sen 1992: 122). Insofern ist die Stärke der Senschen Perspektive, seine Theorie auf unterschiedliche Bereiche von Ungleichheiten - und in diesem Fall auf Genderungleichheiten - anwenden zu können. Hierin ist gleichwohl eine wesentliche Schwäche zu finden: die Offenheit der Theorie kann zu sehr unterschiedlichen Auslegungen führen (für eine detaillierte Zusammenfassung von Sens Theorie sowie einen Überblick über die historische Entwicklung und die Schwächen des HDI s. Stanton (2007) „The Human Development Index. A History").

In Abbildung 3 ist eine Zusammenfassung des Capability Approachs dargestellt. In Anlehnung an Hobson lässt sich das Capability Set, d. h. die Gesamtmenge an Verwirklichungschancen, aus individuellen, kulturellen und institutionellen Faktoren im Europäischen Kontext abbilden. Für die Transformation von Ressourcen in verwirklichbare Handlungsmöglichkeiten und weiterführend in verwirklichte Funktionsweisen („Achieved Functionings") werden sogenannte Umwandlungsfaktoren („Conversion Factors") zur Vermittlung benötigt (s. Kapitel 4.5.). Dass im Sinne einer dynamischen Perspektive erreichte Funktionsweisen grundsätzlich wiederum eine Rückkopplung auf Ressourcen und individuelle Umwandlungsmöglichkeiten haben können, soll ergänzend durch den gestrichelten Pfeil visualisiert werden.

\subsection{Eigene Erweiterung: Macht im Capability-Ansatz als Fähigkeit und Befähigung}

Für die Konzeption einer Theorie der Macht werden Sens Capabilities als Power-Capabilities verstanden, die für die Realisierung einer Handlungsalternative innerhäuslicher Arbeitsteilungsarrangements zur Verfügung stehen. Capabilities beziehen sich im Allgemeinen auf die Freiheit der Handlungswahl als Befähigung, die hier im Sinne einer Ermächtigung (Empowerment) den Handlungsspielraum festlegen (Sens Befähigung entspricht hier der Empowerment). Das Power-Capability Set umfasst die Gesamtheit an PowerCapabilities, die auf unterschiedlichen Ebenen (individuell, kulturell, institutionell und strukturell) verortet werden können (s. Kapitel 9.). Auf individueller Ebene sind Ressourcen zwar für die Machtfähigkeit (im Sinne einer Verhandlungsmacht) von Bedeutung, doch so wie Sen eine ressourcenorientierte Fokussierung der Messung des Wohlergehens feststellen konnte, ist auch eine ressourcenorientierte Analyse der innerhäuslichen Arbeitsteilung 
Abbildung 3: Zusammenfassende Darstellung des Capability Approachs

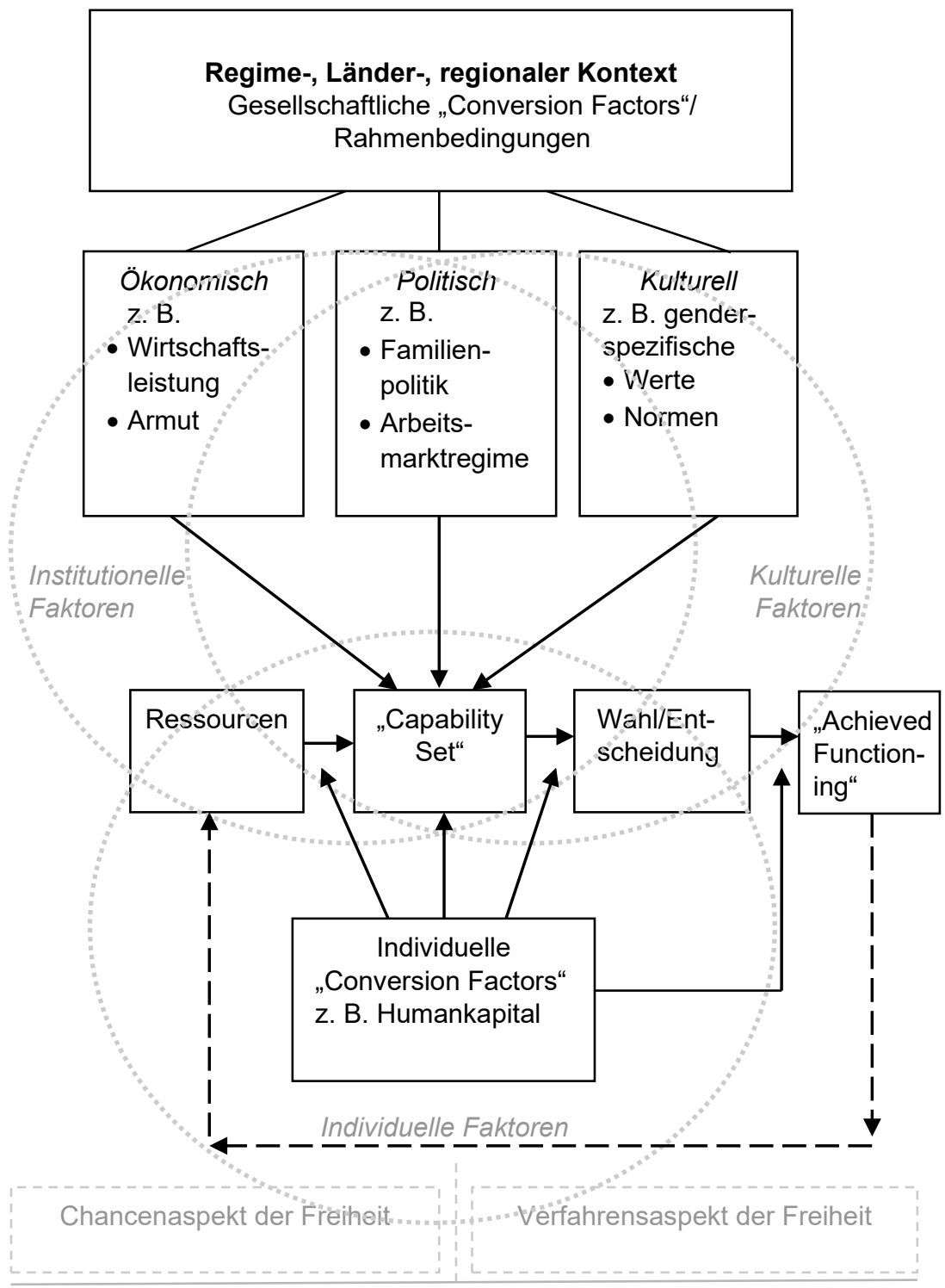

Quelle: modifiziert nach Bartelheimer et al. 2008: 10; Hobson/Fahlén 2009: 230 . 
festzustellen ${ }^{64}$. Darüber hinaus sind die theoretischen Ansätze der Gendertheorien und ihre empirische Analyse zunehmend en vogue. Sens Ansatz bietet den notwendigerweise komplexen und multidimensionalen theoretischen Rahmen für Genderungleichheiten der Power-Capabilities, um diese Theorien in einen argumentativen Zusammenhang innerhalb eines makrostrukturellen Kontextes zu bringen und die Barrieren eines Pluralismus innerhäuslicher Arbeitsteilungsarrangements aufzeigen zu können.

Ähnlich wie auch bei Krügers/Levys (2000) institutionenorientierten Lebenslaufansatzes wird ,[...] ein Modell [konstruiert], das subjektives Handeln und die biographisch zeitgleiche Teilhabe an verschiedenen Institutionen [u. a Bildungssystem, Arbeitsmarkt] miteinander verbindet und hierüber biographisch wechselnden Handlungschancen von Personen Rechnung tragen kann“" (Krüger/Levy 2000: 382). Zwei zentrale Vorteile dieses mehrdimensionalen Verfahrens bestehen darin, dass erstens die Egalitätskonzeption der Gleichstellung nicht, wie in vielen anderen Studien, normativ-egalitär (u. a. Coverman (1985), Ott (1992), Schulz/Blossfeld (2006)), sondern pluralistisch erfolgt. Zweitens kann mittels der Capabilities eine Kontextualisierung vorgenommen werden: „The capabilities paradigm is a normative framework for life quality that can be adopted to different contexts" (Hobson 2011: 153). Sowohl Hobson als auch Esping-Andersen sowie Korpi vertreten eine Orientierung am skandinavischen Wohlfahrtsstaatsmodell, die von Ostner (2004b) als „hegemonialer Wohlfahrtsstaatsfeminismus“ bezeichnet werden würde bzw. bezugnehmend auf Hobson und Orloff auch bezeichnet wird (Ostner 2004b: 45). Bevor auf diese Debatte, einschließlich Ostners Kritik detailliert eingegangen wird, erfolgt zunächst eine Beschreibung der klassischen Typologie von Esping-Andersen.

64 „I would argue that the question of gender inequality in the advanced societies - no less than in the developing countries - can be understood much better by comparing those things that intrinsically matter (such as functionings and capabilities), rather than just the means like primary goods or resources. The issue of gender inequality is ultimately one of disparate freedoms" (Sen 1992: 125). 


\section{Zur systematischen Ausklammerung der innerhäuslichen Arbeitsteilung in der Wohlfahrtsstaatsforschung}

Da die Frage der Sozialpolitik immer auch eine Eigenart der Macht respektive der Ermächtigung darstellt, werden sowohl die Argumente EspingAndersens als auch Korpis im Rahmen der „Power Ressource School“ kategorisiert ${ }^{65}$. "The issue of consensus in social policy is, accordingly, also a question of power" (Esping-Andersen/Korpi 1987: 73). Das Argument lautet: „politics can be used against markets“ und bezieht sich auf die Dimension des Kräfteverhältnisses zwischen Markt und Staat (vgl. Esping-Andersen 1990: 23). Zunahmen von Staatseingriffen drängen den Markt zurück, woraus eine Verschiebung von ökonomischer Macht hin zu politischer Macht resultiert. Die „Power Resource School“ betrachtet den Konflikt zwischen ArbeiterInnen und KapitalistInnen als zentral und erachtet die Sozialpolitik als eine kritische Arena für die Entwicklung, Verteilung und Verbesserung von Machtressourcen. BefürworterInnen dieser Schule - wie u. a. Korpi (1978; 1983) - argumentieren, dass die ArbeiterInnenklasse Möglichkeiten hat, die Sozialpolitik in eine Richtung zu lenken, die ihren Interessen zweckdienlich ist, um ihre Ressourcen weiter auszubauen. Es werden zwei Arten von Machtressourcen identifiziert: marktbasierte Machtressourcen (in Form von Kapital und ökonomischen Ressourcen), die in kapitalistischen Systemen inhärent und zwischen sozialen Klassen und Interessensgruppen ungleich verteilt sind und das Recht zu wählen sowie kollektives Handeln (politisch) zu organisieren, wobei angenommen wird, dass das Wahl- und Vereinigungsrecht in einer Demokratie jedem Bürger/jeder Bürgerin aufgrund der Staatsbürgerschaft offen steht, d. h. gleichmäßig verteilt ist (vgl. Korpi 1989: 312). KapitalistInnen verfügen über größere Ressourcen auf dem Markt, während ArbeiterInnen aufgrund ihrer größeren Anzahl über mehr Ressourcen im politischen Geschehen verfügen. ArbeitnehmerInnen - so die Argumentation der „Power Resource School AnhängerInnen“ - werden politische Ressourcen nutzen, um Marktprozesse zu beeinflussen und Sozialleistungen auszuweiten (vgl. Orloff 1993b: 66). „In short, politics can be used against markets (to borrow from the title of Esping-Andersen's 1985 book)" (Orloff 1993b:

65 „Die leitende Idee war, dass Klasseninteressen mit der sozialen Lage korrelieren. Die Arbeiter[Innen]klasse und die politische Linke unterstützten die Wohlfahrtsreform gleichsam naturgemäß, die Rechte und die Mittelklassen waren zwangsläufig dagegen. Das Kräfteverhältnis zwischen beiden galt als maßgeblich für den Ausgang der Auseinandersetzung um die soziale Frage. So etwa lässt sich die Argumentation zusammenfassen, die von der labouristischen bzw. sozialdemokratischen, auf Machtressourcen abstellenden Wohlfahrtsstaatstheorie vertreten wird“ [wie u. a. von Korpi (1978) und Esping-Andersen (1985; 1990)] (Baldwin, P. 2003: 50). 
66). Da die Entwicklung von Wohlfahrtsstaaten nicht allein auf ArbeiterInnenbewegungen zurückgeführt werden könne (wie jedoch von neomarxistischen „left power resource“-Ansätzen angenommen wird), argumentiert Esping-Andersen: ,[...] the history of political class coalitions [is] the most decisive cause of welfare state variations" (Esping-Andersen 1990: 1). Die unterschiedlichen Ausprägungen der ArbeiterInnenbewegung könne nicht allein die Variationen der Ausgestaltung von Sozialstaaten erklären, weshalb aus der Perspektive Esping-Andersens die Geschichte von Wohlfahrtsstaaten durch politische Klassenkoalitionen zu rekonstruieren sei (vgl. Manow 2007: 416). In Folge der Argumentation von Esping-Andersen (1985) etablierte sich eine Diskussion über die Pluralität von Wohlfahrtstaatlichkeit, weshalb Esping-Andersen (1990) seine These vom „erstrebenswerten sozialdemokratischen Regime“ als Leitbild und den restlichen „residualen Wohlfahrtsstaaten" modifiziert, indem er

,[...] die schlichte manichäische Dualität zugunsten einer neutestamentarischen Trinität aufgegeben [hat]. Statt einer einzigen Achse mit zwei Polen, auf der die Wohlfahrtsstaaten entsprechend ihrer jeweiligen Abweichung vom Ideal verortet werden, sah er nun drei getrennte Pfade wohlfahrtsstaatlicher Entwicklung mit jeweils verschiedenen Zielrichtungen“" (Baldwin, P. 2003: 55).

Die Wohlfahrtsstaatsforschung, die für die Analyse von Ungleichheiten ein wesentlicher Forschungszweig ist, hat hierbei lange Zeit versäumt, innerhäusliche Arbeitsteilungsarrangements $\mathrm{zu}$ berücksichtigen, wie anhand der folgenden Forschungsdebatte ersichtlich wird.

\subsection{Die „Power Resource School“" nach Esping-Andersen und Korpi und ihre feministische Kritik}

Eines der grundlegenden Werke innerhalb der Wohlfahrtsstaatsforschung, auf das immer wieder Bezug genommen wird, ist Esping-Andersens ,The three Worlds of Welfare Capitalism“. Esping-Andersen (1990) entwickelte im Rahmen seiner Staats-Markt-Typologie drei Idealtypen von Wohlfahrtsstaaten, die sich in ihrem Zusammenspiel zwischen Staat, Markt und Familie unterscheiden: den liberal-angelsächsischen, den konservativkontinentaleuropäischen und den sozialdemokratisch-skandinavischen Wohlfahrtsstaat.

„As we survey international variations in social rights and welfare-state stratification, we will find qualitatively different arrangements between state, market and the family. The welfare-state variations we find are therefore not linear distributed, but clustered by regime-types" (Esping-Andersen 1990: 26). 
Im Hinblick auf die Erklärung der Regime seien die Mobilisierung der ArbeiterInnenklasse, politische Koalitionen und nationale Entwicklungspfade der Wohlfahrtsstaatsbildung von Bedeutung, wobei der Mobilisierung der ArbeiterInnenklasse eine deutlich geringere Relevanz ${ }^{66}$ zugesprochen wird (vgl. Esping-Andersen 1990: 29). Für einen kurzen Überblick sind nachfolgend die wesentlichen Charakteristika der Regime aufgeführt:

1. Liberal-angelsächsisch (USA, Australien, Kanada, Schweiz, Japan):

- Marktfreiheit als zentrales Prinzip

- geringe Umverteilung ${ }^{67}$

- minimale Dekommodifizierung ${ }^{68}$

- Arbeit wird als Ware gehandelt

- private Fürsorge/Absicherung

- Markt als zentrale Instanz des Bedarfsausgleichs: geringe Sozialleistungen, die an strenge Bedürftigkeitsüberprüfungen gekoppelt sind

- hohe sozioökonomische Ungleichheiten

2. Konservativ-kontinentaleuropäisch (Österreich, Belgien, Frankreich, Deutschland, Italien, Niederlande $\left.{ }^{69}\right)$ :

66 „It is a historical fact that welfare state construction has depended on political coalitionbuilding. The structure of class-coalitions is much more decisive than are the power resources of any single class" (Esping-Andersen 1990: 30).

67 Die Dimension der Stratifizierung (Schichtung) führt Esping-Andersen auf die Möglichkeit von Sozialstaaten zurück, Umverteilungen vorzunehmen. Sowohl ökonomische Gleichheit als auch Differenz kann durch das Agieren des Staates hergestellt werden. Stratifizierung meint folglich die politische Möglichkeit des Staates eine Strukturierung sozialer Differenzen vorzunehmen. Ferner unterscheiden sich die drei Wohlfahrtsstaatsregime in ihrer Stratifizierungswirkung: Das sozialdemokratische Regime basiert überwiegend auf universalen Wohlfahrtsleistungen, woraus eine egalisierende Stratifizierungswirkung resultiert. Die Leistungen im konservativen Regime sind primär erwerbsabhängig, was Statusungleichheiten verfestigt. Im liberalen Regime sind Leistungen abhängig von einer materiellen Bedarfsüberprüfung, was zu verschärften Ungleichhheiten führt.

68 Die Dimension der Dekommodifizierung minimiert die Abhängigkeit von bezahlter Erwerbstätigkeit durch sozialstaatliche Leistungen respektive es entsteht eine Entkopplung von Erwerbstätigkeit und Sozialleistungen. „De-commodification occurs when a service is rendered as a matter of right, and when a person can maintain a livelihood without reliance on the market" (Esping-Andersen 1990: 21f.).

69 Über die Schwierigkeiten der Klassifizierung der Niederlande wird im Rahmen der Fallbeschreibung ausführlich diskutiert. Vorwegzunehmen ist, dass Goodin/Smitsman auf EspingAndersens Fehlklassifizierung der Niederlande verweisen: „Within his own coding categories and (alas, now dated) data, the Dutch welfare state should have been classed squarely within his "social democratic welfare regime" type. [...] Esping-Andersen himself was reluctant to accept this conclusion, though. For him, it seems, program structures of the sort analyzed in Three Worlds of Welfare Capitalism are only part of the story. While in terms of program structures and policy instruments the Netherlands may have looked like a hightax, high-transfer social democratic welfare state, it was not, in his eyes, "really" social democratic because those policies and programs came about via the wrong historical route. Owing to fundamentally corporatist features of its pillarized past, Esping-Andersen still 
- Familie als zentrale Instanz

- Sicherheit als übergeordnetes Ziel

- geringe Umverteilung, erwerbsorientiert

- Sozialleistungen sind abhängig von Dauer und Höhe zuvor geleisteter Beiträge/Unterstützung korrespondiert mit ehemaligen Beiträgen aus Erwerbstätigkeit

- mittlere Dekommodifizierung

3. Sozialdemokratisch-skandinavisch (Schweden, Norwegen, Dänemark, Finnland):

- Staat als zentrale Instanz

- übergeordnetes Ziel: Gleichheit

- hohe Umverteilung

- hohe Dekommodifizierung

- egalisierende Stratifizierungswirkung

Die Wohlfahrtsregime können anhand der Staats-Markt-Familien-Triade differenziert werden, inwiefern sie welche Einheit gewichten. Diese Typologie hat sich zwar zu einem Grundlagenkonzept in der Wohlfahrtsstaatsforschung etabliert, jedoch finden osteuropäische Länder keine Berücksichtigung und auch die südeuropäischen Länder argumentierten vehement, dass sie eigenständige Wohlfahrtsstaaten darstellen, selbst wenn sie Wohlfahrtsstaatlichkeit nicht in derselben Weise gestalten, wie sie dem nordeuropäischen Modell entspricht (vgl. Baldwin, P. 2003: 56). In Folge eines ausführlichen wissenschaftlichen Diskurses etablierte sich das Argument der neueren Wohlfahrtsstaatsforschung, von einer Vielfalt historischer Wohlfahrtsstaatstypen auszugehen, was maßgeblich von P. Baldwin (1990) vorangetrieben wurde.

Darüber hinaus ist insbesondere seitens des Feminismus auch Fundamentalkritik geübt worden, dass der Bereich der Familie nur oberflächlich thematisiert und vornehmlich die unbezahlte Hausarbeit vernachlässigt würde.

„Die feministische Wohlfahrtsstaatsforschung hat Esping-Andersen $[\ldots] \mathrm{zu}$ Recht vorgeworfen, dass sein Verständnis von Familie oberflächlich und seine Konzeption des Verhältnisses zwischen Familie und Staat in der Wohlfahrtsproduktion unterkomplex sei“ (Leitner 2013: 19).

persists in classifying the Netherlands as a "conservative Continental corporatist" welfare state" (Goodin/ Smitsman 2000: 41). 


\subsection{Feministische Kritik an Mainstream-Typologien}

Das Konzept der Dekommodifizierung ${ }^{70}$ nimmt nahezu ausschließlich Bezug auf das männliche Erwerbsmodell (weil nicht alle in gleichem Ausmaß am Erwerbsleben teilnehmen, Frauen sind weniger kommodifiziert): ,[...] any further development of the concept of 'welfare regime' must incorporate the relationship between unpaid as well as paid work and welfare" (Lewis 1992: 159). Das Konzept der Stratifizierung richtet das Hauptaugenmerk auf Machtressourcen durch Klassenbeziehungen. Die Typologie ist insgesamt auf eine Staats-Markt-Relation begrenzt und vernachlässigt Genderungleichheiten, wobei eine genderdifferenzierte Betrachtung von Wohlfahrtsstaaten einschließlich der innerhäuslichen Hausarbeiten/unbezahlten Arbeit vornehmlich von Jane Lewis, Ilona Ostner und Ann Shola Orloff angeregt wurde.

„Its [mainstream comparative research] concepts are explicitly gender-neutral but the categories of workers, state-market relations, stratification, citizenship, and decommodification are based on a male standard; moreover, gender relations and their effects are ignored" (Orloff 1993a: 307).

Ann Shola Orloff wirft dies insbesondere Esping-Andersen (1985; 1989; 1990) und Korpi (1989 sowie Esping-Andersen/Korpi 1987) vor und leitet aus ihrem Vorwurf einen integrativen Ansatz von Mainstreamkonzepten der Power Resource School und der Genderdimension ab (vgl. Orloff 1993a: 303):

1. Die Staats-Markt-Relation wird zur Staats-Markt-Familien-Relation ausgeweitet, wodurch wohlfahrtsstaatliche Leistungen auch für die Familie berücksichtigt werden. Ein für diese Dissertation zentraler Kritikpunkt ist, dass „Power resource analysts simply do not discuss power relations within the family" (Orloff 1993a: 313). Ferner sind „[...] gender hierarchies, power relations within families, and the social organization of caring and domestic labor" in diese Dimension zu integrieren (Orloff 1993a: 322).

2. Die Dimension der Stratifizierung wird durch die Berücksichtigung von staatlicher sozialer Absicherung in Bezug auf die Genderrelation, insbesondere die Behandlung von bezahlter und unbezahlter Arbeit, erweitert. Orloff übt vornehmlich an der impliziten Annahme von Angehörigen der Power Resource School Kritik, dass Frauen zwischen

70 „Thus concepts such as 'decommodification' or 'dependency' have a gendered meaning that is rarely acknowledged. While Esping-Andersen (1990) writes of decommodification as a necessary prerequisite for workers' political mobilization, the worker he has in mind is male and his mobilization may depend as much on unpaid female household labour as state policies“"(Lewis 1992: 161). 
unbezahlter Hausarbeit und bezahlter Erwerbstätigkeit gewählt hätten (vgl. Orloff 1993a: 317). Teilzeitarbeit sei doch gerade der Versuch, Erwerbsarbeit und Hausarbeiten miteinander zu vereinbaren, sodass diese Tätigkeiten nicht als einander ausschließende Bereiche verstanden werden können.

3. Zugang zu bezahlter Arbeit und die Fähigkeit, einen autonomen Haushalt zu führen (Freiheitsargument der Individualisierung) ${ }^{71}$ werden zusätzlich innerhalb der Dimension der De-kommodifizierung thematisiert.

Ein Wandel der paternalistischen Vorstellungen von führenden WohlfahrtsstaatsforscherInnen kann nach Orloff durch eine Evaluierung von

„(1) the extent to which the state has taken over the provision of welfare services (an aspect of state-family-market relations), (2) the relative treatment of paid and unpaid workers (an aspect of stratification), (3) the bases of people's claims to services (an aspect of social citizenship rights), (4) women's access to paid work, and (5) women's capacities to form an maintain autonomous households", erreicht werden (Orloff 1993a: 323).

Durch die feministische Auseinandersetzung Ostners (1998) mit der Wohlfahrtsstaatstypologie Esping-Andersens (1990) wurde das Konzept der DeFamilialisierung ${ }^{72}$ entwickelt.

„Frauen, so die These, müssten, um dem Mann vergleichbar in den Arbeitsmarkt integriert sein zu können, erst wie dieser „,kommodifiziert“ (beschäftigungsfähig) werden, konkret: von der Pflicht, zuhause für Kinder und ältere Familienangehörige zu sorgen, befreit, also „de-familialisiert“ werden“ (Leitner et al. 2004: 16). „Die anvisierte (Re-)Kommodifizierung erfordert langfristig eine DeFamilialisierung von Frauen. Gleichzeitig bedeutet ein Mehr an DeFamilialisierung ein Weniger an Familialisierung, wobei allerdings höchst ambivalente Entwicklungen und Differenzierungen zwischen unterschiedlichen Gruppen von Frauen festzustellen sind“" (Leitner et al. 2004: 17).

Negative De-Familialisierungsmaßnahmen bzw. Re-Kommodifizierungsmaßnahmen schränken das Spektrum an möglichen Lebensentwürfen ein, positive erweitern den Optionsrahmen durch Möglichkeiten zur Vereinbarkeit von Beruf und Familie (vgl. Leitner et al. 2004: 17; Leitner 2013: 26). Neben De-Familialisierungstendenzen können auch Familialisierungstendenzen in Deutschland festgestellt werden. Positive Maßnahmen fördern insbesondere

71 Die Handlungsoption eine Beziehung zu beenden und eine autonome Haushaltsführung zu übernehmen - das „Right of Exit” nach Albert O. Hirschmann (1970) - könnte auch das Machtverhältnis in Paarbeziehungen beeinflussen.

72 Die De-Familialisierung bezeichnet den „Prozess der Auslagerung von Pflege- und Betreuungsarbeit aus der Familie“ im Unterschied zur Re-Familialisierung, die den „Prozess der Rückverlagerung von Pflege- und Betreuungsarbeit in die Familie“ beschreibt (Leitner 2013: 16). 
verheiratete Eltern, die es sich leisten können, nicht erwerbstätig zu sein (vgl. Leitner et al. 2004: 17).

„Während also kinderlose Ehepartner zu Erwerbstätigen werden sollen, wird Ehepaaren mit Kindern weiterhin die Türe zum Ernährermodell offen gehalten bei gleichzeitiger Verbesserung der Vereinbarkeit von Familie und Beruf" (Leitner et al. 2004: 17).

Negative Maßnahmen der Re-Familialisierungstendenzen betreffen Kürzungen im Sozialversicherungsbereich, wodurch die Existenzsicherung für NiedrigeinkommensbezieherInnen prekär wird (vgl. Leitner et al. 2004: 19) ${ }^{73}$.

„Beide Entwicklungen treffen Frauen in besonderer Weise: Zum einen werden Lebensentwürfe selektiv eingeschränkt (und gleichzeitig erweitert), zum anderen sind Frauen aufgrund geschlechtsspezifischer Arbeitsmarktdisparitäten von sozialen Kürzungsmaßnahmen stärker betroffen und damit schneller auf familiale Unterstützung (oder Sozialhilfe) angewiesen, obwohl mit dieser Unterstützung angesichts gesamtgesellschaftlicher Individualisierungstendenzen nicht mehr ohne weiteres gerechnet werden kann" (Leitner et al. 2004: 19).

Esping-Andersen reagierte (1999) auf die Kritik, indem er die Differenzierung von familialistischen und de-familisierenden Regimen ${ }^{74}$ einführte. Sozialdemokratische Regime werden folglich als de-familisierend definiert, liberale Regime werden über marktabhängige Dienstleistungen de-familisiert und konservative Regime werden als familialistisch klassifiziert.

„Man kann dieser Analyse zunächst entgegen halten, dass (1) auch in den sozialdemokratischen Wohlfahrtsstaaten die Familie immer noch der Hauptdienstleister im Pflege- und Betreuungsbereich ist und (2) die De-Familisierung in den liberalen Wohlfahrtsstaaten einen starken schichtsspezifischen Bias enthält, da sich nur ein Teil der Bevölkerung de-familisierende Dienste leisten kann und die Qualität der erlangten De-Familisierung nach Einkommen variiert. Der Hauptkritikpunkt betrifft jedoch das zweite Indikatorenset, das Esping-Andersen zur Bestimmung des Familialismusgrads einsetzt [Anteil älterer Menschen, die bei ihren Kindern wohnen; arbeitslose Jugendliche, die bei ihren Eltern wohnen und die Anzahl der wöchentlich von Frauen verrichteten Stunden für Hausarbeiten]. Dieses bildet Ergebnisse wohlfahrtsstaatlicher Rahmenbedingungen ab, enthält aber keine Informationen zur strukturellen Ausgestaltung dieser Rahmenbedingungen und kann auch nichts zum Verhältnis zwischen strukturellen Rahmenbedingungen und Ergebnissen aussagen. Wenn es aber, wie in der oben zitierten Definition [s. u. Fußnote 74], um diejenigen sozialpolitischen Maßnahmen geht, die als struktu-

73 Die Differenzierung zwischen positiven/negativen De-Familisierungsmaßnahmen (=ReKommodifizierungsmaßnahmen) und Re-Familisierungsmaßnahmen (=De-Kommodifizierungsmaßnahmen) wurde bereits von Ostner (2003) eingeführt (vgl. Ostner 2003: 54).

74 Ein familialistisches Regime ,[...] is one in which public policy assumes - indeed insists that household must carry the principal responsibility for their members' welfare. A defamilizing regime is one which seeks to unburden the household and diminish individuals' welfare dependence on kinship" (Esping-Andersen 1999: 51). 
relle Rahmenbedingungen familisierend oder de-familisierend wirken, d. h. der Familie als Ort der Wohlfahrtsproduktion mehr oder weniger Gewicht beimessen, sollten diese sozialpolitischen Maßnahmen auch ins Zentrum der Analyse gestellt werden“" (Leitner 2013: 23).

In Abgrenzung zu Esping-Andersen und unter Berücksichtigung dieser Kritik hat Leitner (2003) eine Familialismustypologie ${ }^{75}$ entwickelt, die auf der Gegenüberstellung von familisierenden und de-familisierenden Maßnahmen beruht (vgl. Leitner 2013: 37):

Der erste Typus, der explizite Familialismus, klassifiziert familisierende Maßnahmen, die Familien in ihren Betreuungs- und Pflegeaufgaben unterstützen. Darüber hinaus wird ein Fehlen von de-familisierenden Maßnahmen konstatiert, wodurch keine andere Wahl als die familiale Pflege und Betreuung ermöglicht wird.

Im Rahmen des optionalen Familialismus werden sowohl familisierende als auch de-familisierende Maßnahmen typologisiert. Familiale Pflege und Betreuung werden ermöglicht, ohne hierbei verpflichtend zu sein.

Im impliziten Familialismus werden weder familisierende noch defamilisierende Maßnahmen gewährt. Gleichwohl werden Pflege und Betreuung hauptsächlich von der Familie übernommen, weil es keine anderen Alternativen gibt.

Der De-Familialismus ist durch seine de-familisierenden Maßnahmen gekennzeichnet. Es bestehen keinerlei familisierende Maßnahmen, weshalb die Familie in Bezug auf Pflege und Betreuung zwar entlastet wird, jedoch auch kein Recht auf familiale Pflege und Betreuung besteht (vgl. Leitner 2003: 358f.; Leitner 2013: 26f.). Die Typologie Leitners wird im Rahmen der theoriegeleiteten Fallauswahl in Bezug auf die Beschreibung und Differenzierung der konservativen Länder nochmals aufgegriffen, wobei ihre empirischen Fallbeispiele vertieft werden.

Aufgrund der feministischen Kritik an der international vergleichenden Wohlfahrtsstaatsforschung hat Korpi (2000) einen Ansatz entwickelt, Klassenbeziehungen und Genderungleichheiten als integrative Dimensionen zu analysieren. Folglich gehen Korpi (2000) wie auch Orloff davon aus, dass eine Kommodifizierung von Frauen einen Weg der Unabhängigkeit vom männlichen Ernährer für Frauen darstellt.

„Two additional dimensions are proposed to capture the effects of state social provision on gender relations: access to paid work and capacity to form and maintain an autonomous household" (Orloff 1993a: 303).

Insofern sei es von Relevanz, Frauen den Zugang zum Arbeitsmarkt zu ermöglichen. Das bevorzugte Modell nach Korpi (ebenso nach Esping-

75 Für eine tabellarische Übersicht der Familialismustypen nach Leitner (2003, 2013) s. Tabelle 27 im Anhang. 
Andersen) ist eindeutig das „Adult Worker Model“, das eine modernisierungstheoretisch-egalitaristische Perspektive erzielt. Doch ist eine Integration in den Arbeitsmarkt zwar wichtig, aber nicht um jeden Preis. Es gilt zumindest eine Differenzierung der Arbeitsbereiche respektive die Segregation sowie die Flexibilität des Arbeitsmarktes zu berücksichtigen (z. B. Dienstleistungssektor in Schweden).

\subsection{Weiterentwicklung der „Faces of Inequality“}

\subsubsection{Reaktion von Korpi}

Walter Korpi (2000) nimmt an, dass Forschungen über Wohlfahrtsstaaten und Ungleichheiten entweder auf sozioökonomische Klassen oder Genderungleichheiten fokussieren. „Research on welfare states and inequality has tended to be bifurcated, focusing either on class or on gender" (Korpi 2000: 1). Im Rahmen seiner Synthese wird eine Kombination von „gender“ und „class“ mittels einer Analyse von Mustern von Ungleichheiten in unterschiedlichen Wohlfahrtsstaaten vorgenommen.

Forschungsleitend sind die Fragen, welche Aspekte der ,agency“ im Hinblick auf Genderungleichheiten zu berücksichtigen sind, wie Gender und Klasse mit unterschiedlichen Wohlfahrtsstaaten zusammenhängen und inwiefern spezifische Wohlfahrtsstaatstypen Unterschiede von Ungleichheiten in Bezug auf Gender und Klasse hervorrufen (vgl. Korpi 2000: 2). Korpis Ziel ist die Entwicklung einer Typologie von Wohlfahrtsstaaten, die auf institutionellen Strukturen basiert, die für Klassen- und Genderungleichheiten von Bedeutung sind. „The paper develops a new typology of welfare states based on institutional structures of relevance for gender inequality as well as class inequality" (Korpi 2000: 1). Es wird in Anlehnung an Sen eine Fokussierung auf durch Welfare Policies beeinflusste ,,agency inequalities“ vorgenommen. Sens Argumentation, dass unterschiedliche Aspekte der „,agency“ die „,capability" reflektieren, „functionings" zu erreichen, die die Freiheit, ,well-being“ zu haben, darstellen, gilt es nach Korpi in die Analyse von Gender und Klassenungleichheiten einzubeziehen. Vornehmlich seien drei relevante Dimensionen der ,gendered agency inequality“ relevant: demokratische Politics, Zugang zum tertiären Bildungssystem und Zugang zum Arbeitsmarkt (vgl. Korpi 2000: 6). Bezüglich des Zugangs zum Arbeitsmarkt wird die Annahme vertreten, dass vom Arbeitsmarkt ausgeschlossene Individuen insbesondere im Hinblick auf materiellen Lebensstandard und soziale Rechte (die meist vom Beschäftigungsausmaß abhängen, wie z. B. die Leistungen des konservativen Wohlfahrtsstaats) benachteiligt sind (vgl. Korpi 2000: 8). Wesentlich sei außerdem, die Wahl zwischen bezahlter und unbezahlter Arbeit für ,gen- 
dered agency inequalities" in sozioökonomischen Stratifizierungsprozessen zu erkennen. Das Wollstonecraft-Dilemma (benannt nach Mary Wollstonecraft) nimmt Bezug auf die Debatte über Gleichheit und Differenz der Geschlechter. Ferner wird die Streitfrage zwischen bezahlter und unbezahlter Arbeit aufgegriffen. Die Differenz-Argumentation besagt, dass die unbezahlte Arbeit von Frauen die Basis ihrer Rechte und Positionen bilden sollte (gleichermaßen wie bezahlte Arbeit für Männer). Hingegen erstrebt die Gleichheitsargumentation Policies, die Frauen im gleichen Maße befähigen, am Arbeitsmarkt teilnehmen zu können wie Männer (vgl. Korpi 2000: 8). Korpi vertritt die Gleichheitsargumentation und begründet diese folgendermaßen:

Partizipation am Arbeitsmarkt determiniert nicht nur die Verteilung von Ressourcen (einschließlich Eigenverantwortlichkeit für die Existenzsicherung von Individuen (vgl. Leitner et al. 2004: 10)) als Basis materieller Ungleichheiten, sondern auch die Identität einer Person wie auch den Handlungsrahmen, der Capabilities und Freiheiten in vielen anderen Lebensbereichen beeinflusst. Eine Veränderung der Verteilung der innerfamiliären Verhandlungsmacht ist eine Vorbedingung für einen Wandel der Arbeitsteilungsarrangements innerhäuslicher Tätigkeiten. Zugleich ist der Transfer von innerfamiliärer Betreuung zum außerfamiliären Arbeitsmarkt (insbesondere zum Pflegesektor) mit einem bedeutenden Wandel intrafamiliärer Verhandlungsmacht verbunden (vgl. Korpi 2000: 8f.). Folglich bilden in westlichen Gesellschaften Genderungleichheiten in Bezug auf die Teilhabe am Arbeitsmarkt eine zentrale Domäne der ,gendered agency inequalities“. Oder wie Sen sagen würde: die „capability“, um „functionings“ zu wählen und zu erreichen, konstituiert die individuelle Freiheit, sodann sind ,agency inequalities“ als Hauptprozess sozio-ökonomischer Stratifizierung durch den Arbeitsmarkt von besonderer Bedeutung für Genderungleichheiten.

Im Rahmen der Entwicklung seiner Typologie übt Korpi zunächst (insbesondere in Anlehnung an die bereits angeführte feministische Argumentation von Orloff (1993)) $)^{76}$ Kritik an der Typologie Esping-Andersens (1990). Die Typologie nach Esping-Andersen (1990) sei als Basis für Korrelationen zwischen multidimensionalen Indikatoren für allgemeine deskriptive Zwecke hilfreich, doch könne der Nutzen einer Typologie als analytisches Tool für Kausalanalysen gesteigert werden, wenn Indikatoren auf die Reflexion der Strukturen von Wohlfahrtsstaatsinstitutionen begrenzt werden. MainstreamTypologien, wie die von Esping-Andersen, würden Fragen von Genderoutcomes und Beschäftigungsquoten von Frauen in unterschiedlichen Ländern nicht ausreichend berücksichtigen. Ferner sei eine wesentliche Kritik, dass Mainstream-Typologien auf die Staats-Markt-Relation begrenzt sind und

76 siehe hierzu auch die weiterführende Literatur von Bussemaker/van Keesbergen (1994); Lewis (1992; 1997); Lewis/Ostner (1995); Hobson (1991); Sainsbury (1994; 1996); Shaver (1989). 
unbezahlte Arbeit von Frauen vernachlässigen. Auch staatliche Sozialleistungen, die maßgeblich für das Ausmaß von Genderungleichheiten sind, würden vernachlässigt. Aus der Kritik wird geschlussfolgert, dass Social Policies und staatliche Sozialleistungen, die einen direkten Einfluss auf Familien oder die Mutter-Kind-Beziehung haben, von Bedeutung für Korpis Typologisierung sind (vgl. Korpi 2000: 9f.). Die Argumentation verdeutlicht, dass es unmöglich ist, eine triviale Kategorisierung von Wohlfahrtsstaaten zu finden, die Genderungleichheiten und sozio-ökonomische Ungleichheiten berücksichtigt. Vielmehr können Gender- und Klassenungleichheiten asymmetrisch verlaufen: hohe Genderungleichheit und geringe Klassenungleichheit oder vice versa, sodass entsprechende Indikatoren, die diese Komplexität multidimensional aufgreifen, erforderlich sind (vgl. Korpi 2000: 10).

Korpi entwickelt eine theoretisch gestützte Typologie breit konzipierter Policy Institutionen, die hauptsächlich für „gendered agency Ungleichheiten“ in Bezug auf die Partizipation am Arbeitsmarkt von Relevanz sind (vgl. Korpi 2000: 11ff.):

1. General Family Support: hohe staatliche Unterstützung der Kernfamilie, geringe Unterstützung der Dual Career Couples;

2. Dual Earner Support: geringe Unterstützung der Kernfamilie, hohe staatliche Unterstützung von Dual Career Couples, die die Teilhabe von Frauen am Arbeitsmarkt einschließt;

3. Market Oriented Policies: liberales Modell, weder Dual Career Couples noch Kernfamilie wird staatl. unterstützt, stattdessen regelt der Markt entsprechende Leistungen.

Für die Analyse von ökonomischen Klassenungleichheiten (im Spezifischen Einkommensungleichheiten und Armut) wird auf eine von Korpi und Palme (1998) entwickelte Typologie von Sozialversicherungsinstitutionen zurückgegriffen: the targeted, the state corporatist, the basic security und the encompassing model (vgl. Korpi 2000: 22f.) ${ }^{77}$.

Korpi (2000) analysiert letztlich den Zusammenhang der beiden Typologien zwischen Klassen- und Genderungleichheiten, was ihn zu seinem zentralen Ergebnis führt: „Faces of Inequality“ (Korpi 2000: 28). Soziale Ungleichheiten können in unterschiedliche Dimensionen ausdifferenziert werden, die

77 Targeted Model: minimale Leistungen, die erst nach einer strengen Bedürftigkeitsprüfung gewährt werden;

State Corporatist Model: leistungsberechtigt sind ArbeitnehmerInnen; Leistungen variieren nach Berufsgruppen/sind einkommensabhängig;

Basic Security Model: eher niedrige Sozialleistungen; es gilt das Universalitätsprinzip, d. h. leistungsberechtigt sind alle BürgerInnen (Berechtigung basiert entweder auf der Staatsbürgerschaft oder auf einzelnen Zahlungen von Beiträgen);

Encompassing Model: Entgeltbezogene Leistungen des state corporatist models werden mit der Universalität des basic security models vereint. Folglich sind alle BürgerInnen versichert, doch haben Erwerbstätige zusätzlich einen einkommensabhängigen Vorteil. 
nicht immer einer symmetrischen Entwicklung folgen. Ferner haben soziale Ungleichheiten ,viele Gesichter“. „Gender inequalities as well as class inequalities appear with many faces" (Korpi 2000: 28). Korpi konnte zeigen, dass Gender und Klassenungleichheiten auch asymmetrisch verlaufen können (hohe Klassenungleichheiten bei gleichzeitigen mittelmäßig ausgeprägten Genderungleichheiten; mittelmäßig ausgeprägte Klassenungleichheiten bei gleichzeitig hohen Genderungleichheiten ${ }^{78}$ ). Lediglich die Kombination „Encompassing Model und Dual Earner" (niedrige Klassen- und Genderungleichheiten) ist als symmetrisch zu betrachten. Die divergierenden Wege der Entwicklung von Ungleichheiten in Bezug auf Klasse und Geschlecht zeigen, dass diese nicht einfach als linearer Trend der Modernisierung erachtet werden können.

\subsubsection{Der politökonomische Ansatz (Walby)}

Auch für Sylvia Walby (2009) ist die Analyse von multiplen komplexen Ungleichheiten (einschließlich der sich überschneidenden Dimensionen gender, class und ethnicity) von zentraler Bedeutung. Insbesondere in ihrem Werk "Globalization and Inequalities: Complexity and Contested Modernities" widmet sie sich der zentralen Frage, wie die Globalisierung soziale Ungleichheiten verändert. Sie untersucht hierbei die vielen sich wandelnden Formen sozialer Ungleichheit und ihre Intersektionalitäten auf nationaler und globaler Ebene, basierend auf Analysen der EU und der USA. Ihr Ziel ist nicht nur, Veränderungen und Fortschritte in der globalen Ära aufzuzeigen, sondern auch wesentliche theoretische Konzepte zu überdenken und eine komplexe Gesellschaftstheorie zu entwickeln (vgl. Walby 2009: 1).

Das von Walby $(2004,2009)$ entwickelte Genderregime-Konzept weist eine starke Policy-Orientierung auf. Der Wandel von Genderregimen wird unter Berücksichtigung des politischen Systems (Polity), der Produktionsund Reproduktionssphäre (Ökonomie), der Zivilgesellschaft und der körperlichen Integrität bzw. Gewalt (Violence) betrachtet. Gewalt gegen Frauen in die Konzeption und Analyse von Genderregimen einzubeziehen, ist eine Besonderheit dieses Ansatzes, was auch an die Arbeit internationaler Organisationen anschließt. Erst in den neueren Arbeiten von Walby stellt die Gewaltdimension eine eigene Sphäre dar, während diese in ihrem 2004 erschienenen Aufsatz noch unter der Sphäre der Zivilgesellschaft (interpersonale Gewalt, Sexualität und soziale Bewegungen) subsummiert wurde. Außerdem wird zwischen einem öffentlichen (public) und einem privaten (domestic) Genderregime differenziert. Die Unterscheidung zwischen öffentlich und privat bezieht sich sowohl auf die gesamten Genderregime, als auch auf die

78 Eine Übersicht der Hypothesen Korpis (2000), die weitgehend durch seine Analyse bestätigt wurden, ist Tabelle 28 im Anhang zu entnehmen. 
einzelnen Sphären des politischen Systems, der Wirtschaft, der Zivilgesellschaft und der Gewalt (vgl. Walby 2009: 304). Walby nimmt an, dass es eine langfristige historische Entwicklung zu einem öffentlichen Genderregime in industrialisierten Ländern gibt (vgl. Walby 2004: 10; Walby 2009: 115). Diese Transformation kann über unterschiedliche Pfade erfolgen, deren Antriebskräfte über den Markt (market-led), den Staat (welfare state-led) oder die Politik (regulatory polity-led) reguliert werden können, wobei die verschiedenen Pfade zu einem unterschiedlichen Grad an (Un-)Gleichheit führen (vgl. Walby 2004: 10; Walby 2009: 302). Innerhalb Europas seien Walby (2004, 2009) zufolge insbesondere Polity-Regulationen in Form der Gleichstellungspolitik der EU als supranationaler Akteur wesentlich für die Entwicklung eines öffentlichen Genderregimes.

\subsection{Das Pendant zur Power Resource School: Ein kulturalistischer Ansatz zur Sozialpolitik und der Entwicklung von „Care Arrangements“}

Die grundlegende Annahme kulturalistisch vergleichender Ansätze, wie sie von Hochschild (1995) ${ }^{79}$ oder Pfau-Effinger $(2005)^{80}$ vertreten werden, ist, dass gesellschaftliche Werte ursächlich für die Sozialpolitik sind. Das zentrale Argument Pfau-Effingers ist die Überführung familialer Werte in wohlfahrtsstaatliche Policies, ferner die Bedeutung kultureller Leitbilder für die Entwicklung von „care policies“. Auf explizite Weise wird die Bedeutung von „care“ für den Vergleich von Wohlfahrtsstaaten konstatiert. PfauEffinger ergänzt Hochschilds Care-Modelle, indem sie eine Differenzierung von ,family and welfare values“ einführt. „Family Values“ sind „[...] cultural values and notions with respect to the structure of the family and the gender division of labour“ (im Sinne familialer Leitbilder); „Welfare values” beziehen sich auf , , [...] cultural values and notions with respect to the main sphere for the provision of welfare in society" (im Sinne wohlfahrtsstaatlicher Leitbilder) (Pfau-Effinger 2005: 328). Pfau-Effinger führt einen historischen Vergleich zwischen acht europäischen Ländern (Schweden, Finnland, Norwegen, Frankreich, Niederlande, England und Westdeutschland) durch und stellt in Bezug auf die familialen Werte zwei kulturelle Modernisierungspfade fest:

79 Hochschild differenzierte vier Idealtypen: „Traditional, Postmodern, Cold-modern and Warm-modern Ideals of Care" (Hochschild 1995: 331).

80 „My central thesis is that cultural differences are an important factor understanding the varying paths of development of care policy“"(Pfau-Effinger 2005: 330). 
1. "Modernisation of the male breadwinner model of the family“ (PfauEffinger 2005: 331): In Großbritannien, Norwegen, den Niederlanden und Westdeutschland hat lange Zeit das ,housewife model of the male breadwinner family ${ }^{81 “,}$, nach dem die Pflege und Betreuung spezifische Hausfrauentätigkeiten waren, dominiert. Dieses Modell basiert auf einer Trennung zwischen privater und öffentlicher Sphäre: Während der Mann primär in der öffentlichen erwerbstätigen Sphäre agierte, war die Frau für den privaten Bereich und die Kinderbetreuung zuständig (vgl. Pfau-Effinger 2005: 331). Es galt vornehmlich die Leitvorstellung eines Bedarfs von Kindern an mütterlicher Fürsorge innerhalb des privaten Haushalts. Doch diese Leitvorstellung widersprach zunehmend aufgrund der kulturellen Prämisse einer Ungleichheit von abhängigen Hausfrauen im Vergleich zu erwerbstätigen Männern der innerhalb von modernen Industriegesellschaften aufkommenden Leitvorstellung von Autonomie und Gleichheit (vgl. Pfau-Effinger 2005: 331). Dieser Widerspruch hat gemäß PfauEffinger insofern zu einer Transformation geführt, als das ,housewife model of the male breadwinner family“ entweder durch das ,male breadwinner/female part-time career model ${ }^{82 \text { “ }}$ oder durch das „dual breadwinner/external model" ${ }^{\prime 83}$ ersetzt wurde. Ersteres zeichnet sich durch eine Teilzeiterwerbstätigkeit von Frauen aus, die durch eine Teilauslagerung von Betreuungsaufgaben an Dritte ermöglicht wird, während im letzteren Modell sowohl Männer als auch Frauen gleichermaßen Vollzeit erwerbstätig sind und Betreuungsaufgaben hauptsächlich ausgelagert werden.

2. ,the dual breadwinner family model" (Pfau-Effinger 2005: 333): In Frankreich, Dänemark, Schweden und Finnland hat sehr früh das ,dual breadwinner/external model" dominiert, das durch die geschlechtsunabhängige Vollzeiterwerbstätigkeitsnorm und ausgelagerte (überwiegend staatliche) Kinderbetreuung gekennzeichnet ist, wobei diese Länder je nach geschlechteregalitären Leitbildern variieren (vgl. PfauEffinger 2005: 333f.).

Zusammenfassend schlussfolgert Pfau-Effinger aus ihrer Studie, dass:

$81,[\ldots]$ the housewife model of the male breadwinner family in which care was seen as the specific tasks of the housewife which did not need specific skills but was seen as based on quasi natural skills" (Pfau-Effinger 2005: 329).

$82,[\ldots]$ the male breadwinner/female part-time carer model which is based on the idea that the family, and particularly women inside the family, should share the task of caring with other institutions outside the family like the welfare state, the market or the non-profit sector" (Pfau-Effinger 2005: 329).

83, ,...] the dual breadwinner/external model which is based on the assumption that care should mainly be provided by institutions outside the family, like the welfare state, the market or the non-profit sector" (Pfau-Effinger 2005: 329). 


\begin{abstract}
„West-European care arrangements have by and large changed along two different cultural paths, and these differ fundamentally according to the cultural situation found in different countries after the Second World War. Important cultural differences exist today surrounding the value of informal family childcare by the mother or both parents and question of whether mothers should work part or fulltime" (Pfau-Effinger 2005: 335).
\end{abstract}

Bühlmann et al. (2010) fassen die bis dato geführte Diskussion prägnant zusammen: Die strukturelle Möglichkeit eines egalitären Anteils an Arbeit innerhalb eines Paares wird zunehmend durch Wohlfahrtspolitiken geprägt, die entweder die Integration von Hausarbeit und Berufsarbeit behindern oder erleichtern. Politische Maßnahmen, wie der Elternurlaub oder öffentliche Kinderbetreuungsdienste, können die asymmetrische Arbeitsteilung zwischen den Geschlechtern verstärken oder abschwächen. Um die Auswirkungen einer solchen institutionellen Politik auf das individuelle Verhalten zu erfassen, wurden in den letzten zwei Jahrzehnten auf der Grundlage der ersten Typologie von Esping-Andersen (1990) mehrere Typologien von Wohlfahrtsstaatsregimen entwickelt. Nach den Kritiken der Geschlechterblindheit der frühen Typologien integrieren die jüngsten Revisionen die Familien- und Geschlechterverhältnisse explizit (vgl. Bühlmann et al. 2010: 52).

Für eine zusammenfassende Übersicht einiger der hier angeführten relevanten Typologien, die im späteren Verlauf für die theoriegeleitete Fallauswahl nochmals von Bedeutung sein werden, s. Tabelle 6.

\title{
5.5. „Between Equalization and Marginalization“: Diversität und Dynamik von Teilzeitarbeitsmodellen im historischen Entwicklungsprozess unterschiedlicher moderner Gesellschaften
}

Die Tatsache, dass Frauen nicht in gleicher Weise wie Männer am Arbeitsmarkt kommodifiziert sind sowie unterschiedliche Präferenzen in Bezug auf Erwerbsarbeit und Familie aufweisen, führt zu einer notwendigen Berücksichtigung von Teilzeiterwerbsmodellen. Teilzeiterwerbsarbeit weist im europäischen Ländervergleich eine sehr starke Heterogenität auf, die in den 1990er Jahren mittels konkurrierender, ambivalenter Theorien zu erklären versucht wurde.

„The female part-time workforce is almost as diverse as the female full-time workforce, and there is of course a fair degree of traffic between the two. This diversity, and the close links with the full-time workforce, underlie ambivalent, even contradictory theoretical approaches to date" (Blossfeld/ Hakim 1997: 2). 
Tabelle 6: Genderspezifische Wohlfahrtsregime nach Familienpolitik

\begin{tabular}{|c|c|c|c|c|}
\hline \multirow{2}{*}{$\begin{array}{l}\text { AutorInnen } \\
\text { Lewis (1992), } \\
\text { Ostner (1995) }\end{array}$} & & & \multicolumn{2}{|c|}{ Regimetypen } \\
\hline & $\begin{array}{l}\text { Weak male- } \\
\text { breadwinner } \\
\text { model: } \\
\text { Schweden }\end{array}$ & $\begin{array}{l}\text { Modified } \\
\text { male- } \\
\text { breadwinner } \\
\text { model: } \\
\text { Frankreich }\end{array}$ & $\begin{array}{l}\text { Strong male- } \\
\text { breadwinner } \\
\text { model: } \\
\text { Irland } \\
\text { Groß- } \\
\text { britannien }\end{array}$ & \\
\hline $\begin{array}{l}\text { Esping- } \\
\text { Andersen } \\
\text { (1999) }\end{array}$ & $\begin{array}{l}\text { Sozial- } \\
\text { demokratisch: } \\
\text { Dänemark } \\
\text { Finnland } \\
\text { Norwegen } \\
\text { Schweden }\end{array}$ & $\begin{array}{l}\text { Liberal: } \\
\text { Australien } \\
\text { Kanada } \\
\text { Irland, Groß- } \\
\text { britannien } \\
\text { USA }\end{array}$ & $\begin{array}{l}\text { Konservativ: } \\
\text { Österreich } \\
\text { Belgien } \\
\text { Frankreich } \\
\text { Deutschland } \\
\text { Niederlande }\end{array}$ & $\begin{array}{l}\text { Süd- } \\
\text { europäisch: } \\
\text { Italien } \\
\text { Portugal } \\
\text { Spanien }\end{array}$ \\
\hline Korpi (2000) & $\begin{array}{l}\text { Dual Earner } \\
\text { Support: } \\
\text { Norwegen } \\
\text { Dänemark } \\
\text { Finnland } \\
\text { Schweden }\end{array}$ & $\begin{array}{l}\text { Market } \\
\text { Oriented: } \\
\text { Japan } \\
\text { Australien } \\
\text { Schweiz } \\
\text { Groß- } \\
\text { britannien } \\
\text { Neuseeland } \\
\text { USA } \\
\text { Kanada }\end{array}$ & $\begin{array}{l}\text { General } \\
\text { Family } \\
\text { Support: } \\
\text { Irland } \\
\text { Italien } \\
\text { Niederlande } \\
\text { Belgien } \\
\text { Deutschland } \\
\text { Österreich } \\
\text { Frankreich }\end{array}$ & $\begin{array}{l}\text { Erweiterung } \\
\text { durch } \\
\text { Hobson/Oláh } \\
\text { (2006): } \\
\text { Transition } \\
\text { Post-Socialist } \\
\text { Countries: } \\
\text { Tschechien } \\
\text { Ungarn }\end{array}$ \\
\hline Fux $(2002)^{84}$ & $\begin{array}{l}\text { Etatistic } \\
\text { Regime: } \\
\text { Ostdeutsch- } \\
\text { land } \\
\text { Schweden }\end{array}$ & $\begin{array}{l}\text { Individualistic } \\
\text { Regime: } \\
\text { Schweiz } \\
\text { Niederlande } \\
\text { Groß- }\end{array}$ & $\begin{array}{l}\text { Familialistic } \\
\text { Regime: } \\
\text { Frankreich } \\
\text { Italien, West- } \\
\text { deutschland }\end{array}$ & $f$ \\
\hline
\end{tabular}

84 „A first factor (etatism) is marked with high loadings for decommodification, socialist regime attribute, and high family allowances as well as public child-care for children under 3. Also the duration of maternity leave benefits is positively correlated with this factor. On the other side, there is a sharp contrast to conservative and liberal regime attributes. The second factor (familialism) is characterized by high loadings for conservative regime attributes, the regulation of child-care leave, and the percentage of children between age 3 and school age enrolled in public institutions. As concerning public child-care, one has to distinguish two different historical roots: on the one side, corresponding provisions aimed at an integration of children into the Catholic milieu (e.g. in Belgium, France) on the other hand, public child-care provisions intended to lower the burdens of employed mothers and are therefore driven by emancipatory interest. The third factor (individualism) has a high loading on liberal regime attributes and shows negative associations to maternity leave benefits, as well as day-care enrolment for children age 3 and under. Liberal regimes [...] define social security as a private matter of individuals. It seems plausible therefore that this factor shows negative loadings for most of the family policy incentives" (Fux 2002: 387f., Hervorhebungen im Original; die Verf.). 


\begin{tabular}{|c|c|c|c|c|}
\hline & Dänemark & britannien & Irland & \\
\hline Leitner & Optionaler & Expliziter & $D e-$ & Impliziter \\
\hline \multirow[t]{6}{*}{ (2003) } & Familialismus: & Familialismus: & Familialismus: & Familialismus: \\
\hline & Belgien & Österreich & Irland & Griechenland \\
\hline & Dänemark & Deutschland & Groß- & Portugal \\
\hline & Finnland & Italien & britannien & Spanien \\
\hline & Frankreich & Luxemburg & & \\
\hline & Schweden & Niederlande & & \\
\hline \multirow[t]{9}{*}{ Walby (2004) } & Pfade zum & market-led: & regulatory & \\
\hline & öffentlichen & USA & polity-led: & \\
\hline & Genderre- & & & \\
\hline & gime: welfare & & (abgesehen & \\
\hline & state-led: & & von skandi- & \\
\hline & Schweden & & navischen & \\
\hline & Norwegen & & Ländern) & \\
\hline & Finnland & & & \\
\hline & Dänemark & & & \\
\hline \multirow{8}{*}{$\begin{array}{l}\text { Pfau-Effinger } \\
\text { (2005) }\end{array}$} & Dual bread- & Modernisation & & \\
\hline & winner family & of the male & & \\
\hline & model: & breadwinner & & \\
\hline & Frankreich & model: Groß- & & \\
\hline & Dänemark & britannien & & \\
\hline & Schweden & Norwegen & & \\
\hline & Finnland & Niederlande & & \\
\hline & & $\begin{array}{l}\text { Westdeutsch- } \\
\text { land }\end{array}$ & & \\
\hline
\end{tabular}

Quelle: modifiziert nach Schleutker 2014: 163.

Im renommierten Sammelband von Blossfeld/Hakim (1997) „Between Equalization and Marginalization“ über Diversität und Dynamik der Teilzeiterwerbsarbeit in Europa und den USA werden drei konkurierende Thesen diskutiert: Die „equalization thesis“ besagt, ,[...] that women's increased labour-force participation will reduce their dependence on men and ultimately lead to greater equality between men and women in the labour market and the family“ (Blossfeld/Hakim 1997: 2). Im Rahmen der „marginalization thesis" wird argumentiert, dass Teilzeitarbeit eine Form von Arbeit mit minderwertigem Status ist, während Vollzeitarbeit die Norm darstellt. Da Teilzeitarbeit zwar nicht ausschließlich, aber doch zu großen Teilen von Frauen dominiert wird, werden diese gemäß der zweiten These auf dem Arbeitsmarkt benachteiligt und marginalisiert (vgl. Blossfeld/Hakim 1997: 3). Die dritte These besagt Folgendes: 
„In between the equalization and marginalization theses, a third approach sets part-time workers (and others with non-standard contracts) within the context of the family and the sexual division of labour in the family to show how part-time jobs and other low-paid or non-career jobs, including full-time jobs, can be not merely tolerated but even enthusiastically appreciated by dependent wives [...]. This stream of research distinguishes between primary earners (breadwinners) and secondary earners, and recognizes that the work orientations and needs of the two groups differ qualitatively" (Blossfeld/Hakim 1997: 3).

Obwohl alle drei Thesen konfligierende Argumente implizieren, finden sie Unterstützung in unterschiedlichen Ländern und zwischen spezifischen Gruppen von (Teilzeit-)Arbeitnehmer-Innen oder spezifischen Formen von Teilzeitarbeit. Ferner wird im Rahmen des Sammelbandes von Blossfeld/Hakim (1997) versucht, diese gegensätzlichen Thesen zu kontextualisieren, indem langfristige historische Entwicklungen von diversen Teilzeiterwerbsmustern in unterschiedlichen modernen Gesellschaften und der Stellenwert von Teilzeitarbeit innerhalb des Lebenslaufes von Frauen beleuchtet wird (vgl. Hakim/Blossfeld 1997: 3). Hierbei werden folgende Forschungsfragen fokussiert (vgl. Blossfeld/Hakim 1997: 4):

1. Welche Nachkriegstrends können in Bezug auf Teilzeit- und Vollzeitarbeit von Frauen in Europa und den USA verzeichnet werden und wie können diese Veränderungen erklärt werden?

2. Wie hängen Teilzeit- und Vollzeitarbeit mit dem Familienzyklus in unterschiedlichen industrialisierten Ländern zusammen?

3. In welchem Ausmaß haben sich Teil- und Vollzeitarbeit von Frauen zwischen Kohorten in unterschiedlichen modernen Gesellschaften verändert?

4. Wie wichtig ist der steigende Bildungszugang von Frauen für gleichzeitige Veränderungen der Erwerbstätigkeit von Frauen, ist er Ursache oder Wirkung?

5. Welche institutionellen, strukturellen, und soziokulturellen Kontextfaktoren sind von Bedeutung, um die länderspezifische Variation der Teilzeitarbeit erklären zu können?

6. Wie nützlich sind die drei Perspektiven institutioneller, struktureller, und soziokultureller Kontexte, um das gesamte Spektrum der Teilzeitarbeit zwischen westlichen Gesellschaften und ehemaligen sozialistischen Ländern in Zentral- und Osteuropa zu berücksichtigen? Ist eine Synthese der drei Perspektiven möglich? Welche neuen Erkenntnisse ergeben sich aus einer umfassenden Bewertung der Beweise und Argumente?

Die theoretischen Perspektiven basieren auf 1. (a) historischen Entwicklungen, die die gesamte Nachfrage von Arbeit umfassen, (b) Unterschieden der Struktur von Berufen und Industrie, 2. Angebotsfaktoren, die eine Relevanz 
für Bildungsexpansion, Veränderungen der Fertilität und Haushaltsstruktur haben und 3. weiteren politischen und ideologischen Länderkontexten (vgl. Blossfeld/Hakim 1997: 7). In Bezug auf die gesamte Nachfrage von Arbeit sowie einer Veränderung der Erwerbsbeteiligung von Frauen in Teilzeit- und Vollzeitpositionen sind zwei konträre Hypothesen zu konstatieren: Auf der einen Seite geht die „Reserve-Army-These“ davon aus, dass verheiratete Frauen als ,reserve army“ dienen, sodass Veränderungen ihrer Erwerbsarbeit im Allgemeinen und ihrer Teilzeitarbeit im Spezifischen durch Veränderungen der gesamten Nachfrage für Arbeit erklärt werden können. Im historischen Prozess betrachtet würde eine Zunahme des Wirtschaftswachstums (oder ein Rückgang) zu einem Anstieg (oder einem Rückgang) von Teil- und Vollzeittätigkeiten verheirateter Frauen führen (vgl. Blossfeld/Hakim 1997: 8). Auf der anderen Seite besagt die „Labour-Flexibility-These“, dass Teilzeitarbeit eine dominante Form einer atypischen, unstandardisierten Erwerbsarbeit darstellt, die größtenteils das Ergebnis eines Erwerbsarbeitsmangels sei. Folglich würde eine geringe Nachfrage nach Arbeit zu einem Anstieg von Teilzeitarbeit führen (vgl. Blossfeld/Hakim 1997: 8). Beide Thesen sind insofern konträr, als sie einerseits zu wenig, andererseits zu viel Arbeit im Verhältnis zu den vorhandenen Arbeitsplätzen als Ursache einer Zunahme von Teilzeitarbeit erachten.

„However, the implication of the ,reserve army ${ }^{6}$ thesis is that full-time and parttime work should gain importance primarily for married women (with children) who try to reconcile market work with family roles; while the implication of the labour flexibility thesis is that part-time work should generally be a more widespread phenomenon in the labour market, affecting all different kinds of workers in more or less the same way" (Blossfeld/Hakim 1997: 8f.).

Im Unterschied zu den Argumentationsketten, die sich auf die gesamte Nachfrage von Arbeit beziehen, erörtern eine Reihe von ForscherInnen, dass Veränderungen der (Teilzeit-)Erwerbstätigkeit von Frauen nicht nur auf einen Anstieg oder einen Rückgang der gesamten Erwerbsarbeit zurückzuführen sind, sondern die Konsequenzen einer Expansion bestimmter Arbeitsformen von größerer Bedeutung seien. Im Hinblick auf die Struktur von Berufen und Industrie vertreten Rosenfeld/Birkelund (1995) die ,post-industrial society hypothesis“, die eine Zunahme von Tätigkeiten innerhalb des Dienstleistungssektors, die von TeilzeitarbeiterInnen ausgeführt werden können, beinhaltet. Blossfeld (1987) und Hakim (1993) zufolge ist insbesondere in Zeiten eines Wirtschaftswachstums für nordeuropäische Länder anzunehmen, dass hohe Teilzeitquoten zu einem Anstieg der Arbeitsmarktsegregation geführt haben. Der von Esping-Andersen (1990; 1993) vertretene ,public-sector hypothesis" folgend wurde dieser Trend während der 1960-1970er Jahre durch das Wachstum der Wohlfahrtsstaaten in allen nordeuropäischen kapitalistischen Ländern verstärkt, weil der Dienstleistungssektor einen attraktiven Arbeitsmarkt für Teilzeiterwerbsmöglichkeiten von Frauen bieten würde. Die 
Entwicklungen der Teilzeiterwerbsarbeit in nordeuropäischen Ländern sind im Kontrast zu südeuropäischen und postsozialistischen Ländern zu sehen. In den meisten südeuropäischen Ländern war in den 1960-1970er Jahren kein „Wirtschaftsboom“ zu verzeichnen, wodurch nur ein schwacher, verspäteter und weniger intensiver Trend in Richtung einer post-industriellen Gesellschaft im Allgemeinen sowie im Dienstleistungssektor im Spezifischen beobachtet werden konnte. Der Wandel der Struktur von Berufen war im Vergleich zu nordeuropäischen Ländern bedeutend langsamer, Teilzeitarbeit von Frauen war von geringerer Relevanz, während traditionelle Frauenrollen im Rahmen von unbezahlter Arbeit im Landwirtschaftssektor und anderen kleineren Branchen einen hohen Stellenwert innehatten. Ehemalige sozialistische Länder stellen Spezialfälle dar: In diesen Ländern war bis zum Ende der 1980er Jahre der Trend in Richtung postindustrieller Gesellschaften aufgrund sehr strikter Regulationen von Einstellungs- und Entlassungspraktiken des Arbeitsmarktes, die zu einer hohen Arbeitssicherheit und einer bezeichnenden beruflichen Stabilität geführt haben, vergleichsweise schwach. Jedoch war nach dem Zerfall des kommunistischen Regimes eine massive Umstrukturierung der Berufe zu beobachten, sodass Blossfeld/Hakim (1997) ein Aufholen der Berufsstruktur in Anlehnung an westlich kapitalistische Ökonomien erwarten. Drobnič (1997) geht demzufolge davon aus, dass Ende der 1990er Jahre ein Anstieg der Teilzeitarbeit in postsozialistischen Ländern infolge eines Ausbaus des Dienstleistungssektors zu vermuten wäre (vgl. Blossfeld/Hakim 1997: 10). Wie sich im Zuge der Beschreibung der Länderprofile noch zeigen wird, ist die Teilzeiterwerbsquote von Frauen in Litauen, Polen, Rumänien, Tschechien und Ungarn auch aktuell auf sehr niedrigem Niveau. Gemäß der Bildungsinvestitionshypothese arbeiten höher gebildete Frauen während des Lebenslaufs häufiger kontinuierlich in Vollzeit und treten schneller wieder in eine Voll- oder Teilzeitbeschäftigung ein, falls sie ihre Karriere aufgrund einer Geburt oder Heirat unterbrechen. Doch umso länger Frauen in Bildungsinstitutionen verweilen, umso später treten sie ihre erste Erwerbstätigkeit an. Folglich impliziert die ,thesis of extended educational participation" nach Blossfeld (1995), dass zwischen den Kohorten der Anteil jüngerer (Single-)Frauen, die vollzeiterwerbstätig sind, sinkt, während der Anteil jüngerer teilzeiterwerbstätiger Frauen und Männer steigt, da Teilzeiterwerbstätigkeit mit studentischen Aktivitäten kompatibler ist.

In der Nachkriegsphase ist weiterführend ein Wandel der Angebotsfaktoren für Erwerbsarbeitsmöglichkeiten von Frauen festzustellen: In allen europäischen Ländern sowie in den USA haben Frauen einen starken Anstieg des durchschnittlichen Bildungsniveaus zwischen den Kohorten (Shavit/Blossfeld, 1993), eine Verschiebung der Familiengründungsphase, Veränderungen der Fertilität sowie der Haushaltsstrukturen im Lebenslauf (Blossfeld, 1995) und einen Rückgang des Rentenalters erfahren. In Europa und in den USA sind insbesondere Trends eines steigenden Heiratsalters, 
eines steigenden Alters bei Erstgeburten, steigende Scheidungsraten sowie zumindest in einigen Ländern - niedrige Fertilitätsraten vorzufinden. Diese Trends würden zu einer Re-Evaluation von Frauenrollen in modernen Gesellschaften und einem Anstieg des Angebots von Teil- und Vollzeiterwerbsmöglichkeiten für Frauen während aller Familienzyklusphasen führen, wie die ,thesis of the declining importance of the Family cycle“ besagt. In Bezug auf die Entwicklungen einer Transformation des Familiensystems sind Unterschiede zwischen den Ländern nicht zu vernachlässigen. Anhand eines internationalen Vergleichs von Blossfeld (1995) wird skizziert,

„[...] that Sweden has led the way in terms of marriage and first motherhood age, the level of fertility (which was first declining and then has been rising again since the mid-1980s), the incidence of consensual unions (which have become more or less universal), as well as divorce rates (which are falling slightly today after a steep continuous rise over 30 years); the other northern European capitalist countries and the USA appear to follow this trend with a delay of about fifteen years. In the Southern European countries, however, the family system is still fairly 'conventional' or 'traditional': consensual unions and divorce rates are comparatively low and marriage is still an important event" (Blossfeld/Hakim 1997: 12).

In den 1980er Jahren sind jedoch auch in den südeuropäischen Ländern ein starker Rückgang der Fertilitätsrate und ein Anstieg der Scheidungsrate insbesondere für jüngere Alterskohorten zu verzeichnen. Dies wird von Blossfeld/Hakim (1997) als ein Indikator gedeutet, dass sich auch in südeuropäischen Ländern eine Transformation des Familiensystems abzeichnet, allerdings zwei Jahrzehnte später als es in den nordeuropäischen Ländern der Fall war.

„These results suggest a ,North-South lag' hypothesis in the importance of family cycle events influencing employment-family linkages and conserving a greater sexual division of labour which still leads most wives to concentrate their activities on the home and family" (Blossfeld/Hakim 1997: 12).

Letztlich sei die „thesis of the increasing importance of pensioners“ zu erwähnen: Das Renteneintrittsalter ist in allen modernen westlichen Gesellschaften gesunken, während der Anteil älterer Personen, die gesundheitlich noch fähig sind, ihre Rente durch eine Teilzeiterwerbstätigkeit aufzustocken, wächst (vgl. Blossfeld/Hakim 1997: 12).

Im Hinblick auf politische und ideologische Länderkontexte ist folgendes festzuhalten: Obwohl die von Blossfeld/Hakim (1997) untersuchten europäischen Länder sowie die USA sehr unterschiedlichen Mustern der Teilzeitarbeit folgen, differenzieren die AutorInnen sechs Hauptcluster, die variierende Entwicklungen der (Teilzeit-)Erwerbstätigkeit von Frauen in der Nachkriegsphase aufweisen. Blossfeld/Hakim beziehen sich hierbei in Teilen auf die Wohlfahrtsstaatstypologie Esping-Andersens (1990; 1993), die in modifizier- 
ter Form auf Teilzeitformen angewendet wird (vgl. Blossfeld/Hakim 1997: 12).

$\mathrm{Zu}$ nennen sei erstens das skandinavische sozialdemokratische Regime, das dem Gerechtigkeitsprinzip des Egalitarismus folgt und versucht, Gendersowie soziale Schichtunterschiede zu minimieren. Arbeitsmarktpolicies basieren darauf, Frauen möglichst im Rahmen einer Erwerbstätigkeit gesellschaftlich zu integrieren sowie die ökonomische Abhängigkeit von (Ehe-) Männern zu reduzieren. Folglich erwarten Blossfeld/Hakim (1997), dass im Sinne des politisch-institutionellen Kontextes Frauenerwerbsarbeit - insbesondere eine Vollzeiterwerbstätigkeit - gefördert wird.

Zweitens wird das liberale Regime in Bezug auf Großbritannien und die USA erläutert, das dem Gerechtigkeitsprinzip der individuellen Freiheit eine primäre Beachtung schenkt.

„They accept the distributional consequences of market forces in terms of social class and genderinequalities and do little to stimulate married women's labour supply in particular (Esping-Andersen, 1990). The relatively unregulated labour market tends to create a high proportion of low grade, semi-skilled, and unskilled (part-time) jobs with low pay, poor employment benefits and prospects" (Blossfeld/Hakim 1997: 13).

Der Anteil der Arbeitskräfte im Dienstleistungsbereich ist deutlich geringer als im sozialdemokratischen Regime, weshalb Blossfeld/Hakim (1997) schlussfolgernd erwarten, dass das liberale Regime prekärere Formen von Teilzeiterwerbstätigkeiten begünstigt.

Drittens werden die Niederlande und Westdeutschland als konservativ klassifiziert, da in diesen Ländern Frauen und Mütter darin gefördert werden, ihre Prioritäten familialen Aktivitäten zu widmen.

„National policies generally favour wives' economic dependence on their husbands, and stimulate mother's choices for non-employment against part-time work, and part-time work against full-time employment. Conservative welfare states typically provide low levels of childcare and daycare services for older children" (Blossfeld/Hakim 1997: 13f.).

Im Vergleich zum liberalen Regime ist der Arbeitsmarkt deutlich regulierter und der Anteil von Arbeitskräften im Dienstleistungssektor weitaus höher. TeilzeitarbeitnehmerInnen genießen eine bessere Absicherung und der Anteil prekärer Beschäftigungsverhältnisse ist geringer.

Weiterführend wird Frankreich als vierter Typus aufgeführt, dessen Länderkontext einen besonderen Einfluss auf den Erwerbstätigkeit-Familien-Nexus hat. Vornehmlich werden proaktive politische Rahmenbedingungen geschaffen, um die Fertilität zu erhöhen. Während in vielen anderen Ländern, wie Großbritannien, Westdeutschland, oder in den Niederlanden eine dominante Ideologie besteht, dass Mütter zu Hause bleiben sollen, um sich um den Haushalt und die Kindererziehung zu kümmern, ist in Frankreich das Gegen- 
teil der Fall. Frankreich hat eine der anspruchsvollsten Serviceleistungen der Kinderbetreuung etabliert: Kindertagesstätten sowie für eine Erwerbstätigkeit günstige Schulzeiten ermöglichen es Müttern, vollzeiterwerbstätig zu sein. Ferner nehmen Blossfeld/Hakim an, dass in Frankreich eine ideologische Unterstützung einer Vollzeiterwerbstätigkeit für Frauen und Mütter besteht, während Teilzeitarbeit häufig eher ,unfreiwillig“ ausgeführt wird.

Auch haben die südeuropäischen Länder in Bezug auf Teilzeitarbeit Gemeinsamkeiten aufzuweisen, wie anhand des fünften Typus dargestellt wird. Die Transformation von einer agrarwirtschaftlichen zu einer industriellen Ökonomie ist vergleichsweise verzögert. Zwischen den späten 1950ern und den Mitte 1970ern ist kein „Wirtschaftsboom“ wie in den anderen Ländern zu konstatieren. Arbeitsmarktstrukturen, Familiensysteme und Wohlfahrtsstaatsleistungen würden in südeuropäischen Ländern den nordeuropäischen „hinterherhinken“.

Abschließend unterscheiden sich die ehemaligen sozialistischen Länder fundamental von den westeuropäischen, weshalb Blossfeld/Hakim (1997) einen postsozialistischen Typus klassifizieren. Eine weitreichende Umverteilung von Ressourcen, eine egalitäre Policy im Hinblick auf soziale Schichten und Gender sowie die Ideologie einer Gleichheit der Bedingungen waren die Hauptprinzipien, die von den staatssozialistischen Ländern explizit verfolgt wurden. Die ehemaligen sozialistischen Länder haben relativ großzügige Familienleistungen eingeführt, die eine lange, bezahlte Elternzeit für Mütter umfassten. Die allgemeinen Erwerbsarbeitsmuster basierten auf dem „two full-time earner family model“", das zwei vollzeiterwerbstätige PartnerInnen vorsah und Müttern nach der Entbindung eine Wiederbeschäftigung in Vollzeit ermöglichte. Teilzeitarbeit wurde weder als ,typisch weibliches Phänomen" angesehen noch staatlich gefördert. Nach dem Zusammenbruch des sozialistischen Regimes wurden die rechtlichen Möglichkeiten für befristete Arbeitsverhältnisse und Teilzeitarbeit in diesen Ländern erheblich ausgeweitet, weshalb Blossfeld/Hakim (1997) prekäre, weniger abgesicherte Beschäftigungsformen in der Posttransformationsphase erwarten (vgl. Blossfeld/Hakim 1997: 15).

Aufgrund ihres Vergleiches von Teilzeitarbeitsmustern in europäischen Ländern und den USA, im Rahmen dessen sie die Typologie EspingAndersens modifizieren, auf die Beschreibung einer Entwicklung von Teilzeitmodellen anwenden und hierdurch zugleich Teile der Kritik von Jane Lewis, Ilona Ostner und Ann Shola Orloff umsetzen, ziehen Blossfeld/Hakim (1997) folgende Schlussfolgerung:

„[...] comparisons of part-time work across these dozen labour markets contribute a new understanding of how part-time can take qualitatively different forms in different countries, leading to conflicting accounts for employment practices that share an umbrella label, but often little else" (Blossfeld/Hakim 1997: 17). 


\subsection{Hakims Präferenztheorie: Die Diversität der Präferenzen für Teilzeiterwerbsmodelle zur Vereinbarung von Familie und Beruf}

Hakim (2000) hat eine weiterführende Präferenztheorie entwickelt, die entgegen einer häufigen „feministischen Diskriminierungsthese“ annimmt, dass sich die Wahlmöglichkeiten von Frauen im 21. Jahrhundert durch fünf historische Prozesse ${ }^{85}$ ausgeweitet haben - nämlich durch:

1. die Einführung der Pille,

2. einen gleichen Zugang für Frauen und Männer zu Positionen und Berufen auf dem Arbeitsmarkt,

3. die Ausweitung von Angestelltenberufen im Dienstleistungssektor,

4. die Zunahme von Teilzeitarbeit und Arbeitsformen für „Secondary Earners", deren oberste Priorität nicht die Erwerbsarbeit, sondern andere Interessen darstellen und

5. die zunehmende Bedeutung von Einstellungen, Werten und persönlichen Präferenzen in Bezug auf Lebensstil-Wahlmöglichkeiten von wohlhabenden, liberalen modernen Gesellschaften (vgl. Hakim 2000: $3)$.

„This book proposes a new theory for explaining and predicting current and future patterns of women's choices between family work and market work, a theory that is historically-informed, empirically-based, multidisciplinary, prospective rather than retrospective in orientation, and applicable in all rich modern societies. Our aim is a theory that is genuinely universal" (Hakim 2000: 1).

Frauen haben unterschiedliche Prioritäten und Präferenzen in Bezug auf Familie und Erwerbstätigkeit, die Hakim (2000) zu drei „Work-LifestylePräferenz-Idealtypen“ klassifiziert hat: ,home-centered“ (der Anteil dieser Gruppe betrage im Durchschnitt 20\% der Frauen), „adaptive“ (im Durchschnitt 60\% der Frauen) und „work-centred preferences“ (im Durchschnitt 20\% der Frauen) (Hakim 2000: 6). Die Hauptprioritäten von „homecentered“ Frauen basieren auf dem Familienleben und Kindern, „Adaptive“ versuchen Erwerbsarbeit und Familie durch Teilzeitarbeit zu kombinieren, während „work-centered“ Frauen ihre Hauptprioritäten nach ihrer Erwerbstätigkeit ausrichten und häufig kinderlos sind. Die Heterogenität der Präferenzen von Frauen führe zu konfligierenden Interessen zwischen den verschiedenen Gruppen von Frauen. Hakim zufolge verschaffen diese konfligierenden Interessen Männern einen Vorteil und seien eine Ursache für Patriarchie; Männer würden hingegen vergleichsweise homogene Interessen aufweisen.

85 „The five changes do not necessarily occur in all modern societies, and do not always occur together. Their effects are cumulative" (Hakim 2000: 7). 
Für eine erfolgreichere Policy-Forschung empfiehlt sie, ihre Präferenztheorie respektive die unterschiedlichen Präferenztypen zwischen Familie und Erwerbstätigkeit zu berücksichtigen (vgl. Hakim 2000: 7). Insbesondere für die Gruppe der Adaptiven kommt es entscheidend darauf an, dass entsprechende Teilzeitarrangements ermöglicht und gefördert werden. Dass Teilzeitarbeit sowohl aus feministischer als auch aus ,trade-union-Perspektive“ negativ und als nicht adäquate Alternative zur Vollzeitarbeit erachtet wurde, kritisierte Hakim bereits 1997:

„What can loosely be termed the ,feminist" perspective and the ,trade union" perspective on part-time work have both painted a gloomy picture of part-time jobs, routinely concluding that they are an inadequate alternative to standard" fulltime permanent jobs. [...] This chapter, and the book as a whole, develops an alternative sociological perspective on part-time work, one which differs sharply from the two previous dominant perspectives, is informed by recent empirical research on part-time work, and sets part-time work in the context of work histories and the family lifecycle. [...] The principal argument of this chapter is that parttime employment constitutes a qualitatively different type of workforce involvement from full-time employment, one which gives priority to some other nonmarket activity around which the part-time job must be fitted" (Hakim 1997: 31).

Die Nachkriegstrends der Erwerbsbeteiligung von Frauen und die Zunahme von Teilzeitarbeit seien als eine substantielle Veränderung der Art der Beteiligung von Frauen am Arbeitsmarkt zu verstehen. Hakim thematisiert zwei Prozesse, die sich in den Gewichtungen zwischen Ländern und in ihren Zeitpunkten unterscheiden. In den 1980er Jahren war eine dominante Entwicklung die Substitution von Teilzeitarbeits- durch Vollzeitarbeitsplätze, die zwar den gesamten Arbeitsmarkt, mehrheitlich jedoch erwerbstätige Frauen betraf. Neben der fortgesetzten Ausweitung von Teilzeitarbeitsplätzen war in den 1980er und 1990er Jahren in einigen Ländern ein paralleler Prozess eines Anstiegs der Vollzeitbeschäftigung von Frauen sowie absoluter Vollzeitbeschäftigungsquoten zu verzeichnen. Die Zunahme der Teilzeitarbeit sei vornehmlich ein Hinweis auf eine wesentliche Veränderung der Arbeitsorientierungen von Frauen, die eine Polarisierung der Frauenerwerbsarbeit bewirke (vgl. Hakim 1997: 35). Teilzeitarbeit ist zwar in höchstem Maße segregiert, doch ist es gemäß Hakim bezeichnend, dass Teilzeiterwerbstätige eine höhere Zufriedenheit ihrer Berufstätigkeit aufweisen als Vollzeiterwerbstätige (vgl. Hakim 1997: 35). Aufgrund vorherrschender Normen bezüglich einer genderspezifischen Arbeitsteilung innerhalb der Familie wären Teilzeiterwerbstätigkeiten für Frauen so attraktiv - die Mehrheit der Frauen (ebenso wie die Mehrheit der Männer) würde eine genderspezifische Arbeitsteilung akzeptieren und sogar präferieren. Grundsätzlich würden teilzeiterwerbstätige Frauen traditionellere Einstellungen in Bezug auf Haus- und Erwerbsarbeit vertreten, wobei diese mehr Ähnlichkeiten zu den Einstellungen von Männern und nicht-erwerbstätigen Frauen aufweisen würden, als zur Gruppe der vollzeit- 
erwerbstätigen Frauen (vgl. Hakim 1997: 39). Die Mehrheit vollzeiterwerbstätiger Frauen lehnt hingegen eine genderspezifische Arbeitsteilung ab und präferiert egalitäre Geschlechterrollen. Im Ländervergleich zeigt sich, dass eine egalitäre Arbeitsteilung zwischen den Geschlechtern am häufigsten in Griechenland, Dänemark, Italien und Frankreich, während eine traditionelle genderspezifische Arbeitsteilung am häufigsten in Luxemburg, Irland und Belgien präferiert wird (vgl. Hakim 1997: 40). Obwohl Einstellungsdaten von vielen ForscherInnen mit gewisser Skepsis betrachtet wurden, betont Hakim (1997) die Bedeutung von persönlichen Präferenzen für das Erwerbsverhalten:

„Social attitude survey data are regarded with deep suspicion by many sociolo-
gists, and many reject broadly Weberian theories that treat norms values, and
preferences as having causal power, alongside social structural and economic fac-
tors, in favour of broadly Marxian theories that give primacy to economic and
social structural explanations - in part because much research has found attitudes
to be poor predictors of actual behaviour due to the failure to distinguish between
approval and choice in most studies (Hakim 1996a: $84-5$ ). However, personal
preferences about the appropriate roles of men and women, the role of husbands
and wives, and family relations, are deeply held and change relatively slowly
[...]" (Hakim 1997: 39).

Die Klassifizierung Hakims der Frauen nach ihren Präferenzen in Bezug auf Familie und Erwerbstätigkeit in verschiedene Gruppen (modern, ambivalent, traditionell) sei nicht statisch, sondern stets wandelbar. Die relative Größe der Gruppen möge zwischen den Ländern und im Laufe der Zeit variieren und es mag einige Frauen geben, die während ihres Lebens zwischen den Gruppen wechseln. Doch der entscheidende Punkt sei, dass die Existenz der zwei divergierenden, heterogenen (Rand-)Gruppen, die sich entweder der Familie oder dem Beruf widmen, die Erfahrungen von Frauen auf dem Arbeitsmarkt polarisiert und eine „durchschnittliche“ oder „typische“ berufstätige Frau zu einer fiktiven und irreführenden Illusion wird (vgl. Hakim 1997: 44).

Entgegen der „feministischen Perspektive“ argumentiert Hakim, dass Teilzeitarbeit freiwillig von Frauen gewählt wird, deren Präferenzen vorrangig nicht-erwerbstätigen Aktivitäten gewidmet sind. Die ,feministische Perspektive" skizziert im Gegensatz zur Argumentation Hakims, dass Teilzeitarbeit eine unfreiwillige "Wahl“ ist, die Frauen gezwungenermaßen durch die Verpflichtung auferlegt wird, die Verantwortung für die Kinderbetreuung zu übernehmen (vgl. Hakim 1997: 44f). Trotz der Evidenzen, dass Teilzeitarbeit oftmals präferiert wird, würden viele europäische SozialwissenschaftlerInnen die Ansicht vertreten, dass niemand eine Teilzeiterwerbstätigkeit tatsächlich „wählen“ würde. Dieser Einwand würde nicht nur aus feministischer, sondern auch aus der „trade-union-Perspektive“ vertreten. In Bezug auf Teilzeitarbeit sei der „trade-union-Antagonismus“ auf zwei Ursachen zurückzuführen: Erstens führen patriarchale und sexistische Einstellungen zu einer gedanken- 
losen Priorität der Interessen von Männern, zweitens bestehe eine Norm der Vollzeiterwerbstätigkeit, die dazu führe, dass andere Formen von Erwerbsbeteiligung immer im Gegensatz zur Vollzeitarbeit als Referenz betrachtet werden (vgl. Hakim 1997: 47).

„With their long-standing focus on the interests of main breadwinners (typically men) and hence on 'standard' full-time permanent jobs, it is understandable that trade unions have always viewed part-time (and temporary) work as an inadequate alternative. For decades, part-time jobs have been labelled as not 'real jobs' and thus unacceptable to any serious worker" (Hakim 1997: 49).

Hakim schlussfolgert, dass Teilzeitarbeit einen anderen Typus von Erwerbsarbeit darstellt als Vollzeitarbeit. Teilzeitarbeit ist nicht einfach ,ein bisschen weniger von derselben Sache“, sie ist mehr als eine marginale AmateurInnentätigkeit. Was Teilzeiterwerbstätigkeit zu einer unverwechselbaren Wahl macht, ist ihre Unterordnung im Vergleich zu anderen Lebensbereichen bzw. Interessen (Familie, religiöse Aktivitäten, politische Aktivitäten etc.), wodurch sie eine vollkommen unterschiedliche Form von Arbeit ermöglicht. „[...] we must now stop treating the male working-life profile and male career-committed work orientations as the sole model of work attitudes and behaviour" (Hakim 1997: 62).

Nicht nur, dass Männer vergleichsweise homogene Interessen vertreten würden, auch die Frage einer kausalen Beziehung zwischen Präferenzen und Erwerbsverhalten sowie die Klassifizierung der Präferenzen von Frauen in lediglich drei Typen ist häufig an Hakims Präferenztheorie kritisch hinterfragt worden.

\subsection{Weiterführende feministische Kritik von Ostner}

Auch Ilona Ostner (2004b) greift die Präferenzen von Frauen bezüglich des Erwerbsverhaltens auf und kritisiert weiterführend an Korpi, dass er eine theoretische und empirische Verknüpfung der Ungleichheitsdimensionen Gender, Class, Ethnicity und Alter versäume (vgl. Ostner 2004b: 45). Dieses Fehlen wird von Ostner als „Eigensinnigkeit der Perspektiven“ bezeichnet. Die Integration der Interessen und Wünsche von Frauen müssten theoretischer und empirischer Bestandteil werden, um die Verengung des Blickfeldes auf die Erwerbstätigkeit zu umgehen.

„Mit "agency" sollen die Interessen der Frauen zwar erfasst werden. De facto aber, das zeigt die Analyse Korpis (2000), wird "agency" einsinnig als Erwerbsfähigkeit verstanden, die handlungsleitenden Normen werden allein mit Blick auf diese eine Fähigkeit gewertet. Offensichtlich konfligiert die Heterogenität der Frauen mit der Homogenität der gewählten Perspektive“" (Ostner 2004b: 46). 
Wie bereits gemäß dem Wollstonecraft's Dilemma thematisiert, greift die Gleichheits-Argumentation u. a. die Frage der Abhängigkeit auf. Erwerbstätigkeit von Frauen sei notwendig, um finanzielle Unabhängigkeit vom Partner zu erzielen. Ostner, die bereits 1978 die alltägliche Verschränkung zwischen Hausarbeit und Berufstätigkeit von Frauen thematisierte ${ }^{86}$, argumentiert, dass Abhängigkeit und Unabhängigkeit soziale Konstrukte, d. h. Ergebnis von Interpretationen seien. Vermeintlich Abhängige würden ihre zugeschriebene Abhängigkeit möglicherweise als Freiheit erleben (vgl. Ostner 2004b: 49).

Aus einer spezifischen feministischen Perspektive, von der sich Ostner in dieser Debatte distanziert, solle das skandinavische ZweiverdienerInnenModell auf alle EU-Bürger/innen ausgedehnt werden (vgl. Ostner 2004b: 48).

86 Bereits im Rahmen ihrer Dissertation stellte Ostner (1978) die These des weiblichen Arbeitsvermögens zur Begründung der geschlechtsspezifischen Berufsarbeit auf. Hausarbeit, als naturgebundene, naturwüchsige Tätigkeit, ,[...] basiert auf Erfahrung und der Fähigkeit, Erfahrung intuitiv zu verwenden; sie verlangt Geduld und Beharrlichkeit. Diese Besonderheit $[\ldots]$ der Hausarbeit bestimmt nicht nur das weibliche Arbeitsvermögen [...], sie strukturiert auch die weibliche Berufswahl und Berufspraxis" (Ostner 1978: 239)". Anders formuliert wird die Berufswahl durch das, einer spezifisch weiblichen Sozialisation zugrunde liegende, weibliche Arbeitsvermögen geprägt, weshalb Frauen überwiegend Hausarbeitsund familiennahe Berufe wählen. Ostner konstatiert weiterführend, dass trotz der Integration von Frauen in den Arbeitsmarkt die Verrichtung der Hausarbeiten von ihnen erwartet werden würde, wodurch eine Doppelbelastung resultieren kann. Die Berufstätigkeit von Frauen sei der Logik des Arbeitsmarktes unterworfen: Ist nach der Marktlogik die Erwerbspartizipation von Frauen gefordert, werden sie integriert, andernfalls werden sie vom Arbeitsmarkt ausgeschlossen.

Insbesondere die deutsche Frauenforschung hat nicht nur auf die Geringschätzung und den blinden Fleck der Reproduktionsarbeit in der sozialwissenschaftlichen Forschung hingewiesen, sondern auch darauf aufmerksam gemacht, dass sich Haus- und Lohnarbeit unterscheiden (in der Organisationsform privat/öffentlich und im Arbeitsgegenstand), deren Unterscheidungspraxis die Identität von Frauen (mit) prägt (für einen Überblick des feministischen Diskurses der 1970er und 1980er Jahre s. Gottschall 2000: 137ff.). Hingegen wurden im anglo-amerikanischen Diskurs stärker die „Dual-Approach-Ansätze“ thematisiert, die von einer gleichzeitigen Koexistenz von Kapitalismus und Patriarchat als unterschiedliche, aber interagierende Herrschaftsformen ausgehen. Bei diesen Ansätzen steht deutlicher der Herrschaftscharakter der Verwiesenheit von Frauen auf die Reproduktionssphäre im Vordergrund (vgl. Gottschall 2000: 143; Gottschall 2018: 368f.). Zu nennen ist die renommierte, komplexe Patriarchatskonzeption von Walby $(1989,1990 \mathrm{a})$. In ihrer Theorie begreift sie das Patriarchat als männliche Vorherrschaft anhand der Dimensionen Hausarbeit, Lohnarbeit, Kultur, Sexualität, Gewalt und Staat (vgl. Walby 1989: 220). Des Weiteren differenziert Walby (1990a, 1990b) zwischen einer privaten und einer öffentlichen Form des Patriarchats. Das durch den Kapitalismus entstandene moderne Patriarchat versteht sie als öffentlichen Typus, weil nun der Patriarchalismus in der Lohnarbeit die dominierende gesellschaftliche Struktur vorgebe und nicht mehr primär die männliche Vorherrschaft im Reproduktionsbereich. Im modernen Patriarchat wird männliche Vorherrschaft vermehrt abstrakt und kollektiv ausgeübt und weniger individuell durch einzelne Männer. Frauen werden zwar nicht mehr grundsätzlich aus öffentlichen Sphären, wie der Politik oder der Lohnarbeit, ausgeschlossen, jedoch verläuft ihre Integration über Mechanismen der Unterordnung und Segregation. 
Die Verengung der Perspektive würde auch nicht durch Korpis Versuch, Class und Gender in einem mehrdimensionalen Konzept von Ungleichheiten miteinander zu verbinden, aufgehoben ${ }^{87}$. Eine Erwerbsbeteiligungs-Norm für alle Frauen -unabhängig von subjektiven Interessen, Qualifikation oder gar kulturellen Länderunterschieden - würde erstrebt als wäre sie für alle Frauen von gleicher Bedeutung. Erwerbsbeteiligung wird unreflektiert als Indikator von ,agency“ hingenommen. Um diesen „situativen Partikularismus“ zu umgehen, schlägt Ostner für eine Kontextualisierung des Lebens von Frauen vor, von unterschiedlichen Wünschen und Interessen auszugehen (vgl. Ostner 2004b: 54). Andernfalls würde die Heterogenität von Frauen mit der Homogenität der feministischen Strategie verkoppelt, die Frauen als „Mittel zum Zweck einer egalitaristischen Sozialutopie" mache (Ostner 2004b: 56).

„Sollen Frauen also lieber nicht berufstätig sein, Hausfrau und Mutter vor allem bleiben? Es wird zu schnell übersehen, daß [sic!] die strukturell erzeugte Privatheit der Familie und die damit verbundenen gleichsam symbiotischen Beziehungen der Familienmitglieder, vor allem die von Mutter und Kind und die materielle und emotionale Fixierung der Frau auf den Mann, der Frau ein SELBST verbietet. Die Arbeit der Frau ist nur „Aufopferung“““ (Ostner 1978: 243, Hervorhebungen im Original; die Verf.).

Ostner unterstellt in ihrem Essay, dass sämtliche Aufsätze der vergangenen zehn Jahre in der Zeitschrift „Social Politics“ „Liebe“ als Beziehungscode durch den der „Partnerschaft" abgelöst hätten und verweist auf Koppetschs Erklärung: Der Beziehungscode der Partnerschaft folge allgemeineren gesellschaftlichen Werten, hingegen folge Liebe einer asozialen, ausschließenden Beziehungslogik, die Ungleichheiten zulasse. „Darin liegt für egalitaristische Weltverbesserer das Schockierende an der Liebesbeziehung" (Ostner 2004b: 56). In Ostners - auf den Punkt gebrachten - Worten stellt Koppetsch fest,

„[...] dass nicht nur die "Liebe", sondern auch die "Partnerschaft" zur Reproduktion geschlechtsspezifischer Machtstrukturen beitragen, da gerade die partnerschaftliche Moral der kontraktionellen Reziprozität die Funktionsweise der Gabe (etwas für nichts) außer Kraft setzt ("Dein Problem!"; "Selber schuld"'), was wiederum nur möglich ist, weil das im Partnerschaftsmodell vorgesehene Programm radikaler Individualisierung die Selbstverwirklichung beider Geschlechter nach dem Modell männlicher Autonomie vorsieht, die traditionelle Weiblichkeit also mit defizitärer Individualität gleichsetzt" (Ostner 2004b: 58f., Hervorhebungen durch die Verf.).

87 „Die Verengung wird auch nicht dadurch aufgehoben, dass Autoren wie Walter Korpi (2000) versuchen, soziale Schicht ("class") und Geschlecht durch ein mehrdimensionales und variablenreiches Konzept der sozialen und der Geschlechterungleichheit zu verbinden. Die untersuchten Länder fallen bei Korpi - mit wenigen Abweichungen - wieder in die altbekannten Schubladen. Und die Erwerbsbeteiligung wird fraglos als Indikator für "agency" gewertet. Der politische Impetus bleibt gleich: Berücksichtigung aller Faktoren, die die Erwerbsarbeit von Frauen zu fördern versprechen. Als ob Erwerbsarbeit für alle Frauen das Gleiche bedeutete" (Ostner 2004b: 49). 



\section{Ein Abriss: Reziprozität, Liebe und Solidarität}

\section{1. „Ungleiche“ Liebe und „egalitäre“" Partnerschaft: Koppetschs Differenzierung zwischen Liebe und Partnerschaft}

Koppetschs These ist, „daß [sic!] Liebe und Partnerschaft konkurrierende Prinzipien darstellen, die in der Interaktion von Paarbeziehungen unterschiedliche Aufgaben erfüllen“ (Koppetsch 1998: 111). Sie wie auch Burkart grenzen sich von anderen AutorInnen ab, die Liebe und Partnerschaft als miteinander verschmolzen betrachten (Giddens, 1993) ${ }^{88}$ oder annehmen, dass Partnerschaft die Liebe ersetzt ${ }^{89}$ (vgl. Koppetsch 1998: 111; Burkart 1998: 37). Burkart spitzt dies noch weiter zu, in dem er die These aufstellt, dass die „"Liebe“ aus der Familienforschung verbannt wurde“ (Burkart 1998: 27). Der Kode der Liebe und der Kode der Partnerschaft würden außerdem gemäß Koppetsch unterschiedlichen Formen des Austauschs folgen: Während der Austausch von Liebenden mit einem - jenseits jeder symmetrischen Vorstellung von Gegenleistungen - Gabentausch (Austausch von Geschenken) á la Simmels Geschlechtertheorie verglichen wird ${ }^{90}$ (Nach Simmel kann eine Gabe nicht gänzlich durch eine Gegenleistung erwidert werden, ,,[...] weil in der ersten Leistung eine Freiwilligkeit liegt, die bei der Gegenleistung nicht mehr vorhanden ist" (Simmel 1992: 667). In der Gabe , [...] lebt eine Freiheit, die die Gegengabe, eben weil sie Gegengabe ist, nicht besitzen kann"

88 Giddens hat 1993 im Sinne einer Modernisierungstheorie die Demokratisierung des persönlichen Lebens beschrieben, nach der sich soziale Beziehungen (Liebesbeziehungen, ElternKind-Beziehungen, Verwandtschaftsbeziehungen, Freundschaften) des privaten Lebens zunehmend durch Orientierungen an Gegenseitigkeit und Partnerschaft auszeichnen. Paarbeziehungen, die auf dem Ideal der Geschlechtergleichheit beruhen, beschreibt Giddens fortan auch als „reine Beziehungen“ (Giddens 1993: 148). „Die Möglichkeit von Intimität bedeutet das Versprechen auf Demokratie“ (Giddens 1993: 203). Intimität, verstanden als „Möglichkeit einer dauernden Bindung zwischen gleichberechtigten Personen, [...] impliziert eine durchgreifende Demokratisierung der zwischenmenschlichen Sphäre in einer Weise, die durchaus mit Demokratie in der öffentlichen vereinbar ist" (Giddens 1993: 11). Bezugnehmend auf die Diskussion über Liebe und Partnerschaft argumentiert Giddens im Unterschied zu Koppetsch, dass die romantische Liebe durch eine vollkommene asymmetrische Machtverteilung geprägt ist (vgl. Giddens 1993: 73).

89 Koppetsch grenzt sich im Spezifischen von den individualistischen Annahmen Giddens und Leupolds ab, da sie Liebe als Kommunikationszusammenhang von zwei Individuen erachten würden, die in einem Austauschverhältnis stehen, um ihren individuellen Nutzen zu maximieren (vgl. Koppetsch 1998: 113). Dieses „Prinzip des Individualismus“ würde jegliche Geschlechterrollen vernachlässigen (Koppetsch 1998: 113).

90 Auch Burkart stimmt dem zu: „Liebes-Interaktionen entziehen sich den üblichen sozialen Reziprozitätsnormen. Es geht nicht um Geben und Nehmen, es geht nicht um Tauschwerte“ (Burkart 1998: 34). 
(Simmel 1992: 668).), sei das partnerschaftliche Geben und Nehmen ein rationaler, reziproker Tausch äquivalenter Leistungen (vgl. Koppetsch 1998: 111). „Liebe [sei] in Fragen der Gerechtigkeit eher zur Machtlosigkeit verurteilt [...]: Aus Liebe lassen sich keinerlei Ansprüche ableiten, nicht einmal auf Gegenliebe“ (Koppetsch 1998: 112). Liebe beruht auf der „bedingungslosen und freiwilligen Hingabe“, Partnerschaft auf ,unmittelbare[r] Reziprozität und [dem] Primat individueller Interessen“ (Koppetsch 1998: 113). „Liebe dagegen hat keinen Zweck außer sich selbst"; vielmehr dient sie der Funktion ,[...] zur Aufrechterhaltung der Sozialität, der Liebesgemeinschaft beizutragen“ (Koppetsch 1998: 114; Burkart 1998: 25). Der direkte Tausch der Partnerschaft impliziert rational-kalkulierbare, äquivalente Güter, hingegen wird der Gabe ein symbolischer Wert zugesprochen. An dieser Stelle wird der Bezug von Koppetsch auf Simmels Geschlechtertheorie deutlich, indem sie wie auch Simmel - der Gabe einen tieferen Sinn der Verpflichtung zur Stärkung der Bindung zuweist:

„Die Gabe hat also die Funktion, die Bindung des anderen zu stärken, ihn z.B. in Form von Dankbarkeit ${ }^{91}$ und Zuneigung auf das Gemeinsame hin zu verpflichten und der gemeinsamen Beziehung Ausdruck zu verleihen" (Koppetsch 1998: 115).

Voraussetzung ist, dass der Wunsch der Erwiderung der Gabe (langfristige Perspektive) besteht, die durch die Liebe erfüllt wird. Im Falle einer Gegengabe würde auch diese mit Dankbarkeit und Zuneigung beantwortet, sodass von Koppetsch auch die „economy of gratitude“ nach Hochschild (1989) aufgegriffen wird. Unmittelbarer, auf eine kürzere Zeitperiode bezogener Austausch in Partnerschaften erzielt die individuelle Nutzenmaximierung der Interessen unter Berücksichtigung reziproker Rechte und Pflichten (vgl. Koppetsch 1998: 115f.). Rechte und Pflichten der Partnerschaft folgen ferner den Prinzipien der „Herstellung von Gerechtigkeit und [dem] Abbau von Asymmetrien" (Koppetsch 1998: 116). In der Partnerschaft besteht ein Abhängigkeitsverhältnis der Leistungen von Gegenleistungen (vgl. Koppetsch 1998: 116): bezüglich der innerhäuslichen Arbeitsteilung ergeben sich daraus Erwartungen eines wechselseitigen Austauschs von beispielsweise Kochen als Leistung gegen Wäsche waschen als Gegenleistung. „In der Liebe dürfen

91 Simmels Argumentation über die Dankbarkeit: Dankbarkeit ist ,[...] das subjektive Residuum des Aktes des Empfangens oder auch des Hingebens [...], gleichsam das moralische Gedächtnis der Menschheit [...] [und] ein [...] Weiterbestehen im entschiedensten Sinne, ein ideelles Fortbestehen einer Beziehung, auch nachdem sie etwa längst abgebrochen, und der Aktus des Gebens und Empfangens längst abgeschlossen ist. Obgleich die Dankbarkeit ein rein personaler oder, wenn man so will, lyrischer Affekt ist, so wird sie, durch ihr tausendfaches Hin- und Herweben innerhalb der Gesellschaft, zu einem ihrer stärksten Bindemittel“ (Simmel 1992: 662f.). Sie ist „,...] jenes Motiv, das die Erwiderung der Wohltat von innen heraus bewirkt, wo von äußerer Notwendigkeit [wie z. B. im ökonomischen Tausch der Wirtschaft] nicht die Rede ist" (Simmel 1992: 664). 
solche Erwartungen nicht formuliert werden, soll die Ökonomie des Gabentauschs (der streng genommen kein Tausch ist) nicht zerstört werden“" (Koppetsch 1998: 116). Obwohl Liebe und Partnerschaft als Idealtypen gegensätzlich erscheinen, können sie in der Empirie in Paarbeziehungen wechselseitig auftreten (vgl. Koppetsch 1998: 116). Entwickelt die Paarbeziehung jedoch einen Austausch, der allein auf Partnerschaft beruht, verliert sie ,ihre Bindungskraft, ihre Antriebsstruktur" (Koppetsch 1998: 117). Durch die Partnerschaft wird innerhalb der Beziehung die moralische Willkür der Liebe eingegrenzt, indem Verhandlungen von Interessen und Verteilungen von Leistungen durch den Partnerschaftskode überwacht werden (vgl. Koppetsch 1998: 117).

„Aus diesem Grund scheint Partnerschaft besser als Liebe geeignet, die Gleichheit der Geschlechter, die Reziprozität zwischen Mann und Frau sowie die Individualität der beteiligten Persönlichkeiten zu gewährleisten“ (Koppetsch 1998: 117).

Das Paradox der Partnerschaft besteht nach Koppetsch darin, einerseits Gleichheit zu fordern, wodurch andererseits implizit geschlechtsspezifische Ungleichheiten verstärkt werden. Durch die bestehende Norm der Egalität werden die ausgeführten Hausarbeiten der Frau nicht mehr als Gabe angesehen, sondern verfallen der rationalen Tauschlogik der Partnerschaft. Die ehemalige Gabe wird folglich entwertet, statt Dankbarkeit und Anerkennung wird eine äquivalente Gegenleistung gefordert. Damit setzt , $[. .$.$] die partner-$ schaftliche Moral der kontraktuellen Reziprozität die Funktionsweise der Gabe außer Kraft“ (Koppetsch 1998: 124). Eine weitere Problematik bestehe darin, dass die - durch das Partnerschaftsmodell vorgesehene - Individualisierung eine Selbstverwirklichung beider Geschlechter im Sinne der männlichen Autonomie annimmt. Ferner wird traditionelle Weiblichkeit als defizitäre Individualität erachtet, wodurch die Verrichtung von Hausarbeiten in einen Zusammenhang mit der sozialen Konstruktion von Unterlegenheit gebracht wird (vgl. Koppetsch 1998: 124). Von Frauen verrichtete Hausarbeit wird als mangelnde Emanzipation interpretiert, von Männern verrichtete Hausarbeit wird hingegen als Orientierung an der Gleichheit verstanden. Bezugnehmend auf Hochschilds (1989) Ergebnisse und auf die Ergebnisse von Koppetsch/Maier/Burkart (1997) zieht Koppetsch die Schlussfolgerung, dass ,[...] die Gleichberechtigung bei vielen Paaren etwas ist, das der Mann der Frau zum Geschenk macht, was im übrigen [sic!] das beste Mittel ist, ihre Geltung zu unterlaufen“ (Koppetsch 1998: 118). Argumentiert wird anhand von Statusgefällen, dass in vermeintlich egalitären Paarbeziehungen die innerhäusliche Arbeitsteilung ungleich verteilt ist, weil auf die Gabe des Mannes mit Dankbarkeit (z. B. Wäsche waschen als Heldentat), auf die Gabe der Frau mit Selbstverständlichkeit reagiert werde. Dies sei jedoch keine hegemoniale Strategie weiblicher Abwertung, sondern basiere auf einer Erwartungssicherheit, dass jeder in der Beziehung sein Bestes gebe (vgl. Koppetsch 
1998: 119). Die Gründe, warum auf die Gabe des Mannes anders reagiert wird als auf die Gabe der Frau sind nach Koppetsch auf Gewohnheiten zurückzuführen. Gleichheit und Haushaltspraxis werden auf unterschiedlichen Ebenen verortet: Gleichheit folgt einer reflexiven Diskurslogik, Haushaltspraxis einer praktischen Logik (dies stellen auch Kaufmann (1994; 1999a), Koppetsch/Burkart (1999) und Hochschild (2003) fest. Kaufmann bezeichnet die Diskrepanz jedoch nicht mit den Termini „Diskurs- und praktische Logik“, sondern als Diskrepanz zwischen „Ideen und Gesten“92. Hochschild beschreibt hingegen die Diskrepanz mit „new gender ideology“ und „old reality“ (Hochschild 2003: 135)). So kritisiert auch Burkart, dass ein Großteil der wissenschaftlichen Studien über die Liebe den Diskurs, nicht jedoch die Praxis ins Zentrum der Forschung rückt (vgl. Burkart 1998: 15) und, dass Partnerschaftlichkeit diskursiv anstatt praktisch ist (vgl. Burkart 1998: 38). Alltägliche Aufgaben (Hausarbeiten, Körperpflege, Ankleiden etc.) werden miteinander verknüpft und bilden dadurch ein ,expandierendes System von Praktiken, die sich gegenseitig hervorrufen und stützen, und die, einmal ausgelöst, eine ganze Kette weiterer Praktiken nach sich ziehen. Diese Eigendynamik ist ein Grund dafür, daß [sic!] sich dieses System inkorporierter Praktiken kaum durch Diskurse beeinflussen läßt [sic!]" (Koppetsch 1998: 120f., Hervorhebungen im Original; die Verf.). Dies stellt auch Burkart fest: „Es schleichen sich Routinen ein, Verhaltensweisen und „Gesten“, die nicht mehr reflektiert werden und sich der Macht des Diskurses entziehen“ (Burkart 1998: 42). Ein zweiter Grund sei, dass Reinlichkeits-, Ordnungsvorstellungen und insbesondere der Wunsch, den anderen zu beschenken, stärkere Handlungsmotive darstellen als die Gleichheitsvorstellung. Vordergründig würde die Egalitätsnorm scheinbar befolgt, doch würde die Hausarbeit nach wie vor in Form eines Gabentauschs organisiert, innerhalb dessen sich der Mann über außerordentliche Sonderleistungen profiliert (vgl. Koppetsch 1998: 121). Ferner wird ein mangelndes Interesse der Frau an einer egalitären Arbeitsteilung angenommen, weil sie ,[...] in der Regel höhere Ansprüche an die Haushaltsführung stellt, die Verantwortung für diesen Bereich beibehalten möchte und den Mann in der Rolle des freiwilligen Helfers durchaus schätzt" (Koppetsch 1998: 121). Durch die Rolle des freiwilligen Helfers werde Dankbarkeit ausgedrückt, die Voraussetzung für ihren Antrieb sei. Koppetsch geht von einem Übergang zwischen den Austauschprinzipien aus, sobald sich der Mann als undankbar erweist. Die Frau würde eine egalitäre Arbeitsteilung einfordern, die jedoch aufgrund der oben aufgeführten divergierenden Diskurs- und praktischen Logik wenn überhaupt zu einer kurzfristigen Verhaltensänderung führen würde (vgl. Koppetsch 1998: 122). Das Egalitätsprinzip

92 „Die Gleichheit hat sich in der Welt der Ideen und der moralisch-gesellschaftlichen Referenzen durchgesetzt. [...] Das tägliche Handeln hat eine andere Geschichte. Eine Geschichte, die woanders angesiedelt ist, die aus Trägheiten besteht und notwendig viel langsamer verläuft“"(Kaufmann 1994: 178f.). 
bleibt nach Koppetsch eine Illusion. Sie sieht das Dilemma nicht in der traditionellen Arbeitsteilung, sondern darin, dass , ,...] die im Partnerschaftskode formulierte Norm der konsequenten Aufrechnung einer Honorierung der von ihr eingebrachten Gabe nicht mehr erlaubt" (Koppetsch 1998: 123). Oder in Wimbauers Worten: die Partnerschaftssemantik trägt zur ,, Verschleierung dieser Ungleichheit bei“ (Wimbauer 2003: 274). Ihre verrichteten Hausarbeiten seien folglich ,ein Symbol weiblicher Unterlegenheit“ (Koppetsch 1998: 124).

Bezugnehmend auf das Verhältnis von Partnerschaft und Liebe zur Umwelt, bleibt zu erwähnen, dass Koppetsch wie auch Leupold (1983) ${ }^{93}$ die Funktion von Partnerschaft (Gültigkeit für alle sozialen Beziehungen) in der Anbindung an andere gesellschaftliche Institutionen durch die Übernahme von Wertvorstellungen der Gesellschaft (wie z. B. Egalität, Individualismus etc.) sieht (vgl. Koppetsch 1998: 125). Die Semantik der Liebe zeichne sich dagegen durch eine in Abgrenzung zu anderen Beziehungsformen (und damit in Abgrenzung zu gesellschaftlichen Werten und Normen) eigene Wirklichkeitskonstruktion aus (vgl. Koppetsch 1998: 128). „Paarbeziehungen brauchen deshalb [so Burkart] beides: Liebe und Partnerschaft“ (Burkart 1998: 43). Hieraus ergibt sich die Schwierigkeit für die Soziologie der Liebe, für das Verhältnis von Diskurs und Praxis eine theoretische Erklärung zu finden (vgl. Burkart 1998: 43).

Zusammenfassend besteht nach Koppetsch die Problematik des Partnerschaftmodells darin,

,[...] daß [sic!] sich die in der Alltagspraxis eingespielten Routinen und inkorporierten Verhaltensmuster durch reflexive Prozesse kaum beeinflussen lassen [s.

93 Andrea Leupold hat sich bereits 1983 mit der Liebe und der Partnerschaft als Formen der Codierungen von Ehen befasst. In Anlehnung an Luhmanns Systemtheorie basiert die theoretische Argumentation auf der Liebe als symbolisch generalisiertes Kommunikationsmedium von Intimbeziehungen. „Romantische Liebe wird gesehen als Semantik der PartnerInnenwahl [...], die als funktionsspezifische Semantik den Prozeß [sic!] der Ausdifferenzierung von Intimbeziehungen [fördert]“ (Leupold 1983: 297). Vornehmlich im 18. und 19. Jahrhundert etablierte sich die „freie“ PartnerInnenwahl, die zuvor i. d. R von Elternhaus und Schicht bestimmt wurde. Aufgrund des im 20. Jahrhundert stattfindenden Wandels von einer stratifikatorischen (in der Frauen vorwiegend im sozialen System der Familie agierten) zu einer funktionalen Differenzierung moderner Gesellschaften (die Frauen die Partizipation an unterschiedlichen Systemen ermöglicht) entsteht eine zunehmende Bedeutung der Partnerschaft, weil diese ,[...] den Anschluß [sic!] an die Gesellschaft wiederherstellen will“ (Leupold 1983: 322). ,,Partnerschaft“ ist die sich lange vorbereitende letzte Stufe in der Evolution von Symmetrieidealen für Ehe und Intimbeziehungen“ (Leupold 1983: 314, Hervorhebungen im Original; die Verf.). Partnerschaft bezieht sich auf die Systemgrenze zwischen Ehe und Systemumwelt. Ferner regelt sie die Relation zwischen inner- und außerfamilialen Beziehungen (vgl. Leupold 1983: 315). Das Verhältnis von romantischer Liebe und Partnerschaft ist jedoch nicht ausschließlich: „Partnerschaft ersetzt [...] nicht den binären Schematismus (öffentliche Welt/Privatheit) durch einen äquivalenten Schematismus [...], [sondern] [...] setzt die bereits konstituierte Realität auf Liebe gegründeter Ehen voraus“(Leupold 1983: 324). 
Diskurs- vs. praktische Logik], zum zweiten geraten die im Partnerschaftsmodell verbürgten Prinzipien der Gegenseitigkeit, der Tauschrationalität und des Individualismus mit der bedingungslosen Solidarität der Liebe in Konflikt" (Koppetsch 1998: 125).

Die „Burkart-Schule“, der auch Koppetsch zugehörig ist (Günter Burkart war ihr Lehrer), grenzt sich von Rational-Choice-Ansätzen ab, in dem sie eine stärkere Fokussierung auf Bedürfnisse von Individuen fordert. Diese Argumentation greift auch Ostner (2004b) auf, in dem sie die Wünsche und Interessen von Frauen in den Vordergrund stellt.

„Liebe und Kapitalismus, so die zeitdiagnostische These, durchdringen sich gegenseitig und stehen in einem wechselseitigen Anpassungsprozess" (Burkart 2014: 85). Der Ökonomisierungsdruck, der in einer stärkeren Marktorientiertheit der Liebe Ausdruck findet, steht in Konkurrenz zur romantischen Vorstellung von Liebe (vgl. Burkart 2014: 85). Zu denken sei jedoch an die im 19. Jahrhundert aufkommende Entstehung der romantischen Liebe $^{94}$ in Zusammenhang mit dem Kapitalismus, der eine Romantisierung par Exellence hervorbrachte. Die enge Verschmelzung zwischen einem romantischen Liebesideal und der Ökonomie konnte eindrücklich von Eva Illouz (1997) dargestellt werden. Burkart und Koppetsch nehmen, wie bereits angedeutet, Bezug auf die Argumentation von Simmel:

94 Die romantische Liebe nach Hartmann Tyrell: Nach dem Prinzip „Es ist was es ist-sagt die Liebe"

Hartmann Tyrell - einer der Klassiker auf dem Gebiet der romantischen Liebesforschung hat sich mit der Frage über den Sinn des im 19. Jahrhunderts aufkommenden romantischen Konzepts der Liebe befasst. Romantische Liebe ist nach Tyrell als ein Doppelphänomen konstituiert: „Für die Besonderheit des Erlebens von Liebe ist einerseits konstitutiv eine Selektion unter Personen, genauer: die unbedingte Präferenz für eine bestimmte individuelle Person [...]. Und andererseits: die enthusiastische Besetzung genau dieses Sachverhalts mit höchster persönlicher Relevanz, mit Höchstrelevanz“ (Tyrell 1987: 570, Hervorhebungen im Original; dir Verf.). „Liebe meint das absolute, alternativlose Präferieren des Geliebten“ ein Kriterium der Ausschließlichkeit, das nur auf eine einzige Person zutrifft (Tyrell 1987: 575). „Die Höchstrelevanz erklärt die Liebe für unteilbar; sie zwingt, einem alles geben und sein zu wollen“ (Tyrell 1987: 576). Ferner erfordert die Liebe für ihre Erfüllung Symmetrie und erwidernde Gegenseitigkeit (vgl. Tyrell 1987: 579). Letztlich sei die Liebesbeziehung für Tyrell in Anlehnung an Simmel eine dyadische Konstellation aufgrund von fünf Aspekten (vgl. Tyrell 1987: 583ff.):

1. Das Paar verfügt im Gegensatz zu einer Dreiecksbeziehung über den Vorteil der Komplexitätsreduktion durch eine direkte Verbindung zwischen den PartnerInnen (statt drei Verbindungslinien im Falle einer Dreiecksbeziehung).

2. Sofern Konflikte auftreten ist nur ein Feind vorhanden

3. Das permanente Bedürfnis von Interaktionsnähe, wobei die Interaktion eine dyadischereziproke Tendenz aufweist, beschreibt Tyrell als „konstitutionelle Interaktionsverwiesenheit"

4. Natürliche Komponente der dyadischen Konstellation von Sexualität

5. Voraussetzung der Gegen- und Zweigeschlechtlichkeit 
„In jedem Liebesverhältnis hat der weniger Liebende ein Übergewicht, er kann sozusagen seine Bedingungen stellen, der andere ist ihm ausgeliefert; denn diesen verhindert die innerliche Gebundenheit der Liebe, seine Vorteile zu bemerken, die bemerkten auszunutzen. In der Ehe pflegt, unter sonst gleichen Umständen, der zu herrschen, der das geringere Gefühl einsetzt. Da dies hier, und ebenso in freien Verhältnissen, in der Regel der Mann ist, so scheint mir darin ein wesentlicher Erklärungsgrund für die allgemeine Präponderanz der Männer über die Frauen zu liegen“"(Simmel 1985: 183f.).

Diese Annahmen Simmels wurden häufig zur Begründung der Austauschtheorie herangezogen. Jedoch stellt Burkart fest, dass Simmel kein rationalistischer Austauschtheoretiker ist, ,[...] denn er sieht, neben der MACHT, die der weniger Liebende hat, auch die MACHT, die der Liebende hat: indem er glücklicher ist ${ }^{95 ، " ~(B u r k a r t ~ 1998: ~ 32, ~ H e r v o r h e b u n g e n ~ d u r c h ~ d i e ~ V e r f .) . ~ D i e s ~}$ ist eine sehr romantische Vorstellung der Liebe, weil die Annahme, dass der tiefer Liebende glücklicher ist, rein hypothetisch ist. Es könnte auch der tiefer Liebende unglücklicher sein (zumindest im Sinne der rationalen Austauschlogik). Gleichwohl ist die Reflexivität von Machtverhältnissen zu bedenken - diese Annahme trifft Burkart zu Recht und wird u. a. auch von Wimbauer (2003) ${ }^{96}$ vertreten. Erinnert werden soll an den Ausgangspunkt der vorliegenden Dissertation „Bringing Power Back In“. Das Verhältnis zwischen Macht und Liebe ist keineswegs eine neue Thematik innerhalb der Familiensoziologie, doch soll „Macht“ auch an dieser Stelle eine Reintegration erfahren - wird sie doch nur vorwiegend implizit im Rahmen der aufgeführten Studien erwähnt. Ist es nicht eher die „Macht“" anstatt der Liebe, die aus der Familienforschung verbannt wurde? Burkart hat mit seiner These einer Verbannung der Liebe aus der Familienforschung für eine spezifische Epoche durchaus Recht (vgl. Burkart 1998: 27). Aktuell ist jedoch eher eine Forschungslücke der Macht zu konstatieren. Der Stand der Forschung hat sich lange Zeit auf die Liebe konzentriert, während andere Grundlagenkon-

95 „Vielleicht entbehrt dies doch nicht ganz der Gerechtigkeit. Denn in dem Liebesverhältnis genießt der tiefer Liebende ein soviel [sic!] tieferes Glück, daß [sic!] es am Ende in Ordnung ist, wenn der andere dafür in der Hinsicht des Herrschens und in allem, was sozusagen an der Peripherie des Verhältnisses liegt, im Übergewicht bleibt“" (Simmel 1985: 184).

96 „Nun kann gerade in dieser Konstellation die romantisch Liebende [sic!] Person jedoch keineswegs als >machtlos` bezeichnet werden, denn im Sinne der Wechselseitigkeit aller Machtphänomene ist auch der Partner von ihr abhängig: Zum einen, indem sie maßgeblich an seiner Konstruktion des >Besonderen $<$ mit beteiligt ist und ihn hierbei unterstützt, weil dies genau ihrem Beziehungskonzept entspricht, das eben gerade auf einen spezifischen und einzigartigen Anderen zielt; zum anderen, weil es gerade das von ihr verdiente snormale Alltagsgeld ist, das den Sockel für seine Konstruktion und Praxis des Außergewöhnlichen und Besonderen darstellt. Insofern ist die Abhängigkeit beider Partner eine wechselseitige, wenngleich sich auch die romantisch liebende Person aus einer Außenperspektive in einer ungünstigeren Position befinden mag. Wie sich andeutet, ist es gerade die Konstellation der beiden unterschiedlichen Liebeskonzepte [romantische Liebe und Partnerschaft], die diese Ungleichheiten und Machtdifferenzen einerseits maßgeblich mit konstituieren und andererseits gerade als unproblematisch erscheinen lassen“ (Wimbauer 2003: 281). 
zepte zunehmend vernachlässigt wurden. Liebe ist für ein Verständnis von Familiendynamiken - insbesondere für Bereiche wie Macht und Einfluss eine höchstrelevante Thematik - diese Annahme ist keineswegs in Frage zu stellen. ,[...] love has been an extremely variable for the understanding of family dynamics and, especially, of power and influence" (SafiliosRothschild 1976: 357). Doch wird der Fokus der vorliegenden Dissertation stärker auf die Reintegration der Macht gelegt, wobei die Verbindung der Konzepte lediglich theoretisch angeschnitten wird und zum Weiterdenken anregen soll ${ }^{97}$. Wie können Konzepte wie Liebe, Solidarität, Partnerschaft und Macht miteinander vereinbart werden?

97 Für eine weiterführende Diskussion über die Problematik der ,[...] Integration von sachlich-instrumentellen Dimensionen der austausch-, ressourcen- und haushaltsproduktionstheoretischen Ansätze mit der emotionalen Dimension des Gefühlsmanagements [...], insbesondere unter dem Aspekt, wie der Modus der Liebe, auf dem Paarbeziehungen beruhen, und der Modus des instrumentellen Austauschs, wie er in der Aufteilung der Hausarbeit zu finden ist, miteinander vereinbart werden können" sei auf Röhler et al. verwiesen (Röhler et al. 2000: 21). Sie haben im Rahmen ihrer Studie ein Mehrebenenmodell zur Integration der theoretischen Ansätze zur Verteilung und psychosozialen Bewältigung von Hausarbeiten entwickelt, wobei die Bedeutung der Gefühle für die innerhäusliche Arbeitsteilung im Rahmen von zwei Dimensionen hervorgehoben wird. Erstens liegt dem irrationalen Gefühl der Liebe gemäß Röhler et al. keine Kosten-Nutzen-Kalkulation zugrunde - es ist ein Mechanismus der Festlegung der beiden PartnerInnen, der die Basis einer stabilen Austauschbeziehung erst ermöglicht. Die Charakteristika des Liebesgefühls, nämlich Nichtkalkuliertheit und die Gerichtetheit auf eine bestimmte Person, verdeutlichen, dass Liebe nur eingeschränkt als Ressource beschrieben werden kann. „Liebe ist nur gegen Liebe tauschbar. Erst durch die Gegenliebe des anderen Partners wird die Liebe des einen zur Ressource und umgekehrt" (Röhler et al. 2000: 47). Hierdurch wird ein Problem der handlungstheoretischen Fundierung von diesem Ansatz ersichtlich: „Wie können wir die individuellen Entscheidungen zum Verhalten im Rahmen der partnerschaftlichen Kooperation als Ergebnis von Nutzenerwartungen begründen, die auf sehr verschiedenen Dimensionen angesiedelt sind? Nämlich auf der sachlich-instrumentellen Dimension, die in den austausch-, ressourcen- und haushaltsproduktionstheoretischen Ansätzen betont wird, und der emotionalen Dimension“ (Röhler et al. 2000: 47). Ein möglicher Ausweg soll das situationale Verständnis von Emotionen als auf die Handlungsmöglichkeiten restriktiv wirkende Festlegung sein, der auf die psychosozialen Dispositionen und Präferenzen erweitert werden kann. Voraussetzung ist, dass ein Wissen über die Bedingungen der Veränderung der dispositionalen Größen vorhanden ist, weshalb zweitens die Theorie des Gefühlsmanagements ein Grundelement in der Diskussion über die Hausarbeiten sei. „Prozesse der Selbstmanipulation von Gefühlen sind grundlegend für kognitive Veränderungen der Präferenzen, Werte und Leitbilder und für die Vorbereitung auf die Ausführung einer bestimmten Handlung. Dieser Ansatz zeigt auf, wo mögliche Schwierigkeiten liegen könnten in der Veränderung von traditionalen Geschlechterideologien: Eine solche Veränderung ist mit emotionaler Anstrengung - Gefühlsarbeit - verbunden“ (Röhler et al. 2000: 47). Außerdem würden Geschlechterideologien durch Gefühle verankert, die ihre Wurzeln in der Sozialisation haben und unbewusst wirken. Daher wäre es auch möglich, dass die Gefühle entgegen dem Leitbild wirken. Dies könnte gemäß Röhler et al. (2000) eine Erklärung für Koppetschs/Burkarts (1999) hervorgebrachtes Ergebnis, dass auch bei Paaren, die eine Gleichheitsorientierung vertreten, kaum eine Veränderung der traditionellen innerhäuslichen Arbeitsteilung zu beobachten sei, sein. Die Persistenz traditioneller Muster ginge folglich auf Prozesse des Gefühlsmanagements zurück, ,[...] die es einerseits ermöglichen, ein egalitäres Leitbild mit 


\subsection{Reziprozität, Wohltätigkeit und moralischer Absolutismus - „etwas gegen nichts“ (Gouldner)}

Die These bezüglich des Zusammenhangs zwischen Liebe und Solidarität ist, dass Nächstenliebe der Solidarität immanent ist. Gouldners Wohltätigkeitsnorm ist ein Beispiel dafür:

„Würden Menschen in ihren Handlungen lediglich durch die Reziprozitätsnorm geleitet, so erhielten viele die Hilfe nicht, die sie benötigen, gingen zugrunde oder blieben äußerst unzufrieden. Es wird immer einige Menschen geben können, die erwiesene Wohltaten nicht erwidern können [z. B. Kranke, Kinder, Menschen mit Behinderungen]“" (Gouldner 2005: 110).

Dies erfordert, dass es einen „Moralcode“ gibt, der die Norm solidaristischer Hilfeleistungen enthält - ,die Norm der Wohltätigkeit [im engl. Original „,norm of beneficence“] bzw. der »Güte "“ (Gouldner 2005: 110, Hervorhebungen im Original; die Verf.). „Diese Norm fordert, dass der Mensch anderen Menschen jene Hilfe zukommen lässt, die sie benötigen“" (Gouldner 2005: 110, Hervorhebungen im Original; die Verf.). Während Blau (1964) die Reziprozitätsnorm fokussiert, ist für Gouldner die Wohltätigkeitsnorm von zentraler Bedeutung. Seinen Annahmen zufolge können Gaben für Individuen, die selbst nicht in der Lage sind, Gaben im Sinne der Reziprozität zu erwidern, nur durch die Wohltätigkeitsnorm erklärt werden.

Paradox: „Es gibt kein Geschenk, das einen größeren Vorteil erbringt, als das freiwillige Geschenk - das Geschenk, an dem keine Fallstricke festgemacht sind. Denn das, was die Menschen wirklich freiwillig hergeben, vermag die Empfänger tief zu bewegen und belässt sie tief in der Schuld ihrer Wohltäter. Wenn Reziprozität letzten Endes die Alltagswelt zusammenhält, so ist es andererseits Wohltätigkeit, welche diese Welt zu transzendieren hilft und die Menschen Tränen der Versöhnung weinen lässt. Die Tatsache, dass solche Wunder der sozialen Interaktion höchst selten sind, ist nicht einem Mangel an Wissen darüber geschuldet, wie solche Wunder herbeizuführen wären" (Gouldner 2005: 115).

„Die Reziprozitätsnorm rechtfertigt eine Verpflichtung einem anderen deshalb zu helfen, weil er einem selbst geholfen hat oder helfen wird; die Wohltätigkeitsnorm rechtfertigt die Verpflichtung, einem anderen zu helfen, weil der andere dieser Hilfe bedarf" (Gouldner 2005: 115f., Hervorhebungen im Original; die Verf.).

Zweites, wesentliches Element des Moralcodes ist der moralische Absolutismus:

der abweichenden Realität der Hausarbeit zu versöhnen und damit die Dynamik von Partnerkonflikten zu entschärfen, andererseits aber auch die Durchsetzung des in der Geschlechterideologie formulierten Idealbildes befördern können und damit das Konfliktpotential in Partnerschaften erhöhen“" (Röhler et al. 2000: 48). 


\begin{abstract}
„Der Moralcode muss eine wesentliche Komponente enthalten, die dafür verantwortlich ist, dass die Durchführung bestimmter Dienste und Handlungen für andere unabhängig davon betrachtet wird, was diese für einen selbst getan haben oder tun werden, oder unabhängig von ihrer Hilfebedürftigkeit. [...] Der moralische Absolutismus ist also das »Hauptgebot« eines jeden Moralcodes. Er formuliert eine allgemeine Regel, die festlegt, wie andere moralische Regeln angewandt werden sollen. Als allgemeine Regel sagt er nicht mehr aus als: »Der Code muss befolgt werden«. [...] Der moralische Absolutismus ist eine Dimension der Moralcodes, da er zum einen ein ständig variierendes Kontinuum darstellt; zum zweiten ist er von den anderen Hauptbestandteilen unabhängig und deutlich unterschieden“ (Gouldner 2005: 116, Hervorhebungen im Original; die Verf.).
\end{abstract}

Ohne die Wirksamkeit aller drei Grundelemente des moralischen Systems Reziprozität, Wohltätigkeit und moralischer Absolutismus - ist kein stabiles Sozialsystem möglich, da die von jedem einzelnen Grundelement hervorgebrachten Spannungen, die die anderen beiden Elemente mildern sollen, nach Gouldner (2005) ein notwendiger Faktor sind. Alle drei Normen üben auf die jeweiligen anderen beiden Normen einen Druck aus (vgl. Gouldner 2005: 122).

„In seiner Grundstruktur ist ein moralischer Code ein spannungsreiches System unsicherer Werte und schwieriger Anpassungsvorgänge, wobei Konflikte kein Nebenprodukt, sondern unvermeidlich sind“" (Gouldner 2005: 123).

Betina Hollstein stellt die Annahme Gouldners, dass Gaben für Menschen, die nicht in der Lage sind, auf Gaben durch Gegengaben zu reagieren, nur über die Zusatzannahme einer Wohltätigkeitsnorm einschließlich dem Hauptgebot des moralischen Absolutismus erklärt werden können, in Frage. „Die Frage ist, ob das tatsächlich so ist: Wirkt die Reziprozität nicht in Beziehungen mit Kindern oder alten Menschen“ (Hollstein 2005: 191)? Anhand der familialen Generationenbeziehungen konstatiert Hollstein drei Typen generalisierter ${ }^{98}$, indirekter Reziprozität: das Solidaritätsprinzip, das generative Prinzip und das Stellvertretungsprinzip (vgl. Hollstein 2005: 200). Das Solidaritätsprinzip ist eine Form der Gruppensolidarität, die der Annahme folgt, dass sich gegebene Leistungen über den Lebenslauf ausgleichen werden: „Man soll seinen Angehörigen helfen, wenn sie in Not sind - und man kann von ihnen Hilfe erwarten, wenn man selbst in Not ist" (Hollstein 2005: 200). Im Unterschied dazu ist das generative Prinzip eine Form der vorwärts gerichteten Ketten-generalisierten Reziprozität (A -> B -> C): „Was ich von meinen Eltern erfahren habe, gebe ich an meine Kinder weiter" (Hollstein 2005: 200). Auch das Stellvertreterprinzip wird von Hollstein als Kettengeneralisierte Reziprozität klassifiziert, allerdings rückwärtsgerichtet $(\mathrm{C}<-\mathrm{A}$

98 Generalisierter Tausch zeichnet sich vornehmlich dadurch aus, dass der Tausch zwischen Gabe und Gegengabe auf einen sehr langen Zeitraum angelegt ist, der Wert der Gabe nur sehr schwer zu bestimmen ist, der Fokus der Aufmerksamkeit auf der Beziehung liegt und ein hoher Verpflichtunsgrad besteht (vgl. Hollstein 2005: 195). 
<-B): „Ich betreue meine Schwiegermutter anstelle von meiner Frau“ oder ein weiteres Beispiel „Meine Eltern haben ihre Eltern (nicht) unterstützt, nun unterstütze ich sie (auch nicht)“ (Hollstein 2005: 200). Aus diesen drei Typen der indirekten Reziprozität ergibt sich der Umstand, dass die Erwiderung einer Gabe nicht zwangsläufig an den Gabengeber/die Gabengeberin erfolgen muss, sondern Dritte in die Reziprozitätsverpflichtung einbezogen werden und die Reziprozitätslogik während des gesamten Lebenslaufs zu betrachten ist. Im Gegensatz zu Gouldner ist für Hollstein folglich nicht ausschließlich die Wohltätigkeitsnorm zentral, stattdessen haben für intergenerationale $\mathrm{Be}$ ziehungen vornehmlich indirekte Reziprozitätsformen eine wesentliche Bedeutung.

„Entgegen der These von Alvin W. Gouldner, der bei der Betrachtung von Reziprozität ausschließlich überschaubare Zeiträume im Blick hat, zeigt sich, dass die Verpflichtung zur Reziprozität über sehr lange Zeit wirksam sein kann" (Hollstein 2005: 204).

Dass für Paarbeziehungen der Kode der Liebe und der Partnerschaftskode zentral sind, ist von Koppetsch und Burkart konstatiert worden. Hollsteins Überlegungen, die stärker an einer langfristigen generationenübergreifenden indirekten Reziprozität angelehnt sind, können jedoch auch auf Paarbeziehungen übertragen werden. Es würde dieser Theorie an nichts fehlen, wenn die von Hollstein aufgeführten Beispiele auf Paarbeziehungen angewendet werden würden:

Im Falle des Solidaritätsprinzips hieße dies: „Man soll seinem Partner/seiner Partnerin helfen, wenn er/sie in Not ist und man kann Hilfe von ihm/ihr erwarten, wenn man selbst in Not ist".

Das generative Prinzip impliziere: „Was ich von meinen Eltern erfahren habe, das gebe ich an meinen Partner/meine Partnerin weiter".

Darüber hinaus bestünde eine Anwendungsmöglichkeit für das Stellvertretungsprinzip: ,Mein Vater hat meine Mutter (nicht) unterstützt, nun unterstütze ich meine Partnerin (auch nicht)“.

Die Fokussierung von Solidarität im Rahmen intergenerationaler Beziehungen wird auch anhand der Typologie von Bengtson deutlich. Erneut soll argumentiert werden, dass Solidaritätsformen auf Paarbeziehungen übertragen werden können.

\subsection{Solidaritätstypen nach Bengtson}

Wenn wir die Unterschiede von Menschen berücksichtigen, dass nicht alle gleich sind, d. h. nicht von einer Gleichheit, sondern einer Gleichwertigkeit (der Geschlechter) ausgehen (s. Kapitel 9.4. „Bringing Power Back In“) und 
hinnehmen, dass es einen Rückgang der Nuklearfamilie als soziale Institution sowie eine Pluralisierung von Lebensformen ${ }^{99}$ gibt, dann ist das Konzept der Solidaritätstypen von Bengtson zentral. Bengtsons Forschungsanliegen ist es, die Bedeutung multigenerationaler sozialer Beziehungen hervorzuheben.

„I suggest that family multigenerational relations will be more important [in American society] in the 21 st century for 3 reasons: (a) the demographic changes of population aging, resulting in "longer years of shared lives" between generations; (b) the increasing importance of grandparents and other kin in fulfilling family functions; (c) the strength and resilience of intergenerational solidarity over time" (Bengtson 2001: 1).

Aus den makrosoziologischen Trends der Alterung der Bevölkerung und intergenerationaler Familiendemographie werden sechs mikrosoziologische Dimensionen von Solidaritätstypen abgeleitet (für eine zusammenfassende Übersicht der Reziprozitätsformen von Gouldner, Hollstein und Bengtson s. Tabelle 7). Das theoretische Konstrukt der intergenerationalen Solidarität wird als Mittel genutzt, um die Verhaltens- und die Emotionsdimensionen von Interaktion, Kohäsion, Gefühlen und Unterstützung zwischen Eltern und Kindern, sowie Großeltern und Enkeln im Verlauf langfristiger Beziehungen zu charakterisieren (vgl. Bengtson 2001: 8, Hervorhebungen im Original; die Verf.). 1. „Affectual solidarity“ bezieht sich auf die emotionale Verbundenheit, 2. „associatonal solidarity“ auf die Kontakthäufigkeit zu intergenerationalen Familienmitgliedern, 3. „,consensual solidarity“ auf die Übereinstimmung in Meinungen und Werten, 4. „functional solidarity“ auf das Geben und Nehmen intergenerationaler Hilfe und Unterstützung, 5. „normative solidarity“ auf Erwartungen über familiäre Verpflichtungen und Normen über die Bedeutung familiärer Werte, 6. „structural solidarity“ auf die Möglichkeitsstruktur intergenerationaler Interaktion, wie örtliche Nähe zwischen Familienmitgliedern (Bengtson 2001: 8).

99 Elisabeth Beck-Gernsheim (1994) beschreibt diesbezüglich den Wandel der Familie von der Notgemeinschaft zur Wahlgemeinschaft im Kontext der Diskussion über die Individualisierung. Während die vorindustrielle Familie eine Arbeits- und Wirtschaftsgemeinschaft darstellte, die wenig Möglichkeiten für individuelle Entfaltungen bot, sich vielmehr durch einen Zwang der Solidarität zu einer „Notgemeinschaft“ formierte, zeichnete sich die Familie im Zuge der Industrialisierung durch ihre Differenzierung zwischen Arbeitsmarkt und Familie aus (Beck-Gernsheim 1994: 120f.). „Die Frau wurde abhängig vom Verdienst des Mannes; er wiederum brauchte, um funktionsfähig und einsatzbereit zu sein, ihre alltägliche Arbeit und Versorgung. Der Zwang zur Solidarität, der die vorindustrielle Familie kennzeichnete, setzte sich in modifizierter Form fort“ (Beck-Gernsheim 1994: 121). Heute dominieren nach Beck-Gernsheim die individuellen Lebensentwürfe, die das ehemalige Monopol der traditionellen Kernfamilie auflösen, jedoch nicht ersetzen. Lediglich die Monopolstellung ist durch die Pluralisierung der Lebensformen erodiert. Es entsteht ,[...] ein "ganz normales Chaos" - der Liebe, des Leids, der Beziehungsvielfalt vor allem [...] mehr Zwischenformen und Nebenformen, Vorformen und Nachformen: Das sind die Konturen der »postfamilialen Familie«" (Beck-Gernsheim 1994: 135). 
Tabelle 7: Übersicht der Reziprozitätsformen

\begin{tabular}{ccl}
\hline $\begin{array}{c}\text { Goldner } \\
\text { (Wohltätigkeit }+ \\
\text { moralischer } \\
\text { Absolutismus }+ \\
\text { Reziprozität) }\end{array}$ & $\begin{array}{c}\text { Hollstein } \\
\text { (Generalisierte } \\
\text { Reziprozität) }\end{array}$ & \multicolumn{1}{c}{$\begin{array}{c}\text { Bengtson } \\
\text { (Solidaritätstypen) }\end{array}$} \\
\hline $\begin{array}{c}\text { Wohltätigkeitsnorm } \\
+ \text { moralischer } \\
\text { Absolutismus }\end{array}$ & Solidaritätsprinzip & Affectual Solidarity \\
& Generatives Prinzip & $\begin{array}{l}\text { Consensual } \\
\text { Solidarity }\end{array}$ \\
& Stellvertretungsprinzip & Normative Solidarity \\
\hline & & $\begin{array}{l}\text { Associational } \\
\text { Solidarity }\end{array}$ \\
& & Functional Solidarity \\
Reziprozität & & Structural Solidarity \\
& & \\
\hline
\end{tabular}

Quelle: Eigene Darstellung.

„1. Affectual solidarity: the sentiments and evaluations family members express about their relationship with other members (How close do you feel to your father or mother? How well do you get along with your child or grandchild? How much affection do you feel from them?)

2. Associational solidarity: the type and frequency of contact between intergenerational family members

3. Consensual solidarity: agreement in opinions, values, and orientations between generations

4. Functional solidarity (assistance): the giving and receiving of support across generations, including exchange of both instrumental assets and services as well as emotional support

5. Normative solidarity: expectations regarding filial obligations and parental obligations, as well as norms about the importance of familistic values

6. Structural solidarity: the "opportunity structure" for cross-generational interaction reflecting geographic proximity between family members" (Bengtson 2001: 8).

Im Unterschied zu Bengtson, der in erster Linie intergenerationale Beziehungen betrachtet, werden im Rahmen der vorliegenden Studie die Dimensionen der Solidarität innerhalb von Paarbeziehungen fokussiert. Es wird angenommen, dass Bengtsons Typen auf die Paarebene übertragen werden können. Als Mitglieder der Kernfamilie ist zwischen Frau und Mann innerhalb eines 
Paares eine Solidargemeinschaft anzunehmen, die sich gleichwohl in die von Bengtson aufgeführten Dimensionen ausdifferenzieren lässt und Einfluss auf die innerhäusliche Arbeitsteilung nimmt. Die emotionale Verbundenheit ist eine Solidaritätsform, die auch für Paarbeziehungen von großer Bedeutung ist und nicht zuletzt in der Liebe ihren Ausdruck findet. Auch die Kontaktintensität spielt für Paarbeziehungen eine maßgebliche Rolle, ebenso die Übereinstimmung in Überzeugungen. Das Geben und Nehmen von Hilfeleistungen und Transfers findet abermals im Rahmen von Paarbeziehungen statt und der Verpflichtungsgrad mag zwar je nach Situation zwischen den PartnerInnen variieren, doch insgesamt - das liegt der Paarbeziehung zugrunde - eher hoch sein. Insbesondere vor dem Hintergrund einer zunehmenden räumlichen Mobilität in Folge von Transnationalisierungprozessen, vermag es vornehmlich für hochgebildete Paare häufiger vorkommen, dass sie über keinen gemeinsamen Wohnsitz verfügen und ihre Paarbeziehung als eine „Fernbeziehung" führen, wodurch auch die strukturelle Solidarität nicht außer Acht gelassen werden kann.

Obwohl es immer gängiger zu sein scheint, Solidarität auf die intergenerationalen Beziehungen und damit auf familiale Solidarität zu beziehen - was durchaus sehr wesentlich ist - soll daran erinnert werden, dass es sich hierbei ebenso um ein makrosoziologisches Phänomen handelt - eine Form der gesellschaftlichen Solidarität. Zwischen beiden Formen scheinen Wechselwirkungen zu bestehen. Unter Berücksichtigung eines zunehmenden Ausbaus einiger europäischer Wohlfahrtsstaaten stellt sich unter der Voraussetzung einer Wechselwirkung beider Ebenen die von Ostner aufgeworfene Frage:

„Hat der Wohlfahrtsstaat, indem er mehr Aufgaben der Familien übernahm, die Hilfsbereitschaft zwischen Familienmitgliedern aufgezehrt? Die Substitutionsoder »Crowding-Out-«These behauptet dies: Mit der Entwicklung des Wohlfahrtsstaats nimmt die familiale Unterstützung ab" (Ostner 2004a: 78).

Beispielsweise argumentiert Newman, dass ,In countries with a weaker welfare state [Italy and Spain], the family is the buffer between young people and the relentless market pressures that can reduce their options" (Newman 2012: 155). Hingegen würden in Ländern wie Dänemark und Schweden, die über einen stark ausgeprägten Wohlfahrtsstaat verfügen, gemäß Newmans Ergebnissen familiale intergenerationale Beziehungen durch staatliche Leistungen geschwächt. In Folge der Crowding-Out-These entstanden zahlreiche Studien, die das Gegenteil zu argumentieren suchten:

„Denn nach wie vor fühlen sich die Familienangehörigen verbunden und leisten einander Hilfe. Dabei haben, so die Vertreter der These von Komplementarität und »Crowding-In«, wohlfahrtsstaatliche Leistungen, insbesondere die lebensstandardsichernden Renten, bestimmte Formen des Gebens innerhalb von Familien überhaupt erst ermöglicht und - historisch einmalig - die Richtung der Unterstützung umgekehrt [früher haben Kinder die Altenpflege ihrer Eltern über- 
nommen, heute unterstützen die Eltern ihre Kinder auch noch im Erwachsenenalter]" (Ostner 2004a: 78).

Obgleich beide Thesen annehmen, dass familiale Solidarität prekär ist (staatliche Betreuung kann nicht erzwungen werden, familiale Unterstützung hingegen schon, doch ,[...] der Zwang würde in jedem Fall den Gebenden/die Gebende und den Empfänger/die Empfängerin der Hilfe, somit auch die Gabe und ihr Korrelat, die Dankbarkeit, beschädigen“ (Ostner 2004a: 79)), unterscheiden sie sich in der Annahme über den Einfluss wohlfahrtsstaatlicher Leistungen auf die Hilfebereitschaft in Familien. Während die Komplementaritätsthese annimmt, dass der Wohlfahrtsstaat Freiräume für familiale Solidarität einräumt, geht die Substitutionsthese von einer Einschränkung familialer Solidarität aus (vgl. Ostner 2004a: 79f.). Ostner behauptet in ihrem Artikel, „[...] dass familiale Solidarität [...] in einem gewissen Sinn eine Innovation der modernen Gesellschaft ist" (Ostner 2004a: 80). Doch auch ohne die Unterstützung des Wohlfahrtsstaats fördert die Familie die Gutwilligkeit, ihren Mitgliedern zu helfen - auch dann, wenn lediglich Dankbarkeit erwartet werden kann. Gemeinsame Werte, Normen und Situationsdefinitionen sind Voraussetzungen solidarischen Handelns, die am ehesten in der Familie erfüllt werden (vgl. Ostner 2004a: 86f.). Die Hilfsbereitschaft in Familien und Paarbeziehungen folgt nicht ausschließlich einer rationalen Partnerschaftslogik.

„Intime Beziehungen [...] eröffnen die Möglichkeit, als einmaliges Individuum anerkannt und »zweckfrei« geliebt zu werden. [...] Die andere - negative - Seite der Hilfsbereitschaft ist allerdings ihr Partikularismus: [...] Nicht nur der, der den Arbeitsplatz verliert, auch wer keine Familie hat, ist von »Exklusion« bedroht"“ (Ostner 2004a: 87).

„Die »Liebe« kann sich aber als problematisches Steuerungsprinzip der Familienbeziehungen erweisen. Kaum war sie institutionalisiert, scheitern immer mehr Paarbeziehungen am Anspruch. Etwas zu geben gegen nichts und dies etwa über längere Zeit mit ungewissem Ergebnis - das muss(te) existierende und zukünftige Paare und Eltern überfordern - möglicherweise ein Grund, weshalb Frauen und Männer längerfristige bindende Verpflichtungen aufschieben, wenn nicht gar vermeiden, und ein Grund, weshalb die Gesellschaft die Pflichten zwischen Eltern und Kindern neu gestaltet. Schließlich widerspricht »Liebe« der Norm der Symmetrie und Egalität, die sich - auf den ersten Blick paradox - gleichzeitig mit der Norm, andere nicht als Mittel zum Zweck zu behandeln, durchsetzte. Von daher konfligierten von Anfang an »Liebe« (Einseitigkeit) und »Partnerschaft« (gleiche Gegenseitigkeit) als Steuerungsprinzipien sozialer Interaktion in Familienbeziehungen" (Koppetsch, zitiert nach Ostner 2004a: 87f.).

Ostners Ausgangspunkt war, dass der Modernisierungsprozess sowohl familiale Solidarität als auch den Wohlfahrtsstaat hervorgebracht hat, der familiale Solidarität unterstützt sowie in Teilen auch ermöglicht (vgl. Ostner 2004a: 91). Sofern die familiale Hilfsbereitschaft abnehmen sollte, so sei dies gemäß 
Ostner auch eine Folge der zunehmenden Instabilität moderner, auf Liebe und Freiwilligkeit gegründeter Familien.

„Der Wohlfahrtsstaat würde die familiale Solidarität, so meine Vermutung [...], dann schwächen, wenn er der Familie für eine längere Zeit den Raum nimmt, den sie braucht, um für ihre Hilfebedürftigen selbst zu sorgen“"(Ostner 2004a: 91f.).

Die familiale sowie die makrosoziale Dimension sind nicht nur für intergenerationale Beziehungen von Relevanz, sondern auch für Paarbeziehungen. Zuvor wurde argumentiert, dass Nächstenliebe der Solidarität immanent ist. Mit Fokus auf einer makrosozialen Dimension der Solidarität bedeutet dies weiterführend: Gerechtigkeit braucht Liebe. Als Grundlage für diese Behauptung wird auf Martha Nussbaums Werk „Political Emotions: Why Love matters for Justice“ (2013) und auf die Liste der „Basic Human Functional Capabilities" (s. Anhang) für ein gutes menschliches Leben verwiesen, die unter Punkt fünf die allgemeine Möglichkeit, Liebe, Kummer, Sehnsucht und Dankbarkeit zu empfinden, erläutert. Diese Fähigkeit zu unterstützen heißt weiterführend, Formen des menschlichen Miteinanders zu unterstützen, was wiederum bedeutsam für die menschliche Entwicklung ist. Aus Gründen der Gerechtigkeit entsteht die solidarische Norm andere Menschen zu unterstützen ohne sie persönlich zu kennen - Sozialleistungen basieren auf dieser Idee. 


\section{Zwischenfazit der eigenen Argumentation: Für einen Arbeitsteilungspluralismus}

Wenn wir uns die Frage stellen, inwiefern die Modernisierung zu mehr Gendergleichheit und dadurch auch zu einer Zunahme des Partnerschaftskodes in Paarbeziehungen führt, kann festgehalten werden: Keineswegs ist das skandinavische Modell als erstrebenswertes Modell sämtlicher europäischer Länder zu erachten, gilt es doch zumindest kulturelle Unterschiede zu bedenken. Auch die Frage des Adult Worker Modells ist zu diskutieren: Zweifellos sind die Interessen und Wünsche von Frauen, die durchaus höchst heterogen sind, zu berücksichtigen. Dennoch haben sich in westlichen Industrieländern eine ,adult worker-Norm“ (auch wenn diese zunächst nichts über Ungleichheiten zwischen Erwerbstätigen und die geschlechtsspezifische innerhäusliche Arbeitsteilung aussagt) ebenso wie eine Norm zur egalitären innerhäuslichen Arbeitsteilung etabliert, die nicht entstanden wären, wenn die individuellen Interessen diesen mehrheitlich widersprechen würden (Leitner et al. 2004: 13). Auf der theoretischen Ebene geht es nicht darum, dass alle Frauen einem einzigen Ideal ,hinterherhinken“, sondern darum, dass nach Sens Idealfall die Handlungsmöglichkeiten zur Verfügung stehen, aus denen je nach Interesse gewählt werden kann, wodurch ein pluralistisches Egalitätskonzept ermöglicht wird. Dieses pluralistische Verständnis widerstrebt der (Re)Kommodifizierungspolitik,

„,[...] ohne jede Rücksicht auf die Besonderheiten der zu "kommodifizierenden" Individuen (wie z.B. Qualifikation, Verfügbarkeit für den Arbeitsmarkt, Machtressourcen) deren Integration [in den Arbeitsmarkt] zu erzwing[en], [...] [wodurch] die Möglichkeiten von Lebensentwürfen jenseits der (vollzeitigen) Erwerbsarbeit erheblich eingeschränkt" werden (Leitner et al. 2004: 15).

Stattdessen wird die Freiheit zur Handlungswahl betont, ferner die Wahlfreiheit von Lebensentwürfen. Während beispielsweise für den Einen oder die Eine die Idealvorstellung eine berufliche Karriere ist, ist für den Anderen oder die Andere die Zuwendung zur Familie der idealtypische Lebensentwurf. In dieser Hinsicht kritisiert Ostner zu Recht, dass es einsinnig ist, „agency inequalities“ auf die Erwerbstätigkeit zu beziehen. Ferner stellt sich die Frage, warum überhaupt dieses „Oder" zwischen der Gleichheits- und Differenzargumentation (s. Wollstonecraft-Dilemma) von Nöten ist. Durch Sens Argumentation kann dieser Dualismus überwunden werden. Doch ist die Debatte der Abhängigkeit ausschließlich im Sinne einer zugeschriebenen, interpretativen sozialen Konstruktion $\mathrm{zu}$ verstehen? Ostner argumentiert anhand des Beispiels der USA ${ }^{100}$, dass trotz eines ZweiverdienerInnen-

1001993 argumentierte Ostner, dass kein Wohlfahrtsstaatsregime in der Lage ist, die Problematik zwischen Abhängigkeit und Unabhängigkeit adäquat zu lösen: ,[...] no modern so- 
Modells einkommensarme und überschuldete Haushalte wie auch finanzieller Bankrott zunehmen, sofern eine/ $\mathrm{r}$ der beiden PartnerInnen arbeitslos wird, weshalb dieses Modell beide PartnerInnen ,zusammenzwingen“ würde. Insofern würde das ,,adult worker model“ zu neuen Formen der Abhängigkeiten zwischen den Geschlechtern führen (Ostner 2004b: 57). „Diese sind zwar frei zu handeln, aber bleiben wie bisher auch ökonomisch aufeinander verwiesen“ (Ostner 2004b: 57). Doch kann die empirische Zunahme von ökonomischen Ungleichheiten allein die Grundlage zur Ablehnung einer, ,theoretisch erstrebenswerten Gendergleichheit" darstellen? Ist die Entwicklung, die in den USA zu beobachten ist, nicht vielmehr eine Frage der Umsetzung entsprechender Policies, vornehmlich von minimalen Sozialleistungen? Werden hier nicht sowohl theoretisch als auch empirisch unterschiedliche Dimensionen sozialer Ungleichheit („class“ und „gender") vermengt, die Korpi zwar versuchte, in einen Zusammenhang zu bringen, der jedoch insbesondere durch Asymmetrie gekennzeichnet ist? Kann das Zwei-verdienerInnen-Modell die Hauptursache minimaler Sozialleistungen sein? Wie Ostner annimmt vermag die Abhängigkeit eine soziale Konstruktion, eine Interpretation zu sein, die von Betroffenen womöglich als Freiheit erlebt wird. Doch spätestens im Falle einer Trennung der Paarbeziehung könnte die ,interpretierte Abhängigkeit“ für den nicht-erwerbstätigen Partner bzw. die nicht-erwerbstätige Partnerin zum empirischen finanziellen Problem werden. Davon abgesehen, dass die ehemals im Haushalt tätige Person nach einer längeren Erwerbsunterbrechung womöglich Schwierigkeiten hat, wieder in das Erwerbsleben zurückzukehren, um ihren Lebensunterhalt zu sichern (s. Kritik an der Austauschtheorie von Blau).

Die Egalitätsnorm ist hier zu verstehen als eine Erwartung der Gleichstellung der Geschlechter in sämtlichen Bereichen, wie beispielsweise im Erwerbsleben; sie setzt eine Chancengleichheit der Geschlechter auf - um bei diesem Beispiel $\mathrm{zu}$ bleiben - Erwerbsbeteiligung voraus und ist nicht $\mathrm{zu}$ verwechseln mit der anthropologischen Annahme der Gleichheit der Geschlechter. Ferner sind für die Argumentation des Machtansatzes die Ermächtigungschancen innerhalb eines Handlungsspielraumes sowie die Positionen von Individuen maßgeblich, weil ,[...] die Teilnahme an jedem der sozialen

ciety has really suceeded in solving the problem of dependence and independence: many people will always need to looked after by carers; nobody can live an independent, selfsufficient life from birth to death. The various welfare regimes are different but always flawed with regard to this issue. Each of them has its own contradictions" (Ostner 1993: 115). Zu Recht nimmt sie an, dass im gesamten Lebenslauf betrachtet immer Phasen von Abhängigkeiten bestehen. Es gilt jedoch auch unterschiedliche Abstraktionsniveaus zu berücksichtigen. Ein Abhängigkeitsverhältnis zwischen zwei Personen weist einen niedrigeren Abstraktionsgrad auf als ein Abhängigkeitsverhältnis zwischen einer Person und dem Wohlfahrtsstaat. Im ersten Fall sind konkrete Erwartungen an eine Person gerichtet, die nicht ohne weiteres auf eine andere Person übertragen werden können, wodurch das $\mathrm{Ab}$ hängigkeitsverhältnis verschärft wird. 
Felder heißt, eine Position in dessen Binnenstruktur zu besetzen und die an diese geknüpften Rollenerwartungen beantworten zu müssen“" (Krüger/Levy 2000: 382). Letztlich manifestieren sich Normen je nach Kontext und Interpretation der Individuen teils mehr, teils weniger in Einstellungen.

Wie entsteht die Diskrepanz zwischen egalitärer Einstellungs- und devianter, traditioneller Verhaltensebene? Ein möglicher Erklärungsansatz ist das bereits erläuterte agency gap nach Hobson (2011). Diese Fragestellung zielt außerdem auf die nach Giddens (1992) definierte Strukturierung ab - einen weiteren möglichen Erklärungsansatz bietet das „Cultural Lag“. Der Begriff des „Cultural Lag“ wurde maßgeblich von William F. Ogburn geprägt.

„Eine kulturelle Phasenverschiebung [Cultural Lag] findet statt, wenn von zwei miteinander in Beziehung stehenden Kulturelementen das eine sich eher oder in größerem Maße verändert als das andere, so daß [sic!] der Grad der Anpassung zwischen den beiden Elementen geringer wird als zuvor" (Ogburn 1969: 134).

Vorausgesetzt wird, dass Veränderungen in ungleichen Zeitintervallen auftreten (Ungleichzeitigkeit des strukturellen und kulturellen Wandels), wobei die unabhängige Variable ,technischer, ökonomischer, politischer, ideologischer oder beliebig anderer Natur" sein kann (Ogburn 1969: 140). EspingAndersen et al. (2013) vertreten beispielsweise die These, dass verbesserte Verhütungsmethoden und neue Haushaltstechnologien zu einem ausgeprägten Interesse von Frauen an beruflichen Fähigkeiten geführt haben und letztlich zur Institutionalisierung der Berufstätigkeit von Frauen als Norm beitrugen (vgl. Esping-Andersen et al. 2013: 2f.). Schlussfolgernd aus dem Cultural Lag wird hier die These, dass die Abweichung zwischen Egalitätsnorm und Handlung ausschlaggebend von sozialen Strukturen beeinflusst wird, vertreten. ,[...] Es ist von Bedeutung, soziale Strukturen als Variablen [für die Erklärung der Normentstehung] in Betracht zu ziehen“"(Opp 1983: 103). Aus historischer Perspektive hat die Entwicklung zu modernen marktwirtschaftlichen Gesellschaftsformen eine Trennung von Familien- und Erwerbsleben herbeigeführt (bürgerliches Familienmodell als „Kind des Kapitalismus“), weil der „männliche Arbeiter“ von der „weiblichen Hausfrau“ im Hinblick auf Reproduktionsarbeiten entlastet wurde (obwohl es auch immer erwerbstätige Frauen, vornehmlich in der Arbeiter- und Dienstbotenschaft gab, sowie Kinderarbeit, wenn die finanziellen Verhältnisse nicht ausreichend waren) (vgl. Kreckel 1993: 54f.).

„Then came the industrial revolution. Its most important consequence was to separate the place where a man worked from his home. As a result, the members of the family no longer worked together and, as child-labor laws were passed, children became an economic liability instead of an asset" (Blood/Wolfe 1960: $3)$.

Kaufmann betrachtet die zunehmende Trennung von Haushalts- und Erwerbsarbeit ebenso als Folge der Industrialisierung und bezeichnet die Sepa- 
ration als ,[...] die wohl einschneidenste Veränderung der familialen Lebensverhältnisse" (Kaufmann 1990: 20). Die strukturelle Schwachstelle des Kapitalismus ist, dass die Reproduktion von menschlichen Arbeitskräften marktextern erfolgen muss. Um diese Schwachstelle zu überbrücken, werden Familienhaushalte, allen voran die Spezialisierung der Frauen auf die Reproduktionsarbeit benötigt (vgl. Kreckel 1993: 55). Angenommen wird hier, dass die weiterführende Tertiarisierung (Strukturwandel von Industrie- zu Dienstleistungsgesellschaften) zu einer zunehmenden Erwerbsbeteiligung von Frauen als Trend im Modernisierungsprozess geführt hat ${ }^{101}$, außerdem ein $\mathrm{Zu}-$ sammenhang zwischen außerhäuslicher Erwerbstätigkeit und innerhäuslicher Hausarbeit besteht ${ }^{102}$, folglich genderspezifische Hausarbeitsarrangements als nachhinkender Teil der kulturellen Phasenverschiebung zu erachten sind. Doch ist eine absolute Egalität auf der Verhaltensebene in der Empirie eine Utopie, die nicht ausnahmslos erreicht werden kann, gleichwohl eine Orientierung an der Egalitätsnorm zu bestehen scheint. Modernisierungsprozesse verändern Strukturen, Normen wie auch Werte und sind dennoch nicht als lineare Entwicklungspfade zu betrachten, sondern können multiple Verhaltensweisen hervorrufen. So kann ein Streben nach der Egalitätsnorm zu einem Trend respektive einer Annäherung an egalitäre Arbeitsteilungsarrangements führen, ist jedoch nicht als hinreichende Bedingung zu verstehen. Aus der hier vertretenen Perspektive müsste die Diskrepanz zwischen Einstellungs- und Verhaltensebene im Laufe des sozialen Wandels an Bedeutung verlieren, was nicht zwangsläufig in einer vollkommenen kongruenten Anpassung der beiden Ebenen münden muss. In Beat Fux Worten bedeutet dies:

„Wenn wir den Versuch unternommen haben, die langfristige Entwicklung familialer Leitideen und Lebensformen, der Fertilität und der Familienpolitik mit den Mitteln der quantitativen und qualitativen empirischen Sozialforschung zu rekonstruieren, dann leitete uns der Gedanke, daß [sic!] soziale Prozesse keineswegs gradlinig und ebensowenig konfliktfrei vonstatten gehen" (Fux 1994: 373).

Gershuny/Fisher stellen beispielsweise im Zeitverlauf zwischen 1960 und 2005 eine Annäherung von Arbeitsmustern der Geschlechter (zunehmende Beteiligung von Frauen an bezahlter Arbeit und Abnahme der von Frauen verrichteten unbezahlten Arbeit) in europäischen, nordamerikanischen und

101 Demzufolge ist auch ein Rückgang von Müttern, die dauerhaft nicht erwerbstätig sind sowie ein Rückgang von Frauen mit dauerhaften Erwerbsunterbrechungen zu verzeichnen (vgl. Baumgartner/Fux 2004: 117). Außerdem kommt das traditionelle duale Model respektive Breadwinner-Modell (Mann Vollzeit, Frau nicht erwerbstätig) meist nur noch während der Familienphase mit Kleinkindern zur Anwendung und letztlich steigt der Anteil an teilzeiterwerbstätigen Müttern (vgl. Baumgartner/Fux 2004: 122).

102 Trotz des Trends zur Erhöhung des Teilzeiterwerbspensums ,[...] korrespondiert die Müttererwerbstätigkeit immer mit der familialen Situation und verhält sich komplementär dazu. Der Erwerbsumfang wird dem Alter des Kindes angepasst“ (Baumgartner/Fux 2004: 125). 
pazifischen Demokratien fest (vgl. Gershuny/Fisher 2014: 2). Dennoch wird der größte Anteil der unbezahlten Arbeit von Frauen ausgeführt, wobei sich aus der Summe der Arbeit aus bezahlter und unbezahlter Tätigkeiten (,,isowork") eine ähnliche Arbeitszeit zwischen den Geschlechtern ergibt - eine „unequal sort of equality“ (Gershuny/Fisher 2014: 18). Ferner gilt es zu bedenken, dass Verhaltensweisen auch von dynamischen lebenslaufzyklischen Institutionenstrukturierungen abhängig sind, die ggf. einer egalitären Organisation im Wege stehen. Darüber hinaus sind kulturelle Unterschiede zwischen Regionen zu erwarten, die dazu führen, dass die innerhäusliche Arbeitsteilung in einigen Regionen egalitärer als in anderen organisiert ist. Gleichwohl ist aktuell für europäische Regionen keineswegs eine egalitäre innerhäusliche Arbeitsteilung anzunehmen. Insofern ist auf der Verhaltensebene davon auszugehen, dass traditionelle Rollenbilder ihren Stellenwert trotz Modernisierungsprozessen beibehalten können oder sogar an Bedeutung gewinnen. Ein Verständnis von Modernisierungsprozessen, die Einfluss auf Einstellungen und Verhaltensweisen von Individuen nehmen, erfordert einen komplexeren Bezugsrahmen als eine rein lineare Funktion. Erinnern wir uns an Sen: wenn beiden PartnerInnen innerhalb der Paarbeziehung im Sinne der Pluralität jegliche Handlungsmöglichkeiten eröffnet werden, kann auch eine als traditionell bezeichnete Arbeitsteilung egalitär sein (im Sinne einer pluralistischen Egalitätskonzeption), sofern diese zum well-being beider beiträgt, weil es nicht darauf ankommt, für welche Alternative sich das Paar entscheidet, sondern dass die Auswahlmöglichkeiten für beide zur Verfügung stehen. Stehen die Auswahlmöglichkeiten zur Verfügung, so gilt es den Paaren ihren eigenen Entscheidungsfreiraum zu gewähren, über ihren Lebensweg selbst zu bestimmen. Martha Nussbaum verdeutlicht dies u. a. durch ihre Antwort auf das „Argument von der Kultur":

„Traditionelle Kulturen, so das Argument, haben ihre eigene Norm darüber, wie das Leben von Frauen auszusehen hat [Bescheidenheit, Unterwürfigkeit, Gehorsam, Selbstaufopferung] [...]. Meine vollständige Antwort auf dieses Argument wird aus meinem eigenen Vorschlag hervorgehen, der sicherlich keine Wahlmöglichkeit einer Frau, ein traditionelles Leben zu führen, ausschließt, solange sie ganz sicher bestimmte wirtschaftliche und politische Chancen hat" (Nussbaum 2003: 13).

Die Idee der Selbstbestimmung über den eigenen Lebensweg erfordert sowohl Grundfreiheiten als auch „Formen wirtschaftlicher Ermächtigung, die entscheidend dazu beitragen, dass die Freiheiten für die Menschen wirklich verfügbar sind“" (Nussbaum 2003: 16, Hervorhebungen durch die Verf.).

\section{Eine kritische Betrachtung von Koppetschs Beziehungskodes}

Es wird keineswegs in Abrede gestellt, dass Liebe und Partnerschaft unterschiedlichen Beziehungslogiken folgen und Liebe Ungleichheiten zulässt. Diese Feststellung ändert jedoch nichts an der strukturell schwächeren Posi- 
tion, ,[...] welches auch immer die konkreten Positionen und Ressourcen sein mögen" (Krüger/Levy 2000: 391). Auch wenn eine schwächere Position hingenommen wird, so ist dennoch im Sinne Sens eine strukturell gleichwertige Position zu ermöglichen. Wie und ob diese umgesetzt wird, bleibt letztlich wiederum den individuellen Interessen überlassen. Wiedermal (s. hierzu den bereits beschriebenen Masterstatus nach Levy) wird die innerhäusliche Arbeitsteilung als vermeintlich individuelle Privatentscheidung interpretiert und damit als Verdeckungsphänomen verschleiert - Strukturblindheit gefördert (vgl. Krüger/Levy 2000: 389).

$\mathrm{Ob}$ ein Austausch im Sinne der Liebe oder im Sinne der Partnerschaft erfolgt, ist wenigstens auf die Handlungsmotive der betroffenen Akteure, Handlungsrestriktionen und die Logik der Situation zurückzuführen. Es kommt dabei nicht darauf an, wer, d. h. ob Frau oder Mann, eine Gabe verschenkt. Vielmehr ist von Bedeutung, dass beiden die Verwirklichungschance eines Gabentauschs sowohl in der GeberInnen- als auch in der NehmerInnenposition ermöglicht wird.

Die dargestellte Annahme Koppetschs, dass die von ihr eingebrachte Gabe der innerhäuslichen Hausarbeiten heute nicht mehr erlaubt sei, impliziert die These, dass in der Vergangenheit die durch die Frau verrichteten Hausarbeiten überwiegend Gaben darstellten. Dem ist eine auf Partnerschaft ausgerichtete Tauschlogik - verrichtete Hausarbeiten der Frau gegen finanzielle Absicherung der Frau durch ihren Mann - zu entgegnen, wie sie im bürgerlichen Familienmodell als ,systematische Begleiterscheinung des modernen Kapitalismus" vorgesehen war (Kreckel 1993: 54). Zu hinterfragen bleibt, inwiefern überhaupt eine einzige Austauschlogik für eine spezifische Epoche angenommen werden kann oder ob nicht ohnehin mehrere Austauschtypen anzunehmen wären. Darüber hinaus stellt sich die Frage, inwiefern eine wiederholte Gabe eine Gabe bleibt, selbst wenn sie routiniert wird?

Weiterhin gilt es eine grundlegende Streitfrage, die der Selbstverwirklichung beider Geschlechter nach dem Modell männlicher Autonomie, zu diskutieren. Das Modell männlicher Autonomie, das häufig auf die Berufskarriere bezogen wird, ist ebenso wie die von Ostner thematisierte Abhängigkeit ein soziales Konstrukt. Vielmehr handelt es sich um einen Bereich, der auf zugeschriebener Männlichkeit beruht. Es ist eine Anmaßung, allein diesen Bereich der Männlichkeit zuzuschreiben, wodurch Männern in der Folge die Handlungschance, im innerhäuslichen Feld tätig zu werden, indirekt verwehrt wird. Zugleich wird Frauen qua Semantik die Handlungschance genommen, eine berufliche Selbstverwirklichung zu entfalten. Die geschlechtsspezifische Zuschreibung führt zu einer Einschränkung der Verwirklichungschancen und damit zu einer Verschärfung von Ungleichheiten.

,[...] genauso wenig läßt [sic!] sich aus dem abstrakten Geschlechterverhältnis die eindeutige empirische Zuordnung von Männern oder Frauen zu einer be- 
stimmten Arbeitsform, also: zu Beruf oder Hausarbeit, herleiten“ (Kreckel 1993: 59).

Beiden Geschlechtern sind sowohl der außerhäusliche als auch der innerhäusliche Bereich zu öffnen. Erst wenn diese Voraussetzung erfüllt ist, kann selbst ein Male-Breadwinner-Modell als egalitär (im Sinne einer pluralistischen Egalitätskonzeption) interpretiert werden, sofern ein Pluralismus der Arbeitsteilungsarrangements besteht. Zu wiederholen sei auch nochmals die Argumentation von Korpi (2000), dass die Partizipation am Arbeitsmarkt die Verteilung von Ressourcen als Basis materieller Ungleichheiten, die Identität einer Person, den Rahmen, der Capabilities und Freiheiten in vielen anderen Lebensbereichen beeinflusst und die intrafamiliäre Verhandlungsmacht determiniert. Gershuny und Fisher argumentieren, dass aus einer soziologischen Perspektive ,work [...] is (1) intrinsically enjoyable for some, (2) a psychological necessity for all, (3) an important determinant of individuals' social positions, and (4) an essential constituent of social solidarity“ (Gershuny/Fisher 2014: 5). Insofern ist der Arbeitsmarkt auf einer theoretischen Ebene als geschlechtsunabhängig zu konstatieren. Wie auch Newman (allerdings in Bezug auf intergenerationale Beziehungen) feststellt, gilt es die dichotome Einschätzung der Familienorganisation als richtig oder falsch zu vermeiden:

„We are inclined to think that there is a right and wrong way to organize family relations [...]. It is hard to avoid the temptation to shake our heads when encountering a society in which family relations are either far more distant [in the case of Denmark and Sweden] or, in the case of Spain or Italy, much closer than ours" (Newman 2012: 174).

Im Folgenden wird die Entwicklungsgeschichte einiger prägnanter Ansätze zur Soziologie der Machtverhältnisse dargestellt, bevor aus den oben angeführten theoretischen Argumentationen eine Typologie der Machtverhältnisse (weiter-)entwickelt wird. 



\section{Familiensoziologische Machtansätze „revisited“}

\section{1. Übersicht zur Entwicklungsgeschichte einer Soziologie der Machtverhältnisse in Paarbeziehungen: die Klassiker familiensoziologischer Machtansätze}

Viele Machtansätze, die innerhalb der Familiensoziologie entwickelt wurden, beruhen auf den von den Sozialpsychologen French und Raven (1959) konzipierten „bases of power“: Coercive power (Zwangsmacht: Möglichkeit zur Bestrafung/negative Sanktionen), Reward power (Belohnungsmacht: Möglichkeit zur Belohnung/positive Sanktionen), Legitimate power (legitimierte Macht: auf sozialen Normen und Positionen beruhendes legitimiertes Recht zur Weisung), Expert power (Expertenmacht: basiert auf überlegenen, spezifischen Kenntnissen und besonderen Fähigkeiten), Referent power (Referenzmacht: Identifikation von Unterworfenem mit Machtausübendem bzw. Positionierung des Unterworfenen in Relation zum Machtausübenden) und Informational power (Informationsmacht: wurde später von Raven entwickelt; beruht auf einem Informationsvorsprung, wodurch die Einflussmöglichkeit des Akteurs resultiert, Veränderungen hervorzubringen).

„(a) reward power, based on P's perception that $\mathrm{O}$ has the ability to mediate rewards for him; (b) coercive power, based on P's perception that $\mathrm{O}$ has the ability to mediate punishments for him; (c) legitimate power; based on the perception by $\mathrm{P}$ that $\mathrm{O}$ has a legitimate right to prescribe behavior for him; (d) referent power, based on P's identification ${ }^{103}$ with $\mathrm{O}$; (e) expert power; based on the perception that $\mathrm{O}$ has some special knowledge or expertness“" (French 1959: 263).

Hallenbeck (1966) bezieht sich auf die von French/Raven (1959) entwickelten Machtbasen (vgl. Hallenbeck 1966: 200). Diese werden auf Ehepartnerschaften übertragen, wobei argumentiert wird, dass das Ideal von Rollenbildern (glücklich verheiratete Ehepaare), das beide EhepartnerInnen anstreben, die Basis der Referent power bildet. „It is the contention of this paper that referent power in marriage stems from the desire of the spouses to be like their concepts of the "ideal" husband or wife" (Hallenbeck 1966: 202). Die auf Legitimation beruhende Macht wird durch die kulturelle Definition der Geschlechterrollen ermöglicht. „Das interaktionistische Legitimationskonzept von French \& Raven wird also hier auf die Gesamtkultur ausgeweitet" (Held 1978: 71). Weiterhin nimmt Hallenbeck Bezug auf die coercive power, die sowohl physische Gewalt als auch non-verbal influence nach SafiliosRothschild (1969) umfasst. Wesentlich ist, dass die coercive power wie auch

103 „By identification, we mean a feeling of oneness of $\mathrm{P}$ with $\mathrm{O}$, or a desire for such an identity" (French 1959: 266). 
die reward power durch legitimate power modifiziert werden können. „The powers to reward and coerce become stronger when associated with legitimate power" (Hallenbeck 1966: 202).

Auch Safilios-Rothschild (1975) knüpft an die Machtbasen von French/Raven (1959) an. Die durch gesellschaftliche kulturelle Normen gegebene Macht, von French/Raven (1959) wie auch von Hallenbeck (1966) als legitimate power bezeichnet, wird von Safilios-Rothschild (1975) als authority definiert. Ferner wird zwischen Expert power, Dominance power (die sowohl physischen als auch psychischen Zwang einschließt), Ressource power, Affective power (mehr affective power durch weniger Beziehungsinteresse) und Decision-making power (wer entscheidet? in Abgrenzung zum Einfluss, der als Methode, d. h. als Element der psychischen Zwangsmacht, verstanden wird) unterschieden. Bezugnehmend auf die Decision-making power wird eine Differenzierung zwischen der Orchestrierungsebene (Festlegen von Regeln der Machtausübung) und der Implementationsebene (das Treffen von Entscheidungen in einzelnen Bereichen) vorgenommen (s. hierzu auch Safilios-Rothschild 1976: 359). Das Treffen von Entscheidungen wird seinerseits unterschieden in Entscheidungsebene und Ausführungsebene. Es zeigt sich, dass familiensoziologische Ansätze des Machtkonzepts häufig Bezug auf das Treffen von Entscheidungen nehmen (vgl. Held 1978: 71.). So wird es auch im Machtkonzept von Olson/Cromwell (1975) thematisiert, vielmehr werden Machtgrundlagen sowie -ressourcen erörtert, wobei decision-making power (nach Safilios-Rothschild (1975)) als power outcomes (Machtresultate) beschrieben werden. 1976 widmet sich Safilios-Rothschild den Konzepten von Macht und Liebe unter dem Deckmantel eines Austauschmodells. Ressourcen, die zwischen PartnerInnen getauscht werden können, sind:

„Socioeconomic (Money, Social Mobility, Prestige), Affective (Affection, Love (loving - being loved), Feeling needed - needing the other), Expressive (Understanding, Emotional Support, Special attention), Companionship (Social, Leisure, Intellectual), Sex, Services (Housekeeping Services, Child Care, Personal Services, „Linkage“ Services), Power in relationship“ (Safilios-Rothschild 1976: 356, Hervorhebungen durch die Verf.).

Je abhängiger ein/e (Ehe-)PartnerIn von diesen Ressourcen ist und über keine alternativen Möglichkeiten verfügt, sich diese Ressourcen anderweitig zu beschaffen, desto höher ist der Wert, den er/sie diesen Ressourcen zuschreibt und desto höher sind die Kosten, die er/sie für die Erhaltung aufwenden wird (vgl. Safilios-Rothschild 1976: 356). Wesentlich ist, dass keine ausgeglichene Reziprozität des Tauschs angenommen wird (gleichwohl als möglich und wünschenswert thematisiert), sondern das Tauschverhältnis als asymmetrisch beschrieben wird (vgl. Safilios-Rothschild 1976: 356). Der/die PartnerIn, der/die über erstrebenswerte, hoch wertvolle Ressourcen verfügt, deren $\mathrm{Zu}$ gang dem/der anderen PartnerIn nur über diese Beziehung gewährt werden 
können, genießt Macht. Ist beispielsweise innerhalb der Paarbeziehung der männliche Partner der „mehr Liebende“ und die Frau die „weniger Liebende“, disponiert die Frau über eine wertvolle Ressource, wodurch sie einen Machtvorteil innehat (vgl. Safilios-Rothschild 1976: 357). „Love becomes an important resource for a woman when she is "less in love“ with her husband and is then in the position to exchange her reciprocation to his love in terms of affection and sex for power in the relationship" (Safilios-Rothschild 1976: 360f.). Folglich wird dem Austausch von Liebe gegen Macht eine besondere Bedeutung zugeschrieben.

Blood/Wolfe (1960), die häufig als Begründer der ressourcentheoretischen Argumentation im Hinblick auf familiale Machtverhältnisse bezeichnet werden, widmen sich der Forschungsfrage, ,[...] what factors determine how husbands and wives interact and what are the effects of varying interaction patterns on the general welfare of the husband, the wife, and the family as a whole“" (Blood/Wolfe 1960: 4). Sie nehmen im Rahmen ihrer Detroiter Studie über „Dynamics of Married Living“ in den USA mittels einer ressourcentheoretischen Argumentation eine Reduktion der Machtmittel auf gesamtgesellschaftliche Statuspositionen (Bildungsstand (vgl. Blood/Wolfe 1960: 28), Berufsprestige (vgl. Blood/Wolfe 1960: 31) und Einkommen (vgl. Blood/Wolfe 1960: 31f.), wobei der soziale Status als Index der Variablen Bildung, Beruf, Einkommen und dem Prestige-Ranking der Nationalität erfasst wird (vgl. Blood/Wolfe 1960: 32)) im Vergleich der Geschlechter vor. Sowohl diese Begrenzung als auch die fehlende Integration der austauschtheoretischen Argumentation von Heer (1963) in die ressourcentheoretische Erklärung (Verfügbarkeit von Alternativen außerhalb der bestehenden Paarbeziehung $)^{104}$ und ein Mangel an Berücksichtigung von Kosten werden als grundlegende Kritikpunkte an der Ressourcentheorie hervorgebracht.

„It [resource theory] has been limited because ,resources“ were discussed but not the „cost" involved in receiving the benefit of these resources or the cost incurred from the withdrawal of these resources. Also, despite Heer's (1963) discussion of the exchange principle according to which one spouse in one dyad may be exchanged for a spouse from another dyad, that brought to the forum the important issue of available or potential alternatives to the present marriage, this idea was never incorporated into the „resource theory“““ (Safilios-Rothschild 1976: 355).

104 Den starken Bezug von Blood/Wolfe auf Heer bzw. den engen Zusammenhang zwischen der Ressourcentheorie von Blood/Wolfe und der Austauschtheorie von Heer präzisiert Rodman wie folgt: „Die erstere betont die relativen Ressourcen, die jede Person in die Ehe einführt: Je mehr Ressourcen eine Person im Vergleich zu ihrem Ehepartner besitzt, desto größer ist ihre Macht. David M. Heers Betonung liegt auf einem Vergleich des Wertes der innerhalb der Ehe und der außerhalb der Ehe zu erlangenden Ressourcen. Da er dies explizit in Beziehung setzt zu den Ressourcen, die jeder Ehepartner für den Austausch verfügbar hat, ähnelt seine Auffassung der Theorie der Ressourcen“ (Rodman 1970: 134). 
Darüber hinaus äußert Safilios-Rothschild den Einwand, dass Liebe als relevante Variable für ein Verständnis von familiären Dynamiken, insbesondere von Macht und Einfluss vernachlässigt wird (vgl. Safilios-Rothschild 1976: 357).

Nach Blood/Wolfe wird „Power may be defined as the potential ability of one partner to influence the other's behavior. Power is manifested in the ability to make decisions affecting the life of the family“ (Blood/Wolfe 1960: 11). Relative Ressourcen, die ausschließlich im Außensystem der Gesellschaft erworben werden, beeinflussen die decision making power im Binnensystem Familie. „The balance of power will be on the side of that partner who contributes the greater resources to the marriage" (Blood/Wolfe 1960: 12). Anders formuliert wird innerhäusliche Macht als „The Power to make Decision" definiert, die den Austausch von Familie und Außensystem Gesellschaft regelt (Blood/Wolfe 1960: 11). Derjenige/diejenige, der/die über mehr relative Ressourcen verfügt, genießt einen Machtvorteil innerhalb der Paarbeziehung. Blood/Wolfe (1960) nehmen vornehmlich Bezug auf die im Außensystem der Gesellschaft erworbenen Ressourcen, wodurch der Bezug auf das familiale Binnensystem vernachlässigt wird. Gleichwohl wird zwar eine Differenzierung der Struktur der Familie nach ,the Power to make Decision" (Blood/Wolfe 1960: 11) und ,the Division of Labor" (Blood/Wolfe 1960: 47) vorgenommen, jedoch werden die Machtverteilung und die Arbeitsteilung als weitgehend unabhängig voneinander sowie vorwiegend als durch außerhäuslich erworbene Ressourcen ${ }^{105}$ beeinflusst betrachtet.

"[...] the pattern of task performance is one of marked specialization“ (Blood/Wolfe 1960: 52). [...] ,Power and the division of labor are aspects of marriage which may coexist in almost any combination. As a result, they are best considered separately rather than welded into artificial combinations" (Blood/Wolfe 1960: 54).

Doch ist nicht gerade die, bereits von Held (1978) geforderte, Integration mittels der innerhäuslichen Arbeitsteilung von zentraler Relevanz (da die PartnerInnen nicht direkt vergleichbare Ressourcen einsetzen können), um die gesamtgesellschaftlich geschlechtsspezifische Arbeitsteilung adäquat zu erfassen (vgl. Held 1978: 82)?

Held (1978) verdichtet aus den familiensoziologischen Ansätzen eine Soziologie der ehelichen Machtverhältnisse. Allen klassischen Auffassungen von Macht seien Relationalität, Kausalität und Potentialität gemeinsam (vgl. Held 1978: 61f.): Macht ist 1. relational, weil Macht eine Eigenschaft der

$105, \ldots[\ldots]$ the division of labor is determined by the comparative resourcefulness of the two partners in accomplishing the necessary household tasks" (Blood/Wolfe 1960: 66). [...] the chief resource required is time [but also energy and skill]. Usually the person with the most time is the wife - provided she isn't working outside the home. If she does work, the husband incurs a moral obligation to help her out in what would otherwise be her exclusive task areas“ (Blood/Wolfe 1960: 73). 
Beziehung zwischen Akteuren und nicht der Akteure selbst ist, 2. kausal, weil ein mächtiger Akteur einen anderen Akteur beeinflusst, wobei nur die Interaktionsverhältnisse als Machtverhältnisse bezeichnet werden, die bei längeren, kontinuierlichen Interaktionen (prozesshafte Dynamik) eine Asymmetrie aufweisen und 3. potential, weil die Notwendigkeit besteht, „Akte der Machtausübung (Entscheidungen) von der generalisierten Chance zu solchen Aktionen begrifflich zu unterscheiden“, d. h. eine Unterscheidung zwischen ,decision-making“ und ,non-decision-making“ zu treffen, während es in vielen klassischen Konzepten darüber hinaus um die Abgrenzung von Macht und Autorität (als legitimierte Macht) sowie Macht und Gewalt (entweder verstanden als Machtmittel oder als „Annulierung“ der Macht) geht (Held 1978: 62).

Held entwickelt weiterführend eine multidimensionale Typologie der Machtverhältnisse, die im Kontext von Schichtlage und sozioökonomischer Situation respektive Schichtungs- oder Statustheorie zu verstehen ist, woraus die besondere Bedeutung der strukturellen Macht resultiert (vgl. Held 1978: 81). Mittels der Dynamik der Machtdimensionen sowie Machtebenen verdeutlicht Held zugleich die Unzulänglichkeit einer rein auf Schichtlage und kontextueller Entwicklung basierenden Perspektive der Machtstruktur, wodurch sowohl die Mehrdimensionalität als auch die Veränderungsmöglichkeiten der Machtverhältnisse zum Ausdruck kommen (vgl. Held 1978: 74).

Held kritisiert an den Ressourcen- und Modernisierungstheorien, dass sich ein Trend zur Partnerschaftlichkeit nicht logisch ableiten lässt (vgl. Held 1978: 16) ${ }^{106}$. Es zeigt sich folgender Widerspruch: Gemäß ressourcentheore-

106 Die Kritik bezieht sich vornehmlich auf die Ressourcentheorie nach Blood/Wolfe und ihre durch Rodman hervorgebrachte wichtigste Modifikation: die Theorie der Ressourcen im kulturellen Kontext (vgl. Held 1978: 17). Rodman fand heraus, dass die Ressourcentheorie von Blood/Wolfe (1960) zwar für Paarbeziehungen in Deutschland und den USA zutraf, jedoch für Frankreich sowie Dänemark deutlich weniger zutraf und für Griechenland sowie Jugoslawien stellte er sogar divergierende Ergebnisse fest. Die abweichenden Ergebnisse von Griechenland und Jugoslawien ,[...] schließen die Verallgemeinerung aus, daß [sic!] der Status des Ehemannes in einer positiven Beziehung mit der ehelichen Macht des Ehemannes steht" (Rodman 1970: 128). Aufgrund dieser kulturspezifischen Diskrepanzen hat Rodman eine Theorie der Ressourcen im kulturellen Zusammenhang entwickelt, innerhalb dieser die verschiedenen internationalen Daten über eheliche Macht durch eine Typologie von vier Gesellschaftsformen klassifiziert werden (vgl. Rodman 1970: 135). 1. Das „Patriarchat", in dem starke patriarchale Familienformen in allen sozialen Schichten dominieren. 2. Das „modifizierte Patriarchat" beschreibt ebenfalls einen Gesellschaftstypus mit patriarchalischen Familienformen, diese sind jedoch in der oberen Schicht durch egalitäre Normen ersetzt worden, wodurch die väterliche Autorität negativ mit der Schicht korreliert. 3. Das „Übergangsstadium zum Egalitarismus“, innerhalb dessen patriarchalische Normen durch egalitäre Familiennormen verdrängt werden, wodurch eheliche Machtformen flexibel werden und die väterliche Autorität positiv mit der Schicht korreliert. Ressourcen (wie Bildung, Beruf und Einkommen) verhelfen in dieser Gesellschaftsform zu Macht, weil eine Unsicherheit der Normen in Bezug auf die eheliche Autorität besteht durch die eheliche 
tischen Argumentationen bestimmen individuelle Ressourcen zunehmend die Machtverteilung in Ehen, weshalb sich die damaligen strukturellen Barrieren (Diskriminierung der Frauen in Bezug auf Einkommen, Beruf und Bildung) und die geschlechtsspezifische Arbeitsteilung negativ auf die Stellung von Frauen in der Ehe und Familie auswirken müsste. Zugleich nehmen Moderni-

„Machtkämpfe“ hervorgerufen werden. 4. Der „Egalitarismus“ klassifiziert eine Gesellschaftsform mit starken egalitären Normen sowie einer Machtteilung zwischen Ehemann und Ehefrau in allen Schichten (vgl. Rodman 1970: 136ff.). Rodmans zentrale Erkenntnisse sind, ,[...] daß [sic!] der Austausch von Ressourcen im kulturellen Zusammenhang für die Verteilung der ehelichen Macht sorgt. Dort, wo die Normen flexibel genug sind, einen „Machtkampf“ zu erlauben, fanden wir eine positive Korrelation zwischen dem Status und der Macht eines Ehemannes, wie zum Beispiel in Deutschland, den USA und anderen Gesellschaften. Falls die Normen innerhalb der Gesellschaften stark patriarchal sind, so wird es [...] keine Korrelation zwischen dem Status des Ehemannes und ehelicher Macht geben. Wo die Normen patriarchal, jedoch in der oberen Schicht durch die Beimischung egalitärer Normen modifiziert sind, fanden wir eine negative Korrelation zwischen Status und Macht des Ehemannes, wie in Griechenland und Jugoslawien. Wenn die Normen in der ganzen Gesellschaft betont egalitär sind und falls es ein ausgebautes System progressiver Wohlfahrtsgesetzgebung gibt, so wird [...] eine schwach positive Korrelation zwischen dem Status und der Macht des Ehemannes zu finden sein, wie in Dänemark und Frankreich“ (Rodman 1970: 139). Zusammenfassend wird gemäß Rodmans Erkenntnissen die Balance ehelicher Macht durch die Interaktion zwischen relativen Ressourcen und kulturellen Erwartungen über die Verteilung ehelicher Macht beeinflusst (vgl. Rodman 1970: 133). Held kritisiert hieran, dass sich die geschlechtsspezifische Arbeitsteilung sowie die nach wie vor existierenden strukturellen Barrieren gegen Frauen zu ungunsten ihrer Stellung in der Familie auswirken müssten, wenn - wie Rodman es angenommen hat - in modernen Gesellschaften (mit flexiblen Normen hinsichtlich der geschlechtsspezifischen Rollenbilder) die individuellen Ressourcen tatsächlich die eheliche Machtverteilung bestimmen. „Die modernisierungstheoretischen Konzepte und Trendextrapolationen behaupten aber gerade das Gegenteil“" (Held 1978: 17). Anhand einiger Beispiele erläutert Held die fehlende empirische Basis der Theorie der Ressourcen im kulturellen Kontext. Er führt insbesondere Held/Levy (1974) an, die für die durch traditionelle Normen gekennzeichneten katholischen Kantone der Schweiz einen Zusammenhang zwischen der Stellung des Ehemannes und der ehelichen Macht konstatieren (vgl. Held 1978: 114). Darüber hinaus sei gemäß Held im Hinblick auf die Theorie der Ressourcen im kulturellen Kontext unklar, warum der normative Wandel mit einem Wandel der familialen Machtstruktur zusammenhänge. Durch Rodmans Annahme, dass in egalitären Gesellschaftsformen egalitäre Normen so weit verbreitet seien, dass kein Zusammenhang zwischen dem Status eines Ehemannes und seiner Macht vorzufinden sei, nivelliere er die strukturelle Interpretation familialer Machtverhältnisse (vgl. Held 1978: 115): Die empirisch nachgewiesenen Beziehungen zwischen sozialer Schichtung und der ehelichen Machtstruktur geraten nur zu Phasenverschiebungen in einem modernisierungstheoretisch konzipierten Diffusionsproze $\beta$ [sic!] von (ausschließlich) patriarchalen zu (ausschließlich) egalitären Ehen, wobei kulturelle Normen die unabhängige Größe sind“" (Held 1978: 115). Daher sei nach Rodman die Ressourcentheorie von Blood/Wolfe lediglich im Rahmen flexibler Normen anzuwenden und insofern nur als eine Übergangsphase zu interpretieren. Zusammenfassend übt Held folgende Kritik an Rodman: „Strukturelle Determinanten sollen nur dort wirksam sein, wo kulturelle Determinanten versagen. Dieser Gewichtung liegt letztlich die Vorstellung einer allgemeinen Nivellierung der ehelichen und familiären Verhältnisse zugrunde, die zu einer Vernachlässigung schichtspezifischer Unterschiede und lebenszyklischer Variationen der Machtstruktur führt" (Held 1978: 115). 
sierungstheoretikerInnen das Gegenteil an, dass infolge von Urbanisierung und Industrialisierung ein zunehmender Egalitarismus aufkommt. Diese Diskrepanz der theoretischen Argumentationen nimmt Held zum Anlass, eine Typologie der ehelichen Machtverhältnisse zu entwickeln (vgl. Held 1978: 17). Er begründet seine Perspektive mit der Argumentation von König (1966). König äußert Kritik an Durkheims Annahme einer Herauslösung der Kernfamilie aus der erweiterten Familie (Kontraktionsgesetz) infolge der Industrialisierung insofern, als in jeder Gesellschaft unterschiedliche Familientypen wiederzufinden sind, deren wesentliches Differenzierungsmerkmal die unterschiedliche Stellung zum Eigentum ist. Ferner ist die Coexistenz verschiedener Familientypen strukturell zu interpretieren. Held überträgt die Kritik von König an Durkheim auf verschiedene Formen ehelicher Machtverhältnisse (zumal die Familiengröße mit der Stellung der Frau sowie der Stellung des Mannes in der Ehe verknüpft ist): Weder die Allgemeingültigkeit patriarchaler Machtstrukturen noch die These der Etablierung egalitärer Machtmuster in Folge von Industrialisierung und Urbanisierung könnten beibehalten werden. Stattdessen muss vorausgesetzt werden, dass es in jeder Gesellschaft strukturell bedingte, unterschiedliche Formen ehelicher Machtverhältnisse gibt (vgl. Held 1978: 19). Held leitet daraus sein Forschungsanliegen,

,[...] der Versuch, verschiedene Typen ehelicher Machtverhältnisse mit der sozialen Struktur theoretisch zu verknüpfen, [ab]. Mit anderen Worten: Es soll ein Beitrag zu einer strukturellen Erklärung der ehelichen Machtverteilung geleistet werden, wobei in Analogie zur oben diskutierten Analyse Königs unter Struktur in erster Linie die gesellschaftliche Schichtung bzw. die relative Verfügung über Ressourcen und Macht verstanden werden soll. Daneben soll aber auch die Dimension der sozietalen Entwicklung [...] einbezogen werden“ (Held 1978: 19).

\subsection{Zum Konkurrenzverhältnis von Machtkonzepten und Austauschtheorien}

Die Beziehung zwischen Macht und sozialen Austauschtheorien wurde bereits von David Baldwin (1978) thematisiert. D. Baldwin argumentiert, dass „[...] all exchange relationships can be described in terms of conventional power concepts without twisting the common-sense notions that underlie such concepts" (Baldwin, D. 1978: 1229). Diese Schlussfolgerung zeigt, dass Austauschtheorien einen wichtigen Beitrag zur Typologiekonzeption der Machtverhältnisse leisten, ferner im Sinne einer Synthese zu verstehen sind. Während Homans (1974) darlegt, dass Machtverhältnisse eine Untermenge von Austauschbeziehungen sind, setzt sich Blau (1964) mit Macht und Austauschprozessen als zwei Bereichen auseinander, wobei keiner des anderen 
Untermenge ist. Im Gegensatz dazu sieht Dahl (1968) Austauschprozesse als eine Untermenge von Machtverhältnissen an. Die Synthese dieser drei Thesen wird zu Recht von D. Baldwin (1978) erbracht:

„[The] integration of such basic concepts as power and exchange could be a step toward conceptual unification of the social science, thus facilitating communication among economists, political scientists, sociologists, and psychologists“ (Baldwin, D. 1978: 1241).

Machthaltige Beziehungen werden häufig mit ausbeuterischen Konflikten in Verbindung gebracht, wobei das Machtpotential auf der Möglichkeit zur Ausübung negativer Sanktionen beruht. Hingegen werden Austauschbeziehungen meist mit Kooperation gleichgesetzt, deren Erfüllung auf positiven Sanktionen beruht. Eine Integration von Machtkonzepten und Austauschtheorien verhilft, Macht als relational und reziprok zu verstehen, außerdem die Perspektive auch auf positive Sanktionen auszuweiten, sowie die Bedeutung von Kosten hervorzuheben, anstatt Macht ausschließlich auf Unterdrückung bzw. Konfliktsituationen zu reduzieren (vgl. Baldwin, D. 1978: 1977f.). Ferner ist Macht mehr als nur ein Medium von Austauschprozessen. Die dynamische Reziprozität ist ein zentraler Bestandteil der Studie über familiale Macht von Osmond (1978). Sie kommt zu dem Ergebnis, dass Ressourcen für Handlungsstrategien erforderlich sind, d. h. letztlich für das Machtpotential von elementarer Bedeutung seien. Auch D. Baldwin (1971) verweist auf die Bedeutung von Ressourcen für das Machtpotential, insbesondere wird Geld als Machtressource dargestellt (vgl. Baldwin D., 1971: 583). D. Baldwin (1971) übt Kritik an der Vorstellung Parsons (1963), Macht sei für die Politik ein symbolisch generalisiertes Kommunikationsmedium wie Geld für die Wirtschaft. Die forschungsleitende Frage, ,Is the conception of power as money compatible with other conceptions of power currently in use by political scientists?", steht im Zentrum seiner Argumentation, politikwissenschaftliche und ökonomische Vorstellungen miteinander zu verknüpfen (Baldwin, D. 1971: 581). „Purchaising power is not money" und „Money is neither a necessary nor a sufficient condition for the exercise of purchasing power" sind diesbezüglich die relevanten Thesen, die vertreten werden (Baldwin, D. 1971: 583, Hervorhebungen im Original; die Verf.). Geld wird als eine Machtressource definiert, die das Machtpotential der purchaising power erhöhen kann, aber nicht zwangsläufig muss.

„Money is a power resource (power base or base value) that will very probably allow the possessor to exercise purchaising power that is generalized in scope and domain. [...] [But] Money does not guarantee the exercise of purchaising power. [...] Money is only one of many base values that can be used to exercise purchaising power" (Baldwin, D. 1971: 583).

Es wird betont, dass Geld nicht die einzige Machtbase sei, dennoch wird von D. Baldwin (1971) sowie von Osmond (1978) eine Fokussierung auf ökono- 
mische Macht vorgenommen, die einer Soziologie partnerschaftlicher Machtverhältnisse nicht gänzlich gerecht werden kann.

\subsection{Aktuelle machttheoretische Ansätze}

Auch in der aktuellen Forschung finden eine Fülle von differenten Machtdefinitionen (die hier als einzelne Machtkategorien verstanden werden) Anwendung: Beispiele hierfür sind ein sozialer Einfluss, ein Einfluss auf Ergebnisse, ein geringes Interesse (nach Safilios-Rothschild (1975) affective power), bessere Alternativen sowie eine Kontrolle über Ressourcen (vgl. Grau 2001: 1). Häufig wird hierbei Macht als Aushandlungsprozess mit unterschiedlichen, folgend aufgeführten, Mitteln verstanden.

„[...] Strukturelle Zwänge, [...] geschlechtsspezifische Codierung von Machtmitteln, [...] Sanktionierung von Machteinsätzen bei Frauen, [...] Strategien, Frauen Machtmittel zu verweigern“ (Löw 2009: 7).

Gleichwohl derart viele Definitionen von Macht, ferner Machtkategorien vorzufinden sind, haben im Zuge der Entwicklung einer Enttraditionalisierung auf der Einstellungsebene Analysen geschlechtsspezifischer Machtverhältnisse einen Rückgang erfahren. Doch ist Macht als soziales Phänomen nicht abgeklungen, vielmehr ist die Problembenennung erodiert (vgl. Löw 2009: 7). Machtansätze werden in Frage gestellt, auf reine ökonomische Macht reduziert und als überholt abgetan. Die wenigen Ansätze, die sich dennoch der Macht widmen, argumentieren überwiegend aufgrund von handlungstheoretischen ökonomischen, ggf. gendertheoretischen Konzepten (u. a. Lott (2009)). Nach dem der Aufschwung des Machtkonzeptes in Partnerschaften von Held (1978) seine Blütezeit erreichte, gewannen seither austauschtheoretische Erklärungsansätze sowie Gendertheorien zunehmend an Bedeutung.

Dieses Forschungsdefizit gilt es im Rahmen dieser Dissertation zu überwinden, indem Macht als mehrdimensionales Konstrukt verstanden wird. Macht ist als latentes Phänomen zu erachten, das in verschiedenen, gleichwohl einander ergänzenden Kategorien empirischen Ausdruck findet. Zweifellos sind in den theoretischen Ansätzen Widersprüche zu finden, doch besteht die Herausforderung zur Entwicklung einer umfassenden Theorie der Macht nicht nur darin, diese aufzuzeigen und zu kritisieren (das ist zwangsläufig notwendig), ferner wird daraus der Vorteil gezogen, Überschneidungen zu konstatieren - eine Synthetisierung der bisherigen Ergebnisse zur Soziologie der Machtverhältnisse in Paarbeziehungen herauszuarbeiten. Einzelne Studien aus diesem Forschungsfeld werden strukturiert mit dem Zweck einer Verdichtung der Ergebnisse. Die Kriterien, die zum Theorie-Ein- oder - 
Ausschluss führen, sind, dass die Ergebnisse der jeweiligen Studie zur Erklärung innerhäuslicher Arbeitsteilung relevant sein könnten, darüber hinaus als ein Typus von Macht erachtet werden, wobei wesentliche Indikatoren identifiziert werden. Macht findet in unterschiedlichsten Formen sozialer Beziehungen Ausdruck, deren Unterschiedlichkeit durch Machttypen respektive Machtkategorien auf verschiedenen Dimensionen erfasst wird.

Hierbei wird das Potential darin gesehen, klassische und aktuelle Begriffsvariationen in Beziehung zueinander zu setzen und das Machtkonzept insofern auszuweiten, dass es einem universalen Anspruch - Erklärungsleistung in unterschiedlichen Kontexten zu erbringen - gerecht wird, d. h. auf eine Mehrebenenanalyse angewendet werden kann.

Für die Entwicklung des Rahmens einer Theorie der Macht wird zunächst auf Webers Definition von Macht als mikrosoziologische Erklärung Bezug genommen. Weiterführend wird Foucaults Definition als dispositive, mehrdimensionale Erklärung konstatiert, um dem Anspruch einer Allgegenwart von Macht gerecht zu werden. Die symmetrischen sowie asymmetrischen Ansätze und die Makro-Indizes zur Messung von Genderungleichheiten dienen der Kategorienkonstruktion. Den umfassenden Bezugsrahmen bildet der Capability Approach von Sen, nach dem Machtfähigkeiten als Komponenten der Mikroebene und Befähigungen als Ermächtigungen auf der Makroebene erachtet werden. 


\section{Die Typologie der Machtverhältnisse in Paarbeziehungen: Macht als mehrdimensionale Begriffskonstruktion}

Macht ist ein Begriff, der bereits durch zahlreiche bedeutende Soziologen geprägt wurde; darunter hervorzuheben: Max Weber und Michel Foucault. Ihre soziologischen Definitionen der Macht werden zunächst kurz erläutert. Mit Hilfe der theoretischen Vorarbeiten werden weiterführend die Dimensionen der Macht hergeleitet.

\subsection{Macht als Chance, den eigenen Willen durchzusetzen (Mikro)}

Gemäß der einflussreichen Typologie von Max Weber sei unter Macht folgendes zu verstehen: „Macht bedeutet jede Chance, innerhalb einer sozialen Beziehung den eigenen Willen auch gegen Widerstreben durchzusetzen, gleichviel worauf diese Chance beruht" (Weber 1972: 28). Herrschaft, in Abgrenzung zur Macht, sei „[...] die Chance, für einen Befehl bestimmten Inhalts bei angebbaren Personen Gehorsam zu finden“ (Weber 1972: 28). Folglich sei Herrschaft eine institutionalisierte Form der Macht, die von spezifischen Personen Anerkennung findet. Weber argumentiert in der Tradition der Handlungstheorien aus mikrosoziologischer Perspektive; Machtkonstrukte bestehen innerhalb sozialer Beziehungen, in denen es darauf ankommt, die eigenen Interessen durchzusetzen. Insbesondere, wenn dieser Willen gegen den Willen des anderen bzw. der anderen spricht, ist die Beziehung machthaltig. Eine mehrdimensionale Betrachtung von Macht wird im Gegensatz zu Weber von Foucault betont.

\subsection{Macht als multidimensionales Kräfteverhältnis (Mikro/Makro)}

Während die Argumentation Webers auf der Individualebene anzusetzen ist, ist der Machtansatz von Foucault „dispositiv“107 (Foucault 1978: 119f.).

107 „Was ich unter diesem Titel festzumachen versuche ist erstens ein entschieden heterogenes Ensemble, das Diskurse, Institutionen, architekturale Einrichtungen, reglementierende Entscheidungen, Gesetze, administrative Maßnahmen, wissenschaftliche Aussagen, philoso- 
Foucault beschreibt in seiner „Genealogie des Staates“ drei Prämissen, die für die Konzeption des Machtbegriffes zentral sind. Zum ersten sei Macht ein multidimensionales Kräfteverhältnis, das durch eine Vielzahl von Techniken seinen Ausdruck findet. Beispielsweise greifen Mächte staatlichen Typs (zur Regierung des Staates siehe Foucaults „Gouvernementalität“, zur Regulierung der Bevölkerung den Begriff der „Bio-Macht“108) und Mächte familialen Typs insoweit ineinander, wie sie ihre Spezifität bewahren können (vgl. Foucault 1978: 112). ${ }^{109}$

Zum zweiten bestehe eine „Allgegenwart der Macht: nicht weil sie das Privileg hat, unter ihrer unerschütterlichen Einheit alles zu versammeln, sondern weil sie sich in jedem Augenblick und an jedem Punkt - oder vielmehr in jeder Beziehung zwischen Punkt und Punkt - erzeugt" (Foucault 1983: 94). An dieser Stelle wird deutlich, dass jede soziale Beziehung durch Machtkonstellationen geprägt wird und zugleich Macht die Existenz von sozialen Beziehungen erfordert. ${ }^{110}$ Macht innerhalb sozialer Beziehungen

phische, moralische oder philanthropische Lehrsätze, kurz: Gesagtes ebensowohl wie Ungesagtes umfaßt [sic!]. Soweit die Elemente des Dispositivs. Das Dispositiv selbst ist das Netz, das zwischen diesen Elementen geknüpft werden kann. Zweitens möchte ich mit dem Dispositiv gerade die Natur der Verbindung deutlich machen, die zwischen diesen heterogenen Elementen sich herstellen kann. [...] Kurz gesagt gibt es zwischen diesen Elementen, ob diskursiv oder nicht, ein Spiel von Positionswechseln und Funktionsveränderungen, die ihrerseits wiederum sehr unterschiedlich sein können. Drittens verstehe ich unter Dispositiv eine Art von [...] Formation, deren Hauptfunktion zu einem gegebenen historischen Zeitpunkt darin bestanden hat, auf einen Notstand [...] zu antworten. Das Dispositiv hat also eine vorwiegend strategische Funktion“(Foucault 1978: 199f.).

108 „Die Bevölkerung ist eine Gruppe, die nicht einfach nur aus vielen Menschen besteht, sondern aus Menschen, die von biologischen Prozessen und Gesetzen durchdrungen, beherrscht und gelenkt sind. Eine Bevölkerung hat eine Geburtenrate, eine Alterskurve, eine Alterspyramide, eine Sterblichkeitsrate und einen Gesundheitszustand (Foucault 2015: 231). ,Ich glaube, seit der Entstehung der von mir so genannten Bio-Macht oder anatomischen Politik leben wir in einer Gesellschaft, die dabei ist, nicht länger eine juristische Gesellschaft zu sein. Die juristische Gesellschaft war die monarchische“ (Foucault 2015: 237). Seit dem 19. Jahrhundert setze sich ein Machtmechanismus durch, ,[...] dessen Grundprinzip [...] nicht das Gesetz, sondern die Norm“ darstellt -,,[...] eine Welt der Regulierung“ (Foucault 2015: 237).

109 „Der Staat ist Überbau in Bezug auf eine ganze Reihe von Machtnetzen, die die Körper, die Sexualität, die Familie, die Verhaltensweisen, das Wissen, die Techniken usw. durchdringen, und diese Beziehungen werden ihrerseits von einer Art Übermacht konditioniert und wirken konditionierend auf sie, die im wesentlichen [sic!] um eine gewisse Anzahl großer Verbotsfunktionen herum strukturiert ist; aber diese Über-Macht mit ihren Verbotsfunktionen kann nur insofern wirklich greifen und sich halten, als sie in einer ganzen Reihe vielfältiger, nicht definierter Machtverhältnisse verwurzelt ist, die die notwendige Grundlage dieser großen Formen negativer Macht bilden, und genau das wollte ich deutlich machen“ (Foucault 1978: 39).

110 „Zwischen jedem Punkt eines gesellschaftlichen Körpers, zwischen einem Mann und einer Frau, in einer Familie, zwischen einem [/einer] Lehrer[In] und einem [/einer] Schüler[In], zwischen dem [/der] der weiß und dem [/der] der nicht weiß verlaufen Machtbeziehungen, die nicht die schlichte und einfache Projektion der großen souveränen Macht auf die Indivi- 
wird häufig als Pendant der Unterdrückung definiert, doch dieses Verständnis sei nach Foucault als rein juristische Konzeption begrenzt; ein Gesetz, das Verbot und Gewährung ausspricht (vgl. Foucault 1978: 34f.; vgl. Foucault 2015: 221).

„Wie kommt es, dass unsere Gesellschaft und die westliche Gesellschaft schlechthin Macht so restriktiv, so arm, so negativ versteht? Warum denken wir bei Macht immer an Gesetz und Verbot? Warum diese Privilegierung?“ (Foucault 2015: 222).

Es gilt diese Begrenzung aufzulösen, ferner Macht als ein soziales Phänomen $\mathrm{zu}$ erachten, das Ausdruck in jeglichen Beziehungen findet und einem Anspruch auf Allgegenwart gerecht wird. Zu Recht ist Foucault der Meinung, dass wir uns

„[...] von diesem juristischen Verständnis der Macht, diesem Machtbegriff der auf Gesetz und Souverän, Regel und Verbot aufbaut, [...] befreien [müssen], wenn wir nicht nur die Repräsentation von Macht, sondern deren reale Funktionsweise analysieren wollen" (Foucault 2015: 224).

Nicht nur Formen einer Unterdrückung als negative Produkte der Macht, auch positive Produkte der Macht ${ }^{111}$ seien in sozialen Beziehungen zu beobachten. Foucault führt als Beispiel einer positiven Form von Macht die Sexualität an, die die Funktion der Verknüpfung ,[...] zwischen der individuellen Disziplinierung des Körpers und der Regulierung der Bevölkerung“ einnimmt (Foucault 2015: 232). Insofern sei eine Ausweitung der soziologischen Begriffskonstruktion von Macht durch die Prämisse einer Allgegenwart und Betrachtung von negativen wie positiven Produkten der Macht empfehlenswert.

Zum dritten sieht Foucault einen reflexiven Zusammenhang zwischen Macht und Wissen, d. h. Wissen ist eine zentrale Ressource zur Erzeugung von Macht, zugleich besteht die Möglichkeit, durch Macht Wissen zu erzeugen. ${ }^{112}$

duen sind; sie sind eher der bewegliche und konkrete Boden, in dem die Macht sich verankert hat, die Bedingungen der Möglichkeit, damit sie funktionieren kann“ (Foucault 1978: 110). Die Annahme, dass auf der einen Seite Personen Macht haben und auf der anderen Seite diejenigen stehen, die keine Macht haben, hält Foucault für unzulänglich. „Die Machtbeziehungen sind überall“ (Foucault 2015: 239). Sowohl ProfessorInnen als auch StudentInnen verfügen über Machtpositionen, auch wenn es nicht dieselben sind (vgl. Foucault 2015: 239).

111 „Ich glaube, daß [sic!] genau diese positiven Mechanismen untersucht werden müssen, wobei man sich von dem juristischen Schematismus freimachen muß [sic!], mit dem man bis heute versucht hat, der Macht einen Status zuzuweisen“" (Foucault 1978: 37).

112, ,.... Beziehungen, Strategien und Technologien der Macht, die uns konstituieren, uns durchqueren und ausmachen, (sind) von Formationen des Wissens und der Wahrheit begleitet, die sie ermöglichen und produzieren und die unentbehrlich für sie sind, um sich als evident und naturgegeben zu verfestigen und sich damit zugleich unsichtbar zu machen. Umgekehrt muß [sic!] die Analyse des Wissens, der diskursiven Formationen und ihrer Aussa- 


\subsection{Zur Konzeptualisierung der Typologie der Machtverhältnisse: Die Dimensionen der Macht in Paarbeziehungen}

Die Kategorien respektive Typen der Macht stellen abstrahierte Verallgemeinerungen dar, die den Zweck verfolgen, als ein idealtypisches Gesamtbild von Macht Arbeitsteilungsarrangements in Partnerschaften theoriegeleitet zu erklären. Werden die bisherigen theoretischen Ansätze systematisch betrachtet, können folgende Dimensionen herausgearbeitet werden:

Auf der Mikroebene werden Machtfähigkeiten im Sinne von Sen als Fähigkeiten/Aktivitäten, die spezifische Machtressourcen voraussetzen und von einer Person zur Realisierung einer bestimmten Handlungsalternative genutzt werden, verstanden. Es handelt sich um Machtaspekte, die nach Webers Verständnis zur Durchsetzung des eigenen Willens im Rahmen der möglichen Handlungsalternativen dienen (,So wird etwa in Anlehnung an die bekannte Weber'sche Definition von "Macht“" nur dort gesprochen, wo der machtausübende bzw. „machtbesitzende“ Akteur ein bestimmtes Verhalten des anderen Akteurs intendiert" ) und die gemäß Held unter dem Terminus des ,interaktionistischen Machtbegriffs“ zusammengefasst werden können (Held 1978: 64, Hervorhebungen im Original; die Verf.). Der erste Typus wird bezeichnet als

1. Interaktionelle Macht: Diese dient den unterschiedlichen Einflussmöglichkeiten innerhalb sozialer Beziehungen in Form von Fähigkeiten sowie Ressourcenkonstellationen. Zentral sind hierbei Prozesse der Entscheidungsfindung verbunden mit der Frage, welche Aspekte $\mathrm{zu}$ Gunsten der Durchsetzung des eigenen individuellen Willens zweckdienlich sind. In Anlehnung an die Rational-Choice-Ansätze ist dieser Typus im Sinne einer Verhandlungsmacht zu verstehen, wobei die Verhandlungsmacht maßgeblich durch Machtressourcen, wie das Humankapital, das Einkommen und den Erwerbsstatus, beeinflusst wird.

Mischtypen, die jeweils aus mikro- und makrosoziologischer Perspektive zu betrachten sind, d. h. einerseits als Machtfähigkeiten von Verwirklichungschancen, andererseits als Befähigung bzw. Ermächtigung interpretiert werden können, sind die kulturelle Macht, die institutionelle Macht und die Macht der Teilhabe:

2. Kulturelle Macht: Die kulturelle Macht (nach French/Raven (1959) und Hallenbeck (1966) Legitimate power, nach Safilios-Rothschild

gen in Abhängigkeit von den Machtstrategien durchgeführt werden, die in einer gegebenen Gesellschaft die Körper und die Willen besetzen“ (Foucault 1978: 10). 
(1975) authority, s. Kapitel 8.1.) ist eine Form der Macht, mittels der in Anlehnung an die Gendertheorien nach Bielby/Bielby (1989), Brines (1994) und die Honeymoon-Hypothese nach Künzler (1994) durch Werte sowie Normen legitimierte kulturspezifische Benachteiligungen oder Begünstigungen analysiert werden können. Dieser zweite Typus impliziert kulturelle Leitbilder, die sich als manifeste Muster in Einstellungen ${ }^{113}$ widerspiegeln. Kulturelle Rollenvorstellungen könnten beispielsweise dem Mann (oder der Frau) eine größere Entscheidungsmacht zugestehen (für einen einführenden Überblick s. Huinink/Konietzka (2007): Kapitel „Beziehungsdynamik, Macht und Zufriedenheit in Paarbeziehungen").

3. Institutionelle Macht:

„Institutions in the sense of organizations, and not only « moral » or cultural institutions or ideologies, are doing gender as well as or even more effectively than individual actors, and a gendered functioning of the institutional environment where a couple lives may create strong incentives to accept sexspecific master-statuses even if the partners have other, e.g., egalitarian, normative convictions" (Levy et al. 2002: 33).

Institutionen, seien sie auf der Ebene familialen sozialen Handelns als dauerhafte Regelmäßigkeiten angesiedelt oder auf der Ebene sozialstruktureller Bedingungen, die Ausdruck in organisatorischen Formen finden (Verankerung von Strukturen in Institutionen als Organisationsform nach Levye et al.), sind miteinander zu verknüpfen, insofern als die Teilhabe an unterschiedlichen sozialen Feldern, beispielsweise Erwerbsarbeit, Familie und Kinderbetreuungseinrichtungen (sowohl lebenszyklisch wechselnd als auch die zeitgleiche Teilhabe an unterschiedlichen Institutionen), im Wechselverhältnis zu betrachten ist.

„Sie sind mit unterschiedlicher Strukturierungsmacht untereinander und relationaler Abhängigkeit voneinander ausgestattet und gestalten Lebensläufe hochwirksam geschlechtsspezifisch“ (Krüger/ Levy 2000: 380).

Nach Krüger/Levy schließen Institutionen sowohl handlungsleitende kulturelle Institutionen (Normen, Leitbilder; hier: kulturelle Macht) als auch die organisatorische Verfasstheit von Institutionen (hier: Teilhabe und strukturelle Macht) ein, woraus die zweidimensionale Wirkung der mikro- und makrosoziologischen Perspektive dieser Machtdimension folgt (vgl. Krüger/Levy 2000: 380).

Der Masterstatus (s. Kapitel 3.3.) nach Krüger/Levy (2000) erfährt eine Erweiterung in Form des vorliegenden reintegrativen Machtansat-

113 „Während Einstellungen und damit auch Intentionen primär auf der auf der Mikroebene von Bedeutung sind und individuelle Wahlentscheidung erfordern, verdichten sich Werte auf der Mesoebene zum Wertsystem, das Leitbildcharakter hat. Auf der Makroebene fügen sich Werte und Normen zu einem gesellschaftlich verankerten Wert- und Normsystem zusammen“ (Baumgartner 2008: 37). 
zes, indem die Dimensionen des Masterstatus unter Bezugnahme der Macht herausgearbeitet werden. Den Forderungen, dass

1. (,Bringing the Family back In“) „Familie [...] als eine Kombination unterschiedlich vergesellschafteter Lebensläufe ins Zentrum [rückt]“ und mit den, in der Lebensverlaufsforschung nicht berücksichtigten, Anliegerinstitutionen verknüpft wird,

2. „[...] nicht nur individuelle Verläufe, sondern auch die Relationalität von Lebensläufen untereinander zu thematisieren“" ersucht werden und

3. „Institutionenvernetzungen auf der Basis von Komplementärbeziehungen in der Lebenslaufstrukturierung" in den Mittelpunkt gestellt werden

kann mittels dieser Vorgehensweise nachgegangen werden (Krüger/Levy 2000: 393, Hervorhebungen im Original; die Verf.). Ferner wird nicht nur das Anliegen Bringing the Family back In, sondern auch Bringing Power back In vertreten. Die von Krüger/Levy (2000) konzipierte Mehrdimensionalität der Institutionen erfährt hier eine Anwendung auf die Machtdimensionen, um innerhäusliche Arbeitsteilungsarrangements zu erklären.

Institutionelle Regulierungen werden innerhalb dieses Machttyps vornehmlich auf die unterschiedlichen familialen Formen bezogen - Institutionen „formen“ Beziehungen. Je nach Form verändert sich die Erwartungshaltung. Während beispielsweise in einer Ehe eher traditionelle Rollenbilder erwartet werden können, wären es in einer Kohabitation möglicherweise eher egalitäre Rollenbilder. Diese These ist auf unterschiedliche Erwartungssicherheiten zurückzuführen: Ehepaare empfinden eine höhere Erwartungssicherheit aufgrund ihrer angenommenen Partnerschaftsstabilität als unverheiratete Paare, woraus ein Vertrauen resultiert (nach verhandlungstheoretischer Argumentation ein impliziter Vertrag), irgendwann eine Gegenleistung für die erbrachte Eigenleistung (z. B. Hausarbeit) zu erhalten. Makrosoziologisch gilt es Unterschiede zwischen den europäischen Regionen zu beobachten, die z. B anhand von Eheschließungs-/ sowie Scheidungsraten erfasst werden könnten und die folglich einen Teilaspekt des Ausmaßes der Traditionalisierung einer Region messen.

„[...] der relationale Bezug zwischen Institutionen scheint Familie und Geschlecht weder aus ihren subjektiven Verpflichtungsmustern zu entlassen, noch aus ihrer sozialstrukturellen Vermittler[Innen]rolle zwischen biographischen Optionen und inkompatiblen institutionellen VerfügbarkeitsAnsprüchen“ (Krüger/Levy 2000: 379).

4. Macht der Teilhabe: Auch die Macht der (multiplen) Teilhabe an verschiedenen sozialen Feldern (das Teilhabe-Theorem nach Krü- 
ger/Levy (2000)) kann zum einen als Machtfähigkeit, z. B. in Form der ausgeführten Erwerbsbeteiligung als ökonomische Teilhabechance, aufgefasst werden. Wesentlich ist hierbei die Annahme, dass Individuen zeitgleich an unterschiedlichen sozialen Feldern teilnehmen können, die ggf. konkurrierende Rollenerwartungen auslösen, nach Ausmaß der Verpflichtung differenziert werden können und dynamisch zu betrachten sind (vgl. Krüger/Levy 2000: 382f.). Zum zweiten sind darüber hinaus die Teilhabechancen im Sinne der Ermächtigung relevant, beispielsweise einer politischen Ermächtigung (Empowerment), inwiefern Frauen die Freiheit haben, politisch aktiv zu werden. Eine Operationalisierung dieser Dimension wird beispielsweise gemäß der Makro-Gender-Indizes häufig über den Anteil an Parlamentssitzen, die von Frauen besetzt sind, vorgenommen.

Makroebene: Nach Sen sind es die Befähigungen, die hier als Ermächtigungen verstanden werden, die das Ausmaß an Handlungsfreiheit (anders formuliert: die Verwirklichungschancen, die zur Verfügung stehen) bestimmen. Der eigene Wille, wie er von Weber betont wird, kann aufgrund einer eingeschränkten Handlungsfreiheit von der in die Tat umgesetzten Handlungsalternative abweichen. Foucault beschreibt die Allgegenwart der Macht, deren Ausdruck mikrosoziologisch in sozialen Beziehungen wie auch makrosoziologisch im Kontext von strukturellen Bedingungen (die er als juristische Macht bezeichnet) Ausdruck findet.

5. Strukturelle Macht/Ermächtigung/Empowerment: Die strukturelle Ermächtigung bezieht sich auf ein Normsystem, das den Handlungsspielraum reguliert, wer in welcher Weise durch makrosoziologische Rahmenbedingungen begünstigt wird und die Möglichkeit der Teilhabechance ausschöpfen kann. Insbesondere politische (Frauen-)Rechte, das Wahlsystem und staatliche Kinderbetreuungseinrichtungen sind Indikatoren, die es für die Erklärung innerhäuslicher Arbeitsteilungsarrangements zu berücksichtigen gilt (gleichwohl kein Anspruch auf Vollständigkeit erhoben wird). Vielmehr ist nach Fuwa (2004) ein Einfluss gender-egalitärer Politik anzunehmen.

Während das interaktionelle Machtkonzept, das nach Held (1978) auch als interaktionistisch bezeichnet wird,

,[...] bei aller Differenzierung von einer partikulären Relation zwischen zwei Interaktionspartnern aus[geht] [...] kann nun - nicht mit logischer Ausschließlichkeit, sondern im Sinne eines Kontinuums verschiedener Machtkonzeptionen - ein struktureller Machtbegriff gegenübergestellt werden. [...] Strukturelle Macht beruht auf immateriellen und materiellen Gütern, welche von den Mitgliedern eines Systems nachgefragt werden“ (Held 1978: 64, Hervorhebungen im Original; die Verf.).

Folglich ist auch die ökonomische Stärke eines Landes (z. B. Bruttoinlandsprodukt) als eine strukturelle Dimension der Macht zu verstehen. 
Dem Umstand, dass der strukturelle Machtbegriff den Interaktionsprozess zwischen Akteuren ausblendet, dafür von konkreten Interaktionen abstrahieren kann (vgl. Held 1978: 68), wird über die Konzeptualisierung der Machtdimensionen Rechnung getragen. Hieraus ergibt sich eine dynamische Perspektive, dass nicht nur interaktionelle Machtformen von der kontextuellen Entwicklung, sondern auch Machtstrukturen von situativen Faktoren abhängig sind.

Festzuhalten ist, dass die Ebenen der Macht als reflexiv zu verstehen sind, d. h. sich wechselseitig beeinflussen. Veränderungen der Mikroebene haben als ,bottom-up-Prozess' Auswirkungen auf die strukturellen Komponenten der Makroebene; umgekehrt haben Veränderungen der strukturellen Bedingungen als ,top-down-Prozess' Rückwirkungen auf die individuellen Verhaltensweisen.

„Die Strukturierung sozialer Systeme zu analysieren bedeutet, zu untersuchen, wie diese in Interaktionszusammenhängen produziert und reproduziert werden; solche Systeme gründen in den bewußt [sic!] vollzogenen Handlungen situierter Akteure, die sich in den verschiedenen Handlungskontexten jeweils auf Regeln und Ressourcen beziehen" (Giddens 1992: 77).

Nicht nur die Frage von Struktur und Handlung, von Mikro- und Makroebene, sondern insbesondere die Kontextualisierung ist wesentlich für die Erklärung sozialer Phänomene. „In order to study power, therefore, one must be concerned with the social context within which power relations take place" (Osmond 1978: 51). Schlussfolgernd sind Machtverhältnisse ${ }^{114}$ „dispositiv“: Welche Machtdimension inwiefern wirkt - nach Foucault: das "Spiel von Positionswechseln“ - ist eine Frage des Kontextes (Foucault 1978: 119f.). Kurz: Mittels dieses Machtansatzes wird die Erfassung von Handlung, Kultur und Struktur kontextualisiert. Die Gesamtmenge der Verwirklichungschancen von Macht bildet das „Power-Capability Set“, das über die dargestellten Dimensionen ausdifferenziert werden kann.

Die Ausgangsannahmen, die aus der oben geführten theoretischen Diskussion erschlossen werden, sind zusammenfassend:

1. Macht beeinflusst als ein mehrdimensionales Konstrukt die innerhäusliche Arbeitsteilung in Paarbeziehungen

2. Macht ist allgegenwärtig

3. Macht ist latent, lässt sich jedoch in unterschiedliche Dimensionen ausdifferenzieren

114 Der hier verwendete Begriff des Machtverhältnisses ist in diesem Sinne umfassender als der Begriff der Machtstruktur: Er impliziert, daß [sic!] eine durch die relativen Statuspositionen bzw. Ressourcen gegebene Machtstruktur in der Beziehung zwischen den Akteuren verändert werden kann [Möglichkeit der Modifikation durch interpersonal skills], ohne daß [sic!] die anfängliche Ressourcenverteilung selbst verändert wird“" (Held 1978: 68). 
4. Macht wird sozial konstruiert, d. h. Macht erfordert die Existenz sozialer Beziehungen

5. Machtverhältnisse sind dispositiv

6. Macht ist relational

7. Modernisierungsvorstellung: Jeglicher modernisierungstheoretischer Annahmen über eine Orientierung am liberalen Egalitarismus zum Trotz besteht eine Diskrepanz zwischen der Egalitätsnorm und dem tatsächlichen Verhalten traditioneller Arbeitsteilungsarrangements.

\section{4. „Bringing Power Back In“: Die Verteidigung des Machtansatzes}

Werden Machtansätze entweder einer ökonomischen reduktionistischen Perspektive oder gar einer Banalisierung ausgesetzt, so werden leichtfertige Fehlschlüsse getroffen: Wird beispielsweise argumentiert, dass ausschließlich eine Diskriminierung (nach Foucault die Unterdrückung als rein juristische Machtdimension) von Frauen (schwache, arme Frau im Gegensatz zum starken, faulen Mann) der Kern von Machtansätzen sei, ist missverstanden worden, dass es nicht um normative Schuldzuweisungen geht. Außerdem basiert die Fokussierung auf eine Durchsetzungsrelation innerhalb der Paarbeziehung auf einer unterkomplexen Argumentation, die die verschiedenen Dimensionen von Macht nicht berücksichtigt. Würde das Paar der schwachen, armen Frau und des starken, faulen Mannes isoliert betrachtet, würde die Machtposition des Mannes vollkommen überschätzt werden. „A zero-sum concept of power, in which A is the „victor“ and B is the „victim“, is not adequate for describing many kinds of power relationships" (Baldwin, D. 1978: 1241). Die soziologische Innovation ist in einer Typologie der Macht zu suchen, die Machtstrukturen herausbildet, ferner der Mehrdimensionalität des Begriffes gerecht werden kann. Diese Verknüpfung von ökonomischen, austauschtheoretischen, soziologischen, mikro- und makrosoziologischen Theorien erzielt ein komplexes Gefüge zur Erklärung innerhäuslicher Arbeitsteilungsarrangements.

„Die Multidimensionalität der familiären bzw. ehelichen Machtstruktur und die Existenz verschiedener Machtebenen zeigt, da $\beta$ [sic!] es nicht nur aus methodologischen, sondern auch aus theoretischen Gründen nicht möglich ist, die Machtstruktur allein durch die Verteilung von Entscheidungskompetenzen [s. familiensoziologische Machtkonzepte] zu bestimmen“" (Held 1978: 74).

Durch die Betrachtung des Zusammenhangs von innerhäuslichen Arbeitsteilungsarrangements und mehrdimensionalen Machtverhältnissen werden gesamtgesellschaftlich geschlechtsspezifische Arbeitsteilungen als Differenz 
von Binnensystem Familie und Außensystem Gesellschaft notwendigerweise in die Analyse einbezogen (vgl. Held 1978: 82). Ferner wird das von Krüger und Levy bezeichnete Verdeckungsphänomen familiärer Entscheidungen als individuelle Privatentscheidungen vermieden.

Eine weitere verbreitete Annahme, dass modernisierungstheoretische Perspektiven auf einer Gleichheitsillusion der Geschlechter basieren (s. z. B. Koppetsch/Burkart 1999: Die Illusion der Emanzipation. Zur Wirksamkeit latenter Geschlechtsnormen im Milieuvergleich), ist auf die Ignoranz der Orientierung an einer GleichSTELLUNG der Geschlechter sowie die Fehlinterpretation von Egalitätsnormen als Gleichheitsillusion zurückzuführen. Anthropologische Annahmen (Mann = Frau) und eine Egalitätskonstruktion, basierend auf Positionen (Position Frau = Position Mann), werden unbedacht vermengt. Doch geht es nicht um die Frage der Gleichheit, vielmehr um die Frage der Gleichstellung sowie Chancengleichheit. Mit Platzierungen innerhalb eines sozialen Raumes werden Machtverhältnisse ausgehandelt, wobei je nach Position und damit verbundenen Machtmitteln die Handlungschancen variieren (vgl. Löw 2001: 164). Es vermag WissenschaftlerInnen geben, die aufgrund des Wunsches einer Bestätigung der Gleichheit von Mann und Frau zögern, Unterschiede zu benennen, weil sie dafür benutzt werden könnten, ungleiche Chancen und Behandlungen zu rechtfertigen (vgl. Tannen 1991: 16). Doch Menschen sind nicht alle gleich, sondern unterschiedlich - es gilt diese Prämisse der Unterschiede durch Zugang zur Gleichstellung zu kompensieren; ethisch anthropologisch nicht eine Gleichheit, stattdessen eine Gleichwertigkeit (der Geschlechter) zugrunde zu legen.
„Menschen haben Würde, die Achtung durch Gesetze und soziale Institutionen verdient. Diese Idee geht auf viele Traditionen zurück; sie ist heute der Kern des modernen demokratischen Denkens und Handelns in der ganzen Welt. Die Idee der Menschenwürde umfasst im Allgemeinen die Idee des gleichen Wertes: Rei- che und Arme, Stadt- und Landbewohner, Frau und Mann, alle verdienen sie die gleiche Achtung allein aufgrund ihres Menschseins, und diese Achtung darf nicht willkürlich eingeschränkt werden. Oft ist diese Idee der Gleichwertigkeit auch mit den Ideen Freiheit und Chancengleichheit verbunden: Die Gleichwertigkeit der Personen zu achten, dazu gehört unter anderem, ihre Fähigkeit zu fördern, ein Leben nach ihren ureigensten Vorstellungen zu führen“ (Nussbaum 2003: 12).

Dies verlangt, dass wir nicht von einer absolutistischen Egalitätskonstruktion ausgehen können - so wie es in Simmels Beispiel ausgeführt wird, dass alle BürgerInnen eines Dorfes Rosen bekommen ungeachtet ihrer Fähigkeiten zum Rosenanbau oder der Lichtverhältnisse ihres Gartens - vielmehr die Unterschiede zu berücksichtigen wissen müssen und diese als Basis für Umverteilungen $\mathrm{zu}$ erachten. Vielleicht ist ein/e BürgerIn des Dorfes aus gesundheitlichen Gründen nicht in der Lage, die Rosen selbst anzubauen - so bedarf er/sie weiterer respektive anderer Mittel und Förderungen, um Zugang zu seinem/ihrem Recht auf Rosen zu erhalten. 
„Als Menschen im achtzehnten und neunzehnten Jahrhundert für die Abschaffung der Sklaverei kämpften, arbeiteten sie nicht in der Illusion, dass die Welt durch die Abschaffung der Sklaverei vollkommen gerecht würde. Vielmehr gingen sie davon aus, dass eine Gesellschaft, die Sklaverei duldete, vollkommen ungerecht sei ([...] Adam Smith, Condorcet und Mary Wollstonecraft vertraten diese Sichtweise ausdrücklich). Weil Sklaverei als untragbare Ungerechtigkeit diagnostiziert wurde, erhielt ihre Abschaffung höchste Priorität, und dazu brauchte man keinen Konsens über das Aussehen einer vollkommen gerechten Gesellschaft" (Sen 2010a: 50).

Sen zufolge, dessen Perspektive befürwortet wird, geht es nicht um die Utopie der Definition einer absoluten Gerechtigkeit, sondern um die Frage, ob eine Gesellschaft gerechter oder ungerechter ist bzw. im vorliegenden Beispiel, wie eine Gesellschaft durch die Abschaffung der Sklaverei etwas weniger ungerecht gestaltet werden kann (vgl. Sen 2010a: 37).

Um inkonsistente Argumentationen zu vermeiden, ist die Differenzierung zwischen Gleichheit, Gleichstellung und Chancengleichheit der Geschlechter unumgänglich - ein Plädoyer für die Trennschärfe von Begrifflichkeiten.

Festzuhalten ist außerdem, dass die pluralistische Egalitätskonstruktion, die durch den Capability Approach ermöglicht wird, einer (von Ostner vorgeworfenen) feministischen Homogenität der Perspektive im Wege steht.

Eine der zentralen Erkenntnisse nach Held (1978) ist die Zuschreibung von Gebrauchswerten zu Frauen und Tauschwerten zu Männern, deren Problematik darin besteht, dass kaum ein Markt für Gebrauchswerte existiert. So stellen sich die abschließenden Fragen: Haben wir diese Erkenntnis von Held (1978) schlichtweg vergessen? Gibt es gerechtfertigte Gründe, warum wir diese Position zunehmend ausklammern oder liegt hierin möglicherweise eines der zentralen Ausschlusskriterien des Machtansatzes aus der Familiensoziologie begründet? Schmidt geht sogar soweit, dass er einen Mangel einer Vertiefung der Soziologie ehelicher Machtverhältnisse trotz ihres grundlegenden Charakters seit Held (1978) konstatiert (vgl. Schmidt 2002: $160)$.

\subsection{Zusammenfassende theoretische Modellkonzeption}

Traditionelle Arbeitsteilungsarrangements werden als Ausdruck respektive Resultat unterschiedlicher Machtverhältnisse in der Paarbeziehung analysiert, um herauszufinden, welche Barrieren (individuell, sozietal, institutionell, strukturell) zu einer einseitigen - nämlich traditionellen - Arbeitsteilung führen. Sen zufolge ist im Idealfall eine Pluralität von Handlungsalternativen zu ermöglichen, doch die Feststellung, dass in sämtlichen europäischen Ländern ein traditionelles Muster besteht, verweist auf einen Mangel an Hand- 
lungsoptionen. Im Falle einer Pluralität von Handlungsoptionen würde kein globales Muster dominieren. Gemäß dem Pluralitätsgedanken müsste eine Normalverteilung diverser Arbeitsteilungsarrangements (stark traditional eher traditional - partnerschaftlich/egalitär - eher Rollentausch - Rollentausch) zu beobachten sein, dies ist jedoch keineswegs der Fall. Wesentlich ist, dass die innerhäusliche Arbeitsteilung als erreichte Funktionsweise innerhalb eines auf Macht-Verwirklichungschancen basierenden Handlungsspielraums erfasst wird.

Ein zusammenfassender Überblick der Ideenkonzeption zur Entwicklung des theoretischen Bezugsrahmens der Typologie der Machtverhältnisse in Paarbeziehungen wird in Tabelle 8 gegeben. Die innerhäuslichen Arbeitsteilungsarrangements sind das zu erklärende soziale Phänomen, d. h. bilden die abhängige Variable, während Machtkategorien (sogenannte „PowerCapabilities") als unabhängige/erklärende Variablen auf Individual- und Kontextebene in die Analyse einbezogen werden (das Power-Capability Set umfasst hierbei die Gesamtheit der Power-Capabilities). In Abbildung 4 wird letztlich die Konzeption der Typologie der Machtverhältnisse zusammengefasst.

Die Dimensionen der Macht hängen sowohl vom Kontext (Regimetypen - Länder - Regionen) als auch von der Zeit ab. Ferner wirken nicht alle Mächte in gleichem Umfang; wie stark ihr Effekt auf die innerhäusliche Arbeitsteilung ist, gilt es kontextspezifisch zu differenzieren. Zwischen den Dimensionen nimmt der Masterstatus nach Krüger und Levy (2001) eine theoretische Brückenfunktion ein.

„Krüger and Levy (2001) have discussed the necessity not to focus the problematic of doing gender preferentially or even exclusively on the inter-individual, micro-social level, as the initial ethnomethodological orientation of this concept did, but to apply it to the sometimes macro- but more frequently meso-social, institutional level as well" (Levy et al. 2002: o. S.).

Der Masterstatus ist eine Kombination aus Familien- und Lebenslaufsoziologie respektive eine Verknüpfung zwischen Individuen und institutionenorientierten Lebensläufen. Nach Annahmen des individuellen Masterstatus variiert die Teilhabe an sozialen Feldern nach Geschlecht; der strukturelle Masterstatus impliziert, dass das Geschlecht eine Strukturkategorie darstellt, die u. a. zu einer geschlechtsspezifischen Arbeitsmarktsegregation führt.

Auf kultureller Ebene erfasst das Cultural Lag das Zeitfenster, das erforderlich ist, um strukturelle Veränderungen in Werte zu überführen. In Folge der Tertiarisierung als strukturellem Wandel sind Frauen zunehmend erwerbstätig, wodurch ein Wertewandel von traditionellen zu egalitären Werten entstanden ist. Doch aufgrund des Cultural Lag ist nach wie vor eine Diskrepanz zwischen egalitären Einstellungen und traditionellem Verhalten auf individueller Ebene zu beobachten (die im Zeitverlauf zwar in einer Orientierung, aber nicht zwangsläufig in einer vollkommenen kongruenten Anpas- 
sung beider Ebenen münden muss, da egalitäre Einstellungen keine hinreichende Bedingung für egalitäres Verhalten darstellen. Menschlichen sozialen Handlungen ist schließlich eine Ambivalenz immanent - dies zeichnet das „Menschliche“ aus. Ferner stellen Modernisierungsprozesse keine linearen Entwicklungstrends dar, s. Kapitel 7. „Zwischenfazit der eigenen Argumentation: Für einen Arbeitsteilungspluralismus“).

Abbildung 4: Typologie der Machtverhältnisse

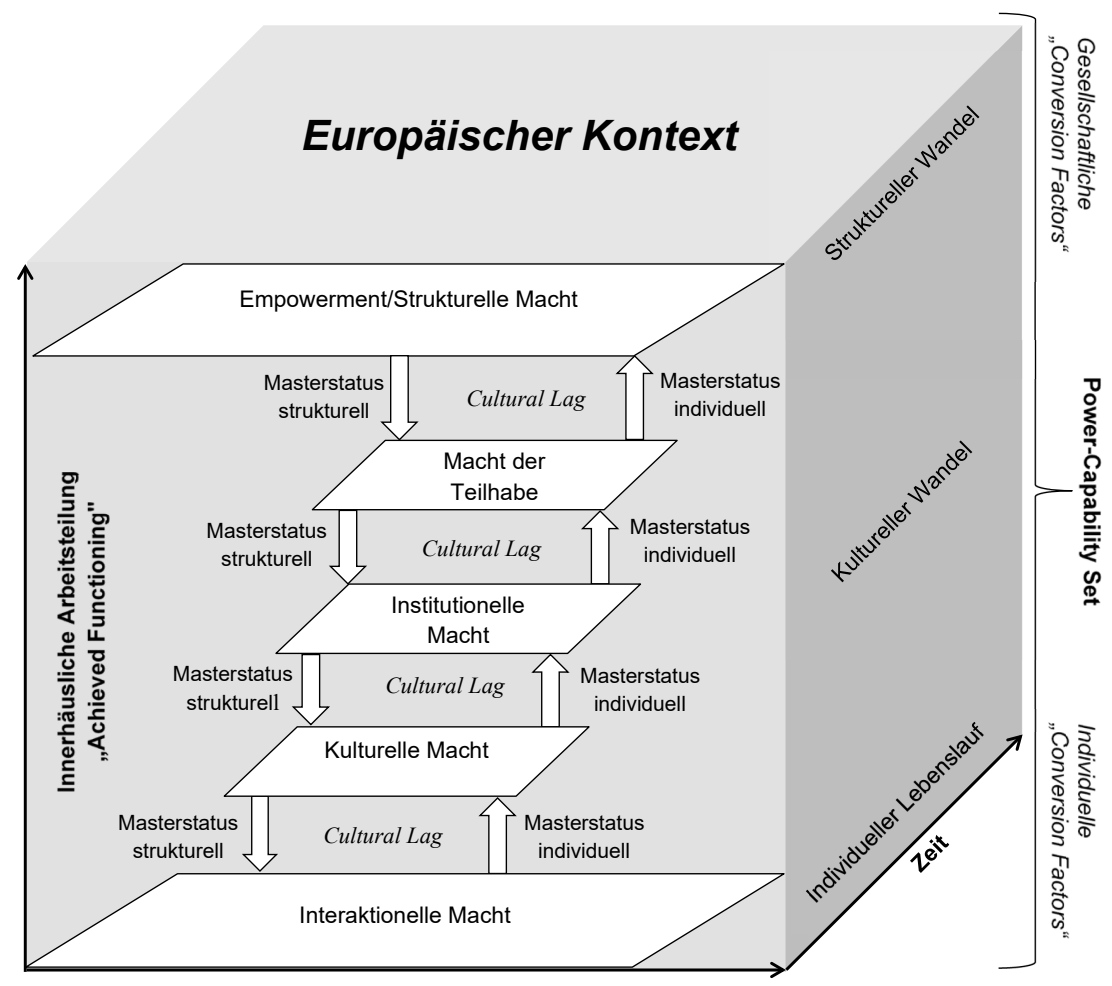

Quelle: Eigene Darstellung. 


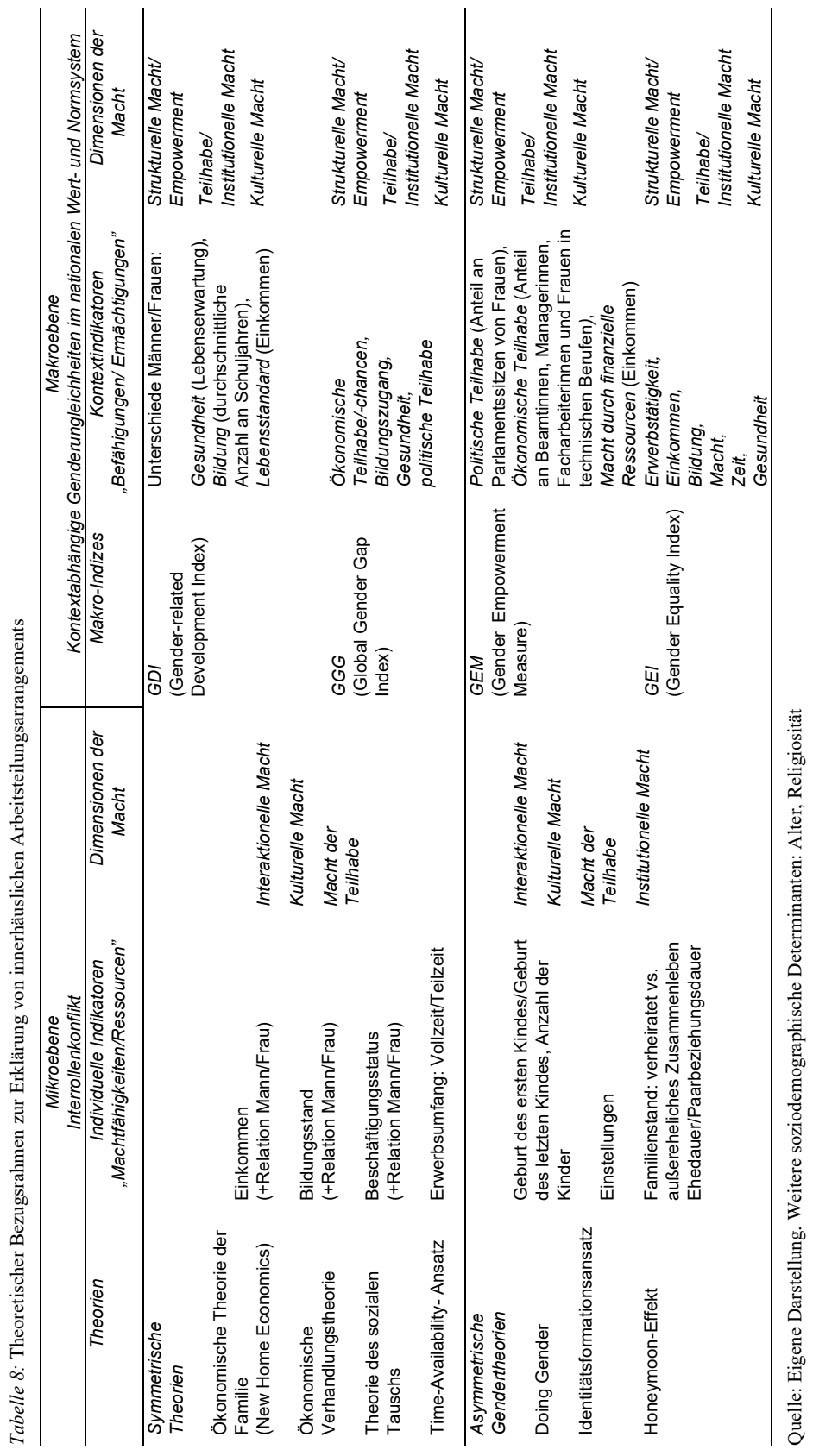




\subsection{Hypothesengenerierung}

Aus der theoretischen Argumentation können eine ganze Reihe von Hypothesen abgeleitet werden. Es ist nicht möglich, jedes kleinste Detail empirisch zu überprüfen, weshalb sich die empirische Umsetzung auf einige wesentliche Aspekte konzentriert. Diejenigen Hypothesen, die für diese Dissertation von zentraler Bedeutung sind, werden nachfolgend angeführt.

Innerhäusliche Arbeitsteilungsarrangements - so die Ausgangsannahme werden insbesondere von strukturellen Bedingungen, Institutionen, Teilhabechancen, kulturellen Werten und individuellen Merkmalen des PowerCapability Sets beeinflusst, was bedeutet, dass Effekte auf Mikro- und Makroebene erwartet werden. Durch die Berücksichtigung einer hierarchischen Datenstruktur können Individual- und Kontexteffekte ex aequo überprüft werden. Die - der Mehrebenenanalyse zugrunde liegende - übergeordnete Idee ist, dass individuelles Handeln stets kontextabhängig ist, wie in Kapitel 12.1.1 im Detail erläutert wird. Im Gegensatz zu klassischen (gepoolten) OLS-Regressionsverfahren wird eine Variation des Niveaus der innerhäuslichen Arbeitsteilung zwischen den Kontexteinheiten angenommen. Zunächst werden die Unterschiede zwischen den Regimetypen skizziert, doch dass diese - wie im Kapitel 10. ausführlich argumentiert wird - zu allgemein sind und auch innerhalb der Regimetypen Diskrepanzen innerhäuslicher Arbeitsteilungsarrangements anzunehmen sind, wird weiterführend vornehmlich anhand der Verwendung der NUTS1-Regionen als Kontexteinheiten berücksichtigt.

H1: Die innerhäusliche Arbeitsteilung variiert zwischen den Kontexteinheiten der Regime, Länder und Regionen

In allen Regimetypen (weiterführend: Ländern und Regionen) dominiert zwar die traditionelle innerhäusliche Arbeitsteilung, jedoch sind geringfügige Unterschiede zwischen den Kontexteinheiten nach folgendem Muster zu erwarten:

Abbildung 5: Erwartete innerhäusliche Arbeitsteilungsarrangements nach Regimetypen

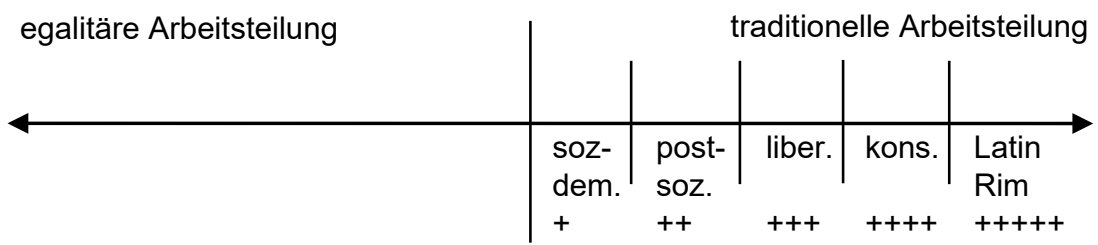

Quelle: Eigene Darstellung. 
Innerhalb einer traditionellen innerhäuslichen Arbeitsteilung sind in skandinavischen Regimen (soz.-dem.) im Vergleich zu den anderen Regimen egalitärere Arbeitsteilungsarrangements dominierend, während in „Latin Rim Regimen" 115 das traditionellste Muster angenommen wird. Postsozialistische Regime (post-soz.) werden innerhalb eines traditionellen Musters als eher egalitär und konservative Regime als eher traditionell erachtet. Liberale Regime bilden die Mittelkategorie. Eine Bestätigung dieses Musters findet sich (mit Ausnahme der postsozialistischen Länder) in der komparativen Analyse Sayers (2010): ,[...] the housework gender gap is smallest in the more egalitarian Nordic countries and largest in the conservative countries, with the gap in English-speaking countries at intermediate levels" (Sayer 2010: 34). Auch Geist (2015) stellt fest, dass die Einteilung der Länder in liberale, konservative und sozialdemokratische Wohlfahrtsstaatsregime auf Unterschiede in der Arbeitsteilung auf der Makroebene hindeutet, die nicht ausschließlich durch Unterschiede individueller Merkmale erklärt werden können. Eine egalitäre Aufteilung der Hausarbeiten ist ihr zufolge in konservativen Ländern selten und unabhängig von relativen Ressourcen, Zeitverfügbarkeit und Geschlechterideologie, was darauf hindeutet, dass die innerhäusliche Arbeitsteilung nicht nur von zwei PartnerInnen verhandelt, sondern auch von kontextuellen Faktoren beeinflusst wird (vgl. Geist 2005: 23). Zwischen den europäischen Regimen sind nationale Differenzen (bzw. nach Kaufmann (2003) ,nationale Eigensinnigkeiten“/Idiosynkrasien) bezüglich der innerhäuslichen Arbeitsteilung aufgrund sozio-kultureller Werte und Traditionen zu konstatieren (vgl. Höpflinger/Fux 2007: 57).

Aus einer Makroperspektive ist in Anlehnung an Sen anzunehmen, dass die Wahlfreiheit in ökonomisch starken Regionen höher ist als in ökonomisch schwachen Regionen. Diese Kontextabhängigkeit nimmt Einfluss auf die individuellen Aushandlungen der innerhäuslichen Arbeitsteilung. Die erste relevante Dimension ist ökonomisch: während Paare in ökonomisch leistungsfähigen Regionen eine höhere Wahlfreiheit genießen, haben Paare in ökonomisch schwächeren Regionen eine eingeschränkte Wahlfreiheit. Es wird erwartet, dass in ökonomisch schwachen Regionen die ökonomischen Theorien (formiert im interaktionellen Machttyp) eine höhere Erklärungskraft haben, weil Paare infolge einer finanziell-strukturell schwächeren Ausgangsposition ,es sich nicht leisten können“ aufgrund von Normen und Werten zu handeln. Beispielsweise ist ein Argument für die hohen Frauenerwerbsquoten in Polen, dass die meisten Frauen das Gefühl haben, arbeiten zu müssen - ob sie wollen oder nicht (vgl. Saxonberg/Szelewa 2007: 367).

In sozialdemokratischen Regimen agiert der Staat als zentraler Akteur, der ZweiverdienerInnen-Modelle und ein hohes Maß an Dekommodifizierung unterstützt. Folglich wird angenommen, dass ZweiverdienerInnen-

115 Die Typen nach Esping-Andersen sind von Leibfried (1992) um einen südeuropäischen Typus, das Latin Rim Regime, erweitert worden. 
Policies, im Sinne Sens Capabilities, einen stärkeren Effekt auf egalitäre Arbeitsteilungsarrangements haben als in den anderen Regimetypen.

Postsozialistische Regime stellen eine Mischform dar, die sowohl liberale, marktorientierte Policies vorweisen als auch Korpis „Dual Earner Typ“ unterstützen. Indem in den ehemaligen sozialistischen Ländern der Zugang für Frauen zur Erwerbstätigkeit ein primäres Ziel war bzw. ist, wurde zwar eine staatliche Gleichstellungspolitik der Geschlechter verfolgt, jedoch basierte diese nicht auf einer Ideologie der Geschlechtergleichstellung, sondern auf einer finanziellen Notwendigkeit, weil die Existenzsicherung vieler Familien von zwei Einkommen abhängig ist (vgl. Batalova/Cohen 2002: 746). Aufgrund des ökonomisch schwachen Marktes ist die Wahlfreiheit eines präferierten Arrangements begrenzt, weshalb Rational Choice Ansätze eine größere Erklärungskraft aufweisen als in den anderen Regimetypen.

Liberale Regime verfügen über marktorientierte Policies und ein geringes $\mathrm{Ma}$ an Dekommodifizierung, ferner hängen Sozialleistungen von der Marktsituation ab. Je nach Marktkonstellation kann die innerhäusliche Arbeitsteilung mehr oder weniger egalitär ausgeführt werden.

Konservative Regime bevorzugen familienunterstützende Policies und ein mittleres Ausmaß an Dekommodifizierung. Aufgrund der starken ökonomischen Marktsituation ist auf struktureller Ebene eine Wahlfreiheit der Arbeitsteilungsarrangements gegeben. Die Fokussierung auf Familienunterstützung führt $\mathrm{zu}$ traditionelleren Mustern als in skandinavischen, postsozialistischen und liberalen Regimen. Insofern wird der kulturellen Argumentation von Gendertheorien die größte Erklärungskraft der innerhäuslichen Arbeitsteilung beigemessen.

Latin Rim Regime bevorzugen ebenfalls ein hohes Ausmaß an familienunterstützenden Maßnahmen sowie konservativen Werten doch, ähnlich wie in postsozialistischen Ländern, ist die ökonomische Situation vergleichsweise prekär. Gleichwohl sind Normen und Werte derart konservativ, dass der soziale Druck in traditionellen Verhaltensweisen mündet.

Die institutionelle Macht und die Macht der Teilhabe sind ihrer Brückenfunktion entsprechend für sämtliche Regime von Bedeutung.

Im Sinne des Dispositivs ist eine Kontextabhängigkeit der Stärke der Effekte von ökonomischen Theorien, Gendertheorien und Policies festzuhalten. Ein wesentlicher Vorteil von Mehrebenenanalysen ist, je nach Kontexteinheit unterschiedliche Individualeffekte annehmen zu können (s. Kapitel 12.1.3.). Dass als Kontextdimensionen die Regimetypen dargestellt werden, ist zunächst eine Vereinfachung der Herangehensweise, die jedoch im Zuge der Argumentation über Regime-, Länder- und Regionenanalysen weiter ausdifferenziert wird. Von besonderer Bedeutung für die Regionenanalyse ist die Forschungshypothese, dass sich die NUTS1-Regionen systematisch im Hinblick auf die innerhäusliche Arbeitsteilung unterscheiden, obgleich eine Dominanz des traditionellen Musters überwiegt. 


\section{Mikroebene - Individualhypothesen zur interaktionellen Macht (ökonomische Argumentation in Anlehnung an Kapitel 3.1.1.)}

Anhand der symmetrischen Theorien über relative Ressourcen und anhand der asymmetrischen Theorien über Geschlechterrollen, die verschiedene latente Formen von Macht darstellen, werden auf der Mikroebene einige Hypothesen aufgestellt. (Für welche Hypothesen in welchen Regimetypen eine unterschiedliche Erklärungsleistung erwartet wird, ist der Tabelle 9: Zum „Spiel von Positionswechseln“ zu entnehmen. Grundsätzlich wird erwartet, dass sich die Effekte der Individualvariablen zwischen den Kontexteinheiten unterscheiden, weshalb Zufallseffekte für die Einkommensrelation, die Kinderanzahl und die Paarbeziehungsdauer in die Mehrebenenmodelle eingeführt werden. Zur methodischen Unterscheidung von Random-Intercept und Random-Slope-Modellen s. Kapitel 12.1.3.) Zu Beginn werden die Hypothesen der Individualebene, die auf die ökonomische Argumentation zurückzuführen sind, angeführt.

\section{Einkommensrelation}

H2: Verfügen beide PartnerInnen über ein vergleichbar hohes Einkommen (interaktionelles Machtgleichgewicht), wird die innerhäusliche Arbeitsteilung eher egalitär ausgeführt.

H3: Wenn das Einkommen in der Paarbeziehung asymmetrisch verteilt ist, insofern als dass das Einkommen der Frau höher ist als das des Mannes (interaktionelles Machtungleichgewicht zugunsten der Frau), wird die innerhäusliche Arbeitsteilung eher egalitär aufgeteilt (im Sinne der Gendertheorien würde in diesem Fall eine traditionelle Arbeitsteilung erwartet, während RCTheorien einen Rollentausch prognostizieren würden. Da ein Rollentausch aufgrund bestehender Werte - die sich zwar an einer Geschlechtergleichstellung orientieren, jedoch nicht an einem Rollentausch - als unwahrscheinlich erachtet wird, ist für diese asymmetrische Konstellation anstelle eines Rollentauschs allenfalls eine egalitäre Aufteilung zu erwarten).

H4: Wenn das Einkommen in der Paarbeziehung asymmetrisch verteilt ist, insofern als dass das Einkommen des Mannes höher ist als das der Frau (interaktionelles Machtungleichgewicht zugunsten des Mannes), wird die innerhäusliche Arbeitsteilung eher traditionell aufgeteilt.

H5: Ein Wandel der Einkommensrelation im Zeitverlauf zwischen den Erhebungswellen zugunsten eines Partners/einer Partnerin führt zu einer Mehrarbeit der Hausarbeit des anderen Partners/der anderen Partnerin. 


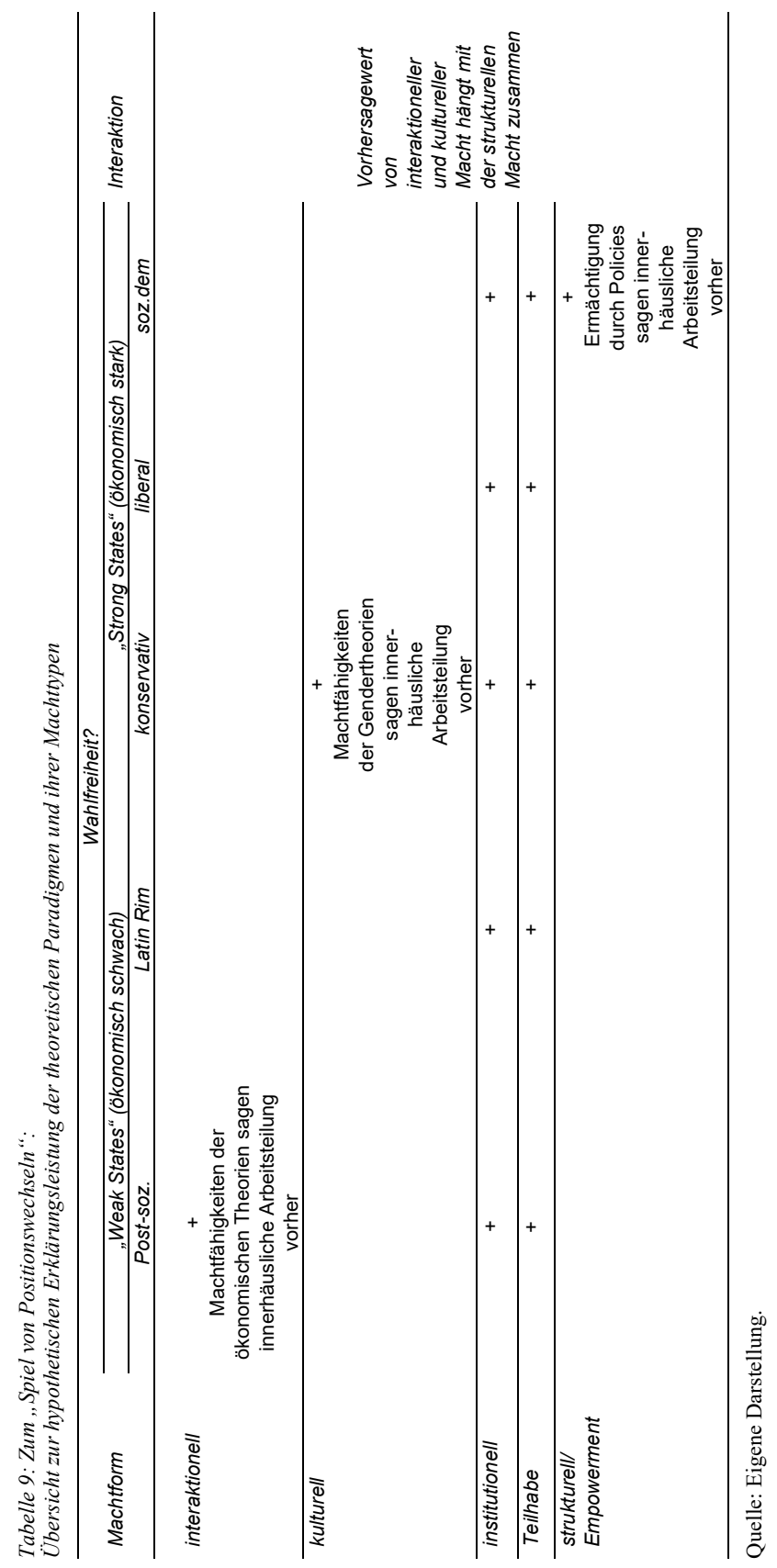




\section{Erwerbsrelation}

H6: Erwerbshomogame Paare (interaktionelles Machtgleichgewicht) verrichten ihre innerhäusliche Arbeitsteilung eher egalitär.

H7: Erwerbshypogame Paare, in denen die Frau mehr Stunden erwerbstätig ist als ihr Partner (interaktionelles Machtungleichgewicht zugunsten der Frau), verrichten ihre innerhäusliche Arbeitsteilung eher egalitär (kein Rollentausch aufgrund der Kritik an ökonomischen Theorien durch gendertheoretische Annahmen, s. H3).

H8: Erwerbshypergame Paare, in denen der Mann mehr Stunden erwerbstätig ist als die Frau (interaktionelles Machtungleichgewicht zugunsten des Mannes), verrichten ihre innerhäusliche Arbeitsteilung eher traditionell.

H9: Ein Wandel der Erwerbsrelation im Zeitverlauf zwischen den Erhebungswellen zugunsten eines Partners/einer Partnerin führt zu einer Mehrarbeit der Hausarbeit des anderen Partners/der anderen Partnerin.

\section{Bildungsrelation}

H10: Bildungshomogene Paare (interaktionelles Machtgleichgewicht) verrichten ihre innerhäusliche Hausarbeit eher egalitär.

H11: Bildungshypogame Paare, in denen die Partnerin über einen höheren Bildungsabschluss als ihr Partner verfügt (interaktionelles Machtungleichgewicht zugunsten der Frau), verrichten ihre innerhäusliche Arbeitsteilung eher egalitär (kein Rollentausch aufgrund gendertheoretischer Annahmen).

H12: Bildungshypergame Paare, in denen der Partner über einen höheren Bildungsabschluss als die Partnerin verfügt (interaktionelles Machtungleichgewicht zugunsten des Mannes), verrichten ihre Arbeitsteilung eher traditionell.

H13: Ein Wandel der Bildungsrelation im Zeitverlauf zwischen den Erhebungswellen zugunsten eines Partners/einer Partnerin führt zu einer Mehrarbeit der Hausarbeit des anderen Partners/der anderen Partnerin.

Zusätzlich zu den Relationshypothesen wird jeweils die Höhe des Einkommens, des Erwerbsumfangs und des Bildungsstandes überprüft.

Individualhypothesen zur kulturellen Macht (in Anlehnung an Kapitel 3.1.2.)

Einkommens- und Erwerbsrelation

Konträr zur ökonomischen Argumentation, die davon ausgeht, dass Einkommens-, Erwerbs- und Bildungsressourcen die Verhandlungsposition innerhäuslicher Arbeitsteilungsarrangements zugunsten des Partners/der Partnerin mit den höheren Ressourcen beeinflussen, argumentieren kulturelle Geschlechterrollenansätze wie folgt: 
H14: Paare, in denen die Frau über ein höheres Einkommen verfügt als ihr Partner, teilen sich die innerhäusliche Arbeitsteilung eher traditionell auf (je stärker seine finanzielle Abhängigkeit, desto weniger Hausarbeiten werden von ihm ausgeführt, d. h. es liegt ein Kompensationseffekt infolge eines kulturellen Machtungleichgewichts zugunsten des Mannes vor).

H15: Ein Wandel der Einkommensrelation im Zeitverlauf zwischen den Erhebungswellen zugunsten der Frau führt dazu, dass der Partner sich weniger an der Ausführung der Hausarbeiten beteiligt.

H16: Paare, in denen die Frau mehr Stunden erwerbstätig ist als der Mann, verrichten ihre innerhäusliche Arbeitsteilung erst recht traditionell (Kompensationseffekt infolge eines kulturellen Machtungleichgewichts zugunsten des Mannes).

H17: Ein Wandel der Erwerbsrelation im Zeitverlauf zwischen den Erhebungswellen zugunsten der Frau führt dazu, dass der Partner sich weniger an der Ausführung der Hausarbeiten beteiligt.

\section{Ehe-/Paarbeziehungsdauer}

H18: „Je länger die Ehe [oder nicht-eheliche Paarbeziehung] andauert, desto mehr verfestigen sich geschlechtsspezifische arbeitsteilige Strukturen im Lebensalltag und desto schwieriger wird es, die Männer dazu zu bewegen, sich stärker zu beteiligen“ (Mikro) (Schulz/Blossfeld 2006: 41). Von besonderer Bedeutung sind Routinen, da diese konstitutiv sind ,[...] sowohl für die kontinuierliche Reproduktion der Persönlichkeitsstrukturen der Akteure in ihrem Alltagshandeln, wie auch für die sozialen Institutionen; Institutionen sind solche nämlich nur kraft ihrer fortwährenden Reproduktion" (Giddens 1992: 111f.). Routinen erfüllen Erwartungssicherheit der Akteure im Alltag und reproduzieren Institutionen.

\section{Kinder}

H19: Die Existenz von kleinen Kindern ( $<6$ Jahre) im Haushalt führt aufgrund kulturell bedingter Geschlechterideologien zu einer traditionellen Arbeitsteilung (vgl. Krüger/Levy 2000: 386; Levy/Ernst 2002: 120; Levy et al. 2002: 24; Schulz/Blossfeld 2006: 43; Lott 2012: 6; Berghammer/Neuwirth 2013: 17; Esping-Andersen et al. 2013: 3; Zabel/Heintz-Martin 2013: 663; Dechant/Schulz 2014: 593ff.).

H20: Eine Erhöhung der Kinderanzahl zwischen den Erhebungswellen führt zu einer Traditionalisierung der innerhäuslichen Hausarbeit.

(Einstellungen: Grundsätzlich wären Einstellungen in Bezug auf Hausarbeitsarrangements und Familienleben an dieser Stelle von Interesse, doch sind im Generations and Gender Survey nicht für alle Länder entsprechende Einstellungsvariablen verfügbar.) 
Hypothesen der institutionellen Macht (in Anlehnung an Kapitel 3.3.)

\section{Familienstand}

H21: Verheiratete Paare führen ihre innerhäusliche Arbeitsteilung traditioneller aus als unverheiratete, weil verheiratete Paare durch die höhere Erwartungssicherheit eines Fortbestehens der Beziehung eine Gegenleistung für ihre Leistung von zeitlichen Tauschaspekten als entkoppelt sehen.

Die institutionelle Macht und die Macht der Teilhabe haben in allen Regimen einen Effekt auf die innerhäusliche Arbeitsteilung, weil diese nach Krüger/Levy eine theoretische Brückenfunktion einnehmen.

Makroebene - Gruppenspezifische Kontexthypothesen ${ }^{116}$ zur strukturellen Macht/Empowerment

Auf der Kontextebene werden ökonomische, politische und kulturelle Empowermentdimensionen erfasst. In Bezug auf die ökonomische Dimension ist festzuhalten, dass sich ökonomische Teilhabechancen für Frauen sowie prosperierende Wirtschaftskontexte in einer höheren Beteiligung von Männern an den Hausarbeiten manifestieren. Auf der politischen Dimension wird angenommen, dass gleichstellungspolitische Instrumente einen egalisierenden Effekt auf die Aufteilung der Hausarbeiten von Paaren haben. Hingegen fördert ein ausgeprägter Traditionalismus der Kontexteinheiten auf der kulturellen Dimension eine traditionelle Arbeitsteilung, während sich ein kultureller Egalitarismus egalisierend auf die Aufteilung der Hausarbeiten auswirken müsste. Im Detail lauten die Kontexthypothesen:

\section{Kontexthypothesen zum ökonomischen Empowerment}

\section{Ökonomische Teilhabechancen}

H22: Je höher die ökonomischen Teilhabechancen (Frauenerwerbsquote) für Frauen, desto egalitärer die innerhäusliche Arbeitsteilung.

H23: In wirtschaftlich prosperierenden Kontexteinheiten beteiligen sich Männer mehr an Hausarbeiten als in nicht-prosperierenden Wirtschaftskontexten (aufgrund ungleicher Capabilities) ${ }^{117}$.

\section{Kontexthypothesen zum politischen Empowerment}

\section{Policies und politische Teilhabechancen}

H24: Je höher die staatliche Förderung von Dual Career Couples, desto egalitärer die innerhäusliche Arbeitsteilung.

116 Kontexthypothesen beziehen sich auf den Zusammenhang zwischen Kollektivmerkmalen (unabhängigen Variablen) und einem Individualmerkmal (abhängige Variable, hier die innerhäusliche Arbeitsteilung).

117 Gupta et al. (2010) stellen außerdem einen Effekt des Gender Wage Gaps fest: „[...] our results show that larger inequalities in women's earnings correspond to bigger economic disparities in housework time among them" (Gupta et al. 2010: 116). 
H25: Je höher die politische Teilhabe von Frauen, desto egalitärer die innerhäusliche Arbeitsteilung.

Kontexthypothesen zum kulturellen Empowerment

Bildungszugang

H26: Je höher der Bildungszugang für Frauen, desto egalitärer die innerhäusliche Arbeitsteilung.

Genderequality (kulturelle Leitvorstellung als aggregierter Makroindikator) H27: Je höher die Genderequality, desto egalitärer die innerhäusliche Arbeitsteilung.

\section{Fertilität}

H28: Je höher die Fertilitätsrate, desto traditioneller die innerhäusliche Arbeitsteilung.

H29: Umso religiös geprägter die Kontexteinheiten, umso traditioneller die innerhäusliche Arbeitsteilung.

Nachdem in diesem Kapitel die aufgestellten Hypothesen dargelegt wurden, die im Rahmen der Analyse überprüft werden, werden die zentralen Determinanten und erwarteten Effekte in Tabelle 10 abschließend zusammengefasst. Im folgenden Kapitel wird die theoretisch bedingte Auswahl der Länder skizziert und die Diversität innerhalb von Regimetypen sowie Ländern diskutiert. 
Tabelle 10: Determinanten der innerhäuslichen Arbeitsteilung

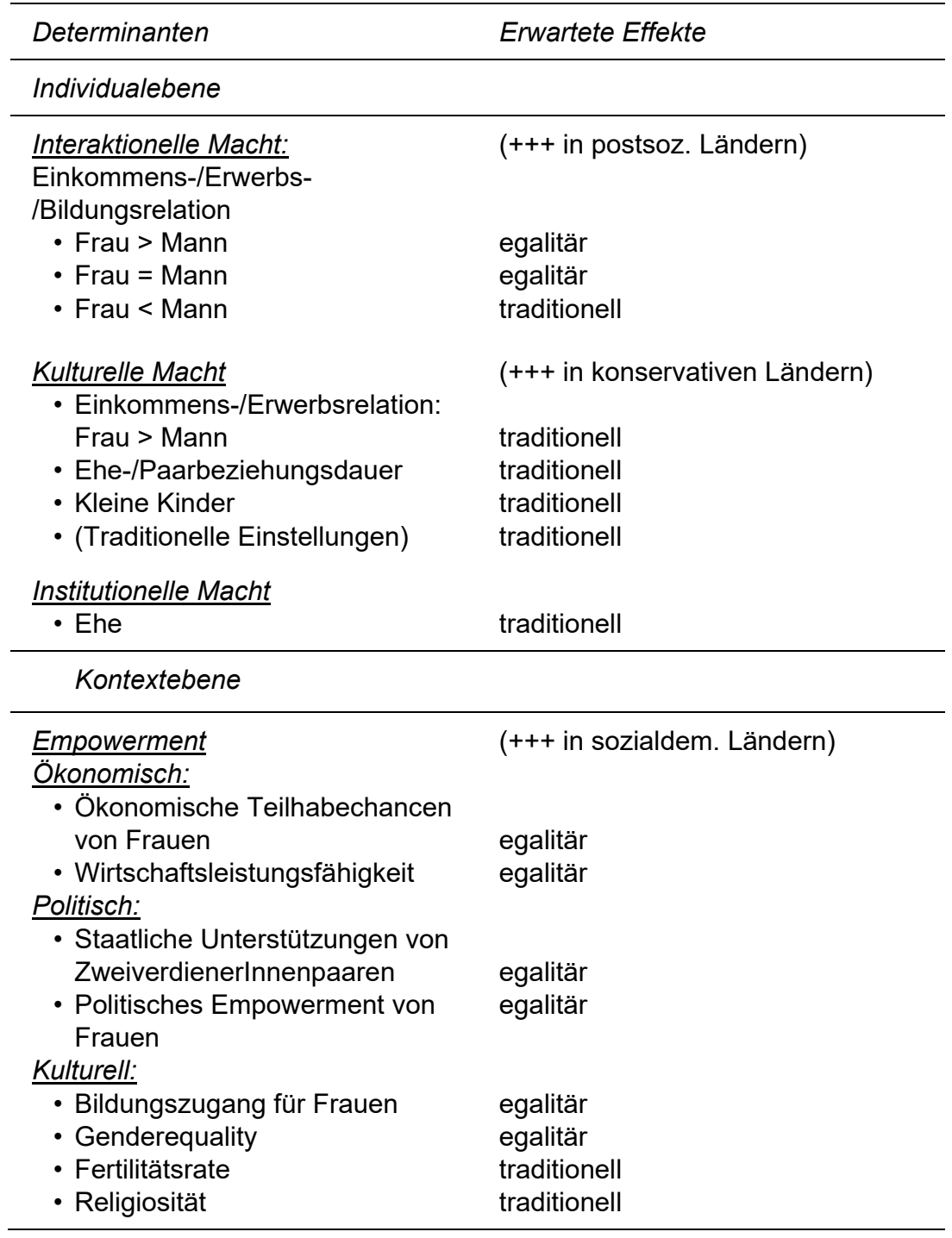

Quelle: Eigene Darstellung. 


\section{0.,,Trouble in Regime Typologies“: \\ Eine auf länderspezifischen Eigenarten von „genderrelevanten Policies“ basierende Fallauswahl}

Die Fallauswahl der Kontexteinheiten, die aus dem Datensatz des Generations and Gender Surveys ausgewählt werden (für die Datensatzbeschreibung s. Kapitel 11.), basiert auf zwei Dimensionen (einer inhaltlich-theoretischen sowie einer empirisch-analytischen): Erstens auf einer theoretisch bedingten Fallauswahl der Länder, die auf den Rahmenbedingungen der Arbeitsteilung - der ,genderrelevanten Welfare Policies“ - basiert und zweitens auf einer methodisch bedingten Auswahl der NUTS1-Regionen aufgrund der in Mehrebenanalysen häufig auftretenden Small-N-Problematik der Kontexteinheiten (für spezifische europäische Themen sind beispielsweise maximal 28 Ländereinheiten vorhanden; s. hierzu Kapitel 11.1.). Dem weiterführenden theoretischen Mehrwert einer Fokussierung auf Regionen im Sinne einer sehr differenzierten und grenzüberschreitenden Perspektive kommt hierbei eine besondere Bedeutung zu. Dass sich die „Welten der Wohlfahrtsstaaten“ durch eine innere Vielfalt und Fragmentiertheit auszeichnen und nicht als homogene Einheiten betrachtet werden können, wie sie einst von EspingAndersen (1990) angenommen wurden, ist bereits durch die Kritik von P. Baldwin (1990) überzeugend erörtert worden. P. Baldwin hat insbesondere an der These der linearen Entwicklung einer Dominanz des sozialdemokratischen Wohlfahrtsstaatsregimes (s. „Skandinavisierung Europas“ im Kapitel 2.5.) Fundamentalkritik geübt.

\subsection{Divergierende Rahmenbedingungen der Arbeitsteilung: ,genderrelevante Welfare Policies ${ }^{66}$}

Der theoretische Ausgangspunkt der Fallauswahl basiert auf der Idee der Differenzierung unterschiedlicher Wohlfahrtsstaatsregime innerhalb Europas, die kritisch hinterfragt und durch eine Beschreibung der inneren Diversität der Regime ergänzt wird. Um dieser Diversität empirisch-analytisch gerecht werden zu können, stellen europäische Regionen (NUTS1) die zentralen Analyseeinheiten dar. Es gilt zunächst möglichst unterschiedliche genderrelevante Welfare Policies zu kontrastieren, die eine Basis unterschiedlicher (struktureller) Machtkonstellationen in europäischen Ländern bilden und im theoretischen Verständnis nach Amartya Sen die Rahmenbedingungen der ,Capabilities' bzw. Verwirklichungschancen darstellen. Ferner ist Familienpolitik konstitutiv u. a. für das well-being. „Family policy can be defined as 
government activities that are designed to support families and their wellbeing" (Robila 2012: 32). Im spezifischen dienen familienpolitische und ökonomische Rahmenbedingungen als Kriterien der Fallauswahl; über kulturelle Rahmenbedingungen wird in einigen Fällen eine kurze Einführung gewährt.

Deutschland und Österreich gelten gemäß Esping-Andersen (1990) als Prototypen des konservativen Wohlfahrtsregimes. Frankreich sowie Belgien werden insbesondere von der feministischen Wohlfahrtsstaatsforschung wegen ihrer früh ausgebauten Kinderbetreuungsstruktur häufig als Sonderfälle des konservativen Wohlfahrtsstaats betrachtet und für die Niederlande hat sich herausgestellt, dass es sich hierbei eher um einen wohlfahrtsstaatlichen Mischtypus mit starken liberalen und sozialdemokratischen Elementen handelt (vgl. Leitner 2013: 15). Goodin/Smitsman (2000) argumentieren für eine Klassifizierung der Niederlande als sozialdemokratisch, wie sie anhand einer Reanalyse der Typologie Esping-Andersens (1990) darlegen. Obwohl die Niederlande in Bezug auf Programmstrukturen und politische Instrumente wie ein sozialdemokratischer Staat mit hohen Steuern und Umverteilungsmechanismen agiert hätten, waren sie aus der Perspektive Esping-Andersen „nicht wirklich sozialdemokratisch“, weil sich politische Inhalte und Programme historisch betrachtet über einen anderen Pfad entwickelt haben, als es in den anderen sozialdemokratischen Ländern der Fall war. Aufgrund der korporatistischen Merkmale der ,versäulten“ Historie (s. Kapitel 10.4.), bestehe Esping-Andersen darauf, die Niederlande dennoch als konservativen kontinental-korporatistischen Wohlfahrtsstaat zu beschreiben (vgl. Goodin/Smitsman 2000: 41). Je nach Regimetypologie wurden die Niederlande in der Vergangenheit häufig als konservativ kategorisiert: so klassifizierten Lewis (1992) sowie Lewis/Ostner (1994) ${ }^{118}$ die Niederlande als „strong male breadwinner country“, Korpi (2000) als „General-FamilySupport-Typus“ und Pfau-Effinger (2005) kategorisierte dieses Land als „Modernisation of the male breadwinner model“. Neueren Erkenntnissen zufolge dominiert jedoch die Klassifizierung der Niederlande als Mischtypus (vgl. Ostner/Schmitt 2008a $\mathrm{a}^{119}$ : 10; Leitner 2013: 15). Bulgarien, Litauen, Polen, Rumänien, Tschechien und Ungarn werden häufig als postsozialistische Länder gruppiert, die Mischformen zwischen liberalen, markorientierten Regimen und dem ZweiverdienerInnen-Modell darstellen (vgl. Hobson/Oláh 2006: 206). Doch auch zwischen den postsozialistischen Ländern zeichnet

118 „Britain, Germany and the Netherlands may be said to be strong male breadwinner countries; all have tended to treat adult women as dependent wives for the purposes of social entitlements; but they differ as to the extent to which they developed policies, transfers or services to promote marriage and family life, thus, compensating for the risks and disadvantages for women in the male centered labour market” (Lewis/Ostner 1994: 18f.).

119 „As is well-known, Esping-Andersen did not care much about hybrid forms. The Netherlands constitute such a hybrid case with respect to (basic and universal) social provisions for its citizens" (Ostner/Schmitt 2008a: 10). 
sich eine Heterogenität in Bezug auf ökonomische Rahmenbedingungen und familienpolitische Kontextfaktoren ab. Schweden und Norwegen als sozialdemokratisch-skandinavische Typen verfügen insbesondere über stark ausgeprägte Policies des Dual Earner-Modells. Bevor landesspezifische Informationen im Detail erläutert werden, erfolgt zunächst eine kurze Einführung in die historisch bedingten zentralen Unterschiede der ,Cleavages' zwischen kontinentaleuropäischen und skandinavischen Ländern, die im Wesentlichen zur Etablierung unterschiedlicher Wohlfahrtsmodelle geführt haben.

\subsection{Ein historischer Abriss über die Gegensätze der gesellschaftlichen Konfliktlinien zwischen kontinentaleuropäischen und sozialdemokratischen Ländern}

Historisch betrachtet unterscheiden sich die gesellschaftlichen Konfliktlinien (Cleavages $)^{120}$ von skandinavischen und kontinentaleuropäischen Ländern in fundamentaler Weise:

„Während in Skandinavien neben der basalen sozioökonomischen Spaltungslinie
zwischen Arbeit und Kapital ein Stadt/Land-Konflikt virulent geworden ist, sind
auf dem Kontinent neben dem grundlegenden Links/Rechts bzw. Arbeit/Kapital-
Konflikt, der sich in allen entwickelten Industrieländern im Parteiensystem mani-
festiert, auch Staat/Kirche-Konflikte politisch prägend geworden. Als Folge un-
terscheiden sich die nordischen und die kontinentalen Parteiensysteme dahin ge-
hend, dass in den Parteiensystemen Schwedens, Dänemarks, Norwegens und
Finnlands Agrarparteien eine wichtige Position eingenommen haben [...], wäh-
rend in den Parteiensystemen der kontinentalen Länder Parteien des ,religious
defence' (Christdemokratische Parteien) eine zentrale Position besetzen. Solche
Parteien spielen wiederum in den nordischen Ländern keine oder eine nur margi-
nale Rolle" (Manow 2007: 415).

Diese Argumentation führt Manow (2007) zu seiner zentralen These, ,[...] dass - pointiert formuliert - der nordische Wohlfahrtsstaat historisch das Resultat einer ,rot-grünen' Koalition aus Sozialdemokratie und Agrarparteien ist, während der kontinentale Wohlfahrtsstaat das Resultat einer ,rotschwarzen" Koalition aus Christ- und Sozialdemokratie ist" (Manow 2007: 416). Dass in den nordischen Wohlfahrtsstaaten kein Staat/Kirche-Konflikt dominiere, sei auf die Homogenität des Protestantismus in den nordischen Ländern zurückzuführen (vgl. Manow 2007: 420). Dafür sei die Spannungslinie zwischen Stadt und Land bzw. agrarisch und industriellem Sektor umso 
stärker ausgeprägt. Manow (2007) erörtert seine Kritik eines Fehlens einer Begründung, warum sich bestimmte Länder in die Regimetypologie EspingAndersens (1990) einordnen lassen, die er durch die Entwicklung eines Arguments zur historisch-kausalen Fundierung der Regimetypologie aufarbeiten möchte. Im Rahmen seiner Argumentation verbindet er die Bedeutung von Wahlsystemen (bezugnehmend auf Iversen/Soskice (2006)) mit unterschiedlichen gesellschaftlichen Spannungslinien (bezugnehmend auf Lipset/Rokkan (1967)) und zieht die Schlussfolgerung:

„[...] dass ein Mehrheitswahlsystem zu einem liberalen Wohlfahrtsstaat führt, während [sich] in den Ländern mit Verhältniswahlsystem entweder Koalitionen aus Sozialdemokratie und Agrarparteien (Skandinavien) oder aus Sozial- und Christdemokratie (Kontinentaleuropa) für den Ausbau der jeweiligen Wohlfahrtsstaaten verantwortlich zeichneten“" (Manow 2007: 414).

Diese Verknüpfung ermögliche eine Erklärung für die Fragen, welche Art von Mittelklasse-Partei in Kombination mit der Sozialdemokratie für den Ausbau des Wohlfahrtsstaates verantwortlich sei sowie zu welchem Wohlfahrtsstaatstyp die unterschiedlichen politischen Klassenkoalitionen geführt haben. Wesentlich sei dabei ein Fehlen des religiösen Cleavages in den homogenen protestantischen Ländern sowie, dass in den skandinavischen Ländern der Stadt-Land-Konflikt politisiert wurde, ebenso wie der ArbeitKapital-Konflikt einen sozioökonomischen Cleavage darstellte. Hingegen führe der Staat-Kirche-Konflikt in den kontinentaleuropäischen Ländern zu einer in den Parteiensystemen repräsentierten nicht-ökonomischen Spaltungslinie. Rokkan zufolge kann daher der skandinavische Wohlfahrtsstaat als Ergebnis des Zusammenwirkens von zwei politischen Kräften interpretiert werden, deren Entstehung auf die industrielle Revolution zurückzuführen sei, während der kontinentale Wohlfahrtstyp ein Resultat von zwei politischen Kräften sei, die sich aus der industriellen und der nationalen Revolution etabliert haben (vgl. Manow 2007: 423). Als Beispiele des Parteieneinflusses auf die Entwicklung der Wohlfahrtsstaatsmodelle erwähnt Manow die skandinavischen Agrarparteien, die für universale und gegen einkommensabhängige Sozialleistungen plädierten, weil kleine Landwirtschaftsbetriebe fürchten mussten, dass sie von einkommensabhängigen Sozialleistungen nicht profitieren würden. Da Christdemokratische Parteien einen parteiinternen Klassenkompromiss zwischen katholischer Mittelschicht und katholischer ArbeiterInnenschaft ausloten mussten, um keine Stimmen an andere sozialdemokratische oder konservative Parteien zu verlieren, gab es für sie keinen Grund, gegen einkommensbasierte Sozialleistungen zu argumentieren. Manow (2007) führt dies darauf zurück, dass beitragsversicherte Sozialleistungen das Umverteilungsausmaß von vorne herein begrenzen und Umverteilungen gruppenintern zu organisieren versprechen (vgl. Manow 2007: 424). 
Gemäß Manow (2007) lässt sich zwar die Entwicklung von Wohlfahrtsstaaten als Reaktion eines aufkommenden Kapitalismus erklären, doch sollen die landesspezifischen Unterschiede aufgezeigt werden, lassen sich diese nicht aufgrund der kapitalistischen Wirtschaftsweise argumentieren, weil sie gerade die Gemeinsamkeit der Entwicklung von westlichen Industriestaaten darstellen würde. Folglich sei der Erkenntnisgewinn neo-marxistischer Sozialstaatstheorien diesbezüglich begrenzt. Stattdessen plädiert Manow (2007) bezugnehmend auf Rokkan (1999) für die Fokussierung auf Staatsbildungsprozessen immanenten gesellschaftlichen Konfliktlinien, die nicht allein auf eine Arbeit-Kapital-Dimension reduziert werden können. Lipset/Rokkan (1967) zufolge weisen die Cleavages langwierige Folgen dort auf, wo sie sich im Parteiensystem manifestiert haben. Wesentlich sei hierbei nach Manow (2007) die Frage, ,[...] ob ein Verhältniswahlsystem eine solche Verfestigung ermöglicht oder ob ein Mehrheitswahlsystem sie unterdrückt und nur die parteipolitische Repräsentation des basalen Links/Rechts-, Arbeit/Kapital-Konflikts zugelassen hat" (Manow 2007: 426). Vornehmlich waren die zwei Konfliktlinien zwischen Kirche und Staat in Kontinentaleuropa sowie zwischen Stadt und Land in Skandinavien wirkungsvoll (in den Ländern von Nord- und Kontinentaleuropa wurde mit Ausnahme von Frankreich nach dem ersten Weltkrieg das Verhältniswahlrecht eingeführt).

„Die deutlichen Spuren dieser Konflikte sind bis heute in den Parteiensystemen des Nordens und Kontinentaleuropa zu finden - in der Form von vormaligen Agrar- und nun Zentrumsparteien in Skandinavien und in Form von Christdemokratischen Parteien in fast allen Ländern Kontinentaleuropas [...]. Wie hier argumentiert wurde, führte dies zur Bildung zweier unterschiedlicher ProWohlfahrtsstaatskoalitionen - einer rot-grünen im Norden und einer schwarzroten auf dem Kontinent" (Manow 2007: 426).

Nachdem die historischen Pfade der Entwicklung fundamental unterschiedlicher Wohlfahrtsstaatsmodelle konstatiert wurde, wodurch die Typologie von Esping-Andersen (1990) durch eine historisch-kausale Argumentation von Manow (2007) erweitert wurde, werden nachfolgend einige Detailinformationen, die für die Fallauswahl maßgeblich sind, landesspezifisch skizziert. Ziel ist, die institutionelle Vielfalt - vornehmlich in Bezug auf familienpolitische und ökonomische Rahmenbedingungen - zwischen den Ländern schrittweise herauszuarbeiten. Hierbei soll kein Anspruch auf Vollständigkeit bestehen, ferner werden einige wesentliche Entwicklungsphasen für die jeweiligen Länder erläutert. 


\subsection{Das „konservative Regime“ kritisch hinterfragt - Zur inneren Diversität}

Leitner (2013) kritisiert an der Typologie Esping-Andersens, dass sich die von ihm als konservativ klassifizierten Länder stärker unterscheiden und hinterfragt folglich die Zusammenfassung zu einem Typus indem sie - so ihre These - innerhalb der konservativen Länder von unterschiedlichen Varianten des Familialismus ausgeht (vgl. Leitner 2013: 14).

\subsection{1. Österreich: „,Der Prototyp des expliziten Familialismus “ (Leitner 2013: 53)}

Die Entwicklung Österreichs zum expliziten Familialismus wird von Leitner anhand der folgenden prägnanten sozialpolitischen Verläufe beschrieben (vgl. Leitner 2013: 80ff.):

Österreich implementierte bereits früh direkte familiale sozialpolitische Maßnahmen zur Unterstützung familialer Betreuung (für einen Überblick der Entwicklung von Karenzurlaub, Karenzgeld, Rentenanrechnung und SonderNotstandshilfe in Österreich s. Leitner 2013: 82f.; Darstellung im Anhang.). Anfang des 20. Jahrhunderts wurden aufgrund der konservativen Familienideologie Ansprüche in Form von Renten- und Krankenversicherung abgeleitet; Ende der 1950er Jahre wurden direkte Transferleistungen für die familiale Betreuung eingeführt. Folglich konstatiert Leitner (2013) einen Wechsel vom indirekten zum expliziten Familialismus. Einige prägende Einführungen familialistischer Transferleistungen sind:

- Der bezahlte Karenzurlaub (1961), der erwerbstätigen alleinerziehenden Müttern eine Erwerbsunterbrechung für exklusive Kinderbetreuungsarbeit ermöglichen sollte, indem sie dieselben Leistungen wie im Falle einer Arbeitslosigkeit erhielten. Hingegen wurden verheiratete Mütter auf ihren Ernährer-Ehemann verwiesen. Das Karenzgeld basierte insofern für alleinerziehende Mütter auf dem Arbeitslosengeld, verheiratete Mütter erhielten hingegen maximal die Hälfte des Arbeitslosengeldes unter Anrechnung des Haushaltseinkommens.

- Die Modifikation des Karenzgeldes sowie die Einführung der SonderNotstandshilfe 1974, durch die alleinerziehende Mütter weiterhin privilegierte Transferleistungen erhielten. Die Sonder-Notstandshilfe ermöglichte es ihnen fortan ihre Erwerbstätigkeit bis zum dritten Lebensjahr des Kindes zu unterbrechen, wobei sich die Höhe in Übereinstimmung mit der Arbeitslosenversicherung am vorangegangenen Erwerbseinkommen orientierte. Hingegen wurde das Karenzgeld in „kleines“ und „großes“ Karenzgeld differenziert und als einkommens- 
unabhängige Pauschale ohne Anrechnung des Haushaltseinkommens konzipiert. Jedoch wurden durch das große Karenzgeld alleinerziehende Mütter und verheiratete Mütter mit gering verdienendem Ehemann gleichgestellt, indem beide im Vergleich zum kleinen Karenzgeld einen 50\% Zuschlag erhielten. Eine Gleichstellung zwischen Alleinerziehenden und verheirateten Müttern mit gering verdienendem Ehemann in Bezug auf die Sonder-Notstandshilfe erfolgte erst 1989; zuvor kam diese Maßnahme lediglich den Alleinerziehenden zugute.

Parteiideologische Konflikte zwischen SozialdemokratInnen, die einen schnellen Wiedereinstieg von Müttern in den Beruf befürworteten und ChristdemokratInnen, die sich für eine möglichst lange Erwerbsunterbrechung zugunsten der Kinderbetreuung einsetzten, wurden lange Zeit durch eine diplomatische Kompromissgesetzgebung besänftigt: Die Kindererziehung wurde nur für zuvor erwerbstätige Mütter abgesichert, Transferleistungen für die Erwerbsunterbrechung wurden mit dem Ernährermodell verknüpft und ein Ausbau institutioneller Kinderbetreuung wurde verhindert. Leitner (2013) erachtet auch die 1990 eingeführte Väterkarenz als Kompromisslösung, die mit einer zeitlichen Verlängerung - vom vormals bis zum ersten Lebensjahr des Kindes auf die Phase bis zum zweiten Lebensjahr des Kindes - einherging. Werden jedoch die empirischen Umsetzungen der wahrgenommenen Karenzurlaube betrachtet, zeigt sich eine konservative Entwicklung in Form eines geschlechtsspezifischen Bias: „Nach wie vor stiegen fast ausschließlich Mütter aus der Erwerbstätigkeit aus, dafür nun aber doppelt so lange wie vorher" (Leitner 2013: 84). Zusätzlich wurden die familialistischen Strukturen durch die Ausweitung der Sonder-Notstandshilfe (1989) auch für verheiratete Frauen mit gering verdienendem Ehemann sowie die 1993 eingeführte Anrechnung von Kinderbetreuungserziehungszeiten auf die Rente gestärkt. Gleichwohl es eine kurze Phase der Kürzungen von familialistischen Maßnahmen aufgrund eines hohen Finanzierungsdrucks in den 1990er Jahren kam, wodurch ein kurzweiliger „Erwerbszwang“ für Mütter entstand, wurde 2002 das Kinderbetreuungsgeld eingeführt. Dieses ermöglichte allen Müttern - sowohl erwerbstätigen als auch nicht erwerbstätigen - die Abgeltung der Betreuung bis zum 30. Lebensmonats des Kindes. 2008/2009 wurde die Möglichkeit gewährt, die Bezugsdauer des Kinderbetreuungsgeldes zu verkürzen und dafür eine höhere Pauschale zu erhalten. Zusätzlich wurde im Sinne eines modernisierten Ernährermodells - die Möglichkeit einer geringfügigen oder Teilzeitbeschäftigung unter Beibehalt des Kinderbetreuungsgeldes geboten. Aktuell ist die Erwerbsquote von Frauen mit Kinder unter sechs Jahren mit 69,8\% im Jahr 2016 ziemlich hoch (EU-Durchschnitt: $61,4 \%$ ), wobei viele Frauen (47,1\% im Vergleich zum EU-Durchschnitt von $31,9 \%$ ) einer Teilzeitbeschäftigung nachgehen. Die derzeitigen Institutionen zur flexiblen Gestaltung der Arbeitszeit ermöglichen den Eltern eine Teilzeitarbeit bis zum siebten Lebensjahr des Kindes, sofern sie in Betrieben mit 
mehr als 20 Beschäftigten angestellt sind und mindestens drei Jahre ununterbrochen in diesem Betrieb beschäftigt waren. Die Verkürzung der Arbeitszeit muss mindestens $20 \%$ der vorherigen Arbeitszeit umfassen. Zusätzlich besteht das Recht, die Arbeitszeit kurzfristig innerhalb eines Tages (beispielsweise von vormittags auf nachmittags) zu ändern ohne den Stundenumfang zu reduzieren sowie das Recht, nach der Teilzeitphase anschließend wieder in Vollzeit erwerbstätig zu sein (vgl. Europäische Kommission 2018: o. S.).

Derzeit ist eine der wichtigsten finanziellen universalen Leistungen in Österreich die Familienbeihilfe, die unabhängig von der Erwerbstätigkeit der Eltern gewährt wird. Je nach Alter des Kindes variiert sie zwischen $114 € \mathrm{im}$ Monat für Neugeborene und 165,10 € im Monat für Kinder ab 19 Jahren. Außerdem wurde das Kinderbetreuungsgeld kürzlich modifiziert, das fortan für Kinder gilt, die ab März 2017 geboren sind (vgl. Europäische Kommission 2018: o. S.). Es kann aus zwei Systemen gewählt werden:

1. Kinderbetreuungsgeld-Konto (Pauschalsystem) oder

2. einkommensabhängiges Kinderbetreuungsgeld

Die Wahl ist bei der erstmaligen Antragsstellung zu entscheiden (eine Änderung ist nur binnen der ersten 14 Tage nach der Antragstellung möglich) und ist auch für den zweiten Elternteil verbindlich (vgl. BMFJ 2018: o. S.).

„Die bisherigen vier Optionen mit festgelegten Zuschüssen werden in ein soge-
nanntes Kinderbetreuungsgeld-Konto umgewandelt. Das einkommensabhängige
Kinderbetreuungsgeld bleibt unverändert. In dem neuen, sehr flexiblen Konten-
schema kann die Bezugsdauer des Kinderbetreuungsgeldes zwischen 365 und
851 Tagen liegen. Wenn beide Elternteile Kinderbetreuungsgeld beanspruchen,
kann die Bezugsdauer des Kinderbetreuungsgeldes zwischen 456 und $1063 \mathrm{Ta}-$
gen liegen. Das bedeutet, dass $20 \%$ der gesamten Anspruchsdauer [91 Tage in
der kürzesten Variante] für ein Kind dem anderen Elternteil vorbehalten sind. Ei-
ne Übertragung dieses Teils der Anspruchsdauer ist nicht möglich. Die Höhe des
Kinderbetreuungsgeldes richtet sich nach dem von den Eltern gewählten Zah-
lungsmodell [und beträgt je nach Variante zwischen 14,53€ und $33,88 €$ am Tag]
"(Europäische Kommission 2018: o. S.).

Das einkommensabhängige Betreuungsgeld beträgt $80 \%$ des vorherigen Einkommens, maximal werden jedoch $66 €$ täglich gestattet (rd. $2000 €$ monatlich). Sofern nur ein Elternteil das einkommensabhängige Betreuungsgeld bezieht, wird im längsten Fall eine Bezugsdauer bis zum 365. Tag ab der Geburt des Kindes gewährt. Nehmen beide Elternteile das einkommensabhängige Betreuungsgeld in Anspruch, verlängert sich die Bezugsdauer um die Zeit, die vom anderen Elternteil bezogen wurde, wobei eine Begrenzung auf maximal 426 Tage ab der Geburt des Kindes festgelegt ist (es können nie mehr als 365 Tage von einem Elternteil einkommensabhängiges Elterngeld bezogen werden). Außerdem ist jedem Elternteil eine unübertragbare Anspruchsdauer von 61 Tagen vorbehalten (vgl. BMFJ 2018: o. S.). Unabhängig 
davon, ob sich die Eltern für das pauschale Kinderbetreuungsgeldkonto oder für die einkommensabhängige Variante entschieden haben, können sich die Eltern beim Bezug maximal zwei Mal abwechseln, wodurch sich höchstens drei Blöcke ergeben, die jeweils mindestens durchgehend 61 Tage eingehalten werden müssen. Grundsätzlich ist es nicht möglich, dass beide Eltern gleichzeitig Kinderbetreuungsgeld beziehen (Ausnahme: beim erstmaligen Bezugswechsel werden beiden Eltern gleichzeitig max. 31 Tage ermöglicht) (vgl. BMFJ 2018: o. S.). Ebenfalls besteht seit März 2017 eine Möglichkeit des „Partnerschaftsbonus“, wenn sich beide Eltern die Anspruchsdauer des Kinderbetreuungsgeldes egalitär aufteilen (50:50/40:60) und das Kinderbetreuungsgeld mindestens im Ausmaß von jeweils 124 Tagen beziehen. In diesem Fall erhalten sie beide einen einmaligen zusätzlichen Bonus von $500 €$ (1000€ insgesamt für beide Elternteile). Des Weiteren gibt es den „Familienzeitbonus" für Väter, die zugunsten der Familie ihre Erwerbstätigkeit unterbrechen. Binnen 91 Tage nach der Geburt wird für 28, 29, 30 oder 31 Tage am Stück ein Bonus von 22,60€ täglich vergütet. Insgesamt belaufen sich die Leistungen für Familien im Jahr 2014 auf 2,8\% des Bruttoinlandsprodukts, die folglich oberhalb des EU-Durchschnitts (2,4\%) liegen (vgl. Europäische Kommission 2018: o. S.). Nach wie vor bleiben trotz dieser Maßnahmen defamilisierende Maßnahmen eher schwach ausgeprägt: die Versorgungsquote mit Betreuungsplätzen für Kinder unter drei Jahren ist gering (für einen Überblick der Versorgungsquoten der 0- bis 2-Jährigen s. Tabelle $30 \mathrm{im} \mathrm{An-}$ hang: im Jahr 2015 beträgt sie gemäß Europäischer Kommission 22,3\% im Vergleich zu 30,3\% im EU-Durchschnitt, jedoch ist die Versorgungsquote von Kindern zwischen drei und sechs Jahren mit 85,3\% etwas höher als der EU-Durchschnitt mit 83,3\%), nur ein Drittel aller Kindergartenplätze werden ganztägig angeboten und der Hortbereich ist ebenfalls schwach ausgeprägt.

„Der explizit familialistische und geschlechtsspezifisch diskriminierende Charakter der österreichischen Kinderbetreuungspolitik hat sich somit zwar im Laufe der Zeit gewandelt, ist aber letztendlich in Form des teilmodernisierten Ernährermodells bis heute erhalten geblieben" (Leitner 2013: 84, Hervorhebungen im Original; die Verf.).

\section{Ökonomische Rahmenbedingungen}

Für eine allgemeine Einordnung der ökonomischen Rahmenbedingungen Österreichs sind einige wesentliche Faktoren, wie das Bruttoinlandsprodukt, Armutsindikatoren, der Gini-Index und der Gender-Pay-Gap zu berücksichtigen (s. Abbildung 21 bis Abbildung 28 im Anhang). Anhand des Bruttoinlandsprodukts pro Kopf (gemessen in Kaufkraftstandards KKS bzw. in engl. PPP $^{121}$, um die unterschiedlichen Preisniveaus zwischen den Ländern zu

121 „GDP per capita based on purchasing power parity (PPP). PPP GDP is gross domestic product converted to international dollars using purchasing power parity rates. An international dollar has the same purchasing power over GDP as the U.S. dollar has in the United 
kompensieren) ist festzustellen, dass die Wirtschaftsleistung Österreichs im Vergleich zu den restlichen Ländern auf sehr hohem Niveau liegt. Im Zeitverlauf zwischen 2001 und 2016 ist ein konstanter Anstieg des Bruttoinlandsprodukts zu konstatieren, wobei lediglich im Zeitraum der Finanzkrise zwischen 2008 und 2009 ein leichter Rückgang zu verzeichnen ist (vgl. Weltbank 2018: o. S.).

Für die Messung von Armut existieren unterschiedliche Kennzahlen, die teils mehr, teils weniger geeignet sind, um europäische Unterschiede zu erfassen: Die von der Weltbank festgelegte Armutsschwelle basiert auf der Idee, dass Personen als absolut arm gelten, die über weniger als 1,90 US\$ am Tag verfügen. Es handelt sich hierbei um ein willkürliches Maß zur Bestimmung des Ausmaßes extremer Armut im weltweiten internationalen Vergleich. Für eine Gewährung der Vergleichbarkeit wird die Kaufkraft des USDollars in die lokale Kaufkraft umgerechnet. Folglich sind nach diesem Indikator als extrem arm definierte Personen nicht fähig, täglich Güter zu erwerben, die in den USA 1,90\$ kosten würden (vgl. BMZ 2018: o. S.) - in Österreich betrifft dies im Jahr 2015 0,5\% der Bevölkerung (vgl. Weltbank 2018: o. S.). Da im europäischen Vergleich nur sehr geringe Unterschiede zwischen den Ländern festzustellen sind und der Anschein erweckt wird, als wäre nur ein sehr geringer Anteil der Bevölkerungen von absoluter Armut betroffen, werden für europäische Darstellungen meist Armutsindikatoren, wie die Quote der von Armut bedrohten Personen nach der Armutsgefährdungsgrenze, die Rate der erheblichen materiellen Deprivation und die von Armut und sozialer Ausgrenzung bedrohte Bevölkerung verwendet.

„Die Armutsgefährdungsquote ist der Anteil der Personen mit einem verfügbaren
Äquivalenzeinkommen (nach Sozialtransfers) ${ }^{122}$ unterhalb der Armutsgefähr-
dungsschwelle, die bei $60 \%$ des nationalen verfügbaren Median äquivalenzein-
kommens [sic!] (nach Sozialtransfers) liegt. Dieser Indikator misst nicht den
Wohlstand oder die Armut, sondern ein (im Vergleich zu anderen Personen im
gleichen Land) niedriges Einkommen, das nicht zwangsläufig mit einem niedri-
gen Lebensstandard gleichzusetzen ist“ (Eurostat 2018a: o. S.).

States. GDP at purchasers prices is the sum of gross value added by all resident producers in the economy plus any product taxes and minus any subsidies not included in the value of the products. It is calculated without making deductions for depreciation of fabricated assets or for depletion and degradation of natural resources. Data are in constant 2011 international dollars" (Weltbank 2018: o. S.).

122 „Das verfügbare Äquivalenzeinkommen ist das Gesamteinkommen eines Haushalts nach Steuern und anderen Abzügen, das für Ausgaben und Sparen zur Verfügung steht, geteilt durch die Zahl der Haushaltsmitglieder, umgerechnet in Erwachsenenäquivalente; zur Ermittlung der Erwachsenenäquivalente wird eine Gewichtung der Haushaltsmitglieder nach ihrem Alter nach der modifizierten OECD-Äquivalenzskala vorgenommen $[1,0=$ für das erste erwachsene Haushaltsmitglied; 0,5 = für das zweite erwachsene Haushaltsmitglied und alle weiteren Personen im Alter von 14 Jahren und älter; 0,3 = für Kinder unter 14 Jahren]“ (Eurostat 2018a: o. S.). 
In Österreich gelten 2017 14,4\% der Bevölkerung als armutsgefährdet, ferner hat sich die Armutsgefährdung seit 2001 (12\%) geringfügig erhöht. Der größte Anstieg ist hierbei während der Finanzkrise zwischen 2007 und 2008 von $12 \%$ auf $15,2 \%$ vorzufinden. Ein ähnlicher Trend ist für die erhebliche materielle Deprivation ${ }^{123}$ festzustellen. Auch in diesem Fall lassen sich die Auswirkungen der Finanzkrise anhand eines Anstiegs der Armut erkennen. 2007 betrug die Rate der erheblichen materiellen Deprivation lediglich 3,3\%, 2008 hingegen 5,9\% (vgl. Eurostat 2018a: o. S.). Insgesamt ist in Österreich sowohl die Quote der von Armut bedrohten Personen nach der Armutsgefährdungsgrenze als auch die Rate der erheblichen materiellen Deprivation auf konstant niedrigem Niveau. Eine weitere Ergänzung ist der Indikator der von Armut und sozialer Ausgrenzung bedrohten Bevölkerung, der die Gruppe der Armutsgefährdeten sowie die Gruppe der erheblich materiell Deprivierten umfasst und zusätzlich Personen in Haushalten mit keiner oder sehr niedriger Erwerbsintensität berücksichtigt. Folglich gelten Personen im Sinne dieses Indikators als von Armut und sozialer Ausgrenzung betroffen, wenn:

1. ihr verfügbares Äquivalenzeinkommen unterhalb der Armutsgefährdungsschwelle liegt,

2. sie vier der festgelegten Merkmale der erheblichen materiellen Deprivation aufweisen,

3. sie im Erwerbsalter zwischen 18 und 59 Jahre alt sind und in einem Haushalt mit geringer Erwerbsintensität leben. Eine geringe Erwerbsintensität liegt in Haushalten vor, in denen im Erwerbsalter be-

123 „Materielle Deprivation umfasst zum einen die wirtschaftliche Belastung und zum anderen den Mangel an langlebigen Gebrauchsgütern und ist definiert als die unfreiwillige Unfähigkeit (im Unterschied zur Wahlfreiheit), für folgende Ausgaben aufkommen zu können: unerwartete Ausgaben, einen einwöchigen Jahresurlaub an einem anderen Ort, jeden zweiten Tag eine Fleisch-, Geflügel- oder Fischmahlzeit, angemessene Beheizung der Wohnung, langlebige Gebrauchsgüter wie Waschmaschine, Farbfernseher, Telefon oder Auto, Schulden (Hypotheken- oder Mietschulden, Rechnungen für Versorgungsleistungen (Strom, Wasser, Gas), Mietkaufraten oder sonstige Kreditzahlungen). Die Quote der materiellen Deprivation ist ein Indikator der EU-SILC, der die Unfähigkeit ausdrückt, sich verschiedene Ausgaben leisten zu können, die von den meisten Menschen als für eine angemessene Lebensführung wünschenswert oder gar notwendig angesehen werden. Bei diesem Indikator wird unterschieden zwischen Personen, die sich bestimmte Waren oder Dienstleistungen nicht leisten können, und Personen, die diese Waren oder Dienstleistungen aus einem anderen Grund nicht besitzen - etwa weil sie sie bewusst nicht wollen oder nicht benötigen. Mit diesem vom Ausschuss für Sozialschutz festgelegten Indikator wird der prozentuale Anteil der Bevölkerung gemessen, der für mindestens drei der neun folgenden Ausgaben nicht aufkommen kann: 1. Hypotheken- oder Mietschulden oder Rechnungen für Versorgungsleistungen; 2. angemessene Beheizung der Wohnung; 3. unerwartete Ausgaben; 4. regelmäßige fleisch- oder eiweißhaltige Mahlzeiten; 5. Urlaubsreisen; 6. Fernseher; 7. Waschkessel; 8. Auto; 9. Telefon.

Die Quote der erheblichen materiellen Deprivation ist definiert als die unfreiwillige Unfähigkeit, für mindestens vier der vorstehend genannten Ausgaben aufkommen zu können“ (Eurostat 2018a: o. S., Hervorhebungen im Original; de Verf.). 
findliche Personen weniger als 20\% ihres Erwerbspotentials erwerbstätig sind (vgl. Eurostat 2018a: o. S.).

Alle drei Merkmale können in Kombination auftreten, weshalb die Anzahl der von Armut und sozialer Ausgrenzung bedrohten Bevölkerung geringer ist als die jeweilige Summe der einzelnen Indikatoren. In Österreich sind aktuell (2017) 18,1\% der Bevölkerung von Armut und sozialer Ausgrenzung betroffen (vgl. Eurostat 2018a: o. S.).

Ein weiteres relevantes Maß für die Einordnung der ökonomischen Rahmenbedingungen, das Auskunft über die Einkommensverteilung innerhalb eines Landes gibt, ist der Gini-Index ${ }^{124}$. In einem allgemeineren Sinne ist der Gini-Koeffizient ein Maß zur Beschreibung von Ungleichheitsverteilungen, dass sich nicht nur für die Darstellung von Einkommens-, sondern beispielsweise auch für Vermögensverteilungen eignet. Der Wertebereich liegt zwischen 0 und 1 , wobei 0 eine maximale Gleichheit und 1 eine maximale Ungleichheit bedeutet. In Österreich hat sich der Gini-Index in den vergangenen Jahren seit 2010 kaum verändert, die Werte schwanken lediglich sehr geringfügig zwischen 30,5\% und 30,8\% und liegen damit im Vergleich zu den anderen Ländern in einem mittleren Bereich (vgl. Weltbank 2018: o. S.).

Im Hinblick auf mögliche Geschlechterunterschiede stellt sich die Frage, wie der durchschnittliche Bruttostundenlohn zwischen den Geschlechtern variiert, was mittels des Gender Pay Gaps ${ }^{125}$ erfasst werden kann. Österreich liegt im Zeitverlauf zwischen 2001 und 2016 auf sehr hohem Niveau, gleichwohl seit 2006 ein rückläufiger Trend (von 25,5\% auf 21,1\%) zu verzeichnen ist. Zwischen 2005 und 2006 kann ein exorbitanter Anstieg des Gender Pay Gaps von $18 \%$ auf 25,5\% beobachtet werden. Wird der mittlere Bruttostundenlohn für das Jahr 2016 betrachtet, ist zu konstatieren, dass der Bruttostundenlohn der Frauen um 20,1\% unterhalb denen der Männer liegt (vgl. UNECE 2018: o. S.).

124 „Gini index measures the extent to which the distribution of income (or, in some cases, consumption expenditure) among individuals or households within an economy deviates from a perfectly equal distribution. A Lorenz curve plots the cumulative percentages of total income received against the cumulative number of recipients, starting with the poorest individual or household. The Gini index measures the area between the Lorenz curve and a hypothetical line of absolute equality, expressed as a percentage of the maximum area under the line. Thus a Gini index of 0 represents perfect equality, while an index of $100 \mathrm{im}$ plies perfect inequality" (Weltbank 2017: o S.).

125 „Gender pay gap is the difference between men's and women's average earnings from employment, shown as a percentage of men's average earnings. [...] Gender Pay Gap in hourly wage rates refers to the gender gap in average hourly earnings. This indicator aims to capture the difference between men's and women's overall position in the labor market. It measures the difference between men's and women's wage rates independent of the number of hours worked, the type of activity or the type of occupation" (UNECE 2018: o. S.). 


\subsubsection{Deutschland: „Vom impliziten über den expliziten zum optionalen Familialismus " (Leitner 2013: 91)}

Leitner (2013) beschreibt in Anlehnung an ihre bereits dargelegte Typologie die Entwicklung der Kinderbetreuungspolitik in folgenden Phasen (vgl. Leitner 2013: 123f.):

Die Epoche bis nach dem zweiten Weltkrieg wird als impliziter Familialismus definiert, weil weder familisierende noch de-familisierende Maßnahmen existiert haben. Es war eine Zeit der geschlechtsspezifischen Diskriminierung, da Kinderbetreuung biologisch-natürlich bedingt reine Frauenarbeit war.

Eine Transformation vom impliziten zum expliziten Familialismus wurde durch die Einführung des Ehegattensplittings und die Reform der Witwenrente in den 50er Jahren ausgelöst. Durch den staatlichen Ausbau der sozialen Rechte nichterwerbstätiger Frauen (ermöglicht durch ein hohes Wirtschaftswachstum) wurde implizit das männliche Ernährermodell gefördert, das sich folglich als dominierendes Gesellschaftsmodell manifestierte. Zugleich wurde von der damaligen Bundesregierung (CDU/CSU) ein konservatives Familienbild vertreten und familienpolitisch umgesetzt. In den 80er Jahren wurde der implizite durch den expliziten Familialismus abgelöst, da zunehmend familisierende Strukturen eingeführt wurden - Kinderbetreuung wurde zur strukturell bedingten Frauenarbeit. Das Motto lautete „Familie oder Beruf“ (Leitner 2013: 123, Hervorhebungen im Original; die Verf.). Gleichwohl sollten Frauen (und Mütter) zunehmend (Teilzeit) Erwerbstätige sein, was den Wandel vom traditionellen zum modernisierten Ernährermodell aufzeigt. Leitner beschreibt den Regierungswechsel 1982 als prägend für einen geschlechtsspezifischen diskriminierenden Familialismus, weil die CDU als neue Koalitionspartei im Rahmen eines Drei-Phasen-Modells der weiblichen Erwerbstätigkeit von einer langen Phase ausschließlicher Kinderbetreuungsarbeit ausging. Hingegen vertrat die SPD als vorherige Regierungspartei der 70er die Ideologie einer Gleichzeitigkeit von Kinderbetreuung und (Teilzeit) Erwerbstätigkeit.

Erst ab den 1990er Jahren wurde zunehmend die Gleichzeitigkeit von Familie und Beruf gefördert, wobei eine Integration der Väter in die Kinderbetreuung durch die Erziehungsurlaubsreform 2001 angestrebt wurde. Die strukturelle Implementation des lohnbezogenen Elterngeldes in Kombination mit einer individualisierten Väter-Elternzeit (für einen Überblick der gesamten bezahlten Elternzeit einschließlich Mutterschutz, Vaterschaftsurlaub und Elternzeit, differenziert nach Geschlecht basierend auf der Datenbank der OECD s. Tabelle 32 im Anhang) spiegelt den Versuch einer Neuordnung der Geschlechterrollen wieder, wobei zugleich indirekt familisierende Leistungen fortbestanden oder auch neu eingeführt wurden, wie z. B. das 2013 eingeführte Betreuungsgeld. Diese Unentschiedenheit zwischen familisierenden 
und de-familisierenden Maßnahmen lässt sich gemäß Leitner durch die unterschiedlichen ideologischen Grundhaltungen der Parteien erklären. Dennoch ist seit den 1990er Jahren ein zunehmender Ausbau de-familisierender Maßnahmen zu verzeichnen. Die Orientierung am sozialdemokratischen Modell wird z. B in der Beschreibung des deutschen Länderprofils der Europäischen Kommission deutlich: „Investitionen in nachhaltige Netzwerke zur Unterstützung der Selbstbestimmung und des Wohlbefindens von Kindern in einem sozialdemokratischen Wohlfahrtsstaat" (Europäische Kommission 2018: o. S.). Das „ElterngeldPlus“ stellt eine solche de-familisierende Maßnahme dar, weil es eine egalitäre Arbeitsteilung zwischen beiden Elternteilen erzielt. Aktuell stehen insgesamt 14 Monate Elterngeldbezug für beide Elternteile gemeinsam zur Verfügung, wenn sich beide an der Betreuung beteiligen und sie durch die Kinderbetreuung Einkommen einbüßen. Pro Elternteil können mindestens zwei und höchstens zwölf Monate in Anspruch genommen werden. Die Höhe des Elterngeldes ist einkommensabhängig (bei niedrigem Einkommen $100 \%$, bei hohem Einkommen $65 \%$ ), wobei sie mindestens 300 $€$ und maximal $1800 €$ monatlich beträgt. Durch das „ElterngeldPlus“ besteht die Möglichkeit, dass Eltern doppelt so lange Elterngeld beziehen (bei maximal halber Höhe des Elterngeldes), wenn sie bereits während des Elterngeldbezugs in Teilzeit arbeiten möchten. Im Falle eines egalitären Zeitarrangements wird ein zusätzlicher Partnerschaftsbonus gewährt: Wenn Eltern gleichzeitig zwischen 25 und 30 Wochenstunden arbeiten, erhalten sie zusätzlich vier ElterngeldPlus-Monate (gilt auch für getrennt erziehende Eltern, die zur gleichen Zeit in Teilzeit arbeiten; Alleinerziehenden steht der gesamte Partnerschaftsbonus zu) (vgl. BMFSFJ 2018: o. S.). Im Hinblick auf öffentliche Kinderbetreuungsmöglichkeiten gilt es nach wie vor Unterschiede zwischen Ost- und Westdeutschland zu berücksichtigen:

„Während die Versorgungsquoten in Ostdeutschland - aufgrund des Erbes der ehemaligen DDR - in allen Altersgruppen vergleichsweise hoch liegen, kann in Westdeutschland nur für die Gruppe der Kindergartenkinder von einer guten, allerdings nur halbtägigen Versorgung ausgegangen werden. Somit weisen die neuen Bundesländer einen optionalen Familialismus auf, während sich die alten Bundesländer noch in der Phase des Übergangs vom expliziten zum optionalen Familialismus befinden" (Leitner 2013: 124, Hervorhebungen im Original; die Verf.).

In Gesamtdeutschland beträgt gemäß den Daten der Europäischen Kommission der Anteil der Kinder im Alter von 0-3 in öffentlichen Kinderbetreuungseinrichtungen im Jahr 2015 25,9\% (EU-Durchschnitt: 30,3\%) und der Anteil der Kinder im Alter von drei Jahren bis zum schulpflichtigen Alter 89,6\% (EU-Durchschnitt: 83,3\%) (vgl. Europäische Kommission 2018: 0. S.). 69,9\% der Frauen und 78,0\% der Männer sind im Jahr 2015 erwerbstätig, wobei die Teilzeitquote der Frauen 46,6\% beträgt. Die Geburtenrate ist 2015 mit 1,5 Kindern je Frau eher niedrig (vgl. Eurostat 2017: o. S.). 


\section{Ökonomische Rahmenbedingungen}

Das durchschnittliche Bruttoinlandsprodukt pro Kopf beläuft sich im Jahr 2016 auf 48860,53 KKS, weshalb die Wirtschaftsleistung Deutschlands als sehr hoch zu klassifizieren ist. Während der Finanz- und Wirtschaftskrise ist lediglich ein sehr leichter Rückgang zwischen 2008 und 2009 zu beobachten, der schnell wieder kompensiert werden konnte (vgl. Weltbank 2018: o. S.). Doch zeigt sich im Zeitverlauf zwischen 2001 und 2015 ein markanter Anstieg der Armutsgefährdung, der in den Jahren 2016 und 2017 geringfügig um 0,6 Prozentpunkte rückläufig ist. Aktuell (2017) gelten 16,1\% der deutschen Bevölkerung als armutsgefährdet. Von erheblich materieller Deprivation sind mit 3,4\% deutlich weniger Personen betroffen. Wird zusätzlich eine geringe Erwerbsbeteiligung berücksichtigt, ist festzustellen, dass im Jahr 2017 19\% der Bevölkerung Armut und soziale Ausgrenzung erleiden (vgl. Eurostat 2018a: o. S.). Im Vergleich zu den anderen - hier dargestellten Ländern liegt Deutschland in Bezug auf den aktuellsten Gini-Index von 31,7\% (2015) im Mittelfeld. Es zeichnet sich seit 2010 ein Trend einer leichten Zunahme der Ungleichverteilung der Einkommen ab (vgl. Weltbank 2018: o. S.). Was die Genderdimension der Bruttostundenlöhne anbelangt, ist ein sehr hoher Gender Pay Gap von 21,5\% zu Ungunsten von Frauen zu verzeichnen (vgl. UNECE 2018: o. S.).

\subsubsection{Frankreich: „Vom strukturellen De-Familialismus zum expliziten und zum optionalen Familialismus “ - ,Optionaler Familialismus als Politikerbe“"(Leitner 2013: 134)}

Leitner fasst für Frankreich vier Entwicklungsphasen der Kinderbetreuungspolitik zusammen (vgl. Leitner 2013: 149):

Bis 1930 ist auf der strukturellen Ebene eine Variante des DeFamilialismus in Frankreich durch die „école maternelle“ (eine kostenlose Vorschuleinrichtung) vorzufinden, indem ein Betreuungsangebot für Vorschulkinder geschaffen wurde, das faktisch jedoch nicht als Betreuungsangebot genutzt wurde. Die Erwerbsquote von Müttern war trotz ,école maternelle" gering und es dominierte ein traditionelles Familienbild, weshalb Leitner die erste Phase als ,geschlechtsspezifisch segregierten impliziten Familialismus" bezeichnet (Leitner 2013: 149, Hervorhebungen im Original; die Verf.). Den Ausbau der école maternelle versteht sie mehr als Bildungshoheit des Staates gegenüber der Kirche, was einer Bildungspolitik anstelle einer Betreuungspolitik gleichkommt.

In den 1930er Jahren wurden familisierende Maßnahmen infolge natalistischer Bewegungen (u. a. angetrieben durch die katholischen Frauenorganisation UFCS) eingeführt, die Müttern eine Nicht-Erwerbstätigkeit ermöglichen sollten. Erzielt wurde hierdurch, 1. dass Frauen ihrer „natürlich- 
biologischen" Rolle als Mutter nachgehen konnten, 2. eine Erhöhung der Fertilität und 3. eine Entlastung des Arbeitsmarktes durch die Erosion von Frauen aus der Erwerbstätigkeit.

In der dritten Phase ab Ende der 1960er fand der indirekte explizite Familialismus durch die Schließung der "allocation des salaire unique“ (die „ASU“ war eine staatliche Förderung des männlichen Alleinverdienermodells, da Familien mit einem Einkommen eine finanzielle staatliche Beihilfe erhielten) einen Wiederaufschwung, der auf struktureller Ebene zunehmend de-familisierende Maßnahmen beinhaltete. Im Ausbau befindliche Kinderbetreuungseinrichtungen wurden vermehrt zur Vereinbarung von Familie und Beruf genutzt und das traditionelle Familienbild erodierte.

Die vierte Phase setzte Mitte der 1980er Jahre ein: Implementationen von familisierenden sowie de-familisierenden Maßnahmen lösten eine Transformation in Richtung eines optionalen Familialismus aus. Einerseits dominierten natalistische und arbeitsmarktpolitische Intentionen die ErziehungsgeldPolitik, andererseits trieben die Reaktionen auf einen vermehrten Bedarf an Kinderbetreuungseinrichtungen für unter dreijährige Kinder in ihrer Kombination mit finanzpolitischen Restriktionen sowie arbeitsmarktpolitischer Ziele den Ausbau von Kinderbetreuungseinrichtungen voran (vgl. Leitner 2013: 150). Vielfältige Betreuungsmöglichkeiten sowie relativ hohe Beschäftigungsquoten von Frauen mit Kindern unter sechs Jahren (61,8\% in Frankreich im Vergleich zu 61,4\% in der EU) sind im Jahr 2016 vorzufinden. Die Erwerbsquote von Frauen entspricht 2016 in etwa dem EU-Durchschnitt (60,9\% in Frankreich, 61,4\% in der EU), wobei $29,8 \%$ der Frauen in Teilzeit beschäftigt sind (EU-Durchschnitt: 31,9\%). Im Jahr 2015 sind 41,7\% der Kinder unter drei Jahren in einer offiziellen Kinderbetreuungseinrichtung untergebracht (EU-Durchschnitt: 30,3\%). Ab drei Jahren (bzw. in einigen benachteiligten Gemeinden bereits ab 2 Jahren) wird ein Zugang zur „Ecole maternelle“ gewährt, ,[...] die von $98 \%$ der Kinder (100\% ab 4 Jahren) genutzt wird, manchmal auch nur auf Teilzeitbasis (vormittags)" (Europäische Kommission 2018: o. S.). Die Unterstützung von Familien durch eine möglichst umfassende Familienpolitik könnte ein Grund für die hohe Fertilitätsrate in Frankreich sein (1,95 Kinder pro Frau im Vergleich zu 1,57 im EUDurchschnitt basierend auf dem Jahr 2016 und den Daten der Europäischen Kommission). Die gesamte bezahlte Elternzeit umfasst im Jahr 2015 in Frankreich für Frauen 42 Wochen, davon sind 16 Wochen für den Mutterschutz und 26 Wochen für die für Frauen zur Verfügung stehende Elternzeit angesetzt. Väter haben einen Gesamtanspruch auf 28 Wochen, davon 2 Wochen für den Vaterschaftsurlaub und 26 für die Elternzeit (vgl. OECD 2018: o. S.). Insgesamt betragen die finanziellen Leistungen für Familien 2,5\% des Bruttoinlandsprodukts (EU-Durchschnitt: 2,4). Im spezifischen ermöglicht das Erziehungsgeld 
„[...] vormals erwerbstätigen Müttern den zeitweisen Ausstieg aus der Erwerbstätigkeit, und der Ausbau der Kleinkinderbetreuung durch Tagesmütter und Kinderfrauen prägt den Charakter des französischen optionalen Familialismus bis heute. Er weist starke schichtspezifische Differenzierungen auf und ist nach wie vor geschlechtersegregierend angelegt" (Leitner 2013: 150).

\section{Ökonomische Rahmenbedingungen}

Die Wirtschaftsleistung Frankreichs gemessen anhand des Bruttoinlandsprodukts pro Kopf in KKS liegt durchaus in einem guten Bereich, wenngleich sie niedriger als in Österreich und Deutschland ist (vgl. Weltbank 2018: o. S.). Im Hinblick auf die Quote der von Armut bedrohten Personen nach der Armutsgefährdungsgrenze trifft dies gemäß den aktuellsten Daten von 2017 auf 13,3\% der Bevölkerung zu. 4,1\% sind im Jahr 2017 sogar von erheblicher materieller Deprivation betroffen, gleichwohl seit 2010 ein leicht rückläufiger Trend zu verzeichnen ist. Für 17,1\% der Bevölkerung lässt sich eine Bertoffenheit von Armut und sozialer Ausgrenzung feststellen (vgl. Eurostat 2018a: o. S.). Der Gini-Index ist in Frankreich vergleichsweise hoch: Die Einkommen sind im Jahr 2015 mit einem Gini-Index von 32,7\% eher ungleich verteilt (vgl. Weltbank 2018: o. S.). Ferner zeigt sich eine Geschlechterdifferenz des Bruttostundenlohns insofern, als Frauen im Durchschnitt 15,2\% weniger verdienen als Männer (vgl. UNECE 2018: o. S.). Gemäß den Eurostat-Daten beträgt der EU-Durchschnitt des geschlechtsspezifischen Lohngefälles 16,3\% (vgl. Europäische Kommission 2018: o. S.), wodurch Frankreich nahe dem EU-Durchschnitt liegt.

\subsubsection{Belgien: Vom De-Familialismus zum optionalen Familialismus - Optionaler Familialismus als (ungeplanter) Nebeneffekt" (Leitner 2013: 158)}

Leitner teilt die Entwicklung der Kinderbetreuungspolitik in Belgien in vier Phasen ein (vgl. Leitner 2013: 180):

Die erste Phase zeichnet sich durch einen geschlechterdiskriminierenden De-Familialismus bzw. einen kulturellen impliziten Familialismus aus. Institutionelle Kinderbetreuungsangebote für Kinder im Vorschulalter wurden ausgebaut und zugleich sank die Frauenerwerbsquote. Diese gegenläufige Entwicklung ist gemäß Leitner wie folgt zu erklären: Der Ausbau institutioneller Kinderbetreuungsangebote war keine Bestrebung, Familie und Beruf vereinbaren $\mathrm{zu}$ wollen, sondern einem „Schulstreit“ zwischen der katholischen Kirche und antiklerikalen Kräften geschuldet. Das männliche Ernährermodell galt in der Gesellschaft als kulturelle Norm, die sich zunehmend durch die Einführung wohlfahrtsstaatlicher Leistungen zur Ermöglichung der Nicht-Erwerbstätigkeit von Frauen in der Realität manifestierte. 
In den 1920er Jahren verschob sich der geschlechterdiskriminierende DeFamilialismus durch die Einführung indirekt familialistischer Strukturen zu einem frühen optionalen Familialismus bzw. auf der kulturellen Ebene zu einem indirekten expliziten Familialismus.

„Weil es sich bei den indirekten familialistischen Maßnahmen um explizit geschlechterdiskriminierende Strukturen handelte, die nur einen geschlechtsspezifischen Gebrauch ermöglichten, wird diese zweite Entwicklungsstufe als geschlechterdiskriminierende Variante eines optionalen (bzw. indirekt expliziten) Familialismus klassifiziert. Sowohl in der ersten als auch in der zweiten Entwicklungsperiode stellte das Ideal der nicht erwerbstätigen VollzeitMutter das kulturell dominante Rollenmodell für Frauen dar" (Leitner 2013: 180, Hervorhebungen im Original; die Verf.).

Der optionale Familialismus wurde in der dritten Phase insbesondere durch zwei Maßnahmen vorangetrieben: Erstens durch den Ausbau der Kinderbetreuungsinfrastruktur für Kinder unter drei Jahren, wobei die institutionellen Betreuungseinrichtungen im Unterschied zur ersten Phase und infolge der Emanzipation auch genutzt wurden, wodurch sich die Frauenerwerbsquote erhöhte. Zweitens gab es politische Bemühungen, Müttern sowohl Erwerbstätigkeit als auch Nicht-Erwerbstätigkeit zu ermöglichen, jedoch konnte diese Wahlfreiheit aufgrund finanzieller Einschränkungen nicht umgesetzt werden.

Die vierte Phase - Mitte der 80er Jahre - war geprägt von familialistischen Strukturen. Durch die ,interruption de carrière“ und dem ,congé parental" wurde das neue Leitbild einer erwerbstätigen Mutter, die ihren Beruf zugunsten der Kinderbetreuung unterbricht, geschaffen. Dies ist gemäß Leitner (2013) nicht nur auf der strukturellen, sondern auch auf der gesellschaftlichen Ebene geschlechterdiskriminierend. Aufgrund der EU-Gesetzgebung zur Geschlechtergleichbehandlung wurden zugleich die sozialen Rechte geschlechterneutral formuliert - ein Schritt in Richtung einer nicht geschlechterdiskriminierenden Variante des optionalen Familialismus. Dieser Schritt konnte jedoch auf der gesellschaftlichen Ebene nicht nachvollzogen werden (vgl. Leitner 2013: 181). 2001 wurde der Vaterschaftsurlaub von max. 10 Tagen innerhalb der ersten 30 Tage nach der Geburt/der Adoption eines Kindes eingeführt.

„Ob dieser erste Versuch der Inklusion von Vätern in die Kinderbetreuung einem geschlechtsspezifisch nicht diskriminierenden optionalen Familialismus den Weg weisen wird, bleibt abzuwarten“ (Leitner 2013: 181).

Für Väter besteht gemäß den aktuellsten Daten aus dem Jahr 2015 ein Anspruch auf Vaterschaftsurlaub von zwei Wochen, anschließend werden für Väter 17,3 Wochen Elternzeit ermöglicht, woraus sich insgesamt 19,3 Wochen ergeben. Der Mutterschutz beträgt in Belgien 15 Wochen. Im Anschluss daran sind lediglich 17,3 Wochen für den Elternurlaub der Mutter vorgesehen. Insgesamt ergibt sich hieraus eine relativ kurze Gesamtphase von 32,3 
Wochen bezahlter Elternzeit für die Mutter (vgl. OECD 2018: o. S.). Derzeit gehört Belgien zu den Spitzenreitern der frühkindlichen Vorschulerziehung: Im Jahr 2015 nutzen 50,1\% der 0- bis 3-jährigen Kinder (davon 23,3\% bis zu 29 Stunden pro Woche, 26,8\% mehr als 30 Stunden pro Woche) eine öffentliche Kinderbetreuungseinrichtung (EU-Durchschnitt: 30,3\%). Die Versorgungsquote der Kinder im Alter von drei bis sechs beträgt 98,8\% (davon 21,7\% weniger als 29 Stunden pro Woche und 77,1 \% mehr als 30 Stunden pro Woche) und liegt damit ebenfalls über dem EU-Durchschnitt $(83,3 \%)$. Doch aufgrund der extrem kurzen Elternzeit sind die Kinderbetreuungseinrichtungen für 0- bis 3-Jährige nicht in der Lage, die Nachfrage vollständig abzudecken. Die Erwerbsquote von Müttern mit Kindern unter sechs Jahren liegt 2016 bei 67,6\% (EU-Durchschnitt: 61,4\%), die der Väter bei 85,8\% (EU-Durchschnitt: 88,2\%). Ferner arbeiten 42,1\% der Frauen in Teilzeit, was mehr als der EU-Durchschnitt ist (31,9\%), während nur 9,5\% der Männer teilzeitbeschäftigt sind (EU-Durchschnitt: 16,3\%). Sowohl die allgemeine Erwerbsquote der Frauen $(58,1 \%)$ als auch die der Männer $(66,5 \%)$ ist in Belgien niedriger als im EU-Durchschnitt (61,4\% und 71,9\%). Insgesamt werden 2,2\% des Bruttoinlandsprodukts in Familienleistungen investiert (vgl. Europäische Kommission 2018: o. S.).

\section{Ökonomische Rahmenbedingungen}

Belgien verfügt mit einem Bruttoinlandsprodukt pro Kopf von 46428,67 KKS im Jahr 2016 im Vergleich zu den anderen Ländern über eine hohe Wirtschaftsleistung (vgl. Weltbank 2018: o. S.). Aktuell sind 15,9\% der Bevölkerung armutsgefährdet. Die Rate der erheblichen materiellen Deprivation ist im Unterschied zu Deutschland, Frankreich und Österreich mit 5,1\% etwas höher, insgesamt aber dennoch auf konstant niedrigem Niveau. Von Armut und sozialer Ausgrenzung betroffen sind derzeit (2017) 20,3\% der Bevölkerung (vgl. Eurostat 2018a: o. S.). Der Gini-Index unterliegt im Zeitverlauf zwischen 2003 und 2015 lediglich geringfügigen Schwankungen und beträgt gemäß den aktuellsten Daten aus dem Jahr 2015 27,7\%, womit er etwas niedriger als in Deutschland, Frankreich und Österreich ist (vgl. Weltbank 2018: o. S.). Das geschlechtsspezifische Lohngefälle des durchschnittlichen Bruttostundenlohns ist im Vergleich zur gesamten EU mit 6,1\% im Jahr 2016 gering (vgl. UNECE 2018: o. S.).

Obwohl Esping-Andersen (1990) Österreich, Deutschland, Belgien und Frankreich als konservativ klassifiziert hat, konnte Leitner (2013) zeigen, dass zwischen diesen konservativen Ländern, die sie als historische Fallbeispiele anführt, zeitweise unterschiedliche Varianten des Familialismus bestanden. Diese Ergänzung wird im Rahmen dieser Dissertation weiterverfolgt, indem all jene Länder im Sinne einer kontrastierenden Fallauswahl ausgewählt werden. Anzumerken ist, dass gemäß Leitner kein spezifisch konservatives Erklärungsmuster des Familialismuswandels empirisch aufgezeigt werden konnte. Jedoch weisen einerseits Deutschland und Österreich, 
andererseits Frankreich und Belgien ähnliche Entwicklungspfade auf (vgl. Leitner 2013: 210). In Österreich und Deutschland folgte auf den impliziten Familialismus eine Phase des indirekt expliziten Familialismus, wobei dieser in Österreich früher einsetzte als in Deutschland. Anschließend wurden innerhalb des expliziten Familialismus direkte Strukturen implementiert (wieder in Österreich früher als in Deutschland) und seit Beginn des 21. Jahrhunderts ist in Deutschland ein Trend zum optionalen Familialismus, d. h. auch eine Abkehr vom konservativen Entwicklungspfad zu verzeichnen (vgl. Leitner 2013: 190). In Belgien und Frankreich war zu Beginn des 19. Jahrhunderts ein struktureller De-Familialismus vorzufinden, der vornehmlich in der frühkindlichen Bildung von Kindern im Vorschulalter zum Ausdruck kam, jedoch nicht von Müttern zur Vereinbarkeit von Familie und Beruf genutzt wurde, wie die niedrigen Erwerbsquoten bis Mitte der 70er zeigten. In beiden Ländern wurde der strukturelle De-Familialismus durch einen indirekt expliziten Familialismus abgelöst - in Belgien früher als in Frankreich, doch hat Frankreich zugleich eine lange Phase direkt familisierender Maßnahmen vorzuweisen. In den 1980er Jahren wurde schließlich in beiden Ländern der optionale Familialismus durch indirekte und direkte familisierende Maßnahmen eingeführt, wobei an den ehemals strukturellen De-Familialismus angeknüpft wurde (vgl. Leitner 2013: 191). Eine Zusammenfassung der Entwicklungspfade der aufgeführten vier konservativen Länder ist in Tabelle 11 und 12 dargestellt.

\subsection{Ein interessanter Mischtypus - die Niederlande}

Da die Niederlande einen Lackmustest der Typologie Esping-Andersens (1990) darstellen, wird im Folgenden zunächst in die Problematik der allgemeinen Klassifizierung eingeführt, bevor die familienpolitischen und ökonomischen Rahmenbedingungen im Detail skizziert werden.

\subsubsection{Einführung in die Diskussion über die Klassifizierung der Niederlande in die Wohlfahrtstypologie Esping-Andersens- ein Lackmustest}

Die Niederlande sind ein Land, das konservative Elemente eines bismarckschen Modells - in erster Linie Absicherungen sozialer Risiken durch beitragspflichtige Sozialversicherungen - mit sozialdemokratischen Elementen in Form von Steuerfinanzierungen mit Grundsicherungscharakter kombiniert. Beide Elemente führen für WohlfahrtsstaatsforscherInnen zu Schwierigkeiten, die Niederlande regimetypologisch verorten zu können (vgl. Haarmann 
Tabelle 11: Zusammenfassende Darstellung der familialistischen Entwicklung in Österreich und Deutschland

\begin{tabular}{|c|c|c|c|c|c|c|c|c|c|c|c|}
\hline \multicolumn{12}{|c|}{ Familialistische Entwicklung der österreichischen Kinderbetreuungspolitik } \\
\hline 1900 & 1910 & 1920 & 1930 & 1940 & 1950 & 1960 & 1970 & 1980 & 1990 & 2000 & 2010 \\
\hline \multicolumn{12}{|c|}{\begin{tabular}{l|} 
Impliziter \\
Familialismus \\
\end{tabular}} \\
\hline & & \multicolumn{10}{|c|}{ Indirekter expliziter Familialismus } \\
\hline & & & & & & \multicolumn{6}{|c|}{ Direkter expliziter Familialismus } \\
\hline \multicolumn{12}{|c|}{ Familialistische Entwicklung der deutschen Kinderbetreuungspolitik } \\
\hline 1900 & 1910 & 1920 & 1930 & 1940 & 1950 & 1960 & 1970 & 1980 & 1990 & 2000 & 2010 \\
\hline \multicolumn{12}{|c|}{ Impliziter Familialismus } \\
\hline & & & & & \multicolumn{7}{|c|}{ Indirekter expliziter Familialismus } \\
\hline & & & & & & & & \multicolumn{4}{|c|}{$\begin{array}{c}\text { Direkter expliziter } \\
\text { Familialismus }\end{array}$} \\
\hline & & & & & & & & & \multicolumn{3}{|c|}{$\begin{array}{c}\text { D-Ost | Ost+West } \\
\text { Optionaler } \\
\text { Familialismus }\end{array}$} \\
\hline
\end{tabular}

Quelle: Leitner 2013: 190.

2012: 368). Dass die Niederlande sehr unterschiedlich kategorisiert werden können, je nach dem, was als Grundlage der Klassifizierung dient, wird u. a. von Goodin/Smitsman (2000) im Rahmen einer Reanalyse der Typologie Esping-Andersens dargestellt. ,Whether typologies are genuinely explanatory or purely heuristic, it matters on what basis cases are assigned to kinds" (Goodin/Smitsman 2000: 39). Typologien können entweder auf der Grundlage historischer Entwicklungen, auf der Grundlage von Programmeigenschaften oder auf der Grundlage von politischen Ergebnissen vorgenommen werden. Im Falle der Niederlande weisen alle drei Dimensionen unterschiedliche Entwicklungen auf, weshalb der niederländische Wohlfahrtsstaat ein Lackmustest für die Kategorisierung von Wohlfahrtsstaaten ist:

„1. Categorizing in terms of the history of policies and institutional ossifications of that history, the Dutch tax-transfer system looks distinctly corporatist.

2. Categorizing instead in terms of program characteristics, the Dutch tax-transfer system looks strikingly social democratic. 
Tabelle 12: Zusammenfassende Darstellung der familialistischen Entwicklung in Frankreich und Belgien

\begin{tabular}{|c|c|c|c|c|c|c|c|c|c|c|c|}
\hline \multicolumn{12}{|c|}{ Familialistische Entwicklung der französischen Kinderbetreuungspolitik } \\
\hline \multirow[t]{6}{*}{1900} & 1910 & 1920 & 1930 & 1940 & 1950 & 1960 & 1970 & 1980 & 1990 & 2000 & 2010 \\
\hline & \multicolumn{7}{|c|}{ Struktureller De-Familialismus } & & & & \\
\hline & & & & \multicolumn{4}{|c|}{$\begin{array}{c}\text { Indirekter expliziter } \\
\text { Familialismus } \\
\end{array}$} & & & & \\
\hline & & & & \multicolumn{3}{|c|}{$\begin{array}{c}\text { Direkter expliziter } \\
\text { Familialismus }\end{array}$} & & & & & \\
\hline & & & & & & & & \multicolumn{4}{|c|}{ Optionaler Familialismus } \\
\hline & \multicolumn{10}{|c|}{ Familialistische Entwicklung der belgischen Kinderbetreuungspolitik } & \\
\hline \multirow[t]{4}{*}{1900} & 1910 & 1920 & 1930 & 1940 & 1950 & 1960 & 1970 & 1980 & 1990 & 2000 & 2010 \\
\hline & & \multicolumn{6}{|c|}{ Struktureller De-Familialismus } & & & & \\
\hline & & \multicolumn{6}{|c|}{ Indirekter expliziter Familialismus } & & & & \\
\hline & & & & & & & & Optic & naler $\mathrm{F}$ & amilialis & mus \\
\hline
\end{tabular}

Quelle: Leitner 2013: 191.

3. Categorizing in terms of policy outputs, programs whose formal features might make the Dutch tax-transfer system seem meanly liberal actually deliver results that make it seem generously post-productivist, well on its way toward effectively realizing (without formally embracing) a "participation wage" version of a "basic income" model" (Goodin/Smitsman 2000: 40).

Historisch betrachtet hat sich die Rolle des Staates in den Niederlanden stark verändert; durch die Wirtschaftskrise in den 1930er Jahren und infolge des zweiten Weltkrieges wurde ein entscheidender Grundstock für die Entwicklung des Wohlfahrtsstaates gelegt. Der niederländische Wohlfahrtsstaat etablierte sich, als sich die „Verzuiling“ (Versäulung) ${ }^{126}$ und der Korporatismus

126 „Diese Art der gesellschaftlichen Organisation, die sich ähnlich auch in Belgien wiederfinden ließ, entfaltete sich mit der Industrialisierung und dem Streit, welche Rechte den nun konkurrierenden konfessionellen Gruppen zustünden. Weite Teile des alltäglichen, kulturellen und politischen Lebens spielten sich in der eigenen Gruppe ab, während Außenkontakte keine größere Bedeutung hatten. Im Kern verliefen die Trennlinien vor allem zwischen den konfessionellen Gruppen, aber auch zwischen den weltanschaulich-politischen, und, weni- 
als zentrale Merkmale des politischen Lebens durchgesetzt haben (vgl. Goodin/Smitsman 2000: 43). Im Rahmen des Säulensystems, das von 1930 bis 1960 dominierte ${ }^{127}$, war jede Säule durch eigene, auf politischen, kulturellen, religiösen und/oder sozioökonomischen „Cleavages“ basierenden Organisationen vertreten, die sich von den anderen Säulen unterschieden. Konfessionelle Parteien, Calvinismus und insbesondere Katholizismus prägten in dieser Phase das politische Leben der Niederlande, was sich in stark divergierenden Parteien (wie die Katholieke Volkspartij, die Anti-Revolutionaire Partij und die Christelijk-Historische Unie) der verschiedenen reformierten Konfessionen ausdrückte (vgl. Haarmann 2012: 367). Jede Säule und ihre verbundenen Organisationen waren souverän; jede war dafür verantwortlich, sich um die eigenen Mitglieder zu kümmern - wodurch die Bildung von Subkulturen begünstigt wurde -, wobei der Staat nicht eingreifen konnte. Finanzielle staatliche Unterstützung war hingegen jederzeit willkommen. Nach den Grundsätzen der Subsidiarität durfte der Staat nur dort agieren, wo „versäulte“ Organisationen nicht tätig waren oder nicht tätig sein konnten. Ferner wurden Sozialleistungen über zwischengeschaltete Organisationen verwaltet, die an die jeweiligen Hauptsäulen gebunden waren. Das niederländische wirtschaftliche und politische Leben zwischen den 1930er und den 1950er Jahren war nicht nur durch die Versäulung, sondern auch durch den Korporatismus gekennzeichnet, der hauptsächlich von KatholikInnen als Reaktion auf liberale Einflüsse angeregt wurde (vgl. Goodin/Smitsman 2000: 43).

Im Vergleich $\mathrm{zu}$ anderen europäischen Ländern bezeichnen Goodin/Smitsman (2000) die Niederlande zunächst als „,welfare lagged“, da fundamentale Sozialleistungen des Wohlfahrtsstaates, wie z. B. Rentenversicherungen, später eingeführt wurden als in den anderen Ländern (in den meisten europäischen Ländern erfolgte die Einführung wohlfahrtsstaatlicher Sicherheitssysteme bereits zwischen 1883 und 1908). In den 1960er Jahren wurden Sozialleistungen, wie Kindergeld, Arbeitslosenversicherung und Invaliditätsrente, etabliert, die einen minimalen Lebensstandard garantieren sollten. In Bezug auf öffentliche Ausgaben haben sich die Niederlande in den 1970er Jahren von einem „Wohlfahrtsnachzügler“ zu einem „welfare leader“ innerhalb der OECD Länder entwickelt. In der gesamten Dekade zwischen 1970 und 1980 waren die Niederlande weltweit unter den Staaten mit den höchsten öffentlichen Ausgaben. Dies hatte Goodin/Smitsman (2000) zufolge zwei Ursachen: Auf der Nachfrageseite entstand in Folge des Zusammenbruchs der alten Säulen ein Konkurrenzkampf um WählerInnenstimmen

ger ausgeprägt, berufsständischen Gruppen“ (Haarmann 2012: 367). Die klassischen Säulen waren die calvinistische, die die katholische, die sozialistische und die neutrale Säule. Zur Theorie der Versäulung s. Lijphart (1968).

127 In den 1960er Jahre löste sich die Versäulung zugunsten einer individualisierteren Gesellschaft nach und nach auf (vgl. Haarmann 2012: 367). 
zwischen konservativ-konfessionellen und sozialdemokratischen Parteien. Die konservativ-konfessionellen Parteien mussten umfangreiche Wohlfahrtsleistungen anbieten, um mit den SozialdemokratInnen um Stimmen aus der ArbeiterInnenklasse konkurrieren zu können. Auf der Angebotsseite wurden die hohen öffentlichen Ausgaben durch Erträge des internationalen Handels und inländischer Erdgasbestände ermöglicht. Institutionell wurde das Wachstum der Sozialausgaben durch korporatistische Traditionen der Selbstverwaltung von Sozialversicherungen begünstigt. Ende der 1970er Jahre trat eine Phase der Stagnation und Rezession ein. In Folge der Wirtschaftskrise 1987 wurden die Ausgaben für Sozialleistungen gekürzt, doch zeigt sich bis heute: „While these reforms certainly pared back the Dutch welfare state in important respects, it remains to this day one of the largest-spending tax-transfer system in the OECD world" (Goodin/Smitsman 2000: 44). Historisch betrachtet weisen die Niederlande und Schweden große Unterschiede auf, da in Schweden die Etablierung des Wohlfahrtsstaates infolge von politischen Mobilisierungen der ArbeiterInnenbewegungen eingeführt wurde (vgl. Goodin/Smitsman 2000: 44). Die historischen Ursprünge des niederländischen Wohlfahrtsstaates sind hingegen fundamental korporatistisch charakterisiert, was Spuren in der Struktur der niederländischen Wohlfahrtsorganisationen und -verwaltung hinterlassen hat. Doch erschöpft sich die historische Entwicklung Goodin/Smitsman (2000) zufolge nicht ausschließlich in ihren Ursprüngen:

„The defining moment for the Dutch welfare state as we know it today was, of course, the point at which vast sums of money started to be pumped through preexisting institutions. And what was going on at that moment was precisely political pressure from the working class, expressed through the threat to abandon old confessional parties in favor of the Social Democrats. It may have been the confessional parties who actually wrote the checks, but it was political pressure from the working classes that made them do it. So in that sense, perhaps we might see the key moment of Dutch welfare state development as substantially of a cloth with certain key features of classically Scandinavian social democratic ones" (Goodin/Smitsman 2000: 45).

Insofern seien trotz der korporatistischen Ursprünge auch sozialdemokratisch-skandinavische Schlüsselmerkmale der Entwicklung des Wohlfahrtsstaates in den Niederlanden zu verzeichnen.

Weiterführend belegen Goodin/Smitsman (2000) durch ihre Reanalyse der Typologie Esping-Andersens, dass die Klassifizierung der Niederlande auf großen Ambivalenzen basiert. Im Rahmen der Typologie von EspingAndersen (1990) weisen die Niederlande im Hinblick auf das LiberalismusCluster einen hoch-moderaten kumulativen Index Score auf, im Hinblick auf das sozialdemokratische Cluster einen vergleichsweise niedrig-starken und im Hinblick auf das korporatistische Cluster einen niedrig-moderaten (vgl. Esping-Andersen 1990: 74). Werden zusätzlich - wie von Goodin/Smitsman 
(2000) erläutert - die Arbeitsmarktmaßnahmen berücksichtigt, zeigt sich, dass die Klassifizierung offenkundig uneindeutig ist. Unabhängig davon, ob das Fazit von Goodin/Smitsman (2000), die Niederlande als sozialdemokratisch zu klassifizieren, befürwortet wird, gäbe es gemäß Haarmann (2012) gute Argumente, die sowohl für den konservativen als auch für den sozialdemokratischen Typus sprechen würden (vgl. Haarmann 2012: 368). Festzuhalten sei, dass die Entstehungsgeschichte und die Verwaltung der Sozialversicherungen einen korporatistischen Charakter aufwiesen, da alle Sozialversicherungen (mit Ausnahme der Rentenversicherung) paritätisch von den SozialpartnerInnen administriert wurden.

\begin{abstract}
„Heute sind die Sozialpartner[Innen] nur noch mit der Administration der Arbeitslosengelder, nicht aber der Arbeitslosenvermittlung vertraut. Die anderen Versicherungen sind in die Hände von durch den Staat bestellte Expertengremien gelegt - wie im Falle der staatlichen Rentenversicherung -, in den Aufgabenbereich der Kommunen und privatwirtschaftlicher Dienstleister[Innen] übergegangen - wie im Falle der Arbeitslosenverwaltung, dem Räte, bestehend aus einfachen Mitgliedern der Versicherungen, zur Seite stehen - oder einem professionellen Management überantwortet - wie im Falle der Krankenversicherung. Diese Entwicklung der Abwendung vom Korporatismus - und damit auch traditioneller Selbstverwaltung - auf Ebene der einzelnen Sozialversicherungen geht mit der Fortexistenz der korporatistischen Beratungsorgane auf nationaler Ebene einher" (Haarmann 2012: 369).
\end{abstract}

\title{
10.4.2. Familienpolitische Momente in den Niederlanden
}

In den Niederlanden wird vornehmlich die Teilzeitbeschäftigung von Müttern (z. B. ist im Jahr 2007 mit 62\% ein extrem hoher Anteil an Müttern, die einer Teilzeitbeschäftigung nachgehen, vorzufinden) in Kombination mit einer relativ kurzen Elternzeit gefördert (vgl. Schleutker 2014: 181). Der bezahlte Mutterschaftsurlaub beträgt in den Niederlanden im Jahr 2015 nur 16 Wochen (s. Tabelle 32 im Anhang: Gesamte bezahlte Elternzeit), was im Vergleich zu den anderen hier aufgeführten Ländern mit Abstand der niedrigste Wert ist. Auch der Vaterschaftsurlaub ist mit 0,4 Wochen sehr niedrig (vgl. OECD 2018: o. S.). Im internationalen Vergleich stechen die Teilzeitquoten von Frauen in den Niederlanden immer wieder hervor, die das familienpolitische Ziel der Vereinbarkeit von Beruf und Familie verfolgen. 76,4\% der Frauen und 26,2\% der Männer gehen im Jahr 2016 einer Teilzeitbeschäftigung nach - was die höchsten Teilzeitquoten in der EU-28 sind. Die aktuellen (2016) Beschäftigungsquoten von Eltern mit Kindern unter sechs Jahren sind mit 75,3\% der Frauen und 93,5\% der Männer deutlich oberhalb des EUDurchschnitts (61,4\% bzw. 88,2\%) (vgl. Europäische Kommission 2018: o. S.). Neben der Förderung zur Teilzeitbeschäftigung ist auch das Angebot an Halbtags-Betreuungseinrichtungen relativ hoch (vgl. Schleutker 2014: 181), 
wobei die Betreuungsangebote überwiegend durch private Kinderbetreuungseinrichtungen (profit oder non-profit) erfolgen (vgl. Ostner/Schmitt 2008b: 210). 46,4\% der Kinder unter drei Jahren werden gemäß den Daten der Europäischen Kommission im Jahr 2015 im Rahmen von Kinderbetreuungseinrichtungen betreut (davon 5,3\% 30 Stunden pro Woche oder mehr und 41,1\% 29 Stunden pro Woche oder weniger), im EU-Durchschnitt sind es hingegen nur 30,3\%. Vom dritten Lebensjahr bis zum schulpflichtigen Alter besuchen 90,7\% der Kinder eine Kinderbetreuungseinrichtung (davon 13,2\% mehr als 30 Stunden pro Woche und 77,5\% bis zu 29 Stunden pro Woche), was ebenfalls deutlich über dem EU-Durchschnitt (83,3\%) liegt (vgl. Europäische Kommission 2018: o. S.). 1991 wurde ein Gesetz zum Elternurlaub verabschiedet, das beiden Eltern bis zum vierten Lebensjahr des Kindes einen Anspruch auf Elternzeit von mindestens 20 Wochenstunden Arbeit für maximal sechs Monate gewährte (vgl. Marold 2009: 69f.). 2006 wurde eine innovative familienpolitische Maßnahme eingeführt - ein , ,...] life-course savings scheme, which rests firmly on the idea of an individualized adult worker model family“ (Lewis et al. 2008: 272). Diese Maßnahme basierte auf der Idee eines erweiterten Handlungsspielraums, je nach individuellen Bedürfnissen ein Lebenslauf-Arrangement innerhalb der Lebensarbeitszeitregelung wählen zu können. Aufgrund der hierauf folgenden Kürzungen einiger Maßnahmen in Bezug auf Erwerbsunterbrechungen zugunsten der Betreuung und einer Einführung individueller Planung einschließlich Finanzierung, sollen Anreize geschaffen werden, die Unterbrechung der Erwerbstätigkeit von Frauen möglichst kurz zu halten (vgl. Marold 2009: 71). Knijn (2008) ${ }^{128}$ und auch Marold (2009) schlussfolgern daraus, dass sich durch die zunehmend flexiblen Arbeitszeitarrangements, marktwirtschaftliche Ansätze der Kinderbetreuung und ein individualisiertes Steuerrecht die niederländische Familienpolitik in einer Transformationsphase - von einer ehemals konservativen Ausrichtung in Richtung einer Fokussierung auf sozialdemokratische und liberale Elemente - befindet und insofern eine Mischform darstellt (vgl. Knijn 2008: 155; Marold 2009: 71). Insgesamt zeigt sich, dass aktuell im Sinne einer liberalen Orientierung nur $0,9 \%$ des Bruttoinlandsprodukts für Sozialschutzleistungen für Kinder und Familien ausgegeben werden (EUDurchschnitt: 2,4\%) (vgl. Europäische Kommission 2018: o. S.).

128 ,Currently, the dominant idea about the relationship between work and care is being transformed by combining its traditional corporatist traits, i.e. the familialisation of care responsibilities, with an even less prominent role of the state. New laws have been introduced, such as The Work and Welfare Act (Wet Werk en Bijstand), the Social Support Act (Wet Maatschappelijke Ondersteuning), a new childcare law (Wet Kinderopvang), and the Life Course Saving Scheme (Levensloopregeling). The overall direction is to encourage Dutch citizens to liberate themselves from the state with respect to work and care: national public policy should not be understood as a problem-solver in this area. Individual citizens are supposed to become more autonomous in how they reconcile work and care obligations" (Knijn 2008: 155). 


\section{Ökonomische Rahmenbedingungen}

Die liberale Orientierung der Niederlande spiegelt sich ebenfalls in einem vergleichsweise sehr hohen Bruttoinlandsprodukt pro Kopf von 50538,61 KKS wieder, das auf eine hohe Wirtschaftsleistungsfähigkeit verweist (vgl. Weltbank 2018: o. S.). Seit 2012 ist ein Anstieg der Quote der von Armut bedrohten Personen von 10,1\% auf 13,2\% festzustellen. Zuvor lag stets ein konstant niedriges Niveau der Armutsgefährdung vor. Auch die Rate der erheblichen materiellen Deprivation ist mit aktuellen 2,6\% sehr niedrig, lediglich in Schweden und Norwegen sind die Raten noch geringer. In Bezug auf die von Armut und sozialer Ausgrenzung betroffene Bevölkerung zeigt sich ein stetiges Muster auf niedrigem Niveau. Im Jahr 2017 sind 17 \% der niederländischen Bevölkerung von Armut und sozialer Ausgrenzung betroffen (vgl. Eurostat 2018a: o. S.). Der Gini-Index beträgt nach aktuellsten Daten aus dem Jahr 2015 29,3\% (vgl. Weltbank 2018: o. S.). Das geschlechtsspezifische Lohngefälle des Bruttostundenlohns sinkt seit 2006 kontinuierlich von ehemals 23,6\% auf 15,6\% im Jahr 2016 (vgl. UNECE 2018: o. S.).

\subsection{Das ,postsozialistische Regime“ kritisch hinterfragt}

Postsozialistische Länder werden häufig in der Wohlfahrtsstaatsforschung nicht - oder zumindest nicht als eigenständiger Typus - berücksichtigt. Weder in der Typologie Esping-Andersens (1990) noch in der Typologie Korpis (2000) werden sie explizit dargestellt. Hobson/Oláh (2006) kritisieren dies zu Recht und integrieren in Korpis Typologie einen vierten postsozialistischen Typus, der als hybride Mischform zwischen liberalen (nach Korpi (2000) „Market Oriented“) und sozialdemokratischen (nach Korpi (2000) „Dual Earner") Regimen beschrieben wird (vgl. Hobson/Oláh 2006: 206) ${ }^{129}$. Dass nicht nur zwischen konservativen Ländern unterschiedliche Varianten des Familialismus bestehen, sondern auch postsozialistische Länder unterschiedliche Familialismusformen aufweisen, legen Szelewa/Polakowski (2008) sowie Szirka/Szelewa (2009) anhand der Anwendung von Leitners (2003) Typologie auf mittel- und osteuropäische Länder dar. Folglich gilt es, ebenso für die postkommunistischen Länder - abgesehen von den Gemeinsamkeiten ihres historischen staatssozialistischen Erbes - die unterschiedlichen Entwicklungspfade des Familialismus zu berücksichtigen.

129 „In our comparative analysis, we use the policy configurations in the Korpi model as ideal types and as a heuristic tool for analyzing similarities and differences among our countries and to suggest divergent patterns in the 1990s. Following but modifying his model, we organize our countries into four clusters: Dual Earner, General Support, and Market-Oriented. Our fourth cluster is the Transition Post-Socialist Countries that are a hybrid between the Dual Earner and Market-Oriented types" (Hobson/Oláh 2006: 206). 


\subsubsection{Familienpolitik in Polen, Tschechien und Ungarn}

Nach dem Mauerfall 1989 und dem Ende der kommunistischen Herrschaft befanden sich osteuropäische Frauen in einer „einzigartigen“ Situation. Auf der einen Seite konnten sie weltweit die höchsten Beschäftigungsquoten vorweisen - nur die skandinavischen sozialdemokratischen Länder kamen diesen sehr nahe. Auf der anderen Seite und im Gegensatz zu den skandinavischen Ländern gab es jedoch wenige Diskussionen über die Notwendigkeit, dass Männer in die Hausarbeiten und Kindererziehung integriert werden sollten. Infolgedessen blieb der Haushalt die Domäne der Frau (vgl. Saxonberg/Sirovátka 2006: 185). Da praktisch alle Frauen während der kommunistischen Ära arbeiteten und es ein gut entwickeltes System der Kinderbetreuung gab, hätte man sich vorstellen können, dass die postkommunistischen Regime dem sozialdemokratischen Modell folgen würden. Saxonberg/Sirovátka (2006) zeigen jedoch, dass postkommunistische Regime versucht haben, das traditionelle Familienregime wiedereinzuführen.

\section{Elternzeit}

In den osteuropäischen Ländern gibt es zwei grundlegende Arten von bezahlter Elternzeit: Erstens gibt es den für die ersten Lebensmonate des Kindes vorgesehenen Mutterschaftsurlaub, der versicherungsbasiert ist und ziemlich großzügig bemessen wird. Zweitens gibt es in jedem Land verschiedene Arten von Elternzeiten, die nicht versicherungsbasiert sind und weniger großzügig bemessen werden, aber dafür über einen längeren Zeitraum in Anspruch genommen werden können und für Väter offen sind. Der Mutterschaftsurlaub umfasste gemäß Saxonberg/Sirovátka (2006) in Tschechien 28 Wochen, in Ungarn 24 Wochen und in Polen 16 Wochen; die gesamte Elternzeit betrug in Tschechien und Polen vier Jahre, in Ungarn zwei Jahre (vgl. Saxonberg/Sirovátka 2006: 186f.). 1996 erhielten polnische Männer erstmals einen Anspruch auf Elternzeit. Da Väter in Polen, Tschechien und Ungarn i. d. R. über ein höheres Einkommen verfügen als Mütter und die wirtschaftliche Situation für viele Paare prekär ist, nehmen bis heute nur wenige Männer die Elternzeit in Anspruch. Das Elterngeld betrug im Jahr 2002 in Tschechien lediglich 14,9\% (82,60€), in Polen 15\% (83,30€) und in Ungarn 22,8\% (82,60 €) des durchschnittlichen Einkommens. In Tschechien war das Elterngeld eine universale Leistung, die pauschal und unabhängig vom Einkommen gewährt wurde. Diese Kombination aus einer langen Elternzeit mit niedrigen Leistungsbeiträgen stellt Saxonberg/Sirovátka (2006) zufolge eine explizite Re-Familisierungspolitik dar, die getrennte Geschlechterrollen für Männer und Frauen fördert, da nur wenige Männer bereit sind, ihr Recht auf Elternzeit unter diesen Bedingungen in Anspruch zu nehmen. Aktuell ist jedoch die Flexibilität des Elternurlaubs eine Stärke der familienpolitischen Maßnahmen in Tschechien. 
„Der Mutterschaftsurlaub kann über eine Dauer von insgesamt 28 Wochen in Anspruch genommen werden: 6-8 Wochen vor dem voraussichtlichen Entbindungstermin und 20-22 Wochen nach der Geburt. Das Mutterschaftsgeld ist einkommensabhängig und belief sich bei Frauen, die 2016 bis zu 901 CZK (35€) pro Kalendertag verdienten, auf $70 \%$ ihres Gehalts. Es wird bei höheren Einkommen teilweise reduziert. Der Höchstbetrag des Mutterschaftsgeldes betrug im Jahr 201733.120 CZK (1.295€) pro Kalendermonat bzw. 1.104 CZK pro Kalendertag (43€)“ (Europäische Kommission 2018: o. S.).

Im Anschluss an den Mutterschaftsurlaub besteht ein Anspruch auf unbezahlten Elternurlaub bis das Kind maximal 3 Jahre alt ist, wobei keine Rückkehr in den gleichen Beruf gewährleistet wird. Der/die ArbeitgeberIn ist lediglich bis zum dritten Lebensjahr des Kindes dazu verpflichtet, eine Stelle anzubieten, die den Qualifikationen des Elternteils entspricht. Die Elternzeit kann von beiden Eltern gleichzeitig genommen werden und zugleich kann ein Elternteil Elterngeld beziehen (der Höchstbetrag des Elterngeldes beläuft sich für den gesamten Zeitraum - die Bezugsdauer kann bis zu einem Kindesalter von 48 Monaten frei gewählt werden - auf $220000 \mathrm{CZK}$, was ca. $8803 €$ entspricht). Während des Bezuges von Elterngeld kann mit einer Einschränkung weiterhin gearbeitet werden: Kinder, die jünger als 2 Jahre alt sind dürfen maximal 46 Stunden pro Monat eine öffentliche Kinderbetreuungseinrichtung besuchen (für Kinder über 2 Jahre gibt es keine zeitliche Einschränkung). Obwohl grundsätzlich Väter gleichermaßen die Elternzeit wahrnehmen und Elterngeld beziehen können wie Mütter, ist ihr Anteil aufgrund des hohen geschlechtsspezifischen Lohngefälles in Tschechien sehr gering (vgl. Europäische Kommission 2018: o. S.).

In Polen war die Elternzeit einer Bedürftigkeitsprüfung unterworfen und auf diejenigen beschränkt, die weniger als das Existenzminimum verdienten. Diese liberale Methode einer Bedürftigkeitsüberprüfung stellte gemäß Saxonberg/Sirovátka (2006) eine implizite Re-Familisierungspolitik dar. Aktuell (seit Januar 2016) wird ein Mutterschaftsurlaub von 20 Wochen gewährt, der bezahlte Elternurlaub umfasst 32 Wochen. Väter haben bis zu einem Kindesalter von 2 Jahren einen Anspruch auf 2 Wochen bezahlten Vaterschaftsurlaub, wobei die Eltern abwechselnd oder gleichzeitig die Elternzeit in Anspruch nehmen können (vgl. Europäische Kommission 2018: o. S.). Im Januar 2016 wurde die Maßnahme „Zlotowka za zlotowke“ (Cent für Cent) eingeführt, die sicherstellen soll,

,[...] dass Familien die meisten Leistungen weiterhin beziehen können, wenn das Familieneinkommen den Grenzwert übersteigt, bis zu dem sie Leistungen beziehen dürfen. Statt auf den Gesamtbetrag der Leistung verzichten zu müssen, erhält die Familie eine Leistung, die nach und nach gekürzt wird. Die Leistung wird um jeden Cent des Einkommens, der den Grenzwert übersteigt, ebenfalls um einen Cent gekürzt“ (Europäische Kommission 2018: o. S.). 
Außerdem wird seit Januar 2016 auch Personen, die keinen Anspruch auf Mutterschaftsgeld haben (Arbeitslose, Studierende, LandwirtInnen etc.), eine neue Form des Elterngeldes in Höhe von 1000 Złoty (ca. $226 €$ ) monatlich gewährt. Die 2015 eingeführte Steuerermäßigung ab dem dritten Kind (2000 Złoty/ca. $453 €$ für das dritte, 2700/ca. $612 €$ für das vierte und folgende Kind pro Jahr) sowie die 2016 eingeführte universelle Leistung „Familie 500 Plus", die monatlich in Höhe von 500 Złoty (ca. $118 €$ ) für das zweite und alle weiteren Kinder finanziert wird, verdeutlichen das familienpolitische Ziel einer Erhöhung der Fertilitätsrate (im Jahr 2015 beträgt die Fertilitätsrate 1,32 Kinder pro Frau) (vgl. Europäische Kommission 2018: o. S.).

„Das Hauptziel der polnischen Familienpolitik besteht darin, zukünftige Eltern davon zu überzeugen, Kinder zu bekommen, um negative demographische Entwicklungen umzukehren" (Europäische Kommission 2018: o. S.).

Ungarn ist ein Ausnahmefall: Die erste konservative postkommunistische Regierung (1990-1994) gewährte - infolge demographischer Überlegungen und dem Ziel einer Erhöhung der Reproduktionsrate (vgl. Liebhart et al. 2003: 423) - eine relativ großzügige zweijährige Elternzeit: Geleistet wurden 70 Prozent des Gehalts mit einer Obergrenze des doppelten Mindestlohns. Darüber hinaus konnten Eltern bis zu drei Jahren Arbeitslosengeld erhalten. Diese Regelung verlieh dem ungarischen Modell einen expliziten Familiencharakter, der ebenfalls getrennte Geschlechterrollen förderte (vgl. Saxonberg/Sirovátka 2006: 189). Im europäischen Vergleich werden Männer in den postsozialistischen Ländern dennoch vergleichsweise deutlich ermutigt, die Elternzeit wahrzunehmen, weshalb auch einige Elemente zur Förderung einer Gleichstellung der Geschlechter vorzufinden sind (vgl. Saxonberg/Sirovátka 2006: 189). In der Regel sind aktuell in Ungarn Frauen mit Kleinkindern 2-3 Jahre lang nicht erwerbstätig, um ihre Kinder zu Hause betreuen zu können - dies könnte eine Hauptursache für die niedrige Erwerbsquote (40\% in Ungarn im Vergleich zu 61,4\% im EU-Durchschnitt) von Frauen mit Kindern unter 6 Jahren sein (vgl. Europäische Kommission 2018: o. S.). Zugleich ist Ungarn bemüht, eine größere Flexibilität im Erziehungsurlaubssystem zu ermöglichen. Im Jahr 2014 werden 2,3\% des Bruttoinlandsprodukts (im Vergleich dazu sind es in Tschechien 1,7\%, in Polen 1,5\% und im EU-Durchschnitt 2,4\% des Bruttoinlandsprodukts) für Familienleistungen und Sozialschutzleistungen ausgegeben. Insgesamt sind 20 verschiedene Arten von Leistungen für Familien zu verzeichnen:

„Einige von ihnen zielen darauf ab, die finanzielle Belastung durch die Erziehung von Kindern zu verringern, indem sie Familien unmittelbar finanzielle oder materielle Unterstützung bieten. Andere zielen darauf ab, Familien in bestimmten Lebenssituationen zu unterstützen, wie z. B. durch zusätzlichen Jahresurlaubsanspruch, bezahlten Krankenstand zur Betreuung von Kindern für berufstätige Eltern und Unterhaltsvorschüsse für Eltern, die sich scheiden lassen“" (Europäische Kommission 2018: o. S.). 


\section{Kindertagesstätten}

Während die postkommunistischen Regime die Elternzeit verlängert haben, wurden zur gleichen Zeit die staatlichen Kinderbetreuungsangebote für Kinder im Alter von 0 bis 3 Jahren drastisch reduziert. Fortan wurde die politische Verantwortung der Betreuungsangebote lokalen Behörden übertragen. Diese haben ihrerseits die Einschreibungsgebühren erhöht und die SchlieBung vieler Kindergärten veranlasst. Ab 1989 ging die Zahl der Kindergärten in der Tschechischen Republik drastisch zurück (die Versorgungsquote von Kindern im Alter von 0 bis 3 Jahren in öffentlichen Betreuungseinrichtungen sank von 20,3\% im Jahr 1989 auf 10,3\% im Jahr 2002; gemäß den aktuellsten Daten der Europäischen Kommission sind es im Jahr 2015 nur noch 2,9\%, im Vergleich zu 30,3\% in der EU), während in Polen die Zahl der Kindergärten bereits 1989 gering war (die Versorgungsquote von Kindern im Alter von 0 bis 3 Jahren in öffentlichen Betreuungseinrichtungen sank hier von 9,1\% im Jahr 1989 auf 5,1\% im Jahr 2002; im Jahr 2015 beläuft sich laut Europäischer Kommission die Versorgungsquote auf 5,4\%) und sich in den kommenden Jahren in geringem Ausmaß reduzierte. Es verwundert nicht, dass die Europäische Kommission in Tschechien im Jahr 2015, ,...] eine der niedrigsten Quoten bezüglich der Bereitstellung offizieller Betreuungsdienste für Kleinkinder unter drei Jahren verzeichnet" (Europäische Kommission 2018: o. S.). Einmal mehr bildet Ungarn die Ausnahme, da kaum Veränderungen der staatlichen Betreuungsangebote nach dem Zerfall des kommunistischen Regimes im Zeitverlauf zwischen 1989 und 2002 vorzufinden sind der Anteil der 0- bis 2-jährigen Kinder in Kindergärten ging lediglich um etwas mehr als ein Prozent zurück (von 11,7\% im Jahr 1989 auf 10,1\% im Jahr 2002; aktuell werden gemäß Europäischer Kommission 15,3 \% der Kinder unter 3 Jahren im Rahmen einer öffentlichen Kinderbetreuungseinrichtung betreut) (vgl. Saxonberg/Sirovátka 2006: 189). In den kommunistischen Ländern konnten Kinder bis zu einem Alter von drei Jahren „Nursery Schools" besuchen. Im Anschluss daran konnten sie bis zu einem Alter von sechs oder sieben Jahren in den Kindergarten gehen. In Tschechien wurde das System der „Nursery Schools“ zugunsten der Kindergärten weitgehend abgeschafft. Obwohl Kindergärten Kinder unter 3 Jahren aufnehmen dürfen, sind sie jedoch nicht dazu verpflichtet (eine Änderung der Gesetzeslage ergab sich 2016 in Form des Rechtes von Kindern im Alter von 2 bis 4 Jahren auf einen Platz in einer Kinderbetreuungseinrichtung). Die Entscheidung über die Aufnahme von unter Dreijährigen hängt im Wesentlichen von der wirtschaftlichen Situation und der Kapazität des jeweiligen Kindergartens ab. Eine Tendenz nach Kindergartenplätzen für jüngere Kindern zu suchen, deutet darauf hin, dass für Kinder unter 3 Jahren noch immer ein Bedarf an Kindertagesstätten besteht (vgl. Saxonberg/Sirovátka 2006: 189f.). ,[...] die Kapazität der Kinderbetreuungseinrichtungen für Kleinkinder [reicht selbst] derzeit nicht aus" (Europäische Kommission 2018: o. S.). Auch für die Kinder 
ab 3 Jahren ist aktuell festzustellen, dass in Tschechien trotz einer hohen Versorgungsquote für diese Kohorte (77,5\% im Jahr 2015) das Angebot nicht der Nachfrage entspricht - 2016/2017 wurden knapp 32000 Anträge auf Kinderbetreuung abgelehnt (vgl. Europäische Kommission 2018: o. S.). Während die Versorgungsraten für Kinder unter 3 Jahren insbesondere in Tschechien im Zeitverlauf stark zurückgegangen sind, sind sie für Kinder im Alter von 3-6 Jahren in Tschechien und Ungarn, aber mit Ausnahme von Polen ${ }^{130}$ (basierend auf dem Jahr 2015 43\% in Polen im Vergleich zum EUDurchschnitt von 83,3\%), hoch geblieben. Die Kombination aus langer Elternzeit und fehlendem Zugang zu Kindertagesstätten für Kinder unter drei Jahren erschwert es Frauen, Beruf und Karriere zu vereinbaren. Tschechien hat gemäß Saxonberg/Sirovátka (2006) auf explizite Weise das konservative geschlechtsspezifische Rollenmodell eingeführt, das aus einer Kombination einer pauschalen Elternzeit und einer unzureichenden staatlichen Unterstützung in Form von Kindertagesstätten beruht. In der Zwischenzeit hat Polen ein liberaleres, implizit familiäreres Modell verfolgt, in dem die meisten Familien keinen Anspruch auf Elternzeit haben und der Staat den Zugang zu Kindergärten nur wenig unterstützt (die Klassifizierung der Kinderbetreuungspolitik von Tschechien als expliziten Familialismus und Polen als impliziten Familialismus wird auch von Szelewa/Polakowski (2008) gestützt ${ }^{131}$ ). Diese eher marktorientierte Politik unterstützt das ,male breadwinner model“", indem es Frauen zur Rückkehr in den Haushalt verleitet, da zu wenig staatliche Betreuungsmöglichkeiten bestehen und kaum Anreize zur Wahrnehmung der Elternzeit für Väter gewährt werden. Die Versorgungsquote von Kindern im Alter zwischen drei und fünf beträgt im Jahr 2002 in Polen lediglich 49,9\%; gemäß aktuellster Daten der Europäischen Kommission beträgt die Einschreibungsrate für Kinder ab 3 Jahren 43\%. Hingegen sind in Tschechien im Jahr 2002 94,7\% und in Ungarn 87,8\% vorzufinden (basie-

130 Obwohl die Einschreibungsraten für Kinderbetreuungsplätze in Polen sehr niedrig sind, werden staatliche Maßnahmen eingeführt, um den Zugang zur Kinderbetreuung zu verbessern. Dadurch hat sich die Zahl der Kinderbetreuungseinrichtungen von 511 im Jahr 2010 auf $3451 \mathrm{im} \mathrm{Jahr} 2016$ erhöht. 2011 wurde beispielsweise das Programm „Maluch“ eingeführt, ,,[...] um Kommunalregierungen zu ermutigen, Kinderkrippen und Kinder-Clubs einzurichten. Die für das Programm verfügbaren Mittel sind kontinuierlich von 15,2 Mio. Złoty (ca. 3,4 Mio. €) im Jahr 2011 auf 151 Mio. Złoty (ca. 34 Mio. €) im Jahr 2016 gestiegen. Bis 2015 wurden durch diese Maßnahme 12.500 neue Kinderbetreuungsplätze geschaffen. Die Regierung plant, die „Maluch“-Maßnahme bis 2018 um weitere 300 Mio. Złoty zu erweitern“ (Europäische Kommission 2018: o. S.). Außerdem wird die Ermöglichung frühkindlicher Bildung für Kommunen zunehmend verpflichtet. „Seit 2009 hat jedes 5-jährige Kind Anspruch auf einen Platz im Kindergarten, seit 2015 jedes 4-jährige Kind und seit 2017 jedes 3-jährige Kind“" (Europäische Kommission 2018: o. S.).

131 Die gesamte Klassifizierung von Szelewa/Polakowski (2008) umfasst Tschechien, die Slowakei und Slowenien als dem expliziten Familialismus zugehörig, Estland und Lettland als „female mobilizing type“, Litauen und Ungarn als „,comprehensive support model“ und Polen als dem impliziten Familialismus zugehörig (Szelewa/ Polakowski 2008: 128). 
rend auf den aktuellen Daten der Europäischen Kommission sind es im Jahr 2015 in Tschechien 77,5\% der Kinder im Alter von 3 bis 6 Jahren und in Ungarn sogar 89,1\% im Vergleich zum EU-Durchschnitt von 83,3\%). Obwohl Ungarn dem konservativen - ausdrücklich auf Re-Familisierung ausgerichteten - Weg folgt, förderte es Anfang des 21. Jahrhunderts nicht so stark geschlechtsspezifische Rollen wie Tschechien, da es den Vätern mehr Anreize gab, die Elternzeit wahrzunehmen und einen besseren Zugang zu Kindergärten bot (vgl. Saxonberg/Sirovátka 2006: 190f.). Dies mögen Gründe sein, warum Ungarn in der Phase nach 2002 von Szelewa/Polakowski (2008) als optional familialistisch klassifiziert wird. Für eine detaillierte Differenzierung zwischen Ungarn - nach seiner Umbruchsphase vom expliziten Familialismus zum optionalen Familialismus - und Polen als strikt dem impliziten Familialismus angehörig sei außerdem auf den Artikel von Szikra/Szelewa (2009) verwiesen. Hierin wird konstatiert, dass beide Länder zwar die Gemeinsamkeit des staatssozialistischen Erbes aufweisen, doch trotzdem große Unterschiede im Hinblick auf die Familienpolitik bestehen. Ungarn bietet ab 2002 nicht nur diverse familialistische finanzielle Unterstützungen, sondern zugleich öffentliche Kinderbetreuungsdienstleistungen an, während in Polen das Angebot öffentlicher Kinderbetreuungseinrichtungen weiterhin sehr gering ist und finanzielle Leistungen lediglich bedarfsorientiert sind, d. h. nur der ärmere Teil der Bevölkerung davon profitiert (vgl. Szikra/Szelewa 2009: 113). Anzumerken ist, dass sich in Ungarn nach dem Ende der Regierung Viktor Orbáns im Jahre 2002 das „,comprehensive support model“ durchgesetzt hat. Zuvor - zwischen 1989 und 1999 - ist die Kinderbetreuungspolitik Ungarns als explizit familialistisch kategorisiert (vgl. Szelewa/Polakowski 2008: 128). Aufgrund der Wiederwahl Orbáns im Jahr 2010, der auch aktuell noch das Amt des Ministerpräsidenten innehat, und dem rechtskonservativen Kurs einschließlich einer pronatalistischen Parteiideologie seiner amtierenden Regierungspartei Fidesz (Regierungskoalition: Fidesz/KDNP) ist ein Wiederaufleben des expliziten Familialismus in Ungarn nicht auszuschließen. Aktuell können berufstätige Eltern zwar für die Betreuung ihrer Kinder im Alter von 20 Wochen bis 3 Jahren öffentliche Kindertagesstätten in Anspruch nehmen, jedoch wird dies nur für $15,3 \%$ der Kinder unter 3 Jahren genutzt (EU-Durchschnitt: 30,3\%; gleichwohl ist dies deutlich mehr als in Tschechien $(2,9 \%)$ oder in Polen $(5,4 \%))$. In Ungarn wird die Mehrheit der Kleinkinder zu Hause von den Eltern bzw. meist von der Mutter betreut. Im Sinne der Chancengleichheit wurde im September 2015 eine Kindergartenpflicht für alle Kinder ab 3 Jahren implementiert (zuvor war nur das letzte Jahr des Kindergartens verpflichtend), um benachteiligte Kinder durch öffentliche Betreuungseinrichtungen zu fördern. Für Kinder ab 3 Jahren bis zum schulpflichtigen Alter von 6 Jahren zeigt sich, dass Ungarn mit einer Versorgungsquote dieser Gruppe von 89,1\% oberhalb des EU-Durchschnitts von 83,3\% liegt (vgl. Europäische Kommission 2018: o. S.). 


\section{Arbeitsmarktpolitik}

Arbeitsmarktpolitische Maßnahmen können die Möglichkeiten der Vereinbarkeit von Beruf und Familie erleichtern, indem Teilzeitarbeit ermöglicht wird. In Westeuropa sind meist flexible Arbeitsarrangements möglich, wodurch viele Frauen in Teilzeit arbeiten. Da in den osteuropäischen Ländern Kindertagesstätten für Kinder unter 3 Jahren nicht ohne weiteres zur Verfügung stehen, ist gemäß Saxonberg/Sirovátka (2006) zu erwarten, dass Mütter solange in Teilzeit arbeiten wollen, bis ihre Kinder in den Kindergarten kommen, um auf dem Arbeitsmarkt Fuß zu fassen und zugleich die Familie durch ein ergänzendes Einkommen zu unterstützen. Saxonberg/Sirovátka (2006) zufolge haben die osteuropäischen Regierungen jedoch bisher die Richtlinie 98/23/EG des Rates nicht umgesetzt und Rechtsvorschriften erlassen, die Eltern von Kleinkindern die Möglichkeit geben, ihr Recht auf Teilzeitarbeit durchzusetzen. Sie haben auch keine steuerlichen Anreize oder andere Maßnahmen eingeführt, um Teilzeitarbeit zu erleichtern. Folglich arbeiten Frauen in Osteuropa weniger in Teilzeit als in Westeuropa mit Ausnahme von Polen (in Polen ist Teilzeitarbeit von Frauen nach wie vor im europäischen Vergleich selten, doch im Vergleich zwischen den osteuropäischen Ländern weist Polen eine der höchsten Teilzeitquoten von Frauen auf), weil polnische Mütter häufig familiäre Unterstützung für die Kinderbetreuung durch ihre Eltern erhalten (vgl. Saxonberg/Sirovátka 2006: 191). Während der Anteil von Frauen am Arbeitsmarkt nach dem Mauerfall nur einen moderaten Rückgang erfahren hat, ist die Fertilitätsrate in Osteuropa dramatisch - um fast $50 \%$ - eingebrochen. Da sich die meisten osteuropäischen Frauen verpflichtet fühlen, arbeiten zu müssen, um ihre Familie zu unterstützen, wird eine Familienpolitik, die auf eine Erwerbsunterbrechung zugunsten der Kinderbetreuung abzielt, genau das Gegenteil bewirken: Frauen werden schlussfolgern, dass sie es sich nicht leisten können den Arbeitsmarkt zu verlassen, um Kinder zu bekommen (vgl. Saxonberg/Szelewa 2007: 367). Die Einkommen der Väter sind zu niedrig, als sie für die Verpflegung der ganzen Familie ausreichend sein könnten, und staatliche finanzielle Anreize, wie das Eltern- oder das Kindergeld, sind zu gering, um den Verlust eines zweiten Einkommens zu kompensieren (vgl. Saxonberg/Szelewa 2007: 368). Dies könnte ein Grund für die immer noch sehr niedrige Fertilitätsrate in Ungarn (gemäß der OECD liegt sie im Jahr 2015 bei 1,4 womit sie zu den niedrigsten Fertilitätsraten in Europa zählt; dennoch ist dies seit 1997 der höchste Wert Ungarns. Die Fertilitätsrate erreichte 2011 mit einem Wert von 1,2 ihren Tiefpunkt und stieg bis 2015 kontinuierlich an) in Kombination mit dem - von der Europäischen Kommission bezeichneten - DoppelverdienerInnen-Modell sein: Die Erwerbsbeteiligung der 15- bis 64-jährigen Männer (73\%) und Frauen (60,2\%) weist im Jahr 2016 kaum Unterschiede zum EUDurchschnitt auf (Frauen 61,4\%; Männer 66,6\%), wobei, wie bereits be- 
schrieben, Frauen mit Kleinkinder meist 2-3 Jahre den Arbeitsmarkt verlassen (vgl. Europäische Kommission 2018: o. S.).

„Seit nunmehr 50 Jahren ermutigt das ungarische familienpolitische System Frauen, den Arbeitsmarkt für etwa drei Jahre nach der Geburt zu verlassen. Seit der Einführung des bezahlten Elternurlaubs (GYES) im Jahr 1967 ist dies zur gesellschaftlichen Norm geworden und wird von den meisten Müttern und Vätern akzeptiert, wobei letztere in diesen Jahren zum Haupternährer wurden“ (Europäische Kommission 2018: o. S.).

Teilzeitarbeit ist nach wie vor in Ungarn kaum verbreitet, da lediglich $6,8 \%$ der Frauen in Teilzeit arbeiten (EU-Durchschnitt: 31,9\%). Gleichwohl wurde 2014 eine Reform eingeführt, die die Erwerbstätigkeit von Müttern mit Kindern unter 3 Jahren in Form einer Teilzeitbeschäftigung durch finanzielle Anreize vor dem 2. Geburtstag des Kindes fördern soll. Im Sinne eines optionalen Familialismus werden hierdurch mehrere Alternativen geboten, weil es nach wie vor auch möglich und unabhängig von der vorherigen Erwerbstätigkeit ist, mehrere Jahre bezahlte Elternzeit zu Hause zu verbringen, um die Kinder im Alter von bis zu drei Jahren zu betreuen (ab dem dritten Kind 8 Jahre) (vgl. Europäische Kommission 2018: o. S.).

Die Beschäftigungsquoten in Tschechien liegen gemäß den aktuellsten Daten für beide Geschlechter oberhalb des EU-Durchschnitts: 2016 befinden sich 79,3\% der Männer (EU-Durchschnitt: 71,9\%) und 64,4\% der Frauen (EU-Durchschnitt: 61,4\%) in einem Beschäftigungsverhältnis. Jedoch ist die Erwerbsquote von Müttern mit Kindern unter 6 Jahren sehr niedrig (45,2\% in Tschechien, $61,4 \%$ in der EU), hingegen die der Väter sehr hoch $(94,7 \%$ in Tschechien, 88,2\% in der EU). Im Jahr 2015 liegt der Gender Pay Gap mit $22,5 \%$ deutlich über dem EU-Durchschnitt (16,3\%). Auch in diesem Land ist die Teilzeitbeschäftigung kaum verbreitet. 2016 beträgt der Anteil teilzeitbeschäftigter Frauen lediglich 10\% (EU-Durchschnitt: 31,9\%) (vgl. Europäische Kommission 2018: o. S.).

In Polen ist aktuell im Ländervergleich die niedrigste Fertilitätsrate vorzufinden (1,3) (vgl. Weltbank 2018: o. S.). Die derzeitige Erwerbstätigenquote von Männern (71,9\%) entspricht (im Jahr 2016) ungefähr dem EUDurchschnitt (71\%), die der Frauen ist im EU-Vergleich $(61,4 \%$ in der EU) etwas niedriger (58,1\% in Polen). Teilzeitbeschäftigungen sind immer noch selten: lediglich 9,7\% der Frauen (und 3,7\% der Männer) befinden sich in einem Teilzeitbeschäftigungsverhältnis. Hingegen ist die Erwerbsbeteiligung von Eltern mit Kindern unter 6 Jahren $(62,2 \%$ der Mütter und 90,5\% der Väter) im europäischen Vergleich hoch (vgl. Europäische Kommission 2018: o. S). Aufgrund des allgemeinen Ziels einer Optimierung der Lebensbedingungen wurde im Januar 2017 der Mindestlohn auf 2000 Złoty (ca. 467,15€) und im Januar 2018 auf 2100 Złoty (ca. 490,51 €) aufgestockt. Auch der Mindeststundenlohn wurde im Januar 2017 auf 13,00 Złoty (ca. 4,06 €) und 
im Januar 2018 auf 13,70 Złoty angehoben (vgl. Europäische Kommission 2018: o. S.).

Obwohl die postkommunistischen Länder durchaus Gemeinsamkeiten im Hinblick auf einen Re-Familisierungstrend aufweisen, sind die Unterschiede zwischen den Ländern nicht zu vernachlässigen. Saxonberg/Szelewa (2007) greifen dieses Argument auf und kontrastieren die tschechische und die polnische Familienpolitik, um die Unterschiede zwischen beiden Ländern zu vertiefen bzw. zu erklären. Ein oftmals genutztes Argument zur Erklärung der Gemeinsamkeiten postkommunistischer Länder bezieht sich auf den Einfluss der katholischen Kirche. Da Polen streng katholisch und Tschechien vergleichsweise säkularisiert ist, müsste - im Sinne der „Catholic Hypothesis“ - in Polen das konservative ,general family support model“ stärker ausgeprägt sein als in Tschechien. Doch konnten Saxonberg/Sirovátka (2006) sowie Saxonberg/Szelewa (2007) zeigen, dass Tschechien stärker am konservativen Modell orientiert ist und Polen hingegen stärker dem liberalen, marktorientierten Modell näherkommt, nachdem familiale Maßnahmen gemäß dem Markt reguliert und einer Bedarfsüberprüfung unterzogen werden (vgl. Saxonberg/Szelewa 2007: 355). Dies manifestiert sich u. a. in Polen in viel geringeren Investitionen für Kindergärten als in Tschechien (vgl. Saxonberg/Szelewa 2007: 359). Zudem zeigt sich eine historische Pfadabhängigkeit, da Polen auch unter der kommunistischen Herrschaft deutlich weniger Kindergartenplätze zur Verfügung stellte als die Tschechische Republik.

\section{Ökonomische Rahmenbedingungen}

Was die wirtschaftliche Leistungsfähigkeit anbelangt, liegt Tschechien auf deutlich höherem Niveau als Polen und Ungarn. Polen und Ungarn weisen im Zeitraum zwischen 2001 und 2016 einen äußerst ähnlichen konstanten Anstieg des Bruttoinlandsprodukts pro Kopf auf niedrigem Niveau auf. Aktuell beträgt das Bruttoinlandsprodukt pro Kopf in Tschechien 34749,21 KKS, in Polen 27383,25 KKS und in Ungarn hingegen nur 26700,76 KKS (vgl. Weltbank 2018: o. S.). Auch die Armutsgefährdungsquote ist im Jahr 2017 in Tschechien mit 9,2\% der Bevölkerung deutlich niedriger als in Ungarn $(13,4 \%)$ und in Polen $(15 \%)$ - im EU-Vergleich weist Tschechien sogar die niedrigste Armutsgefährdungsquote auf. Im Hinblick auf die erhebliche materielle Deprivation liegt Ungarn mit 14,5\% weit vorne (wobei seit 2013 ein kontinuierlicher Rückgang von ehemals $27,8 \%$ auf $14,5 \%$ zu verzeichnen ist), gefolgt von Polen mit 5,9\% und Tschechien mit lediglich 3,7\% (vgl. Eurostat 2018a: o. S.). Während in Ungarn die Situation folglich sehr prekär erscheint, ist in Tschechien das Armutsniveau auffallend niedrig. Auch die Verteilung der Einkommen ist in Tschechien am homogensten: Der GiniIndex beträgt im Jahr 2015 nur 25,9\%, in Ungarn 30,4\% und in Polen 31,8\% (vgl. Weltbank 2018: o. S.). Selbst im Vergleich zu allen anderen Ländern ist Tschechien Spitzenreiter einer homogenen Einkommensverteilung. Eine weitere Spitzenreiterposition ist für Tschechien in Bezug auf den Gender Pay 
Gap vorzufinden - diesmal jedoch mit Blick auf eine Ungleichverteilung. In keinem anderen der dargestellten Länder sind die Bruttostundenlöhne zwischen den Geschlechtern derzeit so ungleich verteilt wie in Tschechien. Der Gender Pay Gap beträgt im Jahr 2016 in Tschechien 21,8\%, in Ungarn 14\% und in Polen nur 7,2\% (vgl. UNECE 2018: o. S.).

\subsubsection{Familienpolitik in Litauen}

Litauen ist (neben Ungarn) gemäß Szelewa/Polakowski (2008) dem „comprehensive support model“ zuzuordnen, weil dieses Modell unterschiedliche Kombinationen von bezahlter Beschäftigung und Kinderbetreuung zulässt und den Familien dadurch eine individuelle Wahlmöglichkeit in Form von umfassenden Alternativen bietet. Es stehen öffentliche Kinderbetreuungseinrichtungen zur Verfügung und insbesondere DoppelverdienerInnenpaare werden gefördert. Familien und Frauen werden im Hinblick auf die Kinderbetreuung sowohl entlohnt als auch entlastet, was zudem eine Mobilisierung von DoppelverdienerInnen innerhalb der Familie beinhaltet (vgl. Szelewa/Polakowski 2008: 127).

Klein (2013) stellt eine hohe lohnbezogene Unterstützung in Form der Elternzeitregelung fest. Im Jahr 2010 wurden 85\% des vorherigen Gehalts über einen Zeitraum von 24 Monaten gewährt (vgl. Klein 2013: 181). Der Elternurlaub kann für eine maximale Dauer von 3 Jahren genommen werden, jedoch wird nur im ersten Jahr eine stark einkommensabhängige Unterstützung geleistet. Für den 24-monatigen bezahlten Elternurlaub bestehen aktuell zwei Möglichkeiten:

„Entweder die Inanspruchnahme des Elternurlaubs über einen Zeitraum von 12 Monaten (bei $100 \%$ des bisherigen Gehalts) oder über einen Zeitraum von 24 Monaten (bei 70\% des bisherigen Gehalts im ersten Jahr und $40 \%$ im zweiten Jahr). Eltern haben Anspruch auf Elterngeld, wenn sie in den vergangenen 24 Monaten mindestens 12 Monate lang Sozialversicherungsabgaben gezahlt haben. [...] Bei Mehrlingsgeburten erhöht sich das Elterngeld entsprechend der Anzahl der Kinder, jedoch nicht um mehr als das 3,2-fache des durchschnittlichen versicherten Monatseinkommens (derzeit $1.379 €$ )““ (Europäische Kommission 2018: o. S.).

Der Elternurlaub kann auch von anderen Bezugspersonen als der Mutter, wie z. B. von Großmüttern oder Großvätern, genommen werden, wobei diese keinen Anspruch auf Elterngeld haben. Insgesamt werden 1,1\% des Bruttoinlandsprodukts für Sozialschutzleistungen aufgewendet (EU-Durchschnitt: $2,4 \%$ ). Bei der Betrachtung von postsozialistischen Ländern in Bezug auf die Versorgungsquote von 0 - bis 2-jährigen Kindern fällt die vergleichsweise hohe Quote in Litauen auf (die Daten der OECD sind im Zeitverlauf von $2005(17 \%)$ bis $2014(28,8 \%)$ verfügbar). Jedoch ist anzumerken, dass die 
Versorgungsquote der 0- bis 2-jährigen Kinder in Litauen gemäß der OECD großen Schwankungen unterliegt (2005: 17\%, 2006: 8,1\%, 2007: 26,9\%, 2008: 13,7\%, 2009: 14,8\%, 2010: 16,07\%, 2011: 11,8\%, 2012: 9\%, 2013: 12,1\%, 2014: 28,87\%). Die Europäische Kommission stellt für das Jahr 2015 fest, dass die Versorgungsquote der Kinder im Alter von 0-3 Jahren mit 9,7\% im Vergleich zum EU-Durchschnitt von 30,3\% relativ gering ist. Für Kinder im Alter von 3 Jahren bis zum schulpflichtigen Alter beträgt die Versorgungsquote $73,8 \%$ und liegt damit ebenfalls unterhalb des EUDurchschnitts von 83,3\%. Die Vorschulerziehung für 6-Jährige ist seit 2016 verpflichtend. Da die Kinderbetreuungseinrichtungen der Nachfrage nicht gerecht werden können, ist der Ausbau der Betreuungsangebote eine aktuelle Priorität der Kommunen - insbesondere aufgrund der hohen Quote armutsgefährdeter Kinder (32,7\% im Jahr 2015) (vgl. Europäische Kommission 2018: o. S.).

Obgleich die Teilzeitquoten von Frauen postsozialistischer Länder insbesondere im Vergleich zu westeuropäischen Ländern sehr gering sind, so zeigt sich doch eine für postkommunistische Länder verhältnismäßig eher hohe Teilzeitquote in Litauen (lediglich in Polen sind noch höhere Teilzeitquoten vorzufinden) - zumindest zwischen 1998 und 2005 lag sie im zweistelligen Bereich und erreichte 1999 einen Höhepunkt von 15,13\% (vgl. OECD 2017: o. S.). 2016 liegt gemäß Europäischer Kommission die Teilzeitbeschäftigungsquote der 15- bis 64-jährigen Frauen bei 8,8\% und die der Männer bei $5,4 \%$. Für Frauen mit Kindern unter 6 Jahren ist festzustellen, dass diese in Litauen besonders häufig erwerbstätig sind: Im Jahr 2016 gehen 75,2\% einer Beschäftigung nach, hingegen sind es im EU-Durchschnitt nur 61,4\%. Jedoch bestehen Unterschiede zwischen den Gehältern von Männern und Frauen, obwohl das geschlechtsspezifische Lohngefälle mit 14,2\% etwas niedriger als im EU-Durchschnitt (16,3\%) ist. Die aktive Rolle der Frauen auf dem Arbeitsmarkt in Litauen wird nicht zuletzt durch die Erwerbsquote von Frauen im Alter zwischen 15-64 Jahren mit 68,8\% (im Jahr 2016) im Vergleich zu den Männern mit 70\% ersichtlich (EU-Durchschnitt: Männer - 71,9\%, Frauen - 61,4\%) (vgl. Europäische Kommission 2018: o. S.).

\section{Ökonomische Rahmenbedingungen}

Das Bruttoinlandsprodukt pro Kopf beläuft sich in Litauen im Jahr 2016 auf 29862,32 KKS und ist folglich geringfügig höher als in Polen und Ungarn. Aufgrund der Finanzkrise ist zwischen 2008 und 2009 eine Rezession insofern festzustellen, als das Bruttoinlandsprodukt pro Kopf von 20744,07 KKS auf 18142,65 KKS gesunken ist (vgl. Weltbank 2018: o. S.). Seit 2010 ist jedoch wieder ein kontinuierlicher Anstieg der Wirtschaftsleistung Litauens zu konstatieren. Auffallend hoch ist die Armutsgefährdungsquote: Im Jahr 2017 erreicht sie einen Spitzenwert von $22,9 \%$ und liegt damit nur etwas unterhalb von dem vergleichsweise höchsten Armutsgefährdungsniveau von Rumänien und Bulgarien. Ferner ist ein deutlicher Anstieg im Zeitverlauf 
zwischen 2001 und 2017 von $17 \%$ auf $22,9 \%$ zu verzeichnen. Hingegen entwickelt sich die Rate der erheblichen materiellen Deprivation positiv: sie ist seit 2012 rückläufig - insgesamt dennoch mit aktuellen 12,4\% auf hohem Niveau. Auch der Bevölkerungsanteil, der von Armut und sozialer Ausgrenzung bedroht ist, ist mit derzeit 29,6\% hoch (vgl. Eurostat 2018a: o. S.). Zugleich zeigt sich, dass Litauen aktuell gemeinsam mit Russland Spitzenreiter der Einkommensungleichverteilung ist. Der Gini-Index von 37,4\% liegt im Jahr 2017 deutlich oberhalb der Gini-Indizes der anderen Länder (vgl. Weltbank 2018: o. S.). Wie bereits erläutert, ist das geschlechtsspezifische Lohngefälle in Litauen etwas niedriger als im EU-Durchschnitt, gleichwohl seit 2011 ein kontinuierlicher Anstieg von 11,5\% (2011) auf 14,4\% (2016) zu beobachten ist (vgl. UNECE 2018: o. S.).

\subsubsection{Familienpolitik in Bulgarien}

Auch in Bulgarien, das vom orthodoxen Christentum geprägt ist, ist ein Familisierungstrend der Familienpolitik seit der Wende 1989 vorzufinden. Die Fertilitätsrate ist ebenfalls dramatisch eingebrochen: sie lag 1989 bei 1,9 und ist kontinuierlich bis 1997 auf einen Tiefpunkt von 1,09 gefallen (vgl. GGP 2017: o. S.). Derzeit (Daten für 2015) ist gemäß der OECD eine Fertilitätsrate von $1,5 \mathrm{zu}$ verzeichnen. Aufgrund der prekären wirtschaftlichen Situation des Landes wurden nach 1989 die Sozialausgaben gekürzt sowie staatliche Kinderbetreuungseinrichtungen drastisch reduziert, wodurch viele Familien fortan private Kinderbetreuungseinrichtungen in Anspruch nehmen mussten (vgl. Liebhart et al. 2003: 422). 1998 wurde die Elternzeit angeglichen: vor 1989 betrug der Mutterschaftsurlaub für das erste Kind 120 Tage, für das zweite 150 Tage und für alle weiteren Kinder 180 Tage. Der Mutterschaftsurlaub wurde anschließend durch eine einheitliche Gesetzgebung für alle Geburten auf 135 Tage normiert, wovon 45 Tage vor der Geburt genommen werden müssen. Aktuell sticht Bulgarien mit einem Mutterschutz von 58,6 Wochen (Daten für 2015) im Vergleich zu den anderen hier aufgeführten Ländern hervor. Weiterhin stehen 51,9 Wochen bezahlte Elternzeit für die Mutter zur Verfügung, sodass sich insgesamt 110,4 Wochen ergeben (vgl. OECD 2018: o. S.). 2003 wurde das Recht auf unbezahlte Elternzeit modifiziert, indem die sechsmonatige Erziehungszeit nicht mehr von anderen Verwandten, sondern von den Eltern genommen werden muss. Diese Maßnahme erzielte eine Kindererziehung, die primär durch die Eltern und weniger durch die erweiterte Familie erfolgen soll. Doch zugleich löste sie eine Forderung der bulgarischen Frauenbewegung in Form einer Flexibilisierung der Arbeitszeiten aus, da mehrheitlich beide Elternteile in Vollzeit erwerbstätig waren (vgl. Liebhart et al. 2003: 422). An der Teilzeitquote von Frauen änderte sich trotz dieser Forderung nicht viel: sie blieb bis heute konstant auf 
sehr niedrigem Niveau und ist derzeit die niedrigste im europäischen Vergleich. Im Zeitverlauf von 2000 bis 2016 ist ein maximaler Wert von 3,63\% im Jahr 2001 vorzufinden; die aktuellsten Daten basieren auf dem Jahr 2016 mit einer Teilzeitquote der Frauen von 2\% (vgl. OECD 2017: o. S.). 2002 wurde ein Gesetz für den Kinderschutz verabschiedet, mit dem ein neuer Ansatz der Familienpolitik zum Wohl der Kinder und ihrer Rechte zu verzeichnen ist. Sofern das Kindeswohl gefährdet ist, wird dem Staat das Recht eines Eingriffes in die Privatsphäre gewährt. Maßnahmen wie diese erstreben u. a. Problemen wie Kinderprostitution oder dem von den Eltern erzwungenen Betteln von Kindern entgegenzuwirken. Dennoch sollen Kinder möglichst in ihren Familien und nicht in staatlichen Institutionen aufwachsen (vgl. Liebhart et al. 2003: 422) - vielleicht in Erinnerung an die katastrophalen Bedingungen der Kinderheime in Bulgarien (vergleichsweise prekär waren sie auch in Rumänien)? Aktuell liegt ein Schwerpunkt der Familienpolitik Bulgariens in der Bekämpfung von Kinderarmut und sozialer Ausgrenzung (insbesondere für Kinder aus gefährdeten Gruppen, wie z. B. ethnische Minderheiten wie Roma, Kinderflüchtlinge etc.). Im Jahr 2015 sind 43,7 \% der Kinder unter 18 von Armut oder sozialer Ausgrenzung bedroht (im Vergleich zu 27,1\% in der EU; in Tschechien sind es sogar nur 18,5\%). 2017 wurde vom Ministerrat eine entsprechende nationale Strategie zur Bekämpfung von Armut und zur Förderung sozialer Eingliederung 2020 verabschiedet (vgl. Europäische Kommission 2018: o. S). Insgesamt ist für Bulgarien festzuhalten, dass ökonomische Zwänge sowie fehlende öffentliche Kinderbetreuungseinrichtungen auch in diesem postsozialistischen Land auf einen ReFamilisierungstrend hinweisen (vgl. Liebhart et al. 2003: 422). Gleichwohl werden derzeit Maßnahmen zur Förderung der Erwerbstätigkeit von Eltern etabliert. Das Ministerium für Arbeit und Sozialpolitik hat beispielsweise 2017 das Projekt „Eltern in der Erwerbstätigkeit“ gegründet. Außerdem werden die für den/die ArbeitgeberIn anfallenden Kosten für Löhne und Sozialbeiträge für jeden geschaffenen Arbeitsplatz vom Staatshaushalt finanziert, sofern arbeitslose Eltern mit Kindern bis zu 5 Jahren eingestellt werden. Die Frauenerwerbsquote von Frauen mit Kindern unter 6 Jahren ist im Jahr 2016 mit 55,2\% niedriger als im EU-Durchschnitt (61,4\%). Auch die Erwerbsquote von Vätern mit Kindern, die jünger als 6 Jahre alt sind, ist in Bulgarien mit $86,6 \%$ etwas niedriger als im EU-Durchschnitt (88,2\%) (vgl. Europäische Kommission 2018: o. S.). Ein Grund vermag der immer noch schlechte Zugang zu öffentlichen Kinderbetreuungsplätzen sein. Obwohl die Zahl der Kinderbetreuungseinrichtungen bis zum Kindergartenjahr 2016/2017 anstieg (auf 1894 Kindergärten mit insgesamt 237962 Plätzen), ist der Anteil der Kinder, die Zugang zu öffentlichen frühkindlichen Bildungseinrichtungen haben, sehr gering. 8,9\% der bulgarischen Kinder unter 3 Jahren werden im Jahr 2015 durch öffentliche Kinderbetreuungseinrichtungen betreut (im EUDurchschnitt sind es 30,3\%). Hingegen besuchen 71,5\% der Kinder im Alter 
von 3 Jahren bis zum schulpflichtigen Alter eine öffentliche Betreuungseinrichtung (im EU-Durchschnitt 83,3\%) (vgl. Europäische Kommission 2018: o. S.).

\section{Ökonomische Rahmenbedingungen}

Die wirtschaftliche Leistungsfähigkeit Bulgariens ist im Jahr 2016 mit 19242,62 KKS auf sehr niedrigem Niveau - sie stellt im Vergleich zu den anderen europäischen Ländern das Schlusslicht dar (vgl. Weltbank 2018: o. S.). Die prekären ökonomischen Rahmenbedingungen spiegeln sich auch in extremer Armut wider: Bulgarien weist im Jahr 2017 mit 23,4\% eine der höchsten Armutsquoten auf, im Hinblick auf erhebliche materielle Deprivation $(30 \%)$ und Armut und soziale Ausgrenzung $(38,9 \%)$ ist Bulgarien Spitzenreiter (vgl. Eurostat 2018a: o. S.). Doch während die beiden letzten Armutsindikatoren seit 2006 durch einen rückläufigen Trend gekennzeichnet sind, steigt die Armutsgefährdungsquote deutlich an. Weiterhin ist festzustellen, dass die Einkommen im Vergleich zu den anderen Ländern - im Übrigen auch mit deutlichem Unterschied zu Rumänien - sehr ungleich verteilt sind. Gemäß den aktuellsten Daten aus dem Jahr 2014 beträgt der Gini-Index 37,4\%, wobei die Einkommensungleichheit seit 2011 stetig wächst (vgl. Weltbank 2048: o. S.). Das geschlechtsspezifische Lohngefälle beläuft sich im Jahr 2016 auf 14,4\% und weist folglich sehr große Diskrepanzen zu Rumänien auf, dessen Gender Pay Gap das niedrigste Level erreicht (vgl. UNECE 2018: o. S.).

\subsubsection{Familienpolitik in Rumänien}

Fodor et al. (2002) vergleichen die familienpolitischen Rahmenbedingungen in Rumänien, Polen und Ungarn, weil die genderspezifische Armutslücke zwischen diesen Ländern variiert. Frauen, die von Armut betroffen sind, sind im Vergleich zu Ungarn und Polen insbesondere in Rumänien überrepräsentiert (vgl. Fodoer et al. 2002: 476). Auch die Kinderarmut ist in Rumänien weit verbreitet. 49,2\% der Kinder und Jugendlichen unter 18 Jahren sind im Jahr 2016 von Armut bedroht (vgl. Europäische Kommission 2018: o. S.). Das Hauptargument von Fodor et al. ist, dass die drei Länder sich in Bezug auf politische Fördermaßnahmen der Elternschaft unterscheiden (vgl. Fodor et al. 2002: 477). Schon vor dem Zerfall der kommunistischen Regime gab es erhebliche Unterschiede zwischen diesen Ländern in Bezug auf die Grundlagen des Anspruchs auf familienpolitische Leistungen. In Rumänien und in Polen konnten Frauen Mutterschaftsleistungen auf der Grundlage ihrer Erwerbstätigkeit beanspruchen. Da nahezu alle Frauen erwerbstätig waren, sind gemäß Fodor et al. (2002) jedoch keine großen Unterschiede zum ungarischen Regime nach 1985 festzustellen, das unabhängig vom Beschäftigungsstatus allen Müttern Mutterschaftsleistungen gewährte (vgl. Fodor et al. 
2002: 483). Im christlich orthodoxen Rumänien fielen Familienleistungen zwar auf der Grundlage universeller Rechte an, jedoch konnte die Elternzeit nur von Frauen mit einer Erwerbstätigkeit von mindestens sechs Monaten beansprucht werden. Die eigene Kindererziehungsarbeit arbeitsloser Frauen wurde weder bezahlt noch für die Rente berücksichtigt. Rumänien bot universelle Absicherungen für Familien, aber die häusliche Kindererziehung wurde durch diese Einschränkung deutlich abgewertet. Mütter waren folglich entweder von der Erwerbstätigkeit einschließlich der Sozialversicherung oder von ihren Familien abhängig (vgl. Fodor et al. 2002: 483). Fodor et al. (2002) stellen insgesamt große Unterschiede der familienpolitischen Maßnahmen zwischen Polen, Ungarn und Rumänien fest. Der polnische Wohlfahrtsstaat schränkt ihnen zufolge die Berechtigung auf Familien- und Mutterschaftsleistungen ein und ermutigt Frauen, arbeitslos zu werden, wodurch sie von ihren Ehepartnern abhängig werden. Ungarn biete durch seine Fördermaßnahmen ein ausgewogeneres Verhältnis zwischen bezahlter Arbeit und Familie für Frauen. Rumänien gewähre Frauen zwar mehr Freiheiten bezüglich ihrer Erwerbstätigkeit als Polen, jedoch würde nicht ausreichend Lebensunterhalt geboten, um Frauen die Möglichkeit zu eröffnen, unabhängige Haushalte gründen zu können (vgl. Fodor et al. 2002: 477). In Anbetracht des Gender Pay Gaps (s. ökonomische Rahmenbedingungen) zeigt sich, dass Rumänien (ebenfalls Slowenien) einen niedrigen Gender Pay Gap erreicht, während Tschechien und Litauen (sowie Estland und die Slowakei, die in der vorliegenden Arbeit jedoch nicht berücksichtigt werden) einen hohen Gender Pay Gap aufweisen (vgl. Robila 2012: 36f.). Aktuell werden in Rumänien Eltern darin unterstützt, früher als es die Elternzeit vorsieht, wieder in die Erwerbstätigkeit zurückzukehren. Mehrere Kindertagesstätten werden für Kinder zur Verfügung gestellt, jedoch ist die Anzahl an Plätzen begrenzt. Für das Jahr 2015 ist gemäß den Eurostat-Daten festzuhalten, dass 9,4\% der Kinder unter 3 Jahren in Rumänien Zugang zu öffentlichen Kinderbetreuungseinrichtungen hat, wobei dies deutlich unter dem EU-Durchschnitt von 30,3\% liegt. Auch sind zwar viele Frauen in Vollzeit erwerbstätig (im Jahr 2015: 60,3\%), aber im europäischen Vergleich zeigt sich, dass diese Erwerbsquote ebenfalls unterhalb des EU-Durchschnitts liegt (71,4\%). Die Erwerbsquote von Müttern mit Kindern unter sechs Jahren $(54,3 \%)$ ist ebenfalls niedriger als der europäische Durchschnitt (61,4\%) (vgl. Europäische Kommission 2018: o. S.).

„Gemäß eines Berichts aus dem Jahr 2017 über Elternurlaub können bis zu 24 Monate als bezahlter Elternurlaub in Anspruch genommen werden [im Falle eines Kindes mit Behinderung bis zu drei Jahre], und es stehen mindestens 1063 Lei pro Monat (ca. $231 €$ ) zur Verfügung. [...] Es ist Pflicht, dass sich Eltern einen Monat Elternurlaub der Gesamtdauer des zulässigen Elternurlaubs teilen. Wenn die Mutter Elternzeit genommen hat, bedeutet das, dass der Vater einen Monat Elternzeit in Anspruch nehmen muss. Die Regierung bietet Eltern, die vor 
Ablauf des gesamten Elternurlaubs in den Beruf zurückkehren, einen Anreiz (531 Lei, ca. $115 €$ pro Monat bis zu einem Alter von 3 Jahren, bzw. bis zu 4 Jahren für behinderte Kinder)“ (Europäische Kommission 2018: o. S.).

\section{Ökonomische Rahmenbedingungen}

Die wirtschaftliche Leistungsfähigkeit Rumäniens liegt zwar mit einem Bruttoinlandsprodukt pro Kopf von 23027,29 KKS oberhalb derer von Bulgarien und Georgien, ist jedoch insgesamt auf eher niedrigem Niveau (vgl. Weltbank 2018: o. S.). Außergewöhnlich hoch ist die Quote der von Armut bedrohten Personen - aktuell beträgt sie 23,6\%. Die Armutsgefährdungsquote liegt bereits seit 2001 oberhalb des Niveaus der anderen Länder. In Bezug auf die erhebliche materielle Deprivation (im Jahr 2017: 19,7\%) sowie die von Armut und sozialer Ausgrenzung bedrohte Bevölkerung (im Jahr 2017: $35,7 \%$ ) erreicht Rumänien den 2. Platz (lediglich Bulgarien weist höhere Werte auf) - die Tendenz ist jedoch rückläufig (vgl. Eurostat 2018a: o. S.). Alle drei Armutsindikatoren deuten auf eine Armutsproblematik in Rumänien hin. Hingegen ist die Einkommensverteilung im Vergleich zu den anderen dargestellten Ländern mit einem Gini-Index von aktuell 28,3\% (2016) relativ homogen (vgl. Weltbank 2018: o. S.). Positiv anzumerken ist außerdem das geschlechtsspezifische Lohngefälle Rumäniens, das mit derzeit 5,2\% (2016) unschlagbar niedrig ist (vgl. UNECE 2018: o. S.). Diese Führungsposition eines sehr niedrigen Gender Pay Gaps hat Rumänien bereits seit 2013 inne.

\subsubsection{Familienpolitik in Georgien}

Auch in Georgien waren die Auswirkungen des kommunistischen Zusammenbruchs deutlich zu spüren. Viele frühkindliche Kinderbetreuungseinrichtungen wurden nach der Wende geschlossen, wodurch der Anteil der Kinder im Alter zwischen 0-2, die im Rahmen von „Nurseries“ betreut wurden, von 16\% im Jahr 1989 auf 7\% im Jahr 1997 fiel. Der Anteil der 3- bis 6-Jährigen betrug im Jahr 1997 44\% und ist bis 1997 auf 19\% gesunken (vgl. UNICEF 1999: 12; Pascall/Manning 2000: 253). Das Elterngeld wurde in Georgien nach dem Zerfall der Sowjetunion zunächst vorübergehend abgeschafft (Pascall/Manning 2000: 254). Baumann (2012) erläutert einen weiteren wichtigen historischen Einschnitt der Sozialpolitik in Georgien, der durch die Rosenrevolution (2003) ${ }^{132}$ - eine Revolution, die häufig als Forderung zu mehr Demokratie unter der Headline ,going West“ interpretiert wird - ausgelöst wurde (vgl. Baumann 2012: 259). Die Unzufriedenheit mit der Regierung unter der Leitung des ehemaligen Präsidenten Eduard Schewardnadse,

132 Die Bezeichnung der Rosenrevolution geht auf die Stürmung des Parlaments am 22.11.2003 von DemonstrantInnen unter der Anführung Saakaschwilis zurück, die während der Stürmung unter dem Motto „Wir werden Rosen statt Kugeln auf unsere Feinde werfen“ Rosen in den Händen hielten. 
die stark verwurzelte Korruption im Land sowie die hohe Armut mögen Auslöser gewesen sein, warum die „Vereinte Nationale Bewegung“ unter der Leitung von Micheil Saakaschwili (ehemaliger Justizminister) zu Protesten im Land, einem Rücktritt Schewardnadses und dem Wahlsieg Saakaschwilis im Rahmen der neuen Präsidentschaftswahlen im Jahr 2004 geführt haben. Baumann (2012) konstatiert in der Folge eine Entwicklung ultra-liberaler Sozialreformen in Georgien.

„In the aftermath of the Rose Revolution, Micheil Saakashvili's regime hastened a powerful modernisation process of public institutions, with ultra-liberalism, privatisation and downsizing of the state being considered the "best practices" for economic development. This approach is in line with New Public Management methods which consider competitiveness as the key feature of any organisation. Not surprisingly, social policy, too, has been affected by the fervent belief in market mechanisms and the imperative of competitiveness. Consequently, the health care system was privatized and social protection is now focusing on private insurance which is believed to progressively become the core of the system. [...] These mechanisms meet the family centred habitus of Georgian society, thus fostering solidarity based on kinship relations and personal ties. Social networks therefore continue being activated in order to overcome the shortcomings of the public protection system" (Baumann 2012: 279f., Hervorhebungen im Original; die Verf.).

Im Vergleich zur Sowjetunion waren die Sozialleistungen in dieser Periode extrem niedrig, wodurch die Bevölkerung sehr stark auf familiale Solidarität angewiesen war (vgl. Baumann 2012: 260). Ferner zieht sie die Schlussfolgerung, dass , ,...] the Rose Revolution has not been a decisive step towards democratisation in the sense of a development process allowing for egalitarian access to wealth" (Baumann 2012: 260). Dennoch könne ein ,langer, langer Weg“ einer Verlagerung hin zu ,demokratischeren Sozialdienstleistungen" nicht ausgeschlossen werden (vgl. Baumann 2012: 260).

Aktuell (basierend auf den ILO-Daten für 2013) besteht im Rahmen der sozialstaatlichen Leistungen ein Anspruch auf Mutterschaftsgeld in Höhe von $100 \%$ des Durchschnittsgehalts, das bis zu 126 Tage ausbezahlt wird (im Falle von Mehrfachgeburten sowie Geburten mit Komplikationen bis zu 140 Tage) (vgl. DACH 2011: 9; ILO 2014: 138). Der maximale finanzielle Wert beläuft sich auf $600 \mathrm{GEL}$ (ca. $255 €$ ). Bis zu drei Jahren kann außerdem der unbezahlte Mutterschaftsurlaub in Anspruch genommen werden (vgl. DACH 2011: 9f.). Für den Vater besteht hingegen kein gesetzlicher Anspruch auf bezahlte Elternzeit (vgl. Nioradze et al. 2016: 9). Die Fertilitätsrate ist von 2,2 (im Jahr 1990) auf 1,6 (im Jahr 2000) gesunken, ist jedoch seither kontinuierlich angestiegen und weist gemäß aktuellster Daten der Weltbank im Jahr 2015 einen Wert von 2,0 auf (vgl. Weltbank 2018: o. S.). Frauen haben in Georgien Zugang zu Bildung, sind aktiv in zivilgesellschaftlichen Nichtregierungsorganisationen, sind häufig erwerbstätig, des Öfteren sogar Haupt- 
verdienerinnen der Familie - doch zugleich scheint ein hoher gesellschaftlicher Erwartungsdruck (oder wie Baumann es zu sagen pflegt: ein „familienzentrierter Habitus" der georgischen Gesellschaft) zu bestehen, sich um Haushalt und Kinder zu kümmern (vgl. Gunda Werner Institut 2011: o. S.). Gemäß aktuellster Daten der International Labour Organization (ILO) beträgt die Erwerbsquote für Frauen im Jahr 2016 52,9\% und die für Männer 67,1\% (vgl. ILO 2018: o. S.). Die Situation von Frauen in Georgien sollte außerdem im Hinblick auf regionale Unterschiede betrachtet werden, da es in vielen Bereichen große Differenzen zwischen den ländlichen Regionen und der Hauptstadt Tiflis sowie anderen größeren Städten, wie z. B. Batumi, gibt (vgl. DACH 2011: 6). Vor allem in den urbanen Gebieten leben Frauen, die hoch gebildet und erfolgreich im Beruf sind. „Diese Frauen bilden eine Art von Parallelgesellschaft" (DACH 2011: 6). Vornehmlich auch, um dem Genderproblem der häuslichen Gewalt ${ }^{133}$ - einer spezifischen Dimension der Macht - in Georgien entgegenzuwirken und Frauen mehr Chancen zu bieten, wurde 2006 ein Gesetz zur häuslichen Gewalt implementiert und 2010 ein Gesetz zur Gleichstellung der Geschlechter verabschiedet. Letzteres soll einer Benachteiligung von Frauen in den Bereichen der Erwerbstätigkeit, Bildung, Gesundheit und im Sozialsystem entgegenwirken (vgl. DACH 2011: 7). Die DACH-Analyse zieht insgesamt die Schlussfolgerung, dass die Lage von Frauen in Georgien, insbesondere die von alleinstehenden und alleinerziehenden, nicht einfach sei. Eines der Hauptprobleme wäre die traditionelle, sehr konservative (vom georgisch orthodoxen Christentum geprägte) Gesellschaft, weil sie Männern Vorzüge einräumen würde und Frauen das höchste Ansehen erreichen würden, wenn sie verheiratet sind und Kinder haben (vgl. DACH 2011: 12).

\section{Ökonomische Rahmenbedingungen}

Im Vergleich zu allen aufgeführten Ländern ist Georgien das Schlusslicht der wirtschaftlichen Leistungsfähigkeit. 2016 beträgt das Bruttoinlandsprodukt pro Kopf nur 10004,53 KKS (vgl. Weltbank 2018: o. S.). Da Georgien kein EU-Mitglied ist, sind über die Eurostat-Datenbank die Armutsindikatoren der Armutsgefährdungsquote, der erheblichen materiellen Deprivation und der von Armut und sozialer Ausgrenzung bedrohten Bevölkerung nicht verfügbar. Jedoch geht aus dem Armutsindikator der Weltbank hervor, dass 2016 $1 \%$ der georgischen Bevölkerung über weniger als 1,90 US\$ am Tag verwalten kann - was im Vergleich zu den anderen Ländern auf ein hohes Ausmaß an absoluter Armut verweist (vgl. Weltbank 2018: o. S.). Auch der GiniIndex liegt auf sehr hohem Niveau, erreichte im Jahr 2010 einen Höchstwert von 40,1\% und ist seither auf 36,5\% im Jahr 2016 gesunken (vgl. Weltbank 2018: o. S.). Der Gender Pay Gap ist zwar nicht in Bezug auf die Bruttostun-

$133, \ldots[\ldots]$ neben körperlicher Gewalt sind auch psychologische Gewalt und ökonomische Abhängigkeit verbreitet" (Gunda Werner Institut 2011: o. S.). 
denlöhne, doch in Bezug auf die Monatseinkommen ${ }^{134}$ vorhanden. Die Differenz zwischen den durchschnittlichen Bruttomonatseinkommen von Frauen und Männern - ausgedrückt als prozentualer Anteil der durchschnittlichen Bruttomonatseinkommen der Männer - ist in Georgien gemäß aktuellster Daten (aus dem Jahr 2014) mit 36,9\% sehr hoch und deutet folglich auf ein stark ausgeprägtes geschlechtsspezifisches Lohngefälle hin (vgl. UNECE 2018: o. S.).

\subsubsection{Familienpolitik in Russland (insbesondere in Abgrenzung zu Schweden)}

Die Ergebnisse von Motiejunaite/Kravchenko (2008) zeigen, dass sowohl Schweden als auch das christlich orthodox geprägte Russland vornehmlich das DoppelverdienerInnen-Modell unterstützen, dass Schweden jedoch gröBeren Wert auf flexible Arbeitsregelungen für Frauen legt. Dennoch ist für beide Länder festzuhalten: „Both countries base the social security provision for the carer on her/his position as a worker, which limits the individual's opportunity to choose whether or not to care" (Motiejunaite/Kravchenko 2008: 38). Die Unterstützung traditioneller Geschlechterrollen ist jedoch in Russland viel höher als in Schweden (vgl. Motiejunaite/Kravchenko 2008: 38). Die Familienpolitik trägt in Russland und Schweden wesentlich zur Förderung der Beschäftigung von Frauen bei, jedoch sind kaum Veränderungen in Bezug auf Geschlechterrollen zu konstatieren (vgl. Motiejunaite/Kravchenko 2008: 39). Sowohl die schwedische als auch das sowjetische Sozialgesetzgebung sind dem Beveridge-Modell nachempfunden, die großen Wert auf staatliche Regulierung der Wirtschaft und Sozialpolitik legt. In beiden Ländern war die extensive Nutzung von Einkommensumverteilungsmechanismen durch eine hohe Besteuerung und öffentliche Mittel an eine Politik der Vollbeschäftigung gebunden (insbesondere in Russland ist nahezu keine Teilzeitbeschäftigung vorhanden), die die Erwerbstätigkeit der Frauen in Industrie und Wirtschaft erzielte. Viele russische Frauen nahmen traditionell „männliche Arbeitsplätze“ ein, während schwedische Frauen vorwiegend im Dienstleistungssektor tätig wurden. Gleichwohl weisen sowohl Russland als auch Schweden ähnliche Muster der Arbeitsmarktsegregation auf. In Russland zeigten sich nach dem Zerfall der Sowjetunion negative Auswirkungen auf die Erwerbstätigkeit der Bevölkerung in den 1990er Jahren. Die

134 „Gender pay gap is the difference between men's and women's average earnings from employment, shown as a percentage of men's average earnings. [...] Gender Pay Gap in monthly earnings refers to the gender gap in average monthly earnings. This indicator aims to capture the variance between men's and women's earnings over a specific period of time. It reflects differences in time worked and type of work performed, which translates into gender differences in economic autonomy" (UNECE 2018: o. S.). 
Erwerbstätigenquote von Frauen und Männern sank, erreichte 1998 ihren Tiefststand und lag trotz eines langsamen anschließenden Aufschwungs 2002 noch immer um fast 10 Prozent niedriger als 1992 (vgl. Motiejunaite/Kravchenko 2008: 40). Derzeit (ILO-Daten für 2016) beträgt die Erwerbsquote von Frauen 65,2\%. Obwohl in beiden Ländern das DoppelverdienerInnen-Modell dominiert, ist einer der größten Unterschiede zwischen beiden Ländern, dass das ,one-and-a-half-income"-Modell (Mann arbeitet in Vollzeit, Frau in Teilzeit) in Schweden in den Jahren 1994 und 2002 viel häufiger vorkam. Folglich war Teilzeitarbeit in Russland sehr selten (gemäß den aktuellsten ILO-Daten aus dem Jahr 2016 beträgt die Teilzeitquote von Frauen in Russland 10,2\%) und es gab viel mehr Ein- und DoppelverdienerInnenfamilien als in Schweden. Interessanterweise stellen Motiejunaite/Kravchenko (2008) für das Jahr 1994 fest, dass der Anteil von Paaren, in denen die Frau in Vollzeit erwerbstätig ist, während der Mann nicht erwerbstätig ist (,female breadwinner"), in Russland fast doppelt so hoch war wie in Schweden, doch ist dieser Unterschied für das Jahr 2002 in diesem Umfang nicht mehr vorzufinden (vgl. Motiejunaite/Kravchenko 2008: 46).

Die Unterschiede in der Familienpolitik zwischen den Gesellschaften beruhen weitgehend darauf, ob die Rolle von Frauen als Ehefrauen bzw. Mütter und/oder als Arbeitnehmerinnen gefördert werden sollen. In beiden Regimen wurde die Rolle der Frau als berufstätige Mutter durch eine getrennte Besteuerung, öffentliche Kinderbetreuungseinrichtungen sowie Sozialleistungen für einen vorübergehenden Rückzug aus dem Erwerbsleben zugunsten der Kinderbetreuung in Form der Elternzeit gefördert (vgl. Motiejunaite/Kravchenko 2008: 40f.). In Russland sind die Regelungen zum Mutterschutz seit der Sowjetunion trotz einer politischen Transformationsphase relativ unverändert geblieben (aktuelle Änderungen vorbehalten). Schwangere Frauen haben gemäß der ILO-Daten für das Jahr 2013 in Russland einen Anspruch auf 20 Wochen bezahlten Urlaub (zehn Wochen vor und zehn Wochen nach der Geburt) (vgl. ILO 2014: 138). Für die Kalkulation der finanziellen Leistung wird der Durchschnittslohn der Frau der vergangenen 12 Monate zugrunde gelegt, sofern sie mindestens ein Jahr vor der Entbindung erwerbstätig war (diejenigen, die weniger als 12 Monate erwerbstätig waren, erhalten für die fehlenden Monate das gesetzliche Existenzminimum). Anschließend besteht ein pauschaler, sozialversicherungsbasierter Anspruch auf einen teilbezahlten Urlaub von bis zu 70 Wochen. Weiterhin können zusätzliche 78 Wochen unbezahlte Elternzeit genommen werden (vgl. Motiejunaite/Kravchenko 2008: 41). Für Kinder im Alter zwischen 0-7 steht ein relativ hohes Angebot öffentlicher Betreuungseinrichtungen zur Verfügung, die i. d. R. lange Öffnungszeiten haben, um die Erwerbstätigkeit der Eltern zu unterstützen. Im Jahr 2002 besuchten rund $60 \%$ der Kinder im Alter zwischen 0-7 Vorschuleinrichtungen (vgl. Motiejunaite/Kravchenko 2008: 41). Russland und Schweden unterscheiden sich hinsichtlich der Anreize für Männer, sich im 
Rahmen der Kinderbetreuung zu beteiligen. In Russland wird das DoppelverdienerInnen-Modell durch eine öffentliche Kindertagesbetreuung zwar gefördert, doch die Kinderbetreuung während der Elternzeit wird überwiegend von Frauen übernommen, weshalb Motiejunaite/Kravchenko (2008) Russland als „dual-earner/state-female carer“ beschreiben. Die Familienpolitik in Schweden sei stärker auf eine egalitäre Aufteilung der Kinderbetreuungspflichten zwischen den Eltern ausgerichtet und wird folglich von ihnen als ,,dual earner/state-dual carer“ klassifiziert (vgl. Motiejunaite/Kravchenko 2008: 42). Rostgaard (2004) stellt für die osteuropäischen Länder fest, dass zwar in allen Ländern die Fertilitätsrate nach dem Zerfall der Sowjetunion rapide gefallen ist, doch sind Unterschiede in den jeweiligen religiösen Ausrichtungen vorzufinden (katholisch: Polen, Litauen und die Slowakei; katholisch säkularisiert: Tschechien, Ungarn und Slowenien; christlich orthodox: Bulgarien, Rumänien, Russland, Moldawien und die Ukraine; protestantisch: Estland und Lettland): Die Fertilitätsraten sind in den Jahren zwischen 1989 und 2000 am stärksten in christlich orthodoxen Ländern - darunter Russland mit einem Rückgang von rund $40 \%$ - eingebrochen, etwas weniger hingegen in den anderen religiösen Gruppen (vgl. Rostgaard 2004: 6f.). Die Daten der OECD (2016) beinhalten eine aktuelle Fertilitätsrate von 1,8. Aufgrund der Gemeinsamkeit von Russland und Schweden das DoppelverdienerInnen-Modell zu fördern und obwohl zugleich die Einstellungen gegenüber der Geschlechterrollen in Russland viel konservativer als in Schweden sind, ziehen Motiejunaite/Kravchenko (2008) die Schlussfolgerung, dass die Familienpolitik zwar einen Zusammenhang zur Erwerbstätigkeit von Frauen aufweist, aber keinen Einfluss auf normative Orientierungen hat (vgl. Motiejunaite/Kravchenko 2008: 47). Teplova (2007) konstatiert für Russland seit den 1990er Jahren bedeutende strukturelle Reformen des sozialen, politischen und wirtschaftlichen Systems, einschließlich der Kinderbetreuung. Solche Reformen beschränkten sich ihr zufolge nicht nur auf Veränderungen des Wohlfahrtsstaats, sondern beinhalteten auch tiefgreifende ideologische Veränderungen. Diese Umbruchsphasen schließen eine Transformation des russischen Wohlfahrtsregimes ein, das sich zu einem neofamilialen Versorgungsmodell entwickelte (vgl. Teplova 2007: 284).

\section{Ökonomische Rahmenbedingungen}

Das Bruttoinlandsprodukt pro Kopf beläuft sich nach aktuellsten Daten aus dem Jahr 2016 auf 24788,68 KKS, wobei nahezu keine Veränderung zum Vorjahr zu beobachten ist (zwischen 2013 und 2014 ist sogar ein leichter Rückgang zu verzeichnen) (vgl. Weltbank 2018: o. S.). Gemäß dem Armutsindikator der Weltbank sind 0\% der Bevölkerung von absoluter Armut betroffen. Wie anhand des Gini-Index (im Jahr 2015: 37,7\%) festgestellt werden kann, ist die Einkommensungleichheit in Russland jedoch auf höchstem Niveau (vgl. Weltbank 2018: o. S.). Auch das geschlechtsspezifische Lohngefälle ist im Hinblick auf das Bruttomonatseinkommen im Zeitverlauf 
zwischen 2005 und 2011 mit über 30\% stark ausgeprägt (die Daten sind nur bis 2011 verfügbar) (vgl. UNECE 2018: o. S.).

\section{6. „Sozialdemokratisch skandinavisch?“ Zur institutionellen Vielfalt skandinavischer Länder}

In idealtypischer Weise müsste gemäß Leitner (2013) das sozialdemokratische Wohlfahrtsregime aufgrund seiner egalitären Ausrichtung, einschließlich direkter Transferleistungen für Betreuungsarbeit und gleichzeitiger defamilialisierender Strukturen dem optionalen Familialismus zugeordnet werden können (vgl. Leitner 2013: 189). Diese Annahme ist jedoch kritisch zu hinterfragen, da die strikte Erwerbsnorm berücksichtigt werden muss, die wenig „optionalen Freiraum“ zulässt. Darüber hinaus ist auch zwischen den skandinavischen Ländern - so das zentrale Argument - eine institutionelle Vielfalt anzunehmen, was im Hinblick auf die Unterschiede der Arbeitsmärkte und Familienpolitik in Dänemark, Finnland, Norwegen und Schweden von Grönlund et al. (2017) thematisiert wird. Ausgehend von der Idee einer institutionellen Vielfalt dieser skandinavischen Länder erforschen die Autorinnen den Gender Gap der Stundenlöhne sowie den Zugang zu Führungspositionen (als zwei zentrale abhängige Variablen für Genderungleichheiten) und erläutern die Bedeutung von Arbeitsmarktsegregation, Fähigkeiten und Erwerbsunterbrechungen (als unabhängige Variablen) (vgl. Grönlund et al. 2017: 97).

„The results show that gender gaps vary both in size and regarding the mechanisms producing them. In particular, we find that gender segregation has a radically different impact in the four countries. The analysis suggests that the mechanisms linking family policies to labour market outcomes are more complex than envisaged in the current debate and point to the importance of comparing seemingly similar countries" (Grönlund et al. 2017: 97).

Grönlund et al. (2017) zufolge behindern „skandinavische“ familienpolitische Maßnahmen, insbesondere die De-Familisierung durch staatliche Kinderbetreuungseinrichtungen, die Berufs- und Einkommenschancen von Frauen. Während scheinbar „familienfreundliche“ Arbeitsplätze im Dienstleistungssektor etabliert werden, würden ArbeitgeberInnen durch institutionalisierte Rechte auf Erwerbsunterbrechungen dazu ermutigt, Frauen statistisch zu diskriminieren, woraus eine Arbeitsmarktsegregation mit unterschiedlichen Berufsqualitäten und Einkommen für Männer und Frauen resultiere. Die geschlechtsspezifischen Unterschiede könnten durch arbeitsmarktpolitische Institutionen, die eine langfristige Beschäftigung fördern, noch verschärft werden. Diese arbeitsmarktpolitischen Institutionen - insbesondere die Arbeitsschutzgesetzgebung - würden Investitionen der Erwerbstätigkeit fördern, 
doch aufgrund der zu erwartenden Erwerbsunterbrechungen zögern die ArbeitgeberInnen, in Frauen als Arbeitskräfte zu investieren. Festzuhalten sei, dass die „skandinavische Familienpolitik“ zu Ungleichheiten zwischen den Geschlechtern beitrage, indem sie Erwerbsunterbrechungen auf den Arbeitsmärkten erleichtert, die eine langfristige, kontinuierliche Qualifizierungsentwicklung erfordern (vgl. Grönlund et al. 2017: 99). Die „skandinavische Familienpolitik" sei vornehmlich für karriereorientierte Frauen hinderlich, weil für Arbeitsplätze mit hohen Ausbildungskosten für ArbeitgeberInnen vermeintlich produktivere ArbeitnehmerInnen mit stabileren Erwerbsverläufen bevorzugt werden würden. Ferner sei die gläserne Decke, die eine Barriere für Frauen in Bezug auf Führungspositionen und hohe Einkommen darstelle, in Skandinavien besonders ausgeprägt (vgl. Grönlund et al. 2017: 100). Das Kernargument von Grönlund et al. (2017) ist jedoch, dass sich die skandinavischen Länder aller Regimetypologien zum Trotz in Bezug auf Familien- und Arbeitsmarktpolitik voneinander unterscheiden bzw. ein „model with variations" darstellen (Grönlund et al. 2017: 101).

,[...] we argue that the varying combinations of family policies and labour market institutions affect both the gender gaps and the mechanisms behind these gaps differently in the four Scandinavian countries" (Gönlund et al. 2017: 101).

Die von Esping-Andersen (1990) festgestellten Gemeinsamkeiten der skandinavischen Länder im Hinblick auf umfassende Wohlfahrtsregime, die auf universalen Rechten basieren, würde hierbei nicht grundlegend in Frage gestellt, vielmehr wird wie folgt argumentiert: Die nationale Familienpolitik von Dänemark, Finnland, Norwegen und Schweden unterscheide sich im Design und obwohl die Differenzen innerhalb der Regime geringer seien als die Differenzen zwischen den Regimen, seien sie theoretisch relevant. Unterschiedliche Kombinationsmöglichkeiten von Elternurlaubsansprüchen, Kinderbetreuungseinrichtungen und "Cash-for-Care-Systemen“, die in unterschiedliche Arbeitsmarktkontexte eingebettet sind, bieten Eltern zwar diverse Möglichkeiten, aber bringen auch Einschränkungen mit sich (vgl. Grönlund et al. 2017: 101). Die Elternzeiten sind nach Aspekten, wie Dauer, Vergütungsniveau und „Genderneutralität" der Ansprüche, zu differenzieren. Die maximal mögliche Elternzeit, die eine Mutter mit einkommensabhängiger Vergütung erhält - vorausgesetzt, dass der Vater nur die für ihn minimal reservierte Elternzeit in Anspruch nimmt - beträgt in Dänemark etwa 48 Wochen, in Norwegen 49, in Schweden 47 und in Finnland 44 Wochen. In Schweden (und Dänemark) können zusätzliche 13 Wochen (bzw. 14) Elternzeit mit einer geringen Vergütung genommen werden. In Norwegen (und Finnland) werden im Rahmen des „Cash-for-Care“-Programms, das 1999 als Teil des nationalen Sicherheitssystems eingeführt wurde, längere zusätzliche pauschale Elternzeiten im Sinne einer „Flat-Rate-Kompensation“ gewährt. „Cash-for-Care“ kann nach dem Elternurlaub und bis zum zweiten Lebens- 
jahr des Kindes in Norwegen (bis zum dritten Lebensjahr in Finnland) in Voll- oder Teilzeit genutzt werden. In Schweden und Norwegen sind Elternurlaubsregelungen „geschlechtsneutral“ mit gleichen Rechten sowohl für Mütter als auch für Väter geltend. Dies bedeutet, dass eine bestimmte Elternzeit für den Vater und eine ebenso lange Elternzeit für die Mutter reserviert ist (in Dänemark und Finnland ist die für den Vater vorgesehene Elternzeit kürzer als für die Mutter) (vgl. Grönlund 2017: 101). Schweden war das erste Land der Welt, das 1974 eine genderneutrale Elternzeit einführte, die nach der Geburt eines Kindes über sechs Monate vergütet wurde. Norwegen folgte 1978 und gewährte berufstätigen Eltern ein Recht auf 18 Wochen Elternzeit. In Schweden wurde die Elternzeit 1989 auf 15 Monate und 2002 auf 16 Monate verlängert. In Norwegen betrug sie im Jahr 199312 Monate und wurde im Jahr 2009 auf 13 Monate ausgeweitet (vgl. Duvander et al. 2010: 46f.). Norwegen führte 1993 eine Väterquote der Elternzeit von mindestens einem Monat ein, Schweden folgte dieser Maßnahme 1995. Die Elternzeit, die vom Vater genommen werden muss (weil sie andernfalls verfällt), wurde in Schweden 2002 auf zwei Monate und in Norwegen im Jahr 2009 auf zehn Wochen verlängert (vgl. Duvander et al. 2010: 47). Da Frauen auch in den skandinavischen Ländern den überwiegenden Teil der Elternzeit in Anspruch nehmen, sollte dies gemäß Grönlund et al. (2017) zu Unterbrechungen der Erwerbstätigkeit von Frauen führen (vgl. Grönlund et al. 2017: 102).

Sowohl die Möglichkeiten von Erwerbsunterbrechungen als auch deren Folgen für die Gleichstellung der Geschlechter würden von den Arbeitsmarktinstitutionen beeinflusst. Die Ergebnisse von Grönlund et al. (2017) belegen, dass die skandinavischen Länder (Dänemark, Finnland, Norwegen und Schweden) trotz ihrer Gemeinsamkeiten Unterschiede hinsichtlich Familienpolitik und Arbeitsmarktinstitutionen aufweisen. In allen skandinavischen Ländern sind Frauen durch niedrigere Löhne und Barrieren zu Führungspositionen benachteiligt. Der „Gender Authority Gap“ ist in Schweden größer als in Norwegen (am geringsten ist er in Dänemark), während in Norwegen der „Gender Wage Gap“ größer ist (s. u.) (vgl. Grönlund et al. 2017: 106f.). Die Arbeitsmarktsegregation hat in Schweden sowie in Norwegen (allerdings nicht in Dänemark) einen signifikanten Einfluss auf den „Gender Wage Gap“ (in Finnland wurde ein starker Effekt der Arbeitsmarktsegregation auf den „Gender Wage Gap“ und auch auf den „Gender Authority Gap“ festgestellt). Ferner ist Teilzeitarbeit diesen Ergebnissen zufolge eine zentrale Komponente zur Erklärung des „Gender Authority Gaps“ in Schweden und Norwegen (auch in Dänemark, allerdings nicht in Finnland) (vgl. Grönlund et al. 2017: 108). Zusammengefasst leisten Grönlund et al. (2017) drei wesentliche Beiträge zum Stand der Forschung: Erstens erörtern sie theoretisch fundierte Unterschiede von Familienpolitik und Arbeitsmarktinstitutionen in den skandinavischen Ländern. Zweitens belegen sie, dass die Gender Gaps der Einkommen und der Führungspositionen nicht nur in Bezug auf die Größe, son- 
dern auch in Bezug auf die Mechanismen, die sie hervorrufen, variieren. Drittens stellen sie insbesondere fest, dass Arbeitsmarktsegregation und auch Teilzeitarbeit jeweils unterschiedliche Einflüsse auf die Genderungleichheiten in den vier untersuchten skandinavischen Ländern (Dänemark, Finnland, Schweden, Norwegen) haben (vgl. Grönlund et al. 2017: 109).

In den sozialdemokratischen Ländern ist die Familienpolitik grundsätzlich durch ein hohes Ausmaß staatlicher Kinderbetreuungsangebote gekennzeichnet. Sowohl in Schweden als auch in Norwegen (ebenso in Dänemark und Finnland) besteht ein Recht für Kinder auf staatliche Kinderbetreuung, wodurch vorzugsweise für Mütter ein Wiedereinstieg in die Vollzeitbeschäftigung ermöglicht werden soll (vgl. Grönlund et al. 2017: 102). Die Einführung des Rechtes auf einen Kinderbetreuungsplatz, das mit einem Kindesalter von einem Jahr eintritt ${ }^{135}$, wurde in Schweden bereits 1995 eingeführt, in Norwegen ${ }^{136}$ hingegen erst im Jahr 2009. Seit 1975 ist die Zahl der Kinderbetreuungseinrichtungen und -plätze in Norwegen kontinuierlich gestiegen. Für Kinder im Alter von 3 bis 5 Jahren ist eine nahezu vollständige Kinderbetreuung erreicht worden, gleichwohl für die jüngste Altersgruppe der Ein- bis Zweijährigen immer noch eine unbefriedigende Nachfrage mit einer Versorgungsquote von unter 50\% (Verhältnis der Anzahl der verfügbaren Kinderbetreuungsplätze zur Anzahl aller Kinder) zu bestehen scheint (vgl. Ostner/Schmitt 2008b: 209). In Schweden sind öffentliche Kinderbetreuungseinrichtungen für Vorschulkinder zwischen einem und sechs Jahren sowie für Schulkinder bis zwölf Jahren vorhanden. Alle Gemeinden sind dazu verpflichtet, eine Kinderbetreuung zu ermöglichen, wenn die Eltern arbeiten oder studieren (vgl. Ostner/Schmitt 2008b: 209). Die Betreuungsplätze werden ganztägig angeboten, die meisten Vorschuleinrichtungen haben von 6.30 bis 18.30 Uhr geöffnet (vgl. Europäische Kommission 2018: o. S.). 2001/2002 wurde dieser Rechtsanspruch mit einer Beschränkung auf 15 Stunden pro Woche auch für arbeitslose und sich in Karenz befindliche Eltern ausgeweitet (vgl. Dörfler/Wernhardt 2016: 62). Gemäß den aktuellsten Daten aus dem Jahr 2015 werden in Schweden 64\% der 1- bis 3-jährigen Kinder und 96,3\% der Kinder über 3 Jahren in öffentlichen Kinderbetreuungseinrichtungen betreut (EU-Durchschnitt 30,3\% bzw. 83,3\%) (vgl. Europäische Kommission 2018: o. S.).

Aufgrund des hohen Ausmaßes staatlicher Kinderbetreuungsangebote verwundert es nicht, dass Schleutker (2014) in Schweden einen hohen Anteil erwerbstätiger Mütter und nur eine geringe Anzahl an AlleinverdienerInnenpaaren feststellt (vgl. Schleutker 2014: 177). Zu dieser Schlussfolgerung kommt ebenfalls die Europäische Kommission:

135 Siehe hierzu Grönlund et al. (2017) sowie Gislason/Eydal (2011).

136 Zuvor galt für Norwegen: ,[...] there is no childcare guarantee or right of children to day care" (Ostner/ Schmitt 2008b: 209). 
„Die Erwerbstätigenquote von Frauen [74,8\% im Jahr 2016 entspricht fast der der Männer mit 77,5\%] und Müttern [die Erwerbsquote von Müttern mit Kindern unter 6 Jahren betrug 2016 81,5\% und war damit die höchste in der EU] zählen zu den höchsten in der EU, und in Schweden wird eine der niedrigsten Kinderarmutsquoten verzeichnet. Die familienpolitischen Maßnahmen des Landes zielen darauf ab, das Doppelverdiener[Innen]-Familienmodell zu fördern und sicherzustellen, dass Frauen und Männer in der Familie und im Beruf die gleichen Rechte und Pflichten haben“ (Europäische Kommission 2018: o. S.)

Auch in Norwegen (2016) besteht mit 72,8\% eine hohe Erwerbsquote von Frauen (vgl. Eurostat 2017: o. S.). Basierend auf dem Jahr 2016 ist die Teilzeitbeschäftigungsquote der Frauen in Schweden mit 35,6\% im Vergleich zum EU-Durchschnitt $(31,9 \%)$ hoch, wobei das geschlechtsspezifische Lohngefälle (s. u.) unterhalb des EU-Durchschnitts (16,3\%) liegt (vgl. Europäische Kommission 2018: o. S.). In Norwegen ist ebenfalls eine vergleichsweise hohe Teilzeitbeschäftigungsquote von Frauen (38\% im Jahr 2016) zu verzeichnen.

Die vielfältigen Kinderbetreuungsmöglichkeiten zur Förderung der Erwerbstätigkeit von Frauen spiegeln sich in der hohen Fertilitätsrate in Schweden (im Jahr 2008: 1,91, wobei dieser Wert bis 2015 stabil bleibt) und Norwegen (im Jahr 2008: 1,96, wobei bis 2015 ein leichter Rückgang auf 1,7 zu verzeichnen ist) wider. Zugleich bestehen großzügige Elternzeiten. Beruhend auf der OECD-Datenbasis für 2015 beträgt die bezahlte Elternzeit für Väter in Norwegen immer noch zehn Wochen, für Väter in Schweden besteht ein Anspruch auf 1,4 Wochen Vaterschaftsurlaub sowie im Anschluss 12,9 Wochen bezahlte Elternzeit, d. h. insgesamt eine Phase von 14,3 Wochen, die der Vater-Kind-Beziehung zugutekommen soll (vgl. OECD 2018: o. S.). Die Beteiligung der Väter an der Elternzeit wurde 2008 in Schweden durch den „Gender-Equality-Bonus“ zusätzlich gefördert: für jeden Tag, den die Eltern nach diesen zwei Monaten egalitär aufteilen, wird ein Bonus von zusätzlich 5,60€ am Tag gewährt. Mit dem Argument der Wahlfreiheit für Eltern wurde 2008 das Betreuungsgeld, das zuvor in der Periode zwischen 1994-1995 als familisierende Maßnahme gültig war, wiedereingeführt (vgl. Dörfler/Wernhart 2016: 63). Eltern, die die Betreuung ihres Kindes nicht an staatliche Einrichtungen auslagern, bekommen in Norwegen seit 1998 eine finanzielle Unterstützung in Form des Betreuungsgeldes. Sowohl in Schweden als auch in Norwegen kann das Betreuungsgeld anteilig im Falle einer privaten Teilzeitbetreuung, die durch staatliche Einrichtungen ergänzt wird, ausbezahlt werden. Heutzutage sieht die Gesetzeslage in Schweden so aus, dass beide Elternteile Anspruch auf maximal 8 Monate bezahlten Urlaub pro Kind haben,

„[...] sodass pro Kind insgesamt 16 Monate (480 Tage) bezahlter Urlaub möglich sind. Während 13 dieser Monate werden im Jahr 2018 etwa 80\% des letzten Einkommens ausgezahlt, bis zu einer Obergrenze von 455.000 SEK (44.630 €) pro 
Jahr. Um die wirtschaftliche Situation in Haushalten mit niedrigem Einkommen zu verbessern, wurde im Jahr 2016 die Mindesthöhe des bezahlten Urlaubs auf 250 SEK $(25 €)$ pro Tag angehoben. In den restlichen 3 Monaten wird eine Pauschale in Höhe von 180 SEK (18 €) pro Tag gezahlt" (Europäische Kommission 2018: o. S.).

Jeder Elternteil erhält einen nicht übertragbaren Anspruch auf Elterngeld von 3 Monaten (von den insgesamt 16 Monaten), die restlichen 10 Monate können je nach individuellem Belieben zwischen den Eltern aufgeteilt werden. Um die Wahlfreiheit für die Eltern zu vergrößern, können Eltern seit dem 1. Januar 2012 während dem ersten Lebensjahr des Kindes 30 Tage lang gleichzeitig Elterngeld beziehen. Insgesamt sind die Ausgaben für finanzielle Leistungen für Kinder mit 3\% des Bruttoinlandsprodukts im Jahr 2015 im europäischen Vergleich sehr hoch (EU-Durchschnitt: 2,4\%) (vgl. Europäische Kommission 2018: o. S.). Folglich werden nicht nur viele Möglichkeiten von öffentlichen Kinderbetreuungsmöglichkeiten geboten, sondern es ist auch eine großzügige und flexible Elternurlaubsregelung in Schweden zu konstatieren.

\section{Ökonomische Rahmenbedingungen}

Norwegen ist im Vergleich zu den anderen Ländern federführend, was seine ökonomische Leistungsfähigkeit anbelangt. Im Jahr 2016 beläuft sich das Bruttoinlandsprodukt pro Kopf auf 58790,06 KKS und liegt damit auf höchstem Niveau - Schweden liegt mit einem Wert von 48904,55 KKS deutlich darunter. Doch während in Schweden ein stetiger Zuwachs zu beobachten ist, ist der Trend in Norwegen für die vergangenen Jahre (seit 2013) rückläufig. Ferner scheint in keinem anderen Land die Wirtschaftskrise so drastische Auswirkungen auf das Bruttoinlandsprodukt pro Kopf gehabt zu haben, wie es in Norwegen der Fall war. Zwischen 2008 und 2009 ist ein dramatischer Rückgang von 61757,25 KKS auf 55427,58 KKS zu konstatieren (vgl. Weltbank 2018: o. S.). In Schweden zeigt sich seit der Wirtschaftskrise ein konstanter Anstieg der Armutsgefährdungsquote, die aktuell (2017) 16\% beträgt - in Norwegen beträgt sie hingegen lediglich 12\%. Die Rate der erheblichen materiellen Deprivation ist in Norwegen mit 1,9\% geringfügig höher als in Schweden (1,9\%). Im Ländervergleich sind dies die niedrigsten Raten erheblicher materieller Deprivation, was auf effektive Sozialleistungen zur Bekämpfung von materieller Armut verweist. Der Anteil der von Armut und sozialer Ausgrenzung betroffenen Bevölkerung stagniert in Schweden mit rund 18\% (respektive max. 18,6\% im Jahr 2015) im Zeitverlauf zwischen 2009 und 2017, während in Norwegen seit 2014 ein Anstieg von 13,5\% auf 16,1\% festzustellen ist (vgl. Eurostat 2018a: o. S.). Beide Länder weisen eine Zunahme der Einkommensungleichverteilung auf, wobei der Gini-Index auf niedrigem Niveau ist (in Norwegen liegt er im Jahr 2015 mit 27,5\% etwas unterhalb von dem Gini-Index in Schweden mit 29,2\%) (vgl. Weltbank 2018: o. S.). Das geschlechtsspezifische Lohngefälle der Bruttostundenlöhne ist 
derzeit, wie bereits skizziert, in Norwegen (14,9\%) geringfügig höher als in Schweden (13,3\%) (vgl. UNECE 2018: o. S.). Obwohl in den skandinavischen Ländern eine Ideologie des Egalitarismus besteht, sind die Bruttostundenlöhne zwischen den Geschlechtern relativ ungleich verteilt.

Festzuhalten ist, dass zwischen den ausgewählten Ländern durchaus große Unterschiede in Bezug auf die genderrelevanten Welfare Policies, einschließlich der Familien- und Arbeitsmarktpolitik sowie der ökonomischen Rahmenbedingungen bestehen.

Dass darüber hinaus nicht nur zwischen den Ländern markante Diskrepanzen der Kontextfaktoren existieren, führt zu der Entscheidung, Regionen als zentrale Analyseeinheiten zu verwenden: Im Hinblick auf ökonomische, politische und kulturelle Kontextdimensionen zeichnet sich nicht nur eine Heterogenität zwischen den europäischen Ländern ab, sondern auch regionale Heterogenitätsmuster sind von Bedeutung, wie anhand der Darstellung der regionalen Kontextindikatoren im Kapitel 11.4. zur Beschreibung der unabhängigen Variablen festgehalten wird. Strukturelle Unterschiede zwischen urbanen und ländlichen Regionen, historisch unterschiedliche Entwicklungspfade - wie es beispielsweise zwischen den alten und neuen Bundesländern in Deutschland der Fall ist - und ausgeprägte kulturelle Unterschiede, wie z. B. unterschiedliche Sprachregionen in Belgien oder unterschiedliche religiös geprägte Regionen, gilt es zu berücksichtigen. Darüber hinaus weisen Transnationalisierungsprozesse sozialer Ungleichheiten darauf hin, dass eine nationalstaatliche Rahmung im Sinne des methodologischen Nationalismus kritisch zu hinterfragen ist:

„[Es] lassen sich drei Anwendungsbeispiele des methodologischen Nationalismus unterscheiden: nationale Rahmung, international vergleichende Rahmung sowie globale Rahmung. Sie alle beruhen darauf, dass, [...] die politische und rechtliche Grammatik nationaler Grenzen unreflektiert und affirmativ zur Prämisse der Rahmung sozialwissenschaftlicher Ungleichheitsforschung gemacht wird. Davon ist zu unterscheiden der kosmopolitische Blick, der eine aktive transformative, transnationale Politik der Rahmung verfolgt: das Nationalstaatsprinzip beantwortet nicht mehr die Wer-Frage sozialer Ungleichheit. Denn in einer globalisierten Welt verliert die nationalstaatliche Rahmung ihre Aura der Selbstevidenz" (Beck 2008: 26).

„Die These lautet: Das Ende der nationalen Klassengesellschaft ist nicht das Ende sozialer Ungleichheit, sondern genau im Gegenteil die Geburt radikalerer neuer „,kosmopolitischer" Formen sozialer Ungleichheit, denen (bislang) keine institutionalisierten Antworten (Gewerkschaften, Wohlfahrtsstaat) entsprechen" (Beck 2008: 27).

Eine grenzüberschreitende Verflechtung zwischen Ländern ist wesentlich und wird häufig - gerade im Bereich der Sozialpolitik - zu wenig beachtet. Sozialpolitik wird oftmals als nationalstaatliches Gebilde begriffen, ohne zu berücksichtigen, dass ,,[...] die Sozialpolitik eines Landes auf vielfältige Weise durch die sozialen Standards anderer Staaten und durch die politischen, sozia- 
len und wirtschaftlichen Beziehungen mit anderen Ländern beeinflusst wird“ (SFB 1342 2018: 4). Das hochaktuelle Forschungsprojekt „Globale Entwicklungsdynamiken von Sozialpolitik“ (Sonderforschungsbereich „SFB 1342“) arbeitet zurzeit dieses Forschungsdesiderat auf. 


\section{Empirische Untersuchungen}

\section{Datenbasis: Generations and Gender Programme (GGP)}

Für die empirische Analyse der theoretisch diskutierten Wechselbeziehungen zwischen Mikro- und Makroebene werden die Daten des ,Generations and Gender Programmes' verwendet. Das ,Generations and Gender Programme (GGP) enthält einen Kontextdatensatz mit Informationen der Makroebene von Mitgliedsländern und nationale Zufallsstichproben ${ }^{137}$, sogenannte ,Generations and Gender Surveys' (GGS), die weltweite Panelerhebungen darstellen und Informationen der Mikroebene beinhalten (vgl. UNECE 2015: o. S.). Die Mitgliedsländer führen die Bevölkerungsumfragen selbst durch, wobei die United Nations Economic Commission for Europe (UNECE) den GGS koordiniert (vgl. Bundesinstitut für Bevölkerungsforschung 2014: o. S.). Befragt werden Männer und Frauen im Alter zwischen 18 und 79 Jahren (eine Ausnahme ist Österreich; hier liegt die Spannweite des Alters in der ersten Welle zwischen 18 und 45 Jahre) mittels Computer Assisted Personal Interviews (CAPI). Der GGS wurde als Nachfolger des Family and Fertility Surveys erstmals 2002 in den Niederlanden erhoben (für einen Überblick der landesspezifischen Erhebungsjahre der ersten beiden Wellen s. Tabelle 17: Übersicht der Messzeitpunkte des GGS) und ist eine international vergleichende repräsentative Studie mit dem Ziel, Erkenntnisse über Familienwandel in der ersten Dekade des 21. Jahrhunderts, ferner Beziehungen zwischen Eltern und ihren Kindern (Generationen), sowie über Beziehungen zwischen den Eltern (Gender/Geschlechterbeziehungen) zu generieren (vgl. Ette et al. 2007: 7). Zentrale Aspekte, die im GGS erhoben werden, sind en détail:

Auswirkungen der Erwerbstätigkeit von Frauen auf die Fertilität, Rollenverteilungen zwischen PartnerInnen zur Erklärung der Fertilitätsentscheidung, Werte und Einstellungen zwischen den Geschlechtern, Arbeitsteilung im Haushalt, Entscheidungs- und Verwaltungsmacht über das Haushaltseinkommen und die Beziehung zwischen den Generationen.

Die Art des Forschungsdesigns wird als Paneldesign bezeichnet; dieselben Fälle werden über drei Erhebungswellen (Messzeitpunkte) nach derselben Vorgehensweise (CAPI) befragt. Veränderung der abhängigen Variablen können durch die Analyse der verschiedenen Messzeitpunkte festgestellt und erklärt werden. Im vorliegenden Fall liegt die Annahme nahe, dass innerhäus-

137 Die Stichprobenbildung erfolgt in drei Stufen per Random Route nach ADM-Design:

1. Sample Points $->$ Schichten (Bundesländer, Regierungsbezirke, BIK-Gemeindetypen)

2. Haushalte $\rightarrow$ Random-Route

3. Zielperson $->$ Schwedenschlüssel 
liche Arbeitsteilungsarrangements in Paarbeziehungen im Zeitverlauf variieren, weshalb durch eine Panelanalyse wichtige Erkenntnisse über die zeitbezogene Veränderung dieses Forschungsfelds erzielt werden können. Aktuell sind zwei Wellen des GGS verfügbar, die Umfragedaten der folgenden Nationen beinhalten:

Tabelle 13: Aktuelle Mitgliedsländer und Fallzahlen des GGS (Stand April 2018)

\begin{tabular}{lll}
\hline Land & Fallzahl Welle 1 & Fallzahl Welle 2 \\
\hline Australien & $\mathrm{n}=7125$ & $\mathrm{n}=6143$ \\
Bulgarien & $\mathrm{n}=12858$ & $\mathrm{n}=9344$ \\
Deutschland & $\mathrm{n}=10017$ & $\mathrm{n}=3226$ \\
Frankreich & $\mathrm{n}=10079$ & $\mathrm{n}=6533$ \\
Georgien & $\mathrm{n}=10000$ & $\mathrm{n}=8292$ \\
Italien & $\mathrm{n}=9570$ & $\mathrm{n}=6476$ \\
Litauen & $\mathrm{n}=10036$ & $\mathrm{n}=2292$ \\
Niederlande & $\mathrm{n}=8161$ & $\mathrm{n}=6090$ \\
Osterreich & $\mathrm{n}=5000$ & $\mathrm{n}=3912$ \\
Polen & $\mathrm{n}=19987$ & $\mathrm{n}=12294$ \\
Russland & $\mathrm{n}=11261$ & $\mathrm{n}=7783$ \\
Tschechien & $\mathrm{n}=10006$ & $\mathrm{n}=3149$ \\
Ungarn & $\mathrm{n}=13540$ & $\mathrm{n}=10641$ \\
Belgien & $\mathrm{n}=7163$ & - \\
Estland & $\mathrm{n}=7855$ & - \\
Japan & $\mathrm{n}=9074$ & - \\
Norwegen & $\mathrm{n}=14880$ & - \\
Rumänien & $\mathrm{n}=11986$ & - \\
Schweden & $\mathrm{n}=9688$ & - \\
Gesamt & $n=198286$ & $\mathrm{n}=86175$ \\
\hline
\end{tabular}

Quelle: Eigene Darstellung.

Die Fallauswahl, die im Rahmen der Dissertation getroffen wird, liegt begründet in einem „Most dissimilar System Design“, d. h. innerhalb des europäischen Kontextes werden Länder einschließlich ihrer NUTS1-Regionen ausgewählt, die möglichst große Unterschiede in Bezug auf Genderungleichheiten aufweisen (und auch regimetypologisch zu differenzieren sind), um mögliche Gemeinsamkeiten einer traditionellen innerhäuslichen Arbeitsteilung konstatieren zu können. 


\subsection{Zur methodisch bedingten NUTS1-Regionenanalyse mit theoretischem Mehrwert}

\subsubsection{Einschränkungen der Small-N-Problematik}

Methodisch betrachtet gilt - wegen der Voraussetzung für alle Analyseebenen eine adäquate Fallzahl zu garantieren (vornehmlich für möglichst präzise Schätzungen der Parameter, Konfidenzintervalle und Standardfehler) - das Prinzip, möglichst viele Kontexteinheiten, die über Daten der ersten und zweiten Welle des Generation and Gender Surveys verfügen, in die Mehrebenenanalyse einzubeziehen ${ }^{138}$. Im Allgemeinen ist eine geringe Anzahl an Makroeinheiten eine häufig auftretende Problematik von Mehrebenenanalysen, die oft diskutiert und durchaus umstritten ist. Grundsätzlich gilt: je geringer die Anzahl der Kontexteinheiten, umso weniger Variablen können in die Modelle aufgenommen werden bzw. je ,abgespeckter“ die Modelle, desto geringer die Fallzahlproblematik und je höher die Fallzahl, desto präziser die Schätzungen. Als Daumenregel empfehlen Hox et al. im Falle eines Forschungsinteresses von fixen Effekten 30 Kontexteinheiten sowie eine durchschnittliche Fallzahl von 30 Individualeinheiten, im Falle eines Forschungsinteresses von Cross-Level-Interaktionen 50 Kontexteinheiten sowie eine durchschnittliche Fallzahl von 20 Individualeinheiten und im Falle eines Forschungsinteresses von Zufallseffekten 100 Kontexteinheiten sowie eine durchschnittliche Fallzahl von 10 Individualeinheiten (vgl. Hox et al. 2018: 215f.). In der Forschungspraxis werden von dieser Vorgabe deutlich abweichende Analysen durchgeführt. Jedoch sollte nicht unterschätzt werden, dass „For accuracy and high power a large number of groups appears more important than a large number of individuals per group" (Hox et al. 2018: 214). Hox et al. zufolge sind allerdings auch Analysen mit weniger Makroeinheiten möglich, sofern die Schätzung der Parameter mittels „Restricted Maximum Likelihood" durchgeführt wird. Die Full-Information-Maximum-LikelihoodMethode (FIML) schließt sowohl die Schätzer für die Varianzkomponenten als auch die Regressionskoeffizienten in die Likelihoodfunktion ein, hingegen werden für die Maximierung der Likelihoodfunktion im Rahmen der Restricted-Maximum-Likelihood-Methode (REML) nur Varianzkomponenten einbezogen (vgl. Hox et al. 2018: 28).

138 „An important conceptual difference between single-level and multilevel approaches is that the sample size considerations are quite different. With the multilevel approach, a sufficient sample size is required at each level of analysis. In smaller group samples, the difference in estimation between ML [Maximum-Likelihood] and REML [Restricted-MaximumLikelihood] results in a downward bias in variance components estimated with ML compared to REML" (Heck et al. 2014: 20). 
„Erstere [FIML] enthält in ihrer Likelihoodfunktion sowohl die festen Regressionsparameter als auch die Varianzkomponenten der Fehlerterme. Bei letzterer [REML] beinhaltet die Likelihoodfunktion nur die Varianzkomponenten, aber nicht die Regressionsparameter. Während die FIML die Regressionsparameter als bekannte Größen bei der Schätzung der Varianzkomponenten behandelt, betrachtet sie die REML als Schätzer, die mit Unsicherheit behaftet sind. Theoretischer Weise sollte die REML bei wenigen vorliegenden Gruppen zu genaueren Schätzer [sic!] führen, wie [auch] Bryk \& Raudenbush (1992: 46; 2002: 53) festgestellt haben" (Langer 2009: 102f.).

Stegmüller, der durch eine Monte-Carlo-Simulation neuere Erkenntnisse über Mehrebenenanalysen mit wenigen Kontexteinheiten (15-20 Länder) gewinnen konnte, stellt fest,

,[...] that maximum likelihood estimates and confidence intervals can be severely biased, especially in models including cross-level interactions. In contrast, the Bayesian approach proves to be far more robust and yields considerably more conservative tests" (Stegmüller 2013: 748).

„Estimated with 15 or 20 available countries, ML confidence intervals are almost $5 \%$ too short - in other words, researchers are more likely to obtain $90 \%$ confidence intervals rather than the $95 \%$ intervals announced by their software package" (Stegmüller 2013: 758).

Im Falle einer zu erstrebenden Mehrebenenanalyse mit weniger als 20 Makroeinheiten empfiehlt Stegmüller folglich ein bayesianisches Schätzverfahren anzuwenden.

Eine weitere Möglichkeit, die Small-N-Problematik zu umgehen, ist eine feinere Differenzierung der Kontexteinheiten vorzunehmen, d. h. im Rahmen einer europäischen Analyse und, sofern es die Datengrundlage zulässt, anstatt der Länder als Einheiten die NUTS-Klassifizierung zugrunde zu legen. Das Generations and Gender Programme bietet diese Möglichkeit, weshalb im Zentrum der Analyse die auf den NUTS1-Regionen (48 Kontexteinheiten der ersten Welle) basierenden Mehrebenenmodelle stehen.

\subsubsection{Bedeutung der Regionen innerhalb Europas}

In erster Linie besteht der Vorteil einer europäischen Regionenanalyse nicht nur darin, eine methodische Small-N-Problematik zu umgehen, sondern vornehmlich auch darin, den theoretischen Aspekt einer zunehmenden Transnationalisierung durch einen nationenübergreifenden Bezugsrahmen zu berücksichtigen. Ist es nicht ein wesentliches Merkmal Europas, dass es sich durch höchst unterschiedliche kulturelle Traditionen auszeichnet? Die Vielfalt wird häufig als ein wesentliches Charakteristikum Europas beschrieben - Europa gedacht im Sinne einer „Einheit in der Vielfalt“ (Mau/Verwiebe 2009: 24). Insbesondere eine Fokussierung auf geschlechterkulturelle Leitbilder lässt 
deutliche Unterschiede zwischen den europäischen Ländern erkennen (vgl. Pfau-Effinger 2005: 321), die sich gleichwohl auch in den Regionen (u. a. durch Stadt-Land-Gefälle) widerspiegeln könnten (für eine Differenzierung von familienpolitischen und ökonomischen Rahmenbedingungen der Länder s. Kapitel 10.). Auch im Hinblick auf die politische EmpowermentDimension von Frauen zeigen sich deutliche Differenzen zwischen den europäischen Regionen in der politischen Repräsentation von Frauen in nationalen Parlamenten (vgl. Sundström/Stockemer 2015: 254). Nicht zu vergessen sind drastische Unterschiede der ökonomischen Rahmenbedingungen. Mit einer regimetypologischen Analyse sind ForscherInnen fähig, eine „Vogelperspektive“ einzunehmen und Gemeinsamkeiten zwischen Ländern aufzuzeigen. Doch gilt es ebenso, eine detailliertere Perspektive einzunehmen und auch mögliche Unterschiede zu erkennen. Zwischen beiden Sichtweisen liegt ein breites Spektrum - innerhalb dieser Dissertation wird versucht, das Spannungsfeld zwischen nationalen und regionalen Dynamiken zu betrachten, indem unter dem Dach eines übergreifenden Bezugsrahmens (s. Argumentation von Kreckel (2006) im Kapitel 3.4.) zunächst OLS-Regressionsanalysen basierend auf den europäischen Ländern und Regimen durchgeführt werden, die durch eine Mehrebenenanalyse basierend auf den NUTS1-Regionen erweitert werden. Sowohl der theoretische Vorteil einer differenzierteren Perspektive im Sinne der regionalen Vielfalt als auch der methodische Vorteil einer höheren Kontextfallzahl werden durch diese Vorgehensweise wahrgenommen. Die ausgewählten Länder sowie die jeweiligen ausgewählten NUTS1-Regionen, die die Basis der weiterführenden Analyse bilden, sind in Tabelle 14 dargestellt. (Anzumerken ist, dass nicht für alle Länder eine regionale Klassifizierung möglich ist, wodurch sich die Fallzahlen der länderund regionenspezifischen Analysen unterscheiden.) Ferner wird in Abbildung 6 ein Überblick über die geographische Verortung der NUTS1-Regionen gegeben. Allgemeiner Hintergrund der NUTS-Klassifizierung am Beispiel von Deutschland ist folgender:

„Die Klassifikation der Gebietseinheiten für die Statistik (Nomenclature des Unités territoriales statistiques - NUTS) ist eine geografische Systematik, nach der das Gebiet der Europäischen Union in drei Hierarchiestufen eingeteilt wird: NUTS-1, NUTS-2 und NUTS-3. Diese Einordnung ermöglicht den grenzüberschreitenden statistischen Vergleich von EU-Regionen. [...] Die Einteilung lehnt sich eng an die Verwaltungsgliederung der einzelnen Länder an. In der Regel entspricht eine NUTS-Ebene einer Verwaltungsebene oder einer räumlichen Aggregation von Verwaltungseinheiten. In Deutschland sind die NUTS-1-Regionen die Bundesländer. Die Bevölkerung einer NUTS-1-Region liegt in aller Regel zwischen 3 und 7 Millionen Einwohnern. NUTS-2-Regionen haben meist zwischen 800000 und 3 Millionen Einwohnern. In Deutschland ist dies im Regelfall die Ebene der Regierungsbezirke. Die Bevölkerungszahl von NUTS-3-Regionen liegt generell zwischen 150000 und 800000 Einwohnern. NUTS-3 entspricht in 
Deutschland den Kreisen und kreisfreien Städten“ (Statistisches Bundesamt 2018b: o. S.).

Nach Eurostat umfasst die aktuelle, seit 1. Januar 2018 gültige, NUTS-2016Klassifizierung 104 NUTS1-Regionen, 281 NUTS2-Regionen und 1348 NUTS3-Regionen. Die von Eurostat festgelegten Ziele sind, mittels dieser Klassifizierung eine Harmonisierung regionalstatistischer Daten innerhalb der EU zu erreichen, sozioökonomische Analysen auf der Grundlage von sozioökonomischen Großregionen (NUTS1), Basisregionen für regionalpolitische Maßnahmen (NUTS2) und Kleinstregionen für spezifische Diagnosen (NUTS3) durchführen zu können sowie Informationen für die Gestaltung der EU-Regionalpolitik zu sammeln (vgl. Eurostat 2018a: o. S.).

Tabelle 14: Fallauswahl

\begin{tabular}{|c|c|c|c|}
\hline $\begin{array}{l}\text { Regime- } \\
\text { typen }\end{array}$ & $\begin{array}{l}\text { Länder- } \\
\text { vergleich* }^{*}\end{array}$ & NUTS1-Regionenvergleich & \\
\hline \multirow{27}{*}{$\begin{array}{l}\text { Konservativ/ } \\
\text { kontinental- } \\
\text { europäisch }\end{array}$} & Deutschland & Baden-Württemberg (DE1) & \\
\hline & & Bayern (DE2) & \\
\hline & & Berlin (DE3) & \\
\hline & & Brandenburg (DE4) & \\
\hline & & Bremen (DE5) & \\
\hline & & Hamburg (DE6) & \\
\hline & & Hessen (DE7) & \\
\hline & & Mecklenburg- & \\
\hline & & Vorpommern (DE8) & \\
\hline & & Niedersachsen (DE9) & \\
\hline & & Nordrhein-Westfalen (DEA) & \\
\hline & & Rheinland-Pfalz (DEB) & \\
\hline & & Saarland (DEC) & \\
\hline & & Sachsen (DED) & \\
\hline & & Sachsen-Anhalt (DEE) & \\
\hline & & Schleswig-Holstein (DEF) & \\
\hline & & Thüringen (DEG) & \\
\hline & Österreich & Ostösterreich (AT1) & \\
\hline & & Südösterreich (AT2) & \\
\hline & & Westösterreich (AT3) & \\
\hline & Belgien $^{+}$ & Brussels (BE1) & \\
\hline & & Flanders (BE2) & \\
\hline & & Wallonia (BE3) & \\
\hline & Frankreich & Ile-de-France (FR1) & \\
\hline & & Bassin Parisien (FR2) & \\
\hline & & Nord-Pas-de-Calais (FR3) & \\
\hline & & Est (FR4) & f. \\
\hline
\end{tabular}




\begin{tabular}{|c|c|c|}
\hline & & $\begin{array}{l}\text { Ouest (FR5) } \\
\text { Sud-Ouest (FR6) } \\
\text { Centre-Est (FR7) } \\
\text { Méditerranée (FR8) }\end{array}$ \\
\hline $\begin{array}{l}\text { Liberal/ } \\
\text { Mischform }\end{array}$ & Niederlande & $\begin{array}{l}\text { Noord-Nederland (NL1) } \\
\text { Oost-Nederland (NL2) } \\
\text { West-Nederland (NL3) } \\
\text { Zuid-Nederland (NL4) }\end{array}$ \\
\hline $\begin{array}{l}\text { Postsozialis- } \\
\text { tisch }\end{array}$ & $\begin{array}{l}\text { Georgien } \\
\text { Litauen } \\
\text { Polen }\end{array}$ & 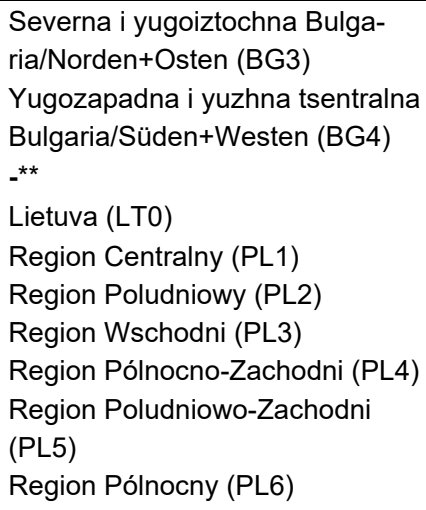 \\
\hline & $\begin{array}{l}\text { Rumänien } \\
\text { Russland } \\
\text { Tschechien } \\
\text { Ungarn }\end{array}$ & $\begin{array}{l}- \\
-* * \\
\text { Ceská republika (CZ0) } \\
\text { Közép-Magyarország (HU1) } \\
\text { Dunántúl (HU2) } \\
\text { Alföld és Észak (HU3) }\end{array}$ \\
\hline $\begin{array}{l}\text { Sozialdemo- } \\
\text { kratisch/ } \\
\text { skandina- } \\
\text { visch }\end{array}$ & $\begin{array}{l}\text { Schweden } \\
\text { Norwegen }^{+}\end{array}$ & $-* *$ \\
\hline & $N_{\text {Kontextebene }}=15$ & $N_{\text {Kontextebene }}=48$ \\
\hline
\end{tabular}

Quelle: Eigene Darstellung.

Anmerkungen:

* Grundsätzlich sind im GGS auch Daten für Italien und Estland verfügbar, jedoch nicht in Bezug auf die innerhäusliche Arbeitsteilung.

** Für die europäischen Nicht-EU-Staaten Georgien und Russland ist keine NUTS-Klassifikation vorhanden. Obwohl Schweden Mitglied der EU ist, sind im GGS keine Informationen bezüglich der NUTS-Klassifizierung enthalten.

+ Für Belgien und Norwegen sind keine Daten für die zweite Welle verfügbar. 
Abbildung 6: NUTS 1 Regionen in Europa

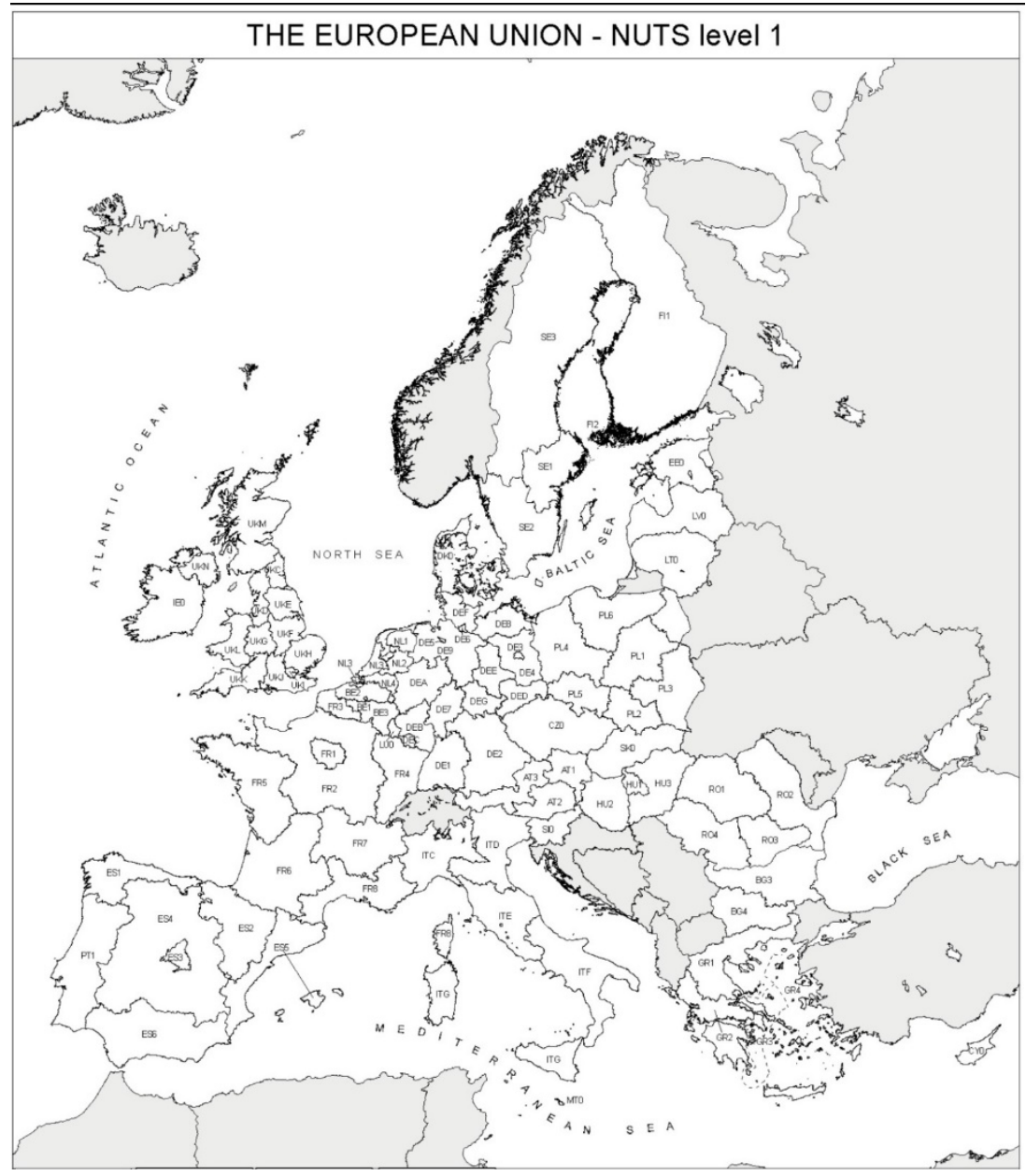

Quelle: Eurostat 2007: 18. 


\subsection{Stichprobenbildung}

Die Stichprobe umfasst heterosexuelle ${ }^{139}$ (verheiratete und nicht verheiratete) Paare mit und ohne Kinder, die ausschließlich in einem gemeinsamen Haushalt leben. Das Generations and Gender Survey ist zwar keine Paarbefragung, dennoch besteht die Möglichkeit, auf die Antworten der PartnerInnen zu schließen, weil die Befragten nicht nur Fragen zur eigenen Person, sondern auch zum/zur PartnerIn gestellt bekommen. Die weiterführende Analyse basiert in der vorliegenden Forschungsarbeit daher auf den Antworten all jener Frauen im Alter zwischen 18 und 79 Jahren, die Angaben über die Aufteilung der Hausarbeiten zwischen ihnen und ihrem Partner gemacht haben. Zum Zeitpunkt der ersten Welle trifft dies auf 41336 Frauen im Ländervergleich und auf 34044 Frauen im NUTS1-Regionenvergleich zu (da nicht für alle Länder die NUTS1-Klassifikation vorgenommen werden kann, variiert die Fallzahl, s. Tabelle 14).

\subsection{Operationalisierung der innerhäuslichen Arbeitsteilung}

Die abhängige Variable „Task-Participation-Index“ - dessen Grundidee auf die Studie von Blood/Wolfe „Husbands and Wives - The Dynamics of Married Living" zurückzuführen ist - umfasst den Aufwand für Hausarbeiten, wobei diejenigen Frauen ausgewählt wurden, die auf die folgende Frage geantwortet haben: ,Jetzt würde ich Ihnen gerne ein paar Fragen dazu stellen, wer in Ihrem Haushalt welche Aufgaben erledigt. Sagen Sie mir bitte, wer die folgenden Aufgaben bei Ihnen im Haushalt verrichtet". Hierbei wurden die Kategorien Kochen, Einkaufen, Staubsaugen/Putzen ${ }^{140}$ und Reparaturen

139 Aus forschungspraktischen Gründen wurde die Heteronormativitätsannahme getroffen, da der Anteil homosexueller Paare im Vergleich zu heterosexuellen Paaren sehr gering ist, zumal der Aspekt der Traditonalität von Arbeitsteilungsarrangements im Vordergrund steht. Eine quantitative Analyse der Aufteilung von Hausarbeiten in homosexuellen Paaren ist aufgrund der zahlenmäßig schlechten Datenlage nach wie vor schwierig, wäre jedoch inhaltlich höchst interessant.

140 Was das Wäsche waschen anbelangt, hat bereits Jean-Claude Kaufmann (1994) die besondere Bedeutung der Wäsche für die Konstruktion des Alltags von Paarbeziehungen und die Konstruktion von Geschlechterrollen analysiert. ,[...] mit der schmutzigen Wäsche verbindet sich die Erinnerung an die ursprüngliche Rolle der Frau innerhalb des Paares, aber zugleich auch der Anspruch auf ihre Veränderung im Sinne einer Gleichberechtigung beider Partner. Gleichzeitig ist die Wäsche ein wesentliches Kennzeichnen persönlicher Identität, das von der Paarbeziehung unweigerlich beeinflußt [sic!] wird“ (Kaufmann 1994: 9). Weitere Indikatoren, wie z. B. die Geldverwaltung oder das Organisieren von sozialen Aktivitä- 
berücksichtigt, die im Rahmen einer fünfstufigen Likert-Skala ${ }^{141}$ mit den Ausprägungen überwiegend ich, meist ich, PartnerIn und ich gleich, meist PartnerIn, überwiegend PartnerIn ${ }^{142}$ erfasst wurden (s. Tabelle 15: Indikatoren der abhängigen Variable). Insofern handelt es sich um eine subjektive Einschätzung von Frauen über die jeweiligen Items, welche/r der beiden PartnerInnen mehr Hausarbeiten übernimmt. Ferner kann für die Analyse der Veränderung der Arbeitsteilung erfasst werden, ob die innerhäusliche Arbeitsteilung zwischen den beiden Messzeitpunkten konstant geblieben ist, sich traditioneller oder egalitärer entwickelt hat. Der „Task-ParticipationIndex" ist ein additiver Summenscore, dessen einzelne Indikatoren grob nach zeitlichem Umfang gewichtet wurden (Kochen mit dem Faktor 1,0, Einkaufen mit dem Faktor 0,5, Staubsaugen/Putzen mit dem Faktor 0,5 und Reparaturen mit dem Faktor 0,25). Cronbachs (1951) Alpha weist insgesamt für die gepoolten Daten einen zwar nicht optimalen, aber gerade noch akzeptablen Wert von 0,67 auf (anzustreben ist ein Wert von 0,8, wobei ein niedrigerer Wert kein absolutes Ausschlusskriterium für eine Skala ist, sofern inhaltlich sinnvolle Argumente für die Skala sprechen). Länderspezifisch sind Werte größer 0,7 in Litauen, Polen, Russland, Schweden und Tschechien aufzuweisen, Werte zwischen 0,6 und 0,69 in Belgien, Bulgarien, Deutschland, Österreich, Rumänien, Ungarn, Werte zwischen 0,5 und 0,59 in den Niederlanden und Werte bis 0,5 in Georgien, Frankreich und Norwegen. Die sehr niedrigen Werte in Frankreich und Norwegen könnten durch ein Entfernen des Indikators „Reparaturen“ verbessert werden, der in diesen Ländern einen Mittelwert größer 4 umfasst, was verdeutlicht, dass diese Tätigkeit überwiegend von Männern ausgeführt wird. Doch handelt es sich hierbei um einen wesentlichen Indikator der Hausarbeiten, der aus inhaltlichen Gründen insofern nicht entfernt werden sollte, zumal die Anzahl der für alle Länder verfügbaren Hausarbeitsitems ohnehin sehr gering ist. In Georgien weist der „TaskParticipation-Index" grundsätzlich keine interne Konsistenz auf, wie anhand der Berücksichtigung der stark unterschiedlichen Mittelwerte der einzelnen Indikatoren (s. Abbildung 12: Mittelwerte der Hausarbeiten im Ländervergleich, Kapitel 13.1.) zu erwarten ist. Die innerhäusliche Arbeitsteilung ist in Georgien am stärksten geschlechtsspezifisch segregiert. Festzuhalten ist auBerdem, dass die Streuung des „Task-Participation-Index“ am niedrigsten in Deutschland (Standardabweichung $=0,90$ ) und am größten in Belgien (Stan-

ten, sind für Hausarbeitsteilungsarrangements grundsätzlich zu berücksichtigen, doch sind nicht für alle Länder entsprechende Daten im Generations and Gender Survey enthalten.

141 Ferner können diese Indikatoren als quasimetrisch erachtet werden: ,[...] in der Forschungspraxis [hat sich] auch gezeigt, dass metrische bzw. als metrisch zu definierende Variablen ab 5 Ausprägungen für eine sinnvolle OLS-Regression geeignet sein können“ (Urban/Mayerl 2011: 14).

142 Die Antwortkategorien $6=$,,always or usually other persons in the household" und $7=$ ,always or usually someone not living in the household" wurden von der Analyse ausgeschlossen. 
dardabweichung $=1,86$ ) ist, wodurch die vergleichsweise deutlichsten regionalen Differenzen der innerhäuslichen Arbeitsteilungsarrangements in Belgien anzunehmen sind. Grundsätzlich ist dieser Vorgehensweise eines gewichteten additiven Indexes eine akribische Erfassung der Hausarbeiten über den zeitlichen Umfang, wie er in Zeitbudgeterhebungen erfasst wird, vorzuziehen. Neben den Schwierigkeiten einer Berücksichtigung des zeitlichen Umfangs stellen die Abhängigkeit des Indizes, welche Tätigkeiten-Items als Hausarbeiten verwendet werden, sowie eine fehlende Auskunft des Partners/der Partnerin (im Sinne einer Paarbefragung) weitere Herausforderungen dar (vgl. Künzler 1994: 56ff; Klaus/Steinbach 2002: 29). So sind nach Künzler die Zeitbudgetforschung und die Befragung beider PartnerInnen der ,Königsweg', der dem Task-Participation-Index vorzuziehen ist" (vgl. Künzler 1994: 58).

„Allerdings ist die Verwendung derartiger relativer Indizes aus mindestens drei Gründen nicht unproblematisch. Erstens wurde der Index in der vorliegenden Analyse nur aus den Angaben der Ehefrauen gebildet. [Studien zum Bamberger Ehepaar-Panel haben jedoch bei zusätzlicher Verwendung der Daten der Männer gezeigt, dass die Ergebnisse weitgehend unverändert bleiben.] [...] Zweitens handelt es sich um einen Summenscore, der jede Tätigkeit mit dem gleichen Gewicht versieht, was insofern problematisch ist, da die Haushaltstätigkeiten unterschiedlich viel Zeit in Anspruch nehmen. Drittens kann eine Veränderung des relativen Anteils immer aufgrund verschiedener absoluter Veränderungen zustande kommen; so kann ein Rückgang des Anteils der Frau, um nur zwei Beispiele zu nennen, aus einer gleichbleibenden absoluten Beteiligung der Frau bei gleichzeitiger Erhöhung der absoluten Beteiligung des Mannes resultieren oder aus einer gleichbleibenden Beteiligung des Mannes bei gleichzeitiger Reduktion der Beteiligung der Frau. Für eine ausführliche Diskussion der Problematik relativer Maßzahlen, wie dem [...] Task-Participation-Index, vgl. vor allem die Arbeit von Künzler (1994)““(Schulz 2010: 190).

Diese Vorgehensweise ist mit dem Generations and Gender Survey jedoch nicht möglich, da weder das präzise Zeitausmaß der Hausarbeiten in Stunden enthalten ist noch die PartnerInnen befragt wurden. Im Hinblick auf die Einzelindikatoren der Hausarbeiten wurden diejenigen ausgewählt, deren Daten für alle Länder verfügbar sind. In den Index gehen weiterführend keine Indikatoren ein, die die Kinderbetreuung umfassen, da aktuelle Studien empfehlen, zwischen den Dimensionen der Routinehausarbeiten und Elternaufgaben $\mathrm{zu}$ differenzieren, weil sie partiell unterschiedlichen Mechanismen folgen (vgl. Wengler et al. 2009: 57). Dies befürworten beispielsweise auch Dechant/Blossfeld (2015):

„Anhand der qualitativen Interviews [aus der Studie von Dechant/Blossfeld] wird ersichtlich, dass Erklärungsmuster, die im Bereich der unbezahlten Arbeit zwischen Kinderbetreuung und Hausarbeit unterscheiden, besser die Realität der Paare erfassen“" (Dechant/Blossfeld 2015: 373). 
Tabelle 15: Indikatoren der abhängigen Variable

Variablen $\begin{gathered}\text { Metrik } \\ \text { Fragestellung: }\end{gathered}$
Jetzt würde ich Ihnen gerne ein paar Fragen dazu stellen, wer in Ihrem Haushalt welche
Aufgaben erledigt. Sagen Sie mir bitte, wer die folgenden Aufgaben bei Ihnen im
Haushalt verrichtet./Now I would like to ask you some questions about who does what in
your household. Please tell me who does the following tasks in your household.

Kochen

Einkaufen

Staubsaugen/Putzen

Reparaturen

(Rechnungen bezahlen)*

*Der Indikator Rechnungen bezahlen ist nicht für Norwegen verfügbar, weshalb er nicht in den Task Participation Index eingeht, jedoch im Rahmen einiger deskriptiver Darstellungen enthalten ist.
1 bis $5 \quad$ Who does hh tasks:

1 always respondent Preparing daily meals/

2 usually respondent Die täglichen Mahlzeiten

3 respondent and zubereiten partner equally

4 usually partner

5 always partner

1 bis 5

1 always respondent

2 usually respondent

3 respondent and partner equally

4 usually partner

5 always partner

1 bis 5

1 always respondent

2 usually respondent

3 respondent and partner equally

4 usually partner

5 always partner

\section{1 bis 5}

1 always respondent

2 usually respondent

3 respondent and partner equally

4 usually partner

5 always partner 1 bis 5

1 always respondent

2 usually respondent

3 respondent and partner equally

4 usually partner

5 always partner
Who does hh tasks:

Shopping for food/

Essen einkaufen

Who does hh tasks:

Vacuum-cleaning the

house/ Staubsaugen

(Für die polnischen Daten

fehlt dieses Item, daher

wurde für diese Ausnahme

der Indikator „Who does

hh tasks: Cleaning the

house" verwendet.)

Who does hh tasks:

Small repairs in, around the house/

Kleinere Reparaturen rund

ums Haus

(Who does hh tasks:

Paying bills, financial

records/

Rechnungen bezahlen und sich um finanzielle Angelegenheiten kümmern)

Quelle: Eigene Darstellung. 


\subsection{Beschreibung der unabhängigen Variablen}

Auf individueller Ebene werden eine Reihe von unabhängigen Variablen überprüft, die die theoretischen symmetrischen und asymmetrischen Ansätze auf Basis der interaktionellen und kulturellen Form von Macht testen sollen: Das Bildungsniveau, die Bildungsrelation innerhalb des Paares, die Einkommenshöhe, die Einkommensrelation, das Erwerbspensum, die Erwerbsrelation, der Familienstand (verheiratet), die Existenz von kleinen Kindern im Haushalt, die Anzahl der Kinder, die Paarbeziehungsdauer sowie die Kontrollvariablen des Alters und der Geburtskohorten (einige detaillierte Informationen zur Operationalisierung der unabhängigen Variablen sind in Tabelle 16 zusammengefasst). Für alle Individualvariablen wird das Generations and Gender Survey als Datenbasis verwendet.

\section{Kurzbeschreibungen der Individualvariablen}

Bildungsniveau

Das Bildungsniveau ist im GGS gemäß der „International Standard Classification of Education" (ISCED) $)^{143}$ erhoben worden. Die ISCEDKlassifizierung wurde so umkodiert, dass eine niedrige Bildung auf ISCED 0-2 basiert, eine mittlere Bildung auf ISCED 3-4 und eine hohe Bildung auf ISCED 5-6. Weiterführend wurden zwei Dummyvariablen für einen hohen und einen mittleren Bildungsstand erstellt, deren Referenzkategorie einen niedrigen Bildungsstand umfasst.

\section{Bildungsrelation}

Für die Messung der Bildungsrelation ist der Bildungsstand der Frau vom Bildungsstand des Mannes zunächst subtrahiert worden. Negative Werte bedeuten, dass die Frau über einen höheren Bildungsstand verfügt als ihr Partner, 0 bedeutet, dass eine Bildungshomogamie besteht und positive Werte verweisen darauf, dass der Mann über einen höheren Bildungsstand verfügt als seine Partnerin. Aus diesen Informationen wurden zwei Dummyvariablen gebildet: im ersten Fall betrifft dies die Relation „Frau > Mann“ (Kodierung $=1$ ), was bedeutet, dass die Frau über einen höheren Bildungsstand verfügt als ihr Partner (Bildungshypogamie). Im zweiten Fall ist die Relation „Frau = Mann“ (Kodierung = 1) gemeint, was bedeutet, dass eine Bildungshomogamie innerhalb des Paares besteht. Die Relation „Frau $<$ Mann“ (Kodierung =

143 Für Belgien, Bulgarien, Deutschland, Georgien, Litauen, Niederlande, Norwegen, Österreich, Polen, Rumänien, Russland, Schweden, Tschechien und Ungarn gilt folgende Kodierung: isced 0 - pre-primary education, isced 1 - primary level, isced 2 - lower secondary level, isced 3 - upper secondary level, isced 4 - post secondary non-tertiary, isced 5 - first stage of tertiary, isced 6 - second stage of tertiary.

Frankreich hat den Bildungsstand über ISCED 97 erhoben: 0 - isced97, 1-2 - isced97, 3A - isced97, 3B - isced97, 3C - isced97, 5A-6 - isced97, 5B - isced 97. 
0), nach der die Frau über einen niedrigeren Bildungsstand verfügt als ihr Partner (Bildungshypergamie), ist Referenzkategorie.

\section{Einkommenshöhe}

Weitere Dummyvariablen wurden für ein mittleres (1500,01 bis 4000,00 $€$ ) sowie ein hohes Einkommen $(4000,01 €$ und mehr) erstellt, deren Referenzkategorie ein niedriges Einkommen (bis 1500,00 €) ist. Aufgrund einiger Schwierigkeiten der Vergleichbarkeit der Einkommensangaben (s. u.) zwischen den Ländern werden Dummyvariablen bevorzugt, anstatt das Einkommen als metrische Variable einzubeziehen.

\section{Einkommensrelation}

Die Bildung der Einkommensrelation ist etwas komplexer, weil nicht alle Variablen für alle Länder verfügbar sind. Während für die Länder, die die Höhe des monatlichen Nettoeinkommens differenziert nach unterschiedlichen Einkommensquellen erhoben haben, eine Summe aller Nettoeinkünfte gebildet wurde, wurden für Deutschland sowie Österreich Gruppenmittelwerte der unterschiedlichen Einkommenstypen gebildet und diese addiert, weil die Einkommenstypen lediglich gruppiert verfügbar sind. Norwegen enthält zwar das Nettoeinkommen pro Einkommensquelle, jedoch sind dies jährliche statt monatlicher Angaben. Insofern wurden diese Werte durch 12 dividiert und anschließend eine Summe aller Quellen gebildet. Für Polen ist das Nettogesamteinkommen aller Einkommenstypen vorhanden, das ohne Modifikation verwendet werden konnte, weil die Informationen der differenten Einkommensquellen durch die Summenbildung ohnehin verloren gehen. Für Schweden - dessen Daten jedoch aufgrund fehlender Informationen zur NUTSKlassifizierung im GGS nicht in die Mehrebenenmodelle eingehen - ist das Nettogesamteinkommen aller Einkommenstypen gruppiert vorhanden, weshalb hierfür die Gruppenmittelwerte verwendet wurden und zusätzlich eine Umrechnung in Euro erfolgte. Die Umrechnung in Euro basierte auf dem Wechselkurs des Jahres 2012 (Stichtag 31.12.12; die erste Welle Schwedens wurde ebenfalls 2012 erhoben) und war erforderlich, da Schweden das einzige Land ist, in dem die Einkünfte nicht in Euro, sondern in schwedischen Kronen erhoben wurden.

Durch die Information der Summenvariable aller monatlichen Nettoeinkünfte je PartnerIn kann die Einkommensrelation gebildet werden, welche/r der beiden PartnerInnen ein höheres bzw. niedrigeres Einkommen verdient. Die Relation „Frau > Mann“ (Kodierung = 1) bedeutet, dass die Frau über ein höheres Nettoeinkommen (diverser Einkommensquellen) verfügt, „Frau = Mann“ (Kodierung = 1) bedeutet, dass beide über ein ähnlich hohes Einkommen verfügen (eine Einkommenshomogamie wurde bei Abweichungen +- 250 Euro angenommen), Referenzkategorie ist „Frau < Mann“ (Kodierung $=0$ ), d. h. dass die Frau über weniger Nettoeinkommen verfügt als ihr Partner. 


\section{Erwerbsumfang}

Da die Verwendung der Teilzeit- und Vollzeit-Klassifizierung der Stunden zwischen den Ländern variiert (z. B. ist in Frankreich eine 35-StundenWoche als eine Vollzeitbeschäftigung definiert, in Deutschland sind es hingegen 40 Stunden), wurde die Grenze zur Vollzeitbeschäftigung ab 35 Stunden gewählt (für eine Differenzierung unterschiedlicher Typen von Teilzeitarbeit s. Hakim (1997) ${ }^{144}$ ). Einen Stundenumfang ab 35 Stunden erfasst die Dummyvariable „Vollzeit“, deren Referenzkategorie eine Teilzeitbeschäftigung im Ausmaß von 1 bis 34,99 Stunden darstellt.

\section{Erwerbsrelation}

Wie auch im Falle der Bildungs- und Einkommensrelation wird die Erwerbsrelation zunächst über die Differenz des Erwerbspensums der Frau abzüglich des Erwerbspensums des Mannes gebildet. Ferner werden aus dieser Differenzvariablen zwei Dummyvariablen gebildet, die eine Erwerbshypogamie beinhalten, sofern die Frau mehr Stunden erwerbstätig ist als ihr Mann sowie eine Erwerbshomogamie, wenn beide gleichermaßen erwerbstätig sind (Abweichungen von rund \pm 2 Stunden liegen hierbei im Toleranzbereich der Erwerbshomogamie). Referenzkategorie ist die Relation „Frau < Mann“, die für Paare mit einer eher traditionellen Aufteilung der außerhäuslichen Erwerbsbeteiligung gilt.

\section{Familienstand}

Ein verheirateter Familienstand wird durch eine Dummyvariable für verheiratete Paare berücksichtigt, deren Referenzkategorie nicht verheiratete heterosexuelle Paare darstellen.

Kleine Kinder (<6 Jahre)

Sofern mindestens ein Kind im gemeinsamen Haushalt lebt, das jünger als sechs Jahre alt ist, wird dies über die Dummyvariable „Kleine Kinder $(<6$ Jahre)“ erfasst, die in Bezug zur Referenzkategorie „kein Kind unter sechs Jahren" interpretiert werden kann.

\section{Anzahl der Kinder}

Die Variable „Anzahl der Kinder“ umfasst alle im gemeinsamen Haushalt lebenden Kinder, unabhängig ihres Alters (im Falle der Mehrebenenmodelle wird diese Variable grand-mean-zentriert; für methodische Aspekte der Zentrierung s. Kapitel 12.1.4.).

144 „Given that part-time work means such different things even within European labour markets, cross-national comparisons are often not comparing like with like" (Hakim 1997: 90). Hakim differenziert drei Typen der Teilzeitarbeit: „,reduced hours work” (umfasst Tätigkeiten, die zeitlich etwas weniger umfangreich sind als eine Vollzeitarbeit, i. d. R. 30-36 oder 30-39 Stunden pro Woche), ,half-time jobs” (15-29 Stunden pro Woche) und ,,marginal jobs“" (15 Stunden pro Woche und weniger) (vgl. Hakim 1997: 25). 


\section{Paarbeziehungsdauer}

Die Paarbeziehungsdauer basiert auf Angaben in Monaten seit Beginn der Paarbeziehung, die ebenfalls im Rahmen der Mehrebenenanalyse grandmean-zentriert wird.

\section{Kurzbeschreibungen der Kontextvariablen}

Auf der regionalen Kontextebene werden zusätzlich acht Kontextvariablen herangezogen, die jeweils nach ökonomischer, politischer und kultureller Empowerment-Dimension differenziert werden können und die auf den regionalen Statistiken von Eurostat sowie dem European Social Survey basieren: Das Bruttoinlandsprodukt pro Kopf, die Erwerbsquote von Frauen (ökonomische Dimension), die politische Links-Rechts-Verortung, die Teilnahmerate der 4-Jahre-Altersgruppe an Bildungsprogrammen (politische Dimension), der auf dem European Social Survey basierende aggregierte Kontextindikator „Genderequality“, die Fertilitätsrate, die Religiosität und der Zugang für Frauen zum tertiären Bildungssektor (kulturelle Dimension). Die L2Prädiktoren wurden auf Basis der Erhebungswellen des GGS jahresspezifisch zusammengefügt (wurde beispielsweise die erste Welle des GGS in Bulgarien 2004 durchgeführt, ist das BIP für 2004 als Kontextindikator ergänzt worden). Ausnahmen sind die Kontextindikatoren auf Basis des European Social Surveys: hier wurden alle Indikatoren (mit Ausnahme der Teilnahmerate der 4-Jahre Altersgruppe an Bildungsprogrammen, die auf dem Jahr 2013 basiert) aus dem Jahr 2008 verwendet, weil keine Daten für die vorhergehenden Jahre verfügbar sind. Detaillierte Angaben über die jahresspezifischen Messzeitpunkte des GGS sind Tabelle 17 zu entnehmen.

\section{Bruttoinlandsprodukt pro Kopf (KKS)}

Um die unterschiedlichen Preisniveaus zwischen den Ländern zu berücksichtigen, wird für die Erfassung der Wirtschaftsleistung der NUTS1-Regionen das in Kaufkraftstandards gemessene Bruttoinlandsprodukt pro EinwohnerInnen auf Basis von Eurostat verwendet.

\section{Erwerbsquote Frauen}

Die Frauenerwerbstätigkeitsquote wird ebenfalls von Eurostat bezogen, deren Definition jedoch auf der International Labour Organisation beruht ${ }^{145}$ :

"The labour force participation rate is defined as the ratio of the labour force to the working age population [15-64], expressed in percentages. According to the International Labour Organization (ILO), the total labour force, or currently active population, comprises all persons who fulfil the requirements for inclusion

145 „The source for the regional labour market information [...] is the EU Labour Force Survey (EU-LFS). This is a quarterly household sample survey conducted in all Member States of the EU and in EFTA and Candidate countries. The EU-LFS survey follows the definitions and recommendations of the International Labour Organisation (ILO)" (Eurostat 2018a: o. S.). 
among the employed or the unemployed during a specified brief reference period" (GGP 2018: o. S.).

Politische Links-Rechts-Verortung ${ }^{146}$

Auf der Datenbasis des European Social Surveys wurde der Indikator „Placement on left right scale“, der einen Wertebereich von $0=$ links bis $10=$ rechts umfasst, für die NUTS1-Regionen aggregiert, wodurch er als Kontextvariable der politischen Verortung der jeweiligen Regionen verwendet werden kann.

\section{Frühkindliche Kinderbetreuung}

Ein relevanter Kontextindikator, der ein zentrales familienpolitisches Instrument der (staatlichen) frühkindlichen Kinderbetreuung im regionalen Kontext darstellt, ist die - von Eurostat ${ }^{147}$ zur Verfügung gestellte - Teilnahmerate der 4-Jahre-Altersgruppe an Bildungsprogrammen.

\section{Fertilitätsrate}

Die Fertilitätsrate, deren Daten auf der regionalen Datenbank von Eurostat basieren, ist wie folgt definiert:

„Total fertility rate (TFR) is the mean number of children that would be born alive to a woman during her lifetime if she were to pass through her childbearing years conforming to the fertility rates by age of a given year" (Eurostat 2018a: o. S.).

\section{Genderequality}

Einen auf NUTS1-Regionen aggregierten Kontextindikator stellt die Variable „Genderequality” dar, die anhand des Items „Men should have more right to job than women when jobs are scarce" mit den Antwortkategorien von 1=,,stimme sehr zu“ bis 5=,,stimme überhaupt nicht zu“ im Rahmen des European Social Surveys erhoben wurde (European Social Survey 2018: o. S.).

\section{Tertiärer Bildungszugang für Frauen}

Der Zugang für Frauen zum tertiären Bildungssektor wird anhand des Eurostat-Indikators „Frauen im Alter von 25-64 mit tertiärem Bildungsabschluss (ISCED 5-8) in \%" erfasst.

146 Ein Indikator, wie der für Länder verfügbare Political Empowerment Index (Subindex des Global Gender Gap Index), der die politische Repräsentation von Frauen umfasst, ist nicht für die NUTS1-Regionen vorhanden, weshalb die politische Links-Rechts-Verortung als Proxy-Variable des politischen Empowerments auf regionaler Eben zu verstehen ist. Sundström/Stockemer (2015) haben für viele (aber nicht für alle der hier zu betrachtenden NUTS1-Regionen) europäische Regionen auf Basis der NUTS-Klassifikation Daten zur politischen Repräsentation zusammengetragen.

147 Die Daten dieses Indikators basieren ausschließlich auf dem Jahr 2013, da sie für vorherige Jahre nicht verfügbar sind. 


\section{Religiosität}

Der auf der regionalen Kontextebene aggregierte Indikator „How often attend religious services apart from special occasions" gibt Aufschluss über den religiös-kulturellen Hintergrund der NUTS1-Regionen und basiert auf den Daten des European Social Survey (European Social Survey 2018: o. S.).

In den Tabellen 16 bis 19 sind detaillierte Informationen zur Operationalisierung der unabhängigen Variablen, zu den Messzeitpunkten des Generations and Gender Surveys, zu den Mittelwerten der Individualvariablen sowie zu den Kontextfaktoren zusammengefasst.

Tabelle 19 bildet einige wesentliche Kennzahlen (Minimum, Maximum, arithmetisches Mittel und Standardabweichung) der Kontextfaktoren für den NUTS1-Regionenvergleich ab. Festzuhalten ist, dass die bulgarische Region Severna i yugoiztochna Bulgaria (BG3) die geringste Wirtschaftsleistung in Kaufkraftstandards aufweist, die NUTS1-Region Brüssel (BE1) hingegen die größte. Während die Erwerbsquote von Frauen in der ungarischen Region Alföld és Észak (HU3) mit 42,4\% am niedrigsten ist, ist sie mit 70,6\% in Norwegen am höchsten. Brandenburg (DE4) ist die Region, in der die Bevölkerung zur stärksten politischen Links-Verortung neigt, Wschodni (PL3) ist die Region mit dem tendenziell höchsten Bevölkerungsanteil einer politisch eher rechten Verortung. In Bezug auf die Teilnahmerate der 4-JahreAltersgruppe an Bildungsprogrammen stellt die Region Pólnocny (PL6) das Schlusslicht dar, hingegen weisen insbesondere niederländische (NL1, NL2, NL4) und französische Regionen (FR1, FR3, FR7, FR8) eine Teilnahmerate von $100 \%$ auf. Brüssel (BE1) ist im Vergleich der Regionen Spitzenreiter was die Fertilitätsrate anbelangt; indessen ist die Fertilitätsrate in der ungarischen Region Közép-Magyarorszá (HU1) und in der polnischen Region Poludniowo-Zachodni (PL5) auf sehr niedrigem Niveau. Der Bevölkerungsanteil, der das Item ,wenn Arbeitsplätze knapp sind, sollten Männer größere Anrechte auf Arbeitsplätze haben als Frauen" befürwortet, ist in Alföld és Észak (HU3) am größten, in Ile-de-France (FR1) am geringsten. Einen Zugang zu tertiärer Bildung haben Frauen vornehmlich in Brüssel (BE1), während ihr Zugang zum tertiären Bildungssektor in Tschechien sehr gering ist $(10,8 \%)$. Sachsen-Anhalt (DEE) ist die säkularisierteste Region - ganz im Gegensatz zu Wschodni (PL3), wo Religiosität nach wie vor eine zentrale Rolle im Alltagsleben der Bevölkerung spielt. Insgesamt zeigen sich einige nicht zu vernachlässigende Unterschiede der Kontextfaktoren zwischen den NUTS1-Regionen. 
Tabelle 16: Operationalisierung der unabhängigen Variablen

\begin{tabular}{|c|c|c|}
\hline Variablen & Metrik & Operationalisierung \\
\hline \multicolumn{3}{|c|}{ Individualebene (L1) } \\
\hline \multicolumn{3}{|c|}{ Interaktionelle und kulturelle Macht } \\
\hline Bildungsstand & Dummys: & \\
\hline \multirow[t]{3}{*}{ (Quelle: GGS) } & hohe Bildung (1) & ISCED 5-6 \\
\hline & mittlere Bildung (1) & ISCED 3-4 \\
\hline & niedrige Bildung $(0)$ & ISCED 0-2 \\
\hline Bildungsrelation & Dummys: & Bildung Mann-Frau: \\
\hline \multirow[t]{3}{*}{ (Quelle: GGS) } & Frau > Mann (1) & Bildungshypogamie \\
\hline & Frau = Mann (1) & Bildungshomogamie \\
\hline & Frau $<$ Mann (0) & Bildungshypergamie \\
\hline Einkommen & Dummys: & \\
\hline (Quelle: GGS) & $\begin{array}{l}\text { hohes Einkommen (1) } \\
\text { mittleres Einkommen (1) } \\
\text { niedriges Einkommen (0) }\end{array}$ & $\begin{array}{l}4000,01 € \text { und mehr } \\
1500,01 \text { bis } 4000,00 € \\
\text { bis } 1500,00 €\end{array}$ \\
\hline Einkommensrelation & Dummys: & Einkommen Mann-Frau: \\
\hline \multirow[t]{3}{*}{ (Quelle: GGS) } & Frau > Mann (1) & Einkommenshypogamie \\
\hline & Frau $=$ Mann (1) & Einkommenshomogamie \\
\hline & Frau $<$ Mann $(0)$ & Einkommenshypergamie \\
\hline Erwerbsarbeitsstunden & Dummy: & \\
\hline \multirow[t]{2}{*}{ (Quelle: GGS) } & Vollzeit (1) & 1-34,99 Stunden \\
\hline & Teilzeit (0) & ab 35 Stunden \\
\hline Erwerbsrelation & Dummy: & Stunden Mann-Frau: \\
\hline \multirow[t]{3}{*}{ (Quelle: GGS) } & Frau $>$ Mann (1) & Erwerbshypogamie \\
\hline & Frau $=$ Mann (1) & Erwerbshomogamie \\
\hline & Frau $<$ Mann (0) & Erwerbshypergamie \\
\hline \multirow{3}{*}{$\begin{array}{l}\text { Kind jünger als } 6 \text { Jahre alt } \\
\text { (Quelle: GGS) }\end{array}$} & Dummy: & \\
\hline & kleine Kinder $<6$ Jahre (1) & $\begin{array}{l}\text { mind. ein kleines Kind } \\
\text { im Haushalt, das jünger } \\
\text { als sechs Jahre alt ist }\end{array}$ \\
\hline & $\begin{array}{l}\text { kein Kind unter sechs } \\
\text { Jahren }(0)\end{array}$ & $\begin{array}{l}\text { kein Kind, das jünger als } \\
\text { sechs Jahre alt ist und } \\
\text { im Haushalt wohnt }\end{array}$ \\
\hline $\begin{array}{l}\text { Kinderanzahl } \\
\text { (Quelle: GGS) }\end{array}$ & Kinderanzahl, metrisch & $\begin{array}{l}\text { Alle Kinder, die im } \\
\text { gleichen Haushalt leben }\end{array}$ \\
\hline $\begin{array}{l}\text { Paarbeziehungsdauer } \\
\text { (Quelle: GGS) }\end{array}$ & $\begin{array}{l}\text { Paarbeziehungsdauer in } \\
\text { Monaten, metrisch }\end{array}$ & $\begin{array}{l}\text { Paarbeziehungsdauer in } \\
\text { Jahren seit Beginn der } \\
\text { Beziehung }\end{array}$ \\
\hline \multicolumn{3}{|c|}{ Institutionelle Macht } \\
\hline $\begin{array}{l}\text { Verheiratet } \\
\text { (Quelle: GGS) }\end{array}$ & $\begin{array}{l}\text { Dummy: } \\
\text { verheiratet }(1) \\
\text { nicht verheiratet }(0)\end{array}$ & $\begin{array}{l}\text { verheiratete Paare } \\
\text { nicht verheiratete Paare }\end{array}$ \\
\hline
\end{tabular}




\begin{tabular}{|c|c|c|}
\hline \multicolumn{3}{|c|}{ Kontrollvariablen } \\
\hline Geburtskohorten & Dummys: & Geburtskohorten von \\
\hline (Quelle: GGS) & 1939 und älter (1) & „1939 und älter“ bis \\
\hline & $1940-49(1)$ & „1980-1993“ \\
\hline & $1950-59(1)$ & \\
\hline & $1960-69(1)$ & \\
\hline & 1979-79 (1) & \\
\hline & 1980-93 (0) & \\
\hline $\begin{array}{l}\text { Alter } \\
\text { (Quelle: GGS) }\end{array}$ & $\begin{array}{l}\text { Alter in Jahren, } \\
\text { metrisch }\end{array}$ & Alter in Jahren \\
\hline \multicolumn{3}{|c|}{ Kontextebene (NUTS1, L2) } \\
\hline \multicolumn{3}{|c|}{ Ökonomisches Empowerment } \\
\hline $\begin{array}{l}\text { Bruttoinlandsprodukt } \\
\text { (Quelle: Eurostat) }\end{array}$ & $\begin{array}{l}\text { BIP pro Kopf in KKS, } \\
\text { metrisch }\end{array}$ & $\begin{array}{l}\text { "The regional gross } \\
\text { domestic product (GDP) } \\
\text { is used in order to } \\
\text { measure and compare } \\
\text { the economic activity of } \\
\text { regions. It is the most } \\
\text { important indicator for } \\
\text { the selection of regions } \\
\text { eligible for support } \\
\text { under the investment for } \\
\text { growth and jobs goal of } \\
\text { the EU's regional policy. } \\
\text { [...] The conversion to } \\
\text { purchasing power } \\
\text { standards (PPS) is } \\
\text { based on national pur- } \\
\text { chasing power parities } \\
\text { (PPP) which are also } \\
\text { regularly calculated and } \\
\text { released by Eurostat. } \\
\text { Regional PPP are not } \\
\text { available. All regional } \\
\text { accounts data published } \\
\text { by Eurostat are based } \\
\text { on PPP for the EU28" } \\
\text { (Eurostat } 2018 a: \text { o. S.). }\end{array}$ \\
\hline
\end{tabular}




\begin{tabular}{|c|c|c|}
\hline $\begin{array}{l}\text { Frauenerwerbsquote } \\
\text { (Quelle: Eurostat) }\end{array}$ & $\begin{array}{l}\text { Erwerbsquote von Frauen, } \\
\text { metrisch }\end{array}$ & $\begin{array}{l}\text { "The source for the } \\
\text { regional labour market } \\
\text { information [...] is the } \\
\text { EU Labour Force Survey } \\
\text { (EU-LFS). This is a } \\
\text { quarterly household } \\
\text { sample survey conduct- } \\
\text { ed in all Member States } \\
\text { of the EU and in EFTA } \\
\text { and Candidate coun- } \\
\text { tries. The EU-LFS sur- } \\
\text { vey follows the defini- } \\
\text { tions and recommenda- } \\
\text { tions of the International } \\
\text { Labour Organisation } \\
\text { (ILO)” (Eurostat 2018a: } \\
\text { o. S.). }\end{array}$ \\
\hline \multicolumn{3}{|c|}{ Politisches Empowerment } \\
\hline $\begin{array}{l}\text { Politische Links-Rechts- } \\
\text { Verortung } \\
\text { (Quelle: } \\
\text { European Social Survey) }\end{array}$ & $\begin{array}{l}\text { Politische Links-Rechts- } \\
\text { Verortung auf regionaler } \\
\text { Ebene } \\
0=\text { links bis } 10=\text { rechts }\end{array}$ & $\begin{array}{l}\text { „Placement on left right } \\
\text { scale”, } \\
\text { aggregierter Indikator } \\
\text { auf NUTS1 } \\
\text { (European Social } \\
\text { Survey 2018: o. S.) }\end{array}$ \\
\hline $\begin{array}{l}\text { Frühkindliche } \\
\text { Kinderbetreuung } \\
\text { (Quelle: Eurostat) }\end{array}$ & $\begin{array}{l}\text { Teilnahmeraten der 4- } \\
\text { Jahre-Altersgruppe an } \\
\text { Bildungsprogrammen auf } \\
\text { regionaler Ebene, met- } \\
\text { risch }\end{array}$ & $\begin{array}{l}\text { Teilnahmeraten der 4- } \\
\text { Jahre-Altersgruppe an } \\
\text { Bildungsprogrammen } \\
\text { auf NUTS1 }\end{array}$ \\
\hline \multicolumn{3}{|c|}{ Kulturelles Empowerment } \\
\hline $\begin{array}{l}\text { Fertilität } \\
\text { (Quelle: Eurostat) }\end{array}$ & Fertilitätsrate, metrisch & $\begin{array}{l}\text { "Total fertility rate (TFR) } \\
\text { is the mean number of } \\
\text { children that would be } \\
\text { born alive to a woman } \\
\text { during her lifetime if she } \\
\text { were to pass through } \\
\text { her childbearing years } \\
\text { conforming to the fertility } \\
\text { rates by age of a given } \\
\text { year" (Eurostat } 2018 \mathrm{a} \text { : } \\
\text { o. S.). }\end{array}$ \\
\hline
\end{tabular}




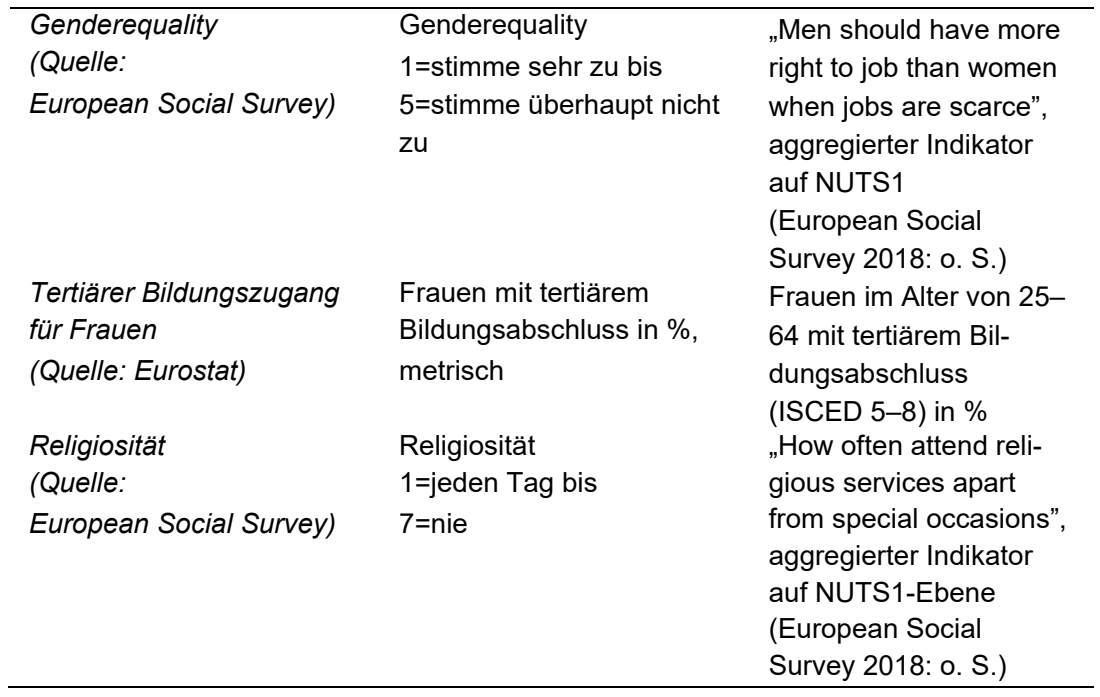

Quelle: Eigene Darstellung. 
Tabelle 17: Übersicht der Messzeitpunkte des GGS

\begin{tabular}{lcc}
\hline Land & $\begin{array}{l}\text { Erhebungsjahr der } \\
\text { 1. Welle }\end{array}$ & $\begin{array}{l}\text { Erhebungsjahr der } \\
\text { 2. Welle }\end{array}$ \\
\hline Niederlande & $2002,2003,2004$ & 2006,2007 \\
Italien & 2003 & 2007 \\
Bulgarien & 2004 & 2007 \\
Russland & 2004 & 2007 \\
Estland & 2004,2005 & - \\
Tschechien & 2004,2005 & 2008 \\
Ungarn & 2004,2005 & 2008,2009 \\
Deutschland & 2005 & 2008,2009 \\
Frankreich & 2005 & 2008 \\
Rumänien & 2005 & - \\
Georgien & 2006 & 2009 \\
Litauen & 2006 & 2009 \\
Norwegen & 2007,2008 & - \\
Österreich & 2008,2009 & 2012 \\
Belgien & $2008,2009,2010$ & - \\
Polen & 2010,2011 & 2014,2015 \\
Schweden & 2012,2013 & - \\
\hline
\end{tabular}

Quelle: Eigene Darstellung.

Anmerkung: Im Rahmen der Mehrebenenmodelle für Längsschnittdaten wird die Zentrierung um den Nullwert eine Rolle spielen, wie dem Kapitel 12.2.2. „Vorgehensweise für längsschnittliche Mehrebenenmodelle“ zu entnehmen ist. Die Nullwert-Zentrierung der Zeit basiert auf folgender Umkodierung: $2002=0,2003=1,2004=2,2005=3,2006=4,2007=5,2008=6,2009$ $=7,2010=8,2011=9,2012=10,2013=11,2014=12,2015=13$ 


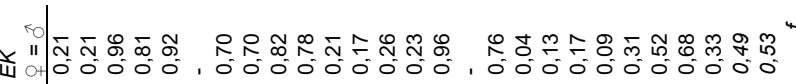

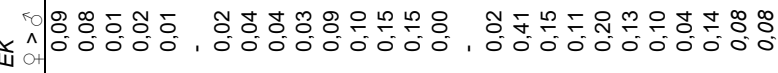

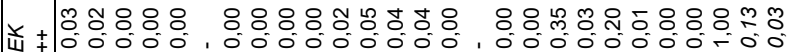

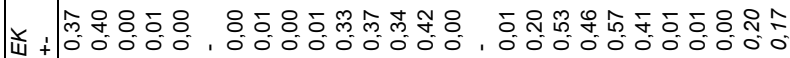

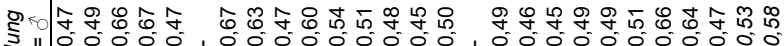
응

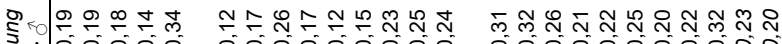

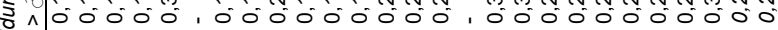
흐아

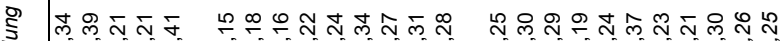

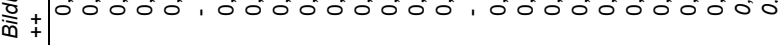

ริ to

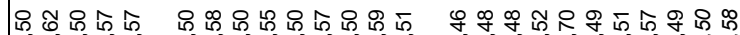

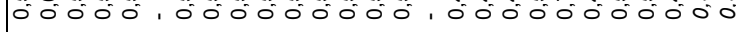

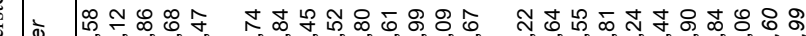

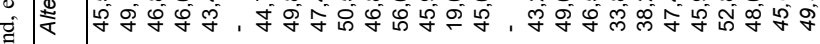

-

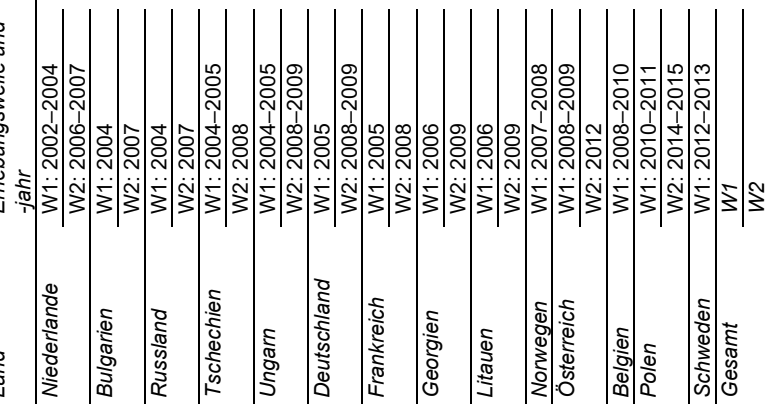




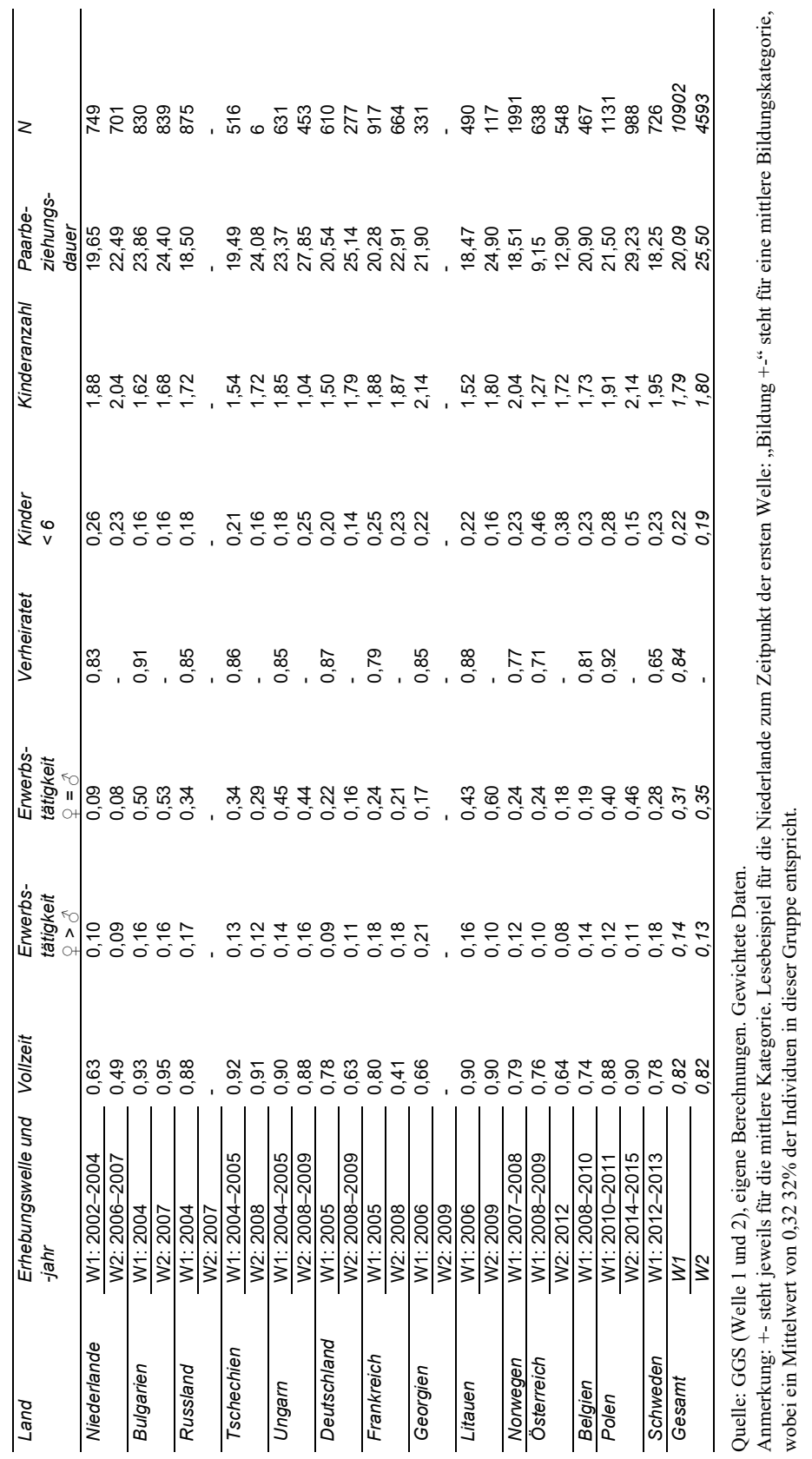


Tabelle 19: Kontextfaktoren für den NUTS1-Regionenvergleich

\begin{tabular}{|c|c|c|c|c|}
\hline $\begin{array}{l}\text { Kontext- } \\
\text { variablen }\end{array}$ & Min & Max & Mittelwert & $\begin{array}{l}\text { Standard- } \\
\text { abweichung }\end{array}$ \\
\hline $\begin{array}{l}\text { - BIP pro Kopf } \\
\text { (KKS) } \\
\text { (Eurostat) }\end{array}$ & $\begin{array}{l}6500,00 \\
\text { (Severna i } \\
\text { yugoiztochna } \\
\text { Bulgaria/Nord } \\
\text { und Ost- } \\
\text { bulgarien, BG3) }\end{array}$ & $\begin{array}{l}56100,00 \\
\text { (Brüssel, } \\
\text { BE1) }\end{array}$ & 23270,53 & 12280,90 \\
\hline $\begin{array}{l}\text { - Frauen- } \\
\text { erwerbsquote } \\
\text { (Eurostat) }\end{array}$ & $\begin{array}{l}42,4 \% \\
\text { (Alföld és Észak, } \\
\text { HU3) }\end{array}$ & $\begin{array}{l}70,6 \% \\
\text { (Norwegen) }\end{array}$ & $56,2 \%$ & 6,92 \\
\hline $\begin{array}{l}\text { - Politische } \\
\text { Links- Rechts- } \\
\text { Verortung } \\
\text { (ESS) }\end{array}$ & $\begin{array}{l}3,56 \\
\text { (Brandenburg, } \\
\text { DE4) }\end{array}$ & $\begin{array}{l}5,98 \\
\text { (Region } \\
\text { Wschodni, } \\
\text { PL3) }\end{array}$ & 5,2 & 0,40 \\
\hline $\begin{array}{l}\text { - Teilnahme- } \\
\text { raten der 4- } \\
\text { Jahre- } \\
\text { Altersgruppe } \\
\text { an Bildungs- } \\
\text { programmen } \\
\text { (Eurostat) }\end{array}$ & $\begin{array}{l}56,4 \% \\
\text { (Region } \\
\text { Pólnocny, PL6) }\end{array}$ & $\begin{array}{l}100 \% \\
\text { (NL1, NL2, } \\
\text { NL4 und } \\
\text { FR1, FR3, } \\
\text { FR7, FR8) }\end{array}$ & $88,1 \%$ & 11,55 \\
\hline $\begin{array}{c}\text { - Fertilitätsrate } \\
\text { (Eurostat) }\end{array}$ & $\begin{array}{l}1,18 \\
\text { (Közép- } \\
\text { Magyarorszá, } \\
\text { HU1 und Region } \\
\text { Poludniowo- } \\
\text { Zachodni, PL5) }\end{array}$ & $\begin{array}{l}2,1 \\
\text { (Brüssel, } \\
\text { BE1) }\end{array}$ & 1,5 & 0,27 \\
\hline $\begin{array}{l}\text { - Gender } \\
\text { Equality (ESS) }\end{array}$ & $\begin{array}{l}2,9 \\
\text { (Alföld és Észak, } \\
\text { HU3) }\end{array}$ & $\begin{array}{l}4,45 \\
\text { (lle-de- } \\
\text { France, } \\
\text { FR1) }\end{array}$ & 3,6 & 0,46 \\
\hline $\begin{array}{l}\text { - Frauen mit } \\
\text { tertiärem } \\
\text { Bildungsab- } \\
\text { schluss in \% } \\
\text { (Eurostat) }\end{array}$ & $\begin{array}{l}10,8 \% \\
\text { (Tschechien, } \\
\text { CZO) }\end{array}$ & $\begin{array}{l}43,1 \% \\
\text { (Brüssel, } \\
\text { BE1) }\end{array}$ & $25,4 \%$ & 8,40 \\
\hline $\begin{array}{l}\text { - Religiosität vs. } \\
\text { Ausmaß der } \\
\text { Säkularisie- } \\
\text { rung (ESS) }\end{array}$ & $\begin{array}{l}3,53 \\
\text { (Region } \\
\text { Wschodni, PL3) }\end{array}$ & $\begin{array}{l}6,36 \\
\text { (Sachsen- } \\
\text { Anhalt, DEE) }\end{array}$ & 5,4 & 0,76 \\
\hline
\end{tabular}

Quellen: Eurostat, European Social Survey, eigene Darstellung. 


\section{Methodische Erläuterungen der Mehrebenenanalyse}

Sollen Datenstrukturen als hierarchisch berücksichtigt werden, außerdem Individual-, Kontexteffekte sowie deren Wechselwirkung modelliert werden, ist eine Mehrebenenanalyse als geeignetes methodisches Verfahren zu erachten. Mehrebenenanalysen beschäftigen sich, allgemein formuliert, mit $\mathrm{Zu}-$ sammenhängen zwischen Individual- und Kollektivmerkmalen sowie der Überprüfung von Kontexthypothesen (vgl. Diekmann 2016: 137). Eine hierarchische Betrachtung impliziert, dass die Messwerte der Erhebungswellen Personen zugeordnet werden, weiterführend diese Personen bestimmten Clustern (Regime/Ländern/Regionen) zugeordnet werden können. Durch die Anwendung einer Mehrebenenanalyse (auch als hierarchisch lineare Modelle [HLM] nach Bryk/Raudenbush (1992) und Mehrebenenregression [FSE] bezeichnet) wird es möglich, Effekte von einer höheren (Makroebene) auf eine niedrigere Ebene (Mikroebene) zu bewerten. Die Mehrebenenanalyse ist eine ,[...] Erweiterung der multiplen Regression um Effekte [Zufallseffekte/Random-Effects], die zwischen den Ebenen variieren können" (Hosaya et al. 2014: 191).

\subsection{Grundlagen der Mehrebenenanalyse}

Grundsätzlich sind zwei allgemeine Argumente für die Anwendung von Mehrebenenanalysen zu berücksichtigen (vgl. Jäckle/Schärdel 2017: 153f.): Erstens ist dieses Verfahren notwendig, sofern es die theoretische Argumentation verlangt und Hypothesen lediglich über eine Mehrebenenanalyse überprüft werden können, weil Effekte auf Mikro- und Makroebene erwartet werden. Zweitens sollte eine Mehrebenenanalyse durchgeführt werden, sofern die statistische Analyse des Intraklassenkorrelationskoeffizienten ${ }^{148}$ (der ICC misst den Anteil der Varianzaufklärung auf der höheren gruppierten Ebene an der Gesamtvarianz; je höher der ICC, desto größer der Anteil der Gesamtvarianz, der durch die Unterschiede zwischen den Makroeinheiten hervorgebracht wird) die statistische Notwendigkeit der Mehrebenenanalyse repräsentiert (der ICC wird im Rahmen des Intercept-Only-Modells berechnet, das im Zuge der allgemeinen Vorgehensweise von Mehrebenenanalysen vertieft wird). Mehrebenenanalysen bieten folglich die Möglichkeit, Mikro-

148 „The degree of resemblance between micro-units belonging to the same macro-unit can be expressed by the intra correlation coefficient. The use of the term 'class' is conventional here and refers to the macro-units in the classification system under consideration" (Snijders/Bosker 2012: 17). 
und Makrozusammenhänge zugleich zu untersuchen. Ein weiterer relevanter Vorteil ist, dass die Mehrebenenanalyse gegen den ökologischen Fehlschluss widerstandsfähig ist,

„[...] da sie ihre Daten nicht auf der höchsten sondern auf der niedrigsten Aggregationsebene erhebt und die Variabilität der kontextabhängigen Effekte durch die Merkmale selbst erklärt. Anstatt vom Aggregat auf das Individuum zu schließen, stellt der Mehrebenenansatz hierdurch die analytische Perspektive wieder vom Kopf auf die Füße. Durch diese Veränderung des Blickwinkels vermeidet sie ebenfalls den individualistischen Fehlschluss, indem sie die Kontextabhängigkeit der Effekte ausdrücklich im zu schätzenden Modell berücksichtigt" (Langer 2009: 22).

\subsubsection{Allgemeine Kausalzusammenhänge in Mehrebenenanalysen}

Welche Kausalzusammenhänge im Allgemeinen im Rahmen von Mehrebenenmodellen analysiert werden können, soll anhand der Abbildungen 7 und 8 skizziert werden (Großbuchstaben visualisieren Kontextmerkmale, Kleinbuchstaben Individualmerkmale). Die Grundidee der Mehrebenenanalyse basiert auf einem Makro-Mikro-Zusammenhang unter Kontrolle einer weiteren Individualvariable:

\section{Abbildung 7:}

Die Grundidee der Mehrebenenanalyse - ein Makro-Mikro-Zusammenhang

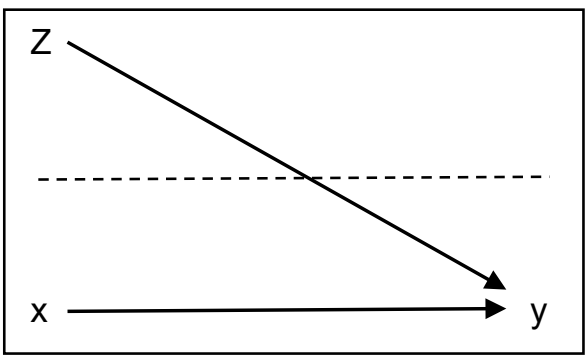

Quelle: Snijders/Bosker 2012: 10.

Ein ausschließlicher Mikroebenenzusammenhang zwischen zwei Individualmerkmalen ist in Abbildung 8.1 dargestellt. Die gestrichelte Linie verdeutlicht in diesem Fall, dass zwar grundsätzlich auch die Makroebene von Bedeutung ist, weil sie für die Stichprobenauswahl benötigt wird, jedoch im Rahmen der Hypothesen keine expliziten Kontexteffekte berücksichtigt werden. Abbildung 8.2 bezieht sich indessen auf einen reinen Makro-EbenenZusammenhang, wie beispielsweise ein Zusammenhang zwischen der ge- 
samtgesellschaftlichen Erwerbsquote von Frauen und der frühkindlichen Versorgungsquote (die gestrichelte Linie separiert Makro- und Mikroebene, wobei die Mikroebene in dieser Darstellung nicht weiter betrachtet wird). Denkbar ist außerdem, dass ein Individualmerkmal Einfluss auf einen Kontexteffekt nehmen kann (Abbildung 8.3) - so könnte sich die innerhäusliche Arbeitsteilung auf die Erwerbsquote von Frauen auswirken - doch kann ein Effekt eines individuellen Merkmals auf eine abhängige Variable, die auf einer höheren Analyseebene verortet wird, nicht mittels Mehrebenenanalysen überprüft werden. Hingegen sind die weiteren Abbildungen 8.4, 8.5 und 8.6 Paradebeispiele für Anwendungsfelder im Bereich der Mehrebenenanalysen, da sie sich auf den Makro-zu-Mikro-Zusammenhang beziehen. Erstens könnte eine Kontextvariable Einfluss auf eine Individualvariable nehmen (MakroMikro-Zusammenhang, 8.4). Zweitens könnte dieser Zusammenhang zusätzlich unter Kontrolle einer weiteren Individualvariable getestet werden (Makro-Mikro-Zusammenhang unter Kontrolle einer weiteren Mikrovariable), was der bereits thematisierten Grundidee der Mehrebenenanalyse entspricht und drittens könnte es sein, dass der Zusammenhang von $\mathrm{x}$ und $\mathrm{y}$ abhängig von $\mathrm{Z}$ ist. Letzterer Fall wird als Makro-Mikro-Interaktion respektive als CrossLevel-Interaktion bezeichnet und ist insofern ähnlich wie ein Interaktionseffekt zu deuten, der jedoch zwischen den Ebenen verortet wird. Ferner kann analysiert werden, inwieweit der Effekt einer Individualvariablen in Abhängigkeit von der Ausprägung einer Kontextvariablen variiert (vgl. Lois 2015: 61). Ein Beispiel hierfür ist, dass der individuelle Einfluss der Kinderanzahl (x) auf die innerhäusliche Arbeitsteilung (y) von der Erwerbsquote der Frauen in der Gesellschaft $(Z)$ konditioniert werden könnte.

Abbildung 8:

Zusammenhang zwischen Variablen der Mikro- und Makroebene

8.1 Mikro-Mikro

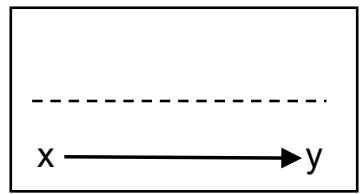

8.4 Makro-Mikro

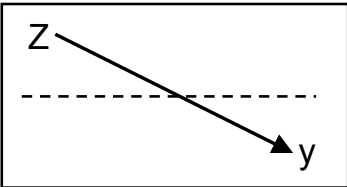

8.2 Makro-Makro

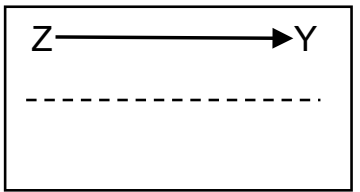

8.5Makro-Mikro+Mikro 8.6Cross-Level-Interakion

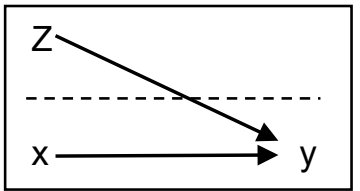

8.3 Mikro-Makro
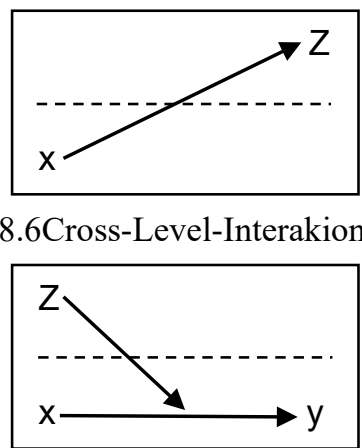

Quelle: vgl. Snijders/Bosker 2012: 10ff.; Jäckle/Schärdel 2017: 152. 
Da die Cross-Level-Interaktion auch umgekehrt mit der Mikro-Variable als konditionierendem Effekt erwägt werden könnte, erweitern Jäckle/Schärdel (2017) das Schaubild 8.6 nach Snijders/Bosker (2012) durch die in Abbildung 8.7 ergänzten gepunkteten Pfeile.

\subsection{Umgekehrte Cross-Level-Interaktion}

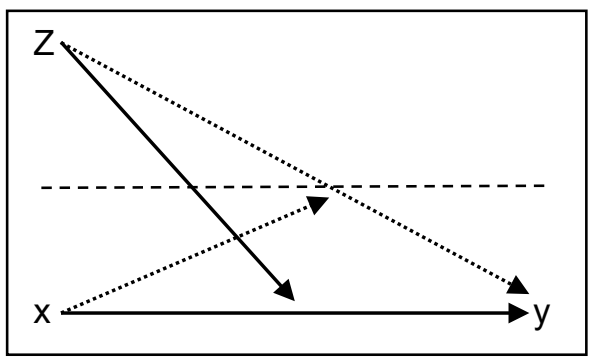

Quelle: Jäckle/Schärdel 2017: 152.

Für das genannte Beispiel ergäbe sich die Interpretation, dass der Effekt, den die Erwerbsquote der Frauen in der Gesellschaft $(Z)$ auf die innerhäusliche Arbeitsteilung (y) nimmt, durch die Kinderanzahl (x) konditioniert wird.

Die Darstellungen bezogen sich bisher auf die Überprüfung einzelner Effekte, doch werden i. d. R. diverse Kombinationen von Interesse sein, die durch Mehrebenenanalysen realisiert werden können. Die berühmte Colemansche Badewanne nach James Samuel Coleman (1990) stellt beispielsweise eine solche Kausalkette dar, die die Beziehung zwischen Makro- (W und $\mathrm{Z}$ ) und Mikrovariablen ( $\mathrm{x}$ und $\mathrm{y}$ ) berücksichtigt (vgl. Snijders/Bosker 2012: 12). Die Kausalkette der Colemanschen Badewanne ist nachfolgend lediglich zu Demonstrationszwecken vereinfacht dargestellt:

8.8 Kausalkette Makro-Mikro-Mikro-Makro (Colemansche Badewanne)

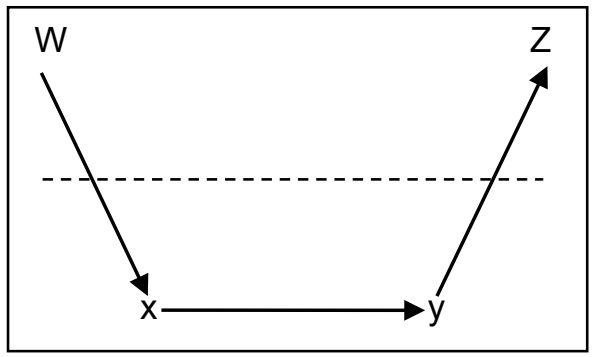

Quelle: Snijders/Bosker 2012: 12. 
Insgesamt ist festzuhalten, dass

„The methodological advances in multilevel modeling are also leading to theoretical advances in contextual research: suitable definitions of context and 'levels', meaningful ways of aggregating variables to higher levels, conceptualizing and analyzing the interplay between characteristics of lower- and higher-level units" (Snijders/Bosker 2012: 12).

\subsubsection{Fallzahl und Schätzverfahren: \\ Die Angemessenheit der Full-Information- (FIML) oder Restricted-Maximum-Likelihood-Methode (REML)}

Da die Fallzahl im Rahmen von Mehrebenenmodellen sowie die notwendigen Schätzverfahren bereits im Kapitel 11.1. „Zur methodisch bedingten NUTS1-Regionenanalyse mit theoretischem Mehrwert" thematisiert wurden, soll nur noch einmal daran erinnert werden, dass Hox et al. idealiter im Falle von fixen Effekten 30 Kontexteinheiten sowie eine durchschnittliche Fallzahl von 30 Individualeinheiten empfehlen, während für Cross-LevelInteraktionen 50 Kontexteinheiten sowie eine durchschnittliche Fallzahl von 20 Individualeinheiten angedacht werden und im Falle von Zufallseffekten 100 Kontexteinheiten sowie eine durchschnittliche Fallzahl von 10 Individualeinheiten ratsam wären (vgl. Hox et al. 2018: 215f.). Von dieser Daumenregel abweichende Analysen sind in der Praxis keine Seltenheit, weil insbesondere Länder häufig gewählte Analyseeinheiten der Kontextebene darstellen, deren Fallzahl meist eher gering ist. Zweifellos gilt: umso höher die Fallzahl, umso präziser die Schätzungen.

Wie bereits erläutert, sind bei kleineren Gruppengrößen gemäß Hox et al. dennoch Mehrebenenanalysen möglich, vorausgesetzt, dass die Varianzschätzung mittels ,Restricted-Maximum-Likelihood“"149 durchgeführt wird.

149 Der Unterschied zwischen der Full-Information- (FIML) und der Restricted-MaximumLikelihood-Methode (REML) sei zur Erinnerung nochmals kurz zusammengefasst: „Erstere enthält in ihrer Likelihoodfunktion sowohl die festen Regressionsparameter als auch die Varianzkomponenten der Fehlerterme. Bei letzterer beinhaltet die Likelihoodfunktion nur die Varianzkomponenten, aber nicht die Regressionsparameter. Während die FIML die Regressionsparameter als bekannte Größen bei der Schätzung der Varianzkomponenten behandelt, betrachtet sie die REML als Schätzer, die mit Unsicherheit behaftet sind. Theoretischer Weise sollte die REML bei wenigen vorliegenden Gruppen zu genaueren Schätzer [sic!] führen, wie Bryk \& Raudenbush (1992: 46; 2002: 53) festgestellt haben. Dennoch fallen die Unterschiede zwischen den beiden Schätzverfahren bei der praktischen Datenanalyse kaum ins Gewicht. Im Vergleich zur REML verfügt die FIML über zwei entscheidende Vorteile. Erstens ist die Berechnung sehr viel einfacher als bei der REML, da die Regressionsparameter ein Bestandteil der zu maximierenden Likelihoodfunktion sind. Zweitens kann der globale Likelihood-Ratio- $\chi^{2}$-Test benutzt werden, um die statistische Signifikanz nicht nur der Varianzkomponenten sondern auch der Regressionsparameter zu überprüfen. 
Aufgrund der eher geringen L2-Einheiten ist im vorliegenden Fall folglich die Restricted-Maximum-Likelihood-Methode ratsam. Diese lässt jedoch keinen Likelihood Ratio Test ${ }^{150} \mathrm{zu}$ :

FML-Schätzungen der Varianzkomponenten sind generell etwas zu klein, weshalb bei geringen Fallzahlen alternativ die RML verwendet wird (vgl. Hox et al. 2018: 28; Windzio 2018: 31). Obwohl die Schätzungen der RML insgesamt etwas besser sind, kann zur Evaluierung des Modellfits (Chiquadrat) kein Likelihood-Ratio Test durchgeführt werden, da ,[...] bei der RML Schätzung [...] die „Deviance“ nur für den random part des Modells berechnet werden [kann], nicht aber für das Gesamtmodell inklusive des fixed part" (Windzio 2018: 31). In der Praxis sind die Unterschiede zwischen FML und RML meist sehr gering, weshalb Windzio (2018) vorschlägt beide Modellvarianten zu schätzen und die geschätzten Varianzen höherer Ebenen miteinander zu vergleichen. Im Rahmen dieser Dissertation wurden folglich beide Varianten geschätzt, wobei für die Mehrebenenmodelle der innerhäuslichen Arbeitsteilung im NUTS1-Regionen-Vergleich der ersten Welle des GGS aufgrund sehr geringer Unterschiede lediglich die Ergebnisse der RML dargelegt werden.

\subsubsection{Zur Unterscheidung von Random-Intercept-und Random-Slope-Modellen}

Im Vergleich zur klassischen Methode der kleinsten Quadrate bzw. der OLSRegression (engl. „Ordinary Least Square Method“) verfügen Mehrebenenanalysen u. a. über den Vorteil, dass je Gruppe ein unterschiedlicher Individualeffekt angenommen werden kann. Insofern stellt die Mehrebenenanalyse eine Erweiterung der klassischen multiplen Regression dar, die zwischen den Ebenen variierende Effekte, sogenannte Zufallseffekte (engl. random effects), berücksichtigt. Es ist daher nicht verwunderlich, dass Robert Bickel (2007) den Untertitel „It's Just Regression!“ für sein Buch zur Einführung in die Mehrebenenanalyse „Multilevel Analysis for Applied Research“ gewählt hat. Ferner kann durch Mehrebenenanalysen im Spezifischen festgestellt werden, ob sich die Steigungskoeffizienten der Individualvariablen z. B. zwischen den Regimen unterscheiden ohne hierbei - wie es im Falle der klassischen OLS-Regression gemacht würde - ein separates Regressionsmodell für jeden Regimetypus schätzen zu müssen oder gar die implizite Annahme einer gepoolten Regression vertreten zu müssen, dass ein L1-Effekt für sämtliche

Bei der REML-Schätzung ist dies nur für die Varianzkomponenten zulässig“ (Langer 2009: 102f.).

150 Für Berechnung des Likelihood-Ratio- $\chi^{2}$-Tests ist die Bestimmung der Anzahl der Freiheitsgerade notwendig: im Fall der Mehrebenenanalyse entspricht die Anzahl der Freiheitsgerade der Anzahl der Parameter im jeweiligen Modell. 
Regime gleich stark ist. Es können zwei Modelltypen von Mehrebenenanalysen differenziert werden: das Random-Intercept und das Random-SlopeModell. Im Rahmen des Random-Intercept-Modells ${ }^{151}$ wird die ausschließliche Annahme eines variierenden Intercepts (Achsenabschnitts) getroffen: Die Regressionslinien verlaufen für die Kontexteinheiten (Länder o. ä.) auf unterschiedlichem Niveau, während die Steigung für alle Gruppen als gleich angenommen wird. Folglich unterscheiden sich hierbei die Individualeffekte zwar in ihrem Niveau, jedoch nicht in ihrer Steigung (s. Abbildung 9.1). Hingegen wird durch Random-Slope-Modelle ${ }^{152}$ (s. Abbildung 9.2) in Erwägung gezogen, dass sich die Effekte der Individualvariablen zwischen den Gruppen unterscheiden könnten, respektive formal betrachtet, dass die Individualvariablen je nach Kontextgruppe über unterschiedliche Steigungen verfügen können. Im Falle des Random-Slope-Modells variieren folglich zusätzlich die Regressionskoeffizienten (vgl. Hadler 2004: 53). Die gepunktete Linie stellt beispielsweise eine Gruppe 1 dar, die mit der mittleren Steigung in Form der fett gedruckten Linie verglichen werden kann, wodurch auffällt, dass Gruppe 1 eine größere Steigung aufweist als das Mittel $\mathrm{u}_{11}$ (vgl. Jäckle/Schärdel 2017: 159).

Während im Falle einer einfachen Regression eine Regressionsgerade mit konstantem Intercept geschätzt wird, wodurch z. B. angenommen wird, dass der Zusammenhang zwischen der Erwerbstätigkeit und den Haushaltstätigkeiten in allen Ländern derselbe ist, wird im Random-Intercept-Modell angenommen, dass zwar der Einfluss der Erwerbstätigkeit in allen Ländern derselbe ist, aber auf unterschiedlichem Niveau anzusetzen ist (graphisch wird dies durch die parallelen Geraden verdeutlicht). Möglich wäre auch, dass sich der Einfluss der Erwerbstätigkeit auf die Hausarbeiten zwischen den Ländern unterscheidet, was durch Abbildung 9.2 visualisiert wird. Im Allgemeinen setzt die einfache Regression voraus, dass die L1-Einheiten (z. B. SchülerInnen) unabhängig voneinander sind, wobei diese Voraussetzung im Falle von hierarchischen Daten verletzt wird. Beispielsweise können im Datensatz SchülerInnen enthalten sein, die die gleiche Schule besuchen, wodurch sie sich überzufällig ähnlich sind, was gegen die Unabhängigkeitsannahme aller Beobachtungen verstößt (vgl. Hox et al. 2018: 12). Ohne eine Berücksichti-

151 Formal: $y_{i j}=b_{0}+b_{1} x_{i j}+u_{j}+e_{i j}$ (wobei $\mathrm{i}=$ Individuum in $\mathrm{j}=$ Gruppe) Beim RandomIntercept-Modell ,[...] wird ausschließlich der Achsenabschnitt (Intercept) als variabel angenommen. Dieser variiert für jede Gruppe um $u_{j} u m$ den mittleren Intercept aller Gruppen $\mathrm{b}_{0}$ herum [...]. Die Steigung $\mathrm{b}_{1}$ hingegen wird für alle Gruppen als gleich angenommen“ (Jäckle/Schärdel 2017: 159).

152 Formal: $y_{i j}=b_{0}+b_{1} x_{i j}+u_{0 j}+u_{l j} x_{i j}+e_{i j}$

„Der Effekt, den eine Level-1-Variable hat, wird relativ zum mittleren Effekt über alle Gruppen betrachtet. Im Unterschied zum vorherigen Random-Intercept-Modell nimmt man allerdings an, dass sich die Gruppen in Bezug auf einen Level-1-Faktor nicht nur in ihren Achsenabschnitten $\mathrm{u}_{0 \mathrm{j}}$, sondern auch in ihren Steigungen $\mathrm{u}_{1 \mathrm{j}}$ unterscheiden können“ (Jäckle/Schärdel 2017: 159). 
gung der hierarchischen Datenstruktur liefern die Signifikanztests verzerrte Ergebnisse (s. hierzu auch die Erläuterungen der Ergebnisse der klassischen OLS-Regression zur Erklärung der innerhäuslichen Arbeitsteilung durch Länderunterschiede) (vgl. Lois 2015: 22).

\section{Abbildung 9:}

Differenzierung des Random-Intercept- und Random-Slope-Modells

9.1 Random-Intercept-Modell

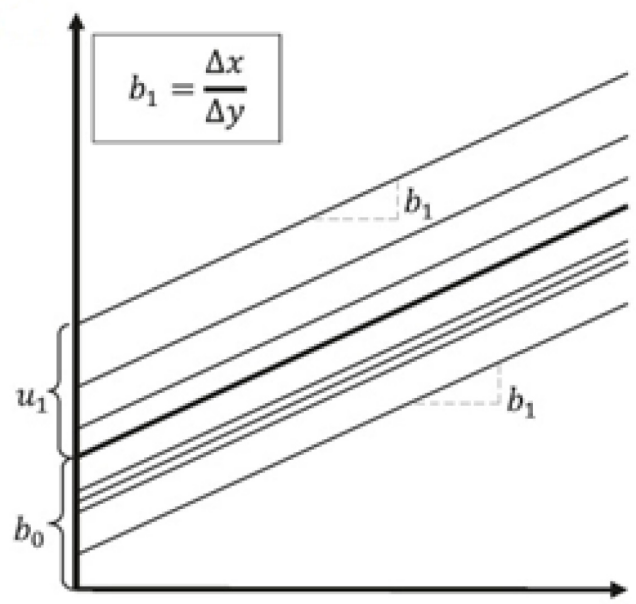

9.2 Random Slope-Modell

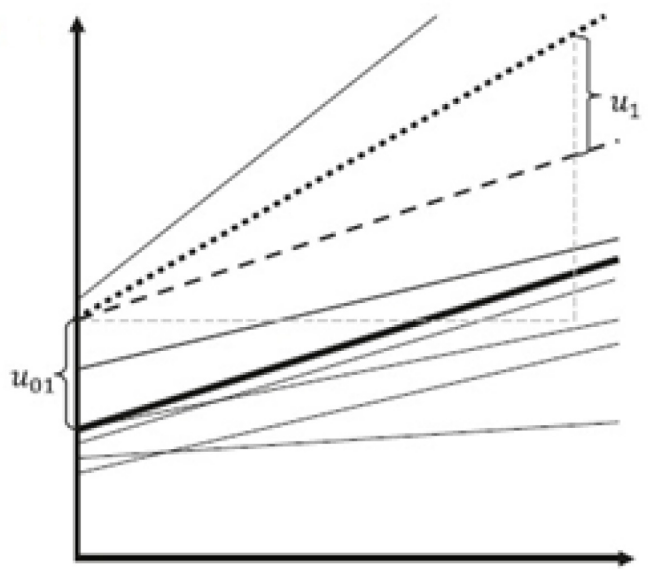

Quelle: Jäckle/Schärdel 2017: 160. 


\subsubsection{Grundlagen der Zentrierung}

Grundsätzlich wird für Mehrebenenanalysen empfohlen, die Variablen zu zentrieren, um eine leichtere Interpretierbarkeit der Ergebnisse zu ermöglichen (vgl. Krause/Urban 2013: 6; Jäckle/Schärdel 2017: 162). Darüber hinaus sind zwei weitere Vorteile der Zentrierung, dass die Problematik der Multikollinearität der Schätzergebnisse sowie unerwünschte Korrelationen zwischen Individual- und Kontextvariablen weitgehend vermieden werden können (vgl. Krause/Urban 2013: 6). Eine Zentrierung metrischer ${ }^{153}$ Variablen am Gesamtmittelwert (engl. ,grand mean centering“") bietet insbesondere die Möglichkeit einer Interpretierbarkeit des Achsenabschnitts: Während im Random-Intercept-Modell (wie auch im Falle der klassischen OLSRegression) ohne Zentrierung der Variablen der Intercept dem Wert entspricht, den die abhängige Variable annimmt, wenn alle anderen unabhängigen Variablen Null sind, ist der Achsenabschnitt im Random-InterceptModell mit grand-mean-zentrierten Variablen so zu interpretieren, dass der Achsenabschnitt dem Wert der abhängigen Variablen entspricht, wenn die grand-mean-zentrierte unabhängige Variable einen durchschnittlichen Fall annimmt (vgl. Jäckle/Schärdel 2017: 162).

„Generell wird durch die Zentrierung eine bessere Interpretierbarkeit der Ergebnisse von MLA-Schätzungen erreicht, denn die Schätzwerte für partielle Effekte sind immer als Veränderungen der abhängigen Variablen unter der Voraussetzung, dass alle anderen Prädiktoren das Ausmaß ihrer Mittelwerte aufweisen, zu verstehen (und nicht, wie bei Variablen mit Rohwerten, einen Wert von 0,00 annehmen)“(Krause/Urban 2013: 7).

Eine weitere Zentrierungsart ist die gruppeninterne Zentrierung (engl. ,group mean centering"), die von Raudenbush (1989) empfohlen wurde. Heutzutage wird diese Zentrierungsform jedoch eher selten verwendet, weil das Basismodell derart verändert wird, dass es nicht äquivalent zum zentrierten Modell ist und gemäß Plewis (1989) die Gefahr besteht, unechte Varianzen auf der Kontextebene zu erzeugen (vgl. Jäckle/Schärdel 2017: 162). Beim groupmean-Zentrieren wird von jedem Fall der Mittelwert der Gruppe subtrahiert, sodass die neue, transformierte Variable in jeder Gruppe einen Mittelwert von Null besitzt. Folglich resultiert ein Informationsverlust über das mittlere Niveau der Gruppen (durch eine zusätzliche Kontextvariable, die diesen Gruppenmittelwert beinhaltet, könnte diese Information allerdings wieder zurückgeholt werden). Da im Falle der grand-mean-Zentrierung ein äquivalentes Modell zum Basismodell mit den Originaldaten erreicht wird, während durch die group-mean-Zentrierung ein Modell mit alternativem Aussagegehalt entsteht, empfehlen Hox et al. (2018) generell die grand-mean-

153 Für Dummyvariablen oder kategoriale Mikroregressoren ist eine Zentrierung i. d. R. nicht notwendig, der Wert Null liegt hier bereits in einem sinnvollen Interpretationsbereich. 
Zentrierung. Lediglich bei einer spezifischen theoretischen Begründung, wie beispielsweise beim „Froschteicheffekt" (ein spezifischer Frosch kann in einem Teich mit vielen, kleinen Fröschen groß aussehen, während er in einem Teich mit großen Fröschen klein aussieht), sei die group-meanZentrierung sinnvoll.

„Group mean centering completely changes the meaning of the entire regression model. If we use grand mean centering, we get different regression slopes for variables that are involved in an interaction, and different variance components, but we have an equivalent model, with identical deviance and residual errors. A formal way to describe this situation is to state that we have a different parameterization for our model; the model is essentially the same, but transformed in a way that makes it easier to interpret. Using straightforward algebra, we can transform the grand mean centered estimates back to the values we would have found by analyzing the raw scores. Group mean centering, on the contrary, is not a simple reparameterization of our model, but a completely different model. We will find a different deviance, and transforming the estimated parameters back to the corresponding raw scores estimates is not possible. The reason is that we have subtracted not one single value, but a collection of different values from the raw scores. [...] Group mean centering of an explanatory variable discards all information concerning differences between groups. It would seem reasonable to restore this information by adding the aggregated group mean again as an additional group-level explanatory variable. But this adds extra information about the group structure, which is not present in the raw scores, and therefore we obtain a model that fits better than the raw score model. [...] Group mean centering and adding the group mean as predictor variable is most useful when the research question requires a clear separation of individual and group effects. This is the case with frog pond theories, with research questions about contextual effects" (Hox et al. 2018: 50f., Hervorhebungen im Original; die Verf.).

\subsubsection{Gütemaße der Mehrebenenanalyse}

Um die Modellgüte zu überprüfen, ist im Allgemeinen (Ausnahme im Falle der RML) die Devianz (2* Log-Likelihood-Wert ${ }^{154}$ ) - auch bezeichnet als Devianz-Test oder Likelihood Ratio Test - ein gängiges Testverfahren (vgl. Snijders/Bosker 2012: 97). Es können hierbei vornehmlich Aussagen über die relative Güte von zwei oder mehreren Modellen getroffen werden, da der jeweilige absolute Wert von der Fallzahl abhängig und daher nicht interpretierbar ist (ein Devianzwert von Null wäre als eine perfekte Modellgüte zu

154 „When parameters of a statistical model are estimated by the maximum likelihood method, the estimation also provides the likelihood, which can be transformed into the deviance defined as minus twice the natural logarithm of the likelihood. This deviance can be regarded as a measure of lack of fit between model and data, but (in most statistical models) one cannot interpret the values of the deviance directly, but only differences in deviance values for several models fitted to the same data set" (Snijders/Bosker 2012: 97). 
interpretieren). Im Falle eines Vergleichs zwischen zwei Modellen und einer signifikanten Differenz der beiden Devianzwerte (der Test basiert auf einer Chi-Quadrat-Verteilung), ist das Modell mit dem niedrigeren absoluten Devianzwert signifikant besser als das Vergleichsmodell (diese Interpretation gilt auch für AIC und BIC-Tests). Da weder Devianz, AIC noch BIC ein absolutes Maß für die Erklärungskraft eines Mehrebenenmodells darstellen, empfehlen Snijders/Bosker (2012) zwei getrennte R-Quadrat-Werte zu bestimmen: ein R-Quadrat für die Makroebene und ein R-Quadrat für die Mikroebene. Das R-Quadrat der Mikroeben ist definiert als , ,...] the principle of proportional reduction of prediction error" (Snijders/Bosker 2012: 111, Hervorhebungen im Original; die Verf.). Indessen bezieht sich das R-Quadrat der Makroebene darauf, um wie viel Prozent sich die Varianz zwischen den Kontextgruppen reduzieren lassen kann (vgl. Jäckle/Schärdel 2017: 163). Sowohl für die Berechnung des R-Quadrats auf der Mikroebene als auch für die Berechnung des R-Quadrats auf der Makroebene werden die Varianzen der Residuen zwischen und innerhalb der Gruppen stets jeweils mit dem Nullmodell verglichen.

Um die Güte des Gesamtmodells zu beurteilen, stehen Maßzahlen wie das Maddala- $\mathrm{R}^{2}$ und das McFadden-Pseudo- $\mathrm{R}^{2}$ zur Verfügung. Diese beiden Kennzahlen unterscheiden sich vom $\mathrm{R}^{2}$ nach Raudenbush und Bryk (2002) sowie vom $\mathrm{R}^{2}$ nach Snijders und Boskers (1999) dadurch, dass sich letztere jeweils für die Individual- und die Kontextebene berechnen lassen (vgl. Braun et al. 2010: 43). In Langer (2009: 148ff.) ist eine umfangreiche Diskussion über die Vor- und Nachteile dieser Gütemaße enthalten. Langer (2009) zufolge sprechen im Gegensatz zum $\mathrm{R}^{2}$ nach Snijders und Boskers (1999) zwei Argumente für die Verwendung der Determinationskoeffizienten nach Bryk und Raudenbush: Erstens sind sie bei Random-Slope-Modellen viel einfacher $\mathrm{zu}$ berechnen wie die Determinationskoeffizienten nach Snijders und Bosker.

„Im Gegensatz zu letzterem führt es auf der zweiten Ebene nicht nur eine Berechnung des mittleren Prognosefehlers für die Gruppenmittelwerte durch, sondern Bryk \& Raudenbush sehen zweitens eine separate Varianzzerlegung für jeden einzelnen kontextabhängigen Effekt (Random-Effect) vor. Hierdurch eignet es sich deutlich besser für eine differenzierte Bestimmung der Effekte der Kontextmerkmale als dasjenige von Snijders \& Bosker" (Langer 2009: 152).

Im Rahmen dieser Dissertation wird folglich auf eine Beurteilung der Modellanpassungen für die jeweiligen Ebenen im Sinne von Bryk/Raudenbush zurückgegriffen. Die Formeln für die Berechnung der R-Quadrate für die Mikro- und Makroebene lauten (vgl. Langer 2009: 150f.; Lois 2015: 71ff.): 
Ebene 2:

Bryk-Raudenbush-R ${ }_{\text {Level2 }}^{2}=\frac{\hat{o}_{u 0 j}^{2}\left(M_{\text {Baseline }}\right)-\hat{o}_{u 0 j}^{2}\left(M_{\text {Comparison }}\right)}{\hat{o}_{u 0 j}^{2}\left(M_{\text {Baseline }}\right)}$

Ebene 1:

Bryk-Raudenbush- $\mathrm{R}_{\text {Level1 }}^{2}=\frac{\hat{o}_{e i j}^{2}\left(M_{\text {Baseline }}\right)-\hat{o}_{\text {eij }}^{2}\left(M_{\text {Comparison }}\right)}{\hat{o}_{e i j}^{2}\left(M_{\text {Baseline }}\right)}$

Für die Beurteilung des Gesamtmodells ist folgende Formel festzuhalten:

$\mathrm{R}^{2}=\frac{\left(\hat{o}_{e i j}^{2}+\hat{o}_{u 0 j}^{2}\right) M_{\text {Baseline }}-\left(\hat{o}_{e i j}^{2}+\hat{o}_{u 0 j}^{2}\right) M_{\text {Comparison }}}{\left(\hat{o}_{e i j}^{2}+\hat{o}_{u 0 j}^{2}\right) M_{\text {Baseline }}}$

Legende:

$\hat{o}_{u 0 j}^{2}$ :

$\hat{o}_{e i j}^{2}$ :

Geschätzte Varianz der Residuen des kontextspezifischen Regressionsparameters $b_{\mathrm{ij}}$ (Varianz der Kontextebene)

Maseline: Geschätzte Varianz der Residuen der Binnenregression (Varianz der Individualebene)

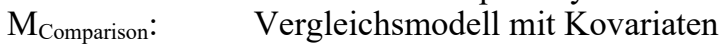

Nachdem in den vorherigen einführenden Methodenkapiteln einige wesentliche Grundlagen der Mehrebenenanalyse erläutert wurden, wird nachfolgend die allgemeine Vorgehensweise der jeweiligen Analyseschritte skizziert, bevor im Spezifischen auf die Analyse von Paneldaten als Mehrebenenmodell eingegangen wird.

\subsubsection{Allgemeine Vorgehensweise für Mehrebenenanalysen}

Hox fasst für die Durchführung von Mehrebenenanalysen fünf Analyseschritte zusammen:

1. „Analyze a model with no explanatory variables“(Hox 2002: 51): Die Schätzung des Null bzw. Intercept-Only-Modells sowie die Berechnung des Intraklassenkorrelationskoeffizienten (ICC).

2. „Analyze a model with all lower level explanatory variables fixed“ (Hox 2002: 51): Das Einbeziehen der L1-Prädiktoren als "fixed effects".

3. „Add the higher explanatory variables“ (Hox 2002: 51): Das Einbeziehen von L2-Prädiktoren als "fixed effects".

„The models in step 2 and 3 are often denoted as variance component models, because they decompose the intercept variance into different variance components for each hierarchical level. In a variance component model, the regression intercept is assumed to vary across the groups, but the regression slopes 
are assumed as fixed. If there are no higher level explanatory variables, this model is equivalent to a random effects analysis of covariance (ANCOVA)" (Hox 2002: 52, Hervorhebungen im Original; die Verf).

4. „Assess whether any of the slopes of any of the explanatory variables has a significant variance component between the groups" (Hox 2002: 52): Die Integration von Level 1-Variablen als "random-effects".

5. „Add cross-level interactions between explanatory group level variables and those individual level explanatory variables that had significant slope variation in step 4" (Hox 2002: 53): Im letzten Analyseschritt werden Cross-Level-Interaktionen in das Modell einbezogen.

Nachfolgend werden einige Details dieser einzelnen Analyseschritte basierend auf zwei Analyseebenen für Querschnittsdaten skizziert.

\section{Ad Schritt 1: Random-Intercept-Only-Modell}

Im ersten Schritt soll lediglich überprüft werden, inwiefern eine Mehrebenenanalyse statistisch notwendig ist, weshalb ein Modell, das nur die Konstante und keine weiteren Variablen enthält - das sogenannte RandomIntercept-Only-Modell (oder auch nur als Intercept-Only-Modell bezeichnet) - berechnet wird, um die Varianzaufteilung zwischen den Ebenen zu ermitteln. Aufgrund dessen, dass nur die Regressionskonstante, die über die Kontexte hinweg variieren darf, enthalten ist, wird dieses Modell auch als das sparsamste Modell beschrieben (vgl. Langer 2009: 107). Formal lässt sich das Random-Intercept-Only-Modell folgendermaßen ausdrücken:

Formale Zwei- und Eingleichungsform ${ }^{155}$ des Intercept-Only-Modells (vgl. Langer 2009: 107; Hox et al. 2018: 12f.):

$\begin{array}{ll}\text { Ebene 1: } & \mathrm{Y}_{\mathrm{ij}}=\beta_{0 \mathrm{j}}+\mathrm{e}_{\mathrm{ij}} \\ \text { Ebene 2: } & \beta_{0 \mathrm{j}}=\gamma_{00}+\mathrm{u}_{0 \mathrm{j}}\end{array}$

Eingleichungsform von (1.2) in (1.1): $\mathrm{Y}_{\mathrm{ij}}=\gamma_{00}+\mathrm{u}_{0 \mathrm{j}}+\mathrm{e}_{\mathrm{ij}}$

wobei die folgende Notation gilt:

i: $\quad 1,2 \ldots$ n Ebene-1-Einheiten

j: $\quad 1,2 \ldots n$ Ebene-2-Einheiten

$Y_{\mathrm{ij}}$ : Messwert der abhängigen Variable $\mathrm{Y}$ von $\mathrm{i}$ im Kontext $\mathrm{j}$

$\gamma_{00}$ : Geschätzter Grand Mean von $\mathrm{Y}$

$\mathrm{u}_{0 \mathrm{j}}$ : Residuum der 2. Ebene, d. h. Abweichung des geschätzten Gruppenmittelwerts $\bar{y}_{j}$ vom geschätzten Grand Mean $\bar{y}$..

$ß_{0 j}$ : Geschätzter Mittelwert von Y der Gruppe $\mathrm{j}$

$\mathrm{e}_{\mathrm{ij}}$ : Residuum der Ebene 1, d. h. Abweichung der Befragten i vom geschätzten Mittelwert seiner Gruppe j

155 Die Formeln können entweder differenziert nach Ebenen oder in integrierter Form dargestellt werden, wobei in dieser Dissertation beide Varianten dargelegt werden. 
Die Gesamtvarianz der abhängigen Variablen wird im Random-InterceptOnly-Modell in die Anteile der verschiedenen Ebenen zerlegt: in die Varianz innerhalb der Gruppen (Abweichung eines jeweiligen individuellen Wertes vom Gruppenmittelwert) und die Varianz zwischen der Gruppen (Abweichung der jeweiligen geschätzten Gruppenmittelwerte vom Gesamtmittelwert) ${ }^{156}$. Für die Bestimmung des Varianzanteils der höheren Ebene wird der Intraklassenkorrelationskoeffizient berechnet.

„The intercept-only model $[\ldots]$ does not explain any variance in Y. It only decomposes the variance into two independent components: $\sigma_{e}^{2}$, which is the variance of the lowest-level errors $\mathrm{e}_{\mathrm{ij}}$, and $\sigma_{u_{0}}^{2}$, which is the variance of the highestlevel errors $\mathrm{u}_{0 \mathrm{j}}$. These two variance sum up to the total variance, hence they are often referred to as variance components. Using this model, we can define the intraclass correlation $\mathrm{p}$ by the equation

$p=\frac{\sigma_{u_{0}}^{2}}{\sigma_{u_{0}}^{2}+\sigma_{e}^{2}}$

The intraclass correlation $\mathrm{p}$ indicates the proportion of the total variance explained by the grouping structure in the population. [The] Equation [...] simply states that the intraclass correlation is proportion of group-level variance compared to the total variance. The intraclass correlation $\mathrm{p}$ can also be interpreted as the expected correlation between two randomly drawn units that are in the same group. In the intercept-only model we defined variance of the lowest-level errors and variance of the highest-level errors. Both terms can be interpreted as unexplained variance on both levels since there are no predictors in the model specified yet. After adding predictors, just like in ordinary regression analyses, the $\mathrm{R}^{2}$, which is interpreted as the proportion of variance modelled by the explanatory variables, can be calculated. In the case of multilevel analyses, however, there is variance to be explained at every level (and also for random slope factors). The interpretation of these separate $\mathrm{R}^{2}$ values are dependent on the ICC-values. For example, if the $\mathrm{R}^{2}$ at the highest level appears to be 0.20 and the ICC is 0.40 , then out of 40 percent of the total variance 20 percent is explained" (Hox et al. 2018: $13)$.

Der ICC misst folglich, wie bereits zu Beginn dieses Kapitels dargestellt, den Anteil der Varianzaufklärung, der auf die Makroebene zurückgeführt werden kann. Sofern der ICC einen Wert von Null aufweist, kann kein Anteil an der Gesamtvarianz durch Unterschiede der Makroeinheiten hervorgebracht werden, wodurch eine Mehrebenenanalyse überflüssig wird, da in diesem Fall ausschließlich die Individualebene von Bedeutung wäre. Als Daumenregel

156 „Dies entspricht exakt dem Vorgehen der klassischen Varianz- und Regressionsanalyse, die beide die Gesamtsumme der Abweichungsquadrate von $\mathrm{Y}$ in ihrer Einzelsumme der Abweichungsquadrate innerhalb der Gruppe sowie zwischen den Gruppen zerlegen. Als Kontingenzmaß zur Bestimmung des Varianzanteils, der auf die Gruppenbildung entfällt bzw. „erklärt“ wird, bieten sich das $\eta^{2}\left(\right.$ eta $\left.^{2}\right)$ und der Determinationskoeffizient $\mathrm{R}^{2}$ an. [...] Ihnen entspricht in der Terminologie der Mehrebenenanalyse der „Intra-class-correlation“Koeffizient" (Langer 2009: 108). 
wird meist empfohlen, ab einem ICC von 5\% eine Mehrebenenanalyse durchzuführen (vgl. Heck et al. 2014: 89f.). Wichtig ist außerdem, dass das Intercept-Only-Modell als Basismodell dem Vergleich mit allen weiteren Modellen dient.

\section{Ad Schritt 2: Das Random-Intercept-Modell}

Im zweiten Schritt werden die unabhängigen Individualvariablen in das Modell eingeführt, wobei die Annahme vertreten wird, dass sich die L1-Effekte für alle Kontexteinheiten gleichermaßen auswirken. Es wird eine Variation der Konstante/des Intercepts zugelassen, während die Steigungen für alle Gruppen als identisch angenommen werden. (In Abbildung 9.1 wurde dies graphisch durch die parallelen Regressionsgeraden visualisiert.) Da RandomIntercept-Modelle bereits in der allgemeinen Unterscheidung zwischen Random-Intercept- und Random-Slope-Modellen umfassend thematisiert wurden, soll lediglich erwähnt werden, dass in diesem Modell die unabhängigen Variablen als fixe Parameter eingeführt werden. Außerdem werden im Gegensatz zu einer klassischen OLS-Regression auf Individualebene Unterschiede der Kontexteinheiten (z. B. Länderunterschiede) berücksichtigt (Länderunterschiede im Rahmen der klassischen OLS-Regression aufzuzeigen ist durch die Einführung von Länderdummies gleichwohl möglich und wäre am ehesten mit diesem Modell zu vergleichen, s. Kapitel 13. „Darstellung und Diskussion der Ergebnisse").

Formal kann das Random-Intercept-Modell folgendermaßen dargestellt werden:

Formale Zwei- und Eingleichungsform des Random-Intercept-Modells (vgl. Langer 2009: 122):

$\begin{array}{ll}\text { Ebene 1: } & Y_{\mathrm{ij}}=\beta_{0 \mathrm{j}}+\beta_{1 \mathrm{j}} X_{\mathrm{ij}}+\mathrm{e}_{\mathrm{ij}} \\ \text { Ebene 2: } & \beta_{0 \mathrm{j}}=\gamma_{00}+\mathrm{u}_{0 \mathrm{j}} \\ & \beta_{1 \mathrm{j}}=\gamma_{10}\end{array}$

Eingleichungsform von (1.5) in (1.4): $\mathrm{Y}_{\mathrm{ij}}=\gamma_{00}+\gamma_{10} \mathrm{X}_{\mathrm{ij}}+\mathrm{u}_{0 \mathrm{j}}+\mathrm{e}_{\mathrm{ij}}$

wobei:

$\mathrm{Y}_{\mathrm{ij}}$ : Messwert der abhängigen Variable $\mathrm{Y}$ von $\mathrm{i}$ im Kontext $\mathrm{j}$

$\gamma_{00}$ : Geschätzter adjustierter Grand Mean von Y

$\gamma_{10}$ : Effekt der kontextunabhängigen Kovariaten $X$

$\mathrm{u}_{0 \mathrm{j}}$ : Residuum der 2. Ebene, d. h. Abweichung des geschätzten adjustierten Gruppenmittelwerts $\bar{y}_{j}$ vom geschätzten adjustierten Grand Mean $\bar{y}$.

$\beta_{0 \mathrm{j}}$ : Geschätzter adjustierter Mittelwert von Y der Gruppe $\mathrm{j}$

$\beta_{1 \mathrm{j}}$ : Geschätzter Effekt des exogenen Merkmals X in Gruppe $\mathrm{j}$

$\mathrm{X}_{\mathrm{ij}}$ : Individualvariable bzw. exogenes Merkmal X

$\mathrm{e}_{\mathrm{ijj}}$ : Residuum der Ebene 1, d. h. Abweichung der Befragten i vom geschätzten adjustierten Mittelwert seiner Gruppe j 
Ad Schritt 3: Random-Intercept-Modell mit Prädiktoren auf Individual- und Kontextebene

Im dritten Schritt, dem Random-Intercept-Modell mit Prädiktoren auf beiden Ebenen, wird das Modell um die Kontextprädiktoren der zweiten Ebene erweitert. Ferner verfügt dieses Modell über Variablen auf der Individual- und auf der Kontextebene. Eine Variation der Kontextvariablen besteht ausschließlich zwischen den Kontexteinheiten, innerhalb der Kontexteinheiten sind diese hingegen konstant. Hieraus ergeben sich folgende Formeln:

Formale Zwei- und Eingleichungsform des Random-Coefficient-Modells bzw. des Random-Intercept-Modells mit Prädiktoren auf beiden Ebenen (vgl. Hox et al. 2018: 10f.):

$\begin{array}{ll}\text { Ebene 1: } & Y_{\mathrm{ij}}=\beta_{0 \mathrm{j}}+\beta_{1 \mathrm{j}} X_{\mathrm{ij}}+\mathrm{e}_{\mathrm{ij}} \\ \text { Ebene 2: } & \beta_{0 \mathrm{j}}=\gamma_{00}+\gamma_{01} Z_{\mathrm{j}}+\mathrm{u}_{0 \mathrm{j}} \\ & \beta_{1 \mathrm{j}}=\gamma_{10}\end{array}$

Eingleichungsform von (1.8) in (1.7): $Y_{i j}=\gamma_{00}+\gamma_{01} Z_{j}+\gamma_{10} X_{i j}+u_{0 j}+e_{i j}$ (1.9)

wobei das Modell um den Kontextprädiktor $Z_{\mathrm{j}}$ erweitert wird.

Ad Schritt 4: Das Random-Slope-Modell

Im Random-Slope-Modell mit Individualvariablen wird die Annahme vertreten, dass der Effekt einer Individualvariablen zwischen den Kontexteinheiten variiert bzw., dass die Individualvariable je nach Kontexteinheit eine unterschiedliche Steigung besitzt. Ferner wird überprüft, inwiefern die erklärenden Individualvariablen je nach Kontexteinheit einen unterschiedlich starken Einfluss auf die abhängige Variable nehmen. Neben den Regressionskonstanten werden in diesem Modell auch die Steigungen als zufällige Effekte modelliert, weshalb Langer (2009) dieses Modell auch als „Random-InterceptRandom-Slope“ bezeichnet (Langer 2009: 131). Da im Rahmen der allgemeinen Differenzierung zwischen Random-Intercept- und Random-SlopeModellen die Vorgehensweise und graphische Visualisierung bereits ausführlich skizziert wurden, gilt es noch die formale Darstellungsform zu spezifizieren:

Formale Zwei- und Eingleichungsform des Random-Slope-Modells (vgl. Langer 2009: 131; Lois 2015: 56):

Ebene 1:

Ebene 2:

$$
\begin{aligned}
& Y_{i j}=\beta_{0 j}+\beta_{1 j} X_{i j}+e_{i j} \\
& \beta_{0 j}=\gamma_{00}+u_{0 j} \\
& \beta_{1 j}=\gamma_{10}+u_{1 j}
\end{aligned}
$$

Eingleichungsform von (2.1) in (2.0): $Y_{i j}=\gamma_{00}+\gamma_{10} X_{i j}+u_{1 j} X_{i j}+u_{0 j}+e_{i j}$

wobei:

$\mathrm{Y}_{\mathrm{ij}}$ : Messwert der abhängigen Variable $\mathrm{Y}$ von $\mathrm{i}$ im Kontext $\mathrm{j}$

$\gamma_{00}$ : Geschätzter Wert von Y, wenn X gleich Null ist 
$\mathrm{u}_{0 \mathrm{j}}$ : Level-2-Residuum der kontextspezifischen Regressionskonstanten $\beta_{0 \mathrm{j}}$ : Abweichung der Regressionskonstante $\beta_{0 \mathrm{j}}$ des Kontextes $\mathrm{j}$ von derjenigen der Population (Random-Part)

$\gamma_{10}$ : Effekt des endogenen Merkmals X

$\mathrm{u}_{1 \mathrm{j}}$ : Level-2-Residuum der kontextspezifischen Regressionskoeffizienten

$\beta_{1 \mathrm{j}}$ : Abweichung der Regressionskoeffizienten $\beta_{1 \mathrm{j}}$ des Kontextes $\mathrm{j}$ von demjenigen der Population (Random-Part)

$\beta_{0 \mathrm{j}}$ : Regressionskonstante der Binnenregression im Kontext $\mathrm{j}$

$\beta_{1 \mathrm{j}}$ : Regressionskoeffizient des Merkmals $X_{\mathrm{ij}}$ der Binnenregression im Kontext $\mathrm{j}$

$\mathrm{e}_{\mathrm{ij}}$ : Residuum der ersten Ebene bei der Vorhersage von Y für ProbandIn i im Kontext $\mathrm{j}$

Ad Schritt 5: Intercepts-and-Slopes-as-Outcome-Modell

Im letzten Schritt werden zusätzlich Cross-Level-Effekte eingeführt, die, wie bereits im Kapitel 12.1.1. über die allgemeinen Kausalzusammenhänge von Mehrebenenanalysen erläutert, Interaktionseffekte zwischen den Ebenen darstellen. Folglich erfasst ein Cross-Level-Effekt, inwiefern ein Individualeffekt (L1) in Abhängigkeit von der Ausprägung eines Kontextprädiktors (L2) variiert (vgl. Lois 2015: 61). Dies lässt sich formal wie folgt veranschaulichen:

Formale Zwei- und Eingleichungsform des Intercepts-and-Slopes-asOutcome-Modells (vgl. Lois 2015: 62; Hox et al. 2018: 11):
Ebene 1:
$Y_{i j}=\beta_{0 j}+\beta_{1 j} X_{i j}+e_{i j}$
Ebene 2:
$\beta_{0 j}=\gamma_{00}+\gamma_{01} Z_{j}+u_{0 j}$
$\beta_{1 j}=\gamma_{10}+\gamma_{11} Z_{j}+u_{1 j}$

Eingleichungsform von (2.4) in (2.3):

$$
Y_{i j}=\gamma_{00}+\gamma_{01} Z_{j}+\gamma_{10} X_{i j}+\gamma_{11} Z_{j} X_{i j}+u_{1 j} X_{i j}+u_{0 j}+e_{i j}
$$

Abschließend werden die integrierten Varianten der Eingleichungsformeln nochmals in Tabelle 20 zusammengefasst. In jedem Schritt sind neu hinzugefügte Terme fett markiert. Aus Gründen der Übersichtlichkeit wurde die Reihenfolge einiger einzelner Terme im Vergleich zu den Eingleichungsformen im Fließtext teilweise angepasst. 
Tabelle 20: Integrierte Mehrebenengleichungen für Querschnittsdaten

\begin{tabular}{|c|c|c|}
\hline & Modell & Eingleichungsformeln \\
\hline 1. Schritt & $\begin{array}{l}\text { Random-Intercept-Only- } \\
\text { Modell }\end{array}$ & $Y_{i j}=y_{00}+u_{0 j}+e_{i j}$ \\
\hline 2. Schritt & $\begin{array}{l}\text { Random-Intercept- } \\
\text { Modell (mit Variablen } \\
\text { der Individualebene) }\end{array}$ & $Y_{i j}=y_{00}+y_{10} X_{i j}+u_{0 j}+e_{i j}$ \\
\hline 3. Schritt & $\begin{array}{l}\text { Random-Intercept- } \\
\text { Modell (mit Variablen } \\
\text { der Individual- und } \\
\text { Kontextebene) }\end{array}$ & $Y_{i j}=y_{00}+y_{10} X_{i j}+y_{01} Z_{j}+u_{0 j}+e_{i j}$ \\
\hline 4. Schritt & $\begin{array}{l}\text { Random-Slope-Modell } \\
\text { (mit Variablen der } \\
\text { Individualebene) }\end{array}$ & $Y_{i j}=y_{00}+y_{10} X_{i j}+u_{1 j} X_{i j}+u_{0 j}+e_{i j}$ \\
\hline 5. Schritt & $\begin{array}{l}\text { Intercepts-and-Slopes- } \\
\text { as-Outcome-Modell } \\
\text { (mit Cross-Level-Effekt } \\
\text { sowie Variablen der } \\
\text { Individual- und } \\
\text { Kontextebene) }\end{array}$ & $Y_{i j}=y_{00}+y_{10} X_{i j}+y_{01} Z_{j}+y_{11} Z_{j} X_{i j}+u_{1 j} X_{i j}+u_{0 j}+e_{i j}$ \\
\hline
\end{tabular}

Quelle: modifiziert nach Braun et al. 2010: 27.

\title{
12.2. Zur Analyse von Paneldaten im Rahmen von Mehrebenenmodellen
}

\begin{abstract}
„Während multivariate Querschnittsanalysen zur Bestimmung des Effekts mehrerer unabhängiger Prädiktoren auf eine abhängige Outcome-Variable allein die Kovarianzen zwischen zeitgleich erhobenen Individualmerkmalen auswerten, basieren Längsschnittanalysen auf Daten zur zeitübergreifenden individuellen Veränderung bzw. zum sozialen Wandel von Individualmerkmalen. Betreffen diese Individualmerkmale die Eigenschaften von einzelnen Personen, so sind zur Längsschnittanalyse nahezu zwangsläufig personengebundene Paneldaten erforderlich, welche bestimmte Merkmale desselben Personenkreises über mehrere Messzeitpunkte hinweg abbilden und somit Wandel und Veränderungen auf der Individualebene dokumentieren können“" (Krause/Urban 2013: 2).
\end{abstract}

Eine Analyse von Paneldaten als Mehrebenenmodell ermöglicht, eine Veränderung der zu erklärenden Variablen (Kriteriumsvariablen) auf Personenebene zu analysieren bzw. Veränderungsprozesse von Individualmerkmalen darzustellen (vgl. Langer 2004: 223). Individuelle Veränderungen der innerhäuslichen Arbeitsteilung können dadurch festgestellt werden. Aufgrund der 
Abbildung 10: Dreiebenenstruktur der Panelstudie des GGP Ebene 3:

\section{Ebene 1:}
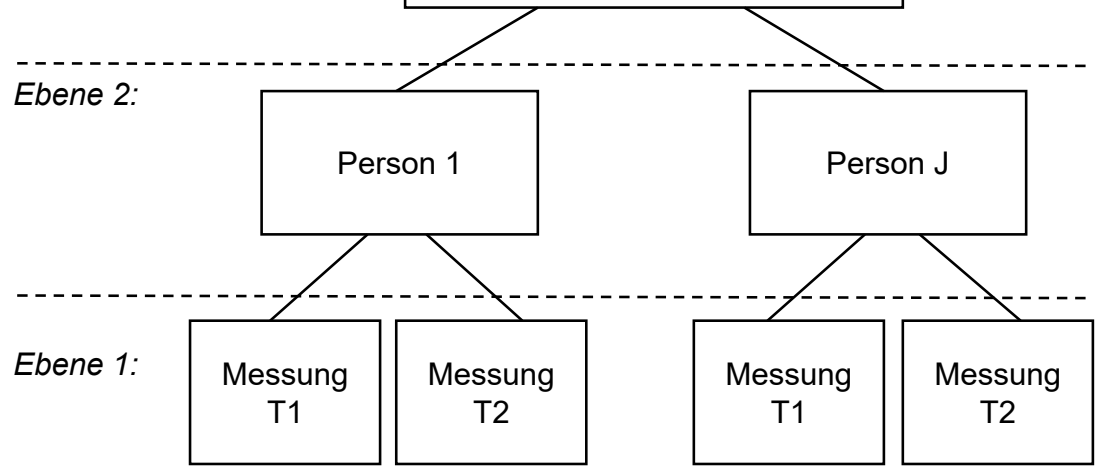

\section{(Nationaler/) Regionaler Kontext}

Quelle:

modifiziert nach Langer 2004: 255; Rabe-Hesketh/Skrondal 2012: 385.

theoretischen Vorannahmen und der Datengrundlage ergibt sich eine Dreiebenenstruktur, wie in Abbildung 10 dargestellt.

Das Drei-Ebenen-Modell berücksichtigt geschachtelte Daten zeitveränderlicher Merkmale zu verschiedenen Zeitpunkten (Ebene 1) von Personen (Ebene 2), die innerhalb eines bestimmten (nationalen bzw.) regionalen Kontextes (Ebene 3) eingebettet sind - die Datenstruktur wird als hierarchisch erachtet (Grundsätzlich wäre auch eine Vierebenenstruktur denkbar: Ebene 4: Länder, Ebene 3: Regionen, Ebene 2: Personen und Ebene 1: Messzeitpunkte, doch ist eine Berücksichtigung von vier Ebenen aufgrund der Fallzahlproblematik der Länder nicht möglich). Hieraus ergeben sich drei zentrale Fragestellungen: Inwiefern kann die Veränderung der innerhäuslichen Arbeitsteilung durch 1. zeitveränderliche Aspekte, 2. Variablen auf der Individualebene, 3. die Zugehörigkeit zu (einem bestimmten Land/) einer bestimmten Region (Aggregatebene) erklärt werden? Bezüglich der Aggregatebene stellt sich weiterführend die Frage, ob sich die innerhäuslicheren Arbeitsteilungsarrangements von Paaren aus unterschiedlichen Kontexteinheiten unterscheiden. Einflüsse des Kontextes wie auch individuelle Merkmale respektive Ressourcen können folglich für die Erklärung der innerhäuslichen Arbeitsteilung in Betracht gezogen werden. Anders formuliert kann mittels einer Mehrebenenanalyse geschätzt werden, inwiefern die Varianz der innerhäuslichen Arbeitsteilung auf unterschiedliche Messzeitpunkte, auf Unterschiede zwischen den Personen und auf Unterschiede zwischen den (Län- 
dern/) Regionen zurückzuführen ist. Die Annahme, dass einerseits die Messungen von verschiedenen Personen innerhalb (eines Landes bzw.) einer Region aufgrund kultureller Werte korreliert sind (interindividuelle Veränderung), andererseits eine Korrelation der Messwerte einer Person zu verschiedenen Zeitpunkten zu erwarten ist (intraindividuelle Veränderung), ist naheliegend und kann mittels einer Mehrebenenanalyse überprüft werden. Analysiert wird, inwiefern die Ausprägungen der zu erklärenden Variable erstens von der Zeitvariation (Ebene 1), zweitens von personenspezifischen Effekten (Ebene 2, random slope) und drittens von den Effekten innerhalb (der Länder/) der Regionen (Ebene 3, random intercept) abhängen (vgl. Hosaya et al. 2014: 202). Cross-level-Interaktionen, d. h. Auswirkungen der Makroebene auf die individuellen Effekte, können ebenfalls kontrolliert werden, sofern ein Effekt der Individualvariablen in Abhängigkeit der Aggregatebene bzw. des Kontextes unterstellt wird (vgl. Beckers 2010: 27). Ohne Berücksichtigung der hierarchischen Datenstruktur würden auch im Falle von Paneldaten die Signifikanztests verzerrte Ergebnisse darstellen. Ferner haben Bryk/Raudenbush (1992) fünf zentrale Argumente für die Analyse von Paneldaten durch hierarchische Mehrebenenmodelle festgehalten:

„First, the [hierarchical linear] model explicitly represents the individual growth at Level 1. In contrast, in an MRM [Multivariate Repeated Measures] model individual variation in growth is not directly modeled but rather appears in the interaction of repeated occasions by subjects. Conceptually, the hierarchical model is more in the spirit of the growth-curve analysis [...].

Second, the hierarchical model is generally more flexible in terms of its data requirements because the repeated observations are viewed as nested within the person rather than as the same fixed set for all persons as in MRM. In an hierarchical model, both the number of observations per person ${ }^{157}$ and the spacing among the observations may vary. The time or age variable may also be continuous rather than a fixed set of points. Although missing observations can now be handled in MRM [...], this poses no special problem in a hierarchical analysis. Other Level-1 predictors besides age or time also can be incorporated [...]. Their effects can be specified as fixed, nonrandomly varying or random.

Third, the hierarchical model permits flexible specification of the covariance structure among the repeated observations and provides methods for direct hypothesis testing about possible determinants of this structure. This is accomplished through specification of both the structural features of the individual growth in Equation 6.1 and the Level-1 and Level-2 random effects. [...]

Fourth, when the restrictive data requirements and assumptions of MRM apply, a hierarchical analysis produces the same point estimates for the fixed effects as in an MRM analysis. The $t$-ratios computed in the hierarchical analysis are also identical to those obtained using a priori contrasts in MRM. In sum, the results of

157 As well as Hox notes: „Since multilevel modeling does not require balanced data, it is not a problem if the number of available measurements is not the same for all individuals. This is an important benefit if there is panel dropout, or other forms of missing measurements within individuals“ (Hox 2002: 73). 
a hierarchical analysis can be formally related to the MRM model when the MRM conditions apply.

Fifth, the formulation of growth models via the hierarchical approach leads naturally to the study of organizational effects on growth [...]" (Bryk/Raudenbush 1992: 133f., Hervorhebungen im Original; die Verf.).

Von den dargestellten Vorteilen der Analyse von Paneldaten als Mehrebenenmodell ist festzuhalten, dass eine Schätzung von Wachstumsmodellen auf Personen- und nicht auf Stichprobenebene möglich ist ${ }^{158}$, dass keine vollständigen Daten für alle Personen und alle Messzeitpunkte vorausgesetzt werden (kein balanciertes Design) ${ }^{159}$, dass im Unterschied zur klassischen Varianzanalyse die Abstände zwischen den einzelnen Messzeitpunkten variieren dürfen, dass die Varianz des personenspezifischen Niveaus der abhängigen Variablen, ihre lineare oder auch quadratische Entwicklungskomponente analysiert werden können und, dass sich die Varianz dieser personenspezifischen Regressionsparameter wiederum aus den Merkmalen der Person erklären lässt (vgl. Langer 2009: 227).

Ein weiterer Vorteil, der von Bryk/Raudenbush hier nicht explizit dargestellt ist, wird von Hox (2002) ergänzt:

„A sixth advantage, not mentioned by Bryk and Raudenbush, is that it is straightforward to include time varying or time constant explanatory variables to the model, which allows us to model both the average group development and the development of different individuals" (Hox 2002: 93).

Werden Paneldaten als Mehrebenenmodelle konstruiert, werden die allgemeinen Vorteile von Längsschnittdaten mit den Vorteilen von Mehrebenenanalysen kombiniert; die wichtigsten seien nachfolgend zusammengefasst. Eine Mehrebenenanalyse von Paneldaten ermöglicht die Überprüfung von (vgl. Krause/Urban 2013: 4f.):

- populationsspezifischen Verlaufsmustern von Variablenveränderungen,

- gruppentypischen Veränderungen,

- zeitabhängigen Messwerten pro Person, auch mit unterschiedlichen Zeitabständen zwischen den Personen (falls nicht für jede Erhebungs-

158 „Im Gegensatz hierzu analysieren klassische Strukturgleichungsmodelle wie LISREL die Veränderung der Messwerte nur auf der Stichprobenebene, wobei sie nur die Stabilität der untersuchten Kriteriumsvariable zwischen den Messzeitpunkten bestimmen. Beim Mehrebenenmodell hingegen gehen wir von [einer] hierarchischen Datenstruktur aus, die es ermöglicht, die Veränderung der Kriteriumsvariablen über die Zeitachse zu bestimmen“ (Langer 2009: 223).

159 Bezüglich des zweiten Vorteils ist anzumerken, dass dies nicht für fehlende Werte der unabhängigen Variablen gilt: „However, this advantage [handle models with varying time points] of multilevel modeling does not extend to missing observations on the explanatory variables. If explanatory variables are missing, the usual treatment is again to remove the case completely from the analysis" (Hox 2002: 95; Hox et al. 2018: 96). 
welle gültige Messwerte einer Person vorliegen; z. B. diese vorzeitig ausgeschieden ist oder zu einem späteren Zeitpunkt aufgenommen wurde) oder zwischen den Erhebungswellen,

- Kovarianzen zwischen den wiederholten Messungen,

- zeitkonstanten (z. B. Geburtsland) und zeitveränderlichen Effekten (z. B. Alter) sowie deren Interaktion,

- geschachtelten Modellen; Kombination von Makro- und Mikroeffekten,

- einer abhängigen Datenstruktur; die Daten werden als hierarchisch berücksichtigt;

- verzerrenden Effekten unbeobachteter Heterogenität ${ }^{160}$,

- Standardfehlern: die Tatsache, dass die Ebenen als statistisch nicht unabhängig voneinander modelliert werden, führt dazu, dass die Standardfehler weitgehend unverzerrt ermittelt werden können.

\subsubsection{Zum Prinzip der Residuen- und Varianzzerlegung in Mehrebenenmodellen}

„A three-level random-intercept model includes error components for nested clusters to allow the total variance to be decomposed into the level-1 variance within clusters, the level-2 variance between clusters within superclusters, and the level-3 variance between superclusters" (Rabe-Hesketh/Skrondal 2012: 418).

Voraussetzungen, die für die Mehrebenenanalyse von Paneldaten erfüllt werden müssen, sind $u$. a. vornehmlich Residuen-Homoskedastizität und die Vermeidung von Autokorrelation der Residuen (weitere Grundannahmen sind gemäß Snijders/Bosker $(1999 ; 2012)$ eine lineare Abhängigkeit zwischen der abhängigen und den unabhängigen Variablen, eine Normalverteilung der Residuen auf beiden Ebenen und eine ausreichende Varianz der Variablen (vgl. Hadler 2004: 67f.)). Die Gültigkeit der Abwesenheit von Residuen-Heteroskedastizität und Residuen-Autokorrelation kann per Vorentscheid über die Festlegung der Struktur der Residuen-Kovarianzmatrix zu Beginn der Mehrebenenanalyse getestet und kontrolliert werden, wie in den nachfolgenden Abschnitten erläutert wird (vgl. Krause/Urban 2013: 8). Zuvor

160 Alle zeitkonstanten (Dritt-)Variablen, die eine relevante Erklärungsleistung aufweisen würden, aber unbeobachtet bleiben, werden als individuelle/einheitsspezifische Heterogenität bezeichnet. Besteht eine Korrelation zwischen unbeobachteten zeitinvarianten Merkmalen und den unabhängigen Variablen, werden die Schätzer von Regressionsfunktionen verzerrt dargestellt. Zur Lösung dieses Problems muss die unbeobachtete einheitsspezifische Heterogenität kontrolliert werden, indem die Daten als Längsschnitt abgebildet, d. h. transformiert werden und geeignete Panelmethoden angewendet werden. Mittels der Datentransformation wird eine zufällige, unstrukturierte Verteilung des Fehlerterms erzeugt (vgl. Giesselmann/Windzio 2012: 28). Diese Kontrollfunktion der unbeobachteten Heterogenität ist einer der relevantesten Vorzüge von Paneldaten. 
wird die für Mehrenenenanalysen bedeutsame Zerlegung des Fehlerterms skizziert.

Im Rahmen einer Mehrebenenanalyse mit drei Ebenen wird die Varianz zerlegt als Varianz der ersten Ebene innerhalb der Personen, Varianz der zweiten Ebene der Prädiktorvariable zwischen den Personen und als Varianz zwischen den Kontextebenen (Ländern, Regionen etc.). Folglich wird auch der Fehlerterm in drei Teile zerlegt: für jeden einzelnen Messwert, für jede Person und für jede Kontexteinheit (vgl. Krause/Urban 2013: 8). In Abbildung 11 wird das Prinzip der Residuen- und Varianzzerlegung graphisch zur Vereinfachung dargestellt. Beispielhaft werden vier Personen, die aus zwei verschiedenen Ländern stammen zu zwei aufeinander folgenden Messzeitpunkten befragt. Der Datenpunkt $Y_{2,1}$ veranschaulicht die Messung von Person 1 zum zweiten Messzeitpunkt und weist einen bestimmten Abstand zum Mittelwert $\bar{y}_{\mathrm{L} 1}$ aller in Land 1 lebenden Personen. „Dieser Mittelwert aller YWerte ist der beste Schätzwert [innerhalb des ersten Landes] für jeden YWert, wenn weder der Zeitpunkt noch die Person bekannt ist, für den/die ein bestimmter Wert von Y geschätzt werden soll" (Krause/Urban 2013: 8). Der Schätzfehler, der hieraus resultiert, ist als schwarze durchgezogene Linie markiert und wird in diesem Fall erheblich unterschätzt. „Werden die Abstände aller beobachteten Y-Werte vom Y-Gesamtmittelwert $\bar{Y}$ [innerhalb des ersten Landes] zu allen Zeiten und von allen Personen addiert, so lässt sich hieraus die Gesamtvarianz von Y berechnen“ (Krause/Urban 2013: 8). Eine Optimierung des Schätzfehlers wird erreicht, wenn ein personenspezifischer Mittelwert $\bar{y}_{1}$, durch gepunktete Linien markiert, spezifiziert wird, der auch als zeitspezifisches L1-Residuum einer bestimmten Person bezeichnet wird. Wird die Summe aller L1-Residuen berechnet, ergibt sich daraus die Varianz innerhalb aller Personen (within). Die personenspezifischen L2-Residuen (s. gestrichelte schwarze Linie) variieren nicht mit den Erhebungszeitpunkten. Vielmehr werden sie ermittelt als Abstand zwischen den personenspezifischen Mittelwerten und dem Gesamtmittelwert. Aus der Summe aller L2Residuen wird die Varianz zwischen den Personen (between) berechnet (vgl. Krause/Urban 2013: 9). Innerhalb des ersten Landes ist der Mittelwert $\bar{y}_{\mathrm{L} 1}$ der beste Schätzer; zur Bestimmung der Varianz zwischen den Ländern wird der Abstand dessen zum Gesamtmittelwert der Länder $\bar{Y}$ betrachtet (s. graue durchgezogene Linie). Wird die Summe aller L3-Residuen gebildet, ergibt sich hieraus die Varianz zwischen den Ländern. Die Bestimmung der Gesamtvarianz erfolgt letztlich mittels der Berücksichtigung der einzelnen Teilvarianzen:

Gesamtvarianz $=$ Varianz innerhalb der Personen, zeitspezifisch

+ Varianz zwischen den Personen innerhalb der Länder

+ Varianz zwischen den Ländern 
Abbildung 11: Zum Prinzip der Residuen- und Varianzzerlegung in Mehrebenenmodellen

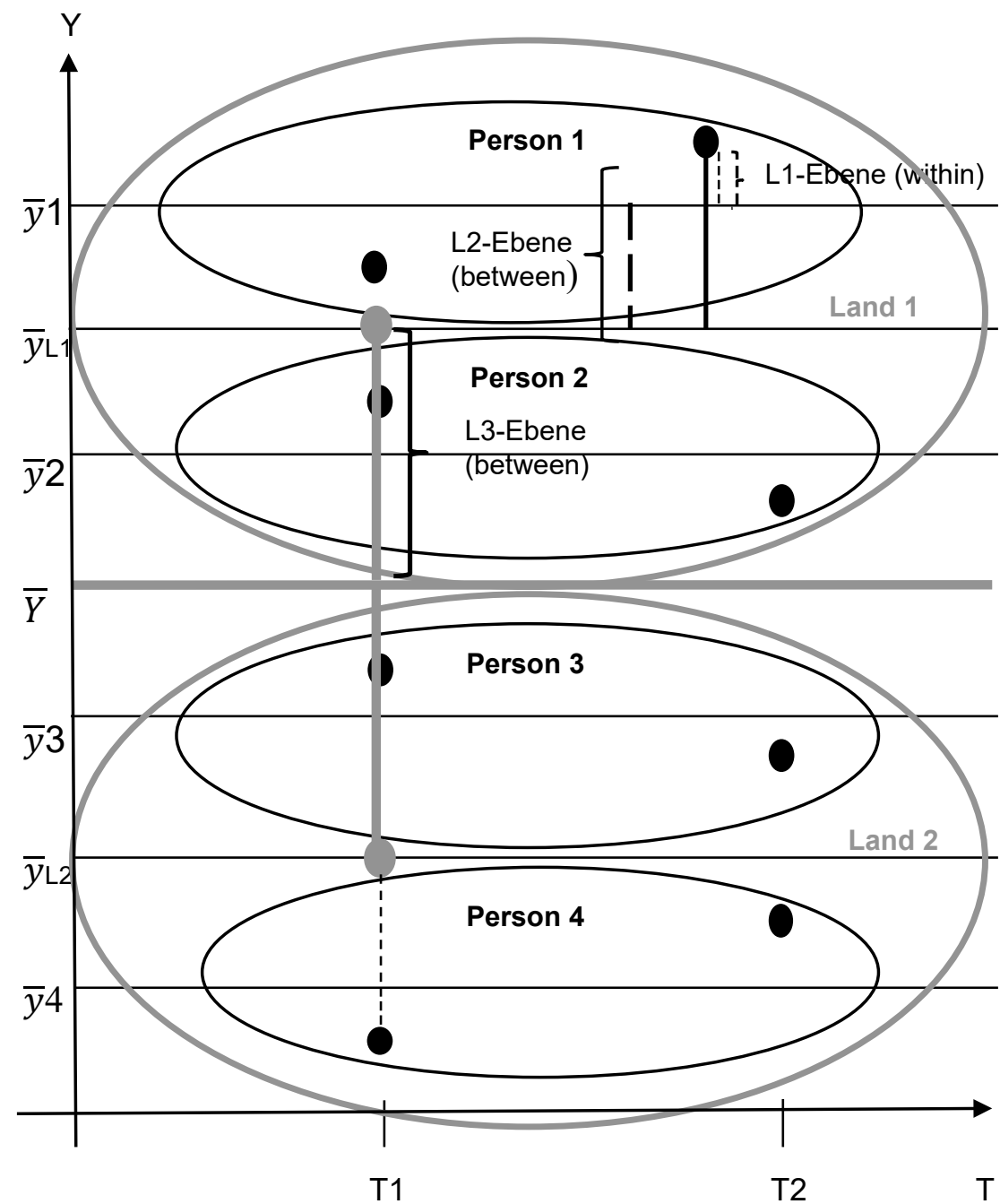

Quelle: modifiziert nach Krause/Urban 2013: 10. 
Nachdem die Zerlegung des Fehlerterms skizziert wurde, soll im Folgenden ein einführender Überblick über die Definition von VarianzKovarianzmatrizen gegeben werden.

\section{Varianz-Kovarianzmatrix}

Für jede Analyse von Paneldaten als Mehrebenenmodell kann eine VarianzKovarianzmatrix für die Residuen der ersten Ebene (L1) und die Residuen der Kontextebene(n) festgelegt werden (vgl. Krause/Urban 2013: 10). Varianten der L1-Matrix sind:

1. Scaled Identity: Die Scaled-Identiy-Matrix folgt der Annahme homogener Residuen-Varianzen, wobei die Resiuden-Varianzen als unkorreliert gelten. Implizit bedeutet dies, dass die Fehlerterme aller Messzeitpunkte unabhängig voneinander sind (vgl. Krause/Urban 2013: 11).

2. Compound Symmetry: Nach der Compound-Symmetry-Matrix sind ebenfalls alle Varianzen gleich, jedoch kovariieren alle Residuen miteinander, wobei alle Kovarianzen vom gleichen Ausmaß sind respektive einen konstanten Wert aufweisen. Insbesondere die Annahme einer identischen Kovarianz aller Messungen scheint eher unplausibel, weil Messwerte von Erhebungszeitpunkten, die zeitlich näher beieinander liegen vermeintlich stärker miteinander kovariieren sollten als Messwerte, deren Erhebungszeitpunkte weit auseinanderliegen (vgl. Krause/Urban 2013: 11).

3. Unstrukturierte Kovarianzmatrix: Im Gegensatz zur CompoundSymmetry-Matrix erfolgt im Falle der unstrukturierten Kovarianzmatrix keine Festlegung in Bezug auf die VarianzKovarianzhomogenität, ferner können alle Elemente frei geschätzt werden.

„Während also die Compound Symmetry-Matrix noch mit der Schätzung von zwei Parametern auskommt (unabhängig davon, wie viele Messzeitpunkte vorhanden sind), müssen bei der unstrukturierten Kovarianzmatrix insgesamt " $p(p+1) / 2 "$ (mit $p=A n z a h l$ der Messzeitpunkte) Parameter geschätzt werden“ (Krause/Urban 2013: 11).

Folglich wird die unstrukturierte Kovarianzmatrix sehr komplex, wenn viele Erhebungswellen vorhanden sind, weshalb ihre Verwendung eher bei sparsamen Modellen in Betracht zu ziehen ist.

4. Diagonal-Matrix: Die Diagonal-Matrix lässt Heteroskedastizität der L1-Residuen zu, indem sie eine freie Schätzung der Varianzen aller L1-Residuen ermöglicht, zugleich fixiert sie jedoch alle Kovarianzen auf 0,00, wodurch jegliche Korrelationen zwischen den Residuen vermieden werden. Die Sinnhaftigkeit des Ausschlusses der Korrelationen zwischen den Residuen ist durch einen Vergleich von Modellen 
mit unterschiedliche Matrixtypen zu überprüfen (vgl. Krause/Urban 2013: 12).

5. Autoregressive Kovarianzen erster Ordnung: Autoregressive Kovarianzen erster Ordnung sind insofern eine Erweiterung der DiagonalMatrix, als die heterogenen Varianzen durch eine Schätzung autoregressiver Kovarianzen der ersten Ordnung ergänzt werden. Die zentrale Annahme ist,

„[...] dass zeitlich benachbarte Residuen mit einer fixen Stärke (r) untereinander korrelieren und dass zeitlich weiter auseinanderliegende Residuen mit einer deutlich reduzierten, aber auch konstanten Korrelation $\left(\mathrm{r}^{2}\right)$ kovariieren. Somit müssen für diesen Matrix-Typ nur $\mathrm{p}+1$ Parameter ( $\mathrm{p}=$ Anzahl der Messzeitpunkte) geschätzt werden“ (Krause/Urban 2013: 12).

L2-Matrix: Ebenso wie für die L1-Residuen kann auch für die L2-Residuen ein Matrix-Typ festgelegt werden. Im Rahmen von Mehrebenenanalysen stellen die Elemente der L2-Matrix die Varianzen und Kovarianzen der Random-Effekte dar (vgl. Krause/Urban 2013: 12). Für die Schätzung der L2Matrix empfehlen Krause/Urban (2013) den Matrixtyp einer unstrukturierten Matrix zu verwenden. Außerdem verweisen sie auf die sinnvolle Möglichkeit den Zeitparameter als Random-Effekt zu spezifizieren, wodurch sich ein äußerst praktischer Kompromiss zwischen den Schätzungen einer restriktiven Compound-Symmetry-Matrix und einer komplexen unstrukturierten Matrix erzielen lässt. Durch diese Vorgehensweise wird eine Kovarianzmatrix erzeugt, deren Varianzen und Kovarianzen eine Variation in Abhängigkeit von der zwischen den Erhebungszeitpunkten abgelaufenen Zeit aufweisen können, ohne hierbei eine Schätzung für jeweils einen eigenen Parameter durchführen zu müssen (vgl. Krause/Urban 2013: 13).

\subsubsection{Vorgehensweise für längsschnittliche Mehrebenenmodelle}

In Anlehnung an die Beschreibung der allgemeinen Vorgehensweise für Mehrebenenanalysen soll in diesem Kapitel die Beschreibung um die spezifische Vorgehensweise für Paneldaten präzisiert werden. Durch die Modellierung von Hybridmodellen, die zeitvariante und zeitinvariante Prädiktoren berücksichtigen, werden die Ideen, die Fixed-Effect-Panel-Modellen und Random-Effect-Panel-Modellen zugrunde liegen, im Rahmen der Mehrebenenanalyse miteinander kombiniert. Die folgenden Modelle basieren zunächst lediglich auf zwei Ebenen (zeitvariierende Messwerte der ersten Ebene und Personen auf der zweiten Ebene). Um Effekte der Makroebene zu berücksichtigen, werden die Modelle abschließend durch eine dritte Ebene erweitert. 


\section{Schritt 1: Random-Intercept-Only-Modell für Paneldaten}

Um die Varianzanteile der verschiedenen Ebenen zu erfassen, wird auch im Rahmen der Panelanalyse mit Mehrebenenmodellen ein Nullmodell geschätzt, das keine erklärenden Variablen enthält. Der ICC, der auf der Varianzzerlegung aufbaut, gibt in diesem Fall Auskunft darüber, wie viel Prozent der Varianz der innerhäuslichen Arbeitsteilung auf Unterschiede zwischen den Befragten zurückgeführt werden kann und wie viel Prozent der Varianz der innerhäuslichen Arbeitsteilung auf Unterschiede zwischen den Messzeitpunkten ${ }^{161}$ zurückzuführen ist. Anders formuliert ist mittels des Intraklassenkorrelationskoeffizieten zu erfassen, ,[...] wie viel Varianz in der abhängigen Variablen auf Personenheterogenität zurückzuführen ist" (Hosaya et al. 2014: 192). Sofern ausreichend Varianz sowohl zwischen den Befragten als auch zwischen den Messzeitpunkten besteht, kann diese weiterführend durch den Einbezug von zeitvarianten und zeitinvarianten Determinanten erklärt werden (vgl. Krause/Urban 2013: 26). Zu beachten ist, dass für die Fehlerterme der beiden Ebenen (Ebene 1: $\mathrm{e}_{\mathrm{ti}}$, Ebene 2: $\mathrm{u}_{0 \mathrm{i}}$ ) angenommen wird, dass diese „[...] unabhängig voneinander sind (z. B. keine Autokorrelationen bestehen), einen Erwartungswert von Null besitzen, normalverteilt sind [und] keine Varianzungleichheiten (Heteroskedastizität) aufweisen“ (Krause/Urban 2013: 24). Formal lässt sich das Random-Intercept-Only-Modell für Paneldaten wie folgt darstellen:

Formale Zwei- und Eingleichungsform des Intercept-Only-Modells (vgl. Krause/Urban 2013: 25; Hosaya et al. 2014: 192):

$\begin{array}{ll}\text { Ebene 1: } & \mathrm{Y}_{\mathrm{ti}}=\beta_{0 \mathrm{i}}+\mathrm{e}_{\mathrm{ti}} \\ \text { Ebene 2: } & \beta_{0 \mathrm{i}}=\gamma_{00}+\mathrm{u}_{0 \mathrm{i}}\end{array}$

Eingleichungsform von (1.2) in (1.1): $\mathrm{Y}_{\mathrm{ti}}=\gamma_{00}+\mathrm{u}_{0 \mathrm{i}}+\mathrm{e}_{\mathrm{ti}}$

wobei für die erste Gleichung gilt:

161 Bezüglich der Messzeitpunkte empfiehlt es sich gemäß Hox (2002) für eine lineare Schätzung, die Wellen „1“ und „2“ in „0“ und „1“ zu transformieren: „In the multilevel regression model, the development over time is often modeled by a linear regression equation, which may have different regression coefficients for different individuals. Thus, each individual can have their own regression curve, specified by the individual regression coefficients that in turn may depend on individual attributes. Quadratic and higher functions can be used to model nonlinear dependencies on time, and both time varying and person level covariates can be added to the model. Although the measurement occasions will usually be thought of as occasion one, two et cetera, it is useful to code the time points $T$ as $t=0,1,2$, $3,4,5$. As a result, the intercept can be interpreted as the expected outcome on the first occasion. Using time points $t=1,2,3,4,5,6$ would be completely equivalent, but more difficult to interpret, because the value zero is not in the range of observed time points" (Hox 2002: 79, Hervorhebungen im Orignial; die Verf.). Auch Krause/Urban (2013) empfehlen diese Vorgehensweise einer Zentrierung um den Nullpunkt für den Zeitparameter, damit der Intercept sinnhaft interpretiert werden kann (vgl. Krause/Urban 2013: 6). 
t: $\quad$ Messzeitpunkt

i: $\quad$ Befragte/r

$\mathrm{Y}_{\mathrm{ti}}$ : Messwert der abhängigen Variablen für jeden i-ten Befragten

$\beta_{0 \mathrm{i}}$ : Intercept, der für alle Befragten und alle Messzeitpunkte identisch ist und lediglich zwischen den Befragten variiert

$\mathrm{e}_{\mathrm{ti}}$ : Zufallswert, der zwischen Erhebungszeitpunkten und zwischen Personen variiert

Im Rahmen der zweiten Gleichung (Ebene 2) wird der Intercept der ersten Ebene als abhängige Variable der zweiten Ebene verwendet. Der Intercept

,[...] wird in seiner Ausprägung bestimmt von einer Konstanten $(\gamma 00)$, die unabhängig vom Messzeitpunkt und unabhängig vom Befragten stets den gleichen Wert aufweist, und von einem Zufallswert ( $\mathrm{u}_{0 \mathrm{i}}$ ), der von Befragtem zu Befragtem unterschiedlich sein kann. Somit wird in diesem Nullmodell das Intercept als "random" behandelt, d.h. in diesem Modell variiert das Intercept zwischen den einzelnen Befragten in Abhängigkeit von einer Zufallsgröße (u) auf der Ebene 2 (=Personenebene)“ (Krause/Urban 2013: 24f.).

Folglich verweist die erste Ebene 1-Gleichung darauf, dass sich der Wert $\mathrm{Y}_{\mathrm{ti}}$ eines/einer Befragten i zum Zeitpunkt $t$ aus einem personenspezifischen Effekt $\beta_{0 \mathrm{i}}$ und einem Ebene 1-Residuum $\mathrm{e}_{\mathrm{ti}}$ zusammensetzt. Im Rahmen der zweiten Gleichung erfolgt eine Zerlegung dieses Effekts in einen globalen Effekt $\gamma_{00}$ und eine personenspezifische Abweichung $\mathrm{u}_{0 \mathrm{i}}$ (Ebene 2-Residuum) (vgl. Hosaya et al. 2014: 193).

Schritt 2: Random-Intercept-Modell mit zeitvariierenden Kovariaten

Im zweiten Schritt werden die zeitvariierenden (oder zeitvarianten) Prädiktoren der ersten Ebene (L1) eingeführt. L1-Kovariaten sind Prädiktoren der untersten Modellebene und können sowohl über die Zeit als auch über Personen hinweg schwanken (vgl. Hosaya et al. 2014: 194). Das RandomIntercept-Modell mit zeitvariierenden Kovariaten kann anhand der folgenden Gleichungen beschrieben werden:

Formale Zwei- und Eingleichungsform des Random-Intercept-Modells mit zeitvariierenden Kovariaten (vgl. Krause/Urban 2013: 26; Hosaya et al. 2014: 194):

Ebene 1:

Ebene 2:

$$
\begin{aligned}
& Y_{t i}=\beta_{0 j}+\beta_{1 \mathrm{i}} X_{1 \mathrm{ti}}+\mathrm{e}_{\mathrm{ti}} \\
& \beta_{0 \mathrm{i}}=\gamma_{00}+\mathrm{u}_{0 \mathrm{i}} \\
& \beta_{1 \mathrm{i}}=\gamma_{10}
\end{aligned}
$$

Eingleichungsform von (1.5) in (1.4): $\mathrm{Y}_{\mathrm{ti}}=\gamma_{00}+\gamma_{10} \mathrm{X}_{1 \mathrm{ti}}+\mathrm{u}_{0 \mathrm{i}}+\mathrm{e}_{\mathrm{ti}}$

wobei ein zeitvariierender Prädiktor $\mathrm{X}_{1 \mathrm{ti}}$ in das Modell eingeführt wird,

,[...] dem ein entsprechender Koeffizient $\beta_{1 i}$ zugeordnet ist. Dieser Koeffizient kann potenziell über die Level-1-Einheiten (Personen) variieren. Allerdings wird dieser Prädiktor in den Level-2-Gleichungen auf $\gamma 10$,fixiert“. Diese restriktive 
Annahme bedeutet, dass der Effekt des Prädiktors $\mathrm{x}_{1 \text { ti }}$ auf die abhängige Variable für alle Individuen identisch ist" (Hosaya et al. 2014: 194).

Schritt 3: Random-Intercept-Modell mit Prädiktoren auf Individual- und der Kontextebene

Dass nicht nur zeitvariierende, sondern auch zeitstabile Eigenschaften von Interesse sein können, wird im dritten Schritt berücksichtigt. Grundsätzlich werden im Falle von Mehrebenenmodellen für Paneldaten zeitkonstante Variablen als Kontextfaktoren betrachtet ${ }^{162}$. Ferner werden innerhalb dieses Modells neben den zeitvariierenden Individualvariablen L2-Kontextfaktoren (Personenebene) hinzugezogen, wie sich gemäß den folgenden Gleichungen darstellen lässt:

Formale Zwei- und Eingleichungsform des Random-Intercept-Modells mit Prädiktoren auf beiden Ebenen (vgl. Krause/Urban 2013: 28):
Ebene 1:
$\mathrm{Y}_{\mathrm{ti}}=\beta_{0 \mathrm{i}}+\beta_{1 \mathrm{i}} \mathrm{X}_{1 \mathrm{ti}}+\mathrm{e}_{\mathrm{ti}}$
Ebene 2:
$\beta_{0 \mathrm{i}}=\gamma_{00}+\gamma_{01} Z_{\mathrm{ti}}+\mathrm{u}_{0 \mathrm{i}}$
$\beta_{1 \mathrm{i}}=\gamma_{10}$

Eingleichungsform von (1.8) in (1.7):

$$
\mathrm{Y}_{\mathrm{ti}}=\gamma_{00}+\gamma_{01} \mathrm{Z}_{\mathrm{ti}}+\gamma_{10} \mathrm{X}_{1 \mathrm{ti}}+\mathrm{u}_{0 \mathrm{i}}+\mathrm{e}_{\mathrm{ti}}
$$

wobei das Modell um den Kontextprädiktor $Z_{\mathrm{ti}}$ erweitert wird.

Da es sich hierbei um Kontextvariablen der Personenebene handelt, fehlt die Betrachtung der Makroebene bisher. Um zeitstabile Effekte der Makroebene (Regionen) berücksichtigen zu können, wäre folglich eine dritte Ebene in die Modelle einzufügen, wie zum Abschluss des Kapitels noch dargestellt werden wird.

\section{Schritt 4: Random-Intercept-Random-Slope-Modell}

Im Unterschied zum Random-Intercept-Modell, das davon ausgeht, dass die zeitvariierenden L1-Prädiktoren für alle Kontexteinheiten konstant sind, wird im Random-Slope-Modell für Paneldaten abermals angenommen, dass die Effekte der L1-Prädiktoren zwischen den Kontexteinheiten (in diesem Fall Personen) variieren können - die L1-Variablen werden als ,random“ betrachtet. Es ergeben sich die folgenden Gleichungen:

Formale Zwei- und Eingleichungsform des Random-Slope-Modells (vgl. Krause/Urban 2013: 30; Hosaya et al. 2014: 195):

Ebene 1:

$$
\mathrm{Y}_{\mathrm{ti}}=\beta_{0 \mathrm{i}}+\beta_{1 \mathrm{i}} X_{1 \mathrm{ti}}+\mathrm{e}_{\mathrm{ti}}
$$

162 Selbstverständlich ist es möglich, dass Kontextfaktoren zeitvariierende Eigenschaften aufweisen, wie beispielsweise die nach Jahren variierende Erwerbsquote von Frauen in unterschiedlichen Regionen. Doch obwohl es sich hierbei um eine Eigenschaft auf Regionenebene handelt, wird sie im Mehrebenenmodell rein technisch betrachtet auf der ersten Ebene modelliert, weil es sich um eine zeitvariierende Variable handelt (vgl. Hosaya et al. 2014: 203). 
$\begin{array}{ll}\text { Ebene 2: } & \beta_{0 \mathrm{i}}=\gamma_{00}+\mathrm{u}_{0 \mathrm{i}} \\ & \beta_{1 \mathrm{i}}=\gamma_{10}+\mathrm{u}_{1 \mathrm{i}}\end{array}$

Eingleichungsform von (2.1) in (2.0):

$$
Y_{t i}=\gamma_{00}+\gamma_{10} X_{1 t i}+u_{1 i} X_{1 t i}+u_{0 i}+e_{t i}(2.2)
$$

Die Einschränkung auf dem Parameter $\beta_{1 \mathrm{i}}$ des Random-Intercept-Modells, dass die L1-Effekte für alle Kontexteinheiten identisch sind, wird im Random-Slope-Modell in der zweiten Gleichung durch eine Variation der zweiten Ebene zugelassen, indem das L2-Residuum $\mathrm{u}_{1 \mathrm{i}}$ hinzugefügt wird. Es ist notwendig, neben der Verteilungsannahme der L1-Residuen eine Verteilungsannahme für die L2-Residuen zu treffen, weil in diesem Modell zwei L2Residuen $u_{0 i}$ und $u_{1 i}$ vorhanden sind (vgl. Hosaya et al. 2014: 195). Eine Normalverteilung der L2-Residuen mit Mittelwerten von Null sowie eine Varianz-Kovarianzmatrix $\sum$ werden von Hosaya et al. (2014) angenommen (für die Grundlagen zur Varianz-Kovarianzmatrix s. Kapitel 12.2.1.).

„Über diese Matrix lässt sich bewerten, ob der Random-Koeffizient $\beta_{0 \mathrm{i}}$ über die Level-2-Einheiten variiert, ob der Effekt des Prädiktors $\mathrm{x}_{1 \mathrm{ti}}$ über die Level-2Einheiten variiert und ob ein Zusammenhang zwischen den variierenden Koeffizienten des Modells besteht" (Hosaya et al. 2014: 195).

Schritt 5: Slope-as-outcome-Modell

Abschließend kann auch für Paneldaten eine Cross-Level-Interaktion in die Mehrebenenmodelle eingeführt werden. Dies dient insbesondere dazu, die Varianz der Random Slopes über Kontextmerkmale (hier: Personenmerkmale) zu erklären (vgl. Krause/Urban 2013: 32). Beispielsweise könnte angenommen werden, dass der Effekt einer Veränderung der Kinderanzahl auf die innerhäusliche Arbeitsteilung zwischen den Geschlechtern variiert.

Formale Zwei- und Eingleichungsform des Intercepts-and-Slopes-asOutcome-Modells (vgl. Krause/Urban 2013: 33):
Ebene 1:
$\mathrm{Y}_{\mathrm{ti}}=\beta_{0 \mathrm{i}}+\beta_{1 \mathrm{i}} \mathrm{X}_{1 \mathrm{ti}}+\mathrm{e}_{\mathrm{ti}}$
Ebene 2:
$\beta_{0 \mathrm{i}}=\gamma_{00}+\gamma_{01} Z_{\mathrm{ti}}+\mathrm{u}_{0 \mathrm{i}}$
$\beta_{1 \mathrm{i}}=\gamma_{10}+\gamma_{11} Z_{\mathrm{ti}}+\mathrm{u}_{1 \mathrm{i}}$

Eingleichungsform von (2.4) in (2.3):

$\mathrm{Y}_{\mathrm{ti}}=\gamma_{00}+\gamma_{01} \mathrm{Z}_{\mathrm{ti}}+\gamma_{10} \mathrm{X}_{1 \mathrm{ti}}+\gamma_{11} \mathrm{Z}_{\mathrm{ti}} \mathrm{X}_{1 \mathrm{ti}}+\mathrm{u}_{1 \mathrm{i}} \mathrm{X}_{1 \mathrm{ti}}+\mathrm{u}_{0 \mathrm{i}}+\mathrm{e}_{\mathrm{ti}}$

Mehrebenengleichungen für Längsschnittdaten mit drei Ebenen

Wie bereits erläutert, fehlt in den vorherigen Gleichungen der Mehrebenenanalyse für Paneldaten die Berücksichtigung der Makroebene. Um dieses Manko auszugleichen, werden nachfolgend die Gleichungen für ein Kontextmodell, das durch eine dritte Ebene erweitert wird (Kennzeichnung: Index j, der sich auf die Makrokontexteinheiten, d. h. die Regionen bezieht), mit aggregierten Variablen der zweiten und dritten Ebene angeführt. Der L1Prädiktor $\mathrm{x}_{\mathrm{tij}}$ sowie die Kontextvariablen (die Bildung der Kontextvariablen erfolgt auf der zweiten Ebene durch Aggregation innerhalb der Personen und 
auf der dritten Ebene durch Aggregation innerhalb der Regionen) werden hierbei am Gesamtmittelwert ${ }^{163}$ zentriert (vgl. Hosaya et al. 2014: 201), d. h. es ,[...] wird um den Gesamtmittelwert einer Variablen in der gesamten Stichprobe zentriert" (Krause/Urban 2013: 7). Auch im Falle einer Analyse von Paneldaten als Mehrebenenmodell ist eine Zentrierung der Daten empfehlenswert. Eine Zentrierung am gruppenübergreifenden Gesamtmittelwert eröffnet, wie bereits thematisiert, die Vorteile, dass die Ergebnisse einfacher interpretiert werden können, Multikollinearität entgegengewirkt werden kann; außerdem werden unerwünschte Korrelationen von Level-1-, -2- und 3-Variablen mit Cross-Level-Interaktionen vermieden (vgl. Krause/Urban 2013: 7). Beispielsweise könnte durch die Zentrierung der unabhängigen Variable ,Erwerbseinkommen der Frau' zum ersten Messzeitpunkt (Prädiktor $\mathrm{x}_{1} \mathrm{ij}$ auf Level-1) der Steigungskoeffizient so interpretiert werden, dass die geschätzte Steigung Auskunft darüber gibt, um wie viele Einheiten sich die innerhäusliche Arbeitsteilung im Vergleich zum ersten Messzeitpunkt ändert, wenn eine Frau einen Euro mehr verdient als von ihr erwartet wird. Der Grand-Mean würde an dieser Stelle interpretiert werden als ,Erwerbseinkommensnorm für Frauen'.

Gleichung Ebene 1: Messwert

Das Basismodell zur Modellierung des Messwertes $\mathrm{y}_{\mathrm{tij}}$ der Person i zum Zeitpunkt $t$ in Region $\mathrm{j}$ lässt sich wie folgt formal darstellen:

Ebene 1: $\quad y_{\mathrm{tij}}=\beta_{0 \mathrm{ij}}+\beta_{1 \mathrm{ij}}\left(\mathrm{x}_{\mathrm{tij}}-\mathrm{x}_{\ldots}\right)+\mathrm{e}_{\mathrm{tij}} \quad$ (2.6)

163 Weitere Zentrierungsoptionen sind die bereits thematisierte Zentrierung um den Nullpunkt und die Zentrierung um gruppenspezifische Mittelwerte; s. hierzu Krause/Urban (2013) und Hosaya et al. (2014). Sollen ein within-Effekt von einem between-Effekt separiert werden, muss der L1-Prädiktor am Gruppenmittelwert zentriert werden (vgl. Hosaya et al. 2014: 199). „Um die Within- und Between-Komponenten zu separieren, müssen die zeitveränderlichen Determinanten in zwei (Teil-)Variablen zerlegt werden. Dabei wird der BetweenAnteil der Determinante (gemessen als seine pro Person beobachtete, zeitlich durchschnittliche Ausprägung: $x i$ ) als Level-2-Variable operationalisiert. Und der Within-Anteil der Determinante wird als Level-1-Variable, welche über die Abweichung vom individuellen Mittelwert gebildet wird $\left(\Delta \mathrm{x}_{\mathrm{ti}}=\mathrm{x}_{\mathrm{ti}}-x_{i}\right)$, berechnet, was rein technisch betrachtet der [...] Group-Mean-Zentrierung entspricht. Beide (Teil-)Prädiktoren werden sodann gemeinsam in die Modellschätzung aufgenommen. Folglich wird der Within-Personeneffekt einer Determinanten über die Spezifikation eines group-mean-zentrierten Prädiktors geschätzt, wohingegen der Between-Personeneffekt über die Spezifikation eines grand-mean-zentrierten Prädiktors ermittelt wird“ (Krause/Urban 2013: 16). Für die formale Gleichung eines Modells für Paneldaten mit drei Ebenen, das dementsprechend einen Effekt der Prädiktorvariable zwischen Personen vom Effekt einer Variable innerhalb der Personen trennt, s. Hosaya et al. 2014: 202 (eine entsprechende Gleichung für Querschnittsdaten ist auf S. 199 zu finden). 
wobei:

$\mathrm{y}_{\mathrm{tij}}$ : $\quad$ Messwert der abhängigen Variable (hier: innerhäusliche Arbeitsteilung) zu verschiedenen Messzeitpunkten von Person i innerhalb einer Region $\mathrm{j}$

$\beta_{0 \mathrm{ij}}$ : Regressionskonstante/Intercept; da die zeitvariierende Prädiktorvariable $\mathrm{x}_{\mathrm{tij}}$ am Gesamtmittelwert $\mathrm{x}_{\ldots} .$. zentriert wurde, ist $\beta_{0 \mathrm{ij}}$ der erwartete Messwert der abhängigen Variablen, wenn die Prädiktorvariable $\mathrm{x}_{\mathrm{tij}}$ einen mittleren Wert aufweist

$\beta_{1 \mathrm{ij}}$ : Regressionssteigung/Slope

$\mathrm{x}_{\mathrm{tij}}: \quad$ Prädiktorvariable

$\mathrm{x}_{\mathrm{tij}}-\mathrm{x}_{\ldots}$ : Zentrierung am Gesamtmittelwert

$\mathrm{e}_{\mathrm{tij}}$ : $\quad$ L1-Residuum/Fehlerterm; $\mathrm{t}=$ Messzeitpunkte; $\mathrm{i}=$ Person; $\mathrm{j}=$ Region

Gleichungen Ebene 2: Person

Schätzung der nach Personen variierenden Koeffizienten $\beta_{0 \mathrm{ij}}$

Ebene 2:

$$
\begin{aligned}
& \beta_{0 i \mathrm{ij}}=\gamma_{00 \mathrm{j}}+\gamma_{01 \mathrm{j}}\left(\mathrm{x}_{\mathrm{.ij}}-\mathrm{x}_{\ldots}\right)+\mathrm{u}_{0 \mathrm{ij}} \\
& \beta_{1 \mathrm{ij}}=\gamma_{10 \mathrm{j}}
\end{aligned}
$$

wobei:

$\beta_{0 \mathrm{ij}}: \quad$ nach Personen variierende Koeffizienten

$\gamma_{00 j}$ : $\quad$ nach Region variierender Intercept

$\gamma_{01 \mathrm{j}}$ : nach Region variierender Effekt

$\mathrm{X} . \mathrm{ij}$ : $\quad$ zentrierter, über die Messzeitpunkte aggregierter stabiler Prädiktor

X...: Gesamtmittelwert

$\mathrm{u}_{0 \mathrm{ijj}}: \quad$ Fehlerterm Ebene 2 (L2-Residuum)

„In der ersten Level-2-Gleichung werden die nach den Personen variierenden Koeffizienten $\beta 0_{i j}$ auf Basis eines nach der Region variierenden Intercepts $\gamma_{00 j}$ und eines nach der Region variierenden Effektes $\gamma_{01 \mathrm{j}}$ des zentrierten, über die Messzeitpunkte aggregierten, zeitstabilen Prädiktors X.ij einer Person i in Region j modelliert" (Hosaya et al. 2014: 202).

Gleichungen Ebene 3: Kontexteinheiten - NUTS-Regionen

Schätzung der nach Region variierenden Koeffizienten $y_{00 j}$

Ebene 3: $\quad \begin{array}{ll}\gamma_{00 \mathrm{j}}=\gamma_{000}+\gamma_{001}\left(\mathrm{x}_{. . \mathrm{j}}-\mathrm{x}_{\ldots}\right)+\mathrm{u}_{00 \mathrm{j}} \quad \text { (2.8) } \\ \gamma_{01 \mathrm{j}}=\gamma_{010} \\ \gamma_{10 \mathrm{j}}=\gamma_{100}\end{array}$

wobei:

$\mathrm{y}_{00 \mathrm{j}}$ : nach Regionen variierende Koeffizienten

$\gamma_{000}$ : feste Konstante

$\gamma_{001}$ : Kontexteffekt der Regionen auf AV (Steigungskoeffizient des Kontextmerkmals) 
$\mathrm{x}_{. \mathrm{j}}$ : $\quad$ zentrierter, über die Personen und Messzeitpunkte aggregierter Prädiktor (variiert ausschließlich zwischen den Regionen)

$\mathrm{x}$...: $\quad$ Gesamtmittelwert

$\mathrm{u}_{00 \mathrm{j}}: \quad$ Fehlerterm Ebene 3 (L3-Residuum)

„Die nach der Region variierenden Koeffizienten $\gamma_{00 j}$ werden auf Level-3 auf Basis einer festen Konstante $\gamma_{000}$ und des zentrierten, über die Personen und Messzeitpunkte aggregierten Prädiktors $\mathrm{x}_{. \mathrm{j}}$ modelliert. $\mathrm{X}_{\mathrm{.} . \mathrm{j}}$ variiert somit lediglich $z w i$ schen den Regionen. uooj ist ein Level-3 Residuum. Der nach den Personen variierende Effekt $\beta_{1 \mathrm{ij}}$ des Prädiktors $\mathrm{x}_{\mathrm{tij}}$ wird der Einfachheit halber über die Level2-Gleichungen auf $\gamma_{100}$ restringiert" (Hosaya et al. 2014: 202, Hervorhebungen im Original; die Verf.).

Gesamtgleichung/Eingleichungsform von (2.8) in (2.7) in (2.6):

$\mathrm{y}_{\mathrm{tij}}=\left[\gamma_{000}+\gamma_{001}\left(\mathrm{x}_{. . \mathrm{j}}-\mathrm{x}_{\ldots} ..\right)+\mathrm{u}_{00 \mathrm{j}}\right]+\gamma_{010}\left(\mathrm{x}_{\mathrm{.ij}}-\mathrm{x}_{\ldots} \ldots\right)+\mathrm{u}_{0 \mathrm{ij}}+\gamma_{100}\left(\mathrm{x}_{\mathrm{tij}}-\mathrm{x}_{\ldots} ..\right)+\mathrm{e}_{\mathrm{tij}}$

$=\gamma_{000}+\gamma_{001}\left(\mathrm{x}_{. \mathrm{j}}-\mathrm{x}_{\ldots} ..\right)+\gamma_{010}\left(\mathrm{x}_{\mathrm{ijj}}-\mathrm{x}_{\ldots}.\right)+\gamma_{100}\left(\mathrm{x}_{\mathrm{tij}}-\mathrm{x}_{\ldots} \ldots\right)+\mathrm{u}_{00 \mathrm{j}}+\mathrm{u}_{0 \mathrm{ij}}+\mathrm{e}_{\mathrm{tij}}$

wobei:

$\gamma_{000}$ : Konstante, d. h. es ergibt sich der erwartete Messwert der abhängigen Variablen, wenn die Prädiktorvariablen einen mittleren Wert der innerhalb der Regionen aggregierten L3-Variablen $\mathrm{x}_{. . j}$, der innerhalb der Personen aggregierten L2-Variablen $\mathrm{x}_{\mathrm{ij}}$ und der innerhalb der Messwerte aggregierten L1-Variablen $\mathrm{x}_{\mathrm{tij}}$ aufweisen

$\gamma_{001}$ : $\quad$ Kontexteffekt der Regionen auf die abhängige Variable/Effekt der Unterschiedlichkeit der Ebene-3-Einheiten

$\gamma_{010}$ : Kontexteffekt der aggregierten Personenvariable/Effekt der Unterschiedlichkeit der Ebene-2-Einheiten

$\gamma_{100}$ : Effekt der Abweichung der individuellen zeitvariierenden Prädiktorvariable vom Gesamtmittelwert/Effekt der Variabilität der zeitvarianten Prädiktorvariable der Ebene-1-Einheiten

Mithilfe dieses Modells soll aufgezeigt werden, inwiefern die Ausprägungen der Kriteriumsvariablen (innerhäusliche Arbeitsteilung) von den Effekten der zeitvariierenden Prädiktoren auf der ersten Ebene, von den Effekten der innerhalb von Personen aggregierten Variablen auf der zweiten Ebene und von den Effekten der innerhalb von Regionen aggregierten Variablen auf der dritten Ebene beeinflusst werden.

„In der Gesamtgleichung zeigt sich, dass $\gamma_{001}$ den Kontexteffekt der Region auf die abhängige Variable darstellt, $\gamma_{010}$ den Effekt der aggregierten Personenvariable und $\gamma_{100}$ denjenigen der Abweichungen der individuellen, zeitvariierenden Prädiktorvariable auf Level-1 vom Gesamtmittelwert" (Hosaya et al. 2014: 202).

Zusammenfassend kann folgende allgemeine Vorgehensweise zur Verknüpfung von längsschnittlichen Modellen auf mehreren Ebenen festgehalten werden (vgl. Langer 2004: 190ff.; Hosaya et al 2014: 191ff.): 
1. Zunächst wird das ,Random-Intercept-Only-Modell' geschätzt (enthält nur die Regressionskonstante), ,[...] mit dem bewertet werden kann, ob eine Personenheterogenität hinsichtlich der längsschnittlich erhobenen abhängigen Variablen vorliegt" (Hosaya et al. 2014: 191).

2. Das Random-Intercept-Only-Modell wird durch eine zeitabhängige Kovariate ergänzt und anschließend bezeichnet als ,RandomIntercept-Modell'.

3. Das Modell wird um einen zeitinvarianten, personenbezogenen Kontextprädiktor erweitert, um Effekte zeitstabiler Eigenschaften der Personen auf die abhängige Variable zu überprüfen.

4. Es folgt eine Erweiterung des Random-Intercept-Modells um einen nach den Personen variierenden Effekt einer zeitvariierenden Prädiktorvariable, das sogenannte ,Random-Intercept-Random-SlopeModell' (je nach Forschungsinteresse kann es sinnvoll sein, die Reihenfolge des dritten und vierten Schritts zu tauschen).

5. Abschließend werden Wechselwirkungseffekte (Cross-LevelInteraktionen) zwischen den Ebenen eingeführt.

Falls ein Forschungsinteresse an Effekten der Makroebene besteht, sollten die Modelle um eine dritte Ebene erweitert werden (s. hierzu Hosaya et al. 2014: 201). 


\title{
13. Darstellung und Diskussion der Ergebnisse
}

\subsection{Deskriptive Darstellung der innerhäuslichen Arbeitsteilung im Ländervergleich}

\begin{abstract}
Abbildung 12 stellt die arithmetischen Mittelwerte der Indikatoren „Rechnungen bezahlen (dieser Indikator ist nicht für Norwegen vorhanden), Einkaufen, Reparaturen, Mahlzeiten zubereiten und Staubsaugen" differenziert nach Ländern zum Zeitpunkt der ersten Erhebungswelle des GGS graphisch dar. Da die Skala einen Wertebereich von eins (Frau erledigt die jeweilige Tätigkeit im Haushalt immer) bis fünf (Mann erledigt die jeweilige Tätigkeit im Haushalt immer) umfasst, würde ein Wert von drei einer durchschnittlichen egalitären Arbeitsteilung entsprechen. Es zeigt sich jedoch, dass in sämtlichen Ländern die Tätigkeiten ,Zubereitung von Mahlzeiten, Staubsaugen, Einkäufe und Rechnungen bezahlen (Ausnahme: Georgien)“ durchschnittlich überwiegend von Frauen verrichtet werden, wobei der Effekt am deutlichsten für die Zubereitung der Mahlzeiten zu erkennen ist. Lediglich die Reparaturen werden meist von Männern ausgeführt - die Darstellung ist insgesamt in höchstem Maße geschlechtsspezifisch segregiert. Auffallend ist, dass in Georgien im Vergleich zu den anderen Ländern durchschnittlich Frauen am häufigsten die Mahlzeiten zubereiten und auch im Falle des Indikators „Rechnungen bezahlen“ stellt Georgien insofern einen Sonderfall dar, als es das einzige Land ist, in dem diese Tätigkeit im Durchschnitt eher von Männern übernommen wird.
\end{abstract}

\subsection{Deskriptive Darstellung der innerhäuslichen Arbeitsteilung im NUTS1-Regionen-Vergleich}

Ähnlich wie in Abbildung 12 werden in Abbildung 13 die arithmetischen Mittelwerte der Indikatoren „Rechnungen bezahlen (dieser Indikator ist nicht für Norwegen vorhanden), Einkaufen, Reparaturen, Mahlzeiten zubereiten und Staubsaugen“ (Wertebereich 1-5) zum Zeitpunkt der ersten Erhebungswelle des GGS graphisch dargestellt, diesmal jedoch nicht differenziert nach Ländern, sondern differenziert nach NUTS1-Regionen. Erneut ist festzuhalten, dass die Tätigkeiten „Zubereitung von Mahlzeiten, Staubsaugen, Einkäufe und Rechnungen bezahlen" durchschnittlich überwiegend von Frauen verrichtet werden (mit Ausnahme des Rechnungen bezahlens in Macroregiunea Patru, Rumänien), wobei sich der Effekt wieder am deutlichsten für die Zubereitung der Mahlzeiten zeigt: Die Mittelwerte schwanken zwischen 
min. 1,5 (in Alföld és Észak, Ungarn) und max. 2,39 (im Saarland, Deutschland). In allen NUTS1-Regionen werden abermals die Reparaturen meist von Männern ausgeführt. Die NUTS1-Region, die einer egalitären Arbeitsteilung (3) der Reparaturen am nächsten kommt, ist Berlin mit einem Mittelwert von 3,31. Relevant für die Interpretation sind an dieser Stelle weniger geringfügige Unterschiede zwischen den Regionen, die ohnehin aufgrund der zu kleinen und zu umfangreichen Darstellung in Abbildung 13 kaum lesbar sind (die Mittelwerte können im Detail Tabelle 21 entnommen werden), als vielmehr die Erkenntnis einer geschlechtsspezifischen innerhäuslichen Segregation, die auch für sämtliche Regionen konstatiert werden kann.

\section{Abbildung 12:}

Mittelwerte der Hausarbeiten im Ländervergleich, GGS Welle 1

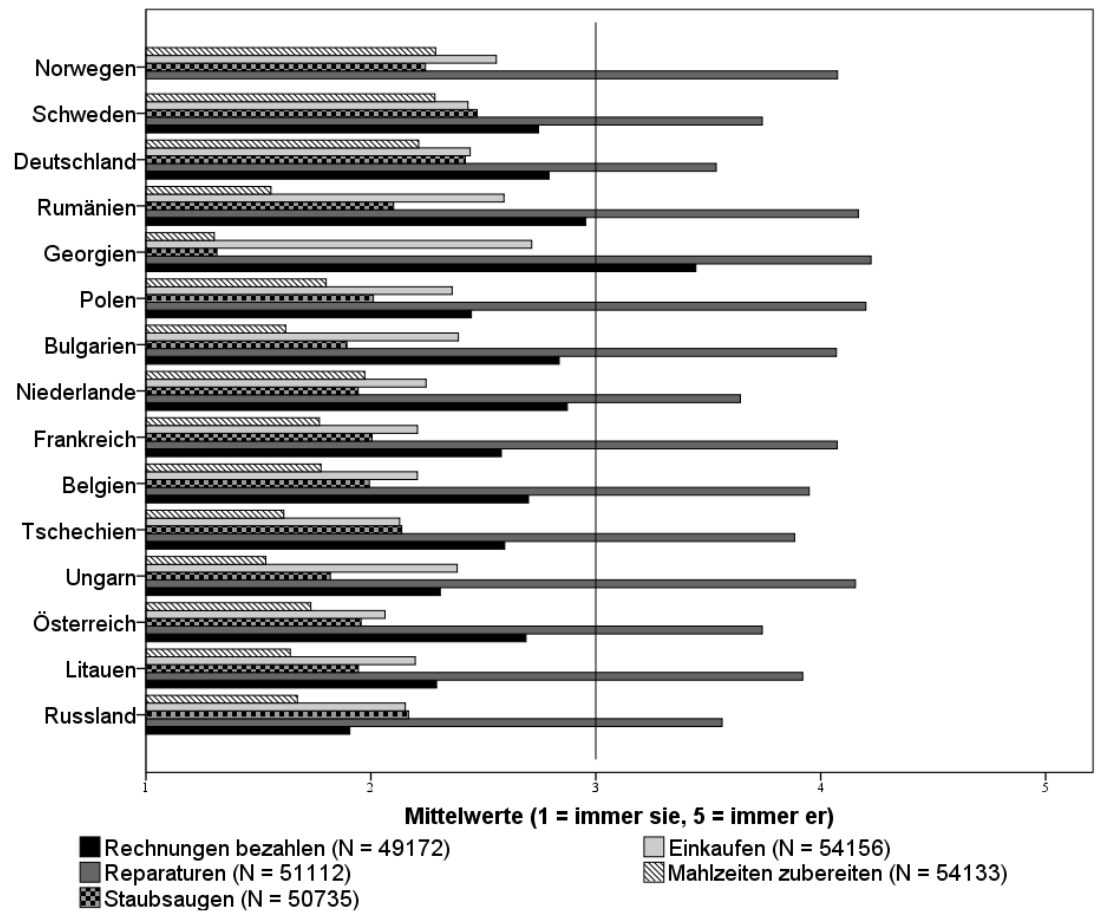

Quelle: GGS Welle 1, eigene Berechnungen. N (L1) = 40581; N (L2) $=15$ 


\section{Abbildung 13: Mittelwerte der Hausarbeiten im regionalen Vergleich (NUTS1), GGS Welle 1}

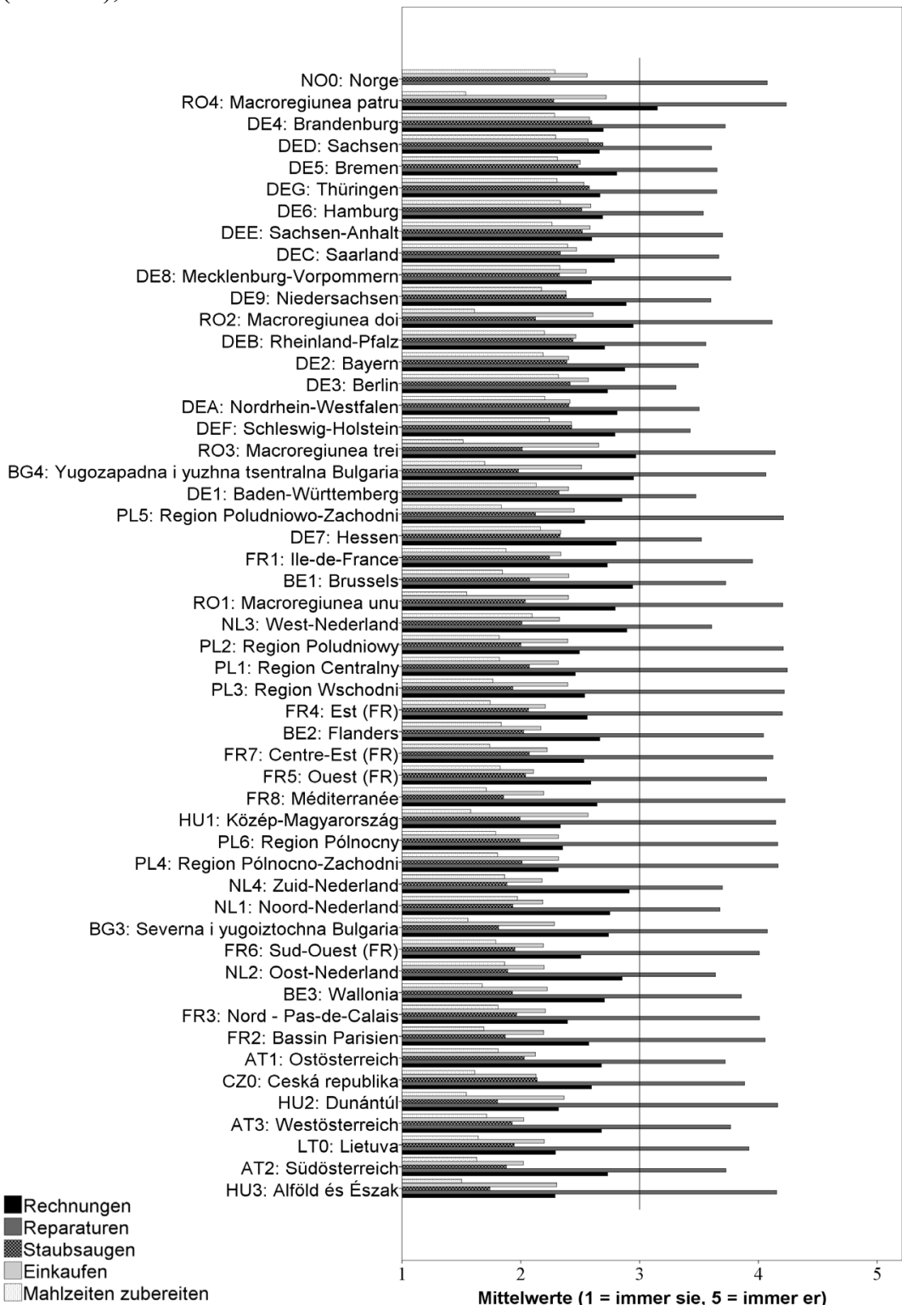

Quelle: GGS Welle 1, eigene Berechnungen. N (L1) = 33380; N (L2) $=52$ 
Tabelle 21: Mittelwerte der Hausarbeiten im regionalen Vergleich (NUTS1), GGS Welle 1

\begin{tabular}{|c|c|c|c|c|c|}
\hline & $\begin{array}{l}\text { Mahlzeiten } \\
\text { zubereiten }\end{array}$ & $\begin{array}{l}\text { Staub- } \\
\text { saugen }\end{array}$ & Einkaufen & $\begin{array}{l}\text { Rechnungen } \\
\text { bezahlen }\end{array}$ & Reparaturen \\
\hline \multicolumn{6}{|c|}{ Deutschland } \\
\hline $\begin{array}{l}\text { Baden- } \\
\text { Württemberg }\end{array}$ & 2,13 & 2,32 & 2,40 & 2,85 & 3,47 \\
\hline Bayern & 2,19 & 2,39 & 2,40 & 2,88 & 3,49 \\
\hline Berlin & 2,32 & 2,42 & 2,57 & 2,73 & 3,31 \\
\hline Brandenburg & 2,28 & 2,60 & 2,58 & 2,69 & 3,72 \\
\hline Bremen & 2,31 & 2,48 & 2,50 & 2,81 & 3,65 \\
\hline Hamburg & 2,33 & 2,51 & 2,59 & 2,69 & 3,54 \\
\hline Hessen & 2,17 & 2,33 & 2,34 & 2,80 & 3,52 \\
\hline $\begin{array}{l}\text { Mecklenburg- } \\
\text { Vorpommern }\end{array}$ & 2,33 & 2,32 & 2,55 & 2,59 & 3,77 \\
\hline Niedersachsen & 2,17 & 2,38 & 2,38 & 2,89 & 3,60 \\
\hline $\begin{array}{l}\text { Nordrhein- } \\
\text { Westfalen }\end{array}$ & 2,20 & 2,40 & 2,41 & 2,81 & 3,50 \\
\hline Rheinland-Pfalz & 2,20 & 2,44 & 2,46 & 2,70 & 3,56 \\
\hline Saarland & 2,39 & 2,33 & 2,47 & 2,79 & 3,67 \\
\hline Sachsen & 2,29 & 2,69 & 2,57 & 2,66 & 3,61 \\
\hline Sachsen-Anhalt & 2,26 & 2,52 & 2,58 & 2,60 & 3,70 \\
\hline Schleswig- & 2,24 & 2,43 & 2,43 & 2,79 & 3,43 \\
\hline Holstein & & & & & \\
\hline Thüringen & 2,30 & 2,58 & 2,53 & 2,67 & 3,65 \\
\hline \multicolumn{6}{|c|}{ Österreich } \\
\hline Ostösterreich & 1,81 & 2,03 & 2,12 & 2,68 & 3,72 \\
\hline Südösterreich & 1,63 & 1,88 & 2,02 & 2,73 & 3,73 \\
\hline Westösterreich & 1,71 & 1,93 & 2,02 & 2,68 & 3,77 \\
\hline \multicolumn{6}{|c|}{ Belgien } \\
\hline Brussels & 1,84 & 2,07 & 2,40 & 2,94 & 3,72 \\
\hline Flanders & 1,84 & 2,02 & 2,17 & 2,67 & 4,04 \\
\hline Wallonia & 1,68 & 1,93 & 2,22 & 2,70 & 3,86 \\
\hline \multicolumn{6}{|c|}{ Frankreich } \\
\hline Ile-de-France & 1,88 & 2,24 & 2,34 & 2,73 & 3,95 \\
\hline Bassin Parisien & 1,69 & 1,87 & 2,19 & 2,57 & 4,06 \\
\hline $\begin{array}{l}\text { Nord-Pas-de- } \\
\text { Calais }\end{array}$ & 1,81 & 1,97 & 2,21 & 2,39 & 4,01 \\
\hline Est & 1,74 & 2,07 & 2,21 & 2,56 & 4,20 \\
\hline Ouest & 1,83 & 2,04 & 2,11 & 2,59 & 4,07 \\
\hline
\end{tabular}




\begin{tabular}{|c|c|c|c|c|c|}
\hline Sud-Ouest & 1,79 & 1,95 & 2,19 & 2,51 & 4,01 \\
\hline Centre-Est & 1,74 & 2,07 & 2,22 & 2,53 & 4,12 \\
\hline Méditerranée & 1,71 & 1,86 & 2,19 & 2,64 & 4,22 \\
\hline \multicolumn{6}{|c|}{ Niederlande } \\
\hline $\begin{array}{l}\text { Noord- } \\
\text { Nederland }\end{array}$ & 1,97 & 1,93 & 2,18 & 2,75 & 3,68 \\
\hline Oost-Nederland & 1,86 & 1,89 & 2,20 & 2,85 & 3,64 \\
\hline West-Nederland & 2,10 & 2,01 & 2,33 & 2,89 & 3,61 \\
\hline West-Nederland & 1,86 & 1,88 & 2,18 & 2,91 & 3,70 \\
\hline \multicolumn{6}{|c|}{ Bulgarien } \\
\hline $\begin{array}{l}\text { Severna i } \\
\text { yugoiztochna } \\
\text { Bulgaria }\end{array}$ & 1,55 & 1,81 & 2,28 & 2,74 & 4,08 \\
\hline $\begin{array}{l}\text { Yugozapadna i } \\
\text { yuzhna tsen- } \\
\text { tralna Bulgaria }\end{array}$ & 1,70 & 1,98 & 2,51 & 2,95 & 4,06 \\
\hline \multicolumn{6}{|c|}{ Litauen } \\
\hline Lietuva & 1,64 & 1,94 & 2,20 & 2,29 & 3,92 \\
\hline \multicolumn{6}{|c|}{ Polen } \\
\hline $\begin{array}{l}\text { Region } \\
\text { Centralny }\end{array}$ & 1,82 & 2,07 & 2,32 & 2,46 & 4,24 \\
\hline $\begin{array}{l}\text { Region } \\
\text { Poludniowy }\end{array}$ & 1,82 & 2,00 & 2,40 & 2,49 & 4,21 \\
\hline $\begin{array}{l}\text { Region } \\
\text { Wschodni }\end{array}$ & 1,76 & 1,93 & 2,40 & 2,54 & 4,22 \\
\hline $\begin{array}{l}\text { Region Pólnoc- } \\
\text { no-Zachodni }\end{array}$ & 1,80 & 2,01 & 2,32 & 2,32 & 4,17 \\
\hline $\begin{array}{l}\text { Region Polud- } \\
\text { niowo-Zachodni }\end{array}$ & 1,84 & 2,12 & 2,45 & 2,54 & 4,21 \\
\hline $\begin{array}{l}\text { Region } \\
\text { Pólnocny }\end{array}$ & 1,80 & 1,99 & 2,32 & 2,35 & 4,16 \\
\hline \multicolumn{6}{|c|}{ Rumänien } \\
\hline $\begin{array}{l}\text { Macroregiunea } \\
\text { unu }\end{array}$ & 1,55 & 2,04 & 2,40 & 2,79 & 4,21 \\
\hline $\begin{array}{l}\text { Macroregiunea } \\
\text { doi }\end{array}$ & 1,61 & 2,12 & 2,61 & 2,95 & 4,12 \\
\hline $\begin{array}{l}\text { Macroregiunea } \\
\text { trei }\end{array}$ & 1,51 & 2,01 & 2,66 & 2,97 & 4,14 \\
\hline $\begin{array}{l}\text { Macroregiunea } \\
\text { patru }\end{array}$ & 1,54 & 2,23 & 2,72 & 3,15 & 4,23 \\
\hline
\end{tabular}




\begin{tabular}{lccccc}
\hline \multicolumn{5}{c}{ Tschechien } \\
\hline Ceská republika & 1,61 & 2,14 & 2,13 & 2,60 & 3,88 \\
\hline \multicolumn{5}{c}{ Ungarn } \\
\hline Közép- & 1,58 & 1,99 & 2,57 & 2,33 & 4,15 \\
Magyarország & \multicolumn{7}{c}{ Norwegen } & & \\
Dunántúl & 1,54 & 1,80 & 2,36 & 2,32 & 4,16 \\
Alföld és Észak & 1,50 & 1,74 & 2,30 & 2,29 & 4,15 \\
\hline \multicolumn{7}{c}{ Norge } & 2,24 & 2,56 & - & 4,07 \\
\hline Norge
\end{tabular}

Quelle: GGS Welle 1, eigene Berechnungen. N (L1) = 33380; N (L2) = 52

Weiterführend werden die Determinanten der innerhäuslichen Arbeitsteilung schrittweise analysiert. Zunächst werden die Ergebnisse klassischer linearer Regressionsanalysen länder- und regimevergleichend zur Erklärung der innerhäuslichen Arbeitsteilung skizziert. Ferner wird diskutiert, dass diese Vorgehensweise - neben der inhaltlichen Argumentation einer notwendigen Fokussierung auf Regionen - auch methodische Grenzen in Bezug auf die Berücksichtigung hierarchischer Daten aufweist.

\subsection{Klassische OLS-Regressionsmodelle zur Erklärung der innerhäuslichen Arbeitsteilung im Länder- und Regimevergleich}

Um auf klassische Weise die Stärke der Kontexteffekte der Länder zu bestimmen, ist die Berechnung eines linearen Regressionsmodells (s. Tabelle 22), dass Dummyvariablen für die jeweiligen Länder enthält, eine Möglichkeit (als Referenzkategorie wurde Schweden ausgewählt).

Durch Kenntnis der Länderzugehörigkeit können 6,4\% der Varianz des „Task-Participation-Index“ statistisch erklärt werden. Frauen, die in Schweden wohnhaft sind, erreichen im Durchschnitt einen „Task-ParticipationIndex" von 5,69. Frauen (fast) aller anderen Länder weichen hoch signifikant negativ, d. h. nach unten von diesem Wert ab, respektive die innerhäusliche Arbeitsteilung ist in (fast) allen anderen Ländern im Vergleich zu Schweden traditioneller (mit zwei Ausnahmen: 1. zwischen Schweden und Norwegen ist kein signifikanter Unterschied zu konstatieren; 2. zwischen Schweden und Deutschland ist der Unterschied zwar nicht hochsignifikant, doch zumindest signifikant). Der größte Unterschied ist für Georgien festzustellen; hier scheint die Arbeitsteilung vergleichsweise am traditionellsten organisiert zu sein. 
Tabelle 22: Klassische OLS-Regression zur Erklärung der innerhäuslichen Arbeitsteilung durch Länderunterschiede

\begin{tabular}{|c|c|c|c|}
\hline \multirow[b]{2}{*}{ Konstante } & \multicolumn{2}{|c|}{ B (Standardfehler) } & \multirow[t]{2}{*}{ [std. B] } \\
\hline & $5,690^{* * *}$ & $(0,032)$ & \\
\hline \multicolumn{4}{|c|}{$\begin{array}{l}\text { Dummyvariablen } \\
\text { (Referenzkategorie: Schweden) }\end{array}$} \\
\hline D_Bulgarien & $-0,891^{* * *}$ & $(0,041)$ & {$[-0,155]$} \\
\hline D_Russland & $-0,818^{* * *}$ & $(0,041)$ & {$[-0,143]$} \\
\hline D_Georgien & $-1,416^{* * *}$ & $(0,046)$ & {$[-0,201]$} \\
\hline D_Deutschland & $-0,126^{\star *}$ & $(0,041)$ & {$[-0,022]$} \\
\hline D_Frankreich & $-0,792^{\star \star *}$ & $(0,042)$ & {$[-0,135]$} \\
\hline D_Ungarn & $-0,991^{* * *}$ & $(0,040)$ & {$[-0,184]$} \\
\hline D_Niederlande & $-0,687^{* * *}$ & $(0,043)$ & {$[-0,113]$} \\
\hline D_Norwegen & $-0,006$ & $(0,039)$ & {$[-0,001]$} \\
\hline D_Österreich & $-0,947^{\star \star *}$ & $(0,051)$ & {$[-0,111]$} \\
\hline D_Belgien & $-0,794^{* * *}$ & $(0,045)$ & {$[-0,115]$} \\
\hline D_Litauen & $-0,919^{\star * *}$ & $(0,045)$ & {$[-0,136]$} \\
\hline D_Polen & $-0,588^{* * *}$ & $(0,037)$ & {$[-0,133]$} \\
\hline D_Tschechien & $-0,972^{* * *}$ & $(0,043)$ & {$[-0,156]$} \\
\hline Korr. $R^{2}$ & & & \\
\hline$N$ & & & \\
\hline
\end{tabular}

Quelle: GGS (Welle 1), eigene Berechnungen.

Sig. $=* * * \mathrm{p}<0,001 ; * * \mathrm{p}<0,01 ; * \mathrm{p}<0,05$

Anmerkung: Die Fallzahl variiert im Vergleich zu Abbildung 12, da der Indikator „Rechnungen bezahlen“ nicht im TPI enthalten ist.

Problematisch an dieser klassischen OLS-Regression ist die Annahme des FTests, dass die Residuen von Frauen i in den Ländern $\mathrm{j}$ nicht miteinander korreliert sind (vgl. Langer 2009: 36). 
„Da der Residualterm $\mathrm{e}_{\mathrm{ij}}$ alle Mess- und Vorhersagefehler erfasst, die auf nicht erhobenen [sic!] exogene Merkmale [...] zurückzuführen sind, ist diese Annahme des F-Tests auf der [Kontextebene] eindeutig verletzt. Daher ist der F-Test im klassischen Sinne bei hierarchischen Datenstrukturen nicht anwendbar" (Langer 2009: 36).

Auch der T-Test unterstellt, dass die Residuen auf der Länderebene nicht korreliert sind, was erneut aufgrund der hierarchischen Daten nicht gewährleistet werden kann. Werden diese Annahmen missachtet, ist eine Verzerrung der Signifikanztests die Folge. Die Voraussetzung, dass Individuen statistisch unabhängig voneinander sind, ist wegen der hierarchischen Datenstruktur nicht erfüllt. Innerhalb der Länder existieren Gruppen, die sich überzufällig ähnlich sind. Würde weiterführend eine unabhängige Individualvariable, wie beispielsweise die Paarbeziehungsdauer, in die Regression aufgenommen, wird implizit angenommen, dass der L1-Effekt für alle Länder gleich stark bzw. identisch ist (vgl. Langer 2009: 37). Doch ist anzunehmen, dass sich abgesehen von den Niveauunterschieden der Konstanten auch die Steigungskoeffizienten zwischen den Ländern unterscheiden und folglich die Paarbeziehungseffekte nicht für alle Länder identisch sind.

,[...] multilevel models are needed because grouped data observations from the same group are generally more similar to each other than the observations from different groups, and this violates the assumption of independence of all observations. The amount of variance can be expressed as a correlation coefficient: the intraclass correlation" (Hox et al. 2018: 12).

Im folgenden Schritt (s. Tabelle 23) soll daher unter Berücksichtigung der Regimetypen untersucht werden, ob sich die Steigungskoeffizienten der Individualvariablen zwischen den Regimen unterscheiden, indem jeweils ein separates Regressionsmodell für jeden Regimetypus geschätzt wird. Die Berechnung von getrennten Regressionen gilt als eine einfache Methode, Unterschiede zwischen den Regimen aufzuzeigen. Zunächst ist bezüglich der Niveauunterschiede der Regressionskonstanten festzustellen, dass sich diese geringfügig unterscheiden: Der höchste $(5,060)$ Wert ist im sozialdemokratischen Typus vorzufinden, gefolgt vom konservativen Regime $(4,692)$ sowie den Niederlanden $(4,672)$, wobei im postsozialistischen Typus die niedrigste Konstante $(4,362)$ aufzuweisen ist. Auch zwischen den unstandardisierten Steigungskoeffizienten sind geringfügige Unterschiede festzustellen. Frauen mit hohem Bildungsstand haben im Vergleich zu Frauen mit niedrigem Bildungsstand in allen Regimetypen eine egalitärere Arbeitsteilung (die unstandardisierten Regressionskoeffizienten variieren zwischen 0,169 und 0,504), wobei der Effekt am stärksten im postsozialistischen Regime $($ Beta $=0,172)$ und am schwächsten im sozialdemokratischen Regime $($ Beta $=0,067)$ ist. Anzumerken ist jedoch, dass Betas i. d. R. einen Wertebereich von -1 bis 1 aufweisen (Ausnahme: im Fall eines Modellverstoßes von Multikollinearität können sich Betawerte größer 1 ergeben), weshalb selbst im postsozialisti- 
schen Regime der Effekt eines hohen Bildungsstandes als schwach zu interpretieren ist. Ein mittleres sowie ein hohes Einkommen erweist sich im Vergleich zur Referenzkategorie der Frauen mit niedrigem Einkommen lediglich im sozialdemokratischen Regime als signifikant. Das postsozialistische Regime ist das einzige, innerhalb dessen eine signifikante Einkommensrelation eines höheren Einkommens der Frau besteht, wobei der Effekt kaum interpretationsfähig ist $($ Beta $=0,052)$. Auffallend ist außerdem die Variation der unstandardisierten Regressionskoeffizienten der Einkommenshomogamie: Während im sozialdemokratischen Regime der Steigungskoeffizient negativ und nicht signifikant ist, ist er in den anderen Regimetypen positiv (die Steigungskoeffizienten variieren zwischen $-0,017$ und 0,453 ), jedoch nur in den Niederlanden und im konservativen Regime signifikant. Im Hinblick auf die Effektstärke ist vergleichsweise der stärkste Effekt in den Niederlanden vorzufinden $($ Beta $=0,108)$. Für die Vollzeiterwerbstätigkeit zeigt sich im Vergleich zur Referenzkategorie der Teilzeiterwerbstätigkeit nur im sozialdemokratischen Regime ein hochsignifikanter, im postsozialistischen Regime ein signifikanter Einfluss (unstandardisierte Regressionskoeffizienten: 0,243 und 0,135), der jedoch in beiden Fällen sehr schwach ist (Betas: 0,094 und 0,036). In allen Regimen sind positive, hochsignifikante Steigungskoeffizienten der Relation einer höheren Erwerbstätigkeit der Frau (Frau > Mann) vorzufinden, die zwischen 0,287 (postsozialistisch) und 0,970 (liberal/Mischform) schwanken, gleichwohl ist der stärkste relative Einfluss für die Niederlande festzustellen (Beta $=0,154)$. Lediglich im konservativen und im postsozialistischen Regime scheint eine Erwerbshomogamie einen positiven, hochsignifikanten, jedoch schwachen Einfluss auf die innerhäusliche Arbeitsteilung zu haben. Die Steigungskoeffizienten der Kinderanzahl sind in allen Regimen negativ und variieren zwischen -0,137 (liberal/Mischform) und - 0,037 (postsozialistisch), die sich im Falle des konservativen als hochsignifikant, im Falle des sozialdemokratischen Regimes und in den Niederlanden als signifikant erweisen. Die standardisierten Regressionskoeffizienten deuten auf sehr schwache Effekte hin. Auch für die Paarbeziehungsdauer sind in sämtlichen Regimen negative Steigungskoeffizienten vorzufinden, die sich kaum zwischen den Regimen unterscheiden (-0,025 bis -0,013). Lediglich in den Niederlanden ist kein signifikanter Paarbeziehungsdauereffekt vorzufinden; der vergleichsweise stärkste Effekt zeigt sich im konservativen Typus (Beta $=-0,158)$. Abschließend unterscheiden sich die unstandardisierten Steigungskoeffizienten des Alters kaum zwischen den Regimen $(0,009$ bis 0,016), jedoch ist ausschließlich im postsozialistischen Regime ein schwacher, signifikanter Effekt aufzuweisen. Die erklärte Varianz schwankt zwischen 4,0\% und 9,1\% und ist folglich insgesamt eher gering.

Ein gravierender Nachteil der Vorgehensweise getrennter Regressionsanalysen ist, dass sie mit steigender Anzahl an Kontexteinheiten kaum durchführbar ist. Bereits eine nach Ländern differenzierte Analyse wird ab einer 
Fallzahl von mehr als zehn Ländern extrem unübersichtlich und es wird schwierig, die geschätzten Parameter miteinander zu vergleichen, weshalb u. a. zu Darstellungszwecken der getrennten Regressionsanalysen hier die Länder zu Regimetypen klassifiziert wurden. Eine derartige Analyse differenziert nach europäischen NUTS1-Regionen ist mittels dieser Vorgehensweise gar unmöglich. Ferner ist die oben beschriebene Problematik der verzerrten Signifikanztests nach wie vor nicht gelöst. Neben den Individualvariablen können darüber hinaus auch weitere Kontextmerkmale für die Erklärung der innerhäuslichen Arbeitsteilung von Bedeutung sein.

Die in den separaten Within-Regressionen festgestellten Unterschiede der Regressionskonstanten und Steigungen könnten zwar auch folgendermaßen innerhalb einer gepoolten Schätzung erfasst werden: Eine Erweiterung der klassischen Regressionsanalyse wäre möglich, indem ein gepooltes Regressionsmodell berechnet wird, das neben den Indvidualvariablen Dummyvariablen der Regimetypen enthält und regimespezifische Interaktionseffekte hinzugefügt werden (durch Multiplikation der Individualvariablen „Kinderanzahl" mit den jeweiligen Regimedummyvariablen), die z. B. die unterschiedlichen Kinderanzahleffekte je nach Regimetypen modellieren ${ }^{164}$. Doch können trotz dieser Erweiterung nicht alle methodischen Probleme gelöst werden. Mehrebenenmodelle verfügen demgegenüber über entscheidende Vorteile: Sie bieten die Möglichkeiten, Analysen mit vielen Kontexteinheiten praktikabel durchzuführen, die Daten als hierarchisch zu betrachten, wodurch die Signifikanztests zu präziseren Schätzungen führen, Unterschiede der Regressionskonstanten und -steigungen aufzuzeigen, sowie eine handhabbare, gleichzeitige Differenzierung zwischen Individual- und Kontexteffekten vorzunehmen. Abgesehen von diesen methodischen Argumenten ist vornehmlich das, bereits ausführlich diskutierte, inhaltliche Argument einer Differenzierung zwischen europäischen Regionen aufgrund ihrer unterschiedlichen ökonomischen, politischen und kulturellen Kontexte, die Einfluss auf die Variation von innerhäuslichen Arbeitsteilungsarrangements nehmen können, von zentraler Bedeutung. Obwohl eine Dominanz traditioneller innerhäuslicher Arbeitsteilungsarrangements anzunehmen ist - die bereits im Rahmen der deskriptiven Darstellungen erkennbar wurde - wird argumentiert, dass innerhalb dieses Traditionalismus Unterschiede in der Ausführung der Hausarbeiten zwischen den Regionen bestehen, die nicht ausschließlich auf Individual, sondern ebenso auf Kontexteffekte respektive regionale Bestimmungsgründe zurückzuführen sind. Folglich ergibt sich auf der inhaltlichen wie auch auf der empirisch-analytischen Dimension, die Notwendigkeit eines Mehrebenendesigns. Inwiefern die empirische Notwendigkeit durch den Intraklassenkorrelationskoeffizienten methodisch abgesichert werden kann, ist dem Random-Intercept-Only-Modell zu entnehmen. 


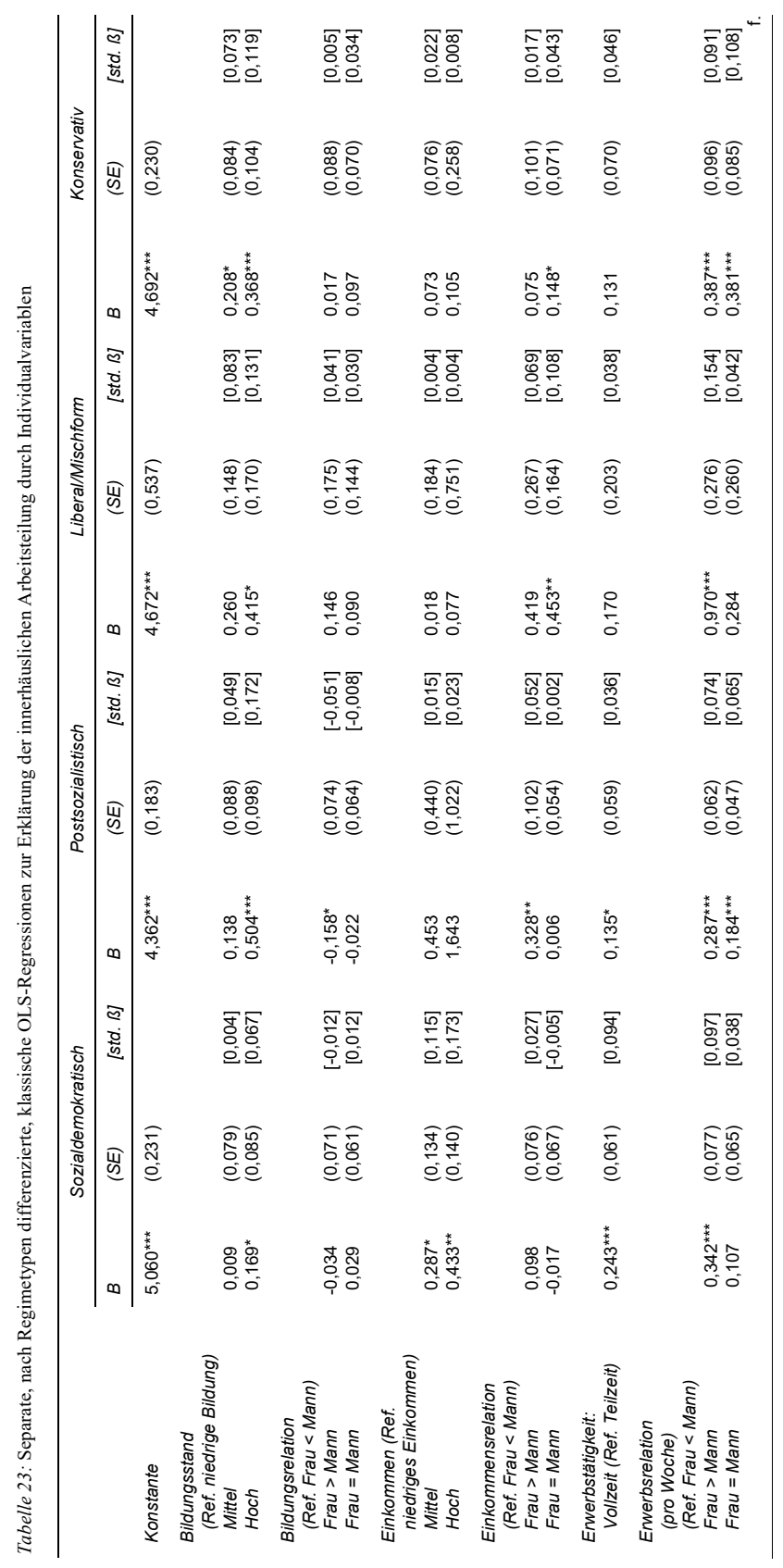




$$
\begin{aligned}
& \text { 琶 } \\
& \text { 10 }
\end{aligned}
$$

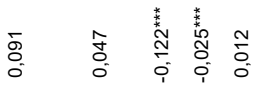

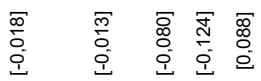

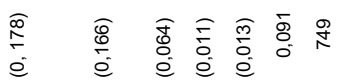

$$
\begin{aligned}
& \text { 苋 }
\end{aligned}
$$

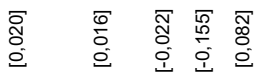

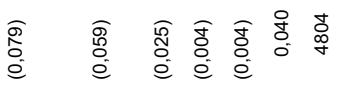

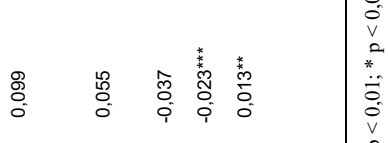

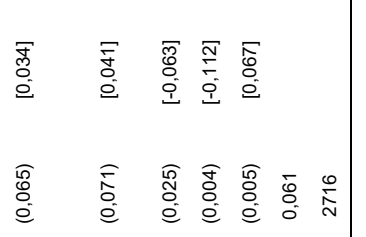

$$
\begin{aligned}
& \text { E }
\end{aligned}
$$

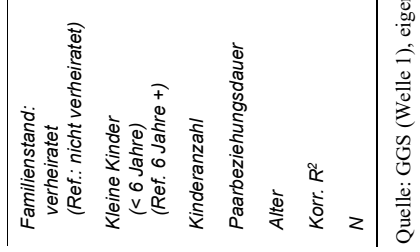




\subsection{Mehrebenenanalyse der innerhäuslichen Arbeitsteilung im NUTS1-Regionen-Vergleich (GGS Welle 1)}

Tabelle 24 stellt die Ergebnisse der Mehrebenenmodelle zur Erklärung der innerhäuslichen Arbeitsteilung dar, wobei die Klassifizierung der Kontexteinheiten fortan auf den NUTS1-Regionen basiert.

Schritt 1: Random-Intercept-Only-Modell

Im ersten Schritt wird mit Hilfe des Nullmodells ohne erklärende Variablen überprüft, ob die Anwendung eines Mehrebenenmodells notwendig und angemessen ist, respektive inwiefern die Gesamtvarianz der innerhäuslichen Arbeitsteilung auf Unterschiede zwischen den NUTS1-Regionen zurückzuführen ist. Die Ergebnisse zeigen, dass eine signifikante Kontextvarianz existiert, da die Intercepts signifikant zwischen den NUTS1-Regionen variieren (das Mehrebenenmodell ist angemessen und notwendig, wenn die Varianz des Random Intercept statistisch signifikant ist) und der ICC einen hochsignifikanten Wert von 0,062 $(* * *$ signifikant $<0,001)$ aufweist (Dieser gibt den Anteil der Level 2-Varianz an der Gesamtvarianz in der abhängigen Variablen wieder. Der ICC misst den Anteil der Varianzaufklärung auf der höheren gruppierten Ebene an der Gesamtvarianz; je höher der ICC, desto größer der Anteil der Gesamtvarianz, der durch die Unterschiede zwischen den Makroeinheiten hervorgebracht wird), d. h. 6,2\% der innerhäuslichen Arbeitsteilung gehen auf Unterschiede zwischen den NUTS1-Regionen zurück und 93,8\% auf Unterschiede innerhalb der NUTS1-Regionen bzw. zwischen Personen. Folglich ist der auf die NUTS1-Regionen zurückzuführende Anteil von 6,2\% zwar gering, jedoch gerade ausreichend, um die Angemessenheit einer Mehrebenenanalyse zu verdeutlichen (die Daumenregel der ,5\%-Hürde“ zur Durchführung von Mehrebenenanalysen wird knapp überschritten). Die Nullhypothese, dass keine signifikanten Unterschiede zwischen den Regionen bestehen, kann verworfen werden, da der Wald-Z-Wert von 4,454 (Berechnung: Intercept variance/Standardfehler) hochsignifikant ist. Ferner kann die erste Hypothese, dass die innerhäusliche Arbeitsteilung zwischen den regionalen Kontexteinheiten variiert, bestätigt werden (für eine detaillierte Übersicht der Hypothesenüberprüfung s. Tabelle 33 im Anhang).

\section{Schritt 2: Random-Intercept-Modell}

Das zweite Modell, das Random-Intercept-Model, beinhaltet die L1Individualvariablen, wobei zunächst angenommen wird, dass es zwar Unterschiede im Mittelwert der innerhäuslichen Arbeitsteilung zwischen den NUTS1-Regionen gibt (variierende Intercepts), sich die Effekte der Ebene 1Variablen jedoch nicht in Stärke und Richtung zwischen den Regionen unterscheiden. Die auf der ersten Ebene eingeführten Individualvariablen umfas- 
sen den Bildungsstand, die Relation der Bildung zwischen den PartnerInnen, die Höhe des Einkommens sowie die Einkommensrelation, den Erwerbsumfang sowie die Erwerbsrelation, den Familienstand (Dummy: 1 = verheiratet/Referenzkategorie: $0=$ nicht verheiratet bzw. Kohabitation), die Existenz von kleinen Kinder (Dummy: 1 = mind. ein Kind $<$ sechs Jahren im Haushalt/Referenzkategorie: Kinder, die älter als sechs Jahre sind und im Haushalt leben), die Anzahl der Kinder und die Paarbeziehungsdauer (die beiden letztgenannten Variablen sind grand-mean zentriert über alle Individuen und alle Regionen).

Für den Regionenvergleich sind signifikante Effekte der Bildungskonstellationen nachzuweisen: Überraschender Weise leisten Frauen mit hohem Bildungsabschluss hochsignifikant mehr Hausarbeiten als Frauen mit niedrigem Bildungsabschluss $(b=-0,326)^{165}$, was an die Ergebnisse des individualisierten Milieus von Koppetsch/Speck (2015) erinnert. Im Falle von Frauen mit mittlerem Bildungsniveau ist ebenfalls ein negativer signifikanter Effekt $\mathrm{zu}$ verzeichnen $(b=0,093)$. Für die Dummyvariablen einer hypogamen Bildungsrelation (nach der die Partnerin über einen höheren Bildungsabschluss als ihr Partner verfügt) und einer homogamen Bildungsrelation zwischen den PartnerInnen sind jedoch keine signifikanten Unterschiede zur Referenzkategorie einer hypergamen Bildungskonstellation (nach der der Partner über einen höheren Abschluss als seine Partnerin verfügt) vorzufinden.

Auch zeigt sich ein signifikanter Unterschied zwischen Frauen mit mittlerem und Frauen mit niedrigem Einkommen: Frauen mit mittlerem Einkommen verrichten mehr Hausarbeiten (der Effekt eines hohen Einkommens ist nicht signifikant). Ferner sind die Einkommensrelationseffekte signifikant: Frauen, die über ein höheres Gehalt verfügen als ihr Partner, müssen mehr im Haushalt leisten $(b=-0,169)$ als Frauen, die weniger als ihr Partner verdienen (Referenzkategorie). In Bezug auf das Einkommen ist der Homogamieeffekt negativ: Frauen, die über ein ähnliches Gehalt verfügen wie ihr Partner, verrichten mehr Hausarbeiten als Frauen, die traditioneller Weise weniger verdienen als ihr Partner.

165 Abgesehen von den nicht-standardisierten b-Koeffizienten sind in Tabelle 24 auch die standardisierten Koeffizienten aufgeführt. Um die Effektstärken der Variablen miteinander vergleichen zu können, muss berücksichtigt werden, dass die erklärenden Variablen unterschiedliche Skalen aufweisen. Für einen Vergleich der Effekte wurden folglich die bKoeffizienten zusätzlich standardisiert, wodurch die Skala der Effekte so transformiert wird, dass sie unabhängig von den ursprünglichen Messeinheiten ist und die Skaleneinheiten fortan in Standardabweichungen ausgedrückt werden (vgl. Snijders/Bosker 2012: 53). Die Standardisierung der Koeffizienten wurde erreicht, indem die Modelle nicht mit den zentrierten Rohwerten, sondern mit den z-transformierten unabhängigen und abhängigen Variablen berechnet wurden (ausgenommen sind Dummyvariablen, die nicht $\mathrm{z}$ transformiert, sondern auch im Rahmen standardisierter Modellschätzungen in ihrer Rohform verwendet werden sollten (vgl. Krause/Urban 2013: 35). „To standardize the regression coefficients $[\ldots]$ one could standardize all variables before putting them into the multilevel analysis" (Hox 2002: 20; Hox et al. 2018: 18). 
Bezüglich einer Vollzeiterwerbstätigkeit ist festzuhalten, dass sich diese negativ auf die Verrichtung der Hausarbeiten auswirkt. Vollzeit erwerbstätige Frauen sind mit hochsignifikantem Effekt einer Doppelbelastung ausgesetzt: Sie übernehmen im Vergleich zu Teilzeiterwerbstätigen mehr innerhäusliche Tätigkeiten. Die Erwerbsrelation zwischen den PartnerInnen stellt einen hochsignifikanten Effekt zur Erklärung der innerhäuslichen Arbeitsteilung dar. Da die Erwerbsrelation als Dummy kodiert wurde, insofern als Paare, in denen die Frau mehr erwerbstätig ist als ihr Mann, mit dem Wert 1 kodiert wurden und Paare, in denen die Frau weniger arbeitet als ihr Partner, mit dem Wert 0, ist der „Task-Participation-Index“ von Paaren mit geschlechtsuntypischer Erwerbsarbeit 'Frau > Mann' im Durchschnitt -0,408 Punkte geringer als der „Task-Participation-Index“ von Paaren mit geschlechtstypischer Erwerbsarbeit 'Frau < Mann'. Paare mit homogener Erwerbsrelation haben im Vergleich zu Paaren mit traditioneller Erwerbsrelation einen durchschnittlich um -0,207 Punkte niedrigeren „Task-Participation-Index“, d. h. eine traditionellere Arbeitsteilung.

Die Ergebnisse der Bildungs-, der Einkommens- und der Erwerbsrelationen verweisen darauf, dass die Hypothesen der relativen Ressourcen ( $\mathrm{H} 2$ bis H4, H6 bis H8, H10 bis H12), die auf einen ökonomischen Aspekt der Verhandlungsmacht verweisen, nicht bestätigt werden können. Im Gegenteil kommt die kulturelle Dimension von Macht zum Tragen, die sich am stärksten in einer hypogamen Erwerbskonstellation (und auch in einer hypogmen Einkommensrelation, s. H14) von Paaren widerspiegelt, weshalb die Hypothese H16 zutreffend ist. Wie bereits Brines (1994) dargelegt hat, wird eine höhere Erwerbstätigkeit der Frau sowie eine finanzielle Abhängigkeit des Mannes durch ein „Nicht-Erledigen“ von Hausarbeiten seitens des Mannes kompensiert. Die Ergebnisse von Koppetsch/Speck (2015) ergänzen diese Perspektive. Ausgerechnet im individualisierten Milieu, das sich in dieser Studie überwiegend durch AkademikerInnen auszeichnet, steht seine Selbstverwirklichung im Vordergrund, wenn anstelle von ihm sie die Familienernährerin ist. Obwohl er aufgrund seiner Arbeitslosigkeit Zeit dafür haben könnte, sich um den Haushalt und die Kinder zu kümmern, ist sie durch Erwerbs- und Hausarbeit doppelt belastet. Die vorliegenden Ergebnisse sowie die Ergebnisse ausgewählter Studien asymmetrischer Gendertheorien sind sich folglich weitgehend einig. Zu betonen sei hier die Bedeutung von Macht, die ein latentes theoretisches Konstrukt darstellt und notwendiger Weise in unterschiedliche Formen auszudifferenzieren ist. Was Werte und Normen anbelangt, können die vorliegenden Ergebnisse belegen, dass eine Normverletzung „weiblicher“ und „männlicher“ Tätigkeiten durch traditionelle innerhäusliche Arbeitsteilungsarrangements ausgeglichen wird - was als ein Ausdruck der kulturellen Dimension von Macht, einer durch gesellschaftliche kulturelle Normen gegebene Macht, interpretiert werden kann. 
Für verheiratete Frauen (H21) und kleine Kinder im Haushalt ${ }^{166}$ (H19) konnte überraschender Weise kein Zusammenhang zur innerhäuslichen Arbeitsteilung nachgewiesen werden.

Steigt die Anzahl der Kinder um eine Standardabweichung (aufgrund der grand-mean-Zentrierung), verringert sich der „Task-Participation-Index“ um $-0,076$ Einheiten. Außerdem ist ein Traditionalisierungseffekt der Paarbeziehungsdauer (H18) vorzufinden: Steigt die Paarbeziehungsdauer um eine Standardabweichung, verringert sich der „Task-Participation-Index“ um 0,019 Einheiten. Weder ein Alters- noch ein Kohorteneffekt können festgestellt werden. Bezüglich der Geburtskohorten scheint dieses Ergebnis jeglichen Modernisierungstheorien zu trotzen. Die genannten L1-Variablen weisen nahezu keine Unterschiede in den anderen Modellen auf (lediglich der negative Effekt eines mittleren Bildungsstandes ist nicht mehr signifikant, sobald die Kontextvariablen im dritten Modell eingeführt werden). Durch alle einbezogenen L1-Prädiktoren können insgesamt 18\% der Binnenvarianz des „Task-Participation-Index“ in den NUTS1-Regionen erklärt werden.

\section{Schritt 3: Random-Intercept-Modell mit Prädiktoren auf Individual- und} Kontextebene

Das Macht ein Phänomen ist, das nicht ausschließlich auf der Mikroebene verortet werden kann, sondern ebenso auf Meso- und Makroebene zu betrachten ist, und diese Dimensionen eine Auswirkung auf die Ausführung der Hausarbeiten haben können, soll weiterführend im dritten Modell dargestellt werden. Der methodischen Vereinfachung ist hierbei geschuldet, dass lediglich zwischen Individual- und Kontexteffekten differenziert werden kann.

Das dritte Modell integriert ferner die Ebene 2-Kontextvariablen: das Bruttoinlandsprodukt in Kaufkraftstandards pro EinwohnerInnen (um das BIP aufgrund unterschiedlicher Währungen international vergleichbar machen zu können basiert die Messung auf Kaufkratfstandards), die Erwerbsquote von Frauen, die politische Links-Rechts-Verortung, die Teilnahmerate 4-Jähriger an Bildungsprogrammen, die Gesamtfertilitätsrate, die Genderegalität, den Anteil von Frauen mit tertiärem Bildungsabschluss und die Religiosität. Während sich das Bruttoinlandsprodukt sowie die Erwerbsquote von

166 In Bezug auf die Fertilitätsbiographien steht eine methodenkritische Anmerkung des Generations and Gender Surveys in Anlehnung an Kreyenfeld et al. (2013) noch aus. Kreyenfeld et al. (2013) haben im Rahmen ihrer Studie versucht, die Fertilitätsbiographien des deutschen GGS zu validieren. „Der zentrale Befund dieser Validierung ist, dass der deutsche GGS die Kinderzahlen der älteren Kohorten unterschätzt und jene der jüngeren Jahrgänge überschätzt. Wir vermuten, dass zwei Mechanismen für dieses Muster verantwortlich sind: Einerseits sind Kinder, die bereits den elterlichen Haushalt verlassen haben, nur lückenhaft erfasst worden. Andererseits sind jüngere Befragte mit kleineren Kindern in der Stichprobe wegen ihrer leichteren Erreichbarkeit überrepräsentiert. Zusammengenommen tragen diese beiden Mechanismen dazu bei, dass die Kinderzahlen der jüngeren Jahrgänge über- und die der älteren Geburtsjahrgänge unterschätzt werden. Die Validierung der Heiratsbiografen offenbart eine ähnliche Schieflage“ (Kreyenfeld et al. 2013: 29). 
Frauen auf die ökonomische Empowerment-Dimension beziehen, der Versorgungsgrad von Kinderbetreuungsplätzen sowie die politische LinksRechts-Verortung Indikatoren der politischen Empowerment-Dimension auf der Makroebene darstellen, erfassen die Kontxtindikatoren der Genderegalität, Zugang zu tertiärer Bildung, die Fertilitätsrate sowie die Religiosität die kulturelle Empowerment-Dimension im Sinne eines gesellschaftlichen „machthaltigen“"Wertesystems.

Die Teilnahmerate 4-Jähriger an Bildungsprogrammen als Indikator für eine staatliche Förderung von Zweiverdienerpaaren (H24) ist signifikant, weshalb der Versorgungsgrad von Kinderbetreuungsplätzen in europäischen Regionen eine egalisierende Wirkung auf die Aufteilung von Hausarbeiten in Paarbeziehungen hat: Mit steigendem Anteil 4-Jähriger an Vorschulbildungsprogrammen erhöht sich der „Task-Participation-Index“ um 0,02 Einheiten. Die Fertilitätsrate stellt einen negativen Effekt dar: Je höher die Fertilitätsrate, desto niedriger ist der „Task-Participation-Index“ (-0,863 Punkte), d. h. desto weniger beteiligen sich Männer an der Hausarbeit (H28). Da es sich im Falle der Religiosität um einen für die Regionen aggregierten Kontextfaktor handelt, der ursprünglich die Häufigkeit des Besuches von Gottesdiensten außerhalb von besonderen Anlässen erfasste und von 1= jeden Tag bis $7=$ nie kodiert war (durch die Aggregation der ursprünglichen Variablen werden regionenspezifische Mittelwerte erfasst) ist interessanter Weise zu konstatieren, dass umso säkularisierter eine Region, umso traditioneller die innerhäusliche Arbeitsteilung von Paaren, die in dieser Region wohnhaft sind, ist. Die Richtung dieses Effekts verläuft insofern genau gegenteilig, wie im Sinne von H29 angenommen wurde. Entgegen eines häufig angenommenen Zusammenhangs zwischen Traditionalismus und Religiosität scheint in Bezug auf innerhäusliche Arbeitsteilungsarrangements das Gegenteil der Fall zu sein. Die anderen L2-Prädiktoren weisen diesem Modell zufolge keinen signifikanten Zusammenhang mit der innerhäuslichen Arbeitsteilung auf (s. hierzu H22, H23, H25 bis H27).

Im Vergleich zum Intercept-Only-Model zeigt sich, dass die Vorhersage des Vergleichsmodells mit L2-Prädiktoren auf der zweiten Ebene um 57\% größer ist als im Nullmodell und, dass die L1-Prädiktoren 18,1\% der Binnenvarianz des „Task-Participation-Index“ erklären. Die proportionale Reduzierung des Vorhersagefehlers durch alle Kovariaten beträgt in diesem Modell $20,5 \%$.

\section{Schritt 4: Random-Slope-Modell}

Unterscheiden sich die Effekte der Einkommensrelationen, Paarbeziehungsdauern und Kinderanzahl zwischen den NUTS1-Regionen? Insbesondere im Sinne des dispositiven Charakters von Macht ist eine Variation der Individualeffekte im Rahmen der Kontexteinheiten zu vermuten. Die zentrale Annahme des Random-Slope-Models ist, dass die Effekte der L1-Prädiktoren zwischen den L2-Einheiten variieren. Die Ergebnisse zeigen, dass die Slope 
Varianzen nicht signifikant sind, was bedeutet, dass die L1-Effekte nicht zwischen den NUTS1-Regionen variieren. Innerhalb der untersuchten NUTS1-Regionen wird hierbei eine Varianzaufklärung von 18,7\% erreicht.

\section{Schritt 5: Intercepts-and-Slopes-as-Outcome-Modell}

Im letzten Schritt wird eine Cross-Level-Interaktion angenommen: „Die Stärke und Signifikanz des Effektes $\gamma 11$ gibt darüber Auskunft, inwieweit der Effekt des Level 1-Prädiktors Xij in Abhängigkeit von der Ausprägung des Level 2-Prädiktors Wj variiert" (Lois 2015: 61). Unterscheidet sich der Kinderanzahleffekt in Abhängigkeit der Erwerbsquote von Frauen? Es konnte keine signifikante Cross-Level-Interaktion $(-0,006)$ nachgewiesen werden.

Die Vorhersage des Vergleichmodells mit den Ebene 1-Prädiktoren ist auf der ersten Ebene um 18,8\% größer als im Random-Intercept-Only-Modell und die Vorhersage des Vergleichsmodells mit Ebene 2-Prädiktoren auf der zweiten Ebene ist um 66,9\% größer als im Nullmodell. Durch die Berücksichtigung aller Kovariaten kann eine proportionale Reduzierung des Vorhersagefehlers von $21,8 \%$ erreicht werden.

Abschließend verweisen die standardisierten Modellschätzungen darauf, dass ein hoher Bildungsstand (-), eine hypogame Erwerbsrelation (-), die Paarbeziehungsdauer (-), die Teilnahmerate 4-Jähriger an Bildungsprogrammen $(+)$ und die Fertilitätsrate (-) vergleichsweise die stärksten Effekte innerhalb der Mehrebenenmodelle darstellen.

Insgesamt verdeutlichen die theoretische Argumentation und die statistische Analyse, dass ein Mehrebenenansatz wesentliche Erkenntnisse erbringt, um die innerhäusliche Arbeitsteilung adäquater als in reinen mikrosoziologischen Studien erklären zu können. Einschränkend ist jedoch anzumerken, dass der Anteil der Gesamtvarianz, der durch die Unterschiede zwischen den Kontexteinheiten (NUTS1-Regionen) hervorgebracht wird, gering ist. Da es sich bei Machtverhältnissen und Arbeitsteilungsarrangements um soziale Phänomene handelt, die sich im Zeitverlauf verändern können, ist eine weiterführende Betrachtung innerhalb des Lebenslaufs unumgänglich, was durch die folgende Panelanalyse mit Mehrebenenmodellen zur innerhäuslichen Arbeitsteilung methodisch umgesetzt werden soll. 


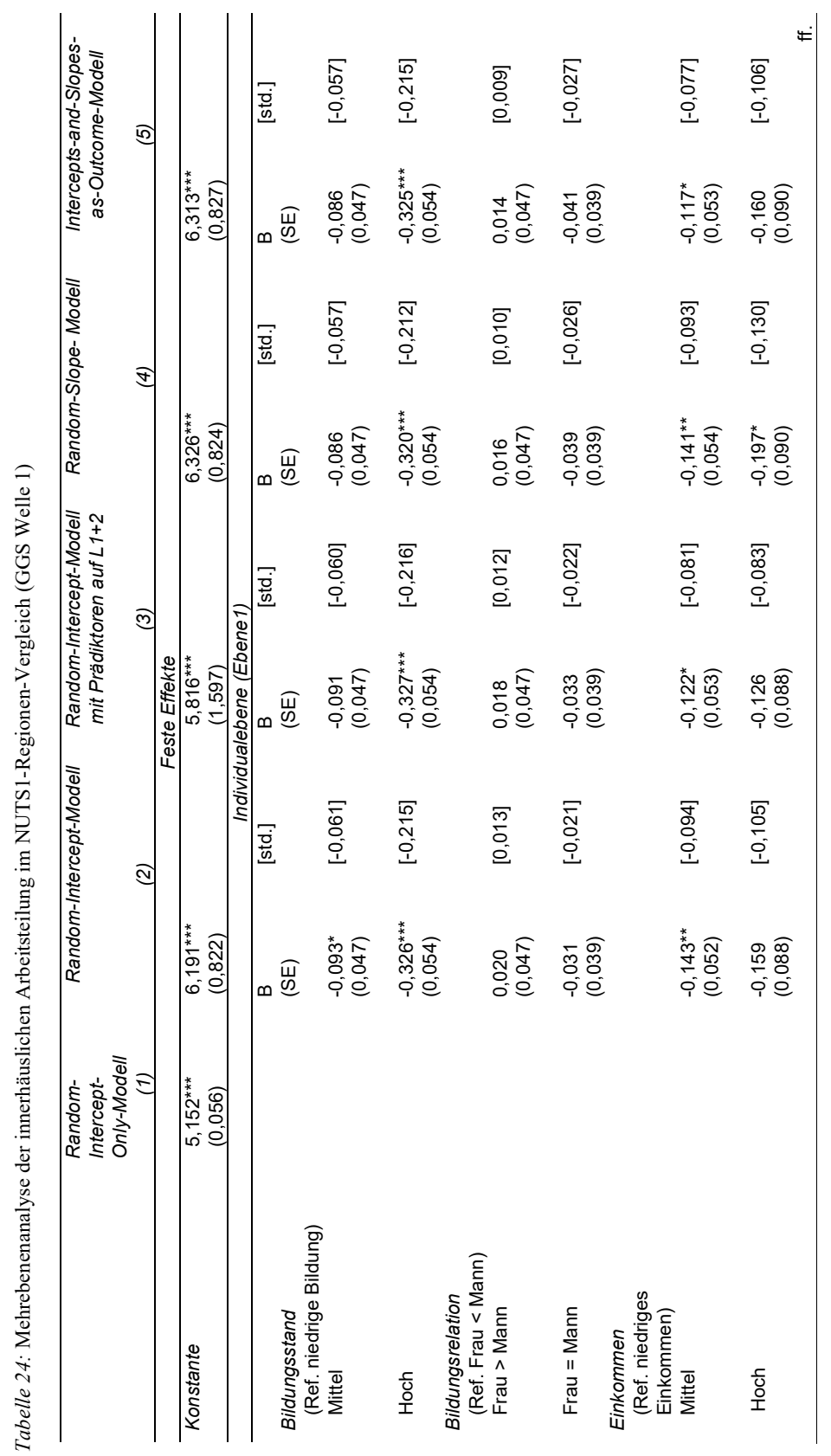




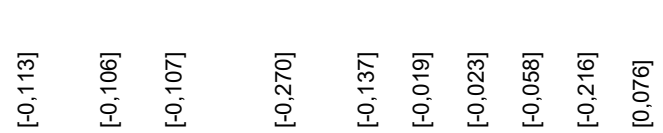

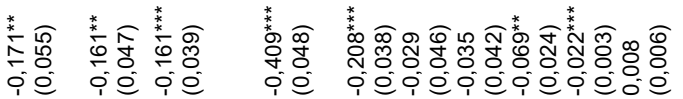

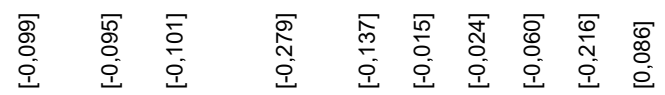

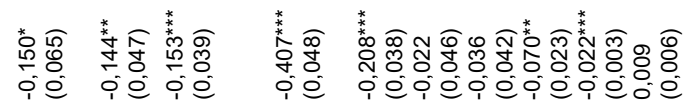

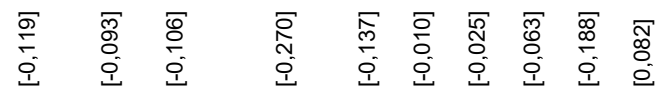

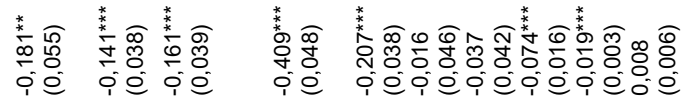

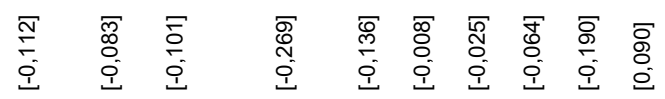

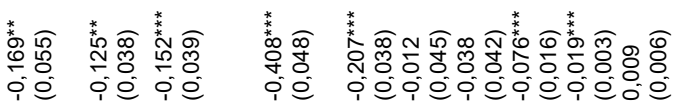

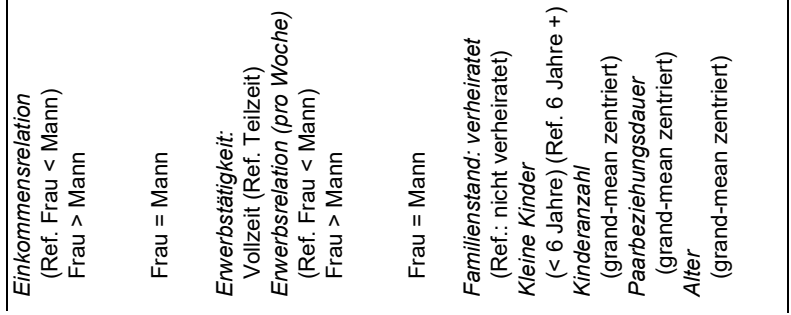




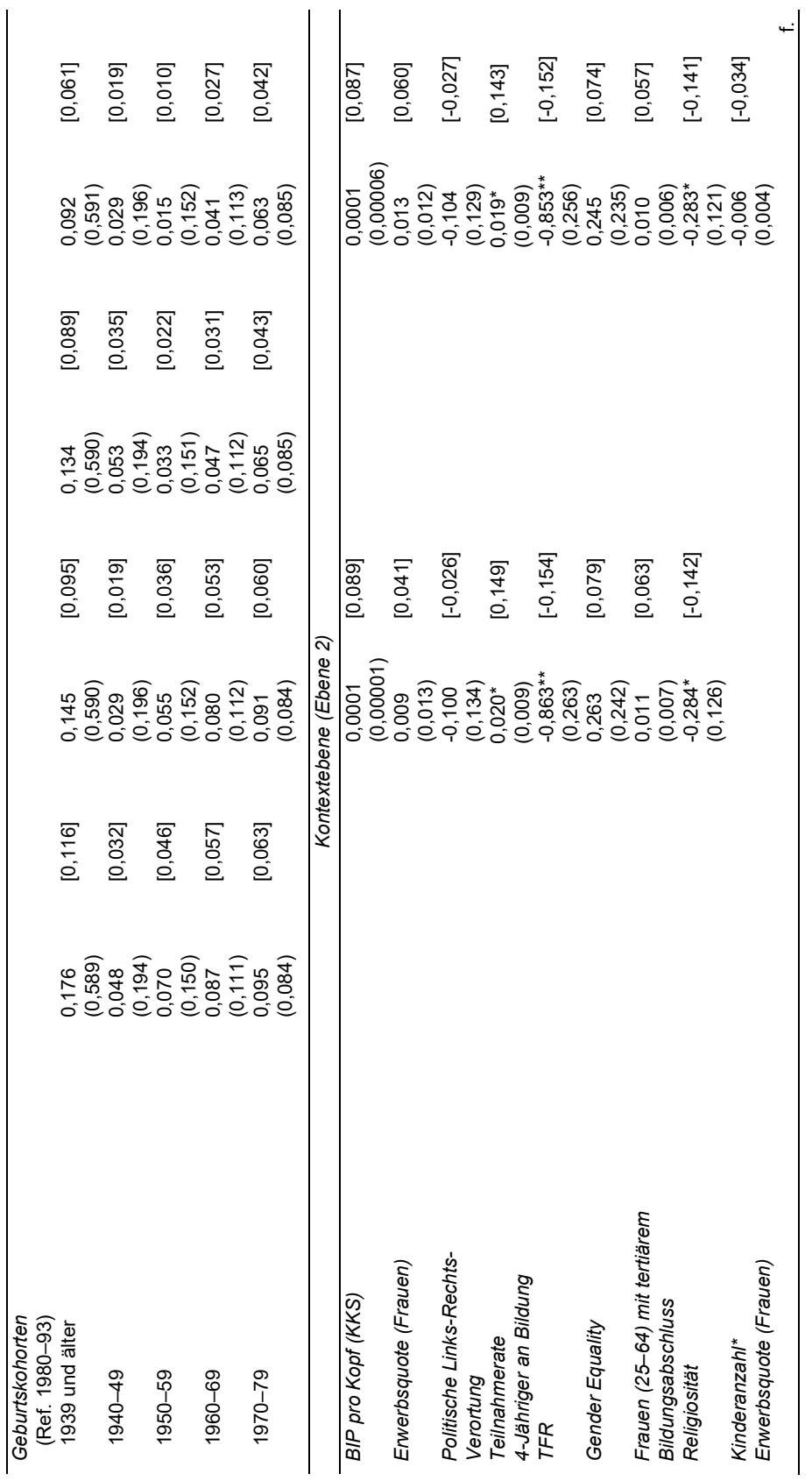




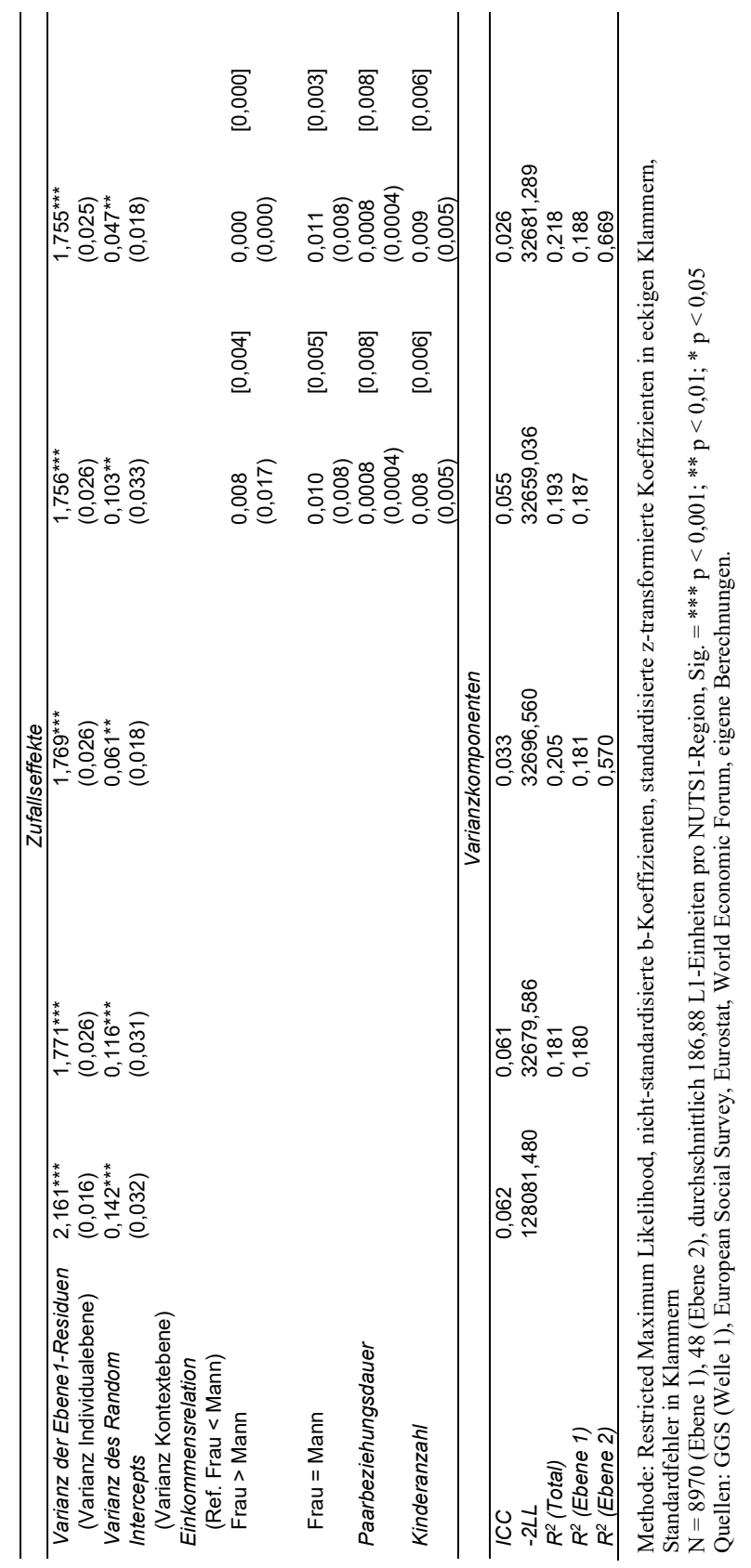




\subsection{Dynamischer Traditionalismus? Eine Panelanalyse mit Mehrebenenmodellen zur innerhäuslichen Arbeitsteilung (GGS Welle 1 und 2)}

Im Folgenden sollen Veränderungen auf der Individualebene durch Mehrebenenmodelle analysiert werden, um die Dynamik von Machtverhältnissen und Arbeitsteilungsarrangement methodisch $\mathrm{zu}$ erfassen. Inhaltlich wäre es grundsätzlich wünschenswert, ein Drei-Ebenen-Modell zu modellieren, das hierarchische Daten zeitveränderlicher Merkmale zu verschiedenen Zeitpunkten (Ebene 1) von Personen (Ebene 2) innerhalb spezifischer regionaler Kontexteinheiten (Ebene 3) berücksichtigt. Aufgrund des massiven Einbruchs der Fallzahl in Deutschland zum Zeitpunkt der zweiten Welle (s. Tabelle 13) resultiert für die Panelanalyse die Schwierigkeit, dass im Rahmen der zweiten Welle zu wenig Einzelbeobachtungen bezüglich der interessierenden Individualvariablen vorhanden sind, um eine Klassifizierung innerhalb der 16 deutschen Bundesländer vorzunehmen. Auch die Alternative, Deutschland aus der Panelanalyse auszuschließen, ist problematisch, da in diesem Fall wiederum die Kontextfallzahl sehr gering wäre. In Folge dieser methodischen Herausforderungen wurde die Schlussfolgerung getroffen, die Mehrebenenmodelle der Paneldaten nicht als Drei-Ebenen-, sondern nur als Zwei-Ebenenmodell zu konzipieren. Ferner wird auf eine Klassifizierung innerhalb der Regionen verzichtet; die Kontextebene basiert insofern lediglich auf der Klassifizierung innerhalb von Personen. In Tabelle 25 sind folglich die Mehrebenenmodelle für die beiden Wellen des Generations and Gender Surveys dargestellt, deren Kontexteinheiten die Personen bilden. Die abhängige Variable ist in allen Modellen der für zwei Messzeitpunkte vorhandene „Task-Participation-Index“. Zeitvariierende unabhängige Individualvariablen sind die Prozesszeit, die dummmy-kodierten Relationsvariablen der Paare in Bezug auf Bildung, Einkommen und Erwerbstätigkeit, die dummy-kodierten Variablen eines hohen und mittleren Einkommens, einer Vollzeiterwerbstätigkeit und einer Existenz von kleinen Kindern im Haushalt sowie die Kovariaten der Anzahl der Kinder und der Paarbeziehungsdauer. Als zeitkonstante Kontextprädiktoren dienen die Geburtskohorten und der Bildungsstand. Im ersten Analyseschritt wird überprüft, inwiefern unter Berücksichtigung der unterschiedlichen Messzeitpunkte eine Personenheterogenität der innerhäuslichen Arbeitsteilung vorzufinden ist.

Schritt 1: Random-Intercept-Only-Modell

Anhand des Random-Intercept-Only-Modells (s. Modell 1) ohne erklärende Variablen können die Varianzanteile auf den verschiedenen Ebenen festgestellt werden. Der ICC weist in diesem Modell einen Wert von 49,9\% auf, was bedeutet, dass 49,9\% der Varianz der innerhäuslichen Arbeitsteilung auf Unterschiede zwischen den Personen zurückzuführen sind und 50,1\% auf 
Unterschiede zwischen den Messzeitpunkten, d. h. auf Unterschiede innerhalb von Personen. Umso höher der Intraklassenkorrelationskoeffizient, umso zeitlich stabiler ist das Explanandum (vgl. Lois 2015: 90). Der Intercept gibt Auskunft über die geschätzte mittlere Ausprägung des „TaskParticipation-Index“, die in diesem Modell 5,02 Punkte beträgt und als eine mittlere traditionelle Arbeitsteilung über alle Personen und Messzeitpunkte hinweg interpretiert werden kann. Festgehalten werden kann außerdem, dass sowohl zwischen den Personen als auch zwischen den Messzeitpunkten ausreichend Varianz besteht, wie der einseitige Wald-Test belegt (für die Varianz der ersten Ebene: Wald $Z^{167}=60,062, p<0,001$; für die Varianz der zweiten Ebene Wald $Z=39,182, \mathrm{p}<0,001)$.

\section{Schritt 2: MEA mit Prozesszeit (fixed)}

Im zweiten Schritt wird die Zeit als fixer, linearer Parameter eingeführt (s. Modell 2: Random-Intercept-Modell mit Prozesszeit), d. h. der Koeffizient wird hier für alle Personen als identisch angenommen (vgl. Krause/Urban 2013: 26). Im Generations and Gender Survey variieren die Messzeitpunkte sehr stark, sodass einer der bereits genannten Vorteile der Mehrebenenanalyse für Paneldaten ausgeschöpft werden kann: die Zeitintervalle zwischen den Messzeitpunkten können nicht nur unterschiedlich lang sein, sondern auch zwischen den Befragten variieren (vgl. Krause/Urban 2013: 5). Der t-Test belegt die Angemessenheit des Zeitparameters $(t=5,218, p<0,001)$. Pro Jahr steigt der TPI um 0,017 Einheiten. Werden die L1-Varianzen und die L2-Varianzen des Random-Intercept-Only-Modells und des RandomIntercept-Modells miteinander verglichen, fällt auf, dass $0 \%$ der Varianz der ersten Ebene erklärt werden können und der Wert des Vergleichs der L2Varianzen ebenfalls rund $0 \%$ beträgt ${ }^{168}$.

\section{Schritt 3: MEA des Within-Personen-Anteils der zeitvarianten Prädiktoren} Im Folgenden werden die zeitveränderlichen Ebene1-Prädiktoren in das Modell aufgenommen (s. Modell 3: Random-Intercept-Modell mit zeitvarianten L1-Prädiktoren). Gemäß der t-Tests wird bestätigt, dass sich ein Wandel der Bildungsrelation (Frau > Mann und Frau $=$ Mann, des Einkommens (mittel), der Einkommensrelation (Frau > Mann, s. H15), der Erwerbsrelation (Frau > Mann und Frau = Mann, s. H17), der Kinderanzahl (H20) und der Paarbeziehungsdauer (H18) signifikant respektive in einigen Fällen hochsignifikant negativ auf die innerhäusliche Arbeitsteilung auswirkt (aufgrund der Dum-

167 Wald Z lässt sich durch den Quotienten der geschätzten Varianz dividiert durch den Standardfehler der geschätzten Varianz ermitteln (vgl. Krause/Urban 2013: 26).

168 Hox (2010) zufolge wäre selbst ein negativer Vergleichswert zwischen Modellen nicht abwegig, sondern ein Resultat des Samplingprozesses. Um in einem solchen Fall die Problematik eines negativen Vergleichswerts zu umgehen, müsste für alle weiteren Schritte das Random-Intercept-Modell mit der Prozesszeit als Referenzmodell verwendet werden, weil das Random-Intercept-Only-Modell die Varianz der Messzeitpunkte überschätzt, hingegen die Varianz zwischen den Personen unterschätzt (vgl. Krause/Urban 2013: 27). 
my-Kodierung der Relationsvariablen sind die geschätzten Koeffizienten im Kontrast zur jeweiligen Referenzgruppe zu interpretieren). Entgegen der Rational-Choice-Argumentation sind die Relations-Effekte geschlechtsspezifisch zu Ungunsten der Frau, weshalb die Hypothesen H5, H9 und H13 nicht bestätigt werden können (eine detaillierte Übersicht der Hypothesenüberprüfung ist Tabelle $34 \mathrm{im}$ Anhang zu entnehmen). Die metrisch skalierten Kovariaten wurden durch die Aufnahme der beiden group- und grand-meanzentrierten (Teil-)Variablen in einen Between- und einen Within-PersonenAnteil separiert (s. Fußnote 163). Die grand-mean-zentrierten Variablen der Kinderanzahl und der Paarbeziehungsdauer stellen als Ausnahmen die einzigen zeitinvarianten Determinanten in diesem Modell dar und verweisen darauf, dass zwischen einer durchschnittlichen Kinderanzahl sowie einer durchschnittlichen Paarbeziehungsdauer und der innerhäuslichen Arbeitsteilung ein hochsignifikant negativer Zusammenhang besteht. Frauen, die sich um eine Einheit in der Paarbeziehungsdauer unterscheiden, unterscheiden sich im Wert der innerhäuslichen Arbeitsteilung um -0,013 Einheiten. Einen Unterschied der innerhäuslichen Arbeitsteilung von -0,07 weisen Frauen auf, die in der Kinderanzahl um ein Kind voneinander abweichen. In Bezug auf die Within-Determinante der Paarbeziehungsdauer ist zu konstatieren, dass eine Verlängerung der Paarbeziehungsdauer um eine Einheit zu einer traditionelleren Aufteilung der Hausarbeiten um -0,034 Einheiten führt, was die Honeymoon-Hypothese (H18) im Längsschnitt betrachtet bestätigt.

Durch Kenntnis des Within-Anteils der innerhäuslichen Arbeitsteilung können 20,9\% der Varianz zwischen den Messzeitpunkten statistisch erklärt werden, während im Hinblick auf die Varianz zwischen den Personen ein negatives R-Quadrat auffällt. Im Allgemeinen kann auch eine Individualvariable zur Erklärung der Kontextvarianz beitragen, vorausgesetzt, dass sich die Gruppen bezüglich dieser Individualvariable unterscheiden. Hingegen kann eine Kontextvariable keine Erklärungsleistung zur Individualvarianz erbringen, weil sie qua definitionem innerhalb der Kontexteinheiten keine Varianz aufweist. Durch das gruppeninterne Zentrieren der Paarbeziehungsdauer und der Kinderanzahl werden Gruppenunterschiede eliminiert, weshalb eine negative Varianzaufklärung der Makroebene resultieren kann, ohne, dass Kontextvariablen im Modell enthalten sind. Grundsätzlich sind negative Varianzanteilschätzungen im Rahmen von Mehrebenenanalysen möglich, was ausführlich von Snijders/Bosker (1999; 2012), Langer (2009) und Hox et al. (2018) diskutiert wird.

\section{Schritt 4: MEA mit zeitinvarianten Kontextmerkmalen}

Das vierte Modell ist eine Erweiterung des dritten Modells um zeitinvariante Kontextmerkmale des Bildungsstandes und der Geburtskohorten (s. Modell 4: Random-Intercept-Modell mit zeitvarianten L1- und zeitinvarianten L2Prädiktoren). Frauen mit mittlerem $(\mathrm{p}<0,01)$ sowie Frauen mit hohem Bildungsstand $(\mathrm{p}<0,001)$ teilen sich die Hausarbeiten traditioneller mit ihrem 
Partner auf als die Referenzgruppe der Frauen mit niedrigem Bildungsstand. Auch die Effekte der 1940-49er- und der 1950-59er-Geburtskohorte sind signifikant und negativ: Frauen dieser Geburtskohorten weisen eine traditionellere Arbeitsteilung auf als Frauen der Referenzkategorie der Geburtskohorte 1980-93.

Die Berücksichtigung der personenspezifischen Kontextvariablen des Bildungsstandes und der Geburtskohorten führt dazu, dass die Varianzanteilschätzung im Vergleich zum Intercept-Only-Modell immer noch negativ ist, doch zumindest zeigt sich eine leichte Verbesserung im Vergleich zum Vormodell, das lediglich die zeitvarianten Individualvariablen enthält. Folglich ist eine Fehlspezifikation des Modells anzunehmen, ferner bleiben wesentliche Kontextprädiktoren in diesem Modell unberücksichtigt. In Anbetracht einer fehlenden Integration von regionenspezifischen Merkmalen, wie sie im Falle eines Drei-Ebenen-Modells möglich gewesen wäre, scheint dies nicht überraschend.

\section{Schritt 5: MEA mit Random Slopes}

Im fünften Schritt werden einige L1-Variablen als Random-Effekte spezifiziert (s. Modell 5: Random-Slope-Modell). Zu erinnern ist an den zu Beginn dieser Dissertation diskutierten Widerspruch, dass weder eine Traditionalisierung noch eine Enttraditionalisierung der innerhäuslichen Arbeitsteilung für sämtliche Kontexte als einheitliche Trendentwicklungen angenommen werden können. Je nach Kontext sind vielmehr unterschiedliche Entwicklungen zu erwägen. Insofern ist es von zentraler Bedeutung, die Prozesszeit methodisch betrachtet als Random-Effekt zu definieren (Modell 5.1), wodurch der zeitliche Wandel zwischen den Kontexteinheiten (hier: Personen) nicht mehr als einheitlicher zeitlicher Wandel angenommen wird. Außerdem wird, wie von Snijders/Boskers (2012) und Krause/Urban (2013) vorgeschlagen, durch diese Vorgehensweise die restriktive Annahme einer Compound-SymmetrieMatrix verworfen, da durch diese Freisetzung der Prozesszeit die Varianzen und Kovarianzen der Messzeitpunkte unterschiedliche Werte annehmen können (vgl. Krause/Urban 2013: 30).

"There are various ways in which the assumption of compound symmetry (which
states that the variance of the observations is constant over time and that the cor-
relation between observations is independent of how far apart they are [...]) can
be relaxed. In the hierarchical linear model framework, the simplest way is to in-
clude one or more random slopes in the model. This makes sense if there is some
meaningful dimension, such as time or age, underlying the measurement occa-
sions" (Snijders/Boskers 2012: 253).

In Bezug auf die Einführung der Prozesszeit als Random-Effekt lässt sich Folgendes festhalten: Die Kovarianz zwischen $\mathrm{u}_{0 \mathrm{i}}$ und $\mathrm{u}_{1 \mathrm{i}}$ der Mittelwertsund Steigungskoeffizienten beträgt $-0,024$ und ist nicht signifikant, weshalb trotz negativem Vorzeichen nicht davon auszugehen ist, dass Frauen mit 
einer vergleichsweise egalitären Arbeitsteilung zum ersten Messzeitpunkt und unter Kontrolle der unabhängigen Variablen eine negativere zeitliche Steigung aufweisen als Frauen mit einem traditionellem Ausgangsniveau der innerhäuslichen Arbeitsteilung (bzw., dass der Einfluss der Prozesszeit auf die innerhäusliche Arbeitsteilung vom durchschnittlichen Hausarbeitsniveau der Frauen abhängig ist). Der Schätzwert der Varianz der personenspezifischen Steigungskoeffizienten beträgt 0,005 , wobei anhand des einseitigen Wald-Tests (Wald $Z=0,2153 ; \mathrm{p}<0,05$ ) bestätigt werden kann, dass eine signifikante Varianz zwischen den Personen in Bezug auf den Zeiteinfluss besteht.

Weiterhin beinhaltet dieses Modell die Einführung des Random-Effekts eines Wandels der Kinderanzahl, um zu überprüfen, ob der Einfluss des Kinderanzahlwandels auf die innerhäusliche Arbeitsteilung signifikant zwischen den befragten Frauen variiert. In Folge der Einführung des Wandels der Kinderanzahl als Random-Effekt umfasst das Modell drei zusätzliche Parameter, nämlich die Varianz des Kinderanzahlwandels und deren Kovarianzen $\mathrm{u}_{0 \mathrm{i}}$ und $\mathrm{u}_{1 \mathrm{i}}$. $\mathrm{Zu}$ konstatieren ist, dass der Einfluss eines Wandels der Kinderanzahl auf die innerhäusliche Arbeitsteilung nicht signifikant über die befragten Frauen variiert.

Während die Erklärungsleistung der Individualvarianz durch die Einführung der Random-Effekte geringfügig ansteigt, ist für die Kontextvarianz im Vergleich zum Intercept-Only-Modell als Referenz erneut ein negatives RQuadrat zu beobachten, wodurch das Gesamtmodell an Erklärungsleistung einbüßt. Ferner kann gemäß dem Likelihood Ratio Test durch die Einführung der Random-Effekte keine signifikante Verbesserung der Modellgüte im Vergleich zum Vormodell erreicht werden.

\section{Standardisierung der Koeffizienten}

Um die einzelnen Effektstärken miteinander vergleichen zu können, werden abschließend die standardisierten Koeffizienten dargestellt. Diesbezüglich erfolgt die Durchführung der jeweiligen Mehrebenenmodelle nicht auf Basis der Rohwerte, sondern auf Basis der z-transformierten Werte der Variablen (ausgenommen sind Dummyvariablen). Festgehalten werden kann, dass auf der ersten Ebene die Erwerbsrelation (Frau > Mann, standardisierter Koeffizient $=-0,280$ ) und die Paarbeziehungsdauer (standardisierter Koeffizient $=$ 0,131) die relevantesten Effekte darstellen, wobei der Effekt der Veränderung der Erwerbsrelation deutlich größer ist. Auf der Kontextebene erweist sich eine hohe Bildung als relevant. Auch für die Geburtskohorte 1940-49 ist ein bedeutender Effekt vorzufinden (der Effekt der Geburtskohorte 1939 und älter weist zwar den stärksten Effekt auf, dieser ist jedoch nicht signifikant). Ausgerechnet für die nach Blücher (1966) als „unbefangene“ bezeichnete Generation der 1940 bis 1949 Geborenen zeigt sich im Vergleich zur Referenzkategorie der 1980 bis 1993 Geborenen ein negativer Effekt der innerhäuslichen Arbeitsteilung. Es ist eine Kohorte, von der nur die Ältesten be- 
wusst etwas von Krieg und Nachkriegszeit erlebt haben, während die Jüngeren zunehmenden Wohlstand, wachsende Konsummöglichkeiten sowie politische Sicherheit erfuhren (vgl. Blücher 1966: 12). Ihre Angehörigen erlebten und gestalteten in ihrer frühen Erwachsenenphase die 68er-Bewegung. Die Jugendgeneration der 68er haben gemäß Blücher keine , ,[...] ideologischen Fixierungen $[\ldots]$ an starke weltanschauliche Bindungen wie Religion, Vaterland, Europa“"inne.

„Wohin wir blicken: Pluralismus, Vielfalt, Offenheit, partielles Engagement, Weltneugier, Vorurteilslosigkeit - Unbefangenheit allem Neuen gegenüber. Damit verbinden ich Experimentierfreudigkeit, Umgänglichkeit mit Anderen, Freude am Leben in allen seinen Rollenangeboten“ (Blücher 1966: 14).

Es ist bezeichnend, dass ausgerechnet diese Kohorte ein noch traditionelleres Muster der innerhäuslichen Arbeitsteilung aufweist als die jüngste Kohorte der 1980-93 Geborenen. Ebenfalls ist für die Kohorte 1950-59 ein Effekt vorzufinden, der jedoch deutlich schwächer zu sein scheint. Alle anderen Geburtskohorten weisen im Vergleich zur Referenzkategorie keine signifikanten Effekte auf.

\subsection{Abschließende Diskussion: Empirische Mehrebenenanalysen im theoretischen Diskurs des „Power-Capability Approachs“}

Am bisherigen Forschungsstand zur Erklärung innerhäuslicher Arbeitsteilungsarrangements in Paarbeziehungen wird insofern Kritik geübt, als es zahlreiche familiensoziologische Studien gibt, die einzelne Teilaspekte von Genderungleichheiten, Macht und traditionellen Rollenbildern untersuchen. Ihre Perspektiven sind meist entweder auf die Mikro- oder auf die Makroebene begrenzt, ohne ihre fundamentale Wechselwirkung zu berücksichtigen. Studien, die eine reflexive Wirkung zwischen Mikro- und Makroebene analysieren, sind in diesem Forschungsfeld eher selten. Darüber hinaus wird eine Benennung von Machtkategorien zunehmend vernachlässigt. Hat der Machtansatz insbesondere einen Aufschwung durch die bahnbrechende Soziologie der ehelichen Machtverhältnisse durch Held (1978) erfahren, so ist seine Relevanz in der heutigen Zeit zunehmend an den Rand gedrängt worden. Zumeist werden Machtansätze auf rein ökonomische Macht reduziert, ohne zu berücksichtigen, dass das Machtkonzept im soziologischen Sinne komplexer ist als eine rein ökonomische Reduktion. Um der Komplexität gerecht werden zu können, ist ein soziologischer Machtansatz, der verschiedene Dimensionen von Macht einschließt, unabdingbar. Ferner soll aufgezeigt 
Tabelle 25: Eine Panelanalyse mit Mehrebenenmodellen zur innerhäuslichen Arbeitsteilung (GGS Welle 1 und 2)

\begin{tabular}{|c|c|c|c|c|c|c|}
\hline & Modell 1 & Modell 2 & Modell 3 & Modell 4 & \multicolumn{2}{|l|}{ Modell 5} \\
\hline \multicolumn{7}{|c|}{ Feste Effekte } \\
\hline Konstante & $\begin{array}{l}5,020 * * * \\
(0,013)\end{array}$ & $\begin{array}{l}4,918^{* * *} \\
(0,024)\end{array}$ & $\begin{array}{l}6,012^{* * *} \\
(0,160)\end{array}$ & $\begin{array}{l}5,781^{* * *} \\
(1,426)\end{array}$ & $\begin{array}{l}5,786^{* * *} \\
(1,442)\end{array}$ & \\
\hline \multicolumn{7}{|c|}{ Individualebene (Ebene1) } \\
\hline & B (SE) & B (SE) & $\mathrm{B}(\mathrm{SE})$ & B (SE) & $\mathrm{B}$ (SE) & [std.] \\
\hline Zeit & & $\begin{array}{l}0,017^{\star * *} \\
(0,003)\end{array}$ & $\begin{array}{l}0,021^{* * *} \\
(0,005)\end{array}$ & $\begin{array}{c}0,032^{* * *} \\
(0,006)\end{array}$ & $\begin{array}{l}0,032^{* * *} \\
(0,006)\end{array}$ & {$[0,079]$} \\
\hline \multicolumn{7}{|c|}{$\begin{array}{l}\text { Bildungsrelation } \\
\quad \text { (Ref. Frau < Mann) }\end{array}$} \\
\hline Frau $>$ Mann & & & $\begin{array}{l}-0,191^{\star \star *} \\
(0,044)\end{array}$ & $\begin{array}{l}-0,041 \\
(0,048)\end{array}$ & $\begin{array}{l}-0,039 \\
(0,048)\end{array}$ & {$[-0,29]$} \\
\hline Frau $=$ Mann & & & $\begin{array}{l}-0,079 * \\
(0,039)\end{array}$ & $\begin{array}{l}-0,010 \\
(0,040)\end{array}$ & $\begin{array}{l}-0,011 \\
(0,041)\end{array}$ & {$[-0,07]$} \\
\hline $\begin{array}{l}\text { Einkommen } \\
\text { (Ref. niedriges } \\
\text { Einkommen) }\end{array}$ & & & & & & \\
\hline Mittel & & & $\begin{array}{l}-0,193^{* * *} \\
(0,042)\end{array}$ & $\begin{array}{l}-0,145^{\star *} \\
(0,042)\end{array}$ & $\begin{array}{l}-0,146^{\star *} \\
(0,043)\end{array}$ & {$[-0,102]$} \\
\hline Hoch & & & $\begin{array}{l}-0,224 \\
(0,127)\end{array}$ & $\begin{array}{l}-0,171 \\
(0,126)\end{array}$ & $\begin{array}{l}-0,169 \\
(0,130)\end{array}$ & {$[-0,121]$} \\
\hline \multicolumn{7}{|c|}{$\begin{array}{l}\text { Einkommensrelation } \\
\quad(\text { Ref. Frau < Mann) }\end{array}$} \\
\hline Frau > Mann & & & $\begin{array}{l}-0,112^{*} \\
(0,053)\end{array}$ & $\begin{array}{l}-0,082 \\
(0,052)\end{array}$ & $\begin{array}{l}-0,090 \\
(0,053)\end{array}$ & {$[-0,058]$} \\
\hline Frau $=$ Mann & & & $\begin{array}{l}-0,005 \\
(0,033)\end{array}$ & $\begin{array}{l}-0,008 \\
(0,033)\end{array}$ & $\begin{array}{l}-0,011 \\
(0,033)\end{array}$ & {$[-0,006]$} \\
\hline Erwerbstätigkeit: & & & & & & \\
\hline $\begin{array}{l}\text { Vollzeit } \\
\text { (Ref. Teilzeit) }\end{array}$ & & & $\begin{array}{l}-0,075 \\
(0,039)\end{array}$ & $\begin{array}{l}-0,086^{*} \\
(0,038)\end{array}$ & $\begin{array}{l}-0,081^{*} \\
(0,039)\end{array}$ & {$[-0,061]$} \\
\hline $\begin{array}{c}\text { Erwerbsrelation } \\
\text { (pro Woche) } \\
\text { (Ref. Frau < }\end{array}$ & & & & & & \\
\hline Frau > Mann & & & $\begin{array}{l}-0,388^{* * *} \\
(0,047)\end{array}$ & $\begin{array}{l}-0,393^{* * *} \\
(0,047)\end{array}$ & $\begin{array}{l}-0,388^{* * *} \\
(0,048)\end{array}$ & {$[-0,280]$} \\
\hline Frau $=$ Mann & & & $\begin{array}{l}-0,155^{\star * *} \\
(0,037)\end{array}$ & $\begin{array}{l}-0,155^{\star \star \star} \\
(0,036)\end{array}$ & $\begin{array}{l}-0,147^{* * *} \\
(0,037)\end{array}$ & {$[-0,111]$} \\
\hline
\end{tabular}




\begin{tabular}{|c|c|c|c|c|}
\hline \multicolumn{5}{|l|}{ Kleine Kinder } \\
\hline (<6 Jahre) & $-0,025$ & $-0,019$ & $-0,019$ & {$[-0,013]$} \\
\hline (Ref. 6 Jahre +) & $(0,038)$ & $(0,039)$ & $(0,040)$ & \\
\hline \multicolumn{5}{|l|}{ Kinderanzahl } \\
\hline (group-mean & 0,045 & 0,013 & 0,012 & {$[0,002]$} \\
\hline zentriert) & $(0,041)$ & $(0,042)$ & $(0,042)$ & \\
\hline \multicolumn{5}{|l|}{ Kinderanzahl } \\
\hline (grand-mean & $-0,070^{\star * *}$ & $-0,052^{\star *}$ & $-0,054^{\star *}$ & {$[-0,036]$} \\
\hline zentriert) & $(0,019)$ & $(0,019)$ & $(0,020)$ & \\
\hline \multicolumn{5}{|c|}{ Paarbeziehungsdauer } \\
\hline (group-mean & $-0,034^{* * *}$ & $-0,045^{\star * *}$ & $-0,046^{\star \star \star}$ & {$[-0,056]$} \\
\hline zentriert) & $(0,009)$ & $(0,009)$ & $(0,009)$ & \\
\hline \multicolumn{5}{|c|}{ Paarbeziehungsdauer } \\
\hline (grand-mean & $-0,013^{* * *}$ & $-0,020^{* * *}$ & $-0,020^{\star * *}$ & {$[-0,131]$} \\
\hline zentriert) & $(0,002)$ & $(0,003)$ & $(0,003)$ & \\
\hline
\end{tabular}

Kontextebene (Ebene 2)

\begin{tabular}{|c|c|c|c|}
\hline \multicolumn{4}{|l|}{ Bildung } \\
\hline \multicolumn{4}{|l|}{ (Ref. niedrige } \\
\hline \multicolumn{4}{|l|}{ Bildung) } \\
\hline \multirow[t]{2}{*}{ Mittel } & $-0,174^{* *}$ & $-0,175^{\star *}$ & {$[-0,124]$} \\
\hline & $(0,053)$ & $(0,054)$ & \\
\hline \multirow[t]{2}{*}{ Hoch } & $-0,489 * * *$ & $-0,488^{* * *}$ & {$[-0,349]$} \\
\hline & $(0,059)$ & $(0,060)$ & \\
\hline \multicolumn{4}{|l|}{ Geburtskohorten } \\
\hline \multicolumn{4}{|l|}{ (Ref. 1980-93) } \\
\hline \multirow[t]{2}{*}{1939 und älter } & 1,043 & 1,028 & {$[0,743]$} \\
\hline & $(1,370)$ & $(1,383)$ & \\
\hline \multirow[t]{2}{*}{$1940-49$} & $-0,501^{* *}$ & $-0,504^{* *}$ & {$[-0,356]$} \\
\hline & $(0,144)$ & $(0,148)$ & \\
\hline \multirow[t]{2}{*}{$1950-59$} & $-0,240^{*}$ & $-0,244^{*}$ & {$[-0,170]$} \\
\hline & $(0,101)$ & $(0,104)$ & \\
\hline \multirow[t]{2}{*}{ 1960-69 } & $-0,137$ & $-0,137$ & {$[-0,096]$} \\
\hline & $(0,082)$ & $(0,085)$ & \\
\hline \multirow[t]{2}{*}{ 1970-79 } & 0,012 & 0,019 & {$[0,010]$} \\
\hline & $(0,070)$ & $(0,073)$ & \\
\hline
\end{tabular}


Zufallseffekte

\begin{tabular}{|c|c|c|c|c|c|}
\hline \multicolumn{6}{|l|}{ Varianz der Ebene1- } \\
\hline $\begin{array}{l}\text { Residuen (Varianz } \\
\quad \text { Individualebene } \mathrm{e}_{\mathrm{ti}} \text { ) }\end{array}$ & $\begin{array}{l}0,992^{* * *} \\
(0,017)\end{array}$ & $\begin{array}{l}0,991^{* * *} \\
(0,017)\end{array}$ & $\begin{array}{l}0,785^{\star * *} \\
(0,023)\end{array}$ & $\begin{array}{l}0,784^{* * *} \\
(0,023)\end{array}$ & $\begin{array}{l}0,763^{\star * *} \\
(0,027)\end{array}$ \\
\hline \multicolumn{6}{|c|}{ Varianz des Random } \\
\hline $\begin{array}{l}\text { Intercepts (Varianz } \\
\quad \text { Kontextebene } \mathrm{u}_{0 \mathrm{i}} \text { ) }\end{array}$ & $\begin{array}{l}0,988^{* * *} \\
(0,025)\end{array}$ & $\begin{array}{l}0,984^{* * *} \\
(0,025)\end{array}$ & $\begin{array}{l}1,098^{* * *} \\
(0,034)\end{array}$ & $\begin{array}{l}1,069^{* * *} \\
(0,034)\end{array}$ & $\begin{array}{l}1,218^{* * *} \\
(0,103)\end{array}$ \\
\hline $\begin{array}{l}\text { Intercept-Zeit } \\
\qquad\left(\text { Kovarianz } \mathrm{u}_{0 \mathrm{i}}, \mathrm{u}_{1 \mathrm{i}}\right)\end{array}$ & & & & & $\begin{array}{l}-0,024 \\
(0,016)\end{array}$ \\
\hline $\begin{array}{l}\text { Zeit } \\
\qquad\left(\text { Varianz } u_{1 i}\right)\end{array}$ & & & & & $\begin{array}{l}0,005^{*} \\
(0,003)\end{array}$ \\
\hline Intercept- & & & & & 0,059 \\
\hline $\begin{array}{l}\text { Kinderanzahl } \\
\qquad\left(\text { Kovarianz } \mathrm{u}_{0 \mathrm{i}}, \mathrm{u}_{2 \mathrm{i}}\right)\end{array}$ & & & & & $(0,074)$ \\
\hline $\begin{array}{l}\text { Zeit-Kinderanzahl } \\
\qquad\left(\text { Kovarianz } \mathrm{u}_{1 \mathrm{i}}, \mathrm{u}_{2 \mathrm{i}}\right)\end{array}$ & & & & & $\begin{array}{l}-0,004 \\
(0,012)\end{array}$ \\
\hline $\begin{array}{l}\text { Kinderanzahl } \\
\qquad\left(\text { Varianz } \mathrm{u}_{2 \mathrm{i}}\right)\end{array}$ & & & & & $\begin{array}{l}0,005 \\
(0,000)\end{array}$ \\
\hline \multicolumn{6}{|c|}{ Varianzkomponenten } \\
\hline ICC & 0,499 & 0,502 & 0,549 & 0,577 & 0,615 \\
\hline$-2 L L$ & 53587,16 & 53550,34 & 32039,56 & 31911,15 & 31933,00 \\
\hline$R^{2}$ (Total) & & 0,003 & 0,049 & 0,069 & 0,00 \\
\hline$R^{2}$ (Ebene 1) & & 0,001 & 0,209 & 0,210 & 0,231 \\
\hline$R^{2}$ (Ebene 2) & & 0,004 & $-0,111$ & $-0,071$ & $-0,233$ \\
\hline
\end{tabular}

Methode: Maximum Likelihood, nicht-standardisierte b-Koeffizienten, standardisierte z-transformierte Koeffizienten in eckigen Klammern, Standardfehler in Klammern

$\mathrm{N}=8660$ Personen (Ebene 2) mit 15801 Einzelbeobachtungen (Ebene 1), Sig. $=* * * \mathrm{p}<0,001 ; * * \mathrm{p}<0,01 ; * \mathrm{p}<0,05$

Quelle: GGS (Welle 1 und 2), eigene Berechnungen.

werden, dass die Familiensoziologie nicht einfach auf ein wesentliches Grundlagenkonzept der Macht verzichten kann. Eine Reintegration der Machtverhältnisse in die soziologische Erklärung innerhäuslicher Arbeitsteilung ist das theoretische Anliegen, das diese Forschungsarbeit leitet. Die größte Herausforderung einer theoretischen Typologie, die den Versuch unternimmt, derart anspruchsvolle Konzepte wie Macht und Verwirklichungschancen miteinander $\mathrm{zu}$ verbinden, ist in ihrer empirischen Umsetzung $\mathrm{zu}$ suchen. Bereits wenn der Capability Approach von Sen bedacht wird, wird allzu häufig kritisiert, dass niemand eine spezifische methodische Herangehensweise zur Überprüfung einfordern kann. Im Falle des Human Development Indexes berief sich die Konzeption zwar auf den Verwirklichungsansatz, doch dass es sich hierbei um eine sehr verkürzte Darstellung handelt und 
Sen Teile seiner Argumentation verwirft, indem er konkrete Indikatoren definiert, die für ein ,gutes Leben“" erforderlich sind, ist in extenso von diversen WissenschaftlerInnen diskutiert worden. Sens Begrifflichkeiten wie „Capability, Capability Set, Functionings, Agency, Conversion Factors und Well-being" bleiben abstrakt, sind kaum messbar, bieten wenig „harte Fakten" und lassen - überspitzt formuliert - viel Raum für Interpretation im Rahmen einer rhetorischen Meisterleistung. Bereits die Messung von Verwirklichungschancen stellt eine Schwierigkeit dar, weil sie hypothetisch sind: Wie kann Wissen darüber generiert werden, welche Wahlmöglichkeiten in ihrer Gänze Individuen zur Verfügung stehen?

„In fact the capability set is not directly observable and has to be constructed on the basis of presumptions [...]. Thus, in practice, one might have to settle often enough for relating well-being to the achieved - and observed - functionings, rather than trying to bring in the capability set (when the presumptive basis of such a construction would be empirically dubious)" (Sen 1992: 52).

Es ist insofern nicht verwunderlich, wenn sich Forschungsarbeiten aus dem Forschungsbereich des Capability Approachs häufig auf die realisierten Funktionsweisen konzentrieren und Vereinfachungen unterworfen sind. Auch die Frage, was Ressourcen und was Umwandlungsfaktoren (Conversion Factors) sind, hängt von der Perspektive und den situativen Gegebenheiten ab. Oftmals kann materieller Wohlstand nicht nur im Sinne von Ressourcen, sondern auch im Sinne von Conversion Factors definiert werden. Beispielsweise kann Geld in die schulische Bildung investiert werden, was als eine Transformation von Ressourcen in Umwandlungsfaktoren interpretiert werden könnte, weil Bildung die Chancen auf dem Arbeitsmarkt erhöht. Zugleich kann jedoch der Bildungsabschluss als Ressource gedeutet werden, da von ihm der weitere Zugang zu Ausbildungsmöglichkeiten oder Universitäten abhängig ist. Weiterführend kritisieren Bartelheimer et al. (2008), dass die Akteure bei Sen ,[...] eher abstrakte Individuen in statisch-stilisierten Positionen als handelnde Personen in sozialen Situationen mit ihren Interaktionsdynamiken“" sind (Bartelheimer et al. 2008: 39). Die Veränderungen von Handlungsmöglichkeiten durch den Handlungskontext, ferner die Fragen, wie und ob diese Veränderungen stattfinden, blieben ausgeblendet. Die Frage, wie die situativen Gegebenheiten der Handlungsoptionen beeinflusst werden könnten, sei ebenso ungeklärt. Dies sei im Hinblick auf Sens Analyserahmen kritisch zu hinterfragen, da sich relevante soziale Bedingungen, vornehmlich Machtverhältnisse, auf kollektive Zusammenhänge beziehen, die innerhalb dieses theoretischen Rahmens nicht skizziert werden könnten.

,Zum andern gerät der für Sens normativen Impetus zentrale Begriff der (positiven) Freiheit eigentümlich steril. Diese besteht nach Simmel wesentlich ,,aus einem Machtverhältnis zu Andren, aus der Möglichkeit, sich innerhalb eines Verhältnisses zur Geltung zu bringen, aus der Verpflichtung oder Unterwerfung Andrer, an der die Freiheit nun erst ihren Wert und ihre Verwertung fin- 
det.“(Simmel 1992: 100) Die Ausblendung der doppelten sozialen Beziehung, in der „,das Subjekt von anderen gebunden wird und andre [sic!] bindet“ (ebd.) bringt den Begriff der Freiheit damit um seine Substanz" (Bartelheimer et al. 2008: 39).

Es ist das zentrale Anliegen der vorliegenden Dissertation, Machtfragen innerhalb von Paarbeziehungen im Rahmen des Capability Approachs unter Einbezug institutioneller Kontextfaktoren $\mathrm{zu}$ erforschen, wodurch Handlungskonstellationen zwar nicht in ihrer Gesamtheit, doch zumindest in Teilen situiert werden.

Die Kritik eines zu hohen Abstraktionsniveaus könnte in ähnlicher Weise für ein soziologisches Konzept der Macht greifen: theoretisch ist es häufig vage, euphemistisch, missverständlich, indirekt. Eine Theorie, die davon ausgeht, dass Macht überall zu finden ist, sagt am Ende nichts mehr aus - der Erklärungsgehalt wird durch eine Allaussage von Macht nivelliert. Zugleich lässt sich Macht nicht auf rein ökonomische Bedingungen reduzieren. Es gilt folglich sowohl der Komplexität des theoretischen Konstrukts gerecht zu werden als auch eine angemessene empirische Erklärungsleistung zu erbringen. Eine adäquate Definition von Macht darf nicht zu weit sein, sondern muss idealiter präzise abgrenzbar sein - zugleich wird eine Flexibilität erfordert, Dynamiken einbeziehen zu können. Auf der theoretischen Ebene ist das Abstraktionsniveau durch die Differenzierung der Dimensionen von Macht in einem ersten Schritt gesenkt worden. Die Überführung der theoretischen Argumentation in empirisch messbare Konstrukte erfolgt während der Forschungsphase der Operationalisierung, die eine der größten und schwierigsten Herausforderungen dieser Forschungsarbeit $\mathrm{zu}$ sein vermag. Die Mehrebenenanalyse stellt hierbei ein methodisches Instrumentarium zur Verfügung, eine Differenzierung zwischen Machtindikatoren auf Individualund Kontextebene in Form der unabhängigen Variablen berücksichtigen zu können und kommt der inhaltlichen Vorstellung, Macht als ein mehrdimensionales Konstrukt zu verstehen, in der methodischen Umsetzung am nächsten. Theoretisch, vielmehr auch empirisch, wurde eine breite Perspektive eingenommen, um das Power-Capability Set soweit wie möglich in seiner Mannigfaltigkeit zu erfassen. Im Sinne des Capability Approachs kann grundsätzlich entweder der Versuch unternommen werden, die Auswahlmenge an Verwirklichungschancen selbst zu messen oder die Auswahlmenge wird in abstrakterer Weise über die unabhängigen Variablen annähernd eruiert. Wie bereits thematisiert, ist die erste Vorgehensweise durch die Problematik eines hypothetischen Rahmens von Verwirklichungschancen gekennzeichnet, weshalb die zweite Vorgehensweise im Zuge der vorliegenden Forschungsarbeit vollzogen und in Anlehnung an die inhaltliche Diskussion über Macht und Arbeitsteilung in Paarbeziehungen gewählt wurde. Die Dominanz traditioneller innerhäuslicher Arbeitsteilungsarrangements, die auf der Paarebene als realisierte Funktionsweise interpretiert werden kann, verweist nicht zuletzt auf 
einen Mangel an Verwirklichungschancen von Männern im familiären Bereich. Eine in Anlehnung an Modernisierungs- oder auch Rational-ChoiceAnsätzen vertretende These einer Erosion traditioneller Arbeitsteilungsarrangements in Paarbeziehungen infolge einer Annäherung der Bildungs- und Erwerbsverläufe zwischen den Geschlechtern hat sich entsprechend der Analyse als keineswegs haltbar erwiesen. Ganz im Gegenteil erscheinen traditionelle innerhäusliche Arbeitsteilungsarrangements als auffallend persistent. Wie die Mehrebenenmodelle belegt haben, sind Erklärungen dieser innerhäuslichen Form von Traditionalität auf der Mikroebene insbesondere im Rahmen von Gendertheorieansätzen - die sich auf einer kulturellen Dimension der Power-Capabilites verorten lassen können - zu finden. Ausgerechnet Frauen mit hohem Bildungsstand tendieren dazu, mehr Hausarbeiten zu leisten als Frauen mit niedrigem Bildungsstand. Dies erinnert an die Ergebnisse von Koppetsch/Speck (2015), die darauf hinweisen, dass gerade im AkademikerInnenmilieu FamilienernährerInnen mehr Hausarbeiten übernehmen, um die Selbstverwirklichung des nicht erwerbstätigen Partners zu unterstützen. Ferner sind den vorliegenden Ergebnissen zufolge - und ganz im Sinne des Gender-Display-Ansatzes nach Brines (1994) - Frauen mit einem höheren Erwerbsumfang als ihr Partner durch Erwerbs- und Hausarbeit doppelt belastet. Umso mehr aus einer modernisierungstheoretischen Perspektive vermutet wird, eine Gleichstellung zwischen den Geschlechtern zu erreichen, umso stärker - das mag für ModernisierungstheoretikerInnen paradox erscheinen - differenzieren sich die Genderungleichheiten aus. Koppetsch/Speck (2015) stellen fest, dass eine egalitäre Arbeitsteilung am ehesten im familistischen, wertkonservativen Milieu erfolgt - dieses Ergebnis kann durch einen niedrigen Bildungsstand von Frauen und einen religiösen Kontext der Regionen ergänzt werden. Solange an einem absoluten Ideal der Gleichheit festgehalten wird, ,schnappt die Genderidentitätsfalle zu“169 anstatt die Konstruktion von Genderrollen und ihre Identitäten als pluralistisch zu begreifen. Geringfügige egalisierende Effekte können durch familienpolitische Instrumente erreicht werden, wie anhand des Indikators frühkindlicher Bildungsprogramme gezeigt werden konnte. Darüber hinaus weist eine aktuelle Studie des RIW Leibniz Instituts für Wirtschaftsforschung (2018) darauf hin, dass Väter, die Elternzeit in Anspruch nehmen, tendenziell mehr Hausarbeiten leisten und mehr Zeit mit ihren Kindern verbringen ${ }^{170}$, was auch noch Jahre nach der Elternzeit beobachtet werden könnte. Auch Schober/Zoch (2015) bestätigen für Deutschland eine höhere Beteiligung an den Routine-

169 Das Argument von Sen (2007), dass eine falsche Illusion einer einzigen Identität einen Krieg der Kulturen vorantreibt und konstruiert, wird an dieser Stelle in einem übertragenen Sinne verwendet. Gemäß Sen (2007) ist die Erkenntnis einer universalen Vielfalt der menschlichen Existenz notwendig, um die Identitätsfalle zu überwinden.

170 „Results show that even short periods of fathers' parental leave may have long-lasting effects on fathers' involvement in childcare and housework" (Tamm 2018: 1). 
Hausarbeiten von Vätern, die Elternzeit in Anspruch genommen haben. Dies gelte jedoch vorwiegend für Väter, die nicht gleichzeitig mit ihrer Partnerin in Elternzeit waren. Eine längere Elternzeit der Mutter führe hingegen zu einer traditionelleren Arbeitsteilung (vgl. Schober/Zoch 2015: 1190). Das vorliegende Ergebnis, dass zwar in skandinavischen Ländern sowie Regionen die innerhäusliche Arbeitsteilung vergleichsweise am egalitärsten organisiert ist - doch jeglicher Egalitarismusideologien zum Trotz keineswegs egalitär! - führt in Kombination mit dem Ergebnis über familienpolitische Maßnahmen zur Schlussfolgerung eines geringfügig egalisierenden Möglichkeitsspielraums sozialpolitischer Steuerungen innerhäuslicher Arbeitsteilungsarrangements.

Dass Macht und das zu erklärende Phänomen der innerhäuslichen Arbeitsteilung im Zeitverlauf betrachtet werden müssen, ist durch die Panelanalyse der Mehrebenenanalyse abgedeckt worden, gleichwohl einschränkend zu bemerken ist, dass es sich lediglich um zwei Messzeitpunkte pro Person handelt. Eine Längsschnittanalyse ist zwingend erforderlich, um die prozesshafte Dynamik, die Machtverhältnissen und Arbeitsteilungsarrangements innerhalb des Lebenslaufs zugrunde liegt, abbilden zu können. So weist Held explizit darauf hin, dass Interaktionsverhältnisse nur bei längeren, kontinuierlichen Interaktionen asymmetrisch sind und als Machtverhältnisse bezeichnet werden können. Die von Bartelheimer et al. beschriebene eigentümliche Statik des Capability Approachs, nach der Entscheidungssituationen keine zeitliche Ausdehnung zu haben vermögen, wird durch diese Vorgehensweise vermieden (vgl. Bartelheimer et al. 2008: 40). Eine zentrale Schwierigkeit ist, dass im Falle der methodischen Umsetzung lediglich differenziert wird, inwiefern die erklärenden Variablen einen egalisierenden oder traditionalisierenden Effekt darstellen, ohne hierbei die theoretische Egalitarismus-Diskussion methodisch präzise umsetzen zu können. Dass Traditionalismus und Egalitarismus nicht einfach zwei gegensätzliche Pole sind, sondern viele verschiedene Abstufungen implizieren und insofern nicht als eine Dichotomie zu begreifen sind, haben Knight/Brinton (2017) ausführlich skizziert. Was methodisch berücksichtigt werden konnte, ist die kontextabhängige Annahme unterschiedlicher Entwicklungspfade zwischen Traditionalisierung und Enttraditionalisierung, indem die Prozesszeit als Random-Effekt modelliert wurde. Wie die Ergebnisse belegen, besteht hinsichtlich des Zeiteinflusses eine signifikante Varianz zwischen den Personen.

Ein Power-Capability Approach kann weiterführend die Gefahr implizieren, politisch instrumentalisiert zu werden. Bartelheimer et al. (2008) interpretieren Sen insoweit, dass er ein individuelles Maß ungleicher Teilhabe suche, an dem sich die gesellschaftliche Wohlfahrt bzw. gesellschaftliche Wohlfahrtsproduzenten messen lassen, wobei es entscheidend darauf ankäme, ob der Ansatz ein politisch positives oder negatives Vorzeichen bekäme. Einerseits könnte eine gesellschaftliche Verantwortung für Teilhabe betont 
werden, die dazu führe, dass ungleiche Teilhabeergebnisse - z. B. in Bezug auf ungleiche Bildungsabschlüsse - als eine mangelnde Chancengleichheit von gesellschaftlichen Institutionen (Bildungssystem) aufgefasst werden können. Andererseits könnte der Mensch als UnternehmerIn seiner Verwirklichungschancen gedeutet werden, wodurch ein individuelles Verschulden seiner Lebenslage zugeschrieben werden könne.

„So könnte Politik sich durch eine Rhetorik der Teilhabechancen von der Verantwortung für Teilhaberergebnisse entlasten. Dass Sen individuelle Verantwortung ermöglichen will, schützt das Konzept nicht vor Deutungen, die Individuen allein für ihre Lage verantwortlich machen. Chancengerechtigkeit lässt sich ungestrafter behaupten als Ergebnisgerechtigkeit" (Bartelheimer et al. 2008: 42).

An dieser Stelle sei abermals auf die in Kapitel 4.6. geführte EgalitarismusDiskussion verwiesen. Wie bereits erläutert, sei es vollkommen unklar, was im Rahmen der Teilhabedimension am gesellschaftlichen Leben zu unterstützende Verwirklichungschancen wären. Folglich könne es nicht darum gehen, die Gesamtmenge an Verwirklichungschancen aufzuzeigen, sondern nur diejenigen darzulegen, die bezüglich des Erkenntnisinteresses von Bedeutung sind. Welche Verwirklichungschancen von Bedeutung sind, müsse dieser Argumentation zufolge einer Bewertung unterzogen werden. Als Anwendungsbeispiel führen Bartelheimer et al. die Beschäftigungsfähigkeit als Verwirklichungschance an, was einer neoliberalen Aktivierungspolitik gleichkäme. Beschäftigungsfähigkeit kann jedoch sowohl in Bezug auf bezahlte als auch in Bezug auf unbezahlte Arbeit interpretiert werden, wobei betont werden soll, dass es sich hierbei nicht um ausschließliche Tätigkeiten handelt und der Capability Approach einen möglichst breiten Handlungsspielraum mit möglichst vielen Verwirklichungschancen erstrebt. Sozialpolitische Leistungen sind insofern mehr nach ihrer Optionalität, denn einer neoliberalen Aktivierungspolitik zu beurteilen. Die Aufgabe einer umfassenden Geschlechterpolitik besteht darin, das Verhältnis zwischen bezahlter und unbezahlter Arbeit zu reflektieren, insbesondere auch der Fürsorgearbeit Anerkennung zu schenken und die Vielfalt unterschiedlicher Lebensmodelle $\mathrm{zu}$ berücksichtigen. Beschäftigungsfähigkeit ist nur eine von mehreren Verwirklichungschancen - wäre dies nicht der Fall, käme Erwerbstätigkeit einem Erwerbszwang ohne Alternativen gleich und müsste als umgesetzte Funktionsweise gedeutet werden. Sen kommt es doch gerade auf das Ausmaß an Verwirklichungschancen an, ohne hierbei eine Verwirklichungschance als die $\mathrm{zu}$ erstrebende zu begreifen. Die jeweilige Bewertung der Verwirklichungschancen obliegt den Individuen. Wohlfahrtsstaatliche Leistungen, die jegliche Tätigkeiten außerhalb der Erwerbstätigkeiten ausblenden, widersprechen der Argumentation von Sen. Menschen sind im Sinne von Sen und auch im Sinne von Nussbaum insofern zu befähigen, entsprechend ihrer Fähigkeiten ein eigenständiges Leben führen und hierbei alle individuellen Gestaltungsmöglichkeiten sowie gesellschaftliche Teilhabemöglichkeiten ausschöpfen zu 
können. Dies verlangt nach einer Berücksichtigung der Diversität von Individuen und ihrer Lebensentwürfe, die eine Teilhabe an unterschiedlichen sozialen Feldern, darunter bezahlte sowie unbezahlte Arbeit für beide Geschlechter, ermöglicht.

Der Komplexität des theoretischen Ansatzes ist geschuldet, dass dieser nicht eins zu eins empirisch umgesetzt werden kann. Dennoch wurde in der vorliegenden Forschungsarbeit der Versuch unternommen, ein Verfahren anzuwenden, das der theoretischen Argumentation möglichst nahekommt. Die Mehrebenenmodelle ermöglichten zumindest einige wesentliche Einblicke in die Dynamik von Macht und innerhäuslicher Arbeitsteilung auf unterschiedlichen Analysedimensionen. Auch wenn die methodische Vorgehensweise niemals erschöpfend sein kann und stets gewissen Regeln unterworfen ist, sind gleichwohl durch sie interessante empirische Erkenntnisse festgestellt worden, die dazu verhelfen, innerhäusliche Arbeitsteilung als keine reine Privatentscheidung, sondern im Kontext gesellschaftlicher Entwicklungsprozesse zu verstehen. 



\section{Fazit und Ausblick: Power matters?}

„Bringing Power Back In“ lautete das Anliegen der vorliegenden Dissertation im Rahmen derer argumentiert wurde, dass im Kontext der liberalen Egalitarismus-Ideologie aktuelle einschlägige Studien den Machtaspekt, der innerhäuslichen Arbeitsteilungsarrangements in Paarbeziehungen zugrunde liegt, zunehmend vernachlässigen. In Anlehnung an den Capability Approach nach Amartya Sen wurde eine Gleichverteilung der Verwirklichungschancen und eine damit verbundene Gleichwertigkeit der Geschlechter in den Fokus der Aufmerksamkeit gerückt, um eine auf Geschlechterdichotomie basierende Argumentation zu überwinden und eine pluralistische Egalitätskonstruktion zu ermöglichen. Eine pluralistische Egalitätskonstruktion zielt darauf ab, dass beide PartnerInnen über die gleichen Wahlmöglichkeiten einer autonomen Lebensführung, einschließlich der Arbeitsteilungsarrangements, verfügen. Die Kontinuität der traditionellen Arbeitsteilung zwischen den Geschlechtern verweist nicht zuletzt auf einen Mangel an Verwirklichungschancen (auch von Männern im familiären Bereich) und damit auf das Wirken strukturell verfestigter Machtpotentiale.

Dass ein theoretisches Konzept von Macht häufig diffus und verwirrend ist, hat bereits Bierstedt (1950) festgestellt: „We all know perfectly well what it [power] is - until someone asks us" (Bierstedt 1950: 730). Macht ist situativ und tritt in unterschiedlichen Formen auf: Seien es die Chance, den eigenen Willen auch gegen Widerstreben durchzusetzen, der soziale Einfluss, der Einfluss auf Ergebnisse, ein geringes Interesse an der Paarbeziehung, bessere Alternativen, der Nachteil, auf eine Leistung keine Gegenleistung erbringen zu können, der Mangel, die eigenen Bedürfnisse nicht ändern oder gewaltsam erzwingen zu können, die Kontrolle über Ressourcen oder Formen der Unterdrückung (die Frauenforschung hat relativ lange an einem vereinfachten Unterdrückungskonzept von Macht festgehalten, s. hierzu Knapp 2012: 226). Im Sinne von Foucault gilt es, diese juristische Konzeption von Macht als Unterdrückung zu überwinden (vgl. Foucault 1978: 34f.; vgl. Foucault 2015: 221). Des Weiteren lässt sich Macht nicht ausschließlich aus einer ökonomischen reduktionistischen Perspektive betrachten. Diese Kritik verlangte nach einem Konzept, das sowohl vertikaler als auch horizontaler Art sein kann und der Mehrdimensionalität von Macht gerecht wird. Das Wirken strukturell verfestigter Machtpotentiale manifestiert sich, wie Kreckel (2006) betont hat, in der Ungleichheit der Verwirklichungschancen (Kreckel 2006: 15). Gleichermaßen vielfältig wie die Macht sind die Gegenstände der Machtanalyse: Regime, Staaten, Regionen, sozialen Klassen, Institutionen, Gruppen, Individuen und das Verhältnis zwischen den Geschlechtern (vgl. Knapp 2012: 226). Ein theoretisches Konzept von Macht sollte insofern zumindest nach zwei Seiten offen sein: einerseits in Bezug auf die gesellschaftlich-historische 
Strukturanalyse und die Verteilung machtrelevanter Ressourcen, andererseits in Bezug auf die Handlungsebene der Geschlechter. Letztere umfasst Machtwirkungen in den Interaktionen, symbolisch-kulturelle sowie materielle Machtrelationen in den Paarbeziehungen (vgl. Knapp 2012: 231f.).

Die zu Beginn gestellte Forschungsfrage, inwiefern Machtstrukturen und Empowerment die innerhäusliche Arbeitsteilung in Paarbeziehungen im Kontext europäischer Regionen determinieren, bedurfte zur Beantwortung insofern einer Unterscheidung der Formen von Macht. Unter Bezug auf KlassikerInnen zur Soziologie (ehelicher) Machtverhältnisse in Paarbeziehungen - wie u. a. die bahnbrechende Studie von Thomas Held (1978) - und im Rahmen des Capability Approachs von Amartya Sen im Sinne eines Ermächtigungskonzepts, wurde eine Typologie der Machtverhältnisse hergeleitet, die zwischen verschiedenen Machtdimensionen (interaktionelle Macht, kulturelle Macht, institutionelle Macht, Macht der Teilhabe, strukturelle Macht) differenziert. Macht wird als ein latentes theoretisches Konstrukt verstanden, das gleichwohl in unterschiedliche Dimensionen ausdifferenziert werden kann. Die, auf den unterschiedlichen Ebenen verorteten, Power-Capabilities bilden in ihrer Gesamtheit das zur Verfügung stehende Power-Capability Set (s. Abbildung 14). Theorien, die aktuell den wissenschaftlichen Diskurs zur Erklärung innerhäuslicher Arbeitsteilungsarrangements dominieren (insbesondere ökonomische Theorien und Gendertheorien), wurden hierbei in die Typologie integriert.

Nachdem eine Einführung in die Thematik, in die forschungsleitende Fragestellung und in den Stand der Forschung über mikro- und makrosoziologische Determinanten der innerhäuslichen Arbeitsteilung erfolgte, wurde Kritik geübt: Aktuelle Studien zur Aufteilung der Hausarbeiten bieten unterschiedliche Perspektiven, doch bleiben diese zumeist entweder auf der Mikroebene oder auf der Makroebene verhaftet, ohne ihre fundamentale Wechselwirkung einzubeziehen. Insbesondere birgt die innerhäusliche Arbeitsteilung die Gefahr, als rein individuelle Privatentscheidung betrachtet zu werden. Ferner werden infolge der aufkommenden liberalen Egalitarismusdebatte Machtaspekte zunehmend aus familiensoziologischen Studien ausgeklammert, was zum Gegenstand einer Reintegration des Machtansatzes in Form eines Mehrebenenkonzepts führte, um die innerhäusliche Arbeitsteilung im europäischen Kontext erklären zu können.

Aufbauend auf der geübten Kritik wurde eine Theorie mehrdimensionaler Machtverhältnisse hergeleitet. Zunächst wurden mikrosoziologische Theorien (Rational Choice Theorien, Bargaining Theorien, Austauschtheorien und Gendertheorien) dargelegt und in Bezug auf ihre Reichweite kritisch beleuchtet. Es folgte eine Beschreibung des allgemeinen Mikro-Makro-Problems in der Soziologie, das weiterführend im Spezifischen in Bezug auf Familie und Geschlecht erläutert wurde und in der makrosoziologischen Theoriedebatte über Gender-Ungleichheiten im sozialstrukturellen Kontext - u.a. von Wohl- 
fahrtsregimen (Wohlfahrtsregime können klassen- und geschlechtsspezifische Handlungsmöglichkeiten beeinflussen) - mündete. Wesentlich für die Argumentation ist der Capability Approach, der die theoretische Rahmung der hergeleiteten Machttypologie darstellt und es möglich machte, Macht als Befähigung zu rekonzeptualisieren sowie Capabilities als Power-Capabilities weiterzuentwickeln. Im Sinne einer Ermächtigung (Empowerment) legen Power-Capabilities den Handlungsspielraum fest. Die in dieser Arbeit dargelegte Verknüpfung von ökonomischen, austauschtheoretischen, soziologischen, mikro- und makrosoziologischen Theorien im Kontext des CapabilityApproachs soll ein komplexes Machtgefüge zur Erklärung innerhäuslicher Arbeitsteilungsarrangements bieten. Unter Berücksichtigung des zentralen Arguments, dass Macht sich in der Ungleichheit der Verwirklichungschancen, d. h. der Capabilities, widerspiegelt, wurden auf der Mikroebene Machtfähigkeiten im Sinne von Sen als Fähigkeiten/Aktivitäten, die spezifische Machtressourcen voraussetzen und von einer Person zur Realisierung einer bestimmten Handlungsalternative genutzt werden, verstanden. Mittels der kulturellen Macht wurden in Anlehnung an Gendertheorien durch Werte und Normen legitimierte kulturspezifische Benachteiligungen oder Begünstigungen der Power-Capabilities erfasst. Auf der nächsten Ebene wurden Institutionen als handlungsleitende kulturelle Institutionen sowie in ihrer organisatorischen Verfasstheit im Rahmen der institutionellen Dimension von Macht diskutiert, von der sich die Macht der Teilhabe in Form von Inklusions- und Exklusionsstrukturen unterscheiden lässt. Über die individuelle Wahlfreiheit zu verfügen, zwischen den Handlungsalternativen einer Teilhabe und NichtTeilhabe an sozialen Feldern frei entscheiden zu können, basiert auf dem Grundgedanken der Verwirklichungschancen. Die strukturelle Macht wurde auf der Makroebene verortet. Hier regulieren Formen von Wohlfahrtsstaatlichkeit Handlungsspielräume, wodurch Individuen entsprechend ermächtigt werden. Ein Teil der Defizite einer begrenzten Reichweite der mikrosoziologischen Theorien konnte durch den argumentativen Zusammenhang innerhalb eines makrostrukturellen Kontextes dazu beitragen, die Barrieren (individueller, sozietaler, institutioneller sowie struktureller Art) eines Pluralismus innerhäuslicher Arbeitsteilungsarrangements umfassender darzulegen. Folglich wurden unterschiedliche Dimensionen interaktioneller, kultureller, institutioneller Macht, Macht der Teilhabe und struktureller Macht hergeleitet. Zwischen diesen Dimensionen wurden sowohl zeitabhängig-dynamische Entwicklungen als auch Interaktionen bedacht. Das System der Machtdimensionen wurde weder als reiner ,top-down-Prozess' noch als reiner ,bottomup-Prozess' verstanden - hingegen vielmehr als das Ergebnis eines komplexen Interdependenzgefüges. Des Weiteren galt es die Machtdimensionen je nach Kontext auszudifferenzieren (für eine zusammenfassende Übersicht der Typologie s. Abbildung 4 in Kapitel 9.5.). 
Auf Grundlage der theoretischen Diskussion wurden explizite Hypothesen abgeleitet, die nach Machtdimension und Kontext systematisch differenziert wurden, um die jeweiligen Wechselwirkungen spezifisch abgrenzbar zu machen.

Es folgte die Beschreibung der theoretisch bedingten Länderauswahl auf Basis genderrelevanter Policies sowie eine kritische Reflexion der Diversität und Fragmentiertheit sowohl von Wohlfahrtsregimen als auch von Ländern, weshalb es den methodologischen Nationalismus zu überwinden gilt. Nicht nur zwischen Regimen und zwischen Ländern bestehen Unterschiede, sondern auch innerhalb dieser Kontexte ließ sich eine innere institutionelle Fragmentiertheit ökonomischer, familienpolitischer und kultureller Rahmenbedingungen der innerhäuslichen Arbeitsteilungsarrangements feststellen.

Der empirische Teil der Arbeit wurde mit der Beschreibung des Datensatzes (Generations and Gender Programme) eingeführt. Da sich die Machtdimensionen auf verschiedenen Ebenen analytisch verorten lassen, wurde methodisch ein Mehrebenendesign gewählt, das eine hierarchische Differenzierung zwischen der individuellen Mikroebene und der kontextuellen Makroebene ermöglicht. Hierdurch waren die Einflüsse der individuellen und kontextuellen Power-Capabilities auf die abhängige Individualvariable der innerhäuslichen Arbeitsteilung überprüfbar (nicht modellierbar sind im Rahmen der Mehrebenenanalyse hingegen Einflüsse von Individualmerkmalen auf Kontextmerkmale). Schließlich galt es auch den dispositiven Charakter von Macht zu bedenken, durch den sich eine Kontextabhängigkeit ergibt, welche Dimension innerhalb welcher sozialen Situation eine Erklärungsleistung für familiale Arbeitsteilungsarrangements erbringen kann. Die Verwendung von 48 NUTS1-Regionen als Kontexteinheiten hat dazu verholfen, Unterschiede zwischen den Regionen in Bezug auf familienpolitische, kulturelle und ökonomische Rahmenbedingungen abzubilden, die sich in innerhäuslichen Arbeitsteilungsarrangements widerspiegeln, sowie eine Überwindung des methodologischen Nationalismus zu erreichen. Die in Abgrenzung zur Typologie Esping-Andersens (1990) einsetzende Diskussion über die innere Vielfalt und Fragmentiertheit von Regimetypen wurde nicht nur inhaltlich thematisiert, sondern auch empirisch durch die Betrachtung von europäischen Regionen in das Forschungsdesign einbezogen.

Die Darlegung der Ergebnisse erfolgte schrittweise: von deskriptiven Darstellungen über klassische OLS-Regressionsmodelle bis hin zu Mehrebenenanalysen. Um die Dynamik, die Machtverhältnissen zugrunde liegt, aufzugreifen, wurden im letzten Analyseschritt zeitliche Veränderungen zwischen den Befragungswellen des Generations and Gender Programms im Rahmen einer Panelanalyse mit Mehrebenenmodellen durchgeführt.

Bezugnehmend auf die makrosoziologische Dimension regionaler Kontextdifferenzen können die folgenden zentralen Erkenntnisse festgehalten werden (Abbildung 14 beinhaltet eine zusammenfassende Gesamtübersicht 
des Power-Capability Approachs, wobei die Effekte, die im Rahmen der Analyse bestätigt werden konnten, jeweils unterstrichen sind): Anhand der Ergebnisse kann belegt werden, dass - aller Modernisierungstheorien und dem Gleichheitsdiskurs zum Trotz - die innerhäusliche Arbeitsteilung in erstaunlichem Maße traditionell organisiert bleibt. Dennoch bestehen - innerhalb dieses traditionellen Musters - geringfügige Unterschiede in der Ausführung der Hausarbeiten zwischen europäischen Regionen. Insgesamt gehen $6,2 \%$ der innerhäuslichen Arbeitsteilung auf Unterschiede zwischen den NUTS1-Regionen zurück. In Regionen mit einem höheren Anteil an frühkindlichen Bildungseinrichtungen (politische EmpowermentDimension/ZweiverdienerInnen Policies) verrichten Frauen weniger Hausarbeiten. Hingegen sind Regionen mit einer hohen Fertilitätsrate förderlich für traditionelle innerhäusliche Arbeitsteilungsarrangements. Überraschender Weise wirkt sich der Säkularisierungsgrad der Regionen (kulturelle Empowerment-Dimension) ebenso als begünstigend für eine traditionelle Aufteilung der Hausarbeiten aus.

Im Hinblick auf die mikrosoziologische Dimension ist anhand der Ergebnisse zu konstatieren, dass relative Ressourcen zugunsten der Frau, die auf einen ökonomischen Aspekt der Verhandlungsmacht in Paarbeziehungen verweisen, keinen egalisierenden Effekt auf die Ausführung der Hausarbeiten haben. Ferner tendieren ausgerechnet Frauen mit hohem Bildungsstand dazu, mehr Tätigkeiten im Haushalt zu übernehmen als Frauen mit niedrigem Bildungsstand. Konträr zur ressourcentheoretischen Argumentation ist die kulturelle Dimension von Macht von zentraler Bedeutung, die sich vorwiegend in einer hypogamen Erwerbskonstellation von Paaren manifestiert. Finanzielle Abhängigkeiten des Mannes werden seinerseits nach wie vor - wie bereits Brines (1994) festgestellt hat - durch ein „Nicht-Erledigen“ von Hausarbeiten kompensiert. Normverletzungen der Erwerbskonstellation werden durch traditionelle innerhäusliche Arbeitsteilungsarrangements ausgeglichen, was als ein Ausdruck einer kulturellen Dimension von Macht, einer durch gesellschaftliche kulturelle Normen legitimierte Macht, gedeutet wird. Schon Held (1978) pflegte zu sagen, dass ,[...] die normative Trennung der Bereiche [zugeschriebene weibliche Hausarbeit und männlich dominierte Erwerbstätigkeit] somit stärker ist als das Gleichheitsideal“" (Held 1978: 71). Durch den Gleichheitsdiskurs werden nicht nur Geschlechterungleichheiten verschärft, wie Koppetsch (1998) festgestellt hatte, sondern darüber hinaus Machtverhältnisse in Paarbeziehungen verschleiert. Der „skandinavische Traum“ einer „Egalitarismusgesellschaft“ bleibt in Bezug auf die Aufteilung von Hausarbeiten eine Utopie, die in keiner der untersuchten Regionen vorgefunden wurde. Familiale Arbeitsteilungsarrangements unterliegen einer weitgehenden Änderungsresistenz; im Zeitverlauf betrachtet, ist nur eine sehr geringe Variabilität aufzuweisen. Mit zunehmender Paarbeziehungsdauer kann vielmehr ein Traditionalisierungsprozess beobachtet werden. Gleichwohl gilt es 
die Annahme einer einheitlichen Trendentwicklung kritisch zu hinterfragen: sowohl Traditionalisierungs- als auch Enttraditionalisierungsprozesse sind als unterschiedliche Entwicklungspfade kontextspezifisch zu betrachten, wie durch die Einführung der Prozesszeit als Random-Effekt analytisch umgesetzt wurde, der sich als signifikant erwies.

Dass im Sinne einer dynamischen Perspektive die erreichte Funktionsweise („Achieved Functioning“) der traditionellen Arbeitsteilung wiederum eine Rückkopplung auf Ressourcenkonstellationen, -relationen und individuelle Umwandlungsmöglichkeiten („Conversion Factors") haben könnte, ist ergänzend durch den gestrichelten Pfeil dargestellt (s. Abbildung 14). Eine Überprüfung dieser Wechselwirkung war im Rahmen der hier durchgeführten Mehrebenenmodelle jedoch nicht möglich.

Werden die Ergebnisse auf die Ausgangsdiskussion bezogen, kann als Fazit dieser Dissertation festgehalten werden, dass eine Annäherung der Bildungs- und Erwerbsverläufe zwischen den Geschlechtern nicht - wie es Rational-Choice-Ansätze und Modernisierungstheorien hätten erwarten lassen - zu einer Auflösung der traditionellen innerhäuslichen Arbeitsteilung führen. Des Weiteren sind der strukturelle und der kulturelle Kontext als zentrale Handlungsspielräume festzuhalten, in denen sich die Dimensionen der Macht auf Mikro- und Makroebene manifestieren.

Die abschließende Diskussion beleuchtete das Abstraktionsniveau des Machtansatzes im Rahmen des Capability-Approachs kritisch, um darzulegen, wie mit dieser Abstraktionsproblematik - nämlich über die kontextabhängigen Ausdifferenzierungen der Formen von Power-Capabilities - in dieser Arbeit umgegangen wurde.

Anstatt eine einzige liberal-egalitaristische Norm der innerhäuslichen Arbeitsteilung zu vertreten, sollten insbesondere Frauen dazu befähigt werden, ihr Leben individuell und autonom gestalten zu können, was eine auf Gleichwertigkeit basierende pluralistische Argumentation von Genderrollen und Identitäten voraussetzt. Um dieses zu Ziel erreichen, bedarf es nicht zuletzt einer höheren Anerkennung und Wertschätzung von Hausarbeiten sowie politischer Rahmenbedingungen, die die Vielfalt unterschiedlicher Lebensentwürfe unterstützen. 
Abbildung 14:

Zusammenfassende Darstellung des Power-Capability Approachs

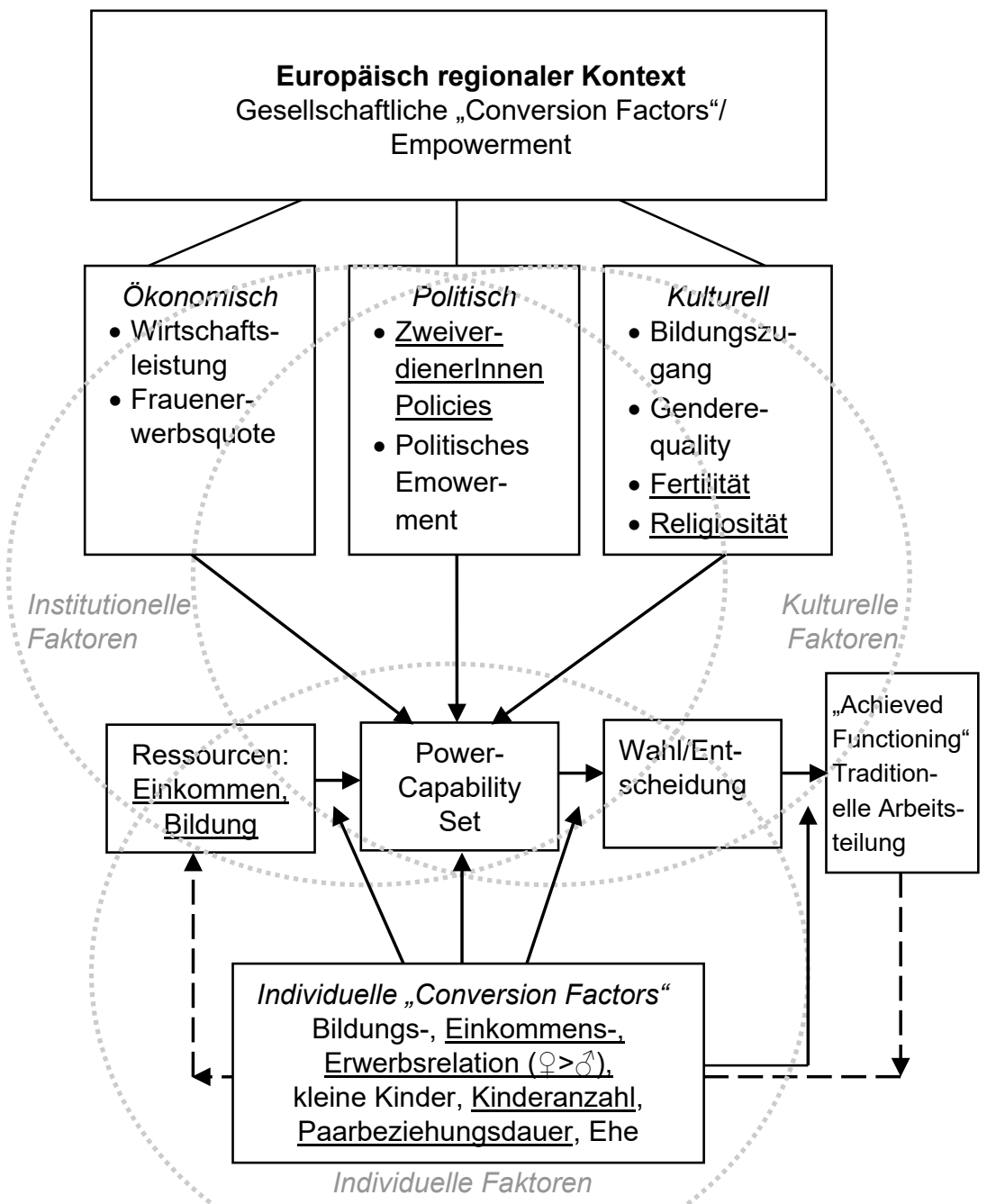

Chancenaspekt đer Freiheit

Verfahî̉ensaspekt der Freiheit

Quelle: modifiziert nach Bartelheimer et al. 2008: 10; Hobson/Fahlén 2009: 230. 
Der empirische Teil dieser Dissertation unterliegt einigen methodischen Einschränkungen. In erster Linie ist die Konstruktion des „Task-ParticipationIndex" in dreierlei Hinsicht kritisch zu beleuchten. Erstens ist dieser höchst selektive Index davon abhängig, welche Einzelindikatoren als Hausarbeitstätigkeiten erfasst werden. Zweitens konnte anhand der Daten des Generations and Gender Surveys keine akribische Betrachtung des zeitlichen Umfangs im Sinne einer Zeitbudgeterhebung vorgenommen werden und drittens handelt es sich bei diesen Daten um keine direkte Paarbefragung, wenngleich die Möglichkeit besteht, auf einige Antworten der PartnerInnen zu schließen, da die Befragten auch Fragen zum/zur PartnerIn gestellt bekommen haben. Ferner ist davon auszugehen, dass sich die kulturelle Bedeutung der jeweiligen Hausarbeitstätigkeiten zwischen den Regionen stark unterscheiden könnte hierüber könnte beispielsweise eine Clusteranalyse der Hausarbeitstätigkeiten einen weiteren Aufschluss geben. Insbesondere für wohlhabendere Haushalte könnte weiterführend die Auslagerung von Hausarbeiten eine zentrale Rolle spielen, die es zukünftig zu berücksichtigen gilt (im Rahmen des GGS war der Anteil an ausgelagerten Hausarbeiten jedoch eher gering). Aufgrund der Tatsache, dass bisher lediglich zwei Panelwellen des GGS zur Verfügung standen, wird sich erst im Laufe der kommenden Jahre die Kohärenz der Ergebnisse zeigen. Folglich müssen die dargestellten Veränderungen zwischen der ersten und zweiten Welle ,[...] daher vorsichtig interpretiert werden, weil sie zufällig sein können oder Teil von Schwankungen, die durch unberücksichtigte Faktoren und Stichprobenfehler zustande kommen“" (Baumgartner/Fux 2004: 117f.). Gewissheit über die Konsistenz der Ergebnisse können lediglich wiederholte Analysen, die ggf. auch auf anderen Datenquellen basieren, liefern. Ein weiteres Forschungsdesiderat der vorliegenden Dissertation ist die fehlende empirische Berücksichtigung südeuropäischer und liberaler Länder, die zwar immer wieder in die theoretische Diskussion einbezogen wurden, doch aufgrund mangelnder Daten im GGS (Italien ist zwar im GGS enthalten, doch fehlen Angaben über die Aufteilung der Hausarbeiten) keinen Eingang in die Analyse gefunden haben. Denkbar wäre, die Analyse durch eine Ergänzung anderer Datenquellen, wie beispielsweise ein Matching des Generations and Gender Surveys mit dem britischen Haushaltspanel, zu erweitern.

Im Rahmen zukünftiger Studien könnte der Fokus des Zusammenspiels von Machtverhältnissen und innerhäuslichen Arbeitsteilungsarrangements in Paarbeziehungen auf transnationale Pflegemigrationsprozesse verschoben werden. Globale Ökonomisierungstendenzen führen $\mathrm{zu}$ einer vermehrten Auslagerung von Hausarbeiten, einer zunehmenden außerhäuslichen Erwerbsbeteiligung von Frauen in westlichen Gesellschaften und zu einer Begünstigung von Pflegemigration (auf europäischer Ebene umfasst dies insbesondere eine Ost-West-Binnenmigration, auf globaler Ebene eine verstärkte Süd-Nord-Migration). Nicht nur innerhäusliche Arbeitsteilungsarrangements 
sind durch eine Persistenz der traditionellen Aufgabenteilung gekennzeichnet, sondern auch die Arbeitskräftemigration im Bereich der Pflege zeichnet sich überwiegend durch weibliche Arbeitskräfte im Kontext globaler Machtverhältnisse aus. Die Perspektivenverschiebung von der innerhäuslichen Arbeitsteilung auf die Pflegemigration verdeutlicht die Intersektionalität von ,Gender and Care', da die private Versorgungsarbeit nicht nur feminisiert, vielmehr auch ethnisiert wird. Die zunehmende Ausdifferenzierung sozialer Ungleichheiten betrifft im Spezifischen den Bereich von Genderungleichheiten, der durch das Ideal einer absoluten Gleichheit vorangetrieben wird welches gleichwohl Machtverhältnisse in Paarbeziehungen verschleiert - und in einer endlosen Spirale von Ressentiments mündet. Genderrollen und Genderidentitäten sind in ihrer Vielfalt zu erfassen, anstatt gemäß der „Genderidentitätsfalle" Gefahr zu laufen, eine liberal-egalitaristische Gleichheitsnorm als universalen Gerechtigkeitsanspruch zu propagieren. 



\section{Literaturverzeichnis}

Alexander, Amy C./Welzel, Christian 2014: Eroding patriarchy. The coevolution of women's rights and emancipative values. In: International Review of Sociology: Revue Internationale de Sociologie, 1-22.

Arpino, Bruno/Esping-Andersen, Gøsta/Pessin, Léa 2015: How Do Changes in Gender Role Attitudes Towards Female Employment Influence Fertility? A Macro-Level Analysis. In: European Sociological Review 0 (0), 113.

Baldwin, David A. 1971: Money and Power. In: The Journal of Politics 33 (3), 578-614.

Baldwin, David A. 1978: Power and Social Exchange. In: The American Political Science Review 72 (4), 1229-1242.

Baldwin, Peter 1990: The Politics of Social Solidarity. Class Bases of the European Welfare State 1875-1975. Cambridge: Cambridge University Press.

Baldwin, Peter 2003: Der europäische Wohlfahrtsstaat. Konstruktionsversuche in der zeitgenössischen Forschung. In: Zeitschrift für Sozialreform 49 (1), 45-63.

Bartelheimer, Peter/Büttner, René/Kädtler, Jürgen 2008: Amartya Sens wohlfahrtstheoretischer Ansatz Verwirklichungschancen als Konzept zur Beurteilung von Arbeitsmarkt und Sozialpolitik. In: http://www.sofigoettingen.de/fileadmin/Textarchiv/Kolloquium/Amartya_Sen_21-112008.pdf, zugegriffen am 20.09.2018.

Batalova, Jeanne A./Cohen, Philip 2002: Premarital Cohabitation and Housework: Couples in Cross-National Perspective. In: Journal of Marriage and Family 64, 743-755.

Baumann, Eveline 2012: Post-Soviet Georgia. It's a Long, Long Way to "Modern" Social Protection... In: Économies et Sociétés 46 (2), 259285.

Baumgartner, Doris A. 2003: Frauen im mittleren Erwerbsalter. Eine Studie über das Potenzial erhöhter Arbeitsmarktpartizipation von Frauen. In: Beiträge zur sozialen Sicherheit. Forschungsbericht 6 (3), 1-71.

Baumgartner, Doris A. 2008: Die flexible Frau. Frauenerwerbsarbeit im Werte- und Struturwandel. Zürich: Seismo Verlag.

Baumgartner, Doris A./Fux, Beat 2004: Und sie bewegen sich doch nicht: die Männer. Zur geschlechtsspezifischen Verteilung der Erwerbsarbeit in Familien. In: Zimmermann, Erwin/Tillmann, Robin (Hrsg.): Leben in der Schweiz 1999-2000. Ein Jahr im Leben der Schweizer Familien und Haushalte. Bern u. a.: Peter Lang Verlag, 109-130. 
Beck, Ulrich 2008: Risikogesellschaft und die Transnationalisierung sozialer Ungleichheiten. In: Berger, Peter A./Weiß, Anja (Hrsg.): Transnationalisierung sozialer Ungleichheit. Wiesbaden: VS Verlag für Sozialwissenschaften.

Becker, Gary S. 1981: A Treatise on the Family. Cambridge/London: Harvard University Press.

Becker, Gary S. 1982: Ökonomische Erklärung menschlichen Verhaltens. In: Boettcher, Erik (Hrsg.): Die Einheit der Gesellschaftswissenschaften. Studien in den Grenzbereichen der Wirtschafts- und Sozialwissenschaften. Tübingen: Mohr Siebeck.

Becker-Schmidt, Regina 1987: Die doppelte Vergesellschaftung - die doppelte Unterdrückung. Besonderheiten der Frauenforschung in den Sozialwissenschaften. In: Unterkircher, Lio/Wagner, Ina (Hrsg.): Die andere Hälfte der Gesellschaft. Wien: Verlag des österreichischen Gewerkschaftsbundes, 10-25.

Beckers, Tilo 2010: Das Vergleichen von Vergleichen als Validierungsstrategie. Ein Vorschlag für die Sekundärdatenanalyse von Umfragedaten. In: http:/www.gesis.org/fileadmin/upload/dienstleistung/fachinformationen/ servicepublikationen/sofid/Fachbeitraege/Methoden_10-1-2_FB.pdf, zugegriffen am 20.07.2015.

Beck-Gernsheim, Elisabeth 1994: Auf dem Weg in die postfamiliale Familie - Von der Notgemeinschaft zur Wahlverwandtschaft. In: Beck, Ulrich/Beck-Gernsheim, Elisabeth (Hrsg.): Riskante Freiheiten. Frankfurt am Main: Suhrkamp, 115-138.

Beck, Ulrich/Grande, Edgar 2010: Jenseits des methodologischen Nationalismus. Außereuropäische und europäische Variationen der Zweiten Moderne. In: Soziale Welt 61 (3/4), 187-216.

Bengtson, Vern L. 2001: Beyond the Nuclear Family. The increasing Importance of Multigenerational Bonds. In: Journal of Marriage and Family 63, 1-16.

Berghammer, Caroline/Neuwirth, Norbert 2013: Veränderung in der Aufteilung der Hausarbeit nach der Geburt des ersten Kindes. In: BuberEnnser, Isabella/Neuwirth, Norbert/Testa, Maria Rita (Hrsg.): Familienentwicklung in Österreich 2009-2013. Partnerschaft, Kinderwunsch, Kinderbetreuung und ökonomische Situation. Wien: AV-Astoria.

Bickel, Robert 2007: Multilevel Analysis for Applied Research. It's Just Regression. New York/London: The Guilford Press.

Bielby, Denise D./Bielby William T. 1989: Family Ties. Balancing Commitments to Work and Family in Dual Earner Households. In: American Sociological Review 54, 776-789.

Blau, Peter M. 1964: Exchange and Power in Social Life. New York/London/Sydney: John Wiley. 
Blau, Peter M. 1989: Exchange and Power in Social Life. Reciprocity and Imbalance. In: http://garfield.library.upenn.edu/classics1989/A1989CA26300001.pdf, zugegriffen am 07.07.2015.

Blau, Peter M. 2005: Sozialer Austausch. In: Adloff, Frank/Mau, Steffen (Hrsg.): Vom Geben und Nehmen. Zur Soziologie der Reziprozität. Frankfurt am Main/New York: Campus, 125-138.

Blau, Peter M. 2008: Exchange and Power in Social Life. 12. Auflage. New Brunswick/New Jersey: Transaction Publishers.

Blood, Robert O./Wolfe, Donald M. 1960: Husbands and Wives. The Dynamics of Married Living. New York: The Free Press.

Blossfeld, Hans-Peter 1995: The New Role of Women. Family Formation in Modern Societies. Boulder u. a.: Westview Press.

Blossfeld, Hans-Peter/Drobnič, Sonja 2001: Careers of Couples in Contemporary Society. From Male Breadwinner to Dual-Earner Families. Oxford: Oxford University Press.

Blossfeld, Hans-Peter/Hakim, Catherine 1997: Introduction. A Comparative Perspective on Part-Time Work. In: Blossfeld, Hans-Peter/Hakim, Catherine (Hrsg.): Between Equalization and Marginalization. Women working Part-Time in Europe and the United States of America. Oxford: Oxford University Press, 1-21.

Blücher, Viggo G. 1966: Die Generation der Unbefangenen. Zur Soziologie der jungen Menschen heute. Düsseldorf, Köln: Eugen Diederichs Verlag.

BMFJ 2018: Bundesministerium für Familien und Jugend. Kinderbetreuungsgeld Geburten ab 01.03.2017. In: https://www.bmfj.gv.at/familie/finanzielle-unterstuetzungen-/kinderbetreuungsgeld-ab-1.3.2017/datenund-fakten.html, zugegriffen am 22.03.2018.

BMFSFJ 2013: Bundesministerium für Familie, Senioren, Frauen und Jugend. Familienernährerinnen - Perspektiven für die Zukunft schaffen. In: https://www.bmfsfj.de/bmfsfj/aktuelles/presse/pressemitteilungen/familie nernaehrerinnen--perspektiven-fuer-die-zukunft-schaffen/82882?view=DEFAULT, zugegriffen am 03.04.2017.

BMFSFJ 2018: Bundesministerium für Familie, Senioren, Frauen und Jugend. Elterngeld und ElterngeldPlus. In: https://www.bmfsfj.de/bmfsfj/themen/familie/familienleistungen/elterngeld/elterngeld-undelterngeldplus/73752, zugegriffen am 23.03.2018.

BMZ 2018: Bundesministerium für wirtschaftliche Zusammenarbeit und Entwicklung. Armut. In: https://www.bmz.de/de/service/glossar/A/armut.html, zugegriffen am 17.10.2018.

Bourdieu, Pierre 2005: Die männliche Herrschaft. Frankfurt am Main: Suhrkamp.

Braun, Daniela/Seher, Nicole/Tausendpfund, Markus/Wolsing, Ansgar 2010: Einstellungen gegenüber Immigranten und die Zustimmung zur Europäi- 
schen Integration. Eine Mehrebenenanalyse. In: Arbeitspapiere des Mannheimer Zentrums für Europäische Sozialforschung 136, 1-56.

Brehmer, Wolfram/Klenner, Christina/Klammer, Ute 2010: Wenn Frauen das Geld verdienen - eine empirische Annäherung an das Phänomen „Familienernährerin“. In: http://www.familienernaehrerin.de/w/gfx/orig/infografiken/130125wegewerk_infografiken-05.png, zugegriffen am 03.04.2017.

Brines, Julie 1994: Economic Dependency, Gender, and the Division of Labor at Home. In: American Journal of Sociology 100, 652-688.

Bryk, Anthony S./Raudenbush, Stephen W. 1992: Hierarchical Linear Models. Applications and Data Analysis Methods. Newbury Park u. a.: Sage.

Bühlmann, Felix/Elcheroth, Guy/Tettamanti, Manuel 2010: The Division of Labour Among European Couples. The Effects of Life Course and Welfare Policy on Value-Practice Configurations. In: European Sociological Review 26 (1), 49-66.

Bundesinstitut für Bevölkerungsforschung 2014: Generations and Gender Survey (GGS). In: http://www.bib-demografie.de/DE/Forschung/6_Surveys/GGS-/ggs_node.html, zugegriffen am 07.09.2014.

Burkart, Günter 1998: Auf dem Weg zu einer Soziologie der Liebe. In: Hahn, Kornelia/Burkart, Günter (Hrsg.): Liebe am Ende des 20. Jahrhunderts. Studien zur Soziologie intimer Beziehungen. Opladen: Leske + Budrich.

Burkart, Günter 2014: Liebe im Kapitalismus zwischen Geschlechtergleichheit und Marktorientierung. In: Gender. Zeitschrift für Geschlecht, Kultur und Gesellschaft 6 (2), 85-101.

Busch-Heizmann, Anne/Bröckel, Miriam 2015: Die Auswirkungen geschlechts(un)typischer Berufstätigkeiten auf die Aufteilung der Hausarbeit in Partnerschaften. In: Kölner Zeitschrift für Soziologie und Sozialpsychologie 67 (3), 475-507.

Bussemaker, Jet/van Kersbergen, Kees 1994: Gender and Welfare States. Some Theoretical Reflections. In: Sainsbury, Diane (Hrsg.): Gendering Welfare States. London u. a.: Sage, 8-25.

Butler, Judith 1999: Gender Trouble. Feminism and the Subversion of Identity. New York/London: Routledge.

Butler, Judith 2009: Die Macht der Geschlechternormen und die Grenzen des Menschlichen. Frankfurt am Main: Suhrkamp.

Carlson, Daniel L./Lynch, Jamie L. 2017: Purchases, Penalties, and Power. The Relationship between Earnings and Housework. In: Journal of Marriage and Family 79 (1), 199-224.

Cesinger, Beate/König, Stefanie/Langhauser, Marc/Leicht, René 2012: Zwei Karrieren - Eine Familie? Ergebnisse aus dem Projekt „Durch Selbstständigkeit zur Doppelkarriere? Berufs-, Partnerschafts- und Lebenskonzept erfolgreicher Gründerinnen“. In: http://www.dcc-selbstaendig.de/vortrag/Broschuere_II.pdf, zugegriffen am 06.07.2015. 
Chesley, Noelle/Flood, Sarah 2017: Signs of Change? At-Home and Breadwinner Parent's Housework and Child-Care Time. In: Journal of Marriage and Family 79 (2), 511-534.

Coleman, James S. 1986: Social theory, social research, and a theory of action. In: American Journal of Sociology 91, 1309-1335.

Coleman, James S. 1990: Foundations of Social Theory. Cambridge/London: Harvard University Press.

Coverman, Shelley 1985: Explaining Husbands' Participation in Domestic Labor. In: The Sociological Quarterly 26 (1), 81-97.

Cranney, Stephen/Miles, Andrew 2016: Desperate Housewives? Differences in Work Satisfaction Between Stay-At-Home and Employed Mothers, 1972-2012. In: https://doi.org/10.1177/0192513X16663253, zugegriffen am 09.12.16.

Cronbach, Lee J. 1951: Coefficient Alpha and the International Structre of Tests. In: Psychometrica 16 (3), 297-334.

DACH 2011: Kooperation Asylwesen Deutschland - Österreich - Schweiz. D-A-C-H-Analyse der Staatendokumentation. Die Lage von Frauen in Georgien (häusliche Gewalt und Sozialleistungen für Bedürftige). In: https://www.sem.admin.ch/dam/data/sem/internationales/herkunftslaende r/europa-gus/geo/GEO-lage-frauen-d.pdf, zugegriffen am 26.03.2018.

Dahl, Robert A. 1968: Power. In: International Encyclopedia of the Social Science. New York: Free Press, 405-415.

Dechant, Anna/Blossfeld, Hans-Peter 2015: Changes in the division of labor within highly educated German couples when the first child is born. In: Zeitschrift für Familienforschung 27 (3), 373-396.

Dechant, Anna/Schulz, Florian 2014: Bedingungsszenarien einer partnerschaftlichen Arbeitsteilung beim Übergang zur Elternschaft in Deutschland. In: Comparative Population Studies - Zeitschrift für Bevölkerungswissenschaft 39 (3), 587-614.

Diekmann, Andreas 2016: Empirische Sozialforschung. Grundlagen, Methoden, Anwendungen. 10. Auflage. Reinbek: Rowohlt.

Dörfler, Sonja/Wernhart, Georg 2016: Die Arbeit von Männern und Frauen. Eine Entwicklungsgeschichte der geschlechtsspezifischen Rollenverteilung in Frankreich, Schweden und Österreich. In: Forschungsbericht des österreichischen Instituts für Familienforschung 19, 1-81.

Drobnič, Sonja 1997: Part-Time Work in Central and Eastern European Countries. In: Blossfeld, Hans-Peter/Hakim, Catherine (Hrsg.): Between Equalization and Marginalization. Women working Part-Time in Europe and the United States of America. Oxford: Oxford University Press, 7189.

Duvander, Ann-Zofie/Lappegård, Trude/Andersson, Gunnar 2010: Family policy and fertility: fathers' and mothers' use of parental leave and con- 
tinued childbearing in Norway and Sweden. In: Journal of European Social Policy 20 (1), 45-57.

Erickson, Rebecca J. 2005: Why Emotion Work Matters: Sex, Gender, and the Division of Household Labor. In: Journal of Marriage and Family 67, 337-351.

Esping-Andersen, Gøsta 1985: Politics Against Markets. The Social Democratic Road to Power. Princeton: Princeton University.

Esping-Andersen, Gøsta 1989: The Three Political Economies of the Welfare State. In: Canadian Review of Sociology and Anthropology 26 (2), 1036.

Esping-Andersen, Gøsta 1990: The Three Worlds of Welfare Capitalism. Princeton: Princeton University Press.

Esping-Andersen, Gøsta 1993: Changing Classes. Stratification and Mobility in Post-industrial Societies. London u. a.: Sage.

Esping-Andersen, Gøsta 1999: Social Foundations of Postindustrial Economies. Oxford: Oxford University Press.

Esping-Andersen, Gøsta 2002: A New Gender Contract. In: EspingAndersen, Gøsta/Gallie, Duncan/Hemerijck, Anton/Myles, John (Hrsg.): Why We Need a New Welfare State. Oxford: Oxford University Press, 68-95.

Esping-Andersen, Gøsta 2016: Families in the 21st Century. Stockholm: SNS Förlag.

Esping-Andersen, Gøsta/Boertien, Diederik/Bonke, Jens/Gracia, Pablo 2013: Couple Specialization in Multiple Equilibria. In: European Sociological Review 0 (0), 1-15.

Esping-Andersen, Gøsta/Korpi, Walter 1987: From Poor Relief to Institutional Welfare States. The Development of Scandinavian Social Policy. In: International Journal of Sociology 16 (4), 39-74.

Esser, Hartmut 1990: „Habits”, „Frames“ und „Rational Choice“. Die Reichweite der Theorie der rationalen Wahl (am Beispiel der Erklärung des Befragtenverhaltens). Zeitschrift für Soziologie 19 (4), 231-247.

Ette, Andreas/Hullen, Gert/Leven, Ingo/Ruckdeschel, Kerstin 2007: Generations and Gender Survey. Dokumentation der Befragung von türkischen Migranten in Deutschland. In: Materialien zur Bevölkerungswissenschaft 121b. Wiesbaden: Bundesinstitut für Bevölkerungsforschung.

Europäische Kommission 2018: Beschäftigung, Soziales und Integration. Länderprofile. In:

http://ec.europa.eu/social/main.jsp?catId=1248\&langId=en, zugegriffen am 30.03.2018.

European Institute for Gender Equality 2015: Gender Equality Index 2015. Measuring gender equality in the European Union 2005-2012. Report. In: $\quad$ http://eige.europa.eu/apps-/gei/content/Gender-Equality-IndexReport-2015.pdf, zugegriffen am 02.07.2015. 
European Social Survey 2018: Data and Documentation. In: https://www.europeansocialsurvey.org/data/, zugegriffen am 26.05.2018.

Eurostat 2007: Regionen in der Europäischen Union Systematik der Gebietseinheiten für die Statistik. In:

http://ec.europa.eu/eurostat/documents/3859598/5902857/KS-RA-07020-DE.PDF/1befb64b-4664-4814-880d-f4d1b537b97c?version=1.0, zugegriffen am 14.06.2018.

Eurostat 2017: Datenbank. In: http://ec.europa.eu/eurostat/de/data/database, zugegriffen am 06.06.2017.

Eurostat 2018a: Datenbank. In: http://ec.europa.eu/eurostat/de/data/database, zugegriffen am 21.06.2018.

Eurostat 2018b: Visualisierungen, Apps und Extraktionstools. In: http://ec.europa.eu/eurostat/help/first-visit/tools, zugegriffen am 21.06.2018.

Flynn, Lindsay 2017: Childcare markets and maternal employment. A typology. In: Journal of European Social Policy 27 (3), 260-275.

Fodor, Eva/Glass, Christy/Kawachi, Janette/Popescu, Livia 2002: Family policies and gender in Hungary, Poland, and Romania. In: Communist and Post-Communist Studies 35, 475-490.

Foucault, Michel 1978: Dispositive der Macht. Berlin: Merve.

Foucault, Michel 1983: Der Wille zum Wissen. Sexualität und Wahrheit 1. Frankfurt am Main: Suhrkamp.

Foucault, Michel 2015: Analytik der Macht. 6. Auflage. Frankfurt am Main: Suhrkamp.

French, John R. P./Raven, Bertram 1959: The Bases of Social Power. In: http://www.communicationcache.com/uploads/1/0/8/8/10887248/the_bas es_of_social_power_-_chapter_20_-_1959.pdf; zugegriffen am 05.08.2015.

Fuwa, Makiko 2004: Macro-level Gender Inequality and the Division of Household Labor in 22 countries. In: American Sociological Review 69 (6), 751-767.

Fux, Beat 1994: Der familienpolitische Diskurs. Eine theoretische und empirische Unterschung über das Zusammenwirken und den Wandel von Familienpolitik, Fertilität und Familie. Berlin: Duncker und Humblot.

Fux, Beat 2002: Which Models of the Family are Encouraged or Discouraged by Different Family Policies? In: Kaufmann, Franz-Xaver/Kuijsten, Anton/Schulze, Hans-Joachim/Strohmeier, Klaus P. (Hrsg.): Family Life and Family Policies in Europe. Volume 2. Problems and Issues in Comparative Perspective. Oxford: Oxford University Press, 363-418.

Fux, Beat/Baumgartner, Doris A. 1998: Wandel von familialen Lebensformen: Lebensverläufe - Lebensentwürfe. Materialienband 3. Zürich: Schlussbericht an den schweizerischen Nationalfonds. 
Galler, Heinz Peter/Ott, Notburga 1990: Zur Bedeutung familienpolitischer Maßnahmen für die Familienbildung - eine verhandlungstheoretische Analyse familialer Entscheidungsprozesse. In: Felderer, Bernhard (Hrsg.): Bevölkerung und Wirtschaft. Berlin: Duncker \& Humblot, 111134.

Geist, Claudia 2005: The Welfare State and the Home. Regime Differences in the Domestic Division of Labour. In: European Sociological Review 21 (1), 23-41.

Gershuny, Jonathan/Fisher, Kimberly 2014: Post-industrious society. Why work time will not disappear for our grandchildren. In: Sociology Working Papers 3, 1-43.

Giddens, Anthony 1992: Die Konstitution der Gesellschaft. In: Joas, Hans/Offe, Klaus (Hrsg.): Theorie und Gesellschaft. Frankfurt am Main/New York: Campus.

Giddens, Anthony 1993: Wandel der Intimität. Sexualität, Liebe und Erotik in modernen Gesellschaften. Frankfurt am Main: Fischer Taschenbuch Verlag.

Giesselmann, Marco/Windzio, Michael 2012: Regressionsmodelle zur Analyse von Paneldaten. In: Sahner, Heinz/Bayer, Michael/Sackmann, Reinhold (Hrsg.): Studienskripte zur Soziologie. Wiesbaden: VS Verlag für Sozialwissenschaften.

Gislason, Ingólfur V./Björk Eydal, Guðný 2011: Parental Leave, Childcare and Gender Equality in the Nordic Countries. TemaNord Copenhagen: Nordic Council of Ministers.

Goodin, Robert E./Smitsman, Anneloes 2000: Placing Welfare States. The Netherlands as a Crucial Test Case. In: Journal of Comparative Policy Analysis. Research and Practice 2 (1), 39-64.

Gottschall, Karin 2000: Soziale Ungleichheit und Geschlecht. Kontinuitäten und Brüche, Sackgassen und Erkenntnispotentiale im deutschen soziologischen Diskurs. Opladen: Leske + Budrich.

Gottschall, Karin 2018: Arbeit, Beschäftigung und Arbeitsmarkt aus der Genderperspektive. In: Böhle, Fritz/Voß, G. Günter/Wachtler, Günther (Hrsg.): Handbuch Arbeitssoziologie. Band 2, 3. Auflage. Wiesbaden: Springer, 671-698.

Gouldner, Alvin W. 2005: Etwas gegen nichts. Reziprozität und Asymmetrie. In: Adloff, Frank/Mau, Steffen (Hrsg.): Vom Geben und Nehmen. Zur Soziologie der Reziprozität. Frankfurt am Main/New York: Campus, $109-124$.

Grau, Ina 2001: Fünf Formen der Macht in Partnerschaften. In: Bielefelder Arbeiten zur Sozialpsychologie 197, 1-15.

Grau, Ina 2004: Machtverhältnisse in Partnerschaften. In: https://www.familienhandbuch.de/partnerschaft/grundlagen-fur-die- 
partnerschaft/machtverhaltnisse-in-partnerschaften, zugegriffen am 07.07.2015.

Grönlund, Anne/Halldén, Karin/Magnusson, Charlotta 2017: A Scandinavian success story? Women's labour market outcomes in Denmark, Finland, Norway and Sweden. In: Acta Sociologica 60 (2), 97-119.

Grunow, Daniela/Schulz, Florian/Blossfeld, Hans-Peter 2007: Was erklärt die Traditionalisierungsprozesse häuslicher Arbeitsteilung im Eheverlauf: soziale Normen oder ökonomische Ressourcen? In: Zeitschrift für Soziologie 36, 162-181.

Gunda Werner Institut 2011: Frauen in Georgien. Gleichberechtigung der Geschlechter wiedererlernen. In: https://www.gwiboell.de/de/2011/01/31/frauen-georgien-gleichberechtigung-dergeschlechter-wiedererlernen, zugegriffen am 27.03.2018.

Gupta, Sanjiv/Evertsson, Marie/Grunow, Daniela/Nermo, Magnus/Sayer, Liana C. 2010: Economic inequality and housework. In: Treas, Judith/Drobnič, Sonja (Hrsg.): Dividing the domestic. Stanford: Stanford University Press, 105-122.

Haarmann, Alexander 2012: Niederlande. Soziale Sicherung zwischen staatlicher Grundsicherung und For-Profit Versicherern. In: Klenk, Tanja/Weyrauch, Philine/Haarmann, Alexander/Nullmeier, Frank (Hrsg.): Abkehr vom Korporatismus? Der Wandel der Sozialversicherungen im europäischen Vergleich. Frankfurt/New York: Campus, 365-434.

Hadler, Markus 2004: Die Mehrebenen-Analyse. Ihre praktische Anwendung und theoretische Annahmen. In: Österreichische Zeitschrift für Soziologie 29 (1), 53-74.

Hakim, Catherine 1996: Key Issues in Women's Work. Female Heterogeneity and the Polarisation of Women's Employment. London: Athlone Press.

Hakim, Catherine 1997: A Sociological Perspective on Part-Time Work. In: Blossfeld, Hans-Peter/Hakim, Catherine (Hrsg.): Between Equalization and Marginalization. Women working Part-Time in Europe and the United States of America. Oxford: Oxford University Press, 22-70.

Hakim, Catherine 2000: Work-Lifestyle Choices in the 21st Century. Preference Theory. Oxford: Oxford University Press.

Hallenbeck, Phyllis N. 1966: An Analysis of Power Dynamics in Marriage. In: Journal of Marriage and Family 28 (2), 200-203.

Heck, Ronald H./Scott, Thomas L./Tabata, Lynn N. 2014: Multilevel and Longitudinal Modeling with IBM SPSS. New York/London: Routledge.

Heer, David M. 1963: The Measurement and Bases of Family Power. An Overview. In: Marriage and Family Living 25 (2), 133-139.

Heidenreich, Martin 1998: Die Gesellschaft im Individuum. In: Schwaetzer, Harald/Stahl-Schwaetzer, Henrieke (Hrsg.): L'homme machine? Anthro- 
pologie im Umbruch. Hildesheim/Zürich/New York: Georg-OlmsVerlag, 229-248.

Held, Thomas 1978: Soziologie der ehelichen Machtverhältnisse. In: Berger, Johannes/Büschges, Günter/Matthes, Joachim/Wippler, Reinhard (Hrsg.): Soziologische Texte 110. Darmstadt/Neuwied: Luchterhand, 1248.

Held, Thomas/Levy, René 1974: Die Stellung der Frau in Beruf und Familie. Eine soziologische Analyse am Beispiel der Schweiz. In: Reihe Soziologie in der Schweiz 1. Frauenfeld/Stuttgart: Verlag Huber.

Hirschmann, Albert O. 1970: Exit, Voice and Loyality. Cambridge/London: Harvard University Press.

Hobson, Barbara 1991: Economic Dependency and Women's Social Citizenship. Some Thoughts on Esping-Andersen's Welfare State Regimes. New Orleans: Paper presented at the Conference on Gender, Citizenship and Social Policy, Social Science History Association Meeting.

Hobson, Barbara 2011: The Agency Gap in Work-Life Balance. Applying Sen's Capabilities Framework Within European Contexts. In: Social Politics 18 (2), 147-167.

Hobson, Barbara/Fahlén, Susanne 2009: Competing Scenarios for European Fathers. Applying Sen's Capabilities and Agency Framework to WorkFamily Balance. In: The Annals of the American Academy of Political and Social Science 624 (1), 214-233.

Hobson, Barbara/Oláh, Livia Sz. 2006: Birthstrikes? Agency and Capabilities in the Reconciliation of Employment and Family. In: Journal of Marriage and the Family Review 39 (3-4), 197-227.

Hochschild, Arlie R. 1989: The economy of Gratitude. In: Franks, David D./McCarthy, E. Doyele (Hrsg.): The Sociology of Emotions. Original Essays and Research Papers. Greenwich/London: Jai Press, 95-113.

Hochschild, Arlie R. 1995: The Culture of Politics. Traditional, Postmodern, Cold-modern and Warm-modern Ideals of Care. In: Social Politics 2 (3), 331-346.

Hochschild, Arlie R. 1997: The Time Bind. When Work Becomes Home and Home Becomes Work. New York: Metropolitan Books.

Hochschild, Arlie R. 2003: The commercialization of intimate life. Notes from home and work. Berkeley u. a.: University of California Press.

Hollstein, Betina 2005: Reziprozität in familialen Generationenbeziehungen. In: Adloff, Frank/Mau, Steffen (Hrsg.): Vom Geben und Nehmen. Zur Soziologie der Reziprozität. Frankfurt am Main/New York: Campus, $109-124$.

Homans, George C. 1974: Social Behavior. Its Elementary Forms. New York: Harcourt. 
Hook, Jennifer L. 2010: Gender Inequality in the Welfare State: Sex Segregation in Housework, 1965-2003. In: American Journal of Sociology 115 (5), 1480-1523.

Hosaya, Georg/Koch, Tobias/Eid, Michael 2014: Längsschnittdaten und Mehrebenenanalyse. In: Kölner Zeitschrift für Soziologie und Sozialpsychologie 66 (1), 189-218.

Hox, Joop J. 2002: Multilevel Analysis. Techniques and Applications. Mahwah: Lawrence Erlbaum Associates.

Hox, Joop J./Moerbeek, Mirjam/van de Schoot, Rens 2018: Multilevel Analysis. Techniques and Applications. Third Edition. New York/London: Routledge.

Huinink, Johannes/Feldhaus, Michael 2008: Beziehungs- und Familienentwicklung - eine konzeptionelle Einführung in ein Forschungsprogramm. In: Feldhaus, Michael/Huinink, Johannes (Hrsg.): Neuere Entwicklungen in der Beziehungs- und Familienforschung. Vorstudien zum Beziehungsund Familienentwicklungspanel (pairfam). Würzburg: Ergon Verlag.

Huinink, Johannes/Konietzka, Dirk 2007: Familiensoziologie. Eine Einführung. Frankfurt am Main/New York: Campus Verlag.

ILO 2014: Maternity and paternity at work. Law and practice across the world. In: http://www.ilo.org/wcmsp5/groups/public/---dgreports/--dcomm/---publ/documents/publication/wcms_242615.pdf, zugegriffen am 27.03.2018.

ILO 2018: Statistics and databases. Key Indicators of the Labour Market. In: http://www.ilo.org/ilostat/faces/oracle/webcenter/portalapp/pagehierarch y/Page21.jspx?_afrLoop $=698951746355045 \&$ afrWindowMode $=0$ \&_afr WindowId=il2qso 1 bn $42 \# ! \% 40 \% 40 \% 3 \mathrm{~F}$ afrW indowId\%3Dil2qsolbn42\%26_afrLoop\%3D698951746355045\%26_afrWindowMode\%3D0\% 26_adf.ctrl-state\%3Dil2qso1bn_95, zugegriffen am 27.03.2018.

Illouz, Eva 1997: Consuming the Romantic Utopia: Love and the Cultural Contradictions of Capitalism. Berkeley u. a.: University of California Press.

Illouz, Eva 2011: Warum Liebe weh tut. Eine soziologische Erklärung. Berlin: Suhrkamp.

Iversen, Torben/Soskice, David 2006: Electoral Institutions and the Politics of Coalitions. Why some Democracies Redistribute More than Others. In: American Political Science Review 100 (2), 165-181.

Jäckle, Sebastian/Schärdel, Julian 2017: Mehrebenenanalyse. In: Jäckle, Sebastian (Hrsg.): Neue Trends in den Sozialwissenschaften. Innovative Techniken für qualitative und quantitative Forschung. Wiesbaden: Springer Fachmedien.

Kaufmann, Franz-Xaver 1990: Zukunft der Familie. Stabilität, Stabilitätsrisiken und Wandel der familialen Lebensformen sowie ihre gesellschaftlichen und politischen Bedingungen. München: C.H. Beck. 
Kaufmann, Franz-Xaver 2003: Varianten des Wohlfahrtsstaats. Frankfurt am Main: Suhrkamp.

Kaufmann, Jean-Claude 1994: Schmutzige Wäsche. Zur ehelichen Konstruktion von Alltag. Konstanz: UVK.

Kaufmann, Jean-Claude 1999a: Mit Leib und Seele. Theorie der Haushaltstätigkeit. Konstanz: UVK.

Kaufmann, Jean-Claude 1999b: Das verstehende Interview. Theorie und Praxis. Konstanz: UVK.

Kelle, Nadiya 2011: Wandel von Erwerbsbeteiligung west-deutscher Frauen nach der Erstgeburt - Ein Vergleich der zwischen 1936 und 1965 geborenen Kohorten. In: SOEPpapers on Multidisciplinary Panel Data Research 406, 1-69.

Kersten, Sarah 2016: Individuelle und kantonale Bestimmungsgründe des Zeitaufwands für Hausarbeit von erwerbstätigen Frauen und Männern in der Schweiz. In: Swiss Journal of Sociology 42 (1), 85-107.

Kessler, Suzanne J./McKenna, Wendy 1978: Gender. An Ethnomethodological Approach. New York: University of Chicago Press.

Klärner, Andreas/Keim, Sylvia 2011: (Re-)Traditionalisierung und Flexibilität. Intergenerationale Unterstützungsleistungen und die Reproduktion von Geschlechterungleichheiten in West- und Ostdeutschland. In: Berger, Peter A./Hank, Karsten/Tölke, Angelika (Hrsg.): Reproduktion von Ungleichheit durch Arbeit und Familie. Wiesbaden: VS Verlag für Sozialwissenschaften, 121-146.

Klaus, Daniela/Steinbach, Anja 2002: Determinanten innerfamilialer Arbeitsteilung. Eine Betrachtung im Längsschnitt. In: Zeitschrift für Familienforschung 14 (1), 21-43.

Klein, Uta 2013: Geschlechterverhältnisse, Geschlechterpolitik und Gleichstellungspolitik in der Europäischen Union. Eine Einführung. 2. Auflage. Wiesbaden: VS Verlag für Sozialwissenschaften.

Knight, Carly R./Brinton, Mary C. 2017: One Egalitarism or Several? Two Decades of Gender-Role Attitude Change in Europe. In: American Journal of Sociology 122 (5), 1485-1532.

Knijn, Trudie 2008: Private Responsibility and Some Support. Family Policies in The Netherlands. In: Ostner, Ilona/Schmitt, Christoph (Hrsg.): Family Policies in the Context of Family Change. The Nordic Countries in Comparative Perspective. Wiesbaden: VS Verlag für Sozialwissenschaften, 155-173.

König, René 1966: Alte Probleme und neue Fragen in der Familiensoziologie. In: Kölner Zeitschrift für Soziologie und Sozialpsychologie 18 (1), $1-20$.

König, Stefanie/Langhauser, Marc 2016: Geschlechterspezifische Hausarbeitsteilung in Deutschland - die Rolle beruflicher Selbständigkeit, rela- 
tiver Ressourcen und traditioneller Rollenorientierung. In: Zeitschrift für Familienforschung 28 (3), 289-304.

Koppetsch, Cornelia 1998: Liebe und Partnerschaft. Gerechtigkeit in modernen Beziehungen. In: Hahn, Kornelia/Burkart, Günter (Hrsg.): Liebe am Ende des 20. Jahrhunderts. Studien zur Soziologie intimer Beziehungen. Opladen: Leske + Budrich, 111-130.

Koppetsch, Cornelia/Burkart, Günter 1999: Die Illusion der Emanzipation. Zur Wirksamkeit latenter Geschlechtsnormen im Milieuvergleich. Konstanz: UVK.

Koppetsch, Cornelia/Maier, Maja/Burkart, Günter 1997: Die Illusion der Emanzipation. Zwischenbericht zum DFG-Projekt „Geschlechtsnormen in Paarbeziehungen im Milieuvergleich“. Freiburg: Manuskript.

Koppetsch, Cornelia/Speck, Sarah 2015: Wenn der Mann kein Ernährer mehr ist. Berlin: Suhrkamp.

Korpi, Walter 1978: The Working Class in Welfare Capitalism. Work, Unions and Politics in Sweden. London: Routledge.

Korpi, Walter 1983: The Democratic Class Struggle. London: Routledge.

Korpi, Walter 1989: Power, Politics, and State Autonomy in the Development of Social Citizenship. Social Rights During Sickness in Eighteen OECD Countries Since 1930. In: American Sociological Review 54 (3), 309-328.

Korpi, Walter 2000: Faces of Inequality. Gender, Class and Patterns of Inequalities in Different Types of Welfare States. In: https://www.researchgate.net/publication-

/31268455_Faces_of_Inequality_Gender_Class_and_Patterns_of_Inequa lities_in_Different_Types_of_Welfare_Sates, ${ }_{-} \quad \overline{\text { zugegriffen }}-\overline{\text { am }}$ 03.03.2016.

Korpi, Walter/Palme, Joakim 1998: The Paradox of Redistribution and the Strategy of Equality. Welfare State Institutions, Inequality and Poverty in the Western Countries. In: American Sociological Review 63 (5), 661687.

Krause, Thomas/Urban, Dieter 2013: Panelanalyse mit Mehrebenenmodellen. Eine anwendungsorientierte Einführung. In: Schriftenreihe des Instituts für Sozialwissenschaften der Universität Stuttgart 1, 1-43.

Krebs, Angelika 2000: Einleitung. Die neue Egalitarismuskritik im Überblick. In: Krebs, Angelika (Hrsg.): Gleichheit oder Gerechtigkeit. Texte der neuen Egalitarismuskritik. Frankfurt am Main: Suhrkamp.

Kreckel, Reinhard 1993: Doppelte Vergesellschaftung und geschlechtsspezifische Arbeitsmarktstrukturierung. In: Frerichs, Petra/Steinrücke, Margareta (Hrsg.): Soziale Ungleichheit und Geschlechterverhältnisse. Sozialstrukturanalyse 3. Opladen: Leske + Budrich, 51-64.

Kreckel, Reinhard 2004: Politische Soziologie sozialer Ungleichheit. 3. erw. Auflage. Frankfurt am Main/New York: Campus. 
Kreckel, Reinhard 2006: Soziologie der sozialen Ungleichheit im globalen Kontext. In: Forschungsberichte des Instituts für Soziologie der MartinLuther-Universität Halle-Wittenberg. Der Hallesche Graureiher (4), 339.

Kreyenfeld, Michaela/Hornung, Anne/Kubisch, Karolin 2013: Der deutsche Generations and Gender Survey. Einige kritische Betrachtungen zur Validität der Fertilitätsverläufe. In: Zeitschrift für Bevölkerungswissenschaft 38 (1), 29-58.

Krüger, Helga/Levy, René 2000: Masterstatus, Familie und Geschlecht. Vergessene Verknüpfungslogiken zwischen Institutionen des Lebenslaufs. In: Berliner Journal für Soziologie 2000 (3), 379-401.

Künzler, Jan 1994: Familiale Arbeitsteilung. Die Beteiligung von Männern an der Hausarbeit. Dissertation: Universität Würzburg.

Künzler, Jan 1999: Arbeitsteilung in Ehen und nichtehelichen Lebensgemeinschaften. In: Klein, Thomas/Lauterbach, Wolfgang (Hrsg.): Nichteheliche Lebensgemeinschaften. Analysen zum Wandel partnerschaftlicher Lebensformen. Opladen: Leske + Budrich, 235-268.

Lam, Chun Bun/McHale, Susan M./Crouter, Ann C. 2012: The Division of Household Labor. Longitudinal Changes and Within-Couple Variation. In: Journal of Marriage and Family 74 (5), 944-952.

Langan, Mary/Ostner, Ilona 1991: Geschlechterpolitik im Wohlfahrtsstaat. Aspekte im internationalen Vergleich. In: Kritische Justiz 24 (3), 302317.

Langer, Wolfgang 2004: Mehrebenenanalyse. Eine Einführung für Forschung und Praxis. Wiesbaden: VS Verlag für Sozialwissenschaften.

Langer, Wolfgang 2009: Mehrebenenanalyse. Eine Einführung für Forschung und Praxis. 2. Auflage. Wiesbaden: VS Verlag für Sozialwissenschaften.

Langfeldt, Bettina 2008: Unterschiede und Determinanten der häuslichen Arbeitsteilung von kinderlosen Paaren mit und ohne Kinderwunsch. In: Bien, Walter/Marbach, Jan H. (Hrsg.): Familiale Beziehungen, Familienalltag und soziale Netzwerke. Ergebnisse der drei Wellen des Familiensurvey. 1. Auflage. Wiesbaden: VS Verlag für Sozialwissenschaften, 81118.

Leibfried, Stephan 1992: Towards a European Welfare State. On Integrating Poverty Regimes into the European Community. In Ferge, Zsuzsa/Kolberg, Jon E. (Hrsg.): Social Policy in a Changing Europe. Frankfurt am Main/New York: Campus, 245-278.

Leitner, Sigrid 2003: Varieties of familialism. The caring function of the family in comparative perspective. In: European Societies 5 (4), 353375.

Leitner, Sigrid 2013: Varianten von Familialismus. Eine historisch vergleichende Analyse der Kinderbetreuungs- und Altenpflegepolitiken in kon- 
tinentaleuropäischen Wohlfahrtsstaaten. In: Klammer, Ute/Leiber, Simone/Leitner, Sigrid (Hrsg.): Sozialpolitische Schriften. Band 91, 1-233.

Leitner, Sigrid/Ostner, Ilona 2000: Von "geordneten" zu unübersichtlichen Verhältnissen. Nachholende Modemisierung des Geschlechterarrangements in der deutschen Sozialpolitik? In: Leibfried, Stephan/Wagschal, Uwe (Hrsg.): Der deutsche Sozialstaat. Bilanzen, Reformen, Perspektiven. Frankfurt am Main/New York: Campus, 199-231.

Leitner, Sigrid/Ostner, Ilona/Schratzenstaller, Margit 2004: Einleitung. Was kommt nach dem Ernährermodell? Sozialpolitik zwischen ReKommodifizierung und Re-Familialisierung. In: Leitner, Sigrid/Ostner, Ilona/Schratzenstaller, Margit (Hrsg.): Wohlfahrtsstaat und Geschlechterverhältnis im Umbruch. Was kommt nach dem Ernährermodell? Jahrbuch für Europa- und Nordamerika-Studien 7. Wiesbaden: Springer Fachmedien, 9-27.

Lesthaeghe, Ron 1992: Der zweite demographische Übergang in den westlichen Ländern. Eine Deutung. In: Zeitschrift für Bevölkerungswissenschaft 18 (3), 313-354.

Leupold, Andrea 1983: Liebe und Partnerschaft. Formen der Codierung von Ehen. In: Zeitschrift für Soziologie 12 (4), 297-327.

Levy, René/Ernst, Michèle 2002: Lebenslauf und Regulation in Paarbeziehungen: Bestimmungsgründe der Ungleichheit familialer Arbeitsteilung. In: Zeitschrift für Familienforschung 14 (2), 103-131.

Levy, René/Widmer, Eric/Kellerhals, Jean 2002: Modern family or modernized family traditionalism? Master status and the gender order in Switzerland. In: Electronic Journal of Sociology 6 (4), 1-42.

Lewis, Jane 1992: Gender and the Development of Welfare Regimes. In: Journal of European Social Policy 2 (3), 159-173.

Lewis, Jane 1997: Gender and Welfare Regimes: Further Thoughts. In: Social Politics 4 (2), 160-177.

Lewis, Jane 2001: The Decline of the Male Breadwinner Model. Implications for Work and Care. In: Social Politics 8 (2), 152-169.

Lewis, Jane/Knijn, Trudie/Martin, Claude/Ostner, Ilona 2008: Patterns of Development in Work/Family Reconciliation Policies for Parents in France, Germany, the Netherlands, and the UK in the 2000s. In: Social Politics 15 (3), 261-286.

Lewis, Jane/Ostner, Ilona 1994: Gender and the Evolution of European Social Policies. In: Zes-Arbeitspapier 4 (94), 1-63.

Lewis, Jane/Ostner, Ilona 1995: Gender and the Evolution of European Social Policy. In: Leibfried, Stephan/Pierson, Paul (Hrsg.): European Social Policy. Between Fragmentation and Integration. Washington, DC: Brookings Institution.

Liebhart, Karin/Peto, Andrea/Schiffbänker, Annemarie/Stoilova, Rumiana 2003: Familienpolitische Maßnahmen in Österreich, Bulgarien und Un- 
garn. In: Österreichische Zeitschrift für Politikwissenschaft 32 (4), 417427.

Lijphart, Arend 1968: The Politics of Accomodation. Pluralism and Democracy in the Netherlands. Berkeley: University of Califonia Press.

Lipset, Seymour M./Rokkan, Stein 1967: Cleavages Structures, Party System, and Voter Alignments. An Introduction. In: Lipset, Seymour M./Rokkan, Stein (Hrsg.): Party Systems and Voter Alignments. CrossNational Perspectives. New York/London: The Free Press, 1-64.

Lois, Daniel 2008: Arbeitsteilung, Berufsorientierung und Partnerschaftsstabilität. Ehen und nichteheliche Lebensgemeinschaften im Vergleich. In: Kölner Zeitschrift für Soziologie und Sozialpsychologie 60 (1), 53-77.

Lois, Daniel 2015: Mehrebenenanalyse mit SPSS. Grundlagen und Erweiterungen. In: https://www.unibw.de/hum-bildungswissenschaft/professuren/swm-/methodenskripte/mehrebenenanalyse-mit-spssgrundlagen-und-erweiterungen.pdf, zugegriffen am 23.10.2015.

Lott, Yvonne 2009: Verwaltung und Entscheidung - Bestimmt das individuelle Einkommen die Machtverteilung in Paarbeziehungen? In: Kölner Zeitschrift für Soziologie und Sozialpsychologie 61 (3), 327-353.

Lott, Yvonne 2012: Who Has It and Who Gets It? The Role of Gender, Resources, and Transitions for Power within Couples. Dissertation: Universität Bremen.

Löw, Martina 2001: Raumsoziologie. Frankfurt am Main: Suhrkamp.

Löw, Martina 2009: Die Machtfrage im Geschlechterverhältnis. Zur Einführung. In: Löw, Martina (Hrsg.): Geschlecht und Macht. Analysen zum Spannungsfeld von Arbeit, Beruf und Familie. 1. Auflage. Wiesbaden: VS Verlag für Sozialwissenschaften, 7-15.

Ludwig-Mayerhofer, Wolfgang/Gartner, Hermann/Allmendinger, Jutta 2006: The Allocation of Money in Couples: The End of Inequality? Allokation von Geld in Paarbeziehungen: Das Ende der Ungleichheit? In: Zeitschrift für Soziologie 35 (3), 212-226.

Manow, Philip 2007: Wahlregeln, Klassenkoalitionen und Wohlfahrtsregime - oder: wie man Esping-Andersen mit Stein Rokkan erklären kann. In: Zeitschrift für Soziologie 36 (6), 414-430.

Marold, Julia 2009: Mütter im Spannungsfeld zwischen Kind und Beruf. Der Weg vom Ernährer- zum Zweiverdienermodell im Spiegel familienpolitischer und geschlechterkultureller Entwicklungen in Deutschland, Dänemark und den Niederlanden. In: Zeitschrift für Familienforschung 21 (1), $54-85$.

Mau, Steffen/Verwiebe, Roland 2009: Die Sozialstruktur Europas. Konstanz: UVK.

McClintock, Elizabeth 2017: Occupational Sex Composition and Gendered Housework Performance. Compensation or Conventionality? In: Journal of Marriage and Family 79 (2), 475-510. 
Meuwly, Nathalie/Wilhelm, Peter/Eicher, Véronique/Perrez, Meinrad 2011: Welchen Einfluss hat die Aufteilung von Hausarbeit und Kinderbetreuung auf Partnerschaftskonflikte und Partnerschaftszufriedenheit bei berufstätigen Paaren? In: Zeitschrift für Familienforschung 23 (1), 37-56.

Motakef, Mona/Wimbauer, Christine 2015: Paardynamiken von Familienernährerinnen im Milieuvergleich. In: https://www.querelles-net.de/index.php/qn/article/view/1199/1314, zugegriffen am 03.04.2017.

Motiejunaite, Akvile/Kravchenko, Zhanna 2008: Family policy, employment and gender-role attitudes. A comparative analysis of Russia and Sweden. In: Journal of European Social Policy 18 (1), 38-49.

Münch, Richard 2002: Soziologische Theorie. Handlungstheorie. 2. Band. Frankfurt am Main/New York: Campus.

Newman, Katherine S. 2012: The Accordion Family. Boomerang Kids, Anxious Parents, and the Private Toll of Global Competition. Boston: Beacon Press.

Nioradze, Ana/Earle, Alison/Bowser, Diana 2016: Strengthening Health Systems by Empowering Women Paternity Leave - Policy Analysis and Development Framework. In: Caucasus Social Science Review 3 (1), 136.

Nitsche, Natalie/Grunow, Daniela 2016: Housework over the course of relationships: Gender ideology, resources, and the division of housework from a growth curve perspective. In: Advances in Life Course Research 29, 80-94.

Nussbaum, Martha C. 1995: Women, Culture and Development. Oxford: Clarendon Press.

Nussbaum, Martha C. 1999: Gerechtigkeit oder das Gute Leben. Frankfurt am Main: Suhrkamp.

Nussbaum, Martha C. 2000: Women and Human Development. The Capabilities Approach. Cambridge: Cambridge University Press.

Nussbaum, Martha C. 2003: Frauen und Arbeit. Der Fähigkeitenansatz. In: Zeitschrift für Wirtschaft und Unternehmensethik 4 (1), 8-31.

Nussbaum, Martha C. 2013: Political Emotions. Why Love matters for Justice. Cambridge/ London: Harvard University Press.

OECD 2017: Part-time employment rate. In: https://ata.oecd.org/emp/parttime-employment-rate.htm, zugegriffen am 30.05.2017.

OECD 2018: Family Database. In: http://stats.oecd.org/Index.aspx?DataSetCode=FAMILY, zugegriffen am 02.03.2018.

Ogburn, William F. 1969: Kultur und sozialer Wandel. In: Maus, Heinz/Fürstenberg, Friedrich (Hrsg.): Soziologische Texte. 56. Band. Neuwied/Berlin: Luchterhand Verlag.

Olson, David H./Cromwell, Ronald E. 1975: Power in Families. In: Olson, David H./ Cromwell, Ronald E. (Hrsg.): Power in Families. New York: Wiley, 3-11. 
Opp, Karl-Dieter 1983: Die Entstehung sozialer Normen. Ein Integrationsversuch soziologischer, sozialpsychologischer und ökonomischer Erklärungen. Tübingen: J. C. B. Mohr.

Orloff, Ann S. 1993a: Gender and the Social Rights of Citizenship. The Comparative Analysis of Gender Relations and Welfare States. In: American Sociological Review 58 (3), 303-328.

Orloff, Ann S. 1993b: The Politics of Pensions. A Comparative Analysis of Britain, Canada, and the United States, 1880-1940. Madison/Wisconsin: University of Wisconsin Press.

Osmond, Marie W. 1978: Reciprocity. A Dynamic Model and a Method to Study Family Power. In: Journal of Marriage and the Family 40 (1), 4961.

Ostner, Ilona 1978: Beruf und Hausarbeit. Die Arbeit der Frauen in unserer Gesellschaft. Frankfurt am Main/New York: Campus.

Ostner, Ilona 1993: Slow Motion. Women, Work and the Family in Germany. In: Lewis, Jane (Hrsg.): Women and Social Policies in Europe. Work, Family and the State. Aldershot: Edward Elgar, 92-115.

Ostner, Ilona 1995: Arm ohne Ehemann? Sozialpolitische Regulierung von Lebenschancen für Frauen im internationalen Vergleich. In: Aus Politik und Zeitgeschichte 36/37, 3-12.

Ostner, Ilona 1998: Soziale Ungleichheit, Ressentiment und Frauenbewegung. Eine unendliche Geschichte? In: Friedrichs, Jürgen/Lepsius, Rainer M./Mayer, Karl U. (Hrsg.): Die Diagnosefähigkeit der Soziologie. Kölner Zeitschrift für Soziologie und Sozialpsychologie. Sonderheft 38, 383-403.

Ostner, Ilona 2003: 'Individualisation' - The Origins of the Concept and Its Impact on German Social Policies. In: Social Policy and Society 3 (1), 47-56.

Ostner 2004a: Familiale Solidarität. In: Beckert, Jens/Eckert, Julia/Kohli, Martin/Streeck, Wolfgang (Hrsg.): Transnationale Solidarität. Chancen und Grenzen. Frankfurt am Main/New York: Campus, 78-94.

Ostner, Ilona 2004b: Review Essay. Aus Anlass eines Geburtstags. „Gender and Welfare Revisited". In: Leitner, Sigrid/Ostner, Ilona/Schratzenstaller, Margit (Hrsg.): Wohlfahrtsstaat und Geschlechterverhältnis im Umbruch. Was kommt nach dem Ernährermodell? Jahrbuch für Europa- und Nordamerika-Studien 7. Wiesbaden: Springer Fachmedien, 44-61.

Ostner, Ilona/Schmitt, Christoph 2008a: Introduction. In: Ostner, Ilona/Schmitt, Christoph (Hrsg.): Family Policies in the Context of Family Change. The Nordic Countries in Comparative Perspective. Wiesbaden: VS Verlag für Sozialwissenschaften, 9-36.

Ostner, Ilona/Schmitt, Christoph 2008b: Conclusion. In: Ostner, Ilona/Schmitt, Christoph (Hrsg.): Family Policies in the Context of Family 
Change. The Nordic Countries in Comparative Perspective. Wiesbaden: VS Verlag für Sozialwissenschaften, 203-212.

Ott, Notburga 1989: Familienbildung und familiale Entscheidungsfindung aus verhandlungstheoretischer Sicht. In: Wagner, Gert/Ott, Notburga/Hoffmann-Nowotny, Hans-Joachim (Hrsg.): Familienbildung und Erwerbstätigkeit im demographischen Wandel. Berlin u. a.: Springer, 97-116.

Ott, Notburga 1992: Intrafamily Bargaining and Household Decision. Berlin u. a.: Springer.

Ott, Notburga 1998: Der familienökonomische Ansatz von Gary S. Becker. In: Pies, Ingo/Leschke, Martin (Hrsg.): Gary Beckers ökonomischer Imperialismus. Konzepte der Gesellschaftstheorie. Tübingen: Mohr Siebeck, 63-90.

Ott, Notburga 1999: The Economics of Gender. Der neoklassische Erklärungsansatz zum Geschlechterverhältnis. In: Dausien, Bettina/Hermann, Martina/Oechsle, Mechtild/Schmerl, Christiane/Stein-Hilbers, Marlene (Hrsg.): Erkenntnisprojekt Geschlecht. Feministische Perspektiven verwandeln Wissenschaft. Opladen: Leske + Budrich, 167-196.

Ott, Notburga 2001: Der Erklärungsansatz der Familienökonomik. In: Huinink, Johannes/Strohmeier, Klaus P./Wagner, Michael (Hrsg.): Solidarität in Partnerschaft und Familie. Zum Stand familiensoziologischer Theoriebildung. Würzburg: Ergon, 129-143.

Pahl, Jan 1983: The allocation of money and structuring of inequality within marriage. Sociological Review 31, 237-262.

Parsons, Talcott 1963: On the Concept of Political Power. Proceedings of the American Philosophical Society 107 (3), 232-262.

Pascall, Gillian/Manning, Nick 2000: Gender and social policy. Comparing welfare states in Central and Eastern Europe and the former Soviet Union. In: Journal of European Social Policy 10 (3), 240-266.

Perrucci, Carolyn C./Potter, Harry R./Rhoads, Deborah L. 1978: Determinants of Male Family-Role Performance. Psychology of Women Quarterly 3 (1), 53-66.

Pfau-Effinger, Birgit 2005: Welfare State Policies and the Development of Care Arrangements. In: European Societies 7 (2), 321-347.

Pfau-Effinger, Birgit/Smidt, Maike 2011: Differences in Women's employment patterns and family policies: eastern and western Germany. In: Community, Work \& Family 14 (2), 217-232.

Plantenga, Janneke/Remery, Chantal/Figueiredo, Hugo/Smith, Mark 2009: Towards a European Union Gender Equality Index. In: Journal of European Social Policy 19 (1), 19-33.

Plewis, Ian 1989: Comment on ,Centering' predictors in multilevel analysis. Choices and consequences. In: Multilevel Modeling Newsletter 1 (2), $10-12$. 
Prokop, Ulrike 1976: Weiblicher Lebenszusammenhang. Von der Beschränktheit der Strategien und der Unangemessenheit der Wünsche. Frankfurt am Main: Suhrkamp.

Rabe-Hesketh, Sophia/Skrondal, Anders 2012: Multilevel and Longitudinal Modeling Using Stata. Volume 1: Continuous Responses. Third Edition. Texas: Stata Press.

Raudenbush, Stephan W. 1989: Centering' predictors in multilevel analysis: Choices and consequences. Multilevel Modelling Newsletter 1 (2), 10 12.

Raudenbush, Steven W./Bryk, Anthony S. 2002: Hierarchial Linear Models. Applications and Data Analysis Methods. 2. Ausgabe. Thousand Oaks u. a.: Sage.

Rawls, John 1971: A Theory of Justice. Cambridge/London: Harvard University Press.

Rawls, John 1993: Political Liberalism. New York: Columbia University Press.

Rawls, John 1996: Eine Theorie der Gerechtigkeit. 9. Auflage. Frankfurt am Main: Suhrkamp.

Robila, Mihaela 2012: Family Policies in Eastern Europe. A Focus on Parental Leave. In: Journal of Child and Family Studies 21 (1), 32-41.

Rodman, Hayman 1970: Eheliche Macht und der Austausch von Ressourcen im kulturellen Kontext. In: Lüschen, Günther/Lupri, Eugen (Hrsg.): Soziologie der Familie. Kölner Zeitschrift für Soziologie und Sozialpsychologie. Sonderheft 14, 121-143.

Röhler, Heiko/Steinbach, Anja/Huinink, Johannes 2000: Hausarbeit in Partnerschaften. Zur Erklärung geschlechtstypischer Arbeitsteilung in nichtehelichen und ehelichen Lebensgemeinschaften. In: Zeitschrift für Familienforschung 12 (2), 21-53.

Rokkan, Stein 1999: State Formation, Nation Building and Mass Politics in Europe. In: Flora, Peter/Kuhnle, Stein/Urwin, Derek (Hrsg.): State Formation, Nation Building and Mass Politics in Europe. The Theory of Stein Rokkan. Oxford: Oxford University Press, 93-401.

Rosenfeld, Rachel A./Birkelund, Gunn E. 1995: Women's Part-Time Employment. A Cross-National Comparison. In: European Sociological Review 11 (2), 111-134.

Rostgaard, Tine 2004: Family Support Policy in Central and Eastern Europe. A Decade and a Half of Transition. In: https://pure.sfi.dk/ws/files/309695/133733e.pdf, zugegriffen am 24.02.2018.

Sainsbury, Diane 1994: Gendering Welfare States. London u. a.: Sage.

Sainsbury, Diane 1996: Gender Equality and Welfare States. Cambridge: Cambridge University Press.

Safilios-Rothschild, Constantina 1969: Patterns of Familial Power and Influence. In: Sociological Focus 2 (3), 7-19. 
Safilios-Rothschild, Constantina 1975: The dimensions of power distribution in the family. In: Christ, Jacob/Grunebaum, Henry (Hrsg.): Marriage Problems and Their Treatment. Boston: Little, Brown.

Safilios-Rothschild, Constantina 1976: A Macro- and Micro-Examination of Family Power and Love. An Exchange Model. In: Journal of Marriage and Family 38 (2), 355-362.

Saxonberg, Steven/ Sirovátka, Tomáš 2006: Failing Family Policy in PostCommunist Central Europe. In: Journal of Comparative Policy Analysis 8 (2), 185-202.

Saxonberg, Steven/Szelewa, Dorota 2007: The Continuing Legacy of the Communist Legacy? The development of family policies in Poland and the Czech Republic. In: Social Politics 14 (3,1), 351-379.

Sayer, Liana C. 2010: Trends in Housework. In: Treas, Judith/Drobnič, Sonja (Hrsg.): Dividing the domestic. Stanford: Stanford University Press, 1938.

Schleutker, Elina 2014: Fertilität, Familienpolitik und Wohlfahrtsregime. In: Comparative Population Studies 39 (1), 157-194.

Schmid, Beat/Schön-Bühlmann, Jacqueline 2003: Auf dem Weg zur Gleichstellung? Frauen und Männer in der Schweiz. Rollenteilung im Haushalt. Neuchâtel: Bundesamt für Statistik.

Schmidt, Uwe 2002: Deutsche Familiensoziologie. Entwicklung nach dem Zweiten Weltkrieg. Wiesbaden: Springer Fachmedien.

Schmitt, Christian/Trappe, Heike 2010: Gender Relations in Central and Eastern Europe - Change or Continuity? Introduction to the Special Issue. In: Zeitschrift für Familienforschung 22 (3), 261-265.

Schneider, Klaus 2010: Wohlstand, Armut und Befähigungschancen. In: Forum für Politik, Gesellschaft und Kultur 295, 17-20.

Schneider, Daniel 2012: Gender Deviance and Household Work. The Role of Occupation. In: American Journal of Sociology 117 (4), 1029-1072.

Schober, Pia/Zoch, Gundula 2015: Kürzere Elternzeit von Müttern - gleichmäßigere Aufteilung der Familienarbeit? In: DIW Wochenbericht 50, $1190-1196$.

Schulz, Florian 2010: Verbundene Lebensläufe. Partnerwahl und Arbeitsteilung zwischen neuen Ressourcenverhältnissen und traditionellen Geschlechterrollen. Wiesbaden: VS Verlag für Sozialwissenschaften.

Schulz, Florian/Blossfeld, Hans-Peter 2006: Wie verändert sich die häusliche Arbeitsteilung im Eheverlauf? Eine Längsstudie der ersten 14 Ehejahre in Westdeutschland. In: Kölner Zeitschrift für Soziologie und Sozialpsychologie 58 (1), 23-49.

Sen, Amartya K. 1970: The Impossibility of a Paretian Liberal. In: Journal of Political Economy 78 (1), 152-157.

Sen, Amartya K. 1987: The Standard of Living. Cambridge: Cambridge University Press. 
Sen, Amartya K. 1992: Inequality Re-Examined. Oxford: Russell Sage Foundation.

Sen, Amartya K. 1994: Economics and the Family. In: Uberoi, Patricia (Hrsg.): Family, Kinship and Marriage in India. Oxford: Oxford University Press, 452-465.

Sen, Amartya K. 1999: Development as Freedom. Oxford: Oxford University Press.

Sen, Amartya K. 2004: Capabilities, Lists, and Public Reason. Continuing the Conversation. In: Feminist Economics 10 (3), 77-80.

Sen, Amartya 2007: Die Identitätsfalle. Warum es keinen Krieg der Kulturen gibt. München: C. H. Beck.

Sen, Amartya K. 2010a: Die Idee der Gerechtigkeit. München: C.H. Beck.

Sen, Amartya K. 2010b: The Idea of Justice. London u. a.: Penguin Books.

SFB 1342 2018: Globale Entwicklungsdynamiken von Sozialpolitik. Zusammenfassung des Forschungsprogramms. In: http://www.socialpolicydynamics.de/f-/f8531f9672.pdf, zugegriffen am 06.10.2018.

Shaver, Sheila 1989: Gender, Class and the Welfare State. The Case of Income Security in Australia. In: Feminist Review 32, 90-110.

Shavit, Yossi/Blossfeld, Hans-Peter 1993: Persistent Inequality. Changing Educational Attainment in Thirteen Countries. Boulder u. a.: Westview Press.

Simmel, Georg 1985: Fragmente aus einer Philosophie der Liebe. In: Dahme, Heinz-Jürgen/Köhnke, Klaus Christian (Hrsg.): Schriften zur Philosophie und Soziologie der Geschlechter. Frankfurt am Main: Suhrkamp, 177182.

Simmel, Georg 1989: Rosen. Eine soziale Hypothese. In: Dahme, HeinzJürgen/Rammstedt, Otthein (Hrsg.): Georg Simmel. Schriften zur Soziologie. 3. Auflage. Frankfurt am Main: Suhrkamp, 169-172.

Simmel, Georg 1992: Soziologie. Untersuchungen über die Formen der Vergesellschaftung. Gesamtausgabe. Band 11. Frankfurt am Main: Suhrkamp.

Snijders, Tom A. B./Bosker, Roel J. 1999: Multilevel Analysis. An Introduction to Basic and Advanced Multilevel Modeling. London u. a.: Sage.

Snijders, Tom A. B./Bosker, Roel J. 2012: Multilevel Analysis. An Introduction to Basic and Advanced Multilevel Modeling. $2^{\text {nd }}$ Edition. Los Angeles u. a.: Sage.

Solga, Heike/Wimbauer, Christine 2005: Wenn zwei das Gleiche tun. Ideal und Realität sozialer Ungleichheit in Dual Career Couples. Opladen: Budrich.

Stafford, Rebecca/Backman, Elaine/Dibona, Pamela 1977: The division of labor among cohabiting and married couples. In: Journal of Marriage and the Family 39 (1), 43-54. 
Stamm, Margrit 2016: Machen Väter tatsächlich einen Unterschied? Zu ihrer Rolle auf dem Weg zum Schulerfolg ihres Kindes. In: $\mathrm{http}: / /$ margritstamm.ch/images-

/Bericht\%20Tarzan\%20Januar\%202016.pdf, zugegriffen am 21.02.2016.

Stanton, Elizabeth A. 2007: The Human Development Index. A History. In: Political Economy Research Institute. Workingpaper Series 127, 1-36.

Statistisches Bundesamt 2015: Qualität der Arbeit. In: https:/www.destatis.de/DE/ZahlenFakten/Indikatoren/QualitaetArbeit/QualitaetDerArbeit.html?cms_gtp=318944_slot\%253D3\&https=1, zugegriffen am 27.08.2015.

Statistisches Bundesamt 2018a: Qualität der Arbeit. Personen in Elternzeit. In:

https://www.destatis.de/DE/ZahlenFakten/Indikatoren/QualitaetArbeit/QualitaetDerArbeit.html?cms_gtp=318944_slot\%253D3\&https=1, zugegriffen am 02.11.2018.

Statistisches Bundesamt 2018b: NUTS-Klassifikation. Die Einteilung der Europäischen Union in EU-Regionen https://www.destatis.de/Europa/DE/MethodenMetadaten-

/Klassifikationen/UebersichtKlassifikationen_NUTS.html, zugegriffen am 14.06.2018.

Stegmüller, Daniel 2013: How Many Countries for Multilevel Modeling? A Comparison of Frequentist and Bayesian Approaches. In: American Journal of Political Science 57 (3), 748-761.

Stiglitz, Joseph/Sen, Amartya/Fitoussi, Jean-Paul 2009: The measurement of economic performance and social progress revisited. Reflections and Overview. In: Sciences Po 33, 1-63.

Sundström, Aksel/Stockemer, Daniel 2015: What determines women's political representation at the local level? A fine-grained analysis of the European regions. In: International Journal of Comparative Sociology 56 (34), 254-274.

Szelewa, Dorota/Polakowski, Michal P. 2008: Who cares? Changing patterns of childcare in Central and Eastern Europe. In: Journal of European Social Policy 18 (2), 115-131.

Szikra, Dorottya/Szelewa, Dorota 2009: Passen die mittel- und osteuropäischen Länder in das „westliche“ Bild? Das Beispiel der Familienpolitik in Ungarn und Polen. In: Klenner, Christina/Leiber, Simone (Hrsg.): Wohlfahrtsstaaten und Geschlechterungleichheit in Mittel- und Osteuropa. Kontinuität und postsozialistische Transformation in den EUMitgliedsstaaten. Wiesbaden: VS Verlag für Sozialwissenschaften, 85120.

Tamm, Marcus 2018: Fathers' Parental Leave-Taking, Childcare Involvement and Mothers' Labor Market Participation. In: Ruhr Economic Papers $773,1-23$. 
Tannen, Deborah 1991: Du kannst mich einfach nicht verstehen. Warum Männer und Frauen aneinander vorbeireden. Hamburg: Ernst Kabel Verlag.

Teplova, Tatyana 2007: Welfare State Transformation, Childcare, and Women's Work in Russia. In: Social Politics 14 (3,1), 284-322.

Trappe, Heike/Pollmann-Schult, Matthias/Schmitt, Christian 2015: The Rise and Decline of the Male Breadwinner Model: Institutional Underpinnings and Future Expectations. In: European Sociological Review 32 (2), 230-242.

Tyrell, Hartmann 1987: Romantische Liebe - Überlegungen zu ihrer »quantitativen Bestimmtheit«. In: Baecker, Dirk/Markowitz, Jürgen/Stichweh, Rudolf/Tyrell, Hartmann/Willke, Helmut (Hrsg.): Theorie als Passion. Niklas Luhmann zum 60. Geburtstag. Frankfurt am Main: Suhrkamp, 570-599.

UNICEF 1999: Women in Transition. A summary. Regional Monitoring Report No. $\quad 6 . \quad$ In: https:/www.unicefirc.org/publications/pdf/monee6sume.pdf, zugegriffen am 26.03.2018.

United Nations Development Programme 2015: Human Development Reports. In: http://hdr.undp.org/en, zugegriffen am 30.06.2015.

United Nations Economic Commission for Europe 2015: Generations and Gender Programme. In: http://www.unece.org/population/ggp.html, zugegriffen am 15.07.2015.

United Nations Economic Commission for Europe 2018: UNECE Statistical Database. In: https://w3.unece.org/PXWeb2015/pxweb/en/STAT/STAT 30-GE 03-WorkAndeconmy/017_en_GE_GPG2 r.px/table-

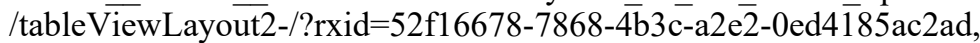
zugegriffen am 17.10.2018.

Urban, Dieter/Mayerl, Jochen 2011: Regressionsanalyse. Theorie, Technik und Anwendung. 4. Auflage. Wiesbaden: VS Verlag für Sozialwissenschaften.

Van de Kaa, Dirk 1987: Europe's Second Demographic Transition. In: Population Bulletin 42 (1), 1-59.

Van der Lippe, Tanja/de Ruijter, Judith/de Ruijter, Esther/Raub, Werner 2011: Persistent Inequalities in Time Use between Men and Women. A Detailed Look at the Influence of Economic Circumstances, Policies, and Culture. In: European Sociological Review 27 (2), 164-179.

Verkerk, Marian/Van Busschbach, Jan/Karssing, Edgar 2001: Health-related quality of life research and the capability approach of Amartya Sen. In: Quality of Life Research 10 (1), 49-55.

Vogel, Claudia 2009: Teilzeitbeschäftigung - Ausmaß und Bestimmungsgründe der Erwerbsübergänge von Frauen. In: Zeitschrift für Arbeitsmarktforschung 42 (2), 170-181.

Walby, Sylvia 1989: Theorising Patriarchy. In: Sociology, 23 (2), 213-234. 
Walby, Sylvia 1990a: Theorising Patriarchy. Oxford/Cambridge: Blackwell. Walby, Sylvia 1990b: From Private to Public Patriarchy. The Periodisation of British History. In: Women's Studies International Forum 13 (1-2), 91104.

Walby, Sylvia 2004: The European Union and Gender Equality. Emergent Varieties of Gender Regime. In: Social Politics 11 (1), 4-29.

Walby, Sylvia 2009: Globalization and Inequalities. Complexity and Contested Modernities. London u. a.: Sage.

Weber, Max 1972: Wirtschaft und Gesellschaft. 5. Auflage. Tübingen: J. C. B. Mohr.

Weltbank 2017: Gini Index (World Bank Estimate). Metadata. In: https://data.worldbank.org/indicator/SI.POV.GINI, zugegriffen am 30.05.2017.

Weltbank 2018: Data. In: https://data.worldbank.org/indicator, zugegriffen am 19.06.2018.

Wengler, Annelene/Trappe, Heike/Schmitt, Christian 2009: Alles wie gehabt? Zur Aufteilung von Hausarbeit und Elternaufgaben in Partnerschaften. In: Zeitschrift für Bevölkerungswissenschaft 34 (1), 57-78.

Wetterer, Angelika 2004: Konstruktion von Geschlecht: Reproduktionsweisen der Zweigeschlechtlichkeit. In: Becker, Ruth/Kortendiek, Beate (Hrsg.): Handbuch Frauen- und Geschlechterforschung. Theorien, Methoden, Empirie. 1. Auflage. Wiesbaden: VS Verlag für Sozialwissenschaften, 122-131.

Wimbauer, Christine 2003: Geld und Liebe. Zur symbolischen Bedeutung von Geld in Paarbeziehungen. Frankfurt am Main/New York: Campus.

Wimbauer, Christine 2012: Wenn Arbeit Liebe ersetzt. Doppelkarriere-Paare zwischen Anerkennung und Ungleichheit. Frankfurt am Main/New York: Campus.

Windzio, Michael 2018: Die Mehrebenenanalyse als Regressionsmodell für hierarchische Daten. In: https://www.migremus.uni-bremen.de/images/stories/pdf/tutoWorkshop.pdf, zugegriffen am 31.07.2018.

World Economic Forum 2014: The Global Gender Gap Report 2014. In: http://reports.weforum.org/global-gender-gap-report-2014/heatmap/, zugegriffen am 06.06.2015.

World Eonomic Forum 2017: The Global Gender Gap Report 2008. In: http://www3.weforum.org/docs/WEF_GenderGap_Report_2008.pdf, zugegriffen am 30.05.2017.

Zabel, Cordula/Heintz-Martin, Valerie K. 2013: Does Children's Age Impact the Division of Housework? In: European Societies 15 (5), 663-685. 

Der Anhang steht auf der Webseite

des Verlages zum kostenlosen Download zur Verfügung:

https://doi.org/10.3224/96665008A 

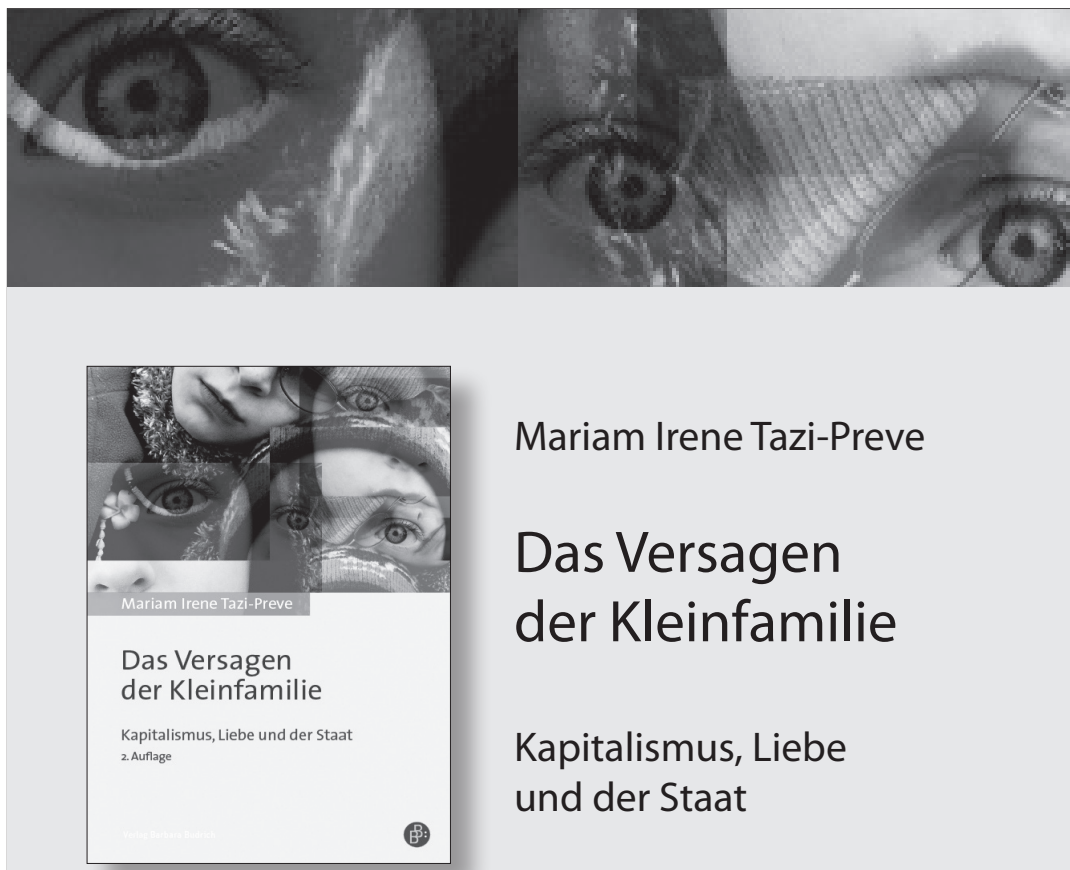

Mariam Irene Tazi-Preve

Das Versagen der Kleinfamilie

2., durchgesehene Auflage 2018 • 225 Seiten • Kart. • $22,90 €(D) \cdot 23,60 €(A) \cdot I S B N$ 978-3-8474-2196-2

Die Autorin geht vom Leiden an den kleinfamilialen Verhältnissen aus und fragt:

Kann das Liebespaar wirklich die Basis einer ganzen Gesellschaftsordnung sein? Sie legt die historischen und ideologischen Ursachen des Dilemmas der Kleinfamilie dar statt einem „individuellen Verschulden" nachzugehen und fordert ein radikales Umdenken des Privaten. Dabei greift sie alle relevanten Themen pointiert und fachkundig auf: das Drama der Mutterschaft, die neue Vaterschaftsdebatte und die Vereinbarkeitsfrage. 


\section{Geschlechterforschung für die Praxis}

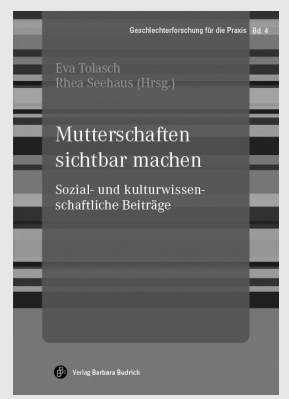

Band 4

2017• 304 Seiten $\bullet$ Kart. • $38,00 €(D) \cdot 39,10 €(A)$

ISBN 978-3-8474-2062-0

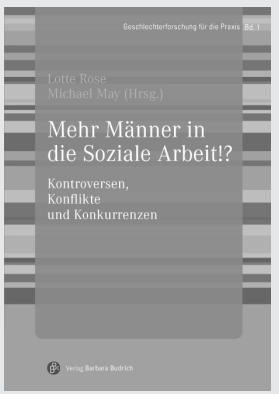

Band 1

2014 320 Seiten $\cdot$ Kart. $36,00 €(D) \cdot 37,10 €(A)$

ISBN 978-3-8474-0057-8

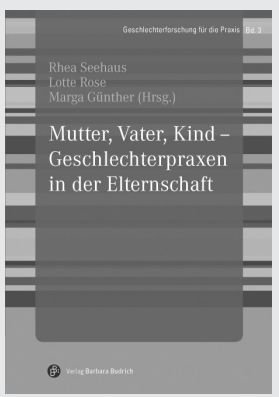

Band 3

$2015 \cdot 283$ Seiten $\bullet$ Kart. $36,00 €(D) \cdot 37,10 €(A)$ ISBN 978-3-8474-0670-9

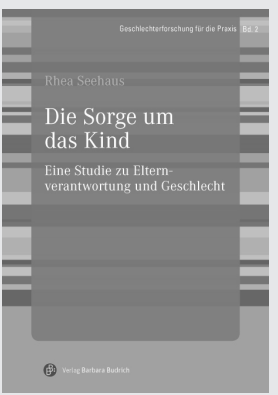

Band 2

$2014 \cdot 278$ Seiten $\bullet$ Kart. $\bullet$ $33,00 €(D) \cdot 34,00 €(A)$ ISBN 978-3-8474-0178-0

Die Publikationsreihe versteht sich als Beitrag zum Theorie-Praxis-Transfer in der Geschlechterforschung. Hierzu werden Ergebnisse aus Forschungsund Entwicklungsprojekten angewandter Wissenschaften präsentiert, in deren Fokus die Kategorie "Geschlecht" steht. Sie will damit nicht nur die Relevanz von Genderfragen in der gesellschaftlichen Praxis dokumentieren, sondern auch Impulse zur geschlechterbezogenen Qualitätsentwicklung in spezifischen Fachdiskursen und in gesellschaftlichen Institutionen liefern.

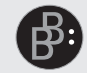

www.shop.budrich.de 


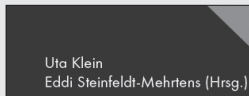

Wegbereiter_innen der Gender

und Queer Studies

Kartenspiel

mit Begleitheft
Uta Klein

Eddi Steinfeldt-Mehrtens

(Hrsg.)

\section{Wegbereiter_innen der Gender und Queer Studies}

Kartenspiel mit Begleitheft

$2018 \cdot 172$ Seiten $\cdot$ Kart. $\bullet 19,90 €(D) \cdot 20,50 €(A)$

ISBN 978-3-8474-2200-6

Einsetzbar für Lehre und Selbststudium: das erste und bisher einzige Kartenspiel zu Gender und Queer Studies!

Auf 39 Wissenskarten werden Schlagwörter, zentrale Werke und Autor_innen, die das Selbstverständnis der Geschlechterund Queerforschung maßgeblich begründet und beeinflusst haben, zusammengetragen. Alle Themen sind in einem Begleitheft aufbereitet und kontextualisiert. 


\section{Ruth Abramowski Das bisschen Haushalt}

Zur Kontinuität traditioneller Arbeitsteilung in Paarbeziehungen ein europäischer Vergleich

„Das bisschen Haushalt": Wer in Paarbeziehungen welche Routine-Hausarbeiten übernimmt, ist nach wie vor eine Frage des Geschlechts. Die Studie setzt sich mit innerhäuslichen Arbeitsteilungsarrangements in Paarbeziehungen im Zusammenhang mit dem von Studien der Familiensoziologie mittlerweile vernachlässigten Machtaspekt auseinander. Um die Kontinuität der traditionellen Arbeitsteilung in europäischen Regionen erklären zu können, wird eine theoretische Macht-Typologie entwickelt, die empirisch anhand einer Mehrebenenanalyse überprüft wird.

Die Autorin: Dr. Ruth Abramowski, wissenschaftliche Mitarbeiterin (Postdoc) am Forschungszentrum für Ungleichheit und Sozialpolitik (SOCIUM), Universität Bremen

ISBN 978-3-96665-008-3

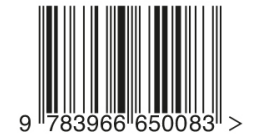

